\title{
Measurement of Charged Current Coherent Pion Production by Neutrinos on Carbon at MINER $\nu \mathrm{A}$
}

\author{
by \\ Aaron Mislivec \\ Submitted in Partial Fulfillment \\ of the \\ Requirements for the Degree of \\ Doctor of Philosophy \\ Supervised by \\ Professor Kevin McFarland \\ Department of Physics and Astronomy \\ School of Arts, Sciences, and Engineering \\ University of Rochester \\ Rochester, New York
}




\section{Table of Contents}

Biographical Sketch vii

Acknowledgements viii

Abstract $\quad x$

Contributors and Funding Sources xi

List of Tables $\quad$ xiii

List of Figures $\quad$ xvi

1 Introduction 1

2 The Standard Model 5

2.1 Quarks and Leptons . . . . . . . . . . . . . . . 5

2.2 Fundamental Interactions _. . . . . . . . . . . . . . . . . 8

2.2.1 Electromagnetic Force . . . . . . . . . . . . . . . . 9

2.2.2 Strong Force . . . . . . . . . . . . . . . . . . . . . . . . 9

2.2.3 Weak Force . . . . . . . . . . . . . . . . . 10

2.3 Electroweak Theory . . . . . . . . . . . . . . . . . 14

2.4 CP Violation ....................... 15 
3 Neutrino Oscillations $\quad 17$

3.1 Theory of Neutrino Oscillations . . . . . . . . . . . . . 18

3.2 Experimental Status of Neutrino Oscillations . . . . . . . . . . . . 21

3.3 Long Baseline Neutrino Oscillation Experiments . . . . . . . . . . . . 23

4 Neutrino-Nucleus Interactions $\quad 25$

4.1 Neutrino-Nucleon Cross Sections . . . . . . . . . . . . . 30

4.2 Nuclear Effects . . . . . . . . . . . . . . . . . . 36

4.3 The GENIE Neutrino Event Generator . . . . . . . . . . . . . . . . 38

5 Coherent Pion Production $\quad 41$

5.1 PCAC Coherent Models ................... 44

5.1.1 Adler's PCAC Theorem ................. . 44

5.1.2 Rein-Sehgal Model . . . . . . . . . . . . . . . . . 48

5.1.3 Berger-Sehgal Model ................ 51

5.2 Microscopic Coherent Models . . . . . . . . . . . . . . . . 52

5.3 Measurements of Neutrino-Nucleus Coherent Pion Production . . . . 53

6 The NuMI Neutrino Beam $\quad 62$

6.1 Proton Acceleration . . . . . . . . . . . . . . . . . . . 62

6.2 NuMI Beamline . . . . . . . . . . . . . . . . . 64

6.3 Neutrino Flux . . . . . . . . . . . . . . . . . . . . . . 68

6.3.1 Flux Simulation .................. 69

6.3.2 Constraints from Hadron Production Data . . . . . . . . . . . 69

6.3.3 Flux Uncertainties .................. . . 72

6.3.4 In situ Constraint From Neutrino-Electron Elastic Scattering • 74 
$\begin{array}{lll}7 & \text { The MINER } \nu \text { A Experiment } & 78\end{array}$

7.1 The MINER $\nu$ A Detector . . . . . . . . . . . . . . 78

7.1.1 Module Assemblies . . . . . . . . . . . . . . . . . . 79

7.1.2 Coordinate System . . . . . . . . . . . . . . . 82

7.1.3 Scintillator...................... 82

7.1.4 Event Display . . . . . . . . . . . . . . . 86

7.1.5 Readout and Electronics . . . . . . . . . . . . . . 87

7.2 The MINOS Near Detector . . . . . . . . . . . . . . . . . . . . . 94

7.3 The Partial MINER $\nu$ A Detector and ArgoNeuT . . . . . . . . . . . . 96

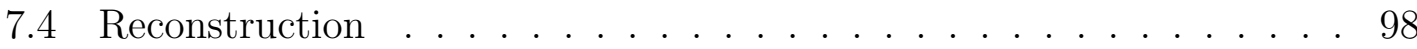

7.4.1 Time Slicing . . . . . . . . . . . . . . . . . . 98

7.4.2 Cluster Formation . . . . . . . . . . . . . . . 99

7.4.3 Track Formation .................... 102

7.4.4 Muon Energy and Charge Reconstruction . . . . . . . . . . . 104

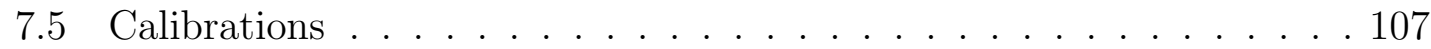

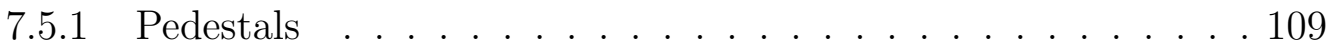

7.5.2 FEB Response .................... 109

7.5 .3 PMT Gains . . . . . . . . . . . . . . . . . . . 110

7.5.4 Attenuation Corrections ................ 111

7.5.5 Scintillator Plane Alignment . . . . . . . . . . . . . . . . 114

7.5.6 Strip Response Corrections . . . . . . . . . . . . . . . . . 115

7.5.7 Detector Energy Scale . . . . . . . . . . . . . . . 119

7.5.8 Timing Corrections . . . . . . . . . . . . . . . . 125

7.5.9 Cross-Talk Measurement . . . . . . . . . . . . . . 130

7.6 MINER $\nu$ A Monte Carlo . . . . . . . . . . . . . . . . . . 131 
7.6 .1 Physics Simulation . . . . . . . . . . . . . . . 132

7.6.2 Detector Simulation . . . . . . . . . . . . . . 133

7.7 The MINER $\nu$ A Test Beam . . . . . . . . . . . . . . . 138

8 Coherent Pion Production at MINER $\nu$ A 144

8.1 Experimental Signature . . . . . . . . . . . . . . . . . . 145

8.2 Fiducial Volume . . . . . . . . . . . . . . . . . . . . . . . 145

8.3 MC Signal and Background Definitions . . . . . . . . . . 146

8.4 Data and MC Samples . . . . . . . . . . . . . . . . . . . 149

8.5 MC Reweighting . . . . . . . . . . . . . . . . . . 151

8.6 Kinematics Reconstruction . . . . . . . . . . . . . . . 155

8.7 Event Selection . . . . . . . . . . . . . . . . . . 168

8.7.1 Reconstruction and Fiducial Volume Cuts . . . . . . . . 168

8.7.2 Muon Charge Cut . . . . . . . . . . . . . . . . . . . 169

8.7 .3 Neutrino Energy Cut . . . . . . . . . . . . . . . . . 170

8.7.4 Proton Score Cut . . . . . . . . . . . . . . . . 170

8.7.5 Vertex Energy Cut . . . . . . . . . . . . . . . . 173

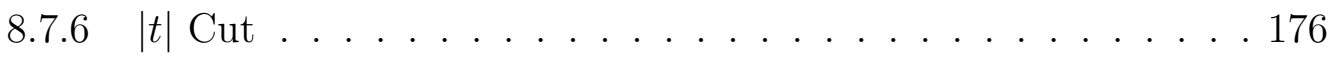

8.8 Background Tuning . . . . . . . . . . . . . . . . . 177

8.8.1 Pion Angle Weighting . . . . . . . . . . . . . . . . 182

8.9 Cross Section Extraction . . . . . . . . . . . . . . . . . . 182

8.9.1 Background Subtraction . . . . . . . . . . . . . 185

8.9 .2 Unfolding . . . . . . . . . . . . . . . . . 190

8.9 .3 Efficiency Correction . . . . . . . . . . . . . . 208

8.9.4 Flux Normalization . . . . . . . . . . . . . . . . . . 213 
8.9.5 Target Number Normalization . . . . . . . . . . . . . . 213

8.10 Signal Model Weighting _. . . . . . . . . . . . . . . . . 215

8.11 Measured Cross Sections _. . . . . . . . . . . . . . . 221

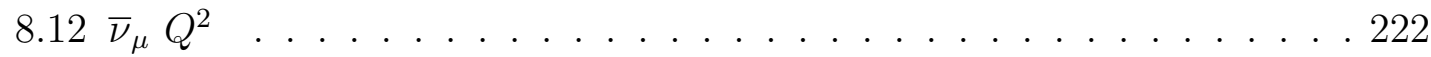

8.13 Systematic Uncertainties . . . . . . . . . . . . . . . . . 232

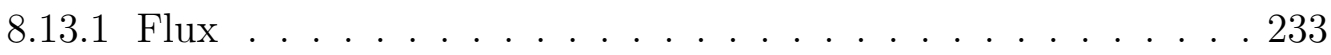

8.13 .2 Neutrino Interaction Model . . . . . . . . . . . . . . 240

8.13.3 Vertex Energy . . . . . . . . . . . . . . . . . 257

8.13 .4 Sideband Model . . . . . . . . . . . . . . . . . . . 258

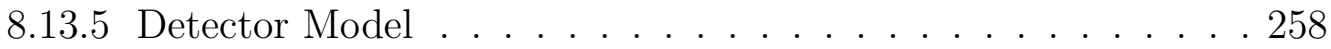

$8.14 \nu_{\mu}-\bar{\nu}_{\mu}$ Cross Section Comparisons . . . . . . . . . . . . 267

8.15 Contribution From Diffractive Scattering . . . . . . . . . . . . 282

8.15.1 Diffractive Acceptance . . . . . . . . . . . . . . . . . 284

8.15.2 Diffractive Cross Section Calculation . . . . . . . . . . . 287

8.15.3 Diffractive Search . . . . . . . . . . . . . . . 292

8.15.4 Diffractive Scattering Conclusions . . . . . . . . . . . . . . 297

9 Conclusions $\quad 298$

$\begin{array}{ll}\text { Bibliography } & 302\end{array}$

Appendix: The MINER $\nu$ A Collaboration 309 


\section{Biographical Sketch}

The author He obtained a Bachelor of Science degree in Mechanical Engineering from North Dakota State University in 2001, after which he worked for three years as a Mechanical Engineer for Emerson Process Management in Chanhassen, MN. In 2004 he enrolled in the Master of Science program in Physics at the University of Minnesota Duluth. As a master student he performed research with the MINOS neutrino oscillation experiment, where he developed an algorithm for selecting quasi-elastic interactions of neutrinos with nuclei.

In 2007, the author enrolled in the Ph.D. program in Physics at the University of Rochester, and joined the MINER $\nu \mathrm{A}$ neutrino-nucleus scattering experiment in 2008 under the supervision of Professor Kevin McFarland. The author has made contributions to several aspects of the MINER $\nu \mathrm{A}$ experiment, which include development of the calibrations, detector simulation, and reconstruction for the MINER $\nu \mathrm{A}$ detector and test beam program, studies of systematic uncertainties, and the physics results reported in this thesis. 


\section{Acknowlegements}

I am grateful for the opportunity to have done my $\mathrm{Ph} . \mathrm{D}$. research with the Rochester Neutrino Group and the MINER $\nu$ A Collaboration. I thank both Kevin McFarland and Steve Manly for their mentorship. I especially thank Kevin for his keen insight and his patience for my perfectionism which, for the record, has borne fruit on several occasions.

I thank my colleagues Phil Rodrigues, Jeremy Wolcott, and Chris Marshall of the Rochester Neutrino Group. Phil provided valuable mentorship as well as consultation in ROOT, which enabled me to emerge victorious from many battles with the data analysis framework. I thank Jeremy for both his analysis advice, particularly on the neutrino flux, and programming advice. Much of my improvement as a programmer is attributable to Jeremy. Chris and I spent countless hours working on calibrations for the MINER $\nu \mathrm{A}$ and test beam detectors and cheering on the Purple and Gold (Skol!). I also want to thank Chris for making spectacular use of the timing calibration.

I want to thank the members of MINER $\nu \mathrm{A}$ collaboration whose collective efforts made the analysis presented in this dissertation possible. I thank Aaron Higuera for his contributions to the analysis. I am grateful for the mentorship I received from Laura Fields, Jorge Morfin, Debbie Harris, Rik Gran, and Gabe Perdue. I also want to thank Howard Budd and the operations group who work tirelessly to ensure that the MINER $\nu \mathrm{A}$ detector runs smoothly and that MINER $\nu \mathrm{A}$ has the highest quality data possible.

My research was also made possible by the administrative staff of the University 
of Rochester. Those in particular I want to thank are Connie Jones, Laura Scuderi, Sue Brightman, and Barbara Warren. I want to give special thanks to Laura Blumkin for her efforts, encouragement, and kindness in helping me finally finish my Ph.D. 


\section{Abstract}

Neutrino-nucleus coherent pion production is a rare neutrino scattering process where the squared four-momentum transferred to the nucleus is small, a lepton and pion are produced in the forward direction, and the nucleus remains in its initial state. This process is an important background in neutrino oscillation experiments. Measurements of coherent pion production are needed to constrain models which are used to predict coherent pion production in oscillation experiments. This thesis reports measurements of $\nu_{\mu}$ and $\bar{\nu}_{\mu}$ charged current coherent pion production on carbon for neutrino energies in the range $2<E_{\nu}<20 \mathrm{GeV}$. The measurements were made using data from MINER $\nu \mathrm{A}$, which is a dedicated neutrino-nucleus scattering experiment that uses a fine-grained scintillator tracking detector in the high-intensity NuMI neutrino beam at Fermilab. Coherent interactions were isolated from the data using only model-independent signatures of the reaction, which are a forward muon and pion, no evidence of nuclear breakup, and small four-momentum transfer to the nucleus. The measurements were compared to the coherent pion production model used by oscillation experiments. The data and model agree in the total interaction rate and are similar in the dependence of the interaction rate on the squared fourmomentum transferred from the neutrino. The data and model disagree significantly in the pion kinematics. The measured $\nu_{\mu}$ and $\bar{\nu}_{\mu}$ interaction rates are consistent, which supports model predictions that the neutrino and antineutrino interaction rates are equal. 


\section{Contributors and Funding Sources}

The defense committee for this dissertation is comprised of Professors Kevin McFarland (advisor), Steven Manly, and Lynne Orr of the Department of Physics and Astronomy, Professor Nickolas Vamivakas of The Institute of Optics and the Department of Physics and Astronomy, and Professor Kara Bren (committee chair) of the Department of Chemistry.

The author performed the work presented in this dissertation as a member of the MINER $\nu \mathrm{A}$ collaboration. The list of MINER $\nu \mathrm{A}$ collaborators is given in the Appendix. The author made several contributions to the MINER $\nu \mathrm{A}$ experiment. The author developed the algorithm that calculates the energy of muons reconstructed in both the MINER $\nu \mathrm{A}$ and MINOS detectors. This algorithm, described in Chapter 7, is used in the majority of MINER $\nu$ A's physics analyses. The author developed the timing and absolute energy scale calibrations for the MINER $\nu \mathrm{A}$ detector which are detailed in Chapter 7. The author implemented the track reconstruction, attenuation corrections, and timing calibration, and developed the absolute energy scale calibration for the MINER $\nu$ A test beam detector described in Chapter 7. The author developed the data-driven response of the optical model in the MINER $\nu \mathrm{A}$ detector simulation described in Chapter 7. The author performed the physics analysis detailed in Chapter 8. An initial version of a subset of this analysis was performed jointly by the author and Aaron Higuera and was published by the MINER $\nu \mathrm{A}$ collaboration.

Funding for the work described in this dissertation was provided by the U.S. 
Department of Energy under grants DE-FG02-91ER40685, DE-FG02-12ER41823, and DE-SC0008475. FNAL is operated by Fermi Research Alliance, LLC under contract no. DE-AC02-07CH11359 with the United States Department of Energy. 


\section{List of Tables}

2.1 Quarks and leptons ................... 6

2.2 Baryons and Mesons . . . . . . . . . . . . . . . . . 7

2.3 Three generations of matter . . . . . . . . . . . . . . . . . 8

2.4 Gauge bosons . . . . . . . . . . . . . . . . . . . . . . . . . . . . . 9

3.1 Neutrino oscillation parameters . . . . . . . . . . . . . . . 21

5.1 Upper limits on the $\nu_{\mu} \mathrm{CC}$ coherent pion production cross section reported by $\mathrm{K} 2 \mathrm{~K}$ and SciBooNE . . . . . . . . . . . . . . . 60

6.1 Neutrino species composition of the NuMI LE fluxes . . . . . . . . . . 68

7.1 Mass fraction of elements in the MINER $\nu \mathrm{A}$ scintillator planes . . . . 84

8.1 Data and MC samples . . . . . . . . . . . . . . . . . 149

8.2 Signal-only MC samples . . . . . . . . . . . . . . . . 150

8.3 Parameters for single pion production in GENIE extracted from the fit of GENIE to re-analyzed $\nu_{\mu}$-deuterium scattering data. . . . . . . 152

8.4 The measured corrections, with statistical uncertainty, to the MINOS muon tracking efficiency in $\mathrm{MC}$. . . . . . . . . . . . . . . 154

8.5 Subdetector passive material corrections . . . . . . . . . . . 158 
8.6 The resolution of the muon and pion angles in the $\mathrm{XZ}$ and $\mathrm{YZ}$ planes for reconstructed $\nu_{\mu}$ and $\bar{\nu}_{\mu} \mathrm{CC}$ coherent $\mathrm{MC}$ interactions inside the

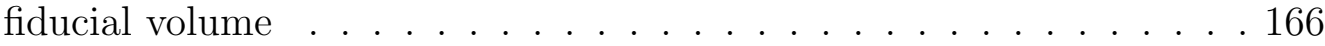

8.7 The $E_{\mu}$ fractional resolution for reconstructed $\nu_{\mu}$ and $\bar{\nu}_{\mu} \mathrm{CC}$ coherent MC interactions inside the fiducial volume . . . . . . . . . . 166

8.8 The $E_{\pi}$ fractional resolution for reconstructed $\nu_{\mu}$ and $\bar{\nu}_{\mu} \mathrm{CC}$ coherent MC interactions inside the fiducial volume . . . . . . . . . . . . 166

8.9 The $E_{\nu}$ fractional resolution for reconstructed $\nu_{\mu}$ and $\bar{\nu}_{\mu} \mathrm{CC}$ coherent MC interactions inside the fiducial volume . . . . . . . . . . 166

8.10 The $Q^{2}$ resolution for reconstructed $\nu_{\mu}$ and $\bar{\nu}_{\mu} \mathrm{CC}$ coherent MC interactions inside the fiducial volume . . . . . . . . . . . . . 167

8.11 The $|t|$ resolution for reconstructed $\nu_{\mu}$ and $\bar{\nu}_{\mu} \mathrm{CC}$ coherent MC interactions inside the fiducial volume . . . . . . . . . . . . . 167

8.12 Background scale factors . . . . . . . . . . . . . . . . . . . . 181

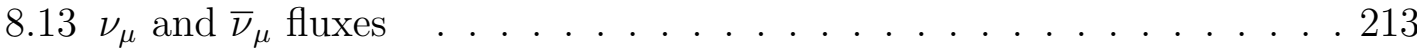

8.14 The nuclear mass number $A$ and estimated number of nuclei $T$ for each nuclear species in the full detector fiducial volume . . . . . . . . 214

$8.15 \chi^{2}$ for the comparisons of the initial measured $\nu_{\mu}$ and $\bar{\nu}_{\mu}$ cross sections to the nominal and weighted GENIE Rein-Sehgal predictions _ . . 217

$8.16 \chi^{2}$ for the comparisons of the measured $\nu_{\mu}$ and $\bar{\nu}_{\mu}$ cross sections to the GENIE Rein-Sehgal and Berger-Sehgal predictions . . . . . . 226

8.17 The statistical $\chi^{2}$, number of degrees of freedom (NDF), and $\chi^{2}$ probability for the difference between the $\bar{\nu}_{\mu}$ cross sections measured from the partial and full detector samples . . . . . . . . . . . . 227 
8.18 The measured and nominal neutrino beam angle deviations in the XZ and YZ planes for the partial detector and full detector data and MC 232

8.19 The default $\pm 1 \sigma$ variations to the GENIE model parameters. . . . . . 241

8.20 The reduced $\pm 1 \sigma$ variations to the $\mathrm{CC}$ resonance production and $\mathrm{CC}$ non-resonant single pion production model parameters in GENIE. . . 242

8.21 The $\chi^{2}$, number of degrees of freedom (NDF), and $\chi^{2}$ probability for the deviation of the measured $\nu_{\mu}-\bar{\nu}_{\mu}$ cross section differences from zero. . . . . . . . . . . . . . . . . . . . . . 272

8.22 Kopeliovich $\frac{d \sigma}{d\left(|t|-|t|_{\text {min }}\right)}$ fit parameters for $E_{\nu}=4.0 \mathrm{GeV} \ldots \ldots . .288$

8.23 The estimated diffractive cross section, the estimated acceptance reduced diffractive cross section, and the GENIE coherent cross section

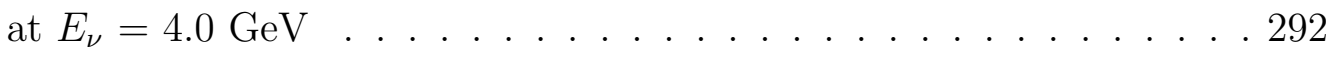


xvi

\section{List of Figures}

2.1 Electromagnetic interaction . . . . . . . . . . . . . . . . . . 9

2.2 Beta Decay . . . . . . . . . . . . . . . . . . 11

2.3 Neutrino-electron elastic scattering . . . . . . . . . . . . . . . 11

2.4 Muon decay ............................ 12

3.1 Mass state composition of the neutrino flavor states . . . . . . . . 22

3.2 Neutrino mass hierarchy . . . . . . . . . . . . . . . . . 22

4.1 Neutrino-nucleus CC quasi-elastic scattering . . . . . . . . . . 27

4.2 Neutrino-nucleus CC resonance production . . . . . . . . . . . . . 27

4.3 Neutrino-nucleus CC deep inelastic scattering . . . . . . . . . . . . 27

4.4 Charged current neutrino-nucleus cross sections . . . . . . . . . . . . 29

4.5 Inverse muon decay . . . . . . . . . . . . . . . . . . 31

5.1 CC and NC neutrino-nucleus coherent pion production in the PCAC

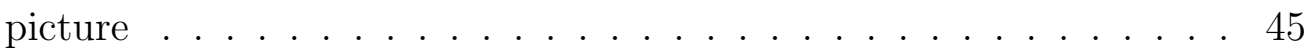

5.2 Rein-Sehgal and Berger-Sehgal pion-carbon elastic scattering cross

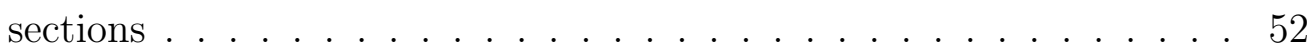

5.3 Early measurements of $\mathrm{NC}$ coherent pion production . . . . . . . 56

5.4 Early measurements of CC coherent pion production . . . . . . . . 58

5.5 Search for $\nu_{\mu} \mathrm{CC}$ coherent pion production at K2K . . . . . . 59 
5.6 Search for $\nu_{\mu}$ CC coherent pion production at SciBooNE . . . . . . . 60

$5.7 \mathrm{~T} 2 \mathrm{~K}$ systematic uncertainties . . . . . . . . . . . . . 61

6.1 Fermilab accelerator complex ............... 63

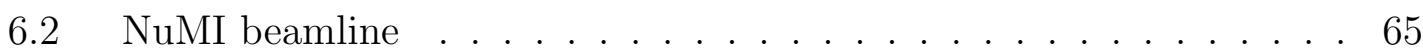

6.3 NuMI target . . . . . . . . . . . . . . . . 66

6.4 NuMI horns ..................... 67

6.5 The average number of hadronic interactions per $\nu_{\mu}$ traversing the MINER $\nu \mathrm{A}$ detector as a function of neutrino energy in the LE FHC beam simulation . . . . . . . . . . . . . . . 70

6.6 Hadron production tuned flux . . . . . . . . . . . . . . 73

6.7 Hadron production tuned flux uncertainty . . . . . . . . . . . . . 74

6.8 Hadron production tuned, neutrino-electron elastic scattering constrained flux and flux uncertainty . . . . . . . . . . . . 77

$7.1 \quad$ MINER $\nu$ A Detector . . . . . . . . . . . . . . . 80

$7.2 \quad$ MINER $\nu \mathrm{A}$ tracking module . . . . . . . . . . . . . . . 81

7.3 Cross section of the MINER $\nu$ A scintillator strips . . . . . . . . 83

7.4 Routing of WLS fibers to clear optical cable connectors . . . . . . . 85

7.5 Orientations of the MINER $\nu \mathrm{A}$ scintillator planes . . . . . . . . . 86

7.6 MINER $\nu \mathrm{A}$ event display . . . . . . . . . . . . . . . 87

$7.7 \quad$ MINER $\nu$ A PMT box .................... 89

7.8 PMT fiber weave . . . . . . . . . . . . . . . . . . . . 90

7.9 An event display of activity in the MINER $\nu \mathrm{A}$ detector during an entire readout gate. . . . . . . . . . . . . . . . 94

7.10 Steel planes and scintillator planes of the MINOS near detector. . . 95 
7.11 Upstream calorimeter and downstream muon spectrometer of the MINOS near detector . . . . . . . . . . . . . . . 96

7.12 The ArgoNeuT detector . . . . . . . . . . . . . . . . . . 97

7.13 Time slices in a MINER $\nu \mathrm{A}$ readout gate . . . . . . . . . . 99

7.14 Cluster types in MINER $\nu \mathrm{A} \ldots \ldots \ldots$. . . . . . . . . . 101

7.15 Tracks reconstructed from activity of a neutrino interaction in the MINER $\nu$ A detector . . . . . . . . . . . . . . . 103

7.16 Position and angle resolution of rock muon tracks . . . . . . . . 104

7.17 Event display of matched MINER $\nu$ A and MINOS tracks . . . . . 106

7.18 Mean energy deposition rate for muons, pions, and protons in various materials per the Bethe-Block formula . . . . . . . . . . . 108

7.19 Pedestal distribution for a single readout chanel . . . . . . . . . . 110

7.20 Measured response for a single FEB channel . . . . . . . . . . . 111

7.21 Measured gains of all PMT channels . . . . . . . . . . . . . . . . . 112

7.22 The MINER $\nu$ A module mapper . . . . . . . . . . . . . . . 113

7.23 Illustration of a rock muon traversing a plane . . . . . . . . . . . 115

7.24 Fits for the transverse displacements and rotations of two scintillator planes . . . . . . . . . . . . . . . . . . 116

7.25 Reconstructed cluster energy along rock muon tracks in data and MC for calibrating the detector energy scale . . . . . . . . . 122

7.26 Average reconstructed energy vs. true energy of clusters on rock muon tracks for calibrating the detector energy scale . . . . . . . 122

7.27 True cluster energy along rock muon tracks in the MINER $\nu \mathrm{A}$ MC . 123

7.28 Cluster PE along rock muon tracks in data and MC for calibrating the light yield in the MC . . . . . . . . . . . . . . . 124 
7.29 Cluster PE and energy along rock muon tracks in data and MC after calibrating the light yield in the MC and the detector energy scale . 124

7.30 Peak cluster PE and calibrated energy vs. time along rock muon tracks in data . . . . . . . . . . . . . . . . . . . 125

7.31 Time slewing . . . . . . . . . . . . . . . . . . . . 128

7.32 Readout channel time offset . . . . . . . . . . . . . . . . . . . . 129

7.33 Calibrated hit time resolution . . . . . . . . . . . . . 130

7.34 Cross talk from a muon track . . . . . . . . . . . . . . . . . . 131

7.35 Time smearing histogram . . . . . . . . . . . . . . . . 139

7.36 The MINER $\nu$ A test beam apparatus . . . . . . . . . . . . 140

7.37 The MINER $\nu$ A test beam detector . . . . . . . . . . . . . . 141

7.38 Event displays of a proton and a pion in the MINER $\nu$ A test beam detector . . . . . . . . . . . . . . . . . . . 142

$8.1 \nu_{\mu}$ coherent scattering candidate $\ldots \ldots \ldots \ldots \ldots \ldots$

8.2 Fiducial volume . . . . . . . . . . . . . . . . . . . . . . . 147

8.3 Correction for the GENIE Rein-Sehgal coherent cross section error . 153

8.4 Reconstructed interaction vertex position resolution . . . . . . . 160

8.5 Reconstructed muon angle resolution _ . . . . . . . . . . . . 161

8.6 Reconstructed pion angle resolution . . . . . . . . . . . . 162

8.7 Reconstructed $E_{\mu}$ and $E_{\pi}$ resolution $\ldots \ldots \ldots \ldots$

8.8 Reconstructed $E_{\nu}$ and $Q^{2}$ resolution $\ldots \ldots \ldots \ldots$

8.9 Reconstructed and true $|t|$ distributions and reconstructed $|t|$ reso-

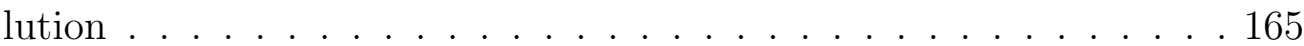

8.10 Event selection: $q / p$ significance distribution $\ldots \ldots \ldots \ldots$ 
8.11 Event selection: $E_{\nu}$ distribution $\ldots \ldots \ldots \ldots \ldots \ldots$

8.12 Event selection: proton score distribution . . . . . . . . . . . . 174

$8.13 \bar{\nu}_{\mu}$ proton score distribution after the $E_{\nu}$ cut and after the vertex

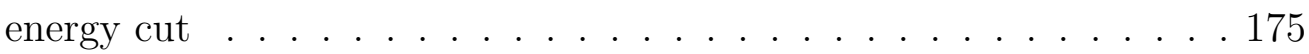

8.14 Event selection: vertex energy distribution . . . . . . . . . 176

8.15 Event selection: $|t|$ distribution . . . . . . . . . . . . . 178

$8.16|t|$ distribution before background tuning $\ldots \ldots \ldots \ldots$

$8.17 \nu_{\mu}$ sideband $E_{\pi}$ and $Q^{2}$ distributions $\ldots \ldots \ldots \ldots \ldots$

$8.18 \bar{\nu}_{\mu}$ sideband $E_{\pi}$ distribution $\ldots \ldots \ldots \ldots \ldots \ldots \ldots$

8.19 Pion angle weighting . . . . . . . . . . . . . 183

8.20 The $E_{\nu}$ distribution for the coherent candidate event samples before and after background subtraction $\ldots \ldots \ldots \ldots$

8.21 The $E_{\pi}$ distribution for the coherent candidate event samples before and after background subtraction . . . . . . . . . . 187

8.22 The $\theta_{\pi}$ distribution for the coherent candidate event samples before and after background subtraction $\ldots \ldots \ldots \ldots$

8.23 The $Q^{2}$ distribution for the coherent candidate event samples before and after background subtraction $\ldots \ldots \ldots \ldots$

8.24 The $E_{\nu}$ unfolding matrices and the unfolded $E_{\nu}$ distributions $\ldots 191$

8.25 The $E_{\pi}$ unfolding matrices and the unfolded $E_{\pi}$ distributions $\ldots 192$

8.26 The $\theta_{\pi}$ unfolding matrices and the unfolded $\theta_{\pi}$ distributions $\ldots . .193$

8.27 The $Q^{2}$ unfolding matrices and the unfolded $Q^{2}$ distributions . . . 194

$8.28 \nu_{\mu} E_{\nu}$ unfolding study $\ldots \ldots \ldots \ldots \ldots \ldots \ldots \ldots \ldots \ldots \ldots$

8.29 Full detector $\bar{\nu}_{\mu} E_{\nu}$ unfolding study $\ldots \ldots \ldots \ldots$. . . . . . 197

8.30 Partial detector $\bar{\nu}_{\mu} E_{\nu}$ unfolding study $\ldots \ldots \ldots \ldots$. . . . . 198 
$8.31 \nu_{\mu} E_{\pi}$ unfolding study . . . . . . . . . . . . . . . 199

8.32 Full detector $\bar{\nu}_{\mu} E_{\pi}$ unfolding study $\ldots \ldots$. . . . . . . . 200

8.33 Partial detector $\bar{\nu}_{\mu} E_{\pi}$ unfolding study . . . . . . . . . . . . 201

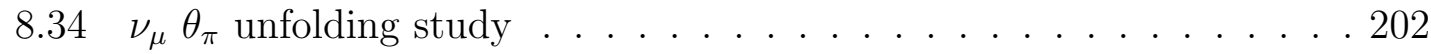

8.35 Full detector $\bar{\nu}_{\mu} \theta_{\pi}$ unfolding study . . . . . . . . . . . . . 203

8.36 Partial detector $\bar{\nu}_{\mu} \theta_{\pi}$ unfolding study . . . . . . . . . . . 204

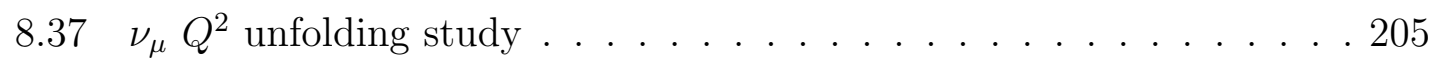

8.38 Full detector $\bar{\nu}_{\mu} Q^{2}$ unfolding study $\ldots \ldots \ldots$. . . . . . . 206

8.39 Partial detector $\bar{\nu}_{\mu} Q^{2}$ unfolding study . . . . . . . . . . . 207

8.40 The selection efficiency as a function of $E_{\nu}$ and the efficiency corrected $E_{\nu}$ distributions $\ldots \ldots \ldots \ldots \ldots$

8.41 The selection efficiency as a function of $E_{\pi}$ and the efficiency corrected $E_{\pi}$ distributions $\ldots \ldots \ldots \ldots \ldots \ldots$

8.42 The selection efficiency as a function of $\theta_{\pi}$ and the efficiency corrected $\theta_{\pi}$ distributions . . . . . . . . . . . . . 211

8.43 The selection efficiency as a function of $Q^{2}$ and the efficiency corrected $Q^{2}$ distributions . . . . . . . . . . . . . 212

8.44 The initial measurements of the $\nu_{\mu}$ and $\bar{\nu}_{\mu} \sigma\left(E_{\nu}\right)$ made without the signal model weighting, and the GENIE prediction with and without the signal model weighting . . . . . . . . . . . . . . . 215

8.45 The initial measurements of the $\nu_{\mu}$ and $\bar{\nu}_{\mu} \frac{d \sigma}{d E_{\pi}}$ made without the signal model weighting, and the GENIE prediction with and without the signal model weighting . . . . . . . . . . . . . 216 
8.46 The initial measurements of the $\nu_{\mu}$ and $\bar{\nu}_{\mu} \frac{d \sigma}{d \theta_{\pi}}$ made without the signal model weighting, and the GENIE prediction with and without the signal model weighting . . . . . . . . . . . . . . 216

8.47 The initial measurements of the $\nu_{\mu}$ and $\bar{\nu}_{\mu} \frac{d \sigma}{d Q^{2}}$ made without the signal model weighting, and the GENIE prediction with and without the signal model weighting . . . . . . . . . . . . . . 217

8.48 The effect of the signal model weighting on the measured $\nu_{\mu}$ and $\bar{\nu}_{\mu}$

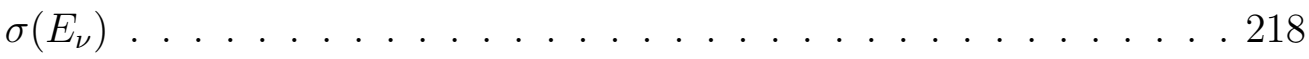

8.49 The effect of the signal model weighting on the measured $\nu_{\mu}$ and $\bar{\nu}_{\mu}$

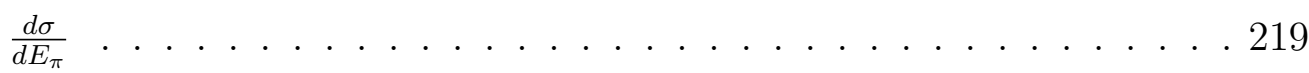

8.50 The effect of the signal model weighting on the measured $\nu_{\mu}$ and $\bar{\nu}_{\mu}$

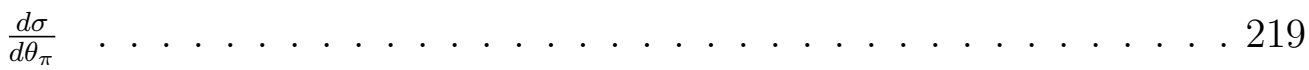

8.51 The effect of the signal model weighting on the measured $\nu_{\mu}$ and $\bar{\nu}_{\mu}$

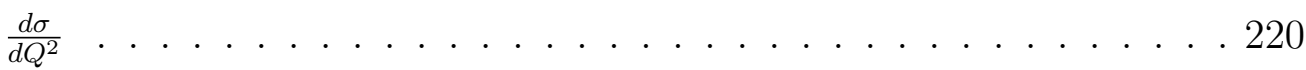

8.52 The measured $\nu_{\mu}$ and $\bar{\nu}_{\mu} \sigma\left(E_{\nu}\right) \ldots \ldots \ldots \ldots \ldots \ldots \ldots \ldots \ldots \ldots$

8.53 The measured $\nu_{\mu}$ and $\bar{\nu}_{\mu} \frac{d \sigma}{d E_{\pi}} \ldots \ldots \ldots \ldots \ldots \ldots \ldots \ldots \ldots \ldots$

8.54 The measured $\nu_{\mu}$ and $\bar{\nu}_{\mu} \frac{d \sigma}{d \theta_{\pi}} \ldots \ldots \ldots \ldots \ldots \ldots \ldots \ldots \ldots \ldots \ldots$

8.55 The measured $\nu_{\mu}$ and $\bar{\nu}_{\mu} \frac{d \sigma}{d Q^{2}} \ldots \ldots \ldots \ldots \ldots \ldots \ldots \ldots \ldots \ldots \ldots \ldots \ldots$

8.56 The measured $\nu_{\mu}$ and $\bar{\nu}_{\mu} \sigma\left(E_{\nu}\right)$ compared with previous measurements at $E_{\nu}<20 \mathrm{GeV} \ldots \ldots \ldots \ldots \ldots \ldots \ldots \ldots$

8.57 Reconstructed $Q^{2}$ distribution for $\bar{\nu}_{\mu}$ coherent candidates in the partial and full detector samples . . . . . . . . . . . . . . . . 229

8.58 Reconstructed $E_{\mu}$ distribution for $\bar{\nu}_{\mu}$ coherent candidates in the partial and full detector samples . . . . . . . . . . . . . . 229 
8.59 Reconstructed $E_{\pi}$ distribution for $\bar{\nu}_{\mu}$ coherent candidates in the partial and full detector samples . . . . . . . . . . . . . . 230

8.60 Reconstructed $\theta_{\mu}$ distribution for $\bar{\nu}_{\mu}$ coherent candidates in the partial and full detector samples . . . . . . . . . . . . . . 230

8.61 Partial to full detector event rate ratio for $\bar{\nu}_{\mu}$ coherent candidates as a function of $Q^{2}, E_{\mu}, E_{\pi}$, and $\theta_{\mu} \ldots \ldots . \ldots 231$

8.62 The statistical and systematic uncertainties on the measured $\nu_{\mu}$ and

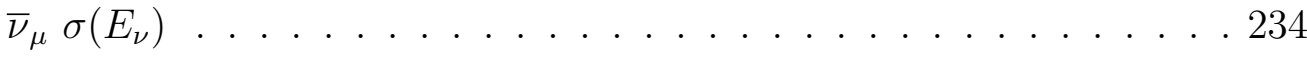

8.63 The statistical and systematic uncertainties on the measured $\nu_{\mu}$ and

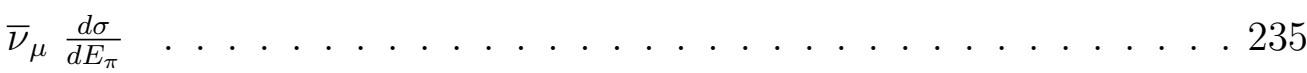

8.64 The statistical and systematic uncertainties on the measured $\nu_{\mu}$ and

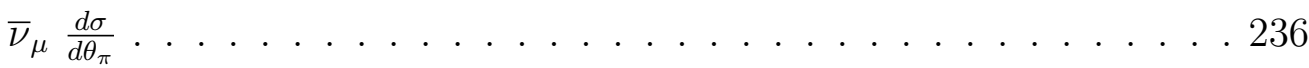

8.65 The statistical and systematic uncertainties on the measured $\nu_{\mu}$ and

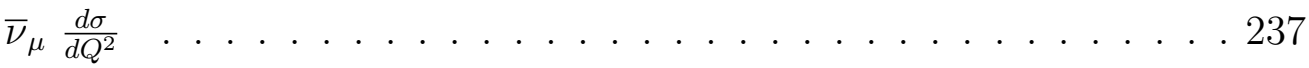

8.66 The effect of a single flux variation on the extraction of the $\nu_{\mu} \sigma\left(E_{\nu}\right) 238$

8.67 The effect of a single flux variation on the extraction of the $\nu_{\mu} \frac{d \sigma}{d E_{\pi}} \quad .238$

8.68 The effect of a single flux variation on the extraction of the $\nu_{\mu} \frac{d \sigma}{d \theta_{\pi}} \quad .239$

8.69 The effect of a single flux variation on the extraction of the $\nu_{\mu} \frac{d \sigma}{d Q^{2}} \quad .239$

8.70 GENIE CCQE and NCEL model uncertainties on the measured $\nu_{\mu}$ and $\bar{\nu}_{\mu} \sigma\left(E_{\nu}\right) \ldots \ldots \ldots \ldots \ldots \ldots \ldots$. . . . . . . . . . . . . . . . . . . . . . . . .

8.71 GENIE CC resonance model uncertainties on the measured $\nu_{\mu}$ and $\bar{\nu}_{\mu} \sigma\left(E_{\nu}\right) \ldots \ldots \ldots \ldots \ldots \ldots \ldots \ldots \ldots$

8.72 GENIE CC and NC non-resonant pion production model uncertainties on the measured $\nu_{\mu}$ and $\bar{\nu}_{\mu} \sigma\left(E_{\nu}\right) \ldots \ldots . \ldots . \ldots . \ldots . \ldots$

8.73 GENIE DIS model uncertainties on the measured $\nu_{\mu}$ and $\bar{\nu}_{\mu} \sigma\left(E_{\nu}\right) .244$ 
8.74 GENIE nucleon FSI model uncertainties on the measured $\nu_{\mu}$ and $\bar{\nu}_{\mu}$

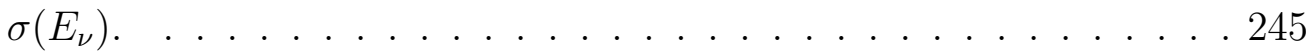

8.75 GENIE pion FSI model uncertainties on the measured $\nu_{\mu}$ and $\bar{\nu}_{\mu}$

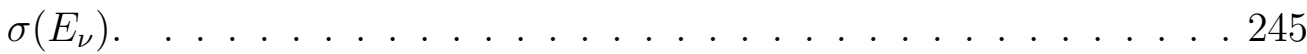

8.76 GENIE hadronization and resonance decay model uncertainties on the measured $\nu_{\mu}$ and $\bar{\nu}_{\mu} \sigma\left(E_{\nu}\right) \ldots \ldots \ldots \ldots \ldots \ldots \ldots \ldots$

8.77 GENIE CCQE and NCEL model uncertainties on the measured $\nu_{\mu}$ and $\bar{\nu}_{\mu} \frac{d \sigma}{d E_{\pi}} \ldots \ldots \ldots \ldots \ldots \ldots \ldots \ldots \ldots \ldots \ldots \ldots \ldots \ldots$

8.78 GENIE CC resonance model uncertainties on the measured $\nu_{\mu}$ and

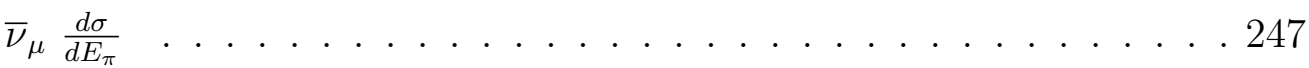

8.79 GENIE CC and NC non-resonant pion production model uncertainties on the measured $\nu_{\mu}$ and $\bar{\nu}_{\mu} \frac{d \sigma}{d E_{\pi}} \ldots \ldots \ldots \ldots 247$

8.80 GENIE DIS model uncertainties on the measured $\nu_{\mu}$ and $\bar{\nu}_{\mu} \frac{d \sigma}{d E_{\pi}}$. . 248

8.81 GENIE nucleon FSI model uncertainties on the measured $\nu_{\mu}$ and $\bar{\nu}_{\mu}$

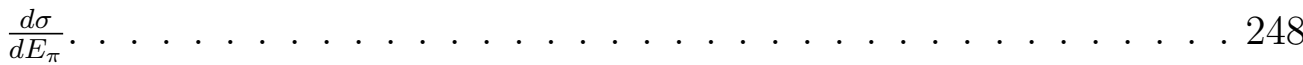

8.82 GENIE pion FSI model uncertainties on the measured $\nu_{\mu}$ and $\bar{\nu}_{\mu} \frac{d \sigma}{d E_{\pi}} \cdot 249$

8.83 GENIE hadronization and resonance decay model uncertainties on

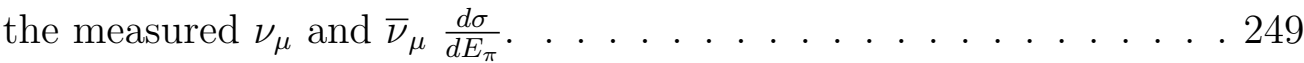

8.84 GENIE CCQE and NCEL model uncertainties on the measured $\nu_{\mu}$

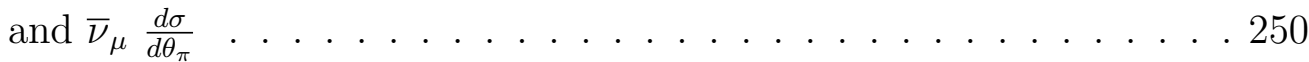

8.85 GENIE CC resonance model uncertainties on the measured $\nu_{\mu}$ and

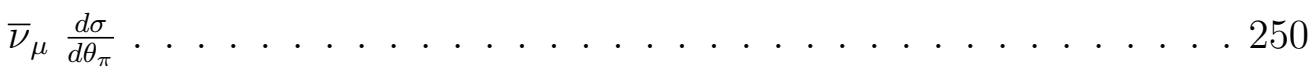

8.86 GENIE CC and NC non-resonant pion production model uncertainties on the measured $\nu_{\mu}$ and $\bar{\nu}_{\mu} \frac{d \sigma}{d \theta_{\pi}} \ldots \ldots \ldots \ldots \ldots \ldots \ldots$

8.87 GENIE DIS model uncertainties on the measured $\nu_{\mu}$ and $\bar{\nu}_{\mu} \frac{d \sigma}{d \theta_{\pi}}$. . . 251 
8.88 GENIE nucleon FSI model uncertainties on the measured $\nu_{\mu}$ and $\bar{\nu}_{\mu}$

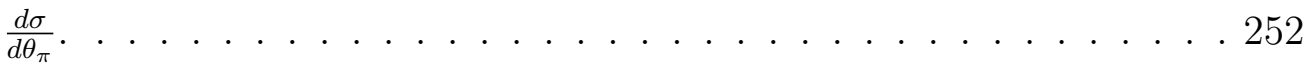

8.89 GENIE pion FSI model uncertainties on the measured $\nu_{\mu}$ and $\bar{\nu}_{\mu} \frac{d \sigma}{d \theta_{\pi}} .252$

8.90 GENIE hadronization and resonance decay model uncertainties on the measured $\nu_{\mu}$ and $\bar{\nu}_{\mu} \frac{d \sigma}{d \theta_{\pi}}$. . . . . . . . . . . . . . . . 253

8.91 GENIE CCQE and NCEL model uncertainties on the measured $\nu_{\mu}$

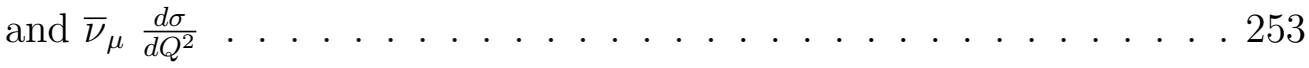

8.92 GENIE CC resonance model uncertainties on the measured $\nu_{\mu}$ and

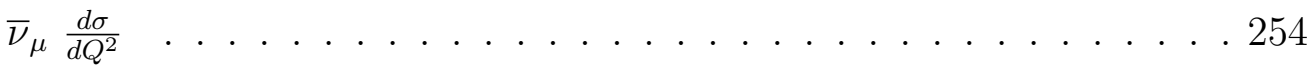

8.93 GENIE CC and NC non-resonant pion production model uncertainties on the measured $\nu_{\mu}$ and $\bar{\nu}_{\mu} \frac{d \sigma}{d Q^{2}} \quad \ldots \ldots . \ldots . . \ldots 254$

8.94 GENIE DIS model uncertainties on the measured $\nu_{\mu}$ and $\bar{\nu}_{\mu} \frac{d \sigma}{d Q^{2}}$. . . 255

8.95 GENIE nucleon FSI model uncertainties on the measured $\nu_{\mu}$ and $\bar{\nu}_{\mu}$

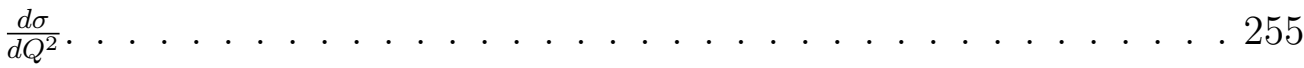

8.96 GENIE pion FSI model uncertainties on the measured $\nu_{\mu}$ and $\bar{\nu}_{\mu} \frac{d \sigma}{d Q^{2}} .256$

8.97 GENIE hadronization and resonance decay model uncertainties on

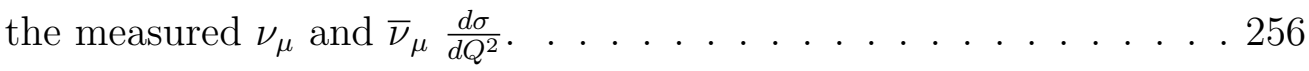

8.98 Detector model uncertainties on the measured $\nu_{\mu}$ and $\bar{\nu}_{\mu} \sigma\left(E_{\nu}\right) \ldots 259$

8.99 Detector model uncertainties on the measured $\nu_{\mu}$ and $\bar{\nu}_{\mu} \frac{d \sigma}{d E_{\pi}} \quad \ldots .259$

8.100 Detector model uncertainties on the measured $\nu_{\mu}$ and $\bar{\nu}_{\mu} \frac{d \sigma}{d \theta_{\pi}} \quad \ldots 260$

8.101 Detector model uncertainties on the measured $\nu_{\mu}$ and $\bar{\nu}_{\mu} \frac{d \sigma}{d Q^{2}} \quad \ldots \quad$. 260

8.102 MINOS muon momentum reconstruction uncertainty . . . . . . . . 262

8.103 The test beam detector response to $\pi^{+}$and $\pi^{-}$in data and MC . . . 263

8.104 The test beam detector response to protons in data and MC . . . . 264 $8.105 \bar{\nu}_{\mu}$-to- $\nu_{\mu}$ flux prediction ratio . . . . . . . . . . . . 267 
8.106 The ratio of the coherent differential cross sections predicted by GENIE for the $\bar{\nu}_{\mu}$ flux to that for the $\nu_{\mu}$ flux, and the ratio of the measured $\nu_{\mu}$ coherent differential cross sections weighted to the $\bar{\nu}_{\mu}$ flux to the unweighted measured $\nu_{\mu}$ coherent differential cross sections . 268

8.107 The measured $\bar{\nu}_{\mu}-\nu_{\mu}$ cross section differences $\ldots \ldots \ldots 270$

8.108 The correlation matrices for the uncertainty on the $\nu_{\mu}$ and $\bar{\nu}_{\mu}$ flux predictions in the measured $\sigma\left(E_{\nu}\right)$ bins $\ldots \ldots . \ldots 273$

8.109 The correlation matrices for the flux uncertainty on the measured $\nu_{\mu}$ and $\bar{\nu}_{\mu} \sigma\left(E_{\nu}\right) \ldots \ldots \ldots \ldots \ldots \ldots \ldots \ldots \ldots \ldots \ldots \ldots \ldots \ldots \ldots \ldots$

8.110 The correlation matrices for the non-flux systematic uncertainties on the measured $\nu_{\mu}$ and $\bar{\nu}_{\mu} \sigma\left(E_{\nu}\right) \ldots \ldots \ldots \ldots \ldots \ldots$

8.111 The correlation matrices for the flux uncertainty on the measured $\nu_{\mu}$ and $\bar{\nu}_{\mu} \frac{d \sigma}{d E_{\pi}} \ldots \ldots \ldots \ldots \ldots \ldots \ldots \ldots \ldots \ldots \ldots \ldots \ldots \ldots \ldots \ldots \ldots$

8.112 The correlation matrices for the non-flux systematic uncertainties on the measured $\nu_{\mu}$ and $\bar{\nu}_{\mu} \frac{d \sigma}{d E_{\pi}} \ldots \ldots \ldots \ldots \ldots \ldots \ldots \ldots$

8.113 The correlation matrices for the flux uncertainty on the measured $\nu_{\mu}$ and $\bar{\nu}_{\mu} \frac{d \sigma}{d \theta_{\pi}} \ldots \ldots \ldots \ldots \ldots \ldots \ldots \ldots \ldots \ldots \ldots \ldots \ldots \ldots$

8.114 The correlation matrices for the non-flux systematic uncertainties on the measured $\nu_{\mu}$ and $\bar{\nu}_{\mu} \frac{d \sigma}{d \theta_{\pi}} \ldots \ldots \ldots \ldots \ldots \ldots \ldots \ldots$

8.115 The correlation matrices for the flux uncertainty on the measured

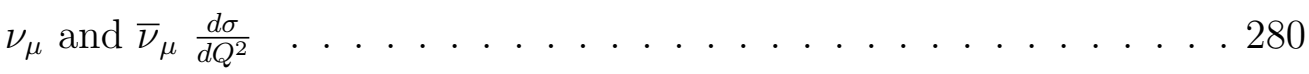

8.116 The correlation matrices for the non-flux systematic uncertainties on the measured $\nu_{\mu}$ and $\bar{\nu}_{\mu} \frac{d \sigma}{d Q^{2}} \ldots \ldots \ldots \ldots \ldots \ldots \ldots \ldots \ldots$

8.117 Diffractive scattering in the PCAC picture . . . . . . . . . 282

8.118 The estimated diffractive acceptance as a function of $|t| \ldots 286$ 
8.119 The Kopeliovich and GENIE predictions of $\frac{d \sigma}{d\left(|t|-|t|_{\text {min }}\right)}$ for inclusive $\nu_{\mu} p \rightarrow \mu^{-} \pi^{+} p$ and $\bar{\nu}_{\mu} p \rightarrow \mu^{+} \pi^{-} p$ on free protons . . . . . . . . 289

8.120 The Kopeliovich and GENIE predictions of $\frac{d \sigma}{d|t|}$ for inclusive $\nu_{\mu} p \rightarrow$ $\mu^{-} \pi^{+} p$ and $\bar{\nu}_{\mu} p \rightarrow \mu^{+} \pi^{-} p$ on free protons . . . . . . . . . . . . 290

8.121 The estimated $\nu_{\mu}$ and $\bar{\nu}_{\mu}$ diffractive $\frac{d \sigma}{d|t|}$ before and after correcting for the diffractive acceptance . . . . . . . . . . . . . . . 291

8.122 The maximum vertex strip energy distribution for the $\nu_{\mu}$ and $\bar{\nu}_{\mu}$ selected coherent candidate samples . . . . . . . . . . . . 294

8.123 The $\nu_{\mu}$ and $\bar{\nu}_{\mu}$ diffractive MC $|t|$ distributions . . . . . . . . . . . 295

8.124 The maximum vertex strip energy distribution for the $\nu_{\mu}$ and $\bar{\nu}_{\mu}$ selected coherent candidate samples with the diffractive prediction . 296 


\section{Chapter 1}

\section{Introduction}

The Standard Model of particle physics describes the fundamental particles that make up matter in the universe and their interactions. Fundamental particles in the Standard Model interact by three fundamental forces, which are the electromagnetic, weak, and strong forces. The Standard model does not describe gravity, which is negligible relative to the other fundamental forces. The Standard Model is considered to be one of the greatest achievements in science due to its predictive power. Electromagnetic interactions are calculated by the Standard Model to high precision. The theory of the combined electromagnetic and weak interactions predicted the existence and properties of the particles that mediate weak interactions, and the field that generates the masses of fundamental particles, prior to being observed by experiment. Prior to their discovery, the top and bottom quarks were proposed to explain the observed differences between the decays of kaons, which are composite particles comprised of quarks, and their antimatter counterparts. Quarks are fundamental particles in the Standard Model, and the top and bottom quarks are heavier versions of the up and down quarks that compose protons and neutrons. 
Despite its predictive power, the Standard Model is known to be incomplete. The Standard Model does not describe gravity. The Standard Model also does not provide answers to unsolved problems of particle physics and the closely related field of cosmology, which include the existence of three generations of matter, the imbalance of matter and antimatter in the universe, and the accelerating expansion of the universe. Dark matter, which was proposed to explain the observed rotation of galaxies, is also not explained by the Standard Model. The Standard Model is both theoretically and experimentally driven, and new experimental clues are needed to improve the model and our understanding of the universe.

One of the most significant recent developments in particle physics is the discovery of neutrino oscillations. Neutrinos are fundamental particles in the Standard Model and three species of neutrinos are known to exist. Neutrino oscillation is a phenomenon where a neutrino, originating as one of the three species, has a non-zero probability of interacting as a different neutrino species after traveling some distance through space or matter. The observation of neutrino oscillations establishes that neutrinos have mass, which contradicts the assumption of the Standard Model that neutrinos are massless. Neutrino oscillations are a window to physics beyond the Standard Model. It is possible for the known neutrino species to oscillate to yet discovered neutrino species. It is also possible that neutrinos and antineutrinos, the antimatter particles to neutrinos, oscillate at different rates, which may have a connection to the origin of the imbalance of matter and antimatter in the universe.

Current and future neutrino oscillation experiments aim to measure neutrino oscillations with the precision necessary to determine the hierarchy of the neutrino masses, the amount by which the neutrino and antineutrino oscillation rates differ, and whether the known neutrino species oscillate to yet discovered species. Preci- 
sion measurements of neutrino oscillations are a significant challenge since neutrinos, ignoring gravity, only interact by the weak force and therefore rarely interact with matter. Oscillation experiments, using large detectors, observe neutrinos only indirectly by their interactions with atomic nuclei. The oscillation rate is dependent on the energy of the neutrino, which must be inferred from the particles produced by the interaction between the neutrino and a nucleus. Comparing an oscillation hypothesis to an oscillation measurement involves predicting the rate and products of neutrino-nucleus interactions. Precise neutrino oscillation measurements therefore require neutrino-nucleus interactions to be well understood.

Neutrinos interact with nuclei by several processes. The processes are distinguished by whether the neutrino interacts with a nucleon (a proton or neutron within the nucleus), a quark within a nucleon, or the nucleus as a whole, and the particles produced by the interaction. Each process is significantly affected by the nuclear medium. For processes where the neutrino interacts with a nucleon or a quark, the nuclear medium can either increase or decrease the interaction rate. The nuclear medium can also modify the particles produced by the interaction. These effects of the nuclear medium are poorly understood. The rates of the neutrinonucleus interaction processes and the effects of the nuclear medium directly affect neutrino oscillation experiments. Predictions of neutrino-nucleus interactions are currently the largest source of uncertainty in neutrino oscillation measurements. Precise measurements of neutrino-nucleus interactions are needed to improve our knowledge of the interactions and the predictions used by oscillation experiments. MINER $\nu \mathrm{A}$, a dedicated neutrino-nucleus scattering experiment, is making precise measurements of the rates of neutrino-nucleus interactions and studying the effects of the nuclear medium at neutrino energies relevant to oscillation experiments. 
This thesis reports measurements of neutrino-nucleus coherent pion production on carbon nuclei from MINER $\nu \mathrm{A}$ data. Coherent pion production is a rare, neutrinonucleus interaction where the neutrino interacts with the nucleus as a whole, a pion is produced, and the nucleus remains in its initial state. A pion is a composite particle comprised of a quark and an antiquark. Depending on the neutrino species, the neutrino may convert to an electron, or a heavier version of the electron such as a muon, in the interaction. The measurements reported in this thesis are of coherent pion production interactions where the neutrino converts to a muon. In order for the nucleus to remain intact in the interaction, the energy and momentum transferred to the nucleus must be small. Compared to neutrino-nucleus interaction processes that break up the nucleus, coherent pion production is energetically disfavored since more energy is required to produce the pion than to break up the nucleus. Despite being rare, coherent pion production is an important background to neutrino oscillation measurements. The measurements reported in this thesis can be used to improve predictions of coherent pion production used by oscillation experiments. 


\section{Chapter 2}

\section{The Standard Model}

The Standard Model of particle physics describes the known fundamental particles in nature and their interactions. In the Standard Model, particles interact via three fundamental forces, which are the electromagnetic force, the strong force, and the weak force. Gravity is not described by the Standard Model. The fundamental particles in the Standard Model are quarks and leptons that form matter, and gauge bosons that are the carriers of the fundamental forces. The Standard Model is based on quantum field theory, which describes particles as excitations of physical fields.

\subsection{Quarks and Leptons}

Quarks and leptons are the fundamental particles in the Standard Model that form matter. Quarks and leptons are differentiated by how they interact. Table 2.3 lists the quark and lepton species along with their masses and electric charges. Quarks and leptons are fermions, which are particles with intrinsic angular momentum, referred to as spin, of $\frac{1}{2}$. Fermions are subject to the Pauli exclusion principle where 
no two identical fermions can simultaneously occupy the same quantum state. An antimatter particle, referred to as an antiparticle, exists for each quark and lepton. For example, the antiparticle to the up quark $u$ is the up antiquark $\bar{u}$, and the antiparticle to the electron $e^{-}$is the positron $e^{+}$. An antiparticle has identical mass and spin, but opposite quantum numbers such as electric charge, as its corresponding particle. The universe consists almost entirely of matter rather than equal amounts of matter and antimatter. The matter-antimatter asymmetry of the universe is one of the unsolved problems of physics.

\begin{tabular}{cccc} 
Quark & Symbol & Charge & Mass $(\mathrm{MeV})$ \\
\hline Up & $u$ & $+2 / 3$ & 2.3 \\
Charm & $c$ & $+2 / 3$ & 1270 \\
Top & $t$ & $+2 / 3$ & 173,200 \\
Down & $d$ & $-1 / 3$ & 4.8 \\
Strange & $s$ & $-1 / 3$ & 95 \\
Bottom & $b$ & $-1 / 3$ & 4660 \\
& & & \\
Lepton & Symbol & Charge & Mass \\
Electron & $e$ & -1 & $0.5 \mathrm{MeV}$ \\
Muon & $\mu$ & -1 & $105.6 \mathrm{MeV}$ \\
Tau & $\tau$ & -1 & $1776.9 \mathrm{MeV}$ \\
Electron Neutrino & $\nu_{e}$ & 0 & $<2 \mathrm{eV}$ \\
Muon Neutrino & $\nu_{\mu}$ & 0 & $<2 \mathrm{eV}$ \\
Tau Neutrino & $\nu_{\tau}$ & 0 & $<2 \mathrm{eV}$
\end{tabular}

Table 2.1: The quarks and leptons. The masses listed were measured in experiments and are given in [1]. The upper limits on the neutrino masses are from tritium decay. Neutrinos are massless in the Standard Model.

Quarks couple to all three fundamental forces in the Standard Model. The strong force combines quarks into composite particles called hadrons, which are grouped into baryons and mesons. Baryons are composed of three quarks or three antiquarks, and mesons are composed of a quark and an antiquark. Examples of baryons are the 
proton $(u u d)$ and neutron $(u d d)$ that make up ordinary matter. Baryons can exist in resonance states where one or more of the constituent quarks are in an excited bound state. A baryon resonance important to the analysis presented in this thesis is the delta baryon resonance $\Delta$. Baryon resonances and mesons are unstable. The baryons and mesons relavent to this thesis are listed in Table 2.2.

\begin{tabular}{cccccc} 
Baryon & Symbol & Quarks & Electric Charge & Spin & Mass $(\mathrm{MeV})$ \\
\hline Proton & $p$ & uud & +1 & $1 / 2$ & 938.3 \\
Neutron & $n$ & udd & 0 & $1 / 2$ & 939.6 \\
\hline \multirow{4}{*}{ Delta Resonances } & $\Delta++$ & uuu & +2 & $3 / 2$ & $\approx 1232$ \\
& $\Delta+$ & uud & +1 & $3 / 2$ & $\approx 1232$ \\
& $\Delta 0$ & udd & 0 & $3 / 2$ & $\approx 1232$ \\
& $\Delta-$ & ddd & -1 & $3 / 2$ & $\approx 1232$
\end{tabular}

\begin{tabular}{cccccc} 
Meson & Symbol & Quarks & Electric Charge & Spin & Mass $(\mathrm{MeV})$ \\
\hline \multirow{3}{*}{ Pions } & $\pi^{+}$ & $u \bar{d}$ & +1 & 0 & 139.6 \\
& $\pi^{-}$ & $\bar{u} d$ & -1 & 0 & 139.6 \\
& $\pi^{0}$ & $\frac{1}{\sqrt{2}}(u \bar{u}-d \bar{d})$ & 0 & 0 & 135.0 \\
\hline \multirow{2}{*}{ Charged Kaons } & $K^{+}$ & $u \bar{s}$ & +1 & 0 & 493.7 \\
& $K^{-}$ & $\bar{u} s$ & -1 & 0 & 493.7
\end{tabular}

Table 2.2: Baryons and mesons relevant to this thesis [1]

The leptons are grouped into charged leptons and neutrinos. Leptons do not couple to the strong force. Charged leptons carry electric charge and couple to the electromagnetic and weak forces. Neutrinos are electrically neutral and couple only to the weak force. Neutrinos are massless in the Standard Model. Each charged lepton has a corresponding neutrino with the same lepton flavor, which is a quantum number that is conserved in Standard Model interactions. For example, the muon $\mu^{-}$and muon neutrino $\nu_{\mu}$ both have muon flavor $L_{\mu}=+1$, the antimuon $\mu^{+}$and antimuon neutrino $\bar{\nu}_{\mu}$ have $L_{\mu}=-1$, and all other particles have $L_{\mu}=0$. [2]

Quarks and leptons form three generations of matter, where each generation 
consists of two quarks, a charged lepton, and a neutrino. In each generation, the charged lepton and neutrino have the same lepton flavor. Particle masses differ between generations, where the first generation particles are the lightest and the third generation particles are the heaviest. First generation particles are stable and form ordinary matter. Quarks and charged leptons in the second and third generations are unstable and decay to lighter particles. The neutrino in each generation is stable. The existence of three generations of matter is another unsolved problem in physics.

\begin{tabular}{cccc} 
& Generation I & Generation II & Generation III \\
\hline \multirow{2}{*}{ Quarks } & $u$ & $c$ & $t$ \\
& $d$ & $s$ & $b$ \\
\hline \multirow{2}{*}{ Leptons } & $e$ & $\mu$ & $\tau$ \\
& $\nu_{e}$ & $\nu_{\mu}$ & $\nu_{\tau}$
\end{tabular}

Table 2.3: The three generations of matter

\section{$2.2 \quad$ Fundamental Interactions}

In the Standard Model, particles interact via three fundamental forces, which are the electromagnetic force, the strong force, and the weak force. These forces are fields whose excitations are fundamental particles called gauge bosons. Gauge bosons are the carriers of the fundamental forces. Particles interact by emitting or absorbing a gauge boson. The electric charges and masses of the gauge bosons are listed in Table 2.4. All gauge bosons have spin 1. 


\begin{tabular}{ccccc} 
Gauge Boson & Symbol & Force & Electric Charge & Mass $(\mathrm{GeV})$ \\
\hline Photon & $\gamma$ & Electromagnetic & 0 & 0 \\
Gluon & $g$ & Strong & 0 & 0 \\
W & $W^{ \pm}$ & Weak & \pm 1 & 80.4 \\
Z & $Z$ & Weak & 0 & 91.2
\end{tabular}

Table 2.4: Gauge bosons

\subsubsection{Electromagnetic Force}

The carrier of the electromagnetic force is the photon. A particle interacts via the electromagnetic force by emitting/absorbing a photon. Only particles with electric charge couple to the electromagnetic force. Figure 2.1 illustrates an electromagnetic interaction between two electrons. Electromagnetic interactions are accurately described within the Standard Model by Quantum Electrodynamics (QED).

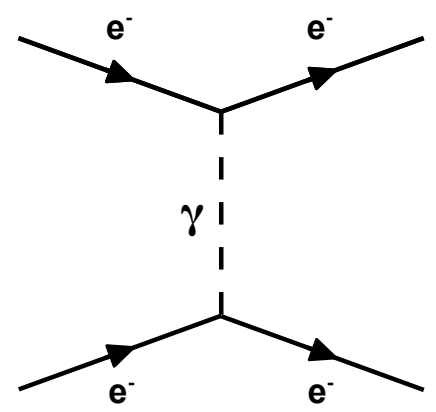

Figure 2.1: An electromagnetic interaction between two electrons. The direction of time is left to right.

\subsubsection{Strong Force}

The carrier of the strong force is the gluon. The charge that particles must possess to couple to the strong force is called color charge. Of the fundamental fermions, only quarks possess color charge. Gluons themselves possess color charge and are 
therefore self coupling. In addition to binding quarks to form baryons and mesons, the strong force binds protons and neutrons to form atomic nuclei. In the Standard Model, strong interactions are described by Quantum Chromodynamics (QCD).

A unique feature of the strong force is that its strength increases with distance. This is in contrast with the electromagnetic and strong forces whose strengths decrease with distance. A consequence of the strength of the strong force increasing with distance is quarks cannot be isolated and are always bound to other quarks, which is referred to as quark confinement. When the distance between two quarks increases the energy of the strong interaction between them increases. At sufficient separation a quark-antiquark pair is created from the the energy of the strong interaction. The strong force then binds each of the created quark and antiquark to one of the original quarks. The strong force becomes asymptotically small with decreasing distance, which is referred to as asymptotic freedom of the strong force.

The self-coupling of the gluon greatly complicates the dynamics of the strong force. As a result, perturbative QCD calculations are accurate only at small distances where strongly interacting particles are quasi free due to asymptotic freedom. Interactions with large momentum transfer, such as those achieved at the Large Hadron Collider (LHC), probe distances small enough where perturbative QCD calculations are accurate [1].

\subsubsection{Weak Force}

The carriers of the weak force are the $W^{+}, W^{-}$, and $Z$ gauge bosons. The $W$ and $Z$ bosons have large masses $(\sim 80-90 \mathrm{GeV})$, while the photon and gluon are both massless. The $W$ bosons have electric charge and mediate charged current weak interactions, and the $Z$ boson is electrically neutral and mediates neutral 
current weak interactions. An example of a charged current interaction is beta decay $n \rightarrow p+e^{-}+\bar{\nu}_{e}$, where one of the down quarks in the neutron converts to an up quark by emitting a $W^{-}$, which decays to an electron and an anti-electron neutrino (Figure 2.2). An example of a neutral current interaction is neutrino-electron elastic scattering $\nu_{\mu}+e^{-} \rightarrow \nu_{\mu}+e^{-}$(Figure 2.4). All quarks and leptons couple to the weak force.

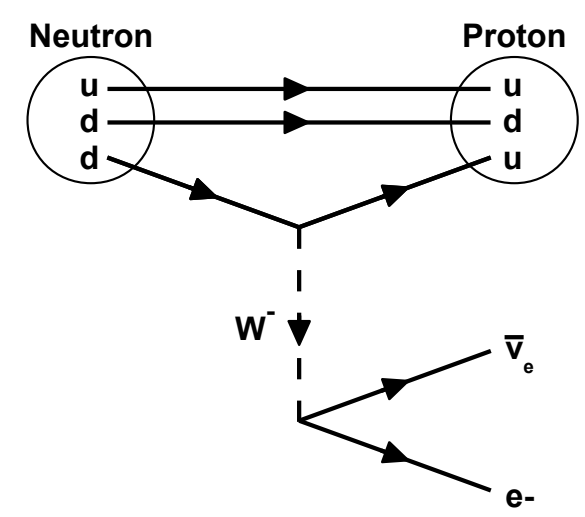

Figure 2.2: Beta decay

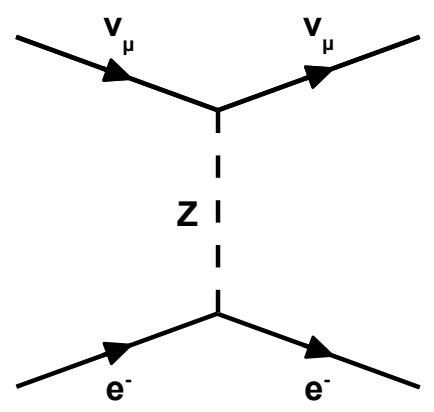

Figure 2.3: Neutrino-electron elastic scattering

The strength, or probability, of weak interactions is typically much smaller than the strengths of the electromagnetic and strong interactions due to the large masses of the $W$ and $Z$ bosons. The probability of a weak interaction is proportional to 
$\sim\left(q^{2}-M^{2}\right)^{-2}$, where $q$ and $M$ are the four momentum and mass of the weak gauge boson. The probability of an electromagnetic interaction is proportional to $q^{-4}$ due to the photon being massless. Therefore, weak interactions are much less probable than electromagnetic interactions at low energies $\left(\left|q^{2}\right| \ll M^{2}\right)$. [3]

Lepton flavor is conserved in weak interactions. For example, in muon decay $\mu^{-} \rightarrow \nu_{\mu}+e^{-}+\bar{\nu}_{e}$, which is a charged current weak interaction, the lepton flavor of the initial and final state is muon flavor +1 (the $e^{-}$and $\bar{\nu}_{e}$ have electron flavor +1 and -1 , respectively). In contrast, the charged current weak interaction mixes quark flavors. The probability of the weak interaction mixing one quark flavor with another is described by the Cabibbo-Kobayashi-Masakawa (CKM) matrix. [2]

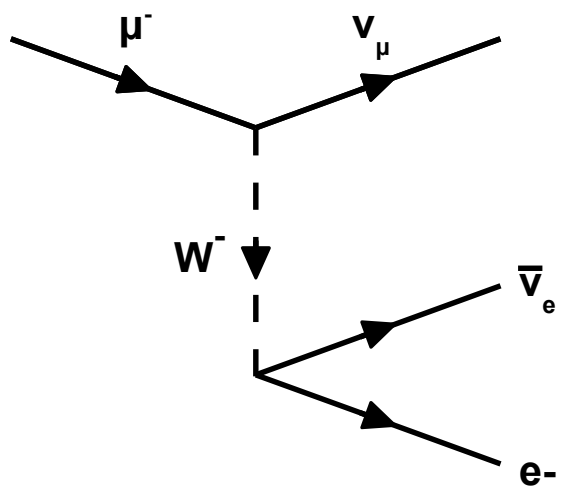

Figure 2.4: Muon decay

A unique feature of the weak force is that it violates parity symmetry. Parity symmetry is the invariance of a physical process under a parity transformation, which is the inversion of all three spatial coordinates. A parity transformation $P$ will flip the direction of a vector $\vec{a}$,

$$
P \vec{a}=-\vec{a},
$$


but will preserve the direction of an axial vector $\vec{a} \times \vec{b}$,

$$
P(\vec{a} \times \vec{b})=(-\vec{a}) \times(-\vec{b})=\vec{a} \times \vec{b}
$$

A parity transformation will therefore flip the direction of a particle's momentum vector $\vec{p}$ while preserving the direction of its spin vector $\vec{s}$, which is an axial vector. The projection of a particle's spin vector onto its momentum vector is the particle's helicity. Right-handed (left-handed) helicity is where the spin and momentum vectors are parallel (antiparallel). The Lorentz invariant (i.e. reference frame invariant) generalization of helicity is chirality. Chirality is the same as helicity only for massless particles. The weak force maximally violates parity by coupling only to particles with left-handed chirality and antiparticles with right-handed chirality.

In the Standard Model, the parity violation in weak interactions arises from vector-axial vector (V-A) structure of the weak current. The weak current for charged current interactions ( $W^{ \pm}$exchange) is given by

$$
J_{\sigma}^{C C}=\bar{u}_{f} \frac{1}{2} \gamma_{\sigma}\left(\mathbb{1}-\gamma^{5}\right) u_{i}
$$

where $u_{i}$ and $u_{f}$ are the Dirac spinors for the initial and final state fermions, respectively; $\bar{u}=u^{\dagger} \gamma^{0}$; and $\gamma^{0}, \gamma_{\sigma}$, and $\gamma^{5}$ are the $4 \times 4$ Dirac matrices. A Dirac spinor has four components and describes the left and right handed chiral states of a fermion and its antiparticle. Under parity transformations, the components $\bar{u}_{f} \gamma^{\sigma} u_{i}$ and $\bar{u}_{f} \gamma^{\sigma} \gamma^{5} u_{i}$ of $J_{\sigma}^{C C}$ transform as a vector and an axial vector, respectively. The matrix $\gamma_{\sigma}\left(\mathbb{1}-\gamma^{5}\right)$ acts as a projection operator which selects the particle state with left-handed chirality and the antiparticle state with right-handed chirality from the Dirac spinors. Likewise, the weak current for neutral current interactions $(Z$ 
exchange) is given by

$$
J_{\sigma}^{N C}=\bar{u}_{f} \frac{1}{2} \gamma_{\sigma}\left(c_{V} \mathbb{1}-c_{A} \gamma^{5}\right) u_{i},
$$

where $c_{V}$ and $c_{A}$ are fermion-dependent coefficients of the vector and axial-vector components, respectively. [2]

\subsection{Electroweak Theory}

Electroweak theory describes the electromagnetic and weak forces as elements of a single force called the electroweak force, and explains how fundamental particles in the Standard Model acquire mass [2]. Electroweak theory postulates that, above an extremely high critical temperature, all particles are massless and the photon, $W^{ \pm}$, and $Z$ existed as four massless gauge bosons of the electroweak force. Below the critical temperature a "Higgs" field acquires a non-zero strength and breaks the symmetry of the electroweak force, where the photon, $W^{ \pm}$, and $Z$ are formed from the original four massless gauge bosons. In the Standard Model, particles acquire mass by coupling to the Higgs field. The $W^{ \pm}$and $Z$ couple to the Higgs field, while the photon does not and remains massless. All fermions, except neutrinos, couple to the Higgs field. It is unknown whether neutrinos acquire mass by this mechanism, but if so their coupling to the Higgs field is dramatically smaller than other fermions.

Electroweak theory is one of the great successes of the Standard Model. It predicted the existence of the $W^{ \pm}$and $Z$ before their discovery in 1983 at CERN [4][5][6]. Electroweak theory also predicted the existence of the Higgs boson, which is an excitation of the Higgs field and is a fundamental particle in the Standard Model [4]. The Higgs boson, discovered at the LHC in 2012 [7][8], is a scalar (spin 0 ) with a mass of $\approx 125 \mathrm{GeV}[1]$. 
Neutrinos are massless in the Standard Model by construction. The assumption that neutrinos are massless is consistent with the $2 \mathrm{eV}$ upper limit on the neutrino mass measured from tritium decay [1]. Neutrinos do not couple to the Higgs field in the Standard Model. The coupling of neutrinos to the Higgs field requires the existence of both left-handed ( $\mathrm{LH})$ and right-handed $(\mathrm{RH})$ chiral neutrino fields. The Standard Model does not include RH neutrinos or LH antineutrinos, and currently there is no compelling evidence for their existence from measurements of neutrino helicity in weak interactions [1]. RH neutrinos and LH antineutrinos in the Standard Model would be "sterile" (i.e. non-interacting), since the weak force couples only to LH particles and RH antiparticles. The Standard Model must be extended to accomodate non-zero neutrino mass.

\subsection{CP Violation}

Charge parity (CP) symmetry is the postulate that all physical processes in nature are invariant under the combination of charge conjugation, which interchanges particles and antiparticles, and parity transformation. CP symmetry was proposed after weak interactions were observed to violate parity symmetry, which was previously believed to be a symmetry of nature. CP symmetry was found to only be an approximate symmetry of nature. Rare instances of CP symmetry violation, referred to simply as $\mathrm{CP}$ violation, have been observed in the decays of neutral kaons [9] and $B$ mesons $[10,11]$ involving the weak interactions of quarks. CP violation is included in the CKM matrix. No evidence of $\mathrm{CP}$ violation has been found in electromagnetic interactions, strong interactions, or the weak interactions of leptons [1]. CP violation is one of the Sakharov conditions necessary for the generation of 
the matter-antimatter asymmetry in the universe [12]. 


\section{Chapter 3}

\section{Neutrino Oscillations}

One of the most significant developments in particle physics over the last few decades was the discovery of neutrino oscillations. A neutrino oscillation is where a neutrino created with one of the lepton flavors $(e, \mu, \tau)$ later interacts as a neutrino with a different lepton flavor. For example, a $\nu_{\mu}$ created from the decay of a pion, $\pi^{+} \rightarrow \mu^{+}+\nu_{\mu}$, may later interact as a $\nu_{e}$ after traveling some distance $L$. The first confirmation of neutrino oscillations was reported in 1998 by the Super-Kamiokande experiment, which measured the disappearance rate of $\stackrel{(-)}{\nu}_{\mu}$ produced by cosmic rays in the atmosphere [13]. Oscillations of neutrinos from the sun $\left(\nu_{e} \rightarrow \nu_{\mu}, \nu_{\tau}\right)$, neutrinos from nuclear reactors $\left(\bar{\nu}_{e}\right.$ disapperance), and neutrinos produced by particle accelerators $\left(\stackrel{(-)}{\nu}{ }_{\mu}\right.$ disapperance, $\stackrel{(-)}{\nu}{ }_{\mu} \rightarrow \stackrel{(-)}{\nu}_{e}$, and $\left.\nu_{\mu} \rightarrow \nu_{\tau}\right)$ have also been measured [1]. The observation of neutrino oscillations establishes that neutrinos have mass, which contradicts the assumption of the Standard Model that neutrinos are massless. The observation of neutrino oscillations also opens the door to possible new discoveries such as CP violation in neutrino oscillations, which may have a connection to the origin of the matter-antimatter asymmetry of the universe. [14]. 


\subsection{Theory of Neutrino Oscillations}

The theory of neutrino oscillations states that neutrino oscillations are the result of the neutrino flavor states $\left|\nu_{\alpha}\right\rangle(\alpha=e, \mu, \tau)$, which couple to the weak force, being superpositions of neutrino mass states $\left|\nu_{j}\right\rangle(j=1,2,3)$,

$$
\left|\nu_{\alpha}\right\rangle=\sum_{j} U_{\alpha j}^{*}\left|\nu_{j}\right\rangle
$$

where $U_{\alpha j}$ are elements of a $3 \times 3$ unitary "mixing" matrix. The neutrino mass states, in turn, are superpositions of the neutrino flavor states,

$$
\left|\nu_{j}\right\rangle=\sum_{\alpha} U_{\alpha j}\left|\nu_{\alpha}\right\rangle
$$

The neutrino flavor states and mass states each form an orthonormal basis,

$$
\begin{aligned}
& \left\langle\nu_{\alpha} \mid \nu_{\beta}\right\rangle=\delta_{\alpha \beta}, \\
& \left\langle\nu_{j} \mid \nu_{k}\right\rangle=\delta_{j k} .
\end{aligned}
$$

Each $\left|\nu_{j}\right\rangle$ has a definite mass $m_{j}$ and propagates as a plane wave,

$$
\left|\nu_{j}(t)\right\rangle=e^{-i\left(E_{j} t-\vec{p}_{j} \cdot \vec{x}\right)}\left|\nu_{j}\right\rangle
$$

where $E_{j}$ and $\vec{p}_{j}$ are the mass state energy and momentum. Neutrinos are typically ultrarelativistic $(E \gg m)$ since the neutrino mass is small $(<2 \mathrm{eV})$. In the ultrarelativistic limit, $t \approx L$, where $L$ is the distance travelled by the neutrino, and the 
magnitude of the mass state momentum $\left|\vec{p}_{j}\right|$ can be approximated as

$$
\left|\vec{p}_{j}\right|=\sqrt{E_{j}^{2}-m_{j}^{2}} \approx E_{j}-\frac{m_{j}^{2}}{2 E_{j}}
$$

The plane wave propagation of the neutrino mass state is approximated in the ultrarelativistic limit as

$$
\begin{aligned}
e^{-i\left(E_{j} t-\vec{p}_{j} \cdot \vec{x}\right)} & \approx e^{-i\left(E_{j}-|\vec{p}|\right) L} \\
& \approx e^{-i\left[E_{j}-\left(E_{j}-\frac{m_{j}^{2}}{2 E_{j}}\right)\right] L} \\
& \approx e^{-i \frac{m_{j}^{2}}{2 E} L}
\end{aligned}
$$

where, for ultrarelativistic neutrinos, $E_{j}$ is approximately equal to the energy $E$ of the neutrino flavor state [14]. In terms of the neutrino mass states, a neutrino flavor state evolves as

$$
\left|\nu_{\alpha}(t \approx L)\right\rangle=\sum_{j}^{3} U_{\alpha j}^{*} e^{-i \frac{m_{j}^{2}}{2 E} L}\left|\nu_{j}\right\rangle .
$$

The mass states of $\left|\nu_{\alpha}\right\rangle$ will propagate at different speeds if their masses are not equal. Since each mass state is itself a superposition of the flavor states, a neutrino created in a pure flavor state will become a mixture of flavor states as it propagates through space. The neutrino will then have a non-zero probability of interacting with a lepton flavor different from its original flavor.

The probability of a neutrino created in flavor state $\left|\nu_{\alpha}\right\rangle$ interacting in flavor 
state $\left|\nu_{\beta}\right\rangle$ after traveling a distance $L$ in a vacuum is given by

$$
\begin{aligned}
P\left(\nu_{\alpha} \rightarrow \nu_{\beta}\right)= & \left|\left\langle\nu_{\beta} \mid \nu_{\alpha}(L)\right\rangle\right|^{2} \\
= & \left|\left(\sum_{j}\left\langle\nu_{j}\right| U_{\beta j}\right)\left(\sum_{j} U_{\alpha j}^{*} e^{-i \frac{m_{j}^{2}}{2 E} L}\left|\nu_{j}\right\rangle\right)\right|^{2} \\
= & \left|\sum_{j} U_{\beta j} U_{\alpha j}^{*} e^{-i \frac{m_{j}^{2}}{2 E} L}\right|^{2} \\
= & \delta_{\alpha \beta}-4 \sum_{j>k} \operatorname{Re}\left(U_{\alpha j}^{*} U_{\beta j} U_{\alpha k} U_{\beta k}^{*}\right) \sin ^{2}\left(\frac{\Delta m_{j k}^{2} L}{4 E}\right) \\
& \quad+2 \sum_{j>k} \operatorname{Im}\left(U_{\alpha j}^{*} U_{\beta j} U_{\alpha k} U_{\beta k}^{*}\right) \sin \left(\frac{\Delta m_{j k}^{2} L}{2 E}\right),
\end{aligned}
$$

where $\Delta m_{j k}^{2}=m_{j}^{2}-m_{k}^{2}[14]$. The unitary matrix $U$ that mixes the neutrino flavor and mass states is called the Pontevorvo-Maki-Nakagawa-Sakata (PMNS) matrix, which can be expressed as

$$
U=\left(\begin{array}{ccc}
1 & 0 & 0 \\
0 & c_{23} & s_{23} \\
0 & -s_{23} & c_{23}
\end{array}\right)\left(\begin{array}{ccc}
c_{13} & 0 & s_{13} e^{-i \delta_{C P}} \\
0 & 1 & 0 \\
-s_{13} e^{i \delta_{C P}} & 0 & c_{13}
\end{array}\right)\left(\begin{array}{ccc}
c_{12} & s_{12} & 0 \\
-s_{12} & c_{12} & 0 \\
0 & 0 & 1
\end{array}\right)
$$

where $c_{j k}=\cos \theta_{j k}, s_{j k}=\sin \theta_{j k}$, and $\delta_{C P}$ is a CP violating phase [15]. A non-zero $\delta_{C P}$ will result in a difference between the oscillation probabilities for neutrinos and antineutrinos. For three neutrino flavors, the oscillation probability $P\left(\nu_{\alpha} \rightarrow \nu_{\beta}\right)$ is dependent on three mass splittings $\left(\Delta m_{21}^{2}, \Delta m_{32}^{2}\right.$, and $\left.\Delta m_{31}^{2}\right)$, three mixing angles $\left(\theta_{12}, \theta_{23}\right.$, and $\left.\theta_{13}\right)$, one $\mathrm{CP}$ violating phase $\delta_{C P}$, and the neutrino energy $E$ and distance traveled by the neutrino $L$. Non-zero $\delta_{C P}$ leads to a difference between the oscillation rates of neutrinos and antineutrinos. 


\subsection{Experimental Status of Neutrino Oscillations}

The current experimental values of the oscillation probability parameters for the three known neutrino flavors are listed in Table 3.1. These values are based on measurements of the oscillations of solar, reactor, atmospheric, and accelerator beam neutrinos. The mass state composition of the three neutrino flavors is illustrated in Figure 3.1 for the current values of the mixing angles. The mass splitting $\Delta m_{31}^{2}$, which is not listed in Table 3.1, is approximately equal to $\Delta m_{32}^{2}$ since $\Delta m_{32}^{2} \gg \Delta m_{21}^{2}$. The CP violating phase $\delta_{C P}$ is currently unknown. Neutrino oscillation experiments have yet to achieve the sensitivity to measure $\delta_{C P}$. Measuring $\delta_{C P}$ is a primary goal of current and future neutrino oscillation experiments.

\begin{tabular}{cc} 
Parameter & Value \\
\hline$\Delta m_{21}^{2}$ & $(7.53 \pm 0.18) \times 10^{-5} \mathrm{eV}^{2}$ \\
$\Delta m_{32}^{2}$ & $(2.44 \pm 0.06) \times 10^{-3} \mathrm{eV}^{2}$ \\
$\sin ^{2}\left(\theta_{12}\right)$ & $0.304 \pm 0.014$ \\
$\sin ^{2}\left(\theta_{23}\right)$ & $0.514_{-0.056}^{+0.055}$ \\
$\sin ^{2}\left(\theta_{13}\right)$ & $(2.19 \pm 0.12) \times 10^{-2}$ \\
$\delta$ & Unknown
\end{tabular}

Table 3.1: Current measured values of the neutrino oscillation paramters [1]

The ordering of the neutrino masses (e.g. $m_{3}>m_{2}>m_{1}$ ), which is referred to as the neutrino mass hierarchy, is also unknown. Solar neutrino oscillation measurements have established that $m_{2}>m_{1}$ [1]. It has yet to be determined whether $m_{3}>m_{2}$ or $m_{3}<m_{1}$, where the former gives the "normal hierarchy" and the latter gives the "inverted hierarchy" (Figure 3.2). Knowing the mass hierarchy is necessary to correctly determine the amount of $\mathrm{CP}$ violation in neutrino oscillations and the sensitivity of direct neutrino mass measurements [16].

Measuring all oscillation parameters to greater precision is another goal of os- 


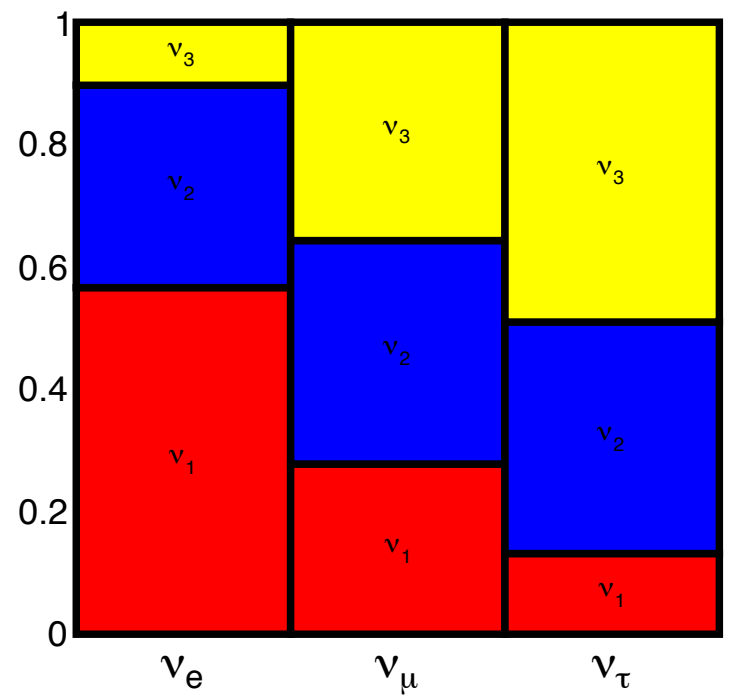

Figure 3.1: Mass state composition of the neutrino flavor states. Figure is from [15].

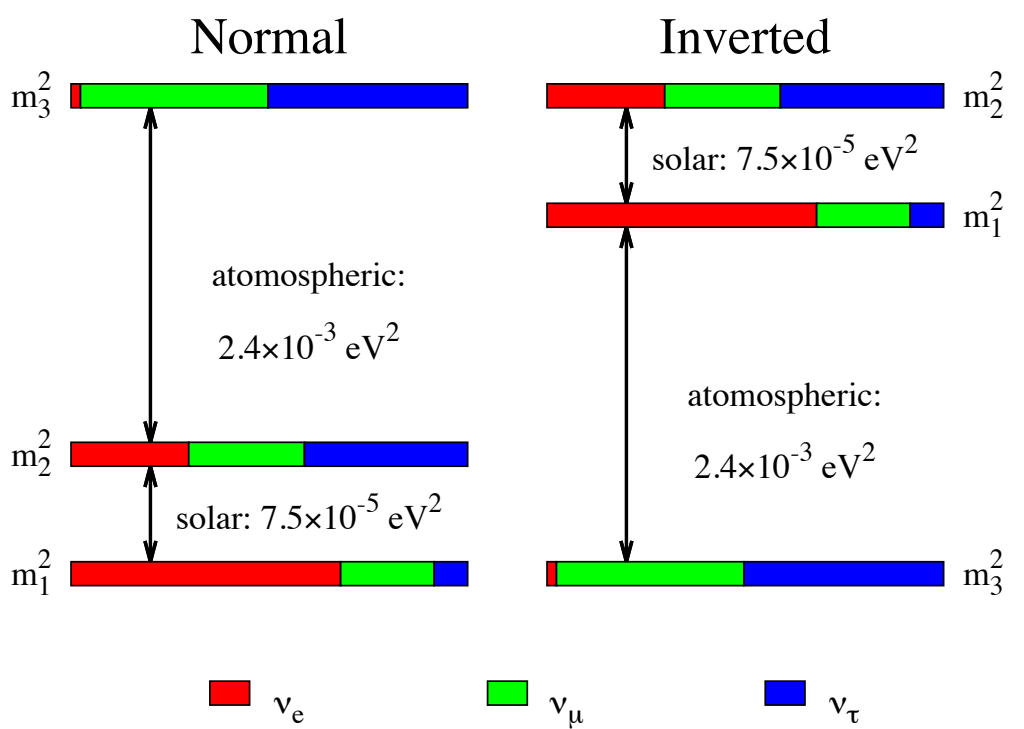

Figure 3.2: The normal and inverted neutrino mass hierarchies. Figure is from [17]. 
cillation experiments. Precise measurements of the oscillation parameters can test the unitarity of the three flavor PMNS matrix. Deviation of the PMNS matrix from unitarity would suggest the existence of additional neutrino species that are participating in the oscillations.

\subsection{Long Baseline Neutrino Oscillation Experi- ments}

Long baseline neutrino oscillation experiments are aiming to make precise measurements of the oscillation parameters, determine the mass hierarchy, and measure $\delta_{C P}$. These experiments measure the oscillation probability $P\left(\nu_{\mu} \rightarrow \nu_{e}\right)$, the survival probability $P\left(\nu_{\mu} \rightarrow \nu_{\mu}\right)$, and their antineutrino counterparts. $\delta_{C P}$ is measured from the difference between $P\left(\nu_{\mu} \rightarrow \nu_{e}\right)$ and $P\left(\bar{\nu}_{\mu} \rightarrow \bar{\nu}_{e}\right)$.

Long baseline experiments consist of an intense beam of $\nu_{\mu}$ or $\bar{\nu}_{\mu}$, a detector near the beam source, and a far detector located hundreds of $\mathrm{km}$ from the beam source. The baseline $L$ is the distance between the near and far detectors. The neutrino beam passes through the earth between the two detectors. The flavor composition of the neutrino beam is measured at the near and far detectors by the charged current weak interactions of neutrinos with nuclei, where the flavor of the incident neutrino is identified by the final state charged lepton.

The mass hierarchy can be determined in long baseline experiments by exploiting how matter in the earth modifies the vacuum oscillation probability (Equation 3.8), which is referred to as the Mikheyev-Smirnov-Wolfenstein (MSW) effect [1]. A derivation of the MSW effect is given in reference [1]. For the normal (inverted) mass hierarchy, the MSW effect enhances (suppresses) $P\left(\nu_{\mu} \rightarrow \nu_{e}\right)$ and suppresses 
(enhances) $P\left(\bar{\nu}_{\mu} \rightarrow \bar{\nu}_{e}\right)[16]$.

The neutrino energy $E$ of the beam and baseline $L$ are the controllable parameters of the oscillation probability and are chosen to maximize the combination of oscillation probability, matter effects, and event rate in the far detector. Long baseline experiments that aim to measure the mass hierarchy and $\delta_{C P}$ include the currently operating $\mathrm{NO} \nu \mathrm{A}(E \approx 2 \mathrm{GeV}, L=810 \mathrm{~km})[16]$ and $\mathrm{T} 2 \mathrm{~K}(E \approx 0.6 \mathrm{GeV}$, $L=295 \mathrm{~km})[18]$ experiments, and the planned DUNE experiment $(E \approx 2.5 \mathrm{GeV}$, $L \approx 1300 \mathrm{~km})[15]$. 


\section{Chapter 4}

\section{Neutrino-Nucleus Interactions}

Ignoring gravity, neutrinos only interact via the weak force. Neutrinos therefore rarely interact and can only be observed indirectly by their weak interactions with other particles. Neutrino oscillation experiments observe neutrinos by their weak charged current $(\mathrm{CC})$ interactions with nuclei. In these interactions, a neutrino $\nu_{l}$ typically scatters off a nucleon $N$ (proton or neutron) within the nucleus, producing a final state charged lepton $l$ and one or more recoil hadrons $X$ :

$$
\nu_{l}+N \rightarrow l+X
$$

The flavor of the final state lepton identifies the flavor of the incident neutrino. Nuclei serve as a dense target with which neutrinos can interact.

Long baseline neutrino oscillation experiments operate at neutrino energies $E_{\nu}$ in

the range $1 \lesssim E_{\nu} \lesssim 10 \mathrm{GeV}$. In this energy range $\mathrm{CC}$ weak interactions of neutrinos with nuclei primarily occur via the following scattering processes:

- Quasi-Elastic Scattering $\left(\nu_{l}+N \rightarrow l+N^{\prime}\right)$ 
A neutrino $\nu_{l}$ interacts with nucleon $N$ bound in a nucleus, producing a charged lepton $l$ and a recoil nucleon $N^{\prime}$ (Figure 4.1). $N$ and $N^{\prime}$ have different electric charge.

- Resonance Production $\left(\nu_{l}+N \rightarrow l+N^{\prime}+\pi\right)$

A neutrino interacts with nucleon bound in a nucleus, producing a charged lepton and a baryon resonance. The resonance decays promptly, usually before exiting the nucleus. A common baryon resonance produced in neutrinonucleus interactions is the $\Delta$, which primarily decays to a nucleon and a pion (Figure 4.2).

- Deep Inelastic Scattering (DIS) $\left(\nu_{l}+N \rightarrow l+X\right)$

A neutrino interacts with a constituent quark of a nucleon bound in a nucleus, producing a charged lepton and multiple hadrons $X$ (Figure 4.3).

- Non-Resonant Background ( $\left.\nu_{l}+N \rightarrow l+X\right)$

A neutrino interacts with nucleon bound in a nucleus, producing a charged lepton and multiple hadrons, which often includes one more pions. Non-resonant background scattering does not produce a baryon resonance and occurs at a lower momentum transfer to the target nucleon than deep inelastic scattering.

The above neutrino-nucleus scattering processes correspond to regions of invariant mass $W$, which is the center of mass energy of the final state hadronic recoil system. The multiplicity of the hadronic recoil (i.e. the number of hadrons in the final state hadronic recoil system) increases with $W$. The deep inelastic scattering region is usually defined as $W>2.0 \mathrm{GeV}$. Resonance production and non-resonant background interactions are thereby defined to have $W<2.0 \mathrm{GeV}$. For quasi-elastic scattering, $W$ is equal to the nucleon mass $m_{N} \sim 1.0 \mathrm{GeV}$. These regions of $W$ are 


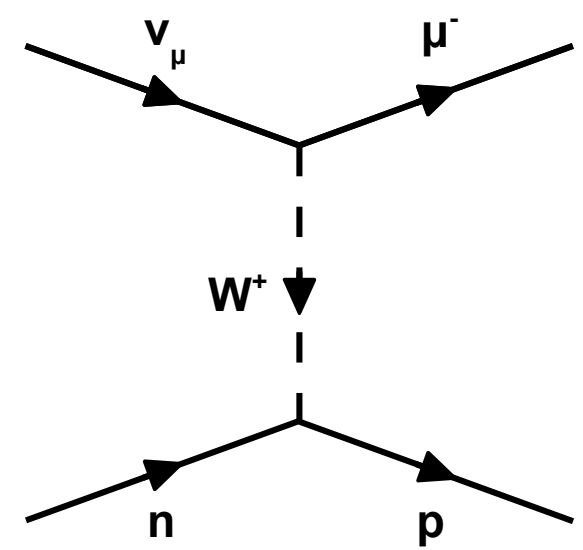

Figure 4.1: Neutrino-nucleus CC quasi-elastic scattering
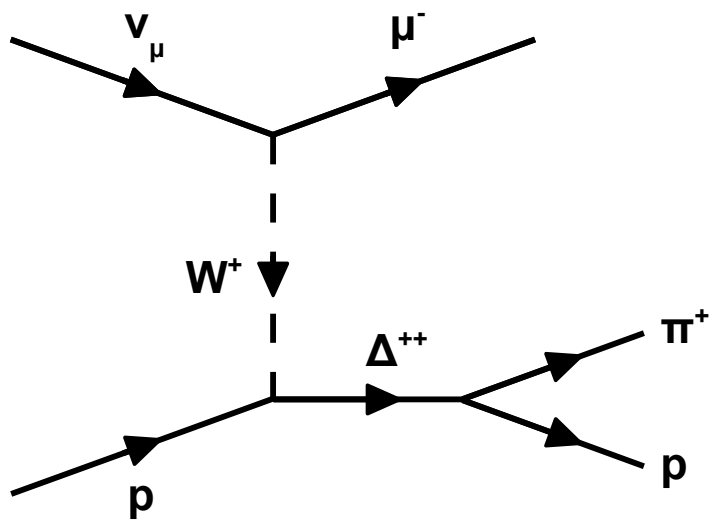

Figure 4.2: Neutrino-nucleus CC resonance production

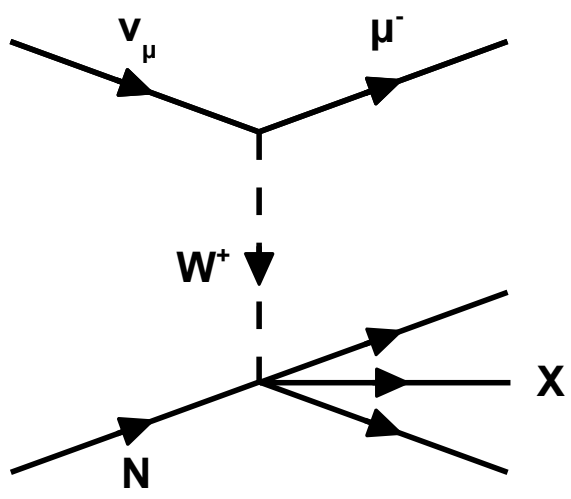

Figure 4.3: Neutrino-nucleus CC deep inelastic scattering 
for interactions with a free nucleon. From conservation of four-momentum, $W^{2}$ for an interaction with a free nucleon is

$$
W^{2}=p_{X}^{2}=\left(p_{\nu}+p_{N}-p_{l}\right)^{2}
$$

where $p_{X}$ is the total four-momentum of the final state hadronic recoil system and $p_{\nu}, p_{N}$, and $p_{l}$ are the four-momentum of the neutrino, target nucleon, and final state charged lepton, respectively. For interactions with a nucleon bound in a nucleus, this calculation must be corrected for the effects of the nuclear medium.

Neutrino oscillation experiments measure the oscillation probability as a function of neutrino energy, which requires knowing the energies of the interacting neutrinos. Oscillation experiments typically use neutrino sources that produce a continuous spectrum of neutrino energies. The energy of an interacting neutrino is therefore unknown and must be reconstructed from the final state particles produced in the interaction. The types and number of hadrons produced in an interaction is dependent on the scattering process, and each hadron species $\left(e . g . p, n, \pi^{+}\right)$produces a different detector response. In addition, the hadrons produced in a neutrino-nucleus interaction can themselves interact within the nucleus. In order to precisely measure the oscillated neutrino energy spectrum, neutrino oscillation experiments need precise knowledge of the rates of the different neutrino-nucleus scattering processes and how the nuclear medium affects the particles produced in neutrino-nucleus interactions.

Neutrino oscillation experiments utilize neutrino-nucleus interaction models. These models calculate the cross section for a particular neutrino-nucleon scattering process, which quantifies the probability for the scattering process to occur. Neutrino- 
nucleus interaction models also calculate effects of the nuclear medium, which modifies both the neutrino-nucleon cross sections and the particles produced in neutrinonucleon interactions. The cross sections and nuclear effects cannot be calculated exactly due to the non-perturbative effects of the strong interactions within the nucleon and nucleus. Neutrino-nucleus interaction models are therefore tuned to measured neutrino-nucleus scattering cross sections. Measurements of the per-nucleon quasi-elastic, resonance production, and deep inelastic scattering cross sections as a function of neutrino energy for $\nu_{\mu}$ and $\bar{\nu}_{\mu}$ CC interactions with isoscalar nuclei (equal numbers of protons and neutrons) are shown in Figure 4.4. Measurements of neutrino-nucleus scattering cross sections tend to have large uncertainties due to small statistics, uncertainties on the neutrino flux, and incomplete knowledge of nuclear effects. Neutrino-nucleus cross sections are typically the largest systematic uncertainty for neutrino oscillation measurements [21, 22]. The MINER $\nu \mathrm{A}$ experiment (Chapter 7) is making precise measurements of neutrino-nucleus scattering cross sections for tuning neutrino-nucleus interaction models and reducing systematic uncertainties in oscillation experiments.
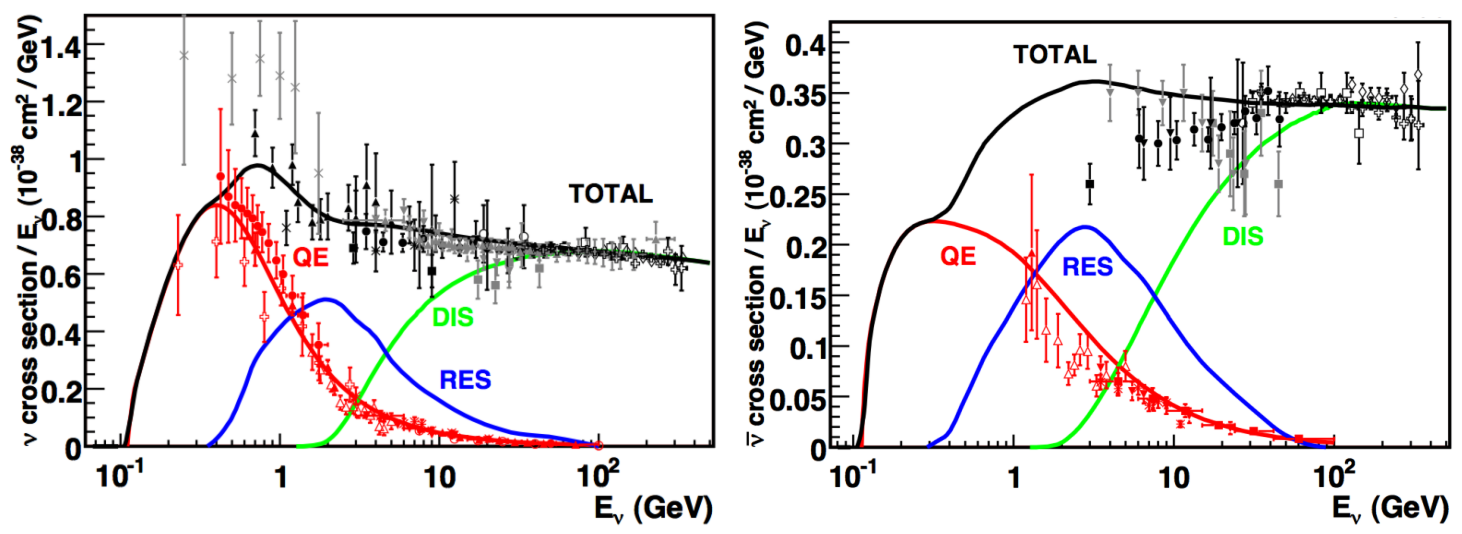

Figure 4.4: Measured and predicted per-nucleon cross sections for $\nu_{\mu}$ and $\bar{\nu}_{\mu} \mathrm{CC}$ interactions with isoscalar nuclei. Figure is from [19]. 


\subsection{Neutrino-Nucleon Cross Sections}

A cross section quantifies the probability for an interaction between particles. Within the Standard Model, the cross section for the interaction of two particles can be calculated by Fermi's golden rule [4]:

$$
\sigma=\frac{1}{4 \sqrt{\left(p_{1} \cdot p_{2}\right)^{2}-\left(m_{1} m_{2}\right)^{2}}} \int \prod_{j=3}^{n} \frac{d^{3} \vec{p}_{j}}{(2 \pi)^{3} 2 E_{j}}(2 \pi)^{4} \delta^{4}\left(p_{1}+p_{2}-\sum_{k=3}^{n} p_{k}\right)|\mathcal{M}|^{2}
$$

where $p$ is four-momentum, $\vec{p}$ is three-momentum, $E$ is energy, $m$ is mass, and $\mathcal{M}$ is the scattering amplitude. The subscripts 1 and 2 denote the two interacting particles, and the subscripts 3-n denote the final state particles. The delta function enforces energy and momentum conservation. The cross section is reduced by a factor of $1 / k$ ! for each group of $k$ final state particles. The scattering amplitude is dependent on the gauge boson and the intial and particle species involved in the interaction.

An example of an interaction with a calculable cross section is inverse muon decay,

$$
\nu_{\mu}+e^{-} \rightarrow \mu^{-}+\nu_{e}
$$

which is a CC weak interaction (Figure 4.5). For $\left|q^{2}\right| \ll M_{W}^{2}$, where $q$ and $M_{W}$ are the four momentum and mass of the $W^{ \pm}$gauge boson, respectively, the scattering amplitude is [4]

$$
\mathcal{M}=\frac{g_{w}^{2}}{8 M_{W}^{2}}\left[\bar{u}_{\mu} \gamma_{\sigma}\left(\mathbb{1}-\gamma^{5}\right) u_{\nu_{\mu}}\right]\left[\bar{u}_{\nu_{e}} \gamma^{\sigma}\left(\mathbb{1}-\gamma^{5}\right) u_{e}\right],
$$


where $g_{w}$ is the weak coupling constant and the two bracketed factors are the CC weak leptonic currents (see Section 2.2.3). Applying trace theorems, the squared scattering amplitude averaged over all spin states is [4]

$$
\left\langle|\mathcal{M}|^{2}\right\rangle=2\left(\frac{g_{w}}{M_{W}}\right)^{4}\left(p_{\nu_{\mu}} \cdot p_{e}\right)\left(p_{\mu} \cdot p_{\nu_{e}}\right) .
$$

In the center of momentum reference frame and ignoring the mass of the electron, $\left\langle|\mathcal{M}|^{2}\right\rangle$ is expressed as

$$
\left\langle|\mathcal{M}|^{2}\right\rangle=8\left(\frac{g_{w} E}{M_{W}}\right)^{4}\left[1-\left(\frac{m_{\mu}}{2 E}\right)^{2}\right],
$$

and the cross section is

$$
\sigma=\frac{1}{8 \pi}\left(\frac{g_{w}}{M_{W}}\right)^{4} E^{2}\left[1-\left(\frac{m_{\mu}}{2 E}\right)^{2}\right]^{2},
$$

where $E$ is the energy of the $\nu_{\mu}$ or $e^{-}$and $m_{\mu}$ is the muon mass [4].

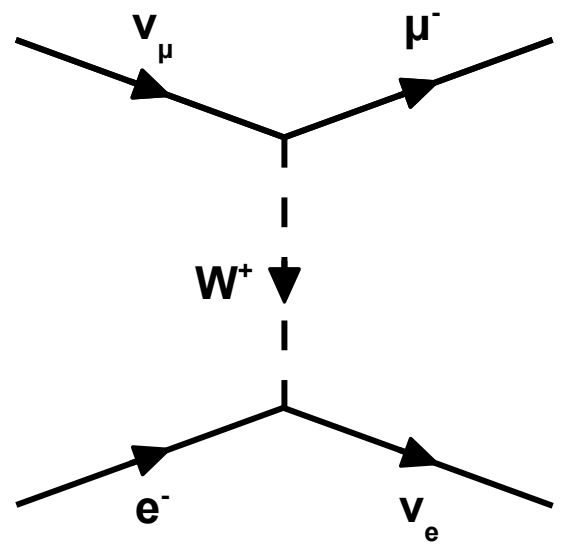

Figure 4.5: Inverse muon decay

The scattering amplitude for a CC weak interaction between a neutrino and a 
nucleon is given by

$$
\mathcal{M} \propto \bar{u}_{l} \gamma_{\sigma}\left(\mathbb{1}-\gamma^{5}\right) u_{\nu_{l}}\left\langle X\left|h_{V}^{\sigma}+h_{A}^{\sigma}\right| N\right\rangle
$$

where $|N\rangle$ is the nucleon initial state, $\langle X|$ is the hadronic system final state, and $h_{V}^{\sigma}$ and $h_{A}^{\sigma}$ are the vector and axial vector weak hadronic currents, respectively [20]. The weak hadronic currents are unknown functions due to the incalculable effects of the strong interactions within the nucleon. In neutrino-nucleon cross section calculations, the components of the cross section from the weak hadronic currents are parameterized and fit to cross section measurements.

The most widely used model of neutrino-nucleon quasi-elastic scattering is the Llewellen Smith model [23]. The CC cross section calculated from the Llewellen Smith model is commonly expressed as [24]

$$
\frac{d \sigma_{C C}^{\nu_{l} n, \bar{\nu}_{l} p}}{d Q^{2}}=\frac{G_{F}^{2}\left|V_{u d}\right|^{2} m_{N}^{4}}{8 \pi\left(p_{\nu} \cdot p_{N}\right)^{2}}\left[A\left(Q^{2}\right) \pm B\left(Q^{2}\right) \frac{s-u}{m_{N}^{2}}+C\left(Q^{2}\right) \frac{(s-u)^{2}}{m_{N}^{4}}\right]
$$

where $G_{F}$ is the Fermi constant defined as

$$
G_{F}=\frac{\sqrt{2}}{8} \frac{g_{w}^{2}}{M_{W}^{2}}
$$

$V_{u d}$ is the CKM matrix element for the transition probability between the up and down quarks in $\mathrm{CC}$ weak interactions, $p_{N}$ and $m_{N}$ are the four-momentum and mass of the initial state nucleon, respectively, and $Q^{2}, s$, and $u$ are the Mandelstam 
variables defined as

$$
\begin{aligned}
Q^{2} & =-q^{2}=-\left(p_{\nu}-p_{l}\right)^{2}, \\
s & =\left(p_{\nu}+p_{N}\right)^{2}, \\
u & =\left(p_{l}-p_{N}\right)^{2} .
\end{aligned}
$$

Collectively, the functions $A\left(Q^{2}\right), B\left(Q^{2}\right)$, and $B\left(Q^{2}\right)$ depend on two nucleon vector form factors $F_{1}\left(Q^{2}\right)$ and $F_{2}\left(Q^{2}\right)$, a nucleon axial vector form factor $G_{A}\left(Q^{2}\right)$, and a nucleon pseudo-scalar form factor $G_{P}\left(Q^{2}\right)$. The vector form factors are determined from measurements of electron-nucleon scattering, which is dominated by the electromagnetic interaction. The electromagnetic interaction has a vector structure. Per the Conserved Vector Current (CVC) hypothesis [39, 40], which is supported by experiment, strong interactions conserve (i.e. do not modify) both the electromagnetic and weak vector hadronic currents. Therefore, the electromagnetic and weak vector hadronic currents are equivalent probes of the vector structure of the nucleon, which allows the nucleon vector vector form factors to be determined from electronnucleon scattering measurements. The nucleon axial vector form factor $G_{A}\left(Q^{2}\right)$ has the dipole form

$$
G_{A} \propto\left(1+\frac{Q^{2}}{M_{A}^{2}}\right)^{-2}
$$

where $M_{A}$ is a free parameter called the axial vector mass [24]. The value of $M_{A}$ extracted from fits to neutrino scattering measurements is [25]

$$
M_{A}=1.026 \pm 0.021 \mathrm{GeV}
$$

The pseudo-scalar form factor is related to the axial vector form factor by the PCAC 
hypothesis (Section 5.1.1) and the Goldberger-Treiman relation [26] as

$$
G_{P}\left(Q^{2}\right)=\frac{2 m_{n}^{2} G_{A}\left(Q^{2}\right)}{m_{\pi}^{2}+Q^{2}}
$$

where $m_{\pi}$ is the pion mass [27].

The most widely used model of baryon resonance production in neutrino-nucleon interactions is the model by Rein and Sehgal [28]. The cross section calculated by the Rein-Sehgal resonance model is

$$
\frac{d^{2} \sigma}{d Q^{2} d \nu}=\frac{G_{F}^{2}}{4 \pi^{2}} \frac{Q^{2}}{|\vec{q}|^{2}}\left(\frac{m_{N}^{2}-M^{2}}{2 m_{N}}\right)\left(u^{2} \sigma_{L}+v^{2} \sigma_{R}+2 u v \sigma_{S}\right)
$$

where $Q^{2}$ is defined per Equation $4.12, \vec{q}$ is the three-momentum of the weak gauge boson, $M$ is the mass of the resonance, and

$$
\begin{aligned}
& \nu=E_{\nu}-E_{l}, \\
& u=\frac{E_{\nu_{l}}+E_{l}+|\vec{q}|}{2 E_{\nu_{l}}}, \\
& v=\frac{E_{\nu_{l}}+E_{l}-|\vec{q}|}{2 E_{\nu_{l}}} .
\end{aligned}
$$

$\sigma_{L}, \sigma_{R}$, and $\sigma_{S}$ in Equation 4.16 are the partial cross sections for the initial state nucleon absorbing a weak gauge boson with left-handed, right-handed, or zero helicity, respectively. $\sigma_{L}, \sigma_{R}$, and $\sigma_{S}$ are functions of $Q^{2}$, invariant mass $W$, and vector and axial vector form factors of the dipole form defined in Equation 4.13. The values of the vector and axial vector masses for the resonance production form factors, $M_{V}^{R E S}$ 
and $M_{A}^{R E S}$, extracted from cross section measurements are [28]

$$
\begin{aligned}
& M_{V}^{R E S}=0.84 \mathrm{GeV}, \\
& M_{A}^{R E S}=0.95 \mathrm{GeV} .
\end{aligned}
$$

The Rein-Sehgal resonance model calculates the amplitudes for 18 baryon resonances with $W<2.0 \mathrm{GeV}$ and sums these to calculate $\sigma_{L}, \sigma_{R}$, and $\sigma_{S}$.

The cross section for deep inelastic scattering in neutrino-nucleon CC interactions is given by [24]

$$
\begin{aligned}
\frac{d^{2} \sigma_{C C}^{\nu_{l} N, \bar{\nu}_{l} N}}{d x d y} & =\frac{G_{F}^{2}}{2 \pi} s\left(1+\frac{Q^{2}}{M_{W}}\right)^{-2} \\
& \times\left[x y^{2} F_{1}\left(x, Q^{2}\right)+(1-y) F_{2}\left(x, Q^{2}\right) \pm x y\left(1-\frac{y}{2}\right) F_{3}\left(x, Q^{2}\right)\right]
\end{aligned}
$$

where $Q^{2}$ and $s$ are defined per Equation 4.12 and

$$
\begin{aligned}
& x=\frac{Q^{2}}{2 p_{N} \cdot q}, \\
& y=\frac{p_{N} \cdot q}{p_{N} \cdot p_{\nu_{l}}} .
\end{aligned}
$$

$x$ is the fraction of the target nucleon four-momentum $p_{N}$ carried by the quark that absorbed the weak gauge boson, and $y$ is the fraction of the neutrino energy transferred to the target nucleon. $F_{i}\left(x, Q^{2}\right)$ in Equation 4.19 are nucleon structure functions, which depend on parton distribution functions $f_{j}(x)$. The parton distribution functions give the probability of quark $j$ within the target nucleon having four-momentum $p_{j}=x p_{N}$ [24]. The nucleon structure functions have a weak logarithmic dependence on $Q^{2}[24]$. The nucleon structure functions and parton 
distribution functions are both fit to cross section measurements.

Different methods are used to model neutrino-nucleon non-resonant background interactions. Rein and Sehgal used a scaled version of their resonance production model that is non-interfering with resonance production interactions [28]. Another method uses a modulated DIS model below the DIS region [29].

\subsection{Nuclear Effects}

Most neutrino-nucleus interactions of interest to neutrino oscillation experiments involve a neutrino interacting with a nucleon bound within a nucleus. The neutrinonucleon interaction can be significantly affected by the nuclear medium. The nuclear medium can suppress or enhance the neutrino-nucleon interaction rate. In addition, recoil hadrons from a neutrino-nucleon interaction can themselves interact with the nuclear medium, thereby altering the final state. Most neutrino-nucleus interaction models calculate the cross section for a neutrino interaction with a free nucleon, and augment the free nucleon cross section for the effects of the nuclear medium.

The nuclear medium can suppress the neutrino-nucleon interaction rate due to

the Pauli exclusion principle. Since the proton and neutron are both spin $\frac{1}{2}$ fermions, the Pauli exclusion principle forbids each nuclear bound state from being occupied by more than one proton or more than one neutron. Each nuclear bound state corresponds to a momentum. A recoil nucleon from a neutrino-nucleus interaction is therefore required to have a momentum greater than the momenta of the occupied nuclear bound states. This leads to a suppression of the neutrino-nucleus interaction rate at low squared four-momentum transfer to the target nucleon $Q^{2}$, which is referred to as Pauli suppression. A simple model of the nucleus that includes 
Pauli suppression is the Relativistic Fermi Gas (RFG) model [30]. The RFG model describes the nucleus as gas of relativistic, non-interacting fermions in a negative potential energy well. Most neutrino-nucleus interaction models utilize the RFG model.

To a good approximation, nucleons within the nucleus can be treated as noninteracting for neutrino-nucleus interactions at high $Q^{2}$. At low $Q^{2}$ the effects of nucleon-nucleon interactions become important. Interactions between nucleons within a nucleus can be described as the exchange of a meson (usually a pion) and are referred to as meson exchange currents (MEC). Also important at low $Q^{2}$ are nucleon-nucleon short range correlations (SRC), which are the overlap of nucleon wave functions at small distances. Both MEC and SRC can result in a neutrino interacting with a pair of nucleons rather than a single nucleon. For quasi-elastic scattering the effects of MEC and SRC can be interpreted as an enhancement to the cross section at low $Q^{2}$, where a neutrino interacts with a pair of nucleons resulting in the ejection of two nucleons from the nucleus [31]. The effects of MEC and SRC have been observed in electron-nucleus scattering, but only recently has evidence been found for MEC and SRC effects in neutrino-nucleus quasi-elastic scattering [121]. The effects of MEC and SRC in neutrino-nucleus resonance production are unknown.

Recoil hadrons produced by neutrino-nucleon interactions within a nucleus often undergo strong interactions with the nuclear medium before exiting the nucleus, which are referred to as final state interactions (FSI). Several different outcomes can result from FSI. Recoil hadrons can loose a portion of their energy through FSI or be absorbed by the nuclear medium. FSI can result in charge exchange of recoil hadrons (e.g. a proton converts to a neutron, a $\pi^{ \pm}$converts to a $\pi^{0}$ ) as well as 
the production of additional hadrons. Since the energy of an interacting neutrino is reconstructed from the observed final state particles and detector response varies by hadron species, all of these outcomes of FSI affect the reconstructed neutrino energy. In addition, FSI can produce hadronic final states that mimic neutrino oscillation signals. Precise reconstruction of the neutrino energy and estimation of neutrino oscillation background therefore requires FSI effects to be well understood. A common approach to modeling FSI is to use a hadronic cascade model, which is a Monte Carlo simulation that steps recoil hadrons through the nucleus and simulates strong interactions according to hadron-nucleon interaction cross sections.

\subsection{The GENIE Neutrino Event Generator}

Neutrino event generators are Monte Carlo simulations of neutrino interactions. Neutrino oscillation experiments use neutrino event generators to simulate data for predicting the observed oscillated neutrino energy spectrum for a given neutrino oscillation hypothesis. Neutrino event generators are also used by neutrino scattering experiments to simulate data for testing neutrino-nucleus interaction models. Although several neutrino event generators exist, the following discussion will focus on the GENIE neutrino event generator [29] which is used in this thesis. GENIE simulates neutrino-nucleus interactions for a variety of nuclei and for neutrino energies ranging from $\sim 1 \mathrm{MeV}$ to $\sim 1 \mathrm{PeV}$.

GENIE uses a RFG model of the nucleus. The RFG model gives the momentum spectrum and binding energy of initial state nucleons in a nucleus as well as Pauli suppression effects. The version of GENIE used in this thesis (release 2.6.2) did not simulate neutrino interactions with nucleon pairs due to meson exchange currents 
and short range correlations.

The primary neutrino-nucleus scattering processes in the neutrino energy range $1 \lesssim E_{\nu} \lesssim 10 \mathrm{GeV}$ are quasi-elastic scattering, resonance production, non-resonance background, and deep inelastic scattering. GENIE models these scattering processes using the Llewellen Smith quasi-elastic scattering model, the Rein-Sehgal resonance production model, and the DIS model by Bodek and Yang [33]. Non-resonance background is modeled in GENIE by modulating the Bodek-Yang DIS model below the DIS region. GENIE models resonance production and non-resonance background up to invariant mass $W=1.7 \mathrm{GeV}$ and deep inelastic scattering for $W>1.7 \mathrm{GeV}$. Sub-dominant scattering processes modeled by GENIE include neutrino-nucleus coherent pion production (Chapter 5). GENIE uses the coherent pion production model by Rein and Sehgal [34].

In deep inelastic scattering interactions, hadrons are produced from the recoil quark and the energy of the recoil quark's strong interactions, which is referred to as hadronization. GENIE models hadronization for both deep inelastic scattering and non-resonance background interactions using the AGKY hadronization model, which is detailed in [29].

GENIE simulates FSI using the data-driven INTRANUKE hadronic cascade model. INTRANUKE steps each recoil hadron through the nucleus and for each step decides whether to simulate an interaction according to the total hadron-nucleon interaction cross section and the nucleon density of the nucleus. The total hadronnucleon interaction cross section is determined from hadron-nucleon scattering data, and the nucleon density of the nucleus is determined from electron-nucleus elastic scattering data. The simulated interaction type (e.g. elastic, inelastic, hadron absorption), particles produced, and the momenta of the produced particles are deter- 
mined from hadron-nucleon scattering data where available, or model calculations where data are not available. Interactions of particles produced by FSI with the nuclear medium are also simulated. 


\section{Chapter 5}

\section{Coherent Pion Production}

Neutrino-nucleus coherent pion production is an inelastic interaction that produces a lepton and pion in the forward direction while leaving the nucleus in its initial state. The initial and final states for charged current (CC) coherent pion production are

$$
\begin{gathered}
\nu_{l}+\mathrm{A} \rightarrow l^{-}+\pi^{+}+\mathrm{A} \\
\bar{\nu}_{l}+\mathrm{A} \rightarrow l^{+}+\pi^{-}+\mathrm{A}
\end{gathered}
$$

and the initial and final states for neutral current (NC) coherent pion production are

$$
\begin{gathered}
\nu_{l}+\mathrm{A} \rightarrow \nu_{l}+\pi^{0}+\mathrm{A}, \\
\bar{\nu}_{l}+\mathrm{A} \rightarrow \bar{\nu}_{l}+\pi^{0}+\mathrm{A},
\end{gathered}
$$

where $\mathrm{A}$ is the nucleus. For the interaction to preserve the initial state of the nucleus, the square of the four-momentum exchanged with the nucleus $|t|$ must be small, $|t| \lesssim 1 / R^{2}$, where $R$ is the radius of the nucleus, and the particle(s) exchanged 
with the nucleus can only carry vacumm quantum numbers [74]. Coherent pion production interactions are rare. For neutrino (antineutrino) interactions on carbon nuclei at neutrino energy $E_{\nu}=1.0 \mathrm{GeV}$, GENIE predicts the rate of coherent pion production to be $\sim 1 \%(\sim 3 \%)$ of the total interaction rate for both $\mathrm{CC}$ and $\mathrm{NC}$ interactions.

Coherent pion production, despite being a small fraction of the total neutrinonucleus interaction rate, is an important background to neutrino oscillation experiments. NC coherent pion production is an important background to $\nu_{\mu} \rightarrow \nu_{e}$ and $\bar{\nu}_{\mu} \rightarrow \bar{\nu}_{e}$ oscillation measurements where the oscillation signal is $\nu_{e}$ and $\bar{\nu}_{e} \mathrm{CC}$ scattering:

$$
\begin{gathered}
\nu_{e}+N \rightarrow e^{-}+X, \\
\bar{\nu}_{e}+N \rightarrow e^{+}+X,
\end{gathered}
$$

where $N$ is a target nucleon and $X$ is the final state hadronic recoil. The only particle detected from a NC coherent pion production interaction is the $\pi^{0}$, which decays via the electromagnetic force as

$$
\pi^{0} \rightarrow \gamma+\gamma
$$

Each $\gamma$ from the $\pi^{0}$ decay can convert to an electron-positron pair,

$$
\gamma \rightarrow e^{-}+e^{+}
$$

which can mimic the final state electron (positron) in $\nu_{e}\left(\bar{\nu}_{e}\right) \mathrm{CC}$ interactions.

CC coherent pion production is a background to measurements of $\nu_{\mu}$ and $\bar{\nu}_{\mu}$ 
disappearance at low neutrino energy $\left(E_{\nu} \lesssim 1 \mathrm{GeV}\right)$ where quasi-elastic scattering,

$$
\begin{gathered}
\nu_{\mu}+n \rightarrow \mu^{-}+p, \\
\bar{\nu}_{\mu}+p \rightarrow \mu^{+}+n,
\end{gathered}
$$

is the primary interaction process. These measurements reconstruct the neutrino energy under a quasi-elastic (QE) scattering assumption, where by two-body scattering kinematics the neutrino energy $E_{\nu}^{Q E}$ can be calculated from the reconstructed muon kinematics only:

$$
E_{\nu}^{Q E}=\frac{m_{r}^{2}-\left(m_{t}-\epsilon_{b}\right)^{2}-m_{\mu}^{2}+2 E_{\mu}\left(m_{t}-\epsilon_{b}\right)}{2\left(m_{t}-\epsilon_{b}-E_{\mu}+\left|\vec{p}_{\mu}\right| \cos \theta_{\mu}\right)},
$$

where $m_{t}$ is the target nucleon mass, $m_{r}$ is the recoil nucleon mass, $\epsilon_{b}$ is the average nucleon binding energy of the target nucleus, and $m_{\mu}, E_{\mu}, \vec{p}_{\mu}$, and $\theta_{\mu}$ are the mass, energy, momentum, and angle (with respect to the direction of the incoming neutrino) of the muon. $E_{\nu}^{Q E}$ will give the incorrect neutrino energy for non-quasi-elastic scattering processes, and coherent pion production can be mistaken for quasi-elastic scattering when the $\pi^{ \pm}$is misidentified as a proton or when the $\pi^{ \pm}$is not detected.

The coherent pion production rate must be well understood in order to precisely predict the background in neutrino oscillation measurements. Coherent pion production is also important to understanding the total rate of pion production in neutrino-nucleus interactions, to which coherent pion production is a signifiant contribution at small scattering angle of the outgoing lepton with respect to the incoming neutrino. 


\subsection{PCAC Coherent Models}

PCAC coherent models $[34,42,43,44,45,46,47,48,49,50]$ are a class of coherent pion production models that are based on the partially conserved axial-vector current (PCAC) hypothesis, which is discussed in Section 5.1.1. In the PCAC picture of coherent pion production (Figure 5.1), the intermediate weak boson fluctuates to a virtual pion, which scatters elastically off the nucleus:

$$
\pi+\mathrm{A} \rightarrow \pi+\mathrm{A}
$$

where the nucleus remains in its initial quantum state. In the Regge theory of strong interactions, the pion-nucleus elastic interaction occurs via the exchange of a pomeron, $\mathbb{P}$, which is a universal trajectory with quantum numbers of the vacuum only [35]. In Quantum Chromodynamics, pomeron exchange can be described as the exchange of confined (zero net color charge) gluons $[35,36,37]$. The $\pi$ A elastic scattering cross section falls exponentially with $|t| \sim \exp (-b|t|)$, where $b \sim R^{2}$ and $R$ is the radius of the nucleus $[34,38,74]$.

\subsubsection{Adler's PCAC Theorem}

PCAC coherent models utilize Adler's PCAC theorem [20], which considers the neutrino interaction

$$
\nu_{l}+\alpha \rightarrow l+\beta
$$

where $\alpha$ is a nucleon or nucleus and $\beta$ is the final state hadronic system. The theorem considers the limit where the incoming neutrino and final state lepton directions are parallel, which is referred to as the parallel configuration, and the final state lepton 


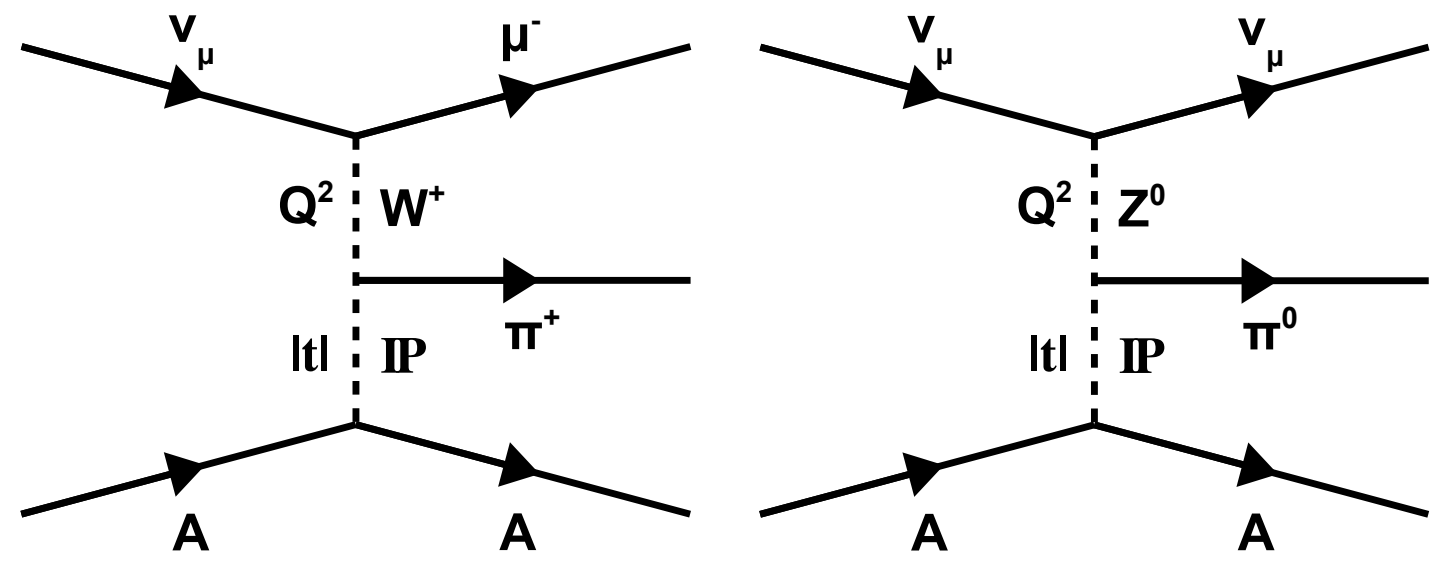

Figure 5.1: CC (left) and NC (right) neutrino-nucleus coherent pion production in the PCAC picture.

mass is ignored. In this limit the squared four-momentum of the weak gauge boson $Q^{2}=0$, which follows from

$$
\begin{aligned}
Q^{2} & =-q^{2}=-\left(p_{\nu_{l}}-p_{l}\right)^{2}, \\
& =2 p_{\nu_{l}} \cdot p_{l}-m_{\nu_{l}}^{2}-m_{l}^{2}, \\
& \approx 2\left(E_{\nu_{l}} E_{l}-\vec{p}_{\nu_{l}} \cdot \vec{p}_{l}\right)-m_{l}^{2}, \\
& \approx 2 E_{\nu_{l}}\left(E_{l}-\left|\vec{p}_{l}\right| \cos \theta_{l}\right)-m_{l}^{2},
\end{aligned}
$$

where $q, p_{\nu_{l}}$, and $p_{l}$ are the four-momenta of the weak gauge boson, neutrino, and final state lepton, respectively; $m_{\nu_{l}}, E_{\nu_{l}}$, and $\vec{p}_{\nu_{l}}$ are the mass, energy, and threemomentum of the neutrino, respectively; $m_{l}, E_{l}$, and $\vec{p}_{l}$ are the mass, energy, and three-momentum of the final state lepton, respectively; and $\theta_{l}$ is the angle between the final state lepton direction and neutrino direction. In the parallel configuration and in the limit where $p_{l} \gg m_{l}, \theta_{l}=0$ and $E_{l}=\left|\vec{p}_{l}\right|$, which gives $Q^{2}=0$. 
The general scattering amplitude for the interaction at all $Q^{2}$ is

$$
\mathcal{M}=\frac{i}{\sqrt{2}} \bar{u}_{l} \gamma_{\sigma}\left(\mathbb{1}-\gamma^{5}\right) u_{\nu_{l}}\left\langle\beta\left|h_{V}^{\sigma}+h_{A}^{\sigma}\right| \alpha\right\rangle,
$$

where $h_{V}^{\sigma}$ and $h_{A}^{\sigma}$ are the weak vector and axial vector hadronic currents, respectively. At $Q^{2}=0$, the squared scattering amplitude averaged over all lepton spin states reduces to

$$
\begin{aligned}
\left\langle|\mathcal{M}|^{2}\right\rangle & =\frac{2 E_{\nu_{l}} E_{l}}{q_{0}^{2}} q_{\sigma} q_{\lambda}\left\langle\beta\left|h_{V}^{\sigma}+h_{A}^{\sigma}\right| \alpha\right\rangle\left\langle\beta\left|h_{V}^{\lambda}+h_{A}^{\lambda}\right| \alpha\right\rangle^{*} \\
& =\frac{2 E_{\nu_{l}} E_{l}}{q_{0}^{2}}\left\langle\beta\left|\partial_{\sigma}\left(h_{V}^{\sigma}+h_{A}^{\sigma}\right)\right| \alpha\right\rangle\left\langle\beta\left|\partial_{\lambda}\left(h_{V}^{\lambda}+h_{A}^{\lambda}\right)\right| \alpha\right\rangle^{*},
\end{aligned}
$$

where $q_{0}$ and $q_{\sigma(\lambda)}$ are the energy and four-momentum of the weak gauge boson. At $Q^{2}=0,\left\langle|\mathcal{M}|^{2}\right\rangle$ depends on the divergences of the weak vector and axial-vector hadronic currents. Per the conserved vector current (CVC) hypothesis [39, 40], strong interactions conserve the weak vector hadronic current [24]:

$$
\partial_{\sigma} h_{V}^{\sigma}=0 .
$$

Therefore, the $\alpha \rightarrow \beta$ transition amplitude at $Q^{2}=0$ only depends on the divergence of the weak axial-vector hadronic current. The partially conserved axial-vector current (PCAC) hypothesis [41] states that strong interactions modify (i.e. do not conserve) the weak axial-vector hadronic current $h_{A}^{\sigma}$, and at small $Q^{2}$ the divergence of $h_{A}^{\sigma}$ is proportional to the pion field $\phi_{\pi}$ :

$$
\partial_{\sigma} h_{A}^{\sigma}=f_{\pi} m_{\pi}^{2} \phi_{\pi}
$$


where $f_{\pi}$ is the pion decay constant and $m_{\pi}$ is the pion mass [24]. Adler's PCAC theorem thereby relates the $\alpha \rightarrow \beta$ transition at $Q^{2}=0$ to the pion scattering process

$$
\pi+\alpha \rightarrow \beta
$$

For coherent pion production, the pion scatters elastically off the nucleus, and Adler's PCAC theorem gives the cross section for CC coherent pion production at $Q^{2}=0$ as

$$
\left.\frac{d \sigma_{c o h}^{C C}}{d Q^{2} d y d|t|}\right|_{Q^{2}=0}=\left.\frac{G^{2} f_{\pi^{ \pm}}^{2}}{2 \pi} \frac{(1-y)}{y} \frac{d \sigma_{e l}^{\pi^{ \pm} \mathrm{A}}}{d|t|}\right|_{E_{\pi}=y E_{\nu}}
$$

where $y=\left(E_{\nu_{l}}-E_{l}\right) / E_{\nu}$ is the inelasticity, $G_{F}$ is the Fermi constant, $f_{\pi^{ \pm}}$is the charged pion decay constant, $\sigma_{e l}^{\pi^{ \pm} \mathrm{A}}$ is the $\pi^{ \pm} \mathrm{A}$ elastic scattering cross section, and $E_{\pi}$ is the pion energy. The $\mathrm{NC}$ cross section is calculated from Equation 5.16 by replacing $f_{\pi^{ \pm}}$by the neutral pion decay constant $f_{\pi^{0}}$, and $\sigma_{e l}^{\pi^{ \pm} \mathrm{A}}$ by the $\pi^{0} \mathrm{~A}$ elastic scattering cross section $\sigma_{e l}^{\pi^{0} \mathrm{~A}}$.

The coherent pion production cross section from Adler's theorem is equal for neutrinos and antineutrinos. This is due to the hadronic transition amplitude depending only on the divergence of the axial-vector hadronic current. In general, for neutrino-nucleon/nucleus interactions at $Q^{2}>0$, the vector and axial-vector hadronic currents interfere, which appears in the cross section calculation as terms containing both vector and axial-vector hadronic currents. This interference is referred to as vector-axial-vector (V-A) interference, and is typically constructive for neutrinos and destructive for antineutrinos. The coherent pion production cross section from Adler's theorem does not contain V-A interference and is therefore equal for neutrinos and antineutrinos.

The $\mathrm{CC}$ and $\mathrm{NC}$ coherent pion production cross sections have a simple relation 
in the PCAC picture. Ignoring the final state lepton mass, the CC and NC coherent pion production cross sections from Adler's theorem are related as

$$
\left.\frac{d \sigma_{c o h}^{N C}}{d Q^{2} d y d|t|}\right|_{Q^{2}=0}=\left.\frac{1}{2} \frac{d \sigma_{c o h}^{C C}}{d Q^{2} d y d|t|}\right|_{Q^{2}=0} .
$$

This relation follows from the difference between the neutral and charged pion decay constants [34],

$$
f_{\pi^{0}}=\frac{1}{\sqrt{2}} f_{\pi^{ \pm}},
$$

and is valid for isoscalar nuclei (equal numbers of protons and neutrons) where the $\pi^{ \pm} \mathrm{A}$ and $\pi^{0} \mathrm{~A}$ elastic scattering cross sections are equal [48]. Therefore, in the PCAC picture, a measurement of $\mathrm{CC}$ coherent pion production provides a constraint on the $\mathrm{NC}$ reaction.

In order to calculate cross sections that are useful for experiments, PCAC coherent models must extrapolate the cross section from Adler's theorem to $Q^{2}>0$ and calculate the $\pi \mathrm{A}$ elastic scattering cross section. PCAC coherent models also apply a correction for the final state lepton mass which becomes important at lower neutrino energies.

\subsubsection{Rein-Sehgal Model}

The Rein-Sehgal PCAC coherent model [34, 42] is the most widely used coherent pion production model in neutrino event generators. The Rein-Sehgal model extrapolates the Adler coherent cross section (Equation 5.16) to $Q^{2}>0$ using a multiplicative axial-vector dipole form factor

$$
F_{A}=\frac{M_{A}^{2}}{Q^{2}+M_{A}^{2}},
$$


where $M_{A} \approx 1 \mathrm{GeV}$ is the axial vector mass. The Rein-Sehgal model assumes no vector current contribution in extrapolating to $Q^{2}>0$, and therefore predicts equal cross sections for neutrinos and antineutrinos.

The Rein-Sehgal model calculates the $\pi^{ \pm} \mathrm{A}$ elastic cross section using charged pion-nucleon $\left(\pi^{ \pm} N\right)$ scattering data. The differential $\pi^{ \pm}$A elastic scattering cross section is parameterized in the Rein-Sehgal model as

$$
\frac{d \sigma_{e l}^{\pi^{ \pm} \mathrm{A}}}{d|t|}=\left.A^{2}\left|F_{N}(|t|)\right|^{2} \frac{d \sigma_{e l}^{\pi^{ \pm} N}}{d|t|}\right|_{|t|=0}
$$

where $A$ is the number of nucleons in the nucleus, $\left|F_{N}(|t|)\right|^{2}$ is the nuclear form factor, and $\sigma_{e l}^{\pi^{ \pm}} N$ is the $\pi^{ \pm} N$ elastic scattering cross section. The differential $\pi^{ \pm} N$ elastic scattering cross section at $|t|=0$ (i.e. in the forward direction where the outgoing pion is parallel with the incoming pion) is calculated via the optical theorem as

$$
\left.\frac{d \sigma_{e l}^{\pi^{ \pm} N}}{d|t|}\right|_{|t|=0}=\frac{1}{16 \pi}\left[\sigma_{\text {tot }}^{\pi^{ \pm}} N\right]^{2}\left(1+r^{2}\right)
$$

where $\sigma_{\text {tot }}^{\pi^{ \pm}} N$ is the total (elastic + inelastic) $\pi^{ \pm} N$ scattering cross section determined from charged pion-deuterium scattering data and is a function of the pion energy, and $r=\operatorname{Re} f(0) / \operatorname{Im} f(0)$ is the ratio of the real and imaginary parts of the $\pi^{ \pm} N$ forward scattering amplitude. The nuclear form factor is parameterized as

$$
\left|F_{N}(|t|)\right|^{2}=\exp (-b|t|) \exp \left(-\frac{9 A}{16 \pi R^{2}} \sigma_{\text {inel }}^{\pi^{ \pm} N}\right)
$$

where the left exponential is the $|t|$-dependence of the $\pi^{ \pm} \mathrm{A}$ elastic scattering cross section and the right exponential is the average attenuation of the pion in the nucleus 
with radius $R$. The nuclear radius is estimated from Fermi gas models to be

$$
R=R_{0} A^{1 / 3}
$$

where $R_{0} \sim 1 \mathrm{fm}$ is the nuclear length scale. The exponential slope $b$ is calculated as

$$
b=\frac{1}{3} R^{2}=\frac{1}{3} R_{0}^{2} A^{2 / 3}
$$

The $\pi^{ \pm} N$ inelastic cross section $\sigma_{\text {inel }}^{\pi^{ \pm} N}$ in the absorption factor is determined from data and is a function of the pion energy.

The differential CC coherent pion production cross section calculated by the Rein-Sehgal model is

$$
\begin{aligned}
\frac{d \sigma_{\text {coh }}^{C C}}{d Q^{2} d y d|t|} & =\frac{G^{2} f_{\pi^{ \pm}}^{2}}{2 \pi} \frac{(1-y)}{y} \frac{M_{A}^{2}}{Q^{2}+M_{A}^{2}} A^{2} \exp \left(-\frac{1}{3} R_{0}^{2} A^{2 / 3}|t|\right) \\
& \times \exp \left(-\frac{9 A^{1 / 3}}{16 \pi R_{0}^{2}} \sigma_{\text {inel }}^{\pi^{ \pm} N}\left(E_{\pi}\right)\right) \frac{1}{16 \pi}\left(\sigma_{\text {tot }}^{\pi^{ \pm}}\left(E_{\pi}\right)\right)^{2}\left(1+r^{2}\right)
\end{aligned}
$$

where $E_{\pi}=y E_{\nu}$. The Rein-Sehgal model calculates the NC differential cross section from Equation 5.25 using $f_{\pi^{0}}=f_{\pi^{ \pm}} / \sqrt{2}$ and assuming the $\pi^{ \pm} N$ and $\pi^{0} N$ cross sections are equal.

The Rein-Sehgal model corrects the CC differential cross section (Equation 5.25) for the mass of the final state lepton [42]. The correction, proposed by Adler [51], is

$$
\begin{aligned}
C & =\left(1-\frac{1}{2} \frac{Q_{\min }^{2}}{Q^{2}+m_{\pi}^{2}}\right)^{2}+\frac{y}{4} \frac{Q_{\min }^{2}\left(Q^{2}-Q_{\min }^{2}\right)}{\left(Q^{2}+m_{\pi}^{2}\right)^{2}} \\
& \times \theta\left(Q^{2}-Q_{\min }^{2}\right) \theta\left(y-y_{\min }\right) \theta\left(y_{\max }-y\right),
\end{aligned}
$$

where $Q_{\min }^{2}=m_{l}^{2} y /(1-y)$ is the kinematic minimum $Q^{2}, y_{\min }=m_{\pi} / E_{\nu}$ and 
$y_{\max }=1-m_{l} / E_{\nu}$ are the kinematic minimum and maximum $y$, and $m_{l}$ and $m_{\pi}$ are the final state lepton and pion masses.

The Rein-Sehgal model predicts both the $\mathrm{CC}$ and $\mathrm{NC}$ cross sections $\sigma_{c o h}^{C C}\left(E_{\nu}\right)$ and $\sigma_{c o h}^{N C}\left(E_{\nu}\right)$ scale with $A$ as $A^{1 / 3}$, which comes from the nuclear coherence, pion absorption, and $|t|$-dependence.

\subsubsection{Berger-Sehgal Model}

The Berger-Sehgal PCAC coherent model [48] is a modification of the Rein-Sehgal model where the parameterization of the $\pi^{ \pm} \mathrm{A}$ elastic scattering cross section is instead fit to charged pion-carbon $\left(\pi^{ \pm} \mathrm{C}\right)$ elastic scattering data and scaled to other nuclei. This approach avoids the uncertainties from modeling nuclear effects (e.g. pion absorption) in the Rein-Sehgal parameterization. The Berger-Sehgal model parameterizes the differential $\pi^{ \pm} \mathrm{C}$ elastic cross section as

$$
\frac{d \sigma_{e l}^{\pi^{ \pm} \mathrm{C}}}{d|t|}=A_{1} e^{-b_{1}|t|}
$$

where the normalization $A_{1}$ and slope $b_{1}$ are functions of $E_{\pi}$ and are fit to data (Figure 5.2). The comparison of Rein-Sehgal and Berger-Sehgal calculations of $\sigma_{e l}^{\pi^{ \pm} \mathrm{C}}$ in Figure 5.2 shows that, while the two calculations agree for $\left|\vec{p}_{\pi}\right| \gtrsim 0.7 \mathrm{GeV}$, the Rein-Sehgal predicts a much larger cross section in the $\Delta$ resonance region $\left(\left|\vec{p}_{\pi}\right| \sim 0.3 \mathrm{GeV}\right)$. 


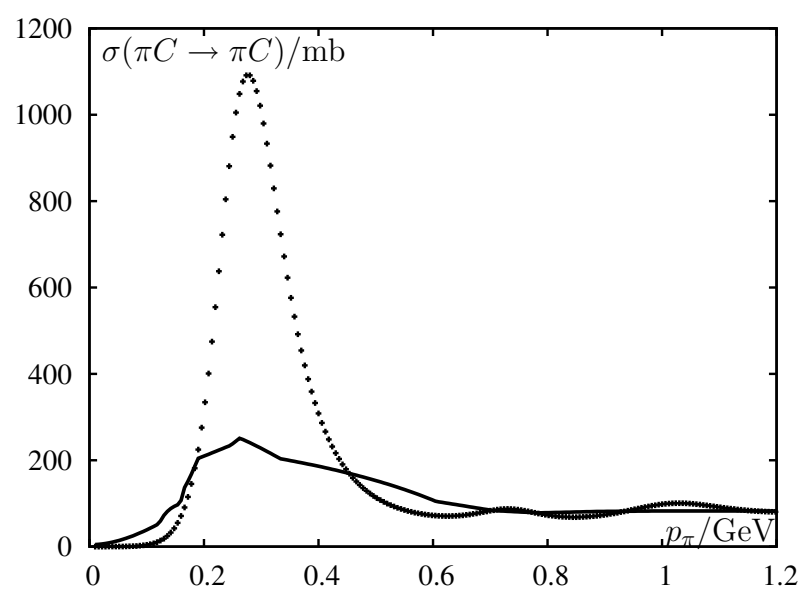

\begin{tabular}{r|r|r}
$\begin{array}{r}T_{\pi} \\
\mathrm{GeV}\end{array}$ & $\begin{array}{r}A_{1} \\
\mathrm{mb} / \mathrm{GeV}^{2}\end{array}$ & $\begin{array}{r}b_{1} \\
1 / \mathrm{GeV}^{2}\end{array}$ \\
\hline 0.076 & 11600 & 116.0 \\
0.080 & 14700 & 109.0 \\
0.100 & 18300 & 89.8 \\
0.148 & 21300 & 91.0 \\
0.162 & 22400 & 89.2 \\
0.226 & 16400 & 80.8 \\
0.486 & 5730 & 54.6 \\
0.584 & 4610 & 55.2 \\
0.662 & 4570 & 58.4 \\
0.776 & 4930 & 60.5 \\
0.870 & 5140 & 62.2 \\
\hline
\end{tabular}

Figure 5.2: The plot on the left is the Rein-Sehgal (dashed line) and Berger-Sehgal (solid line) predictions of the pion-carbon elastic scattering cross section as a function of the pion momentum in the laboratory frame. The table on the right are the parameters for the Berger-Sehgal pion-carbon elastic scattering cross section determined from fitting pion-carbon-elastic scattering data. The plot and table are from [48]

\subsection{Microscopic Coherent Models}

Microscopic coherent models $[52,53,54,55,56]$ describe neutrino-nucleus coherent pion production in terms of neutrino-nucleon scattering processes, summed coherently over all nucleons in the nucleus, that give the final state

$$
\nu_{l}+N \rightarrow l+N+\pi
$$

where the nucleon $N$ is remains in its initial quantum state. In current microscopic model calculations, the dominant neutrino-nucleon scattering process giving the required final state is $\Delta$ resonance production. Microscopic model calculations include modifications of the $\Delta$ resonance properties and distortion of the pion wave function in the nuclear medium. The validity of microscopic model calculations is restricted 
to $E_{\nu} \lesssim 2 \mathrm{GeV}$, since the contribution from neutrino-nucleon scattering processes with invariant mass $W$ (Chapter 4$)$ larger than that of the $\Delta$ resonance $(W \approx 1.2$ $\mathrm{GeV}$ ) become important for $E_{\nu}>2 \mathrm{GeV}$. This thesis presents measurements of $\nu_{\mu}$ and $\bar{\nu}_{\mu} \mathrm{CC}$ coherent pion production on carbon for $E_{\nu}>2 \mathrm{GeV}$. Therefore, microscopic models cannot be tested by the measurements presented in this thesis and will not be discussed further.

\subsection{Measurements of Neutrino-Nucleus Coherent Pion Production}

To measure coherent pion production, experiments must isolate coherent interactions from non-coherent interactions. Ideally, this is done without making assumptions of the physics of coherent pion production and using only its defining characteristics, which are the production of a lepton and pion in the forward direction and the nucleus remaining in its initial state. Per these defining characteristics, the final state of a coherent interaction is distinguished by the absence of particles in addition to a lepton and pion and small $|t|\left(\lesssim 1 / R^{2}\right)$. The presence of additional particles is indicative of nuclear breakup in non-coherent interactions, and $|t|$ must be small for the nucleus to remain intact.

$\mathrm{CC}$ coherent interactions can be isolated by requiring that the observed final state consist only of a charged lepton and pion (the target nucleus is not observed since the energy transferred to the nucleus is small) and small $|t|$. For CC coherent interactions, $|t|$ can be calculated from the measured four-momenta of the charged lepton and pion. From conservation of four-momentum and the assumption that 
the target nucleus is at rest,

$$
\begin{aligned}
|t| & =\left|\left(p_{\nu_{l}}-p_{l}-p_{\pi}\right)^{2}\right|, \\
& \approx\left(\sum_{i=\mu, \pi} E_{i}-p_{i, L}\right)^{2}+\left|\sum_{i=\mu, \pi} \vec{p}_{i, T}\right|^{2},
\end{aligned}
$$

where $p_{\nu_{l}}, p_{l}$, and $p_{\pi}$ are the four-momenta of the neutrino, charged lepton, and pion, respectively, and $\vec{p}_{T}$ and $p_{L}$ are the transverse and longitudinal momenta with respect to the incoming neutrino direction. The approximation made in Equation 5.29 is that zero energy is transferred to the nucleus [74]. Measuring the energy and direction of the charged lepton and pion gives $p_{l}$ and $p_{\pi}$. The neutrino four-momentum can be determined by assuming the neutrino direction is parallel to the direction of the neutrino beam and calculating the neutrino energy from the measured charged lepton and pion energies as

$$
E_{\nu}=E_{l}+E_{\pi}
$$

where the energy transferred to the nucleus is ignored.

For NC coherent interactions the final state lepton (a neutrino) is not observed. Therefore, the observed final state of a NC coherent interaction is a lone $\pi^{0}$. Neither $E_{\nu}$ nor $|t|$ can be measured for NC coherent interactions since only the energy and direction of the pion can be measured. Since $|t|$ is not available, experiments isolate NC coherent interactions using the condition

$$
E_{\pi}\left(1-\cos \theta_{\pi}\right)<\frac{1}{R}
$$

where $\theta_{\pi}$ is the angle between the pion and incoming neutrino directions. This condition is deduced from the $|t| \lesssim 1 / R^{2}$ condition for coherent scattering [38]. However, 
imposing restrictions on the pion kinematics of coherent scattering also imposes restrictions on the lepton kinematics (see Equation 5.29) and $Q^{2}$. Therefore, NC coherent pion production measurements implicitly make assumptions about the $Q^{2}$ dependence of the coherent scattering cross section in correcting for the efficiency in selecting coherent interactions. The $Q^{2}$ dependence of the coherent scattering cross section is model dependent, particularly for PCAC coherent models which must extrapolate the cross section at $Q^{2}=0$ from the Adler theorem to $Q^{2}>0$. Therefore, NC coherent coherent pion production measurements are model dependent. In addition, since $E_{\nu}$ cannot be measured, $\mathrm{NC}$ coherent pion production measurements are averaged over the energy spectrum of a neutrino beam which can span a wide range of $E_{\nu}$. These experimental limitations of NC coherent pion production measurements increase the importance of CC coherent pion production measurements, which in the PCAC picture provide a constraint on the NC reaction.

Several measurements of NC $[57,58,59,60,61,62,63,64]$ and CC $[60,65,66,67$, $68,69,70,71,72,73,74]$ coherent pion production have been made to date. These measurements were made at neutrino energies in the range $1 \lesssim E_{\nu} \lesssim 100 \mathrm{GeV}$ using $\nu_{\mu}$ and $\bar{\nu}_{\mu}$ accelerator neutrino beams and a variety of scattering target materials (carbon, neon, aluminum, argon, Freon, glass, and marble). Prior to the discovery of neutrino oscillations, the motivation for measuring coherent pion production was to study the weak axial vector current. Most "early" measurements of coherent pion production (i.e. those made before the discovery of neutrino oscillations) were made at $E_{\nu}>10 \mathrm{GeV}$, which is larger than the neutrino energies important to oscillation experiments. The discovery of neutrino oscillations renewed interest in coherent pion production. Precise measurements of coherent pion production at $1 \lesssim E_{\nu} \lesssim 10 \mathrm{GeV}$ are needed for constraining background in current and future 
oscillation measurements. "Modern" measurements of coherent pion production use high intensity $\nu_{\mu}$ and $\bar{\nu}_{\mu}$ accelerator neutrino beams that operate at $1 \lesssim E_{\nu} \lesssim 10$ $\mathrm{GeV}$.

Early measurements of the NC coherent pion production cross section as a function of $E_{\nu}[57,58,59]$ are shown in Figure 5.3. The measurements were made using different scattering target materials and, for the purpose of comparison, are scaled in Figure 5.3 to a marble scattering target with an effective $A=20$ using the $A^{1 / 3}$ dependence of the cross section predicted by the Rein-Sehgal model [59]. While the Rein-Sehgal model prediction agrees with the measured cross sections within their uncertainties, the uncertainties are large $(\gtrsim 30 \%$ [62]) and most measurements span a wide range of neutrino energies.

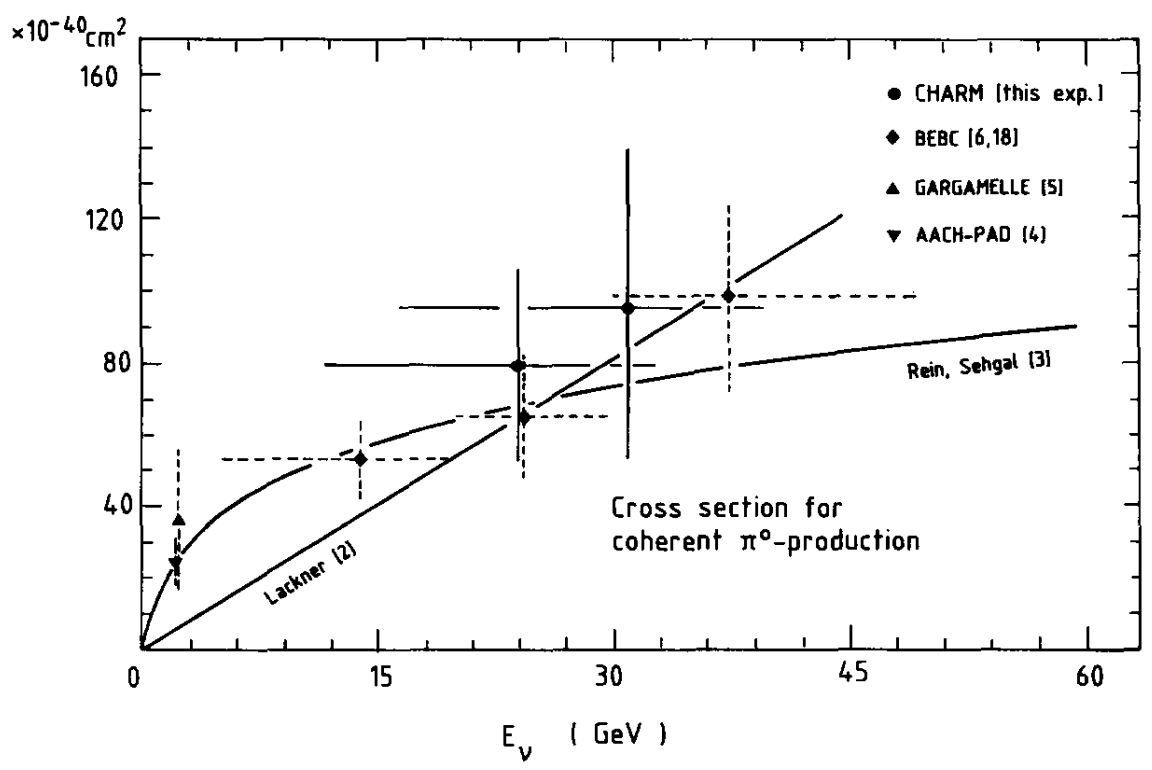

Figure 5.3: Early measurements of the NC coherent pion production cross section. The horizontal error bars represent the range of neutrino energies sampled by the measurement. The figure is from [59].

The early measurements of the $\nu_{\mu}$ and $\bar{\nu}_{\mu} \mathrm{CC}$ coherent pion production cross 
section as a function of $E_{\nu}[60,65,66,67,68,69,70]$, along with the early measurements of the $\mathrm{NC}$ current coherent pion production cross section [57, 58, 59,60], are shown in Figure 5.4. For comparison, the measurements in Figure 5.4 are scaled to a glass scattering target with an effective effective $A=20.1$, and the NC measurements are additionally scaled by a factor of 2 per the relation between the $\mathrm{CC}$ and NC coherent cross sections (Equation 5.17) from Adler's PCAC theorem. The early $\mathrm{CC}$ measurements isolated $\mathrm{CC}$ coherent interactions by requiring a forward $\mu^{\mp}$ and $\pi^{ \pm}$, the absence of additional particles emerging from the interaction vertex, and small $|t|$. The Rein-Sehgal model agrees well with most of the measurements, which supports the predicted $A^{1 / 3}$ dependence of the coherent cross section and the relation between the $\mathrm{CC}$ and $\mathrm{NC}$ coherent cross sections.

The first, and currently only, measurements of $\mathrm{NC}$ coherent pion production at $E_{\nu}<2 \mathrm{GeV}$ were made recently by the MiniBooNE [64] and SciBooNE [63] experiments which use the same $\nu_{\mu} / \bar{\nu}_{\mu}$ beam. The MiniBooNE measurement was made using a mineral oil target $\left(\mathrm{CH}_{2}\right)$ at a peak neutrino energy of $0.7 \mathrm{GeV}$. MiniBooNE measured the NC coherent pion production cross section to be $(19.5 \pm 2.7) \%$ of the total (coherent + non-coherent) NC single $\pi^{0}$ production cross section. The SciBooNE measurement was made using a polystyrene target $\left(\mathrm{C}_{8} \mathrm{H}_{8}\right)$ at an average neutrino energy of $0.8 \mathrm{GeV}$. SciBooNE measured the ratio of the $\mathrm{NC}$ coherent pion production cross section to the $\nu_{\mu} \mathrm{CC}$ total (all scattering processes) cross section to be $(1.16 \pm 0.24) \times 10^{-2}$. These measurements provide strong evidence for NC coherent pion production at $E_{\nu}<2 \mathrm{GeV}$.

The first searches for $\nu_{\mu} \mathrm{CC}$ coherent pion production at $E_{\nu} \lesssim 2 \mathrm{GeV}$ were also performed recently by the K2K [71] and SciBooNE [72] experiments. These experiments used the same detector with a $\mathrm{C}_{8} \mathrm{H}_{8}$ scattering target in two different 

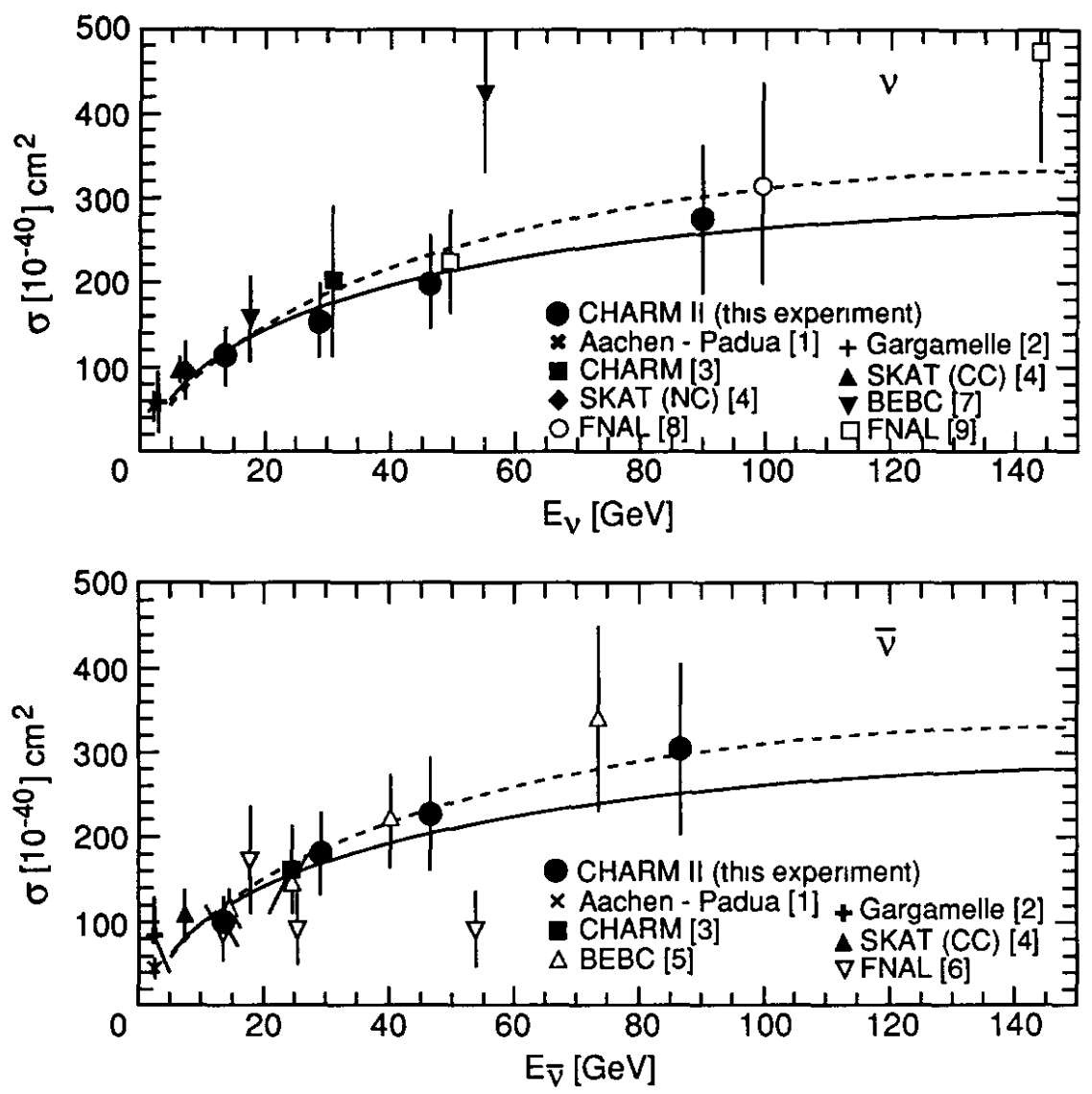

Figure 5.4: Early measurements of the $\nu_{\mu}$ (top) and $\bar{\nu}_{\mu}$ (bottom) CC and NC coherent pion production cross sections. The solid line is the Rein-Sehgal model prediction. The figure is from [70].

neutrino beams. Both searches isolated $\mathrm{CC}$ coherent interactions by requiring a forward muon and pion and the absence of additional particles emerging from the interaction vertex. However, neither experiment was able to measure $|t|$ since the detector did not provide containment of the pion for measuring the pion energy. Both experiments instead searched for $\mathrm{CC}$ coherent interactions in $Q^{2}$, which was calculated per Equation 5.10 from the measured muon energy, the measured muon angle, and the neutrino energy calculated under a quasi-elastic scattering hypothesis (Equation 5.7). Neither experiment found evidence for CC coherent interactions 
after subtracting the predicted non-coherent background (Figures 5.5 and 5.6). The experiments reported upper limits on the ratio of the $\nu_{\mu} \mathrm{CC}$ coherent pion production cross section to the $\nu_{\mu}$ CC total cross section, which are listed in Table 5.1. The non-observation of $\nu_{\mu} \mathrm{CC}$ coherent pion production at $E_{\nu}<2 \mathrm{GeV}$ is in contradiction with the Rein-Sehgal coherent model. It should be noted that a search for coherent pion production in $Q^{2}$ is model dependent. In addition, both the $\mathrm{K} 2 \mathrm{~K}$ and SciBooNE measurements constrained their background prediction using interactions with activity near the interaction vertex in addition to that from the muon and pion. The background prediction is therefore sensitive to the modeling of nuclear effects which are poorly understood (see Section 8.8).

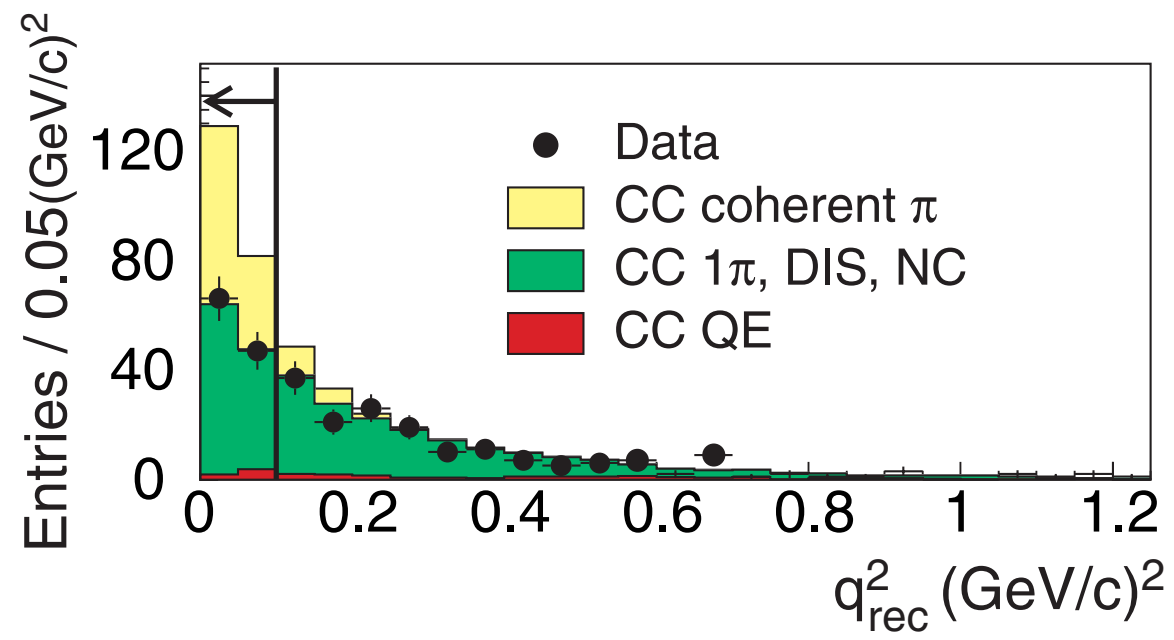

Figure 5.5: The data and simulated $Q^{2}$ distributions for $\nu_{\mu} \mathrm{CC}$ coherent pion production candidates at K2K. The figure is from [71].

The non-observation of $\mathrm{CC}$ coherent pion production at $E_{\nu} \lesssim 2 \mathrm{GeV}$ posed a problem for both theorists and neutrino oscillation experiments. Coherent pion production models are unable to reconcile the observation of the NC reaction with the non-observation of the $\mathrm{CC}$ reaction at $E_{\nu} \lesssim 2 \mathrm{GeV}$. To account for the theoretical 


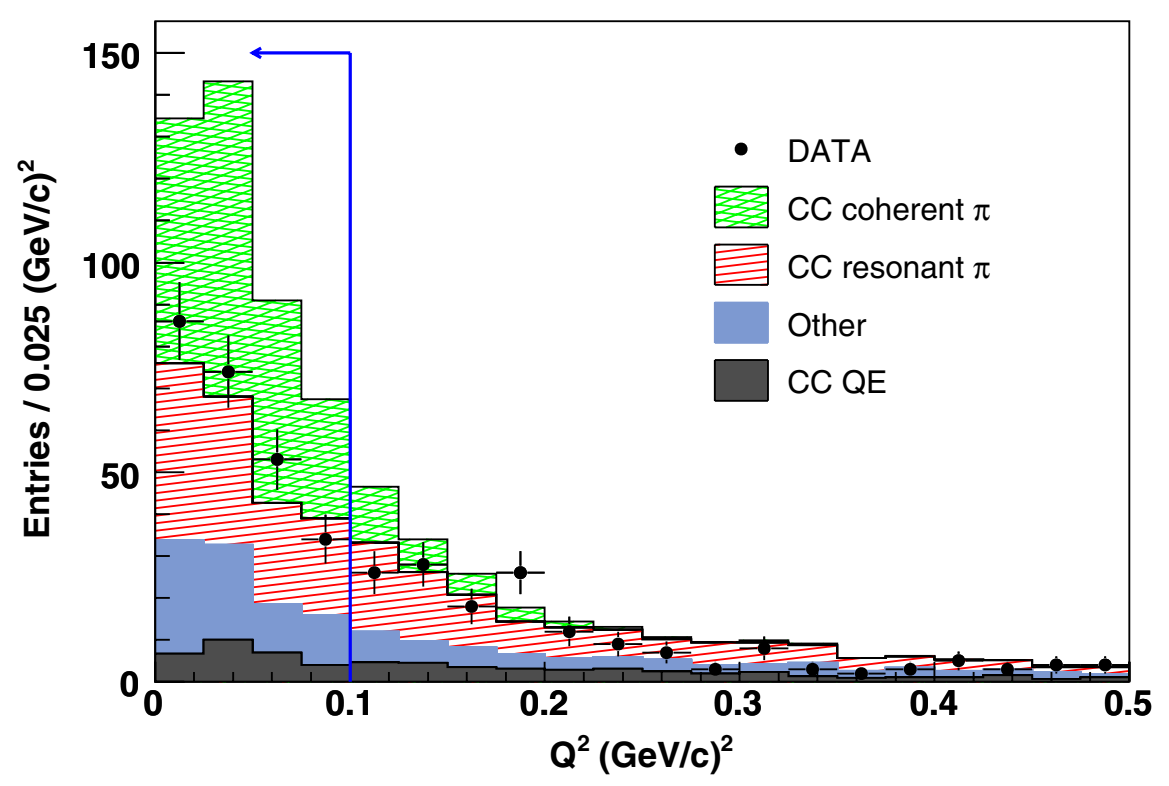

Figure 5.6: The data and simulated $Q^{2}$ distributions for $\nu_{\mu} \mathrm{CC}$ coherent pion production candidates at SciBooNE. The figure is from [72].

\begin{tabular}{c|c|c} 
Experiment & $\sigma_{C C}^{\text {coh }} / \sigma_{C C}^{\text {tot }}(90 \%$ C.L. $)$ & $\left\langle E_{\nu}\right\rangle(\mathrm{GeV})$ \\
\hline K2K & $<0.6 \times 10^{-2}$ & 1.3 \\
SciBooNE & $<0.67 \times 10^{-2}$ & 1.1 \\
SciBooNE & $<1.36 \times 10^{-2}$ & 2.2
\end{tabular}

Table 5.1: The upper limits on the ratio of the $\nu_{\mu} \mathrm{CC}$ coherent pion production cross section to the $\nu_{\mu} \mathrm{CC}$ total cross section, $\sigma_{C C}^{c o h} / \sigma_{C C}^{\text {tot }}$, reported by K2K [71] and SciBooNE [72].

and experimental disagreement, the $\mathrm{T} 2 \mathrm{~K}$ neutrino oscillation experiment, which operates at a peak neutrino energy of $\approx 0.6 \mathrm{GeV}$, applied a $100 \%$ uncertainty on their predicted CC coherent interaction rate, while applying a 30\% uncertainty on their predicted $\mathrm{NC}$ coherent interaction rate [21]. Neutrino-nucleus interactions are the largest source of systematic uncertainty in the T2K $\nu_{\mu} \rightarrow \nu_{e}$ oscillation and $\nu_{\mu}$ disappearance measurements (Figure 5.7), and the predicted rate of coherent interactions is a significant contribution to the uncertainty [21]. 


\begin{tabular}{lcc}
\hline \hline Source of uncertainty & $\nu_{\mu} \mathrm{CC}$ & $\nu_{e} \mathrm{CC}$ \\
\hline Flux and common cross sections & & \\
(w/o ND280 constraint) & $21.7 \%$ & $26.0 \%$ \\
(w ND280 constraint) & $2.7 \%$ & $3.2 \%$ \\
\hline Independent cross sections & $5.0 \%$ & $4.7 \%$ \\
\hline SK & $4.0 \%$ & $2.7 \%$ \\
FSI + SI $(+\mathrm{PN})$ & $3.0 \%$ & $2.5 \%$ \\
\hline Total & & \\
\hline (w/o ND280 constraint) & $23.5 \%$ & $26.8 \%$ \\
(w ND280 constraint) & $7.7 \%$ & $6.8 \%$ \\
\hline \hline
\end{tabular}

Figure 5.7: The systematic uncertainties on the predictions of $\nu_{\mu} \mathrm{CC}$ and $\nu_{e} \mathrm{CC}$ interactions in the Super Kamiokande (SK) far detector of the T2K neutrino oscillation experiment. Constraints on the neutrino beam flux and neutrino interaction predictions are obtained from the ND280 near detector. The table is from [21].

Precise measurements of neutrino-nucleus coherent pion production at $1 \lesssim E_{\nu} \lesssim$ $10 \mathrm{GeV}$ are needed for testing coherent pion production models and reducing systematic uncertainty on neutrino oscillation measurements. This thesis presents a precise measurement of the $\nu_{\mu}$ and $\bar{\nu}_{\mu}$ CC coherent pion production cross sections on carbon for $2<E_{\nu}<20 \mathrm{GeV}$. In addition, the cross sections are measured as a function of the pion energy, pion angle, and $Q^{2}$, which characterize the coherent pion production kinematics. 


\section{Chapter 6}

\section{The NuMI Neutrino Beam}

The Neutrinos at the Main Injector (NuMI) neutrino beam is a high-intensity neutrino beam located at Fermi National Accelerator Laboratory (Fermilab). The NuMI neutrino beam is utilized by neutrino oscillation and neutrino-nucleus scattering experiments. The NuMI neutrino beam is generated from a beam of $120 \mathrm{GeV}$ protons. The energy spectrum of the NuMI neutrino beam peaks at few GeV. This section describes the process of generating the proton and neutrino beams and the determination of the neutrino beam flux.

\subsection{Proton Acceleration}

The Fermilab accelerator complex generates the proton beam that is used to generate the NuMI neutrino beam. The complex consists of an $\mathrm{H}^{-}$ion source, a Linac, and the Booster and Main Injector synchrotrons (Figure 6.1).

The proton beam originates as $\mathrm{H}^{-}$ions from a magneton surface plasma source. $\mathrm{H}_{2}$ gas is injected into the source, which emits a $15 \mathrm{~Hz}$ pulsed beam of $\mathrm{H}^{-}$ions. The 


\section{Fermilab Accelerator Complex}

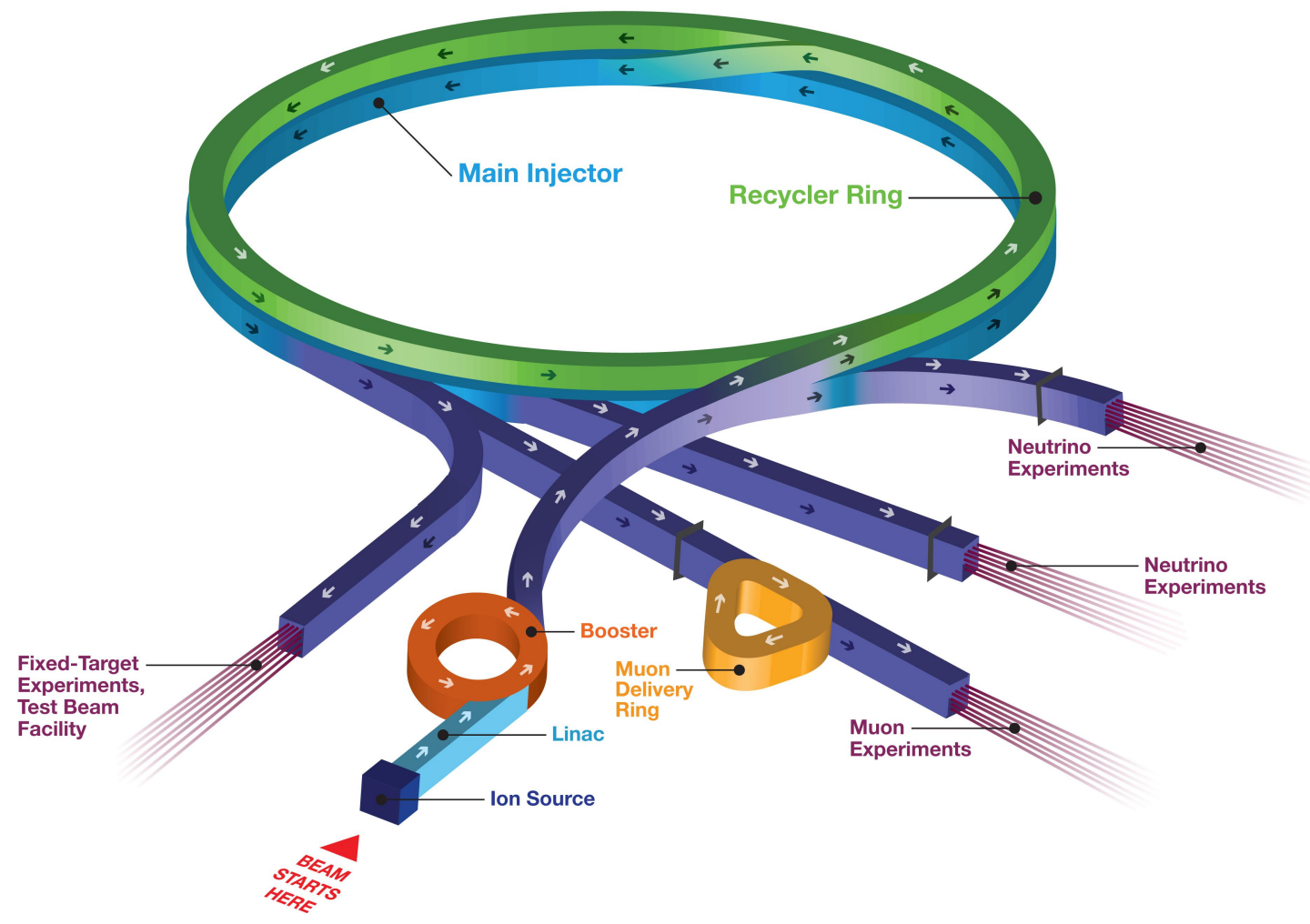

Figure 6.1: Fermilab accelerator complex [76]

ion beam is accelerated to $750 \mathrm{keV}$ by a Cockroft-Walton accelerator. A single gap RF cavity then divides each beam pulse into $\sim 200 \mathrm{MHz}$ bunches for injection into the Linac [77].

The Linac is a two-stage linear accelerator. The first stage is a series of Alvarez drift-tubes that accelerate the ion beam to $116 \mathrm{MeV}$. The second stage is a sidecoupled linac that accelerates the beam to $400 \mathrm{MeV}$. The accelerated beam has a bunch structure with a frequency equal to the $\sim 200 \mathrm{MHz}$ RF frequency of the Linac. An RF cavity at the end of the Linac debunches the beam for injection into the Booster [77]. 
The Booster is a $150 \mathrm{~m}$ diameter synchrotron accelerator containing $84 \mathrm{RF}$ magnets. Upon injection into the Booster, the $\mathrm{H}^{-}$ion beam from the Linac passes through a carbon foil that strips the electrons from the ions. Dipole magnets steer the resulting proton beam into the Booster orbit. After the Booster accumulates $3 \times 10^{12}$ protons, the proton beam is accelerated through multiple orbits to $8 \mathrm{GeV}$. The accelerated protons, referred as a batch, are extracted from the Booster by quadrupole kicker magnets for injection into the Main Injector. The Booster cycles at $15 \mathrm{~Hz}$, where each cycle corresponds to a single proton batch [77].

The final stage of the proton beam acceleration is performed by the Main Injector, which is a $3.3 \mathrm{~km}$ circumference synchrotron accelerator. Each Main injector cycle accelerates multiple proton batches from the Booster to $120 \mathrm{GeV}$. The accelerated protons are extracted from the Main Injector in a single turn (i.e. orbit) by quadrupole kicker magnets. Each extraction, referred to as a spill, contains $35 \times 10^{12}$ protons at full intensity. The spills are extracted at a rate of $0.45 \mathrm{~Hz}$, and each spill is $10 \mu \mathrm{s}$ long. The extracted spills form a pulsed proton beam which is directed to the NuMI beamline [75].

\subsection{NuMI Beamline}

The NuMI beamline consists of a graphite target, magnetic focusing horns, and a decay pipe (Figure 6.2). Protons from the Main Injector interact with carbon nuclei in the target to produce charged pions and kaons. The horns focus the pions and kaons into the decay pipe where they decay to the neutrinos that compose the NuMI neutrino beam.

The proton beam from the Main Injector is directed to the NuMI target, which is 


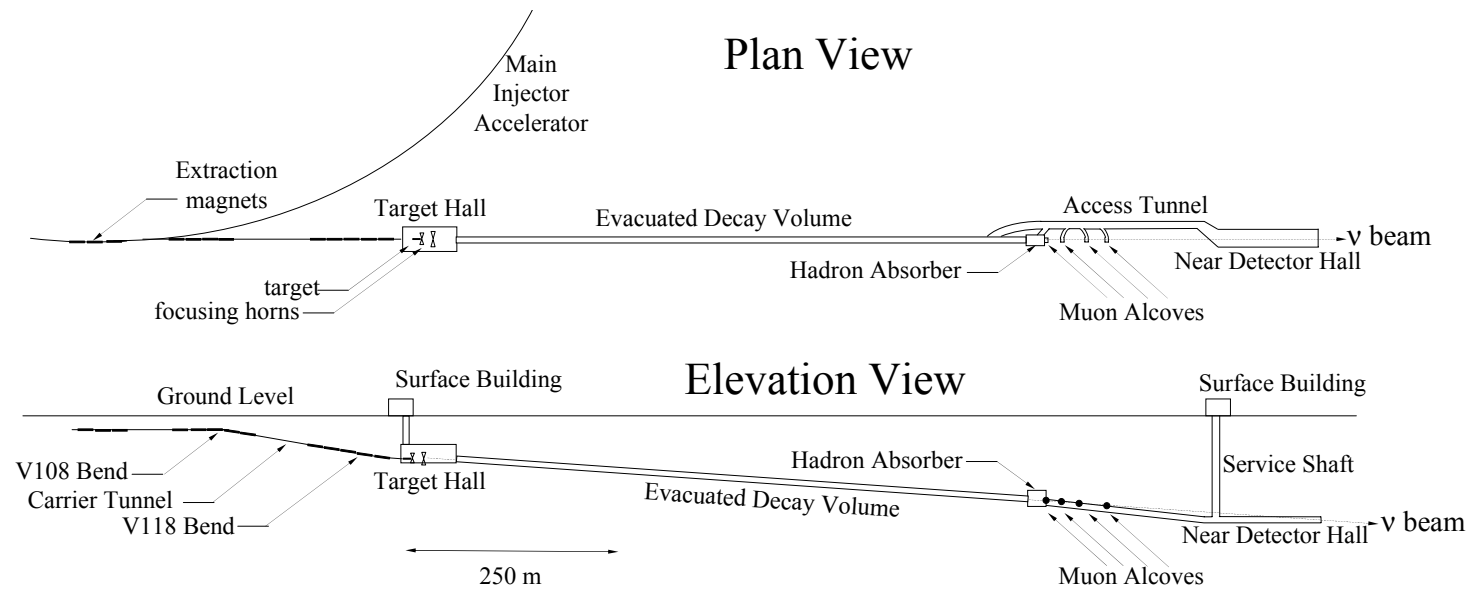

Figure 6.2: NuMI beamline [75]

located $41 \mathrm{~m}$ underground in the NuMI target hall (Figure 6.2). The proton beam and resulting neutrino beam are angled 58 mrad downward to serve the MINOS neutrino oscillation experiment. Before reaching the NuMI target, the proton beam passes through a graphite baffle which collimates the beam and protects the horn inner conductors from beam mis-alignment [79][80].

The NuMI target (Figure 6.3) is composed of 47 graphite fins. Looking along the direction of the proton beam, each fin is $20 \mathrm{~mm}$ long and has a $6.4 \mathrm{~mm} \times 15 \mathrm{~mm}$ rectangular cross section. The proton beam is $1 \mathrm{~mm}$ in diameter at the target. The fins are arranged in series longitudinally with $0.3 \mathrm{~mm}$ spacing between fins, giving a total target length of $95 \mathrm{~cm}$ ( $\sim 2$ interaction lengths). The target is cooled by water pipes running along the fin edges.

The interactions of protons in the beam with carbon nuclei in the target produce hadrons including charged pions $\left(\pi^{ \pm}\right)$, charged kaons $\left(K^{ \pm}\right)$, neutrons, and lowenergy protons. These secondary particles can also interact in the target or other beamline components to produce tertiary particles. The particles emerging from the target, which are primarily $\pi^{ \pm}$, are focused down the decay pipe by two magnetic 


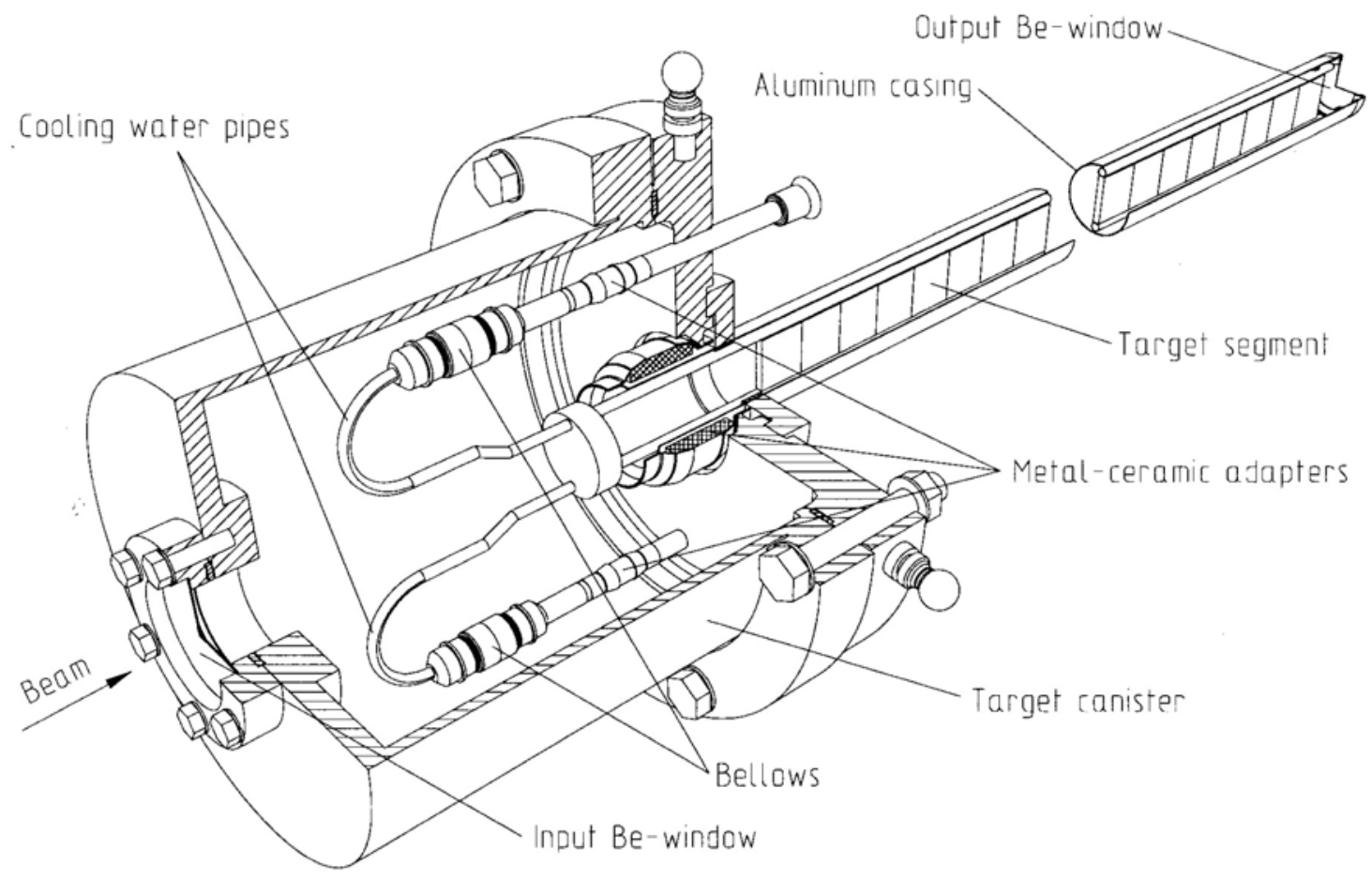

Figure 6.3: NuMI target [78]

horns (Figure 6.4). Each horn is a thin, toroidal, $2 \mathrm{~m}$ long aluminum conductor with a parabolic inner cavity. The magnetic fields are generated by a pulsed current through the horns. The first horn is located immediately downstream of the target, and the second horn is located between the first horn and the decay pipe. The relative positions of the target and horns and the magnitude of the horn current determine the energy spectra of the focused particles and resulting neutrino beam.

The direction of the current in the horns determines the charges of the particles that are focused and defocused, which in turn determines whether the beam is composed primarily of neutrinos or antineutrinos. Forward horn current (FHC) focuses (defocuses) particles with positive (negative) charge, while reverse horn current (RHC) focuses (defocuses) particles with negative (positive) charge. Most neutrinos 


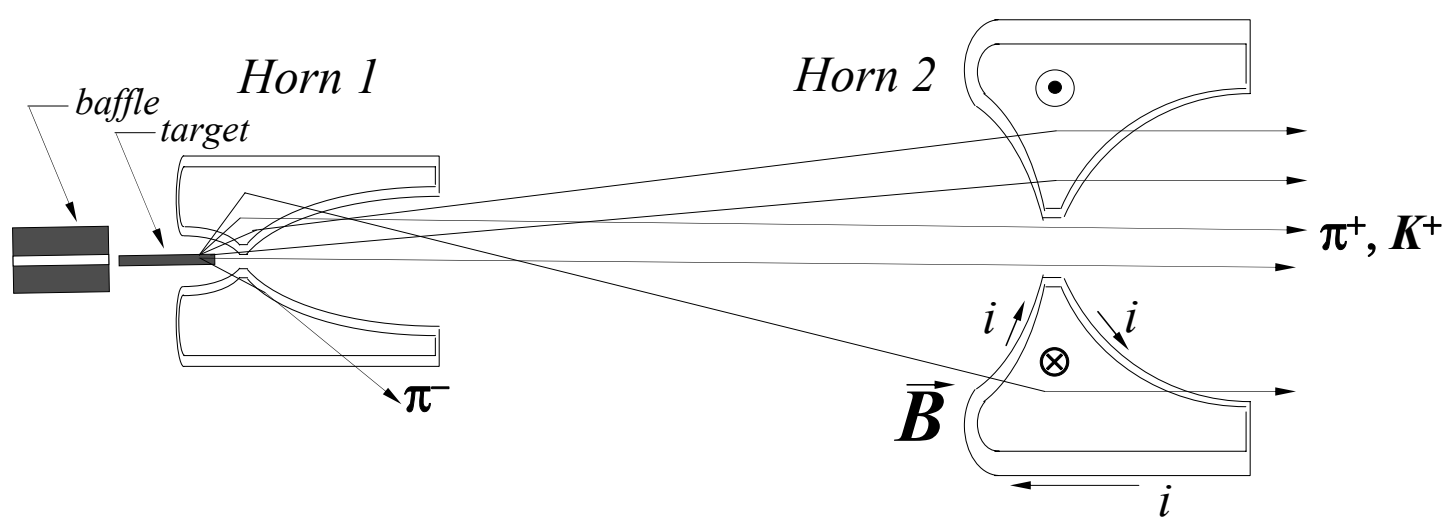

Figure 6.4: NuMI horns [79]

in the NuMI beamline come from $\pi^{+} \rightarrow \mu^{+}+\nu_{\mu}$ and $\pi^{-} \rightarrow \mu^{-}+\bar{\nu}_{\mu}$ decays. The neutrino beam generated with FHC (RHC) is therefore composed primarily of $\nu_{\mu}$ $\left(\bar{\nu}_{\mu}\right)$.

The decay pipe provides a long path along which the focused particles can decay to neutrinos. The decay pipe is iron, $675 \mathrm{~m}$ long, $2 \mathrm{~m}$ in diameter, and filled with helium to minimize hadronic interactions which occur more frequently in air. The beam emerging from the end of the decay pipe consists of neutrinos and undecayed hadrons and muons. These undecayed particles are absorbed by $240 \mathrm{~m}$ of dolomite rock between the end of the decay pipe and the Near Detector Hall. The neutrinos in the beam pass through the rock and enter the Near Detector Hall where the MINER $\nu \mathrm{A}$ detector is situated.

This thesis presents MINER $\nu \mathrm{A}$ data taken while the NuMI beamline was running in its low energy (LE) configuration. In this configuration, "the downstream end of the $95 \mathrm{~cm}$ long target was inserted $57 \mathrm{~cm}$ past the front face of the first horn, and both horns (separated by $10 \mathrm{~m}$ ) were pulsed at $185 \mathrm{kA} "$ [80]. The flux spectra for the FHC and RHC fluxes in the LE configuration both peak at $3.5 \mathrm{GeV}$. The 
predicted neutrino species composition of the LE FHC and RHC fluxes is listed in Table 6.1.

\begin{tabular}{c|c|c} 
Species & FHC (\%) & RHC (\%) \\
\hline$\nu_{\mu}$ & 88.4 & 17.8 \\
$\bar{\nu}_{\mu}$ & 10.1 & 80.8 \\
$\nu_{e}+\bar{\nu}_{e}$ & 1.5 & 1.4
\end{tabular}

Table 6.1: Neutrino species composition of the LE FHC and RHC fluxes [81]

\subsection{Neutrino Flux}

In a fixed target experiment such as MINER $\nu \mathrm{A}$, an interaction cross section $\sigma$ is measured as

$$
\sigma=\frac{N}{T \phi}
$$

where $N$ is the observed number of interactions, $T$ is the number of scattering targets, and $\phi$ is the incident particle flux. The neutrino-nucleus interaction cross sections measured by MINER $\nu \mathrm{A}$ are normalized to the neutrino flux in the NuMI beam. The precision of MINER $\nu$ A's cross section measurements are therefore dependent upon the precision to which the neutrino flux is known.

It is difficult to measure the flux of the neutrino beam directly since neutrinos rarely interact. Instead, a prediction of the neutrino flux is made from a detailed simulation of the NuMI beamline. The flux prediction and its uncertainty are constrained by tuning the production of hadrons leading to neutrinos in the simulation to external hadron production data. The flux prediction is further constrained by an in situ measurement of neutrino-electron elastic scattering in MINER $\nu \mathrm{A}$. 


\subsubsection{Flux Simulation}

The NuMI beamline consists of many components with complex geometries made from a variety of materials. An analytical calculation of the hadron production in the beamline and the resulting neutrino flux that accounts for all these details is not feasible. These details can be accommodated in a Monte Carlo simulation of the flux.

The flux simulation [80] consists of models of the geometry and materials of the beamline components, magnetic fields in the focusing horns, and particle propagation and interactions in the target and other beamline components. Particle propagation and interactions are simulated using Geant4 [82, 83], where hadronic interactions are simulated by the FTFP hadron-nucleon interaction and BERT intranuclear cascade models. Figure 6.5 shows the average number of hadronic interactions per $\nu_{\mu}$ traversing the MINER $\nu \mathrm{A}$ detector as a function of neutrino energy in the LE FHC beam simulation.

\subsubsection{Constraints from Hadron Production Data}

In the hadron interactions in the NuMI beamline, an incident hadron interacts with a nucleon (proton or neutron) within a nucleus. For the hadron energies in NuMI, these interactions are described by non-perterbative QCD. In addition, the effects of the nuclear environment in these interactions are not completely understood. The hadron interaction model in the flux simulation can be inaccurate as a result. This situation is handled by tuning hadron interactions in the simulation that result in neutrinos in MINER $\nu \mathrm{A}$ to hadron production data [80].

The tuning uses data of $\pi^{ \pm}, K^{ \pm}$, and nucleon production rates in proton-carbon 


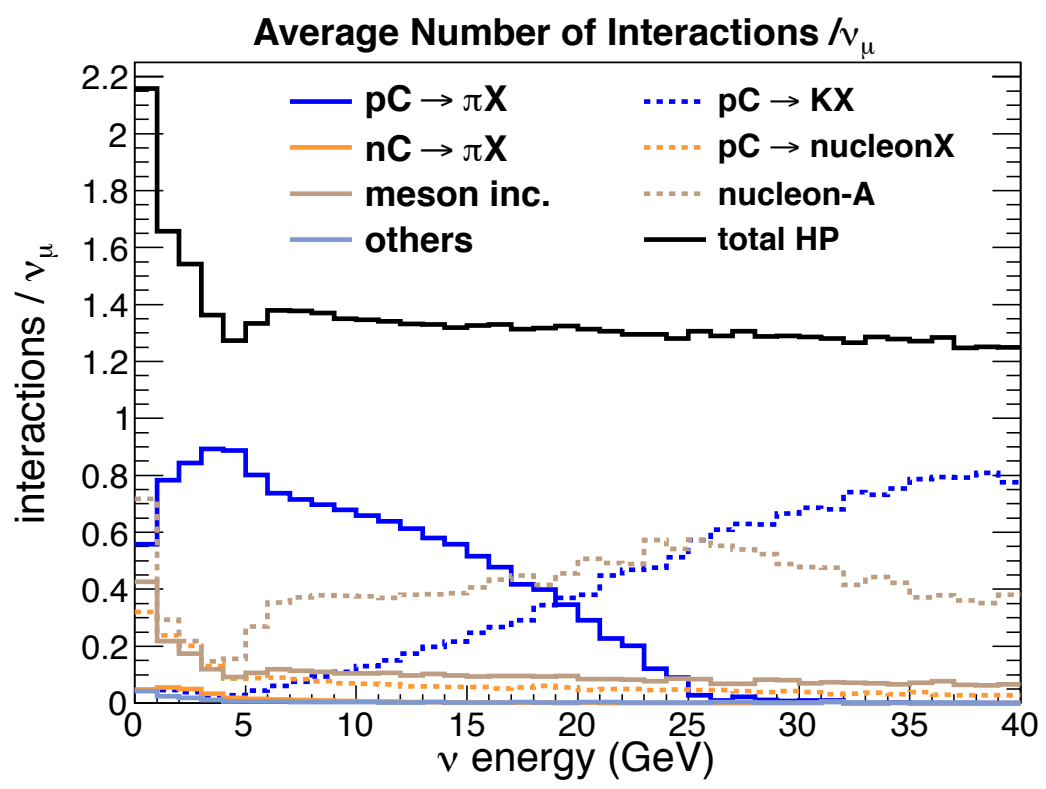

Figure 6.5: The average number of hadronic interactions per $\nu_{\mu}$ traversing the MINER $\nu$ A detector as a function of neutrino energy in the LE FHC beam simulation. The colored lines show the categories of hadronic interactions which are constrained by external data. "meson inc." refers to meson interactions in all beamline materials and "nucleon-A" refers to interactions in non-carbon materials. The figure is from reference [80].

$(p C)$ interactions measured using a thin carbon target (few percent interaction length). The data give the production rate for a fixed incident proton momentum $p_{0}$ as a function of the transverse momentum $p_{T}$ and Feynman scaling factor $x_{F}$ for the produced particle $\left(e . g . \pi^{+}\right)$. The Feynman scaling factor [84] is defined as

$$
x_{F}=\frac{2 p_{L}}{\sqrt{s}},
$$

where $p_{L}$ is the longitudinal momentum of the produced particle and $s$ is the squared interaction center of momentum. The data were measured at different $p_{0}$ and span different regions of $x_{F}$ and $p_{T}$.

The simulation predicts $85 \%$ of the $\nu_{\mu}$ flux in MINER $\nu$ A results from $p C$ in- 
teractions. The majority of hadron interactions that result in beam neutrinos were tuned using data of $\pi^{ \pm}$production in $p C$ interactions from NA49 [85] and Barton et al. [86]. The NA49 data was measured at $158 \mathrm{GeV} / \mathrm{c}$ incident proton momentum and is used to tune interactions with $x_{F}<0.5$. The Barton data was measured at $100 \mathrm{GeV} / \mathrm{c}$ incident proton momentum and is used to tune interactions with $0.5<x_{F}<0.88$ and $0.3<p_{T}<0.5 \mathrm{GeV} / \mathrm{c}$. All other data used in the tuning are given in [80].

A non-negligible fraction of the flux results from non- $p C$ interactions. Proton interaction rates with aluminum, iron, and helium nuclei were tuned to the $p C$ data using an $A$-dependent scaling, where $A$ is the relative atomic mass. Neutron interaction rates with carbon were also tuned to the $p C$ data using isospin symmetry. Few data exist that are applicable to the $\pi^{ \pm}$and $K^{ \pm}$interactions with nuclei in NuMI. The rates of these and all other interactions with no applicable data were not tuned and were assigned a $40 \%$ uncertainty, which was inferred from the agreement of the hadron interaction model (FTFP-BERT) with the $p C$ data.

The rates of simulated hadron interactions with applicable data that resulted in beam neutrinos were tuned by weighting each interaction by

$$
w_{\text {rate }}\left(x_{F}, p_{T}, p_{0}^{\text {sim }}\right)=\frac{f_{\text {data }}\left(x_{F}, p_{T}, p_{0}^{\text {data }}\right)}{f_{\text {sim }}\left(x_{F}, p_{T}, p_{0}^{\text {data }}\right)} \times g\left(x_{F}, p_{T}, p_{0}^{\text {data }}, p_{0}^{\text {sim }}\right)
$$

where $f_{\text {data }}$ and $f_{\text {sim }}$ are the data and simulation production rates, respectively, at the produced particle $x_{F}$ and $p_{T}$ and incoming particle momentum in the data $p_{0}^{\text {data }}$. The factor $g$ scales the production rate at $p_{0}^{\text {data }}$ to the simulated incoming particle momentum $p_{0}^{\text {sim }}$ via Feynman scaling [84].

The tuning of the hadron interaction rates in the simulation did not correct the 
amount of material traversed by the incident particle. The location of interactions in the simulation affects the rate of secondary interactions and the focusing of particles emerging from the target. The amount of material traversed by the incident proton in simulated $p C$ interactions was tuned by weighting the interactions by

$$
w_{a b s}(z)=\exp \left(-z N_{A} \rho\left(\sigma_{a b s}^{\text {data }}-\sigma_{a b s}^{\text {sim }}\right)\right)
$$

where $z$ is the distance traversed by the incident proton, $\rho$ is the nuclear number density, and $\sigma_{a b s}^{d a t a}$ and $\sigma_{a b s}^{\text {sim }}$ are the the absorption cross sections for protons in carbon from external data and the simulation, respectively. These weights correct the survival probability of the incident proton. The incident particle survival probability was corrected for $p C$ interactions only. An uncertainty on the absorption cross section, and thereby the survival probability, for the incident particle was applied to all interactions in the simulation. The proton-carbon absorption cross section data, and the uncertainty on the absorption cross section for all interactions, are given in [80].

Figure 6.6 shows the untuned and hadron production tuned predictions of the $\nu_{\mu} \mathrm{FHC}$ and $\bar{\nu}_{\mu}$ RHC fluxes in the LE configuration. The hadron production tuning reduces both flux predictions near the peak of the spectrum and increases both predictions in the high energy tail.

\subsubsection{Flux Uncertainties}

The uncertainty on the flux prediction (Figure 6.7) consists of uncertainties on the hadron production and beamline models in the flux simulation. Uncertainties on the hadron interactions tuned by data are determined by the data uncertainties, 

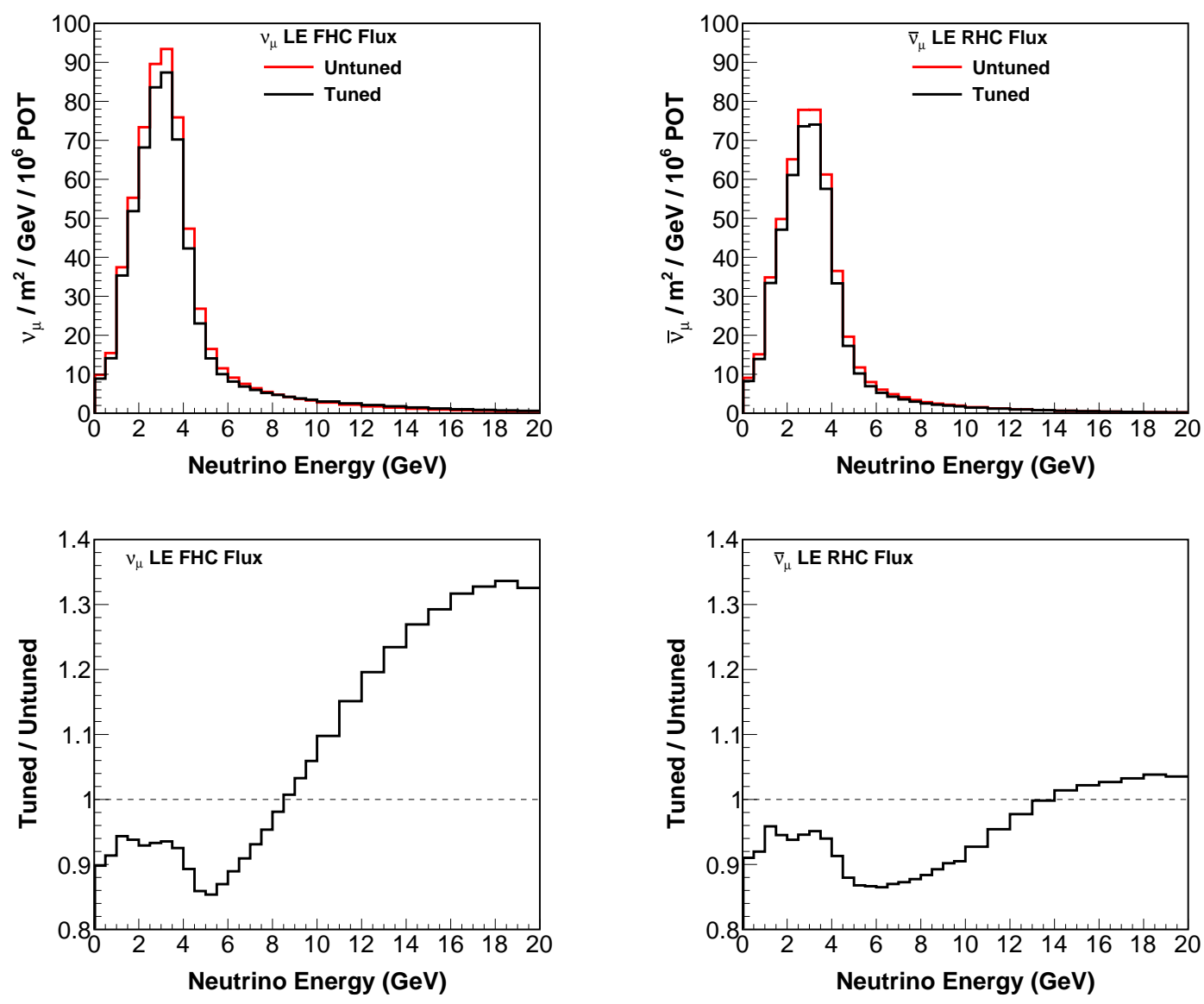

Figure 6.6: The untuned and hadron production tuned predictions of the $\nu_{\mu} \mathrm{FHC}$ (top left) and $\bar{\nu}_{\mu}$ RHC (top right) fluxes in the LE configuration. The bottom plots show the ratio of the hadron production tuned and untuned flux predictions.

and untuned interactions are assigned a $40 \%$ uncertainty as previously discussed. The beamline model uncertainties consist of uncertainties on the alignments of the proton beam trajectory, target, and horns; the geometries of the target and horns; the magnitude and distribution of current in the horns; and the number of protons incident on the target. The hadron production uncertainties dominate the total flux uncertainty except near $4 \mathrm{GeV}$ neutrino energy, where the slope of the falling edge of the neutrino energy spectrum is sensitive to focusing effects. Values of all 
uncertainties in the flux simulation are given in [80].
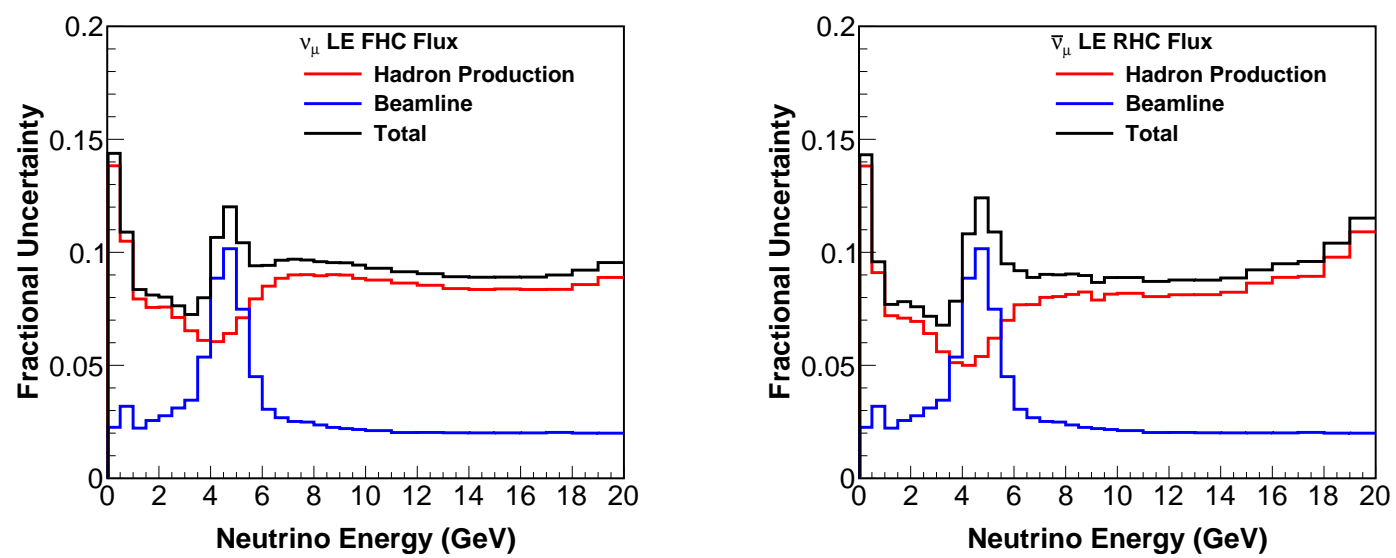

Figure 6.7: The uncertainty on the hadron production tuned predictions of the $\nu_{\mu}$ FHC (left) and $\bar{\nu}_{\mu}$ RHC (right) fluxes in the LE configuration.

The flux uncertainty on MINER $\nu \mathrm{A}$ measurements is evaluated using 100 flux universes (i.e. varied flux predictions). Each flux universe was the result of simultaneously varying the parameters that govern the hadron production and beamline models, where each parameter was varied by a random amount determined from a gaussian distribution centered on the nominal parameter value with a $1 \sigma$ width equal to the parameter uncertainty [80].

\subsubsection{In situ Constraint From Neutrino-Electron Elastic Scat- tering}

An in situ constraint on the flux prediction was provided by a measurement of neutrino-electron elastic scattering $\left(\nu+e^{-} \rightarrow \nu+e^{-}\right)$in the MINER $\nu \mathrm{A}$ detector [87]. The cross section for this process, which involves fundamental particles only, is precisely predicted by the Standard Model. This allows a measurement of the neutrino flux from the rate of $\nu+e^{-} \rightarrow \nu+e^{-}$interactions, the predicted cross 
section, and the detector mass (Equation 6.1). The rate of $\nu+e^{-} \rightarrow \nu+e^{-}$ interactions, corrected for selection efficiency and background, was measured in the LE FHC beam to a total precision (statistical + systematic) of $12 \%$. This precision is comparable to the uncertainty on the flux prediction, and can therefore be used to constrain the flux prediction and uncertainty.

The flux prediction and its uncertainty was constrained using the $\nu+e^{-} \rightarrow \nu+e^{-}$ measurement of the integrated flux as follows. For each flux universe, a likelihood was calculated from a comparison of the measured and universe integrated fluxes. In each neutrino energy bin $i$, the constrained flux prediction $\phi_{i}^{\nu+e}$ was the weighted average of the flux universes,

$$
\phi_{i}^{\nu+e}=\frac{\sum_{k} w_{k} \phi_{i k}}{\sum_{k} w_{k}}
$$

where $\phi_{i k}$ is the predicted flux in bin $i$ in universe $k$, and $w_{k}$ is the likelihood for universe $k$. The constrained flux uncertainty and bin-to-bin covariance was calculated from a weighted covariance matrix $C$, the elements of which were calculated as

$$
C_{i j}=\frac{\sum_{k} w_{k}\left(\phi_{i k}-\phi_{i}^{\nu+e}\right)\left(\phi_{j k}-\phi_{j}^{\nu+e}\right)}{\sum_{k} w_{k}} .
$$

The $\nu+e^{-} \rightarrow \nu+e^{-}$interaction rate was measured in the LE FHC beam only, but was used to constrain both the LE FHC and RHC fluxes. This was permitted by the FHC and RHC flux universes being generated by the same set of flux parameter variations.

Figure 6.8 shows the effect of the $\nu+e^{-} \rightarrow \nu+e^{-}$constraint on the hadron 
production (HP) tuned LE $\nu_{\mu} \mathrm{FHC}$ and $\bar{\nu}_{\mu}$ RHC fluxes and uncertainties. The constraint results in a roughly $2-4 \%$ reduction in the tuned flux prediction, and a $\sim 1 \%$ reduction in the tuned flux uncertainty. The results presented in this thesis were measured using the HP tuned, $\nu+e^{-} \rightarrow \nu+e^{-}$constrained LE $\nu_{\mu} \mathrm{FHC}$ and $\bar{\nu}_{\mu}$ RHC flux predictions. 

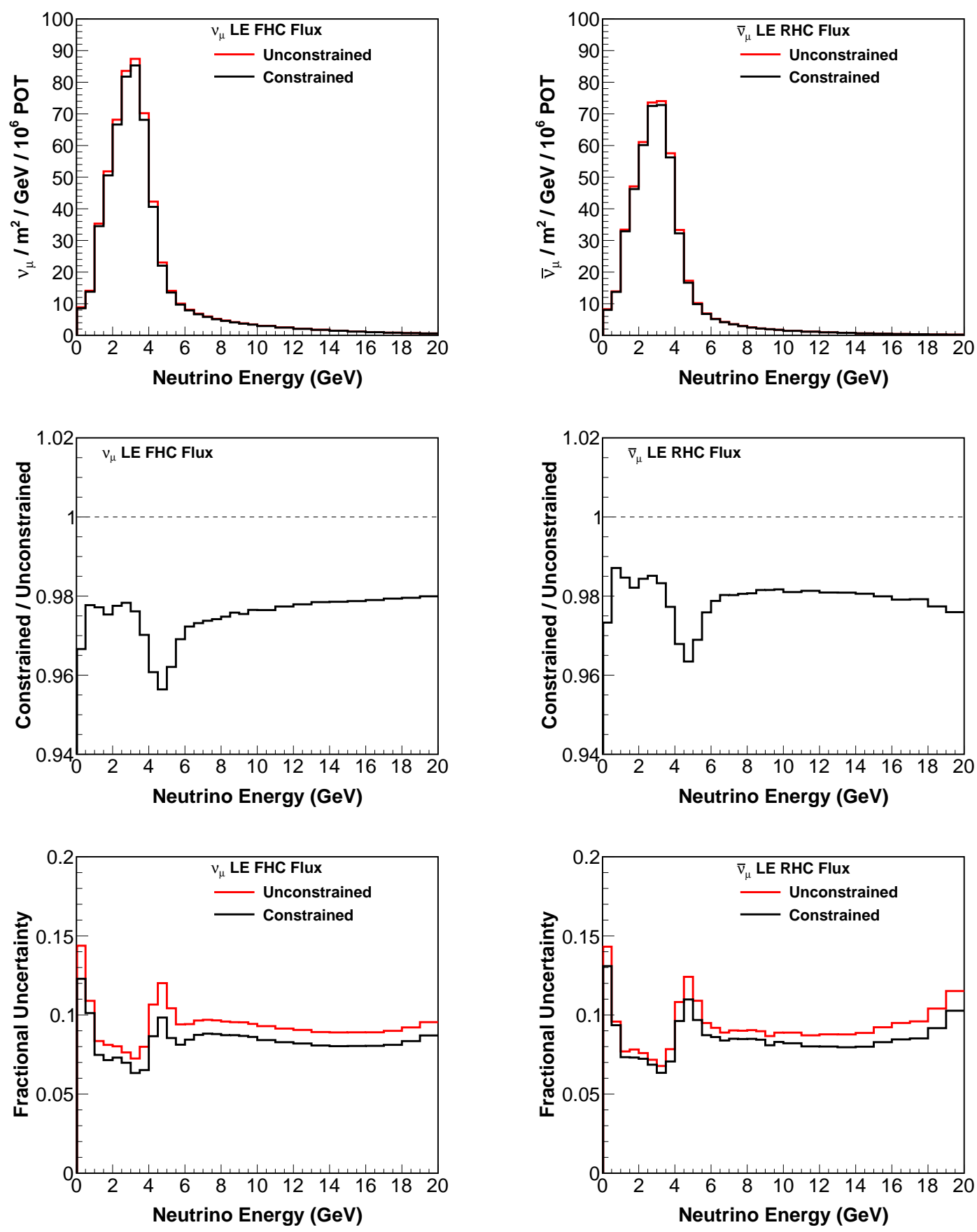

Figure 6.8: The top plots show the hadron production tuned flux prediction with and without the $\nu+e^{-} \rightarrow \nu+e^{-}$constraint. The middle plots show the ratio of the constrained and unconstrained flux predictions. The bottom plots show the total uncertainty on the constrained and unconstrained flux predictions. The left and right plots are for the $\nu_{\mu} \mathrm{FHC}$ and $\bar{\nu}_{\mu} \mathrm{RHC}$ flux predictions in the LE configuration, respectively. 


\section{Chapter 7}

\section{The MINER $\nu$ A Experiment}

$\operatorname{MINER} \nu \mathrm{A}$ (Main Injector Experiment for $\nu$-A) is a dedicated neutrino-nucleus scattering experiment. The physics goals of MINER $\nu \mathrm{A}$ are to make precise measurements of neutrino-nucleus cross sections for neutrino energies $1 \lesssim E_{\nu} \lesssim 20 \mathrm{GeV}$, and to measure the cross sections on different nuclei to study nuclear effects. The measurements made by MINER $\nu \mathrm{A}$ are important to neutrino oscillation experiments for reducing uncertainties from neutrino-nucleus interactions. The MINER $\nu$ A experiment is located at Fermilab and consists of the NuMI neutrino beam (Chapter 6), the MINER $\nu \mathrm{A}$ detector [88], and the near detector of the MINOS neutrino oscillation experiment [89].

\subsection{The MINER $\nu$ A Detector}

The MINER $\nu \mathrm{A}$ detector, described in detail in [88], is located $\approx 100 \mathrm{~m}$ below ground in the NuMI near detector hall, and $\approx 1 \mathrm{~km}$ downstream of the NuMI target (Figure6.2). A schematic of the MINER $\nu \mathrm{A}$ detector is shown in Figure 7.1, where 
the NuMI neutrino beam enters from the left. The MINER $\nu$ A detector consists of a central tracker region surrounded by side and downstream electromagnetic and hadronic calorimeters (ECALs and HCALs, respectively), and an upstream nuclear targets region. The sensitive elements of the MINER $\nu \mathrm{A}$ detector are strips of plastic scintillator, which emit light when traversed by charged particles. The central tracker region is composed entirely of scintillator for precisely measuring the path and energy deposition of charged particles. The ECALs (HCALs) contain layers of lead (iron) interleaved with scintillator for containing and measuring the energy deposition of particles that primarily interact electromagnetically (strongly). The upstream nuclear targets region contains planes of graphite, iron, and lead along with water and liquid helium targets separated by planes of scintillator for measuring the dependence of neutrino-nucleus cross sections on nuclear species. A veto wall, consisting planes of steel and scintillator, sits in front of the MINER $\nu \mathrm{A}$ detector for tagging particles entering the MINER $\nu \mathrm{A}$ detector that originated from neutrino interactions in the rock upstream of the NuMI near detector hall (see Section 6.2). The MINER $\nu \mathrm{A}$ detector sits immediately upstream of the MINOS near detector (Section 7.2), which serves as the muon spectrometer for the MINER $\nu \mathrm{A}$ experiment.

\subsubsection{Module Assemblies}

The MINER $\nu \mathrm{A}$ detector is composed of 120 hexagonal modules (Figure 7.2). The modules are mounted vertically and are stacked facing the neutrino beam. Each module contains a hexagonal steel frame which serves as a support structure for scintillator planes and absorber layers (lead, iron) or a nuclear target plane. The hexagonal steel frames are instrumented with scintillator and comprise the side 


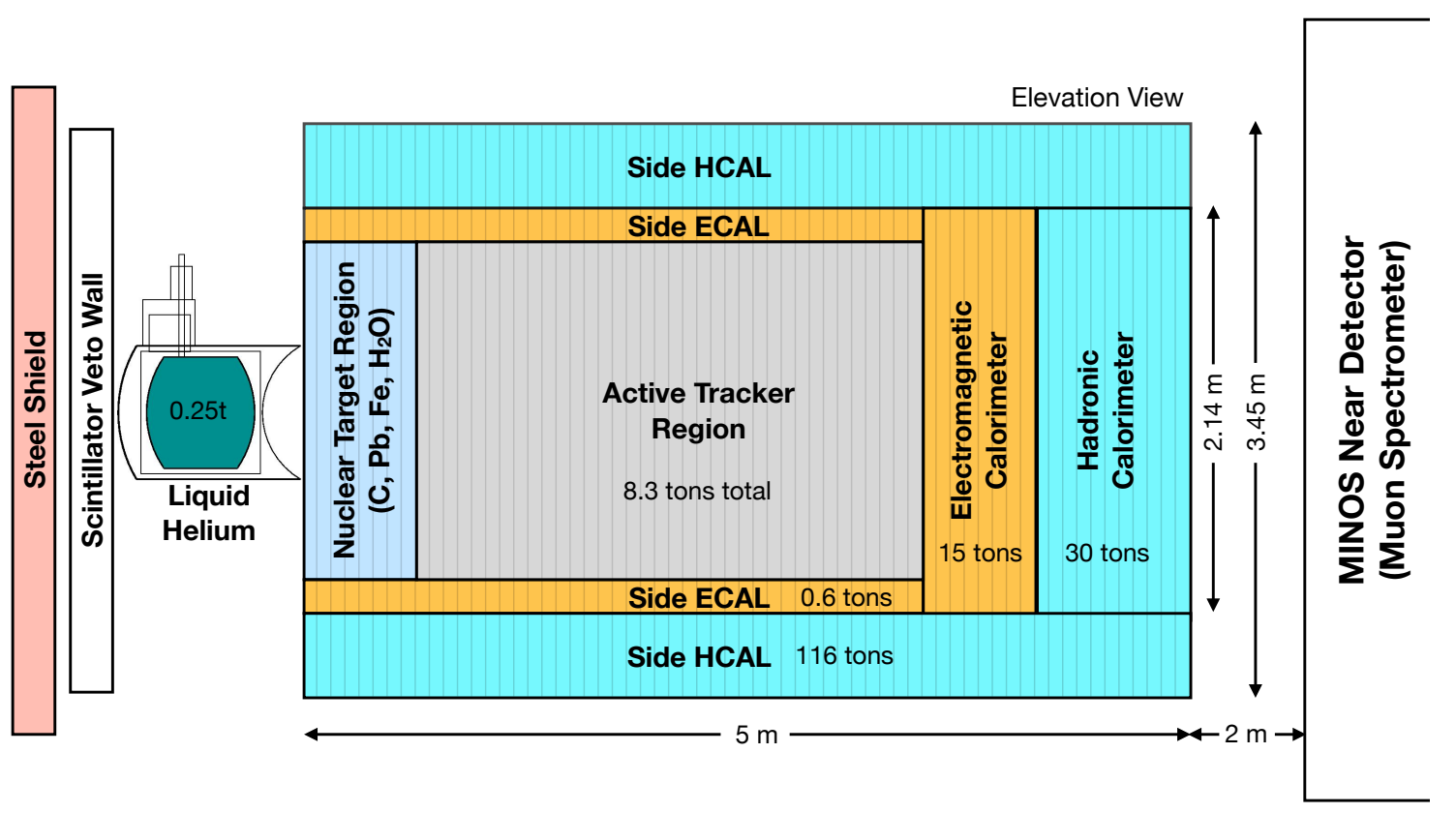

Figure 7.1: Schematic of the MINER $\nu$ A detector. The figure is from [88].

HCAL. There are four types of modules that differ by the components mounted to the hexagonal steel frame. The module types are tracker, ECAL, HCAL, and nuclear target.

Tracker modules have two scintillator planes mounted inside the hexagonal steel frame. The scintillator planes are hexagonal with a $105 \mathrm{~cm}$ apothem and are $\approx 2$ cm thick. Each scintillator plane contains 127 parallel scintillator strips. Each scintillator plane in a tracker module has a lead "collar" mounted to its upstream face (i.e. the scintillator plane surface facing the NuMI target). The lead collars are hexagonal with inner and outer apothems of $90 \mathrm{~cm}$ and $105 \mathrm{~cm}$, respectively, and are $0.2 \mathrm{~cm}$ thick. The lead collars and the underlying scintillator form the side ECAL. The central tracker region is composed of 62 consecutive tracker modules.

The ECAL modules are identical to the tracker modules with the exception that the lead collar is replaced by a $0.2 \mathrm{~cm}$ thick plate covering the entire upstrem face of 


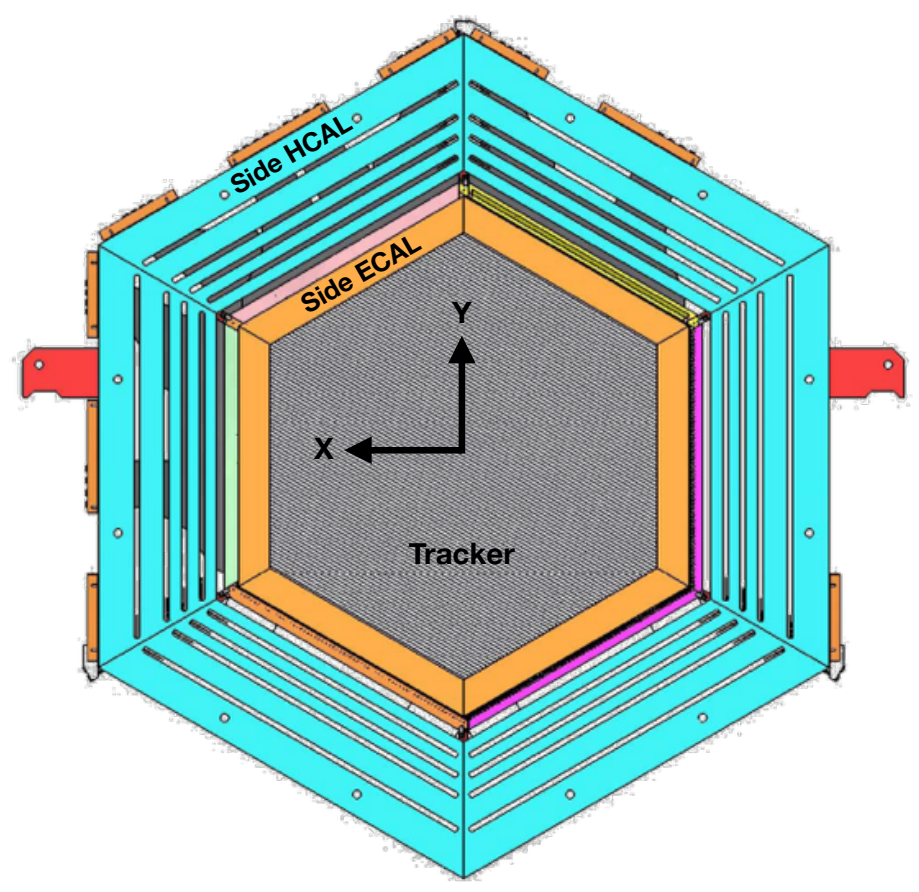

Figure 7.2: Engineering drawing of a tracker module in the MINER $\nu \mathrm{A}$ detector. The $\mathrm{X}$ and $\mathrm{Y}$ axes of the detector coordinate system are shown where the $\mathrm{XY}$ origin is located at the center of the module. The $\mathrm{Z}$ axis points into the page. The perspective is looking downstream. The figure is from [88].

the scintillator plane. The downstream ECAL is composed of 10 consecutive ECAL modules.

The HCAL modules have a steel plate and one scintillator plane mounted inside the hexagonal steel frame. The steel plate is hexagonal with a $105 \mathrm{~cm}$ apothem and $2.54 \mathrm{~cm}$ thick. The steel plate sits upstream of the scintillator plane. The downstream HCAL is composed of $20 \mathrm{HCAL}$ modules.

The nuclear target modules have a nuclear target plate mounted inside the hexagonal steel frame. The nuclear target plates are hexagonal with a $105 \mathrm{~cm}$ apothem and consists of two sections of iron and lead or three sections of iron, lead, and graphite. There are five nuclear target modules with nuclear target plates of dif- 
ferent thicknesses. The nuclear target modules do not contain a scintillator plane. The nuclear targets region is composed of the five nuclear target modules, the liquid helium and water targets, and tracker modules separating each of the solid and liquid targets. Further details of the nuclear targets region are contained in [88].

The hexagonal steel frames of the 120 modules compose the side HCAL. The steel frames are $3.8 \mathrm{~cm}$ thick for HCAL modules and $3.5 \mathrm{~cm}$ thick for all other modules. Each section of the steel frames is $56 \mathrm{~cm}$ wide and has four slots, referred to as stories, that parallel to its inner and outer edges. Each story holds two scintillator strips that are stacked such that one strip sits upstream of the other.

\subsubsection{Coordinate System}

The coordinate system of the MINER $\nu \mathrm{A}$ detector (Figure 7.2) is defined relative to the detector axis, which intersects the centers of the 120 modules. The XY plane is perpendicular to the detector axis and the XY origin is located at the detector axis. $\mathrm{X}$ is the horizontal axis and $\mathrm{Y}$ is the vertical axis. Looking along the direction of the neutrino beam, $+\mathrm{Y}$ points up (towards the surface) and $+\mathrm{X}$ points to the left. The $\mathrm{Z}$ axis is collinear with the detector axis, and $+\mathrm{Z}$ points downstream (away from the NuMI target). The axis of the NuMI beam points downward 3.34 degrees relative to the $\mathrm{Z}$ axis.

\subsubsection{Scintillator}

The sensitive elements of the MINER $\nu \mathrm{A}$ detector are strips of translucent polystyrene scintillator which emit light when traversed by a charged particle [90]. Strips composing the hexagonal scintillator planes have a triangular cross section with a base 
width of $33 \mathrm{~mm}$, a height of $17 \mathrm{~mm}$, and $2.6 \mathrm{~mm}$ hole at the center (Figure 7.3 ). Strips in the side HCAL have a rectangular cross section with a base width of 19 $\mathrm{mm}$, a height of $16.6 \mathrm{~mm}$, and $3.5 \mathrm{~mm}$ hole at the center. The strips were extruded with a white, $0.25 \mathrm{~mm}$ thick coating that is $15 \% \mathrm{TiO} 2$ and $85 \%$ polystyrene by weight for internal reflection. A $1.2 \mathrm{~mm}$ diameter wavelength shifting (WLS) fiber runs through the central hole of each strip. The WLS fiber spans the length of the strip and is optically coupled to the scintillator by optical epoxy. The scintillator is doped with PPO and POPOP fluors and emits blue light when excited by charged particles or by UV light. The WLS fiber, doped with Y11 fluor, absorbs blue light emitted by the scintillator and emits green light, which propagates through the fiber to the ends of the strip. Each strip has a "mirrored" end and a readout end. At the mirrored end of the strip the end of the WLS fiber is coated with aluminum, which reflects light with an efficiency of $85 \%$ for propagation to the readout end of the strip.
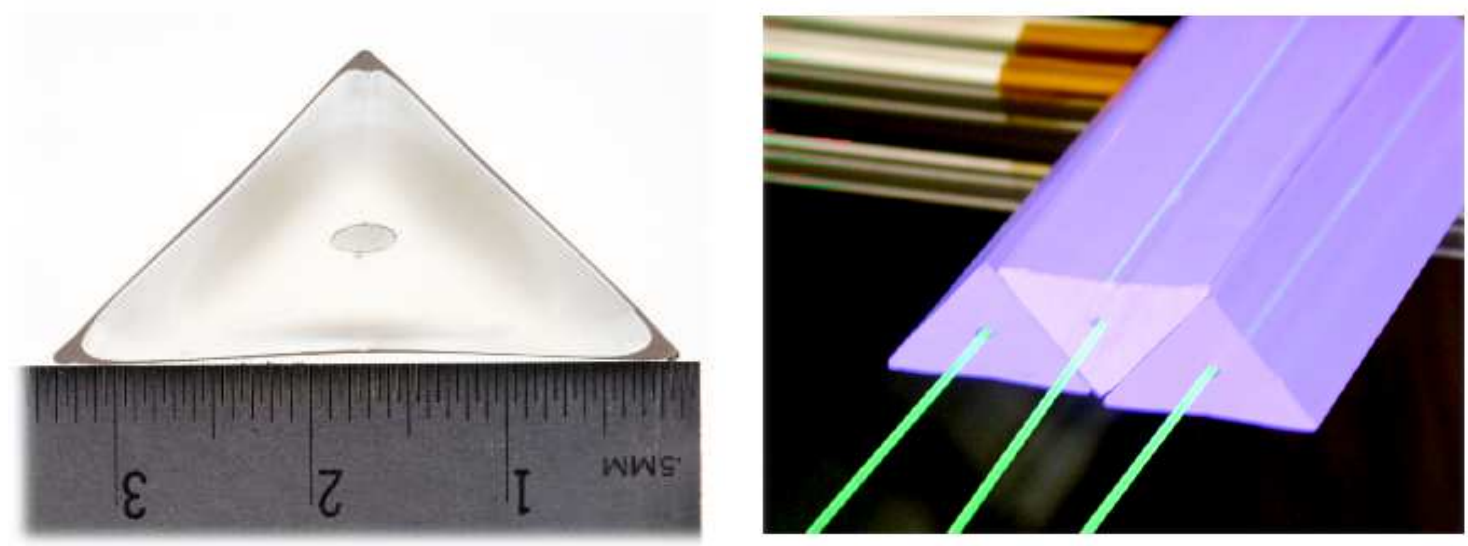

Figure 7.3: Cross section of the MINER $\nu \mathrm{A}$ scintillator strips. The figure is from [88].

The hexagonal scintillator planes are composed of 127 scintillator strips. The strips arranged in parallel with the alternating triangular cross section orientation 
illustrated in Figure 7.3. The strips are glued together with epoxy. To shield the strips from external light, each face of the scintillator plane is covered with a Lexan sheet which is adhered to the plane with epoxy. The chemical composition of the scintillator planes, which is important to the cross section measurement presented in this thesis, is listed in Table 7.1.

\begin{tabular}{c|c} 
Element & Mass Fraction \\
\hline $\mathrm{C}$ & 0.8762 \\
$\mathrm{H}$ & 0.0742 \\
$\mathrm{O}$ & 0.0318 \\
$\mathrm{Ti}$ & 0.0069 \\
$\mathrm{Al}$ & 0.0026 \\
$\mathrm{Si}$ & 0.0027 \\
$\mathrm{Cl}$ & 0.0055
\end{tabular}

Table 7.1: Mass fraction of elements in the MINER $\nu \mathrm{A}$ scintillator planes.

At the readout end of a scintillator plane the WLS fibers extend beyond the ends of the strips for routing to a clear optical cable connector (Figure 7.4). Clear optical fibers transmit light from the WLS fibers to photomultiplier tubes (PMTs) described in Section 7.1.5. WLS fibers are enclosed in a light tight "baggie" between the end of the strips and the connector.

Each scintillator plane provides 2D information of the position of an energy deposition by a particle: the $\mathrm{Z}$ position of the plane in the detector and the position of the strip(s) containing the deposited energy within the plane. A particle traversing a plane will typically traverse and deposit energy in two or more strips due to their overlapping triangular cross sections. This enables an energy weighted average position to be calculated for an energy deposition in a plane for improved position resolution. To enable $3 \mathrm{D}$ reconstruction of the position and path of a particle, the scintillator planes are mounted in three different orientations, referred to as $\mathrm{X}, \mathrm{U}$, 


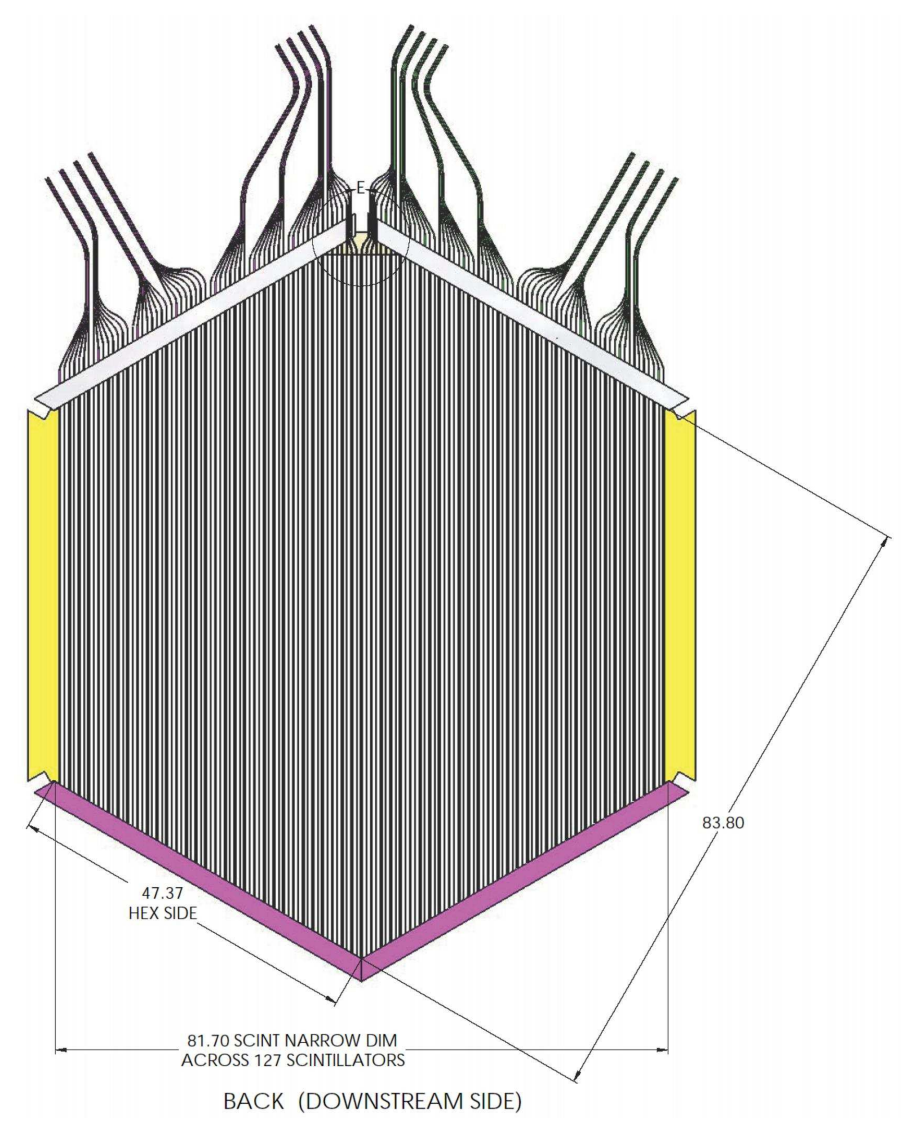

Figure 7.4: An engineer's drawing of a MINER $\nu \mathrm{A}$ scintillator plane showing the WLS fibers (top) extending beyond the readout ends of the scintillator strips for routing to clear optical cable connectors. Clear optical cables transmit light from the WLS fibers to photomultiplier tubes. Each bundle of WLS fibers is enclosed in a light tight "baggie" between the strip ends and the connector. The figure is from reference [91]. 
and $\mathrm{V}$ (Figure 7.5). Planes mounted in the $\mathrm{X}$ orientation are referred to as $\mathrm{X}$ planes, and likewise for planes mounted in the $\mathrm{U}$ and $\mathrm{V}$ orientations. Strips in $\mathrm{X}$ planes are parallel to the $\mathrm{Y}$ axis. $\mathrm{U}(\mathrm{V})$ planes are rotated $+60(-60)$ degrees about the $\mathrm{Z}$ axis relative to $\mathrm{X}$ planes. From upstream to downstream, scintillator planes are mounted in alternating orientations with the repeating pattern XUXV.
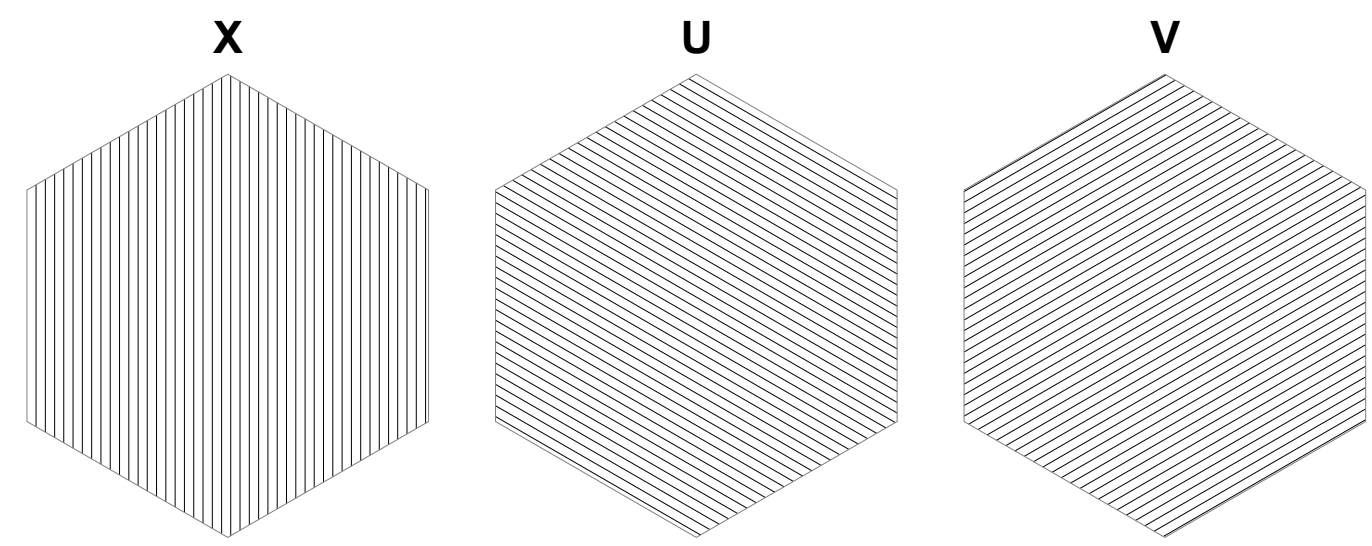

Figure 7.5: Orientations of the MINER $\nu$ A scintillator planes

\subsubsection{Event Display}

A visualization of a neutrino interaction in the MINER $\nu$ A detector, referred to as an event display, is shown in Figure 7.6 [92]. In an event display the neutrino beam enters from the left and $+\mathrm{Z}$ points to the right. The perspective in each pane is looking into the readout end of the scintillator strips, where color denotes the amount of energy deposited in a strip. The left, center, and right sets of panes are the $\mathrm{X}, \mathrm{U}$, and $\mathrm{V}$ "views," respectively. For the $\mathrm{X}$ view, the middle pane displays the $\mathrm{X}$ planes and the top and bottom panes display the strips in the side HCAL that are parallel to the strips in the $\mathrm{X}$ planes, and likewise for the $\mathrm{U}$ and $\mathrm{V}$ views. The 
horizontal and vertical axes in each pane are the module number and strip number, respectively.
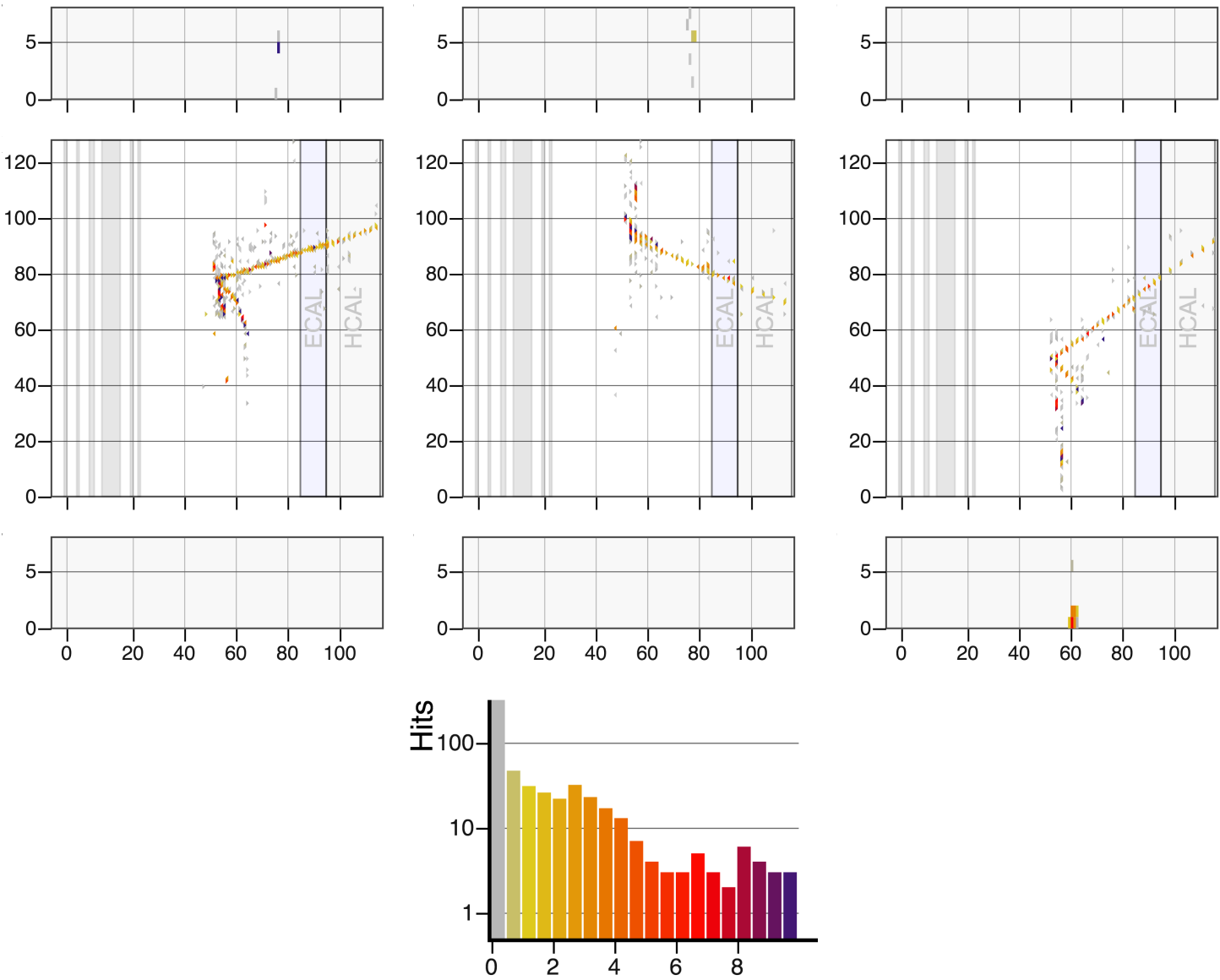

Figure 7.6: An event display of the activity produced by a neutrino interaction in the MINER $\nu \mathrm{A}$ detector. Color denotes the amount of energy deposited in a scintillator strip in $\mathrm{MeV}$.

\subsubsection{Readout and Electronics}

Scintillation light generated by particles in the MINER $\nu \mathrm{A}$ detector is converted to a digitized electrical signal for analysis. Photomultiplier tubes (PMTs) are used to convert the scintillation light to an electrical charge, which is digitized by front end 
boards (FEBs). A data acquisition (DAQ) PC reads out and controls the FEBs through rack mounted electronics. A detailed description of the MINER $\nu \mathrm{A}$ readout electronics and software is given in reference [93].

\section{Photomultiplier Tubes}

For each scintillator strip, the readout end of the WLS fiber is coupled to a clear optical cable which transmits scintillation light to a PMT. Each PMT has an $8 \times 8$ grid of photocathode pixels, with each pixel having an area of $2 \times 2 \mathrm{~mm}^{2}$. Photoelectrons (PE) are ejected from the pixel by incident scintillation light. The quantum efficiency of the photocathode is $\approx 20 \%$. Each pixel has a 12-stage dynode chain which amplifies the charge of the PE by a factor $10^{5}-10^{6}$. A several hundred volt potential is supplied to each dynode chain by a Cockcroft-Walton generator mounted on a FEB. A custom PMT circuit board containing 64 anodes, one for each dynode chain, collects the amplified charge.

Each PMT is housed in an assembly called a PMT box (Figure 7.7). The PMT box has a steel cylindrical housing that shields the PMT from the magnetic field of the MINOS near detector (Section 7.2). The input end of the housing is covered by a plate containing optical connectors for 64 clear optical cables. Each clear optical cable is coupled to an optical fiber contained within the PMT box. The optical fibers are referred to collectively as an Optical Decoder Unit (ODU). The ODU fibers are routed onto a "cookie" which is mounted to the photocathode grid of the PMT. The cookie couples each ODU fiber to a photocathode pixel. The arrangement of the ODU fibers onto the photocathode grid (Figure 7.8), referred to as the ODU fiber weave, is such that adjacent strips in a plane are not read out by adjacent photocathode pixels. This allows identification of PMT cross talk, 
which is the leakage of light and/or charge from PMT channel to another, using the size of hits and their proximity in the strips and on the photocathode pixels (see Section 7.5.9). The PMT circuit board containing the anodes is mounted to the PMT. Another circuit board mounted to the plate covering the output end of the PMT box provides an interface for a FEB.

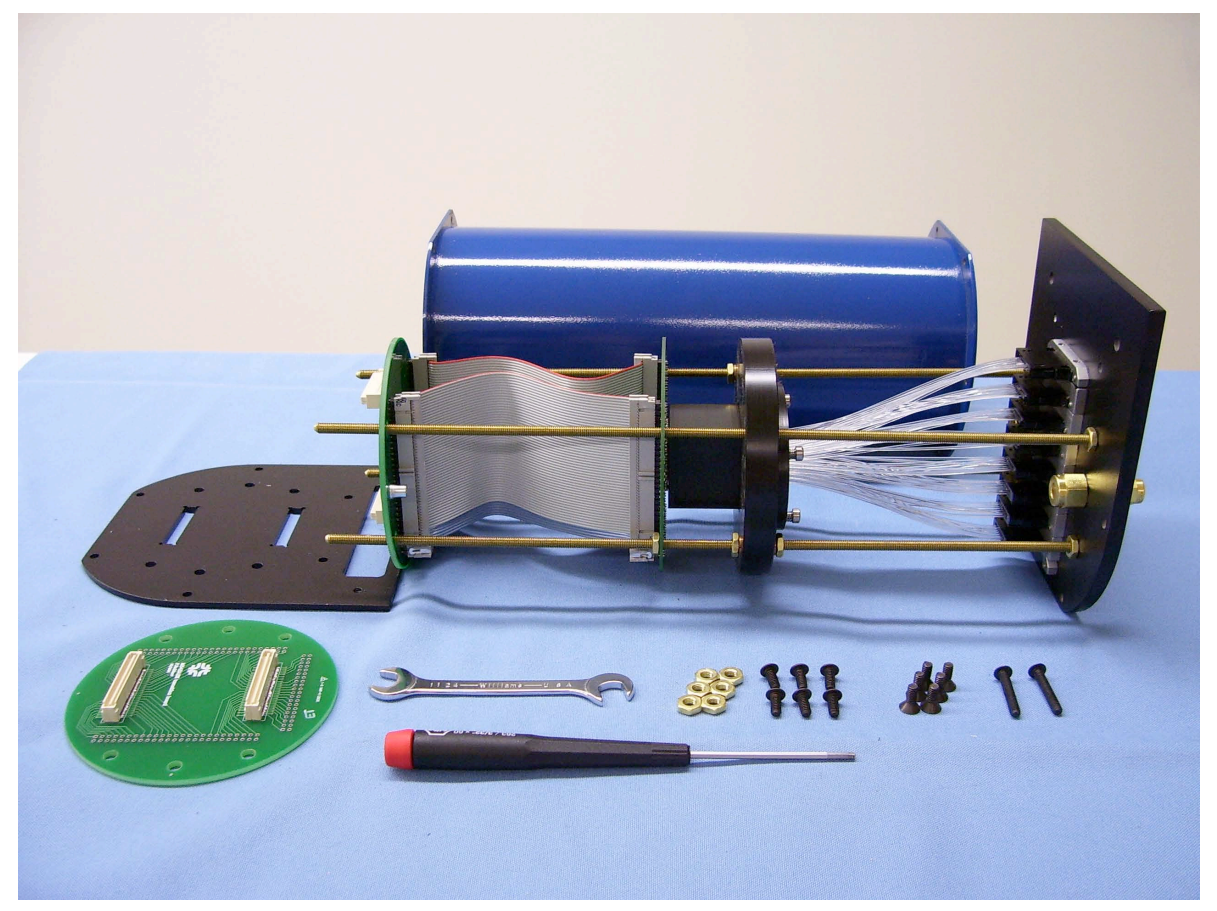

Figure 7.7: A PMT box where the cylindrical steel housing (blue) has been removed to reveal the interior. From right to left are the clear optical cable connectors, ODU fibers, cookie fixture (black disk), the PMT (black rectangular column), and the PMT circuit board. A spare PMT circuit board is shown in the left foreground. The figure is from reference [88].

An undesirable feature of PMTs is afterpulsing, which is a delayed output signal. Afterpulsing is primarily due to ionization of latent gas within the PMT in the dynode chains during PE amplification. The dynode chain potential accelerates positive ions back to the photocathode where they generate PE resulting in a delayed output signal. The time delay of afterpulsing from the initial output signal 


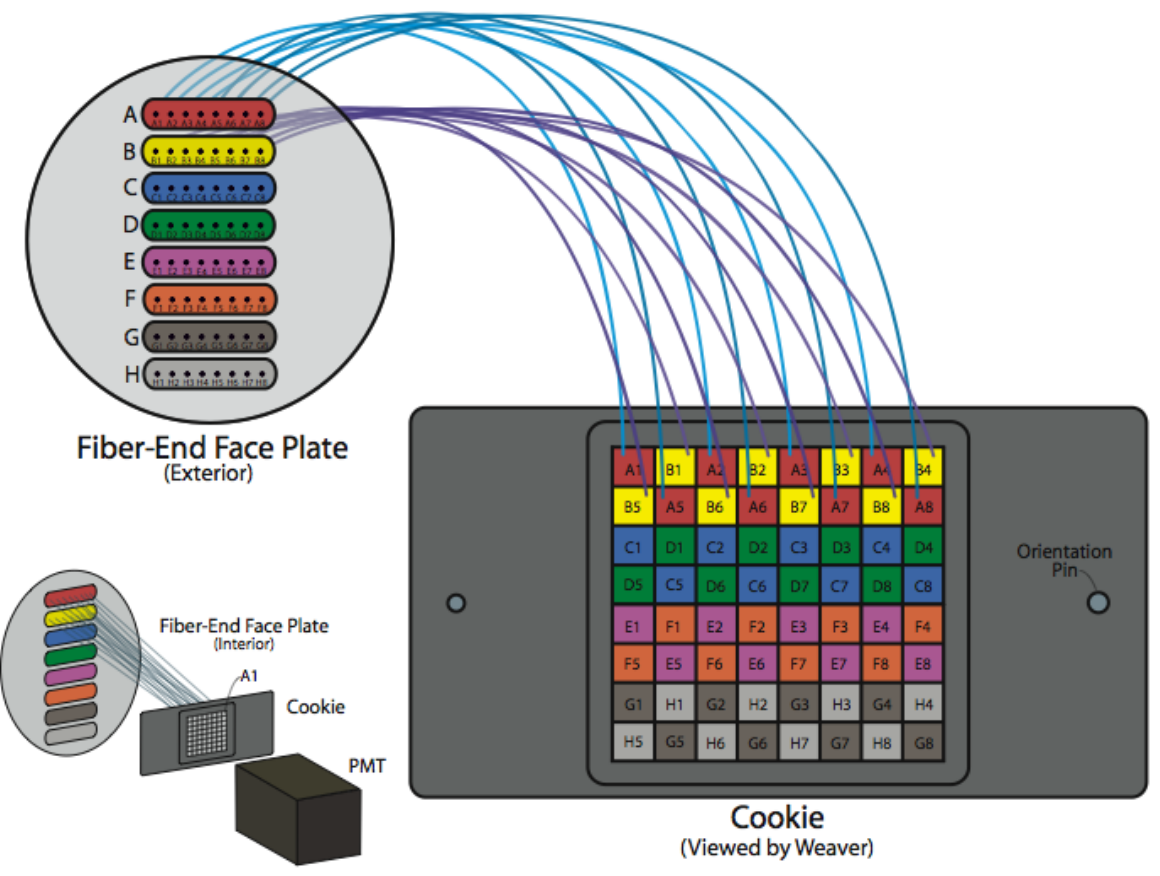

Figure 7.8: A diagram of the ODU fiber weave. The ODU fibers route scintillation light from the clear optical fibers to the photocathode grid on the PMT. Each set of clear optical fiber optical connectors (A-H) serves eight consecutive scintillator strips in a plane. The ODU fiber weave routes adjacent strips in a plane to nonadjacent photocathode pixels, which facilitates PMT cross talk identification. The figure is from reference [88].

ranges from hundreds of nanoseconds to several microseconds. The output charge from afterpulsing is small relative to that of the initial output signal. However, afterpulsing can result in FEB dead time and can fake the activity of a Michel electron, which is the electron from a muon decay. Michel electrons are utilized by several of MINER $\nu$ A's physics analyses, which must account for afterpulsing with selection cuts. 


\section{Front End Boards}

Each PMT is serviced by one FEB, which is mounted to the output end plate of the PMT box. A FEB has 64 channels, one for each PMT anode. In each FEB channel, charge from a PMT anode is capacitively divided into low, medium, and high gain channels to increase the dynamic range. The gain channels are routed to six Application-Specific Integrated Circuit (ASIC) chips [94], referred to as TriP-t chips, mounted on the FEB. Four of the six TriP-t chips each serve 16 high and 16 medium gain channels. The remaining two TriP-t chips each serve 32 low gain channels. The TriP-t chips integrate and digitize charge on each channel into an analog-to-digital converter (ADC) count. A integrated charge in a gain channel is stored in an ADC block, and each gain channel has an ADC pipeline with capacity for 7 time stamped ADC blocks and one non-time stamped ADC block. Each FEB channel has a discriminator that fires when charge in its high gain channel rises above an amount corresponding to one PE at the photcathode. When a discriminator fires the associated low, medium, and high gain ADC blocks in the FEB channel are stamped with the time that the discriminator fired.

FEBs integrate charge in a scheme called a charge integration cycle, where a set of 32 FEB channels served by one low gain TriP-t chip and two high gain TriP-t chips are charge integrated for a duration of 150 ns. A charge integration cycle is initiated by a discriminator firing on one of the 32 channels. The charge integration cycle is immediately followed by a $200 \mathrm{~ns}$ reset period during which the integrated charge in each gain channel is stored and the integrators are reset and reopened. The TriP-t chips digitize the charge in each ADC block at the end of a readout gate described in Section 7.1.5. Charge from the PMTs entering integrated channels during the reset period will be lost. The reset period is referred to as dead time. 
For neutrino interactions occurring closely in time in the detector, which is referred to as event pile-up, dead time from detector activity of the initial interaction can mask activity of the following interaction(s). Dead time is accounted for in Minerva's physics analyses by simulating dead time in the MINER $\nu$ A Monte Carlo simulation (Section 7.6) and applying selection cuts.

Each FEB has an on-board Field-Programmable Gate Array (FPGA) chip that controls the FEB functions and communicates with the VME electronics described below. The FPGA chip receives timing and trigger information from, and sends ADC data to, the VME electronics. The FPGA chip has a $53 \mathrm{MHz}$ clock, which is used to produce a digital signal, with a granularity of $9.4 \mathrm{~ns}$, which is referred to as a clock tick. The FPGA chip uses a quadrature circuit to achieve a finer granularity of $\sim 2.4 \mathrm{~ns}$, referred to as a quarter tick, for discriminator time stamps. The FPGA also regulates the voltage on the PMT dynode chains, which is supplied by a Cockcroft-Walton generator mounted to the FEB.

\section{Rack-Mounted Electronics}

The DAQ PC reads out and controls the FEBs through rack mounted VME electronics. The FEBs are read out in chains, which consist of up to ten FEBs connected in series via ethernet cable. Each chain is connected at both ends to a custom VME module called a Chain Read Out Controller (CROC), with each CROC serving up to four FEB chains. Another custom VME module called a CROC Interface Module (CRIM) provides timing and trigger commands to up to four CROCs. The MINER $\nu \mathrm{A}$ Timing Module (MvTM) relays information from the timing system of the MINOS experiment and information of the state of the Main Injector to the CRIMs. The MvTM has a $53.1 \mathrm{~Hz}$ crystal oscillator clock which serves as the sys- 
tem clock for the MINER $\nu \mathrm{A}$ readout electronics. The FEB clocks are synchronized to the system clock to within 2 clock ticks due to timing offsets in the ethernet cables and VME electronics.

\section{Readout Gate}

A readout gate is a period of time in which activity in the MINER $\nu \mathrm{A}$ detector is recorded. A readout gate is $16 \mu$ s long and begins $0.5 \mu$ s before the beginning of a NuMI beam pulse. Each beam pulse is $10 \mu \mathrm{s}$ long, and a beam pulse arrives every $2.2 \mathrm{~s}$. The trigger that starts the readout gate is determined from the Main Injector timing information received by the MvTM. The trigger is relayed to all FEBs to start the gate. The 0.5 ns gap between the beginning of the readout gate and the beginning of the beam pulse accounts for the time offsets in the MINER $\nu \mathrm{A}$ readout electronics (Section 7.5.8). The extra $5.5 \mu \mathrm{s}$ at the end of the readout gate allows delayed activity, such as the activity from a Michel electron (the electron from a muon decay with a decay time of $2 \mu \mathrm{s}$ ), to be recorded. FEBs undergo charge integration cycles in response to detector activity occurring during the readout gate. At the end of the readout gate, any remaining charge in the FEB channels is integrated and charge in all ADC blocks on the FEBs is digitized. The ADC data is then collected from the FEBs by the DAQ PC and the FEBs are reset for the next readout gate. An event

display of detector activity during an entire readout gate is shown in Figure 7.9. A readout gate often contains activity from several neutrino interactions due to the high intensity of the NuMI neutrino beam. 

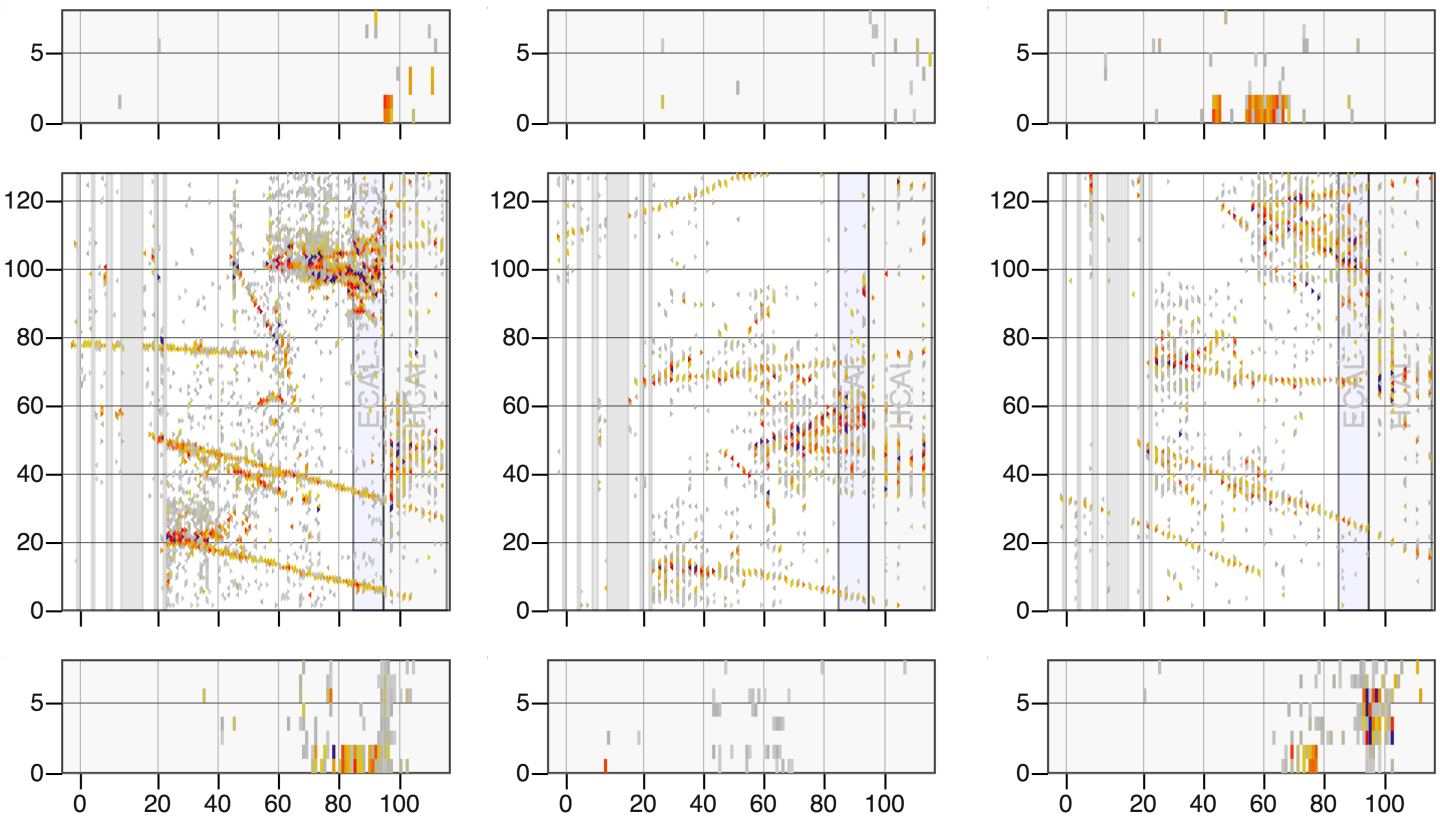

Figure 7.9: An event display of activity in the MINER $\nu \mathrm{A}$ detector during an entire readout gate. Multiple neutrino interactions are visible.

\subsection{The MINOS Near Detector}

The MINER $\nu \mathrm{A}$ detector is situated immediately upstream of the near detector of the MINOS neutrino oscillation experiment. The detectors are separated by a $\approx 2$ m air gap. The MINOS near detector, referred to herein as MINOS, is a magnetized iron sampling calorimeter. MINER $\nu \mathrm{A}$ utilizes MINOS to measure the momentum and charge of muons produced in neutrino interactions that exit the downstream end of MINER $\nu \mathrm{A}$.

MINOS is composed of steel planes interleaved with planes of plastic scintillator strips. The steel and scintillator planes are $2.54 \mathrm{~cm}$ and $1 \mathrm{~cm}$ thick, respectively. The shape and dimensions of the steel and scintillator planes are shown in Figure 7.10. The steel planes are identical throughout the detector. There are two types of 
scintillator planes: full and partial. The full planes cover a larger fraction of the area of the steel planes than the partial planes. MINOS and MINER $\nu$ A use the same scintillator technology and similar PMTs. All MINOS strips are $4.1 \mathrm{~cm}$ wide and 1 $\mathrm{cm}$ thick, and are arranged in parallel within the scintillator planes. To provide 3D position information, the scintillator planes are mounted in alternating +45 degrees ("U") and -45 degrees ("V") orientations with respect to the vertical.

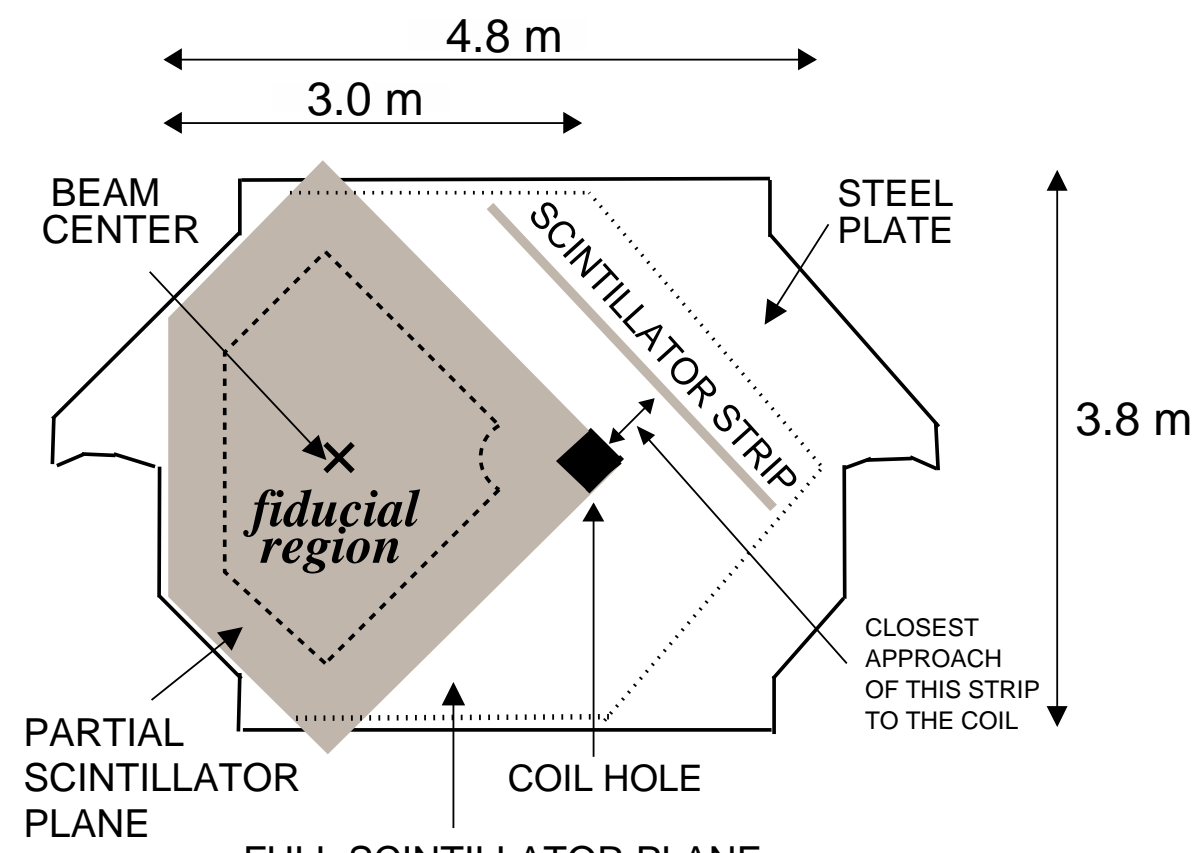

FULL SCINTILLATOR PLANE

Figure 7.10: Steel planes and scintillator planes of the MINOS near detector. The figure is from reference [95].

MINOS is divided into an upstream calorimeter region and downstream muon spectrometer region (Figure 7.11). The calorimeter is composed of 120 steel planes, where each steel plane is followed by a scintillator plane for hadron calorimetry. In the calorimeter region, every fifth scintillator plane is a full plane and all other planes are partial planes. The muon spectrometer is composed 162 steel planes, where a scintillator plane only follows every fifth steel plane. All scintillator planes in the 
muon spectrometer are full planes. The muon spectrometer is partially sampled since its primary function is to measure the long paths of muons.

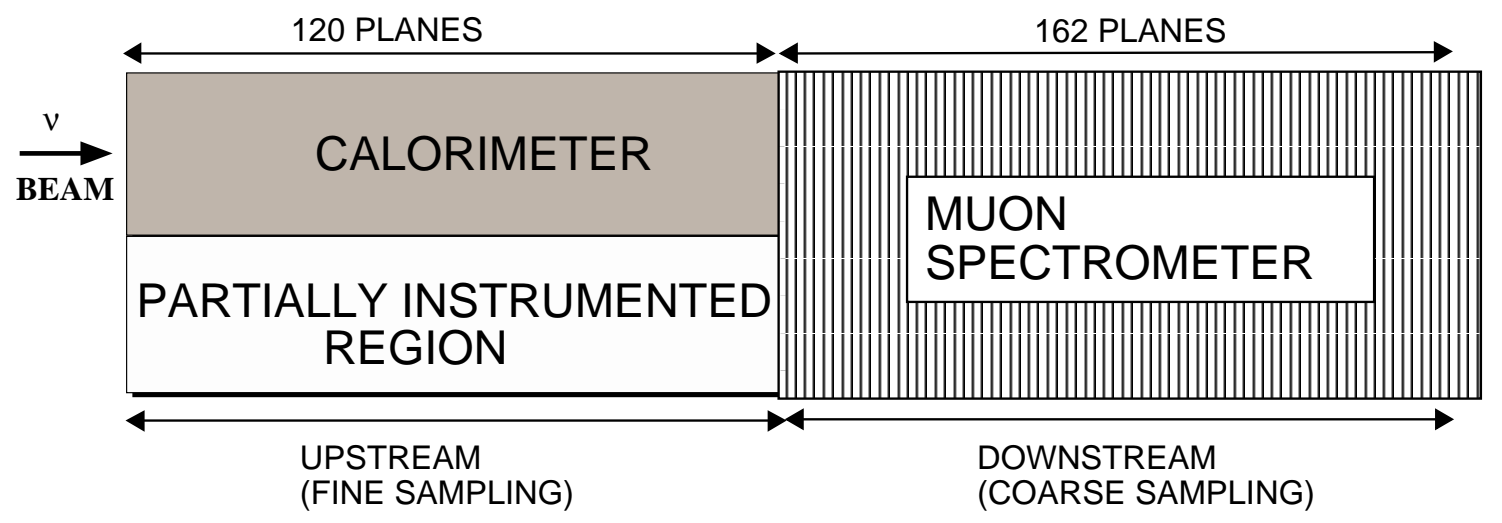

Figure 7.11: A schematic showing an overhead view of the upstream calorimeter and downstream muon spectrometer of the MINOS near detector. The figure is from reference [95].

A current carrying coil passes through the entire length of MINOS. The coil carries a current of $40 \mathrm{kA}$ and generates an average $1.3 \mathrm{~T}$ magnetic field in the steel planes. The direction of the current can be changed to focus particles with either negative or positive charge. Since the coil creates a void in the scintillator coverage, the axis of the coil is offset horizontally from the neutrino beam axis (Figure 7.10). The momentum and charge of a muon in MINOS can be measured by its curvature in the magnetic field. The momentum of muons that stop within MINOS can also be measured by range.

\subsection{The Partial MINER $\nu$ A Detector and ArgoNeuT}

Prior to completing the construction of the MINER $\nu \mathrm{A}$ detector, a data set was collected with the partially constructed MINER $\nu$ A detector, referred to as the partial detector, in the LE RHC ("antineutrino") beam. The partial detector data set 
amounted to $45 \%$ of MINER $\nu$ A's total LE antineutrino beam exposure. The partial detector consisted of all 20 HCAL modules, all 20 ECAL modules, and 36 of the 62 tracker modules. The partial detector did not contain nuclear target modules or either of the liquid targets.

The ArgoNeuT detector [96] was situated between partial detector and MINOS while the partial detector was taking data. ArgoNeuT is a prototype liquid argon time projection chamber. The ArgoNeuT cryostat contained $550 \mathrm{~L}$ (0.77 t) of liquid argon. The presence of ArgoNeuT resulted in muons from MINER $\nu$ A losing energy and scattering in ArgoNeuT before entering MINOS. Neutrino-nucleus cross section measurements that use the partial detector data set, which include the measurements presented in this thesis, account for the effects of ArgoNeuT when correcting for the efficiency in selecting neutrino-nucleus interactions and the resolution of the measured kinematics.
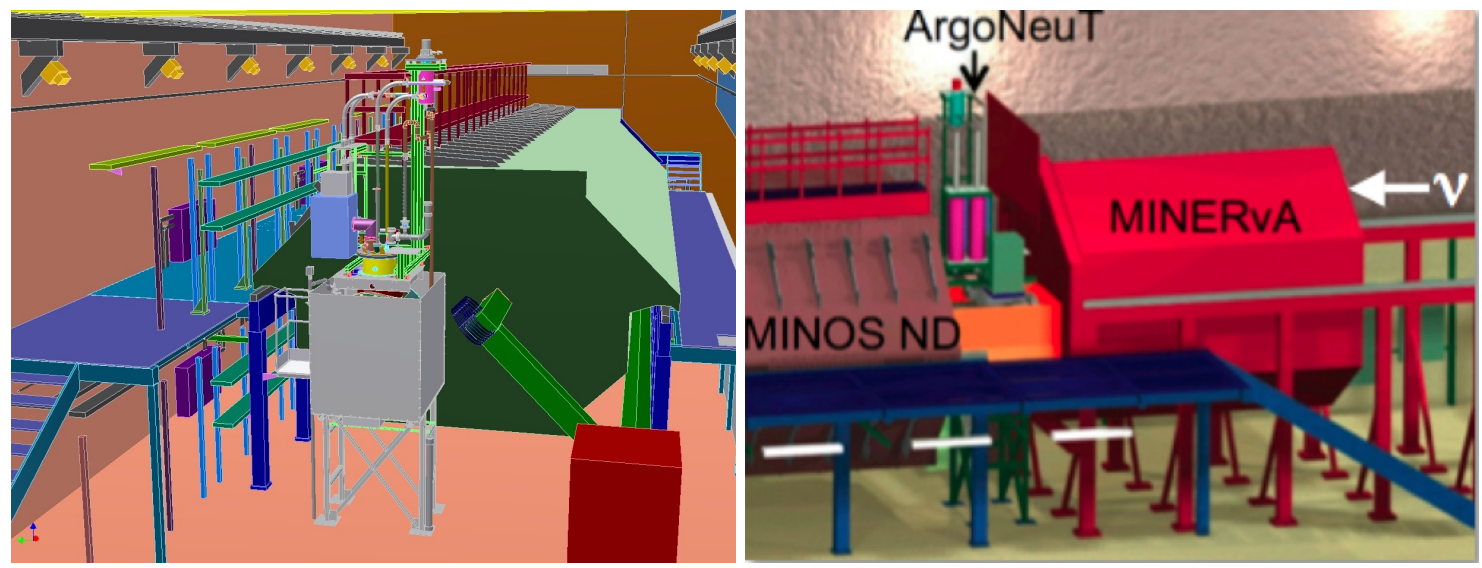

Figure 7.12: Rendering of the NuMI near detector hall. Left: The ArgoNeuT detector (gray) and the MINOS near detector (green) prior to the construction of the partial MINER $\nu \mathrm{A}$ detector. Right: The ArgoNeuT detector sitting between the MINOS near detector and the partial MINER $\nu \mathrm{A}$ detector. The images are from reference [96] 


\subsection{Reconstruction}

The reconstruction is a set of algorithms that analyze activity in the detector to determine the activity associated with each neutrino interaction, the trajectories of particles produced by interactions, and the positions at which the interactions occur. The reconstruction also determines the energy of muons from $\nu_{\mu}$ and $\bar{\nu}_{\mu}$ $\mathrm{CC}$ interactions. The information provided by the reconstruction is foundational to MINER $\nu$ A's cross section measurements.

The discussions in the following sections use the term "hit", which refers to a signal in a single optical readout channel. An optical readout channel consists of a scintillator strip, clear optical fiber, photocathode pixel, dynode chain, PMT anode, and FEB channel. Hit PE and hit energy are the PE at the photocathode pixel and energy deposited in the scintillator strip of a single readout channel, respectively. Hit PE and hit energy are estimated from the ADC counts recorded in the readout channel using a set of calibrations described in Section 7.5. Hits also have a calibrated hit time which is also discussed in Section 7.5.

\subsubsection{Time Slicing}

Several neutrino interactions can occur inside the MINER $\nu \mathrm{A}$ detector within a single readout gate due to the high intensity of the NuMI neutrino beam. The first stage of the reconstruction is time slicing, which separates detector activity within a readout gate into time periods called time slices. Nearly all activity from a neutrino interaction is contained within a single time slice, with the primary exceptions being Michel electrons and PMT afterpulsing.

The time slicing algorithm scans the activity in each readout gate with a $80 \mathrm{~ns}$ 
time window, stepping the window forward in time from the beginning of the gate. At each step the algorithm sums the PE of hits with a fired discriminator and a calibrated hit time within the window. A time slice starts when the summed PE within the window rises above 10 (the approximate amount of PE generated by a muon traversing a single scintillator plane). The time slice ends when the summed $\mathrm{PE}$ in the search window falls below 10. Hits with a fired discriminator and a calibrated hit time within the time slice are added to the slice. Hits without a fired discriminator that share a TriP-t with a hit already in the slice are also added.

A histogram of hit times in a MINER $\nu \mathrm{A}$ readout gate is shown in Figure 7.13. The different colors in the histogram represent time slices. The diffuse black hits are below the activity threshold for time slice formation and are mostly due to detector noise and PMT afterpulsing. A typical time slice is 100-200 ns wide. The width is due to the low-PE time slice threshold, which captures small, delayed hits.

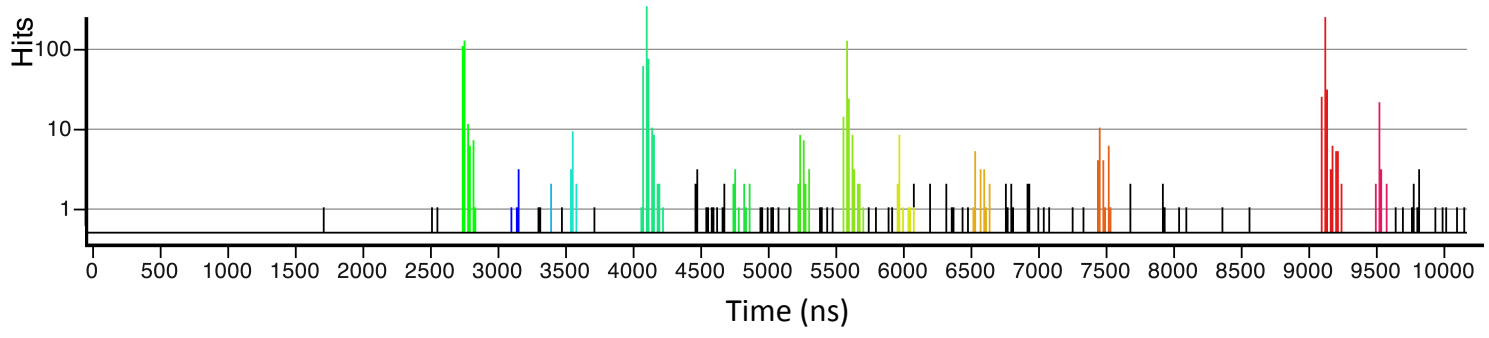

Figure 7.13: A histogram of hit times in a MINER $\nu \mathrm{A}$ readout gate. The different colors represent time slices. The diffuse black hits are below the activity threshold for time slice formation.

\subsubsection{Cluster Formation}

After time slices are formed, hits are combined into clusters. A cluster is a collection of one or more hits in adjacent scintillator strips within the same plane and time 
slice. An initially formed cluster is bound by strips which do not contain a hit. A particle typically traverses at least two strips in a single plane due to the triangular cross section of the strips. Therefore, a cluster represents the energy deposited in a single plane by one or more charged particles. Near the event vertex, clusters often contain energy from more than one particle due to the close proximity of final state particle emerging from the target nucleus. To aid higher level reconstruction (e.g. track formation) and physics analyses, clusters are classified by the number and energies of hits they contain. The cluster classifications are dependent on the density of energy deposition, particle trajectory, and particle content. The cluster classifications, illustrated in Figure 7.14, are:

- Trackable - Clusters that have a total energy between 1 and $12 \mathrm{MeV}$, fewer than 4 hits, and one or more hits with energy greater than $0.5 \mathrm{MeV}$. Hits with energy greater than $0.5 \mathrm{MeV}$ must be adjacent. Trackable clusters are often produced by minimum ionizing particles, such as a muon, that traverse the detector at lower angles with respect to the detector axis.

- Heavy Ionizing - Clusters that are not trackable clusters, have a total energy greater than $1 \mathrm{MeV}$, and have between 1 and 3 hits with energy greater than $0.5 \mathrm{MeV}$. Hits with energy greater than $0.5 \mathrm{MeV}$ must be adjacent. Heavy ionizing clusters are often produced at the end of proton trajectories where the ionization density is large, and by particles with higher angle trajectories.

- Supercluster - Clusters that have a total energy greater than $1 \mathrm{MeV}$ and do not meet the criteria for either trackable or heavily ionizing clusters. Super clusters often are wider (contain a larger number of hits) than trackable and heavy ionizing clusters and contain energy from multiple particles. Super 
clusters are often found near an event vertex. Super clusters are also produced by charged particles with trajectories nearly perpendicular to the detector axis.

- Low Activity - A cluster with a total energy less than $1 \mathrm{MeV}$ that is not a cross talk cluster.

- Cross Talk - Clusters consisting of one or more hits identified as cross talk. The identification of cross talk is discussed in Sections 7.1.5 and 7.5.9.
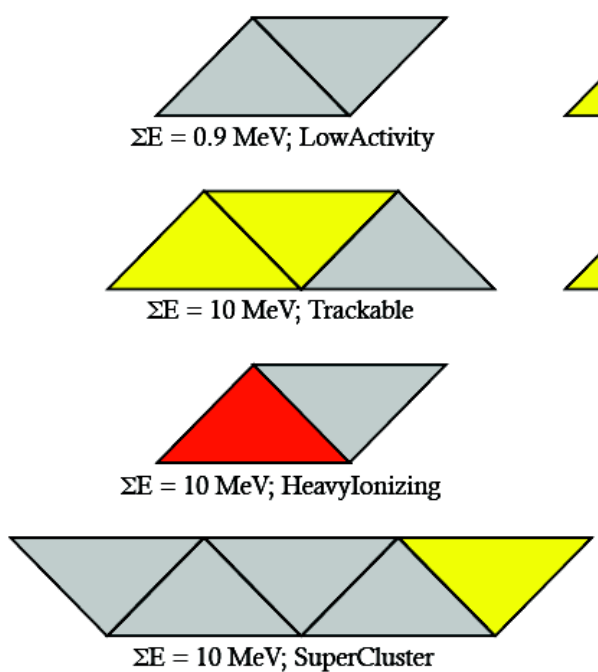

\begin{tabular}{|c|c|}
\hline Low E & $<1 \mathrm{MeV}$ \\
\hline Medium E & $1-8 \mathrm{MeV}$ \\
\hline High E & $8+\mathrm{MeV}$ \\
\hline
\end{tabular}
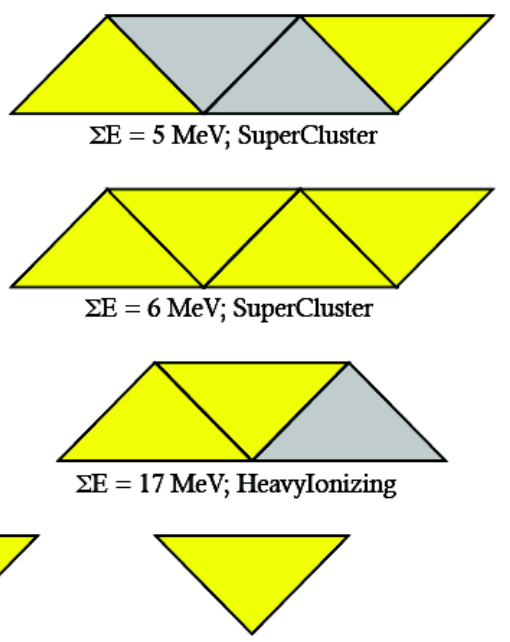

$\Sigma \mathrm{E}=5 \mathrm{MeV} ;$ Trackable

Figure 7.14: Cluster types in MINER $\nu$ A. Figure from G. Perdue of the MINER $\nu$ A collaboration.

Each cluster has a $2 \mathrm{D}$ position. The $\mathrm{Z}$ position of a cluster is the $\mathrm{Z}$ position of the plane containing the cluster. The transverse position (the position in the direction perpendicular to the strips in the XY plane) of a cluster is the energy weighted average transverse position of the strips in the cluster within the plane. 
The cluster time is defined as the calibrated time of the most energetic hit in the cluster.

\subsubsection{Track Formation}

A track is a reconstruction of a particle's 3D trajectory in the detector. Tracks are formed using the $2 \mathrm{D}$ positions of clusters and the three plane orientations. In addition to providing a measure of a particle's position and direction, tracks are used to locate the position at which a neutrino interaction occurred.

The first stage of the track reconstruction forms track candidates, which are 2D tracks in each of the three plane orientations. Track candidates consist of trackable and highly ionizing clusters in the same time slice and orientation. A track candidate is formed from one or more track seeds, which are a set of three clusters in consecutive planes in an orientation that satisfy a straight line fit. Track seeds with consistent slopes and intercepts are merged into a track candidate. Track candidates may not have more than one cluster in a plane, but may cross planes without a cluster. This allows a track candidate to cross dead strips, strips undergoing dead time, and super clusters.

Track candidates in different orientations that are in the same time slice, span similar regions in $\mathrm{Z}$ of the detector, and are consistent with the same 3D line are merged into a track. A track contains two or three track candidates from different plane orientations. The 3D position and direction at each plane along the track, which are grouped into a "node", are fit from the 2D positions of the clusters in the track and their plane orientations. The fit uses a Kalman filter that allows for multiple Coulomb scattering. After the fit, the track is projected onto each plane orientation and untracked clusters, including superclusters, with positions 
consistent with the projection are added to the track. The track reconstruction algorithm requires clusters spanning a minimum of 9 consecutive planes to form a track, which results in only tracks with an angle less than 70 degrees with respect to the $\mathrm{Z}$ axis being formed. Figure 7.15 shows tracks formed from the activity of a neutrino interaction.

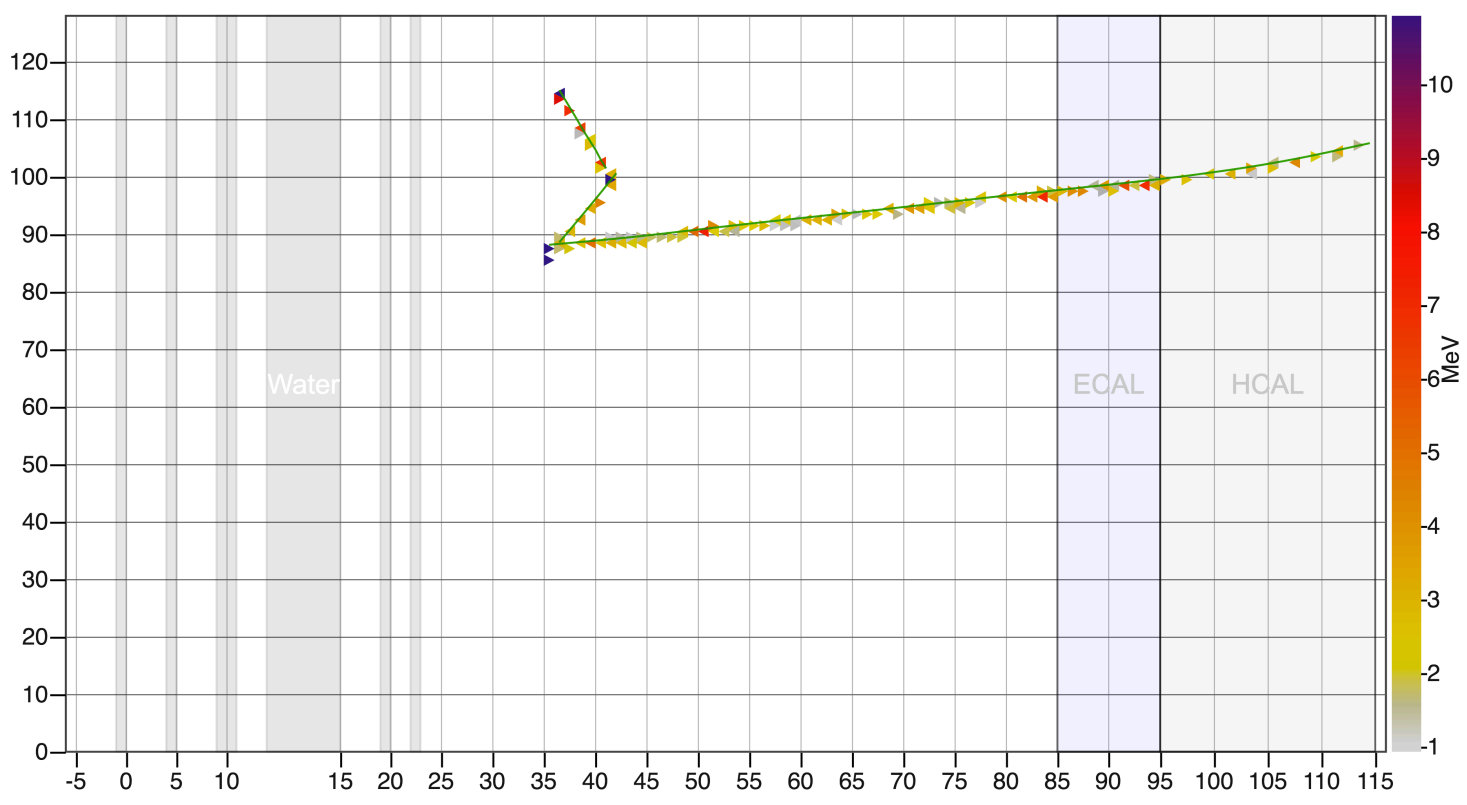

Figure 7.15: Tracks reconstructed from activity of a neutrino interaction in the MINER $\nu \mathrm{A}$ detector. Only the $\mathrm{X}$ planes are shown. The long track exiting the downstream end of the MINER $\nu \mathrm{A}$ detector represents a muon. The two shorter tracks represent a hadron produced in the neutrino interaction that scattered in the detector.

The position and angle resolution of tracks were measured using rock muons, which are muons produced by neutrino interactions in the rock of the detector hall upstream of the MINER $\nu$ A detector. Muons in MINER $\nu$ A deposit energy primarily by ionization and produce long, straight tracks. The range in $\mathrm{Z}$ spanned by each muon was split into an upstream region and a downstream region, and a track was was formed in each region from the clusters produced by the muon. The position 
and angle difference between the tracks at the boundary of the regions served as a measure of the position and angle resolution of the tracks, respectively. The position and angle resolution of rock muon tracks (Figure 7.16) was measured to be $\sim 3 \mathrm{~mm}$ and $\sim 0.02$ radians, respectively, and is consistent between data and the MINER $\nu \mathrm{A}$ Monte Carlo simulation (Section 7.6).
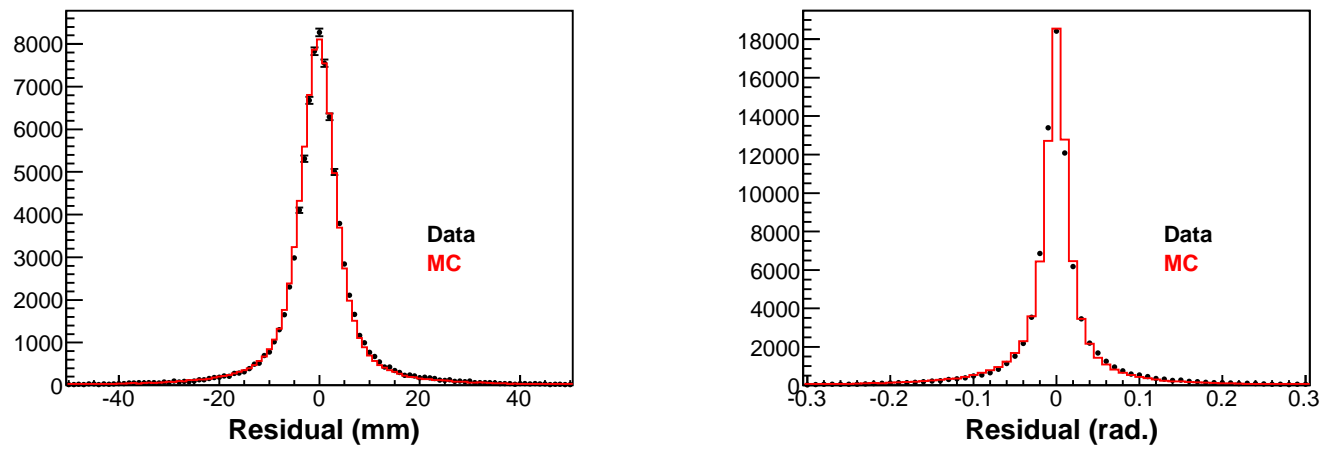

Figure 7.16: Position (left) and angle (right) resolution of rock muon tracks in the MINER $\nu \mathrm{A}$ data and simulation (MC). The figures are from reference [88].

\subsubsection{Muon Energy and Charge Reconstruction}

Muons produced by neutrino interactions in the MINER $\nu \mathrm{A}$ detector usually exit the detector. The MINER $\nu \mathrm{A}$ detector does not have magnetic field, and therefore does not provide a measurement of either the energy or charge of a muon. The MINOS near detector, which is located immediately downstream of the MINER $\nu \mathrm{A}$ detector and has a magnetic field, is used to measure both the energy and charge of muons that originate in MINER $\nu \mathrm{A}$ and enter MINOS. The energy and charge of a muon in MINOS are measured by the curvature of the muon track in the magnetic field. Muon energy is also measured by the range of the track if the muon stops within the fully instrumented upstream calorimeter region (Figure 7.11). Track formation 
in MINOS is described in reference [97].

Reconstructing a muon in both MINER $\nu \mathrm{A}$ and MINOS requires matching the muon track in MINER $\nu \mathrm{A}$ to the muon track in MINOS. Tracks considered for matching are required to be within $200 \mathrm{~ns}$ of each other. The MINER $\nu \mathrm{A}$ track is required to end within the last five modules of MINER $\nu \mathrm{A}$, and the MINOS track is required to begin within the first four scintillator planes of MINOS. The MINER $\nu \mathrm{A}$ track is projected forwards into MINOS and the MINOS track is projected backwards into MINER $\nu \mathrm{A}$. The tracks are matched if the MINOS projection is within $40 \mathrm{~cm}$ of the MINER $\nu \mathrm{A}$ track in the plane containing the end of the MINER $\nu \mathrm{A}$ track and the MINER $\nu \mathrm{A}$ projection is within $40 \mathrm{~cm}$ of the MINOS track in the plane containing the start of the MINOS track.

If matching MINER $\nu \mathrm{A}$ and MINOS tracks are not found using the above projection method, a closest approach method is used. For MINER $\nu \mathrm{A}$ and MINOS tracks considered for matching, the MINER $\nu \mathrm{A}$ track is projected forwards and the MINOS track is projected backwards. The distance of closest approach between the projections is calculated. The tracks are matched if the projections nearly cross. Matching by the closest approach method allows for muon scattering in the passive materials, as well as the ArgoNeuT detector, between the end of the MINER $\nu \mathrm{A}$ track and the beginning of the MINOS track.

An event display of matched MINER $\nu \mathrm{A}$ and MINOS tracks is shown in Figure 7.17. Nearly all $(>99 \%)$ matched MINER $\nu$ A and MINOS tracks represent a muon since other charged particles in MINER $\nu \mathrm{A}$ either interact or are absorbed before reaching the downstream end of the MINER $\nu \mathrm{A}$ detector. MINER $\nu \mathrm{A}$ tracks with an angle greater than 20 degrees with respect to the $\mathrm{Z}$ axis will not have a matching track in MINOS due to the transverse span of the MINOS detector. 

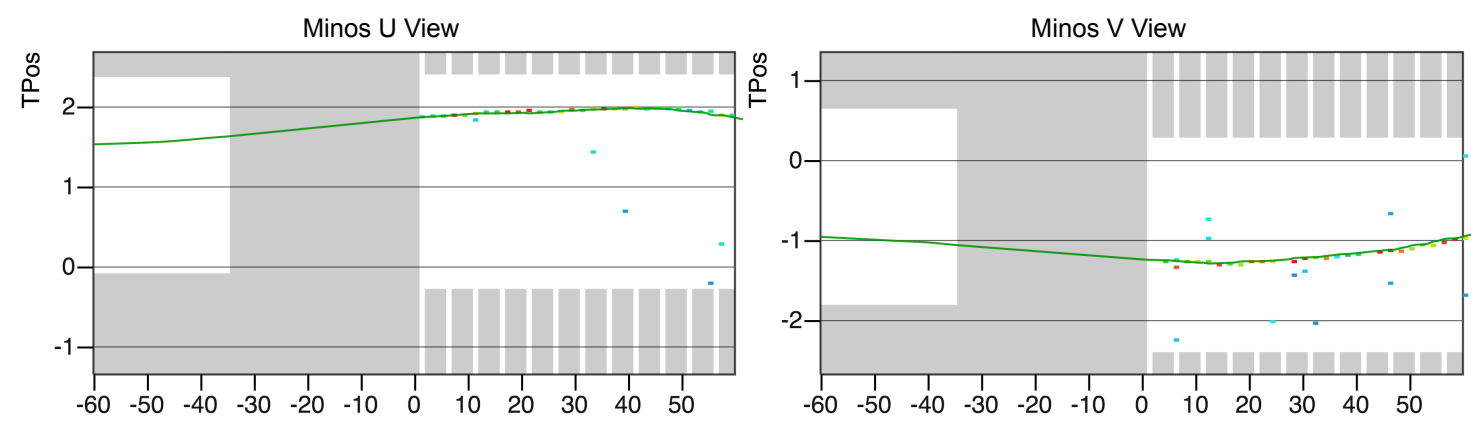

Figure 7.17: Event display of matched MINER $\nu \mathrm{A}$ and MINOS tracks in the U (left) and $\mathrm{V}$ (right) plane orientations of the MINOS near detector

The muon track in MINOS gives the energy of the muon at the front of MINOS. To determine the energy of a muon at its origin in MINER $\nu \mathrm{A}$, the energy deposition along the muon track in MINER $\nu \mathrm{A}$ is calculated and added to the energy of the muon at the front of MINOS [98, 99, 100]. Muons in MINER $\nu$ A deposit energy primarily by ionization, which is accurately described by the Bethe-Bloch formula:

$$
-\frac{d E}{d x}=K z^{2} \frac{Z}{A} \frac{1}{\beta^{2}}\left[\frac{1}{2} \ln \frac{2 m_{e} c^{2} \beta^{2} \gamma^{2} T_{\max }}{I^{2}}-\beta^{2}-\frac{\delta}{2}\right],
$$

where $\frac{d E}{d x}$ is the average energy deposited per length traversed in a material by an incident charged particle, $K=4 \pi N_{A} r_{e}^{2} m_{e} c^{2}, N_{A}$ is Avogadro's constant, $r_{e}$ is the classical electron radius, $m_{e}$ is the electron mass, $c$ is the speed of light, $z e$ is the charge of the incident particle, $Z$ is the atomic number of the material, $A$ is the atomic mass of the material, $T_{\max }$ is the maximum kinetic energy which can be imparted to a free electron in a single collision, $I$ is the mean excitation energy of the material, $\delta$ is the density effect correction to ionization energy loss, and $\beta$ and $\gamma$ are the relativistic kinematic variables [1]. The Bethe-Bloch $\frac{d E}{d x}$ for muons, pions, and protons in various materials is shown in Figure 7.18. The path length of the muon in each layer of the MINER $\nu \mathrm{A}$ detector is determined from the MINER $\nu \mathrm{A}$ track. 
The energy deposited by the muon in each layer is calculated from the Bethe-Bloch formula, starting from the downstream end of the MINER $\nu \mathrm{A}$ detector and ending at the upstream end of the MINER $\nu \mathrm{A}$ track, and added to the energy of the muon at the front of MINOS. The muon energy is updated in each layer since the amount of energy deposited is dependent on the energy of the incident particle. For the partial MINER $\nu$ A detector, muon energy loss in ArgoNeuT, which is typically $\lesssim 100 \mathrm{MeV}$, is not included in the muon energy calculation. Muon energy loss in ArgoNeuT is accounted for when correcting for the muon energy resolution (Section 8.9.2). The resolution and systematic uncertainty of the muon energy reconstruction for the results reported in this thesis are given in Sections 8.6 and 8.13.5, respectively.

\subsection{Calibrations}

Detector activity is recorded in ADC counts, which is digitized charge. In order to be processed by the reconstruction and ultimately be used in physics analyses, digitized charge in a readout channel must be converted to $\mathrm{PE}$ at the photocathode and energy deposited in the strip. This is accomplished using a set of calibrations. The calibrations are dependent on optical readout channel $c$ and change with time t. Hit $\mathrm{ADC}$ is converted to $\mathrm{PE}$ at the photocathode by

$$
\mathrm{PE}=(\mathrm{ADC}-P(c, t)) \times Q(c, \mathrm{ADC}) \times \frac{1}{G(c, t)},
$$

where $P(c, t)$ is the latent channel noise referred to as the pedestal, $Q(c, \mathrm{ADC})$ is the ADC to PMT anode charge conversion factor for the FEB channel, and $G(c, t)$ is the PMT channel gain (the mean amplified charge at the anode per PE generated at the photocathode). PE at the photocathode is converted to energy deposited in 

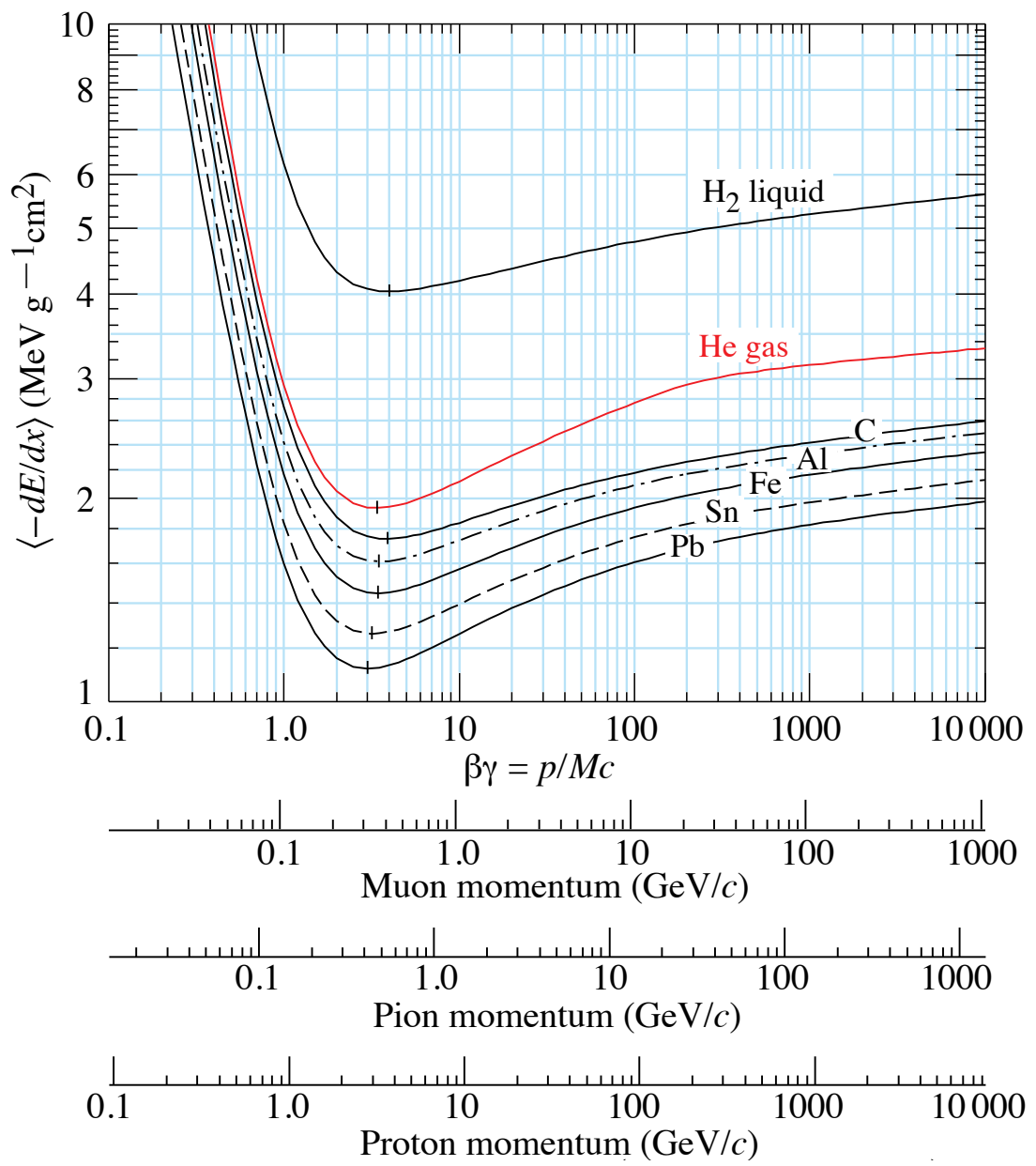

Figure 7.18: Mean energy deposition rate for muons, pions, and protons in various materials per the Bethe-Block formula. Particles in MINER $\nu \mathrm{A}$ typically have momentum $\lesssim 10 \mathrm{GeV} / \mathrm{c}$. The figure is from reference [1]. 
the strip by

$$
E=\mathrm{PE} \times A(c) \times S(c, t) \times M(t)
$$

where $A(c)$ is the correction for the attenuation of scintillation light in the WLS and clear optical fibers, $S(c, t)$ is the strip response correction, and $M(t)$ is the detector energy scale. The raw time of a hit, which is a time stamp from the readout electronics, is also converted to a calibrated time using a set of timing corrections. The calibrations and a measurement of the cross talk are described in the following sections.

\subsubsection{Pedestals}

A pedestal is the latent noise in an electronics channel. Pedestals are measured between neutrino beam pulses when the signals in the detector are primarily pedestals and larger signals due to cosmic ray muons and radiation from the rock of the detector hall. The pedestals for all channels in the detector are measured once every 10 hours for a period of 30 min. An example of a measured pedestal distribution for a channel is shown in Figure 7.19. The measured pedestal for a channel is the mean of the measured pedestal distribution for the channel, where large background signals are excluded using an outlier removal scheme based on Peirce's criterion [101].

\subsubsection{FEB Response}

Prior to installing the FEBs in the detector, the response of each channel on each FEB was measured on a test stand by injecting charge into the channel and measuring the resulting low, medium, and high gain ADC counts. The measured ADC counts as a function of injected charge for each gain was fit with a tri-linear func- 


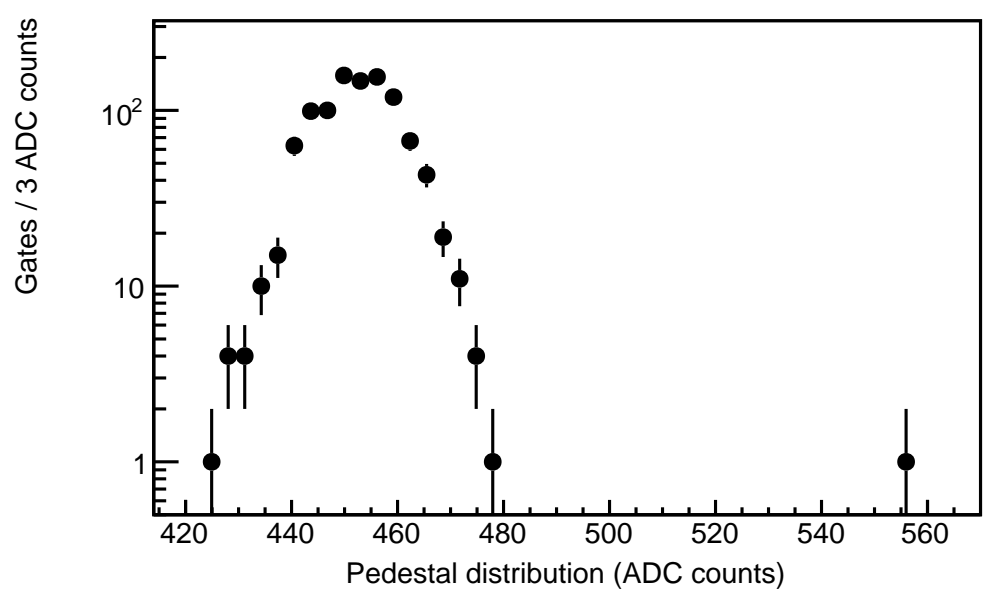

Figure 7.19: An example of a pedestal distribution for a single channel measured from one $16 \mu$ s readout gate. The signal 100 ADC counts above the pedestal level is removed before calculating the pedestal mean. The figure is from reference [88].

tion, which is used to convert ADC counts to PMT anode charge in calculating hit $\mathrm{PE}$ (Equation 7.2). The measured response for a single FEB channel is shown in Figure 7.20.

\subsubsection{PMT Gains}

The gain of a PMT channel is the mean output charge at the anode per PE. Gains vary in time primarily due to PMT aging and changes in the voltage supplied to the dynodes. The gain for each channel in the detector is measured daily by flashing the photocathode pixel with blue LED light, which is referred to as light injection, and measuring the resulting charge at the anode. A light injection box containing blue LEDs sends pulses of light to each PMT box via two optical cables. A diffuser spreads out the light inside the box where the light reaches the photocathode grid through the cookie. Light reaching a pixel generates a small (few PE) signal. Light 


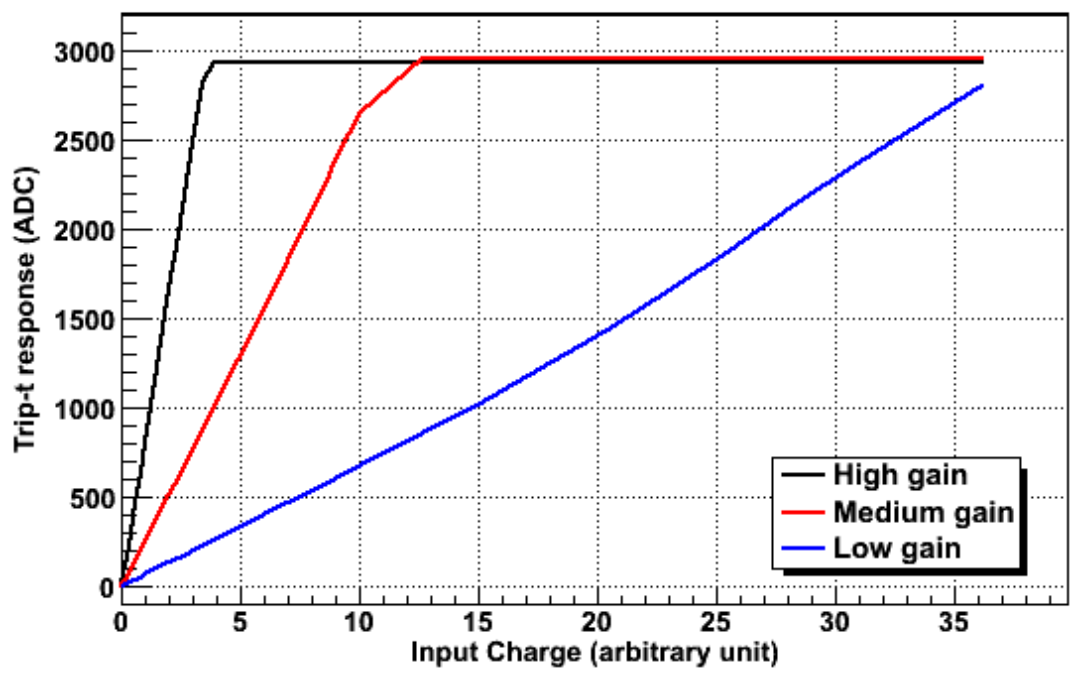

Figure 7.20: The measured low, medium, and high gain response for a single FEB channel. Figure is from reference [88].

injection data is collected between the $10 \mathrm{~ns}$ long neutrino beam pulses that occur every $2.2 \mathrm{~s}$. The LED pulses are $\sim 30 \mathrm{~ns}$ wide, which allows rapid measurements of the gains between beam pulses. The calculation of the gains from the light injection data is given in reference [88]. An example of the measured gains for all PMT channels in the detector is shown in Figure 7.21. The statistical uncertainty for each measured channel is 3-5\%. The measured gains are used to convert analog charge at the PMT anodes to PE at the photocathode pixels.

\subsubsection{Attenuation Corrections}

Scintillation light is attenuated within the WLS and clear optical fibers. The attenuation within each optical channel is factorized into attenuation in the strip, attenuation in the WLS fiber in the baggie, and attenuation in the clear optical cable. 


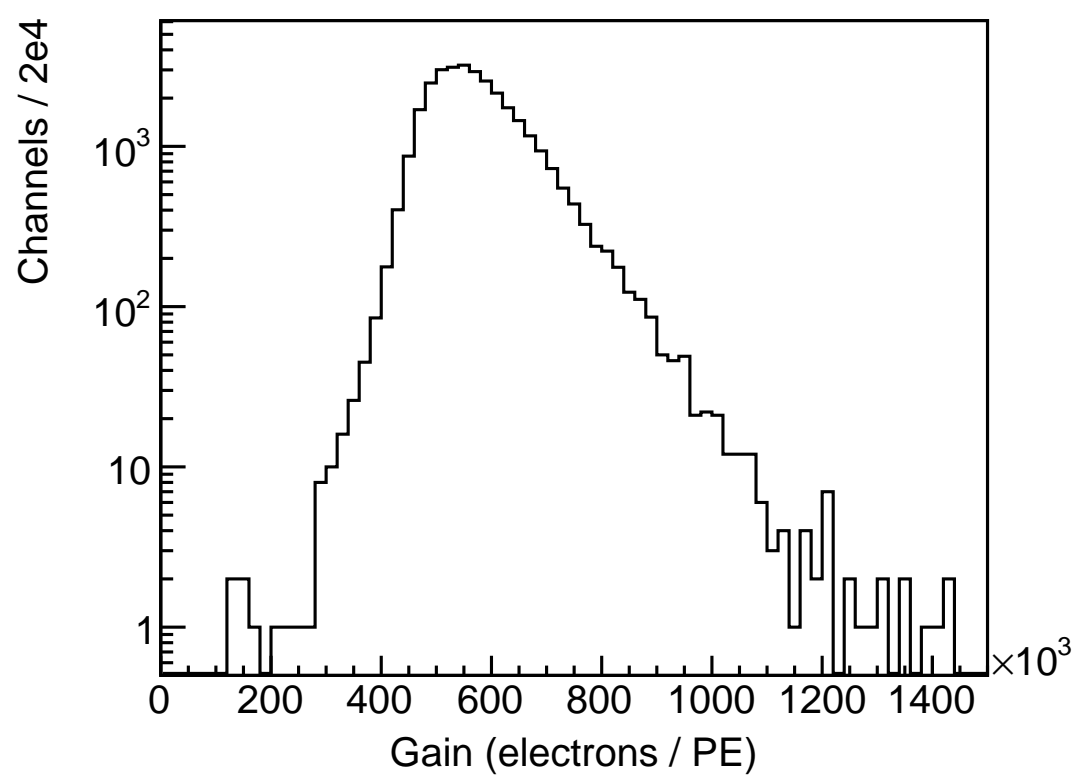

Figure 7.21: Distribution of the measured gains of all PMT channels in the MINER $\nu \mathrm{A}$ detector on April 1, 2010. The figure is from reference [88].

The attenuation in each strip was was measured as a function of position along the strip using a custom "module mapper." Prior to installing a module in the detector, the module was placed in a fixture in the mapper, which scanned the upright face of the module with a Cs-137 source (Figure 7.22). The response of each scintillator strip in the module was measured as function of the source position, from which the attenuation of each strip as a function of position along the strip was measured. The measured strip attenuation includes manufacturing defects such as voids in the optical epoxy coupling the scintillator and WLS fiber.

The attenuation lengths of the WLS and clear optical fibers were measured on a test stand. Attenuation in the WLS fiber in the baggie and in the clear optical cable is estimated as $\exp (-L / \lambda)$, where $L$ is the length of the fiber/cable and $\lambda$ is 


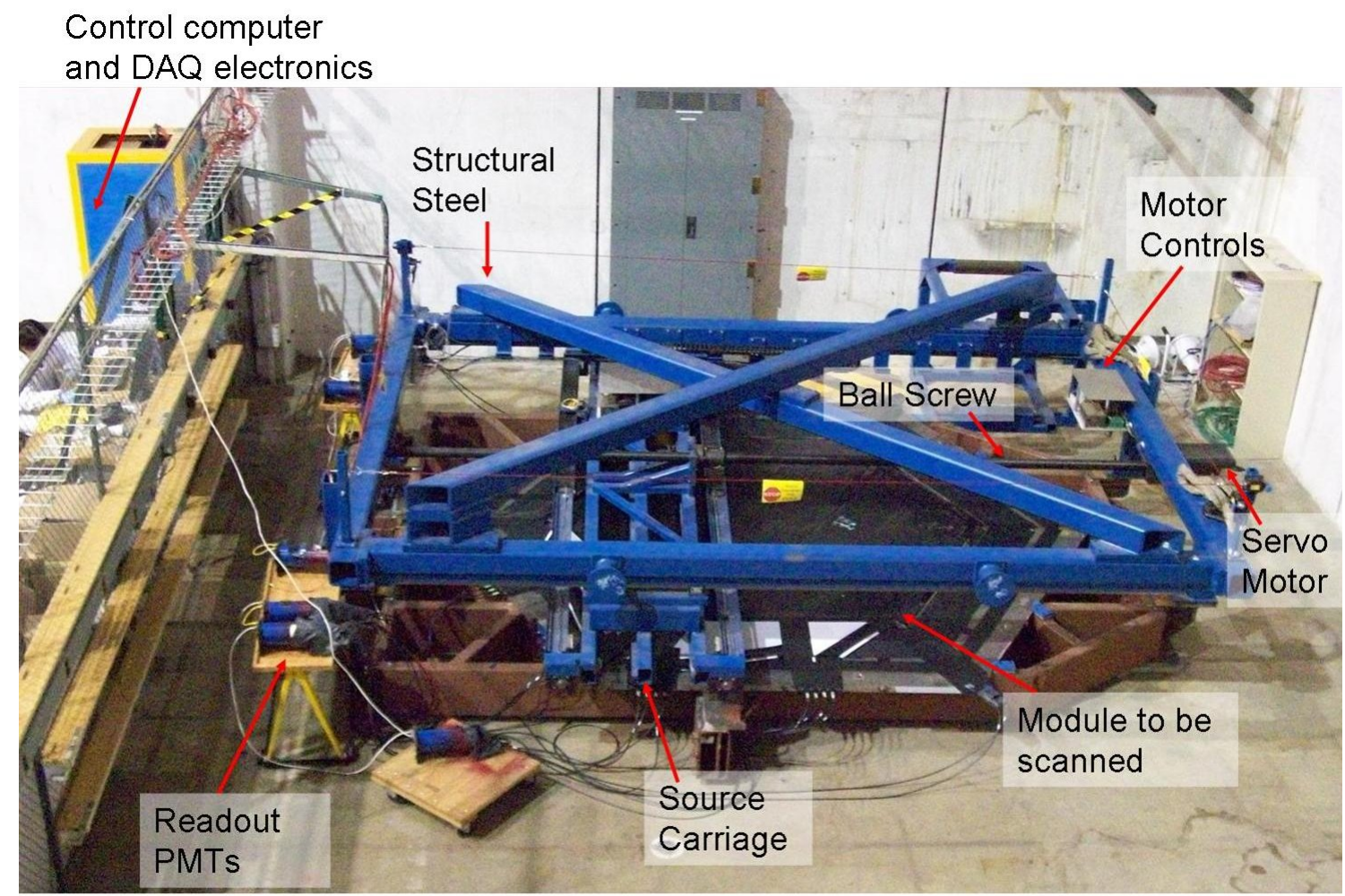

Figure 7.22: The MINER $\nu \mathrm{A}$ module mapper. Each module was placed lying down in a fixture in the mapper. The mapper scanned the upright face of the module with a radioactive (Cs-137) source. The response of each scintillator strip in the module was measured as function of the source position. The figure is from reference [88].

the measured attenuation length.

The estimated total attenuation in an optical channel is the product of the measured strip attenuation and estimated attenuations in the WLS fiber in the baggie and clear optical cable. The attenuation correction is the reciprocal of the estimated total attenuation. For each hit, the attenuation is corrected to the length center of the strip. For each cluster on a track, the cluster energy is corrected for attenuation to the position of the node. 


\subsubsection{Scintillator Plane Alignment}

Corrections are made for misalignments of the scintillator planes as installed in the detector. Misalignments of a plane are rotations about the $\mathrm{X}, \mathrm{Y}$, and $\mathrm{Z}$ axes and displacements in the transverse direction (perpendicular to the strips in the XY plane), the longitudinal direction (parallel to the strips), and Z. Misalignments in the XY plane that affect track reconstruction are rotations about the $\mathrm{Z}$ axis and transverse displacements. Longitudinal displacements do not affect reconstruction since a plane does not give the longitudinal position of an energy deposition. Rotations about the $\mathrm{Z}$ axis and transverse displacements of the installed planes are small, which allows these misalignments to be measured using rock muon tracks.

The procedure for measuring the plane misalignments is as follows. For each hit along a rock muon track, the point at which the muon intersected the triangular base of the strip containing the hit was determined from the track (Figure 7.23). The hit energy was corrected to normal incidence by multiplying the hit energy by $\cos \theta$, where $\theta$ is the angle between the direction of the track in the strip and the normal to the base of the strip. In each strip, the average hit energy is maximum at the transverse center of the strip where the muon path length at normal incidence is greatest (Figure 7.24). Average hit energy in a strip as a function of strip transverse displacement is fit with the strip shape to determine the transverse displacement of the strip. To determine the transverse displacement of a plane, all strips in the plane are fit simultaneously, where the strips are shifted in unison in the fit. To determine the rotation of a plane about the $\mathrm{Z}$ axis, the transverse displacement of the plane is measured in six bins in hit longitudinal position in the plane, where the longitudinal position of a hit is determined from the track (Figure 7.24). The transverse displacement of the plane as a function of longitudinal position is fit with 
a straight line. The rotation of the plane about the $\mathrm{Z}$ axis is determined from the slope of the straight line fit. One iteration of this procedure is sufficient to measure the misalignments of all the planes. Typical transverse displacements are $\lesssim 33 \mathrm{~mm}$ (the width of a strip) and the rotations are on the order of a few mrad. The plane misalignments are corrected during reconstruction.

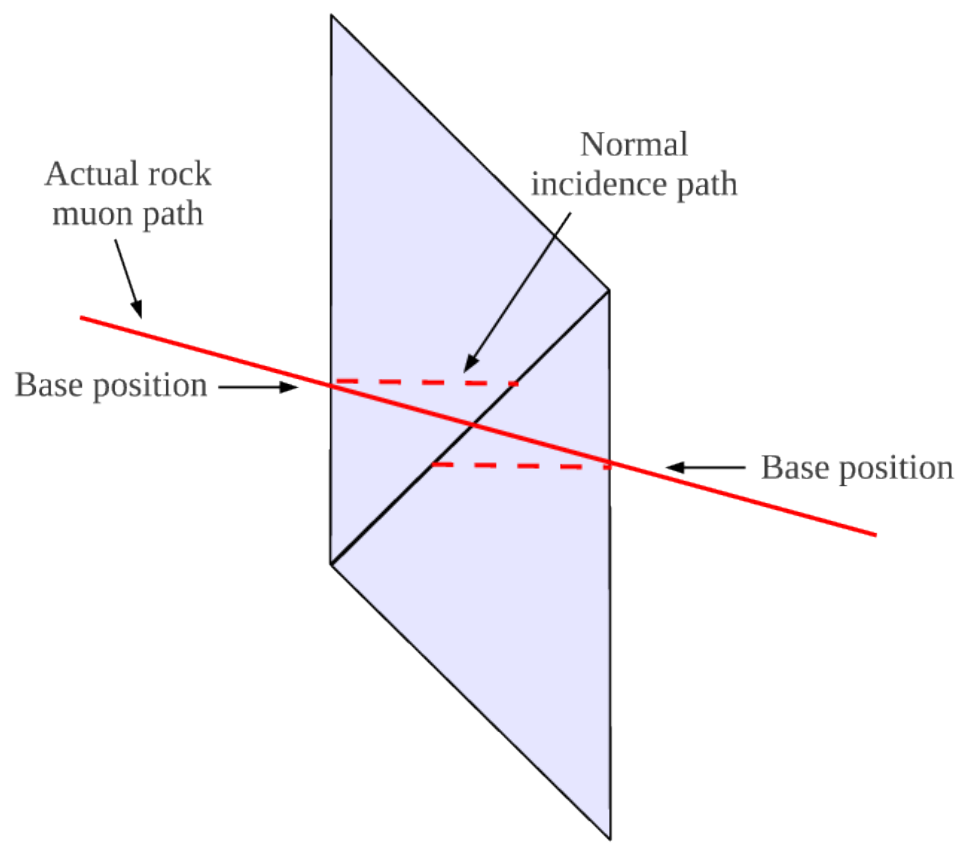

Figure 7.23: Illustration of a rock muon traversing a plane. For measuring scintillator plane misalignment, the muon track is used to determine point at which the muon intersects the triangular base of each strip and the angle between the direction of the muon and the normal to the triangular base. The figure is from reference [102].

\subsubsection{Strip Response Corrections}

The response of the scintillator strips, as seen by the photocathodes, varies across the detector. Strip response variation primarily results from light loss in the optical connectors between the WLS fibers and photocathodes, manufacturing defects, 

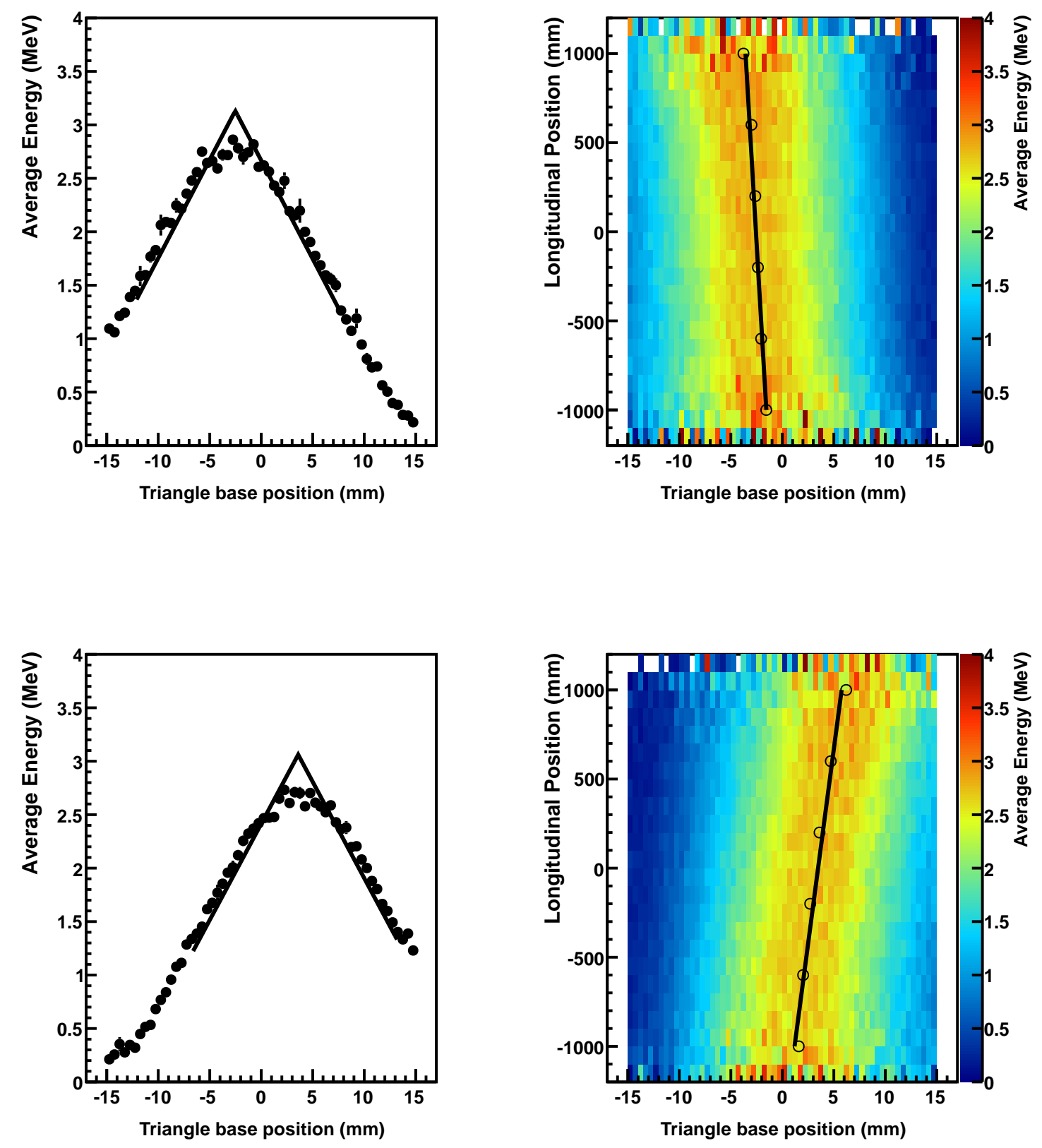

Figure 7.24: Fits for the transverse displacements and rotations of two scintillator planes. The left plot shows the average muon hit energy, corrected to normal incidence, in a strip as a function of strip transverse displacement, which is fit with the strip shape to locate the transverse displacement of the strip. The right plot shows the average muon hit energy, corrected to normal incidence, in all strips in a plane as a function of strip transverse displacement and longitudinal hit position. The straight line is a fit to the transverse displacement of the plane in six bins in longitudinal hit position. The figure is from reference [88]. 
damage during detector construction, and scintillator aging (Section 7.5.7) coupled with the different manufacturing times of the strips. Strip response variation is corrected by a set of strip response corrections which make strip response uniform across the detector and are used in estimating hit energy.

The strip response corrections are measured from the energy deposited per unit length, $\frac{d E}{d x}$, in strips along rock muon tracks. Muons in MINER $\nu \mathrm{A}$ deposit energy primarily by ionization. Mean ionization $\frac{d E}{d x}$ varies with particle energy. Peak (i.e. the most probable) ionization $\frac{d E}{d x}$ is approximately constant [1]. Peak $\frac{d E}{d x}$ in strips along rock muon tracks is used to measure the strip response corrections. $\frac{d E}{d x}$ in a strip is calculated from the hit energy and muon path length in the strip. The path length in a strip is measured from the reconstructed $3 \mathrm{D}$ position and direction of the track in the plane containing the strip. When measuring the strip response constants, hit energy is calculated per Equation 7.3, where the attenuation is corrected to the $3 \mathrm{D}$ track position and the strip response correction is set to unity.

The strip response corrections change with time due to hardware swaps, such as replacing a PMT. It is therefore necessary to maximize the frequency of remeasuring the strip response corrections, which in turn requires minimizing the number of rock muons used to measure the corrections. Measuring peak $\frac{d E}{d x}$ for a strip requires several thousand rock muon hits in the strip. Measuring the truncated mean $\frac{d E}{d x}$ for a strip, which approximates peak $\frac{d E}{d x}$, requires a few hundred rock muon hits in the strip. The latter enables measurement of the strip response corrections from a sample of $\sim 10^{5}$ rock muons, which corresponds to $2-4$ weeks of data taking in the LE neutrino beam. 
The strip response correction $S_{i}$ for strip $i$ is calculated as

$$
S_{i}=\frac{1}{u_{i}}\left(\frac{1}{N_{s}} \sum_{j}^{N_{s}} \frac{1}{u_{j}}\right)^{-1},
$$

where $u_{i}$ is the truncated mean $\frac{d E}{d x}$ in strip $i$ and $N_{s}$ is the number of good (not dead or underperforming) strips in the detector. The definition of $S_{i}$ gives an average strip response correction of unity so the strip response corrections preserve the detector energy scale (Section 7.5.7).

The passive layers in the ECAL, HCAL, and nuclear targets region bias the strip response corrections in their respective regions of the detector. The passive layers absorb delta rays (knock-on electrons) generated by the rock muons, which changes the shape and truncated mean of the $\frac{d E}{d x}$ distribution in the strips immediately downstream of the layers. Peak $\frac{d E}{d x}$, in contrast, is insensitive to delta rays (see Section 7.5.7). The bias is corrected by plane response corrections which are calculated from the truncated mean and peak $\frac{d E}{d x}$ of rock muon hits in the planes, where hit energy is corrected by the measured strip response corrections. Aggregating rock muon hits in all strips of a plane enables the peak $\frac{d E}{d x}$ in the plane to be measured. Peak $\frac{d E}{d x}$ is determined from a fifth order polynomial fit. The plane response correction $P_{k}$ for plane $k$ is calculated as

$$
P_{k}=\frac{u_{k}}{w_{k}}\left(\frac{1}{N_{p}} \sum_{l}^{N_{p}} \frac{u_{l}}{w_{l}}\right)^{-1}
$$

where $u_{k}$ and $w_{k}$ are the peak and truncated mean $\frac{d E}{d x}$ in a plane, respectively, and $N_{p}$ is the number of planes in the detector. The definition of $P_{k}$ gives an average plane response correction of unity so the plane response corrections preserve the 
detector energy scale. The strip response correction $S_{i}$ for strip $i$ is multiplied by the plane response correction $P_{k}$ for plane $k$ containing strip $i$ to give the final strip response correction.

\subsubsection{Detector Energy Scale}

The MINER $\nu$ A detector energy scale $M$ is the deposited energy per observed amount of scintillation light in a strip. $M$ is used to convert an observed amount of scintillation light in a strip, measured in strip response and attenuation corrected PE, to an energy deposition. This section describes the procedure for calibrating the detector energy scale. Since the variation in strip response is corrected, one $M$ is calibrated for all strips in the detector.

The light yield is the number of scintillation photons generated per energy deposited in a strip. The optical model in the Monte Carlo (MC) simulation of the MINER $\nu$ A detector (Section 7.6.2) calculates the amount of scintillation light arriving at the photocathode for a simulated energy deposition in a strip. The light yield factor, $Y$, corrects the light yield in the MC. $Y$ is calibrated to match the light yield in the MC to data, which ensures that statistical fluctuations in the number of PEs generated at the photocathode in data and MC are identical. The scintillator properties are assumed to be uniform across the detector, so one $Y$ is calibrated for all strips in the detector.

The calibration of $M$ and $Y$ uses the response of MINER $\nu$ A's scintillator strips to rock muons in both data and MC. Muons in MINER $\nu$ A deposit energy primarily by ionization, which is accurately described by the Bethe-Bloch formula (Equation 7.1). The MC uses Geant4 $[82,83]$ to simulate particle propagation in the MINER $\nu \mathrm{A}$ detector, and Geant4 uses the Bethe-Bloch formula in simulating the energy deposition 
of muons in MINER $\nu \mathrm{A}$. For calibrating $M$ and $Y$, the simulated energy deposited by rock muons in $\mathrm{MC}$ serves as the expected energy deposition, and hit $\mathrm{PE}$ for strips traversed by rock muons in data serves as the expected light level. Cluster energy along rock muon tracks represents the measured energy deposited per plane by rock muons. $M$ is calibrated by matching cluster energy along rock muon tracks in data to MC. $Y$ is calibrated by matching cluster $\mathrm{PE}$ along rock muon tracks in $\mathrm{MC}$ to data.

The amount of ionization energy loss is dependent upon the muon's energy. For this reason, only momentum-analyzed rock muons are used for the calibration. For the data sample, rock muons that traverse the full length of MINER $\nu \mathrm{A}$ and are reconstructed in both MINER $\nu \mathrm{A}$ and MINOS are selected. The momentum of the MINOS track and the materials traversed in MINER $\nu \mathrm{A}$ are used to reconstruct the rock muon's momentum at its entrance point into MINER $\nu \mathrm{A}$. The reconstructed momentum, position, and direction of the rock muons selected in data are input to $\mathrm{MC}$ to generate a data-driven rock muon $\mathrm{MC}$ sample. This is done so the rock muon momentum and angle spectra in the data and MC samples match.

$M$ is calibrated by matching peak reconstructed cluster energy along rock muon tracks in the data sample to that in the MC sample (Figure 7.25). All planes in the detector are sampled. Reconstructed cluster energy in both data and MC is calculated from the sum of the strip response and attenuation corrected PE of the strips composing the cluster and a trial detector energy scale, $M_{T}$, that is correct to $\sim 15 \%$. Peak reconstructed cluster energy serves as the expected muon energy deposition per plane. Peak, rather than average, reconstructed cluster energy is used to tune $M$ to minimize effects from passive materials and muon energy loss. The absorption and propagation of delta rays (knock-on electrons) is different between subdetectors 
(nuclear targets region, tracker region, electromagnetic and hadronic calorimeters) due to their passive materials, and delta rays contribute primarily to the high-side tail of the cluster energy distribution. Muons loose 2-3 GeV of their energy traversing the full length of MINER $\nu \mathrm{A}$, and while average muon energy deposition changes with muon energy, peak energy deposition is approximately constant [1]. Peak reconstructed cluster energy is determined by fitting the reconstructed cluster energy distribution in the region above half-height with a fifth order polynomial. The MC reconstructed cluster energy, calculated from $M_{T}$, is corrected to the true cluster energy, which is the sum of the simulated energy depositions in the strips composing the cluster. The correction is determined from the slope of reconstructed vs. true cluster energy in the MC sample (Figure 7.26), where the slope determined from a straight line fit with zero intercept. The calibrated detector energy scale is calculated as

$$
M=M_{T} \frac{E_{M C}}{a} \frac{1}{E_{\text {data }}},
$$

where $E_{M C}$ and $E_{d a t a}$ are the data and $\mathrm{MC}$ peak reconstructed cluster energies, respectively, and $a$ is the slope of reconstructed vs. true cluster energy in the MC sample. The calibrated detector energy scale is used to calculate hit energy in both data and MC.

The light yield factor, $Y$, is calibrated by matching peak cluster PE along rock muon tracks in the MC sample to that in the data sample. All planes in the detector are sampled. Cluster PE is the total PE of the strips composing the cluster. The cluster PE distributions in the data and MC samples are shown in Figure 7.28, where the MC sample was simulated with a trial light yield factor, $Y_{T}$, that is correct to $\sim 15 \%$. As with the detector energy scale calibration, peak cluster PE is used to 

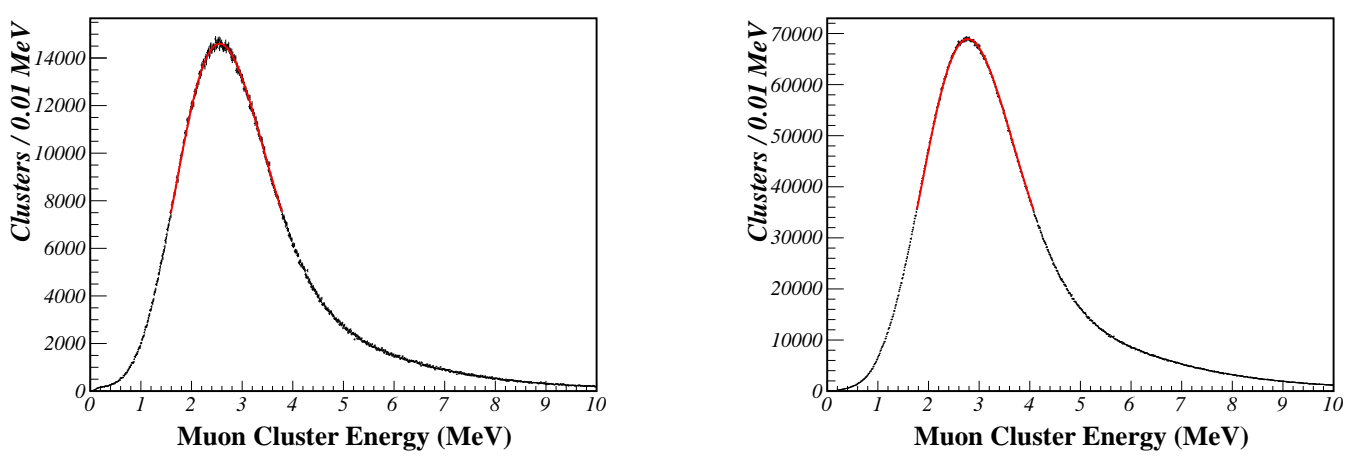

Figure 7.25: Reconstructed cluster energy along rock muon tracks in data (left) and MC (right) for calibrating the detector energy scale. The red line is a fifth order polynomial fit used to determine the peak of the distribution.

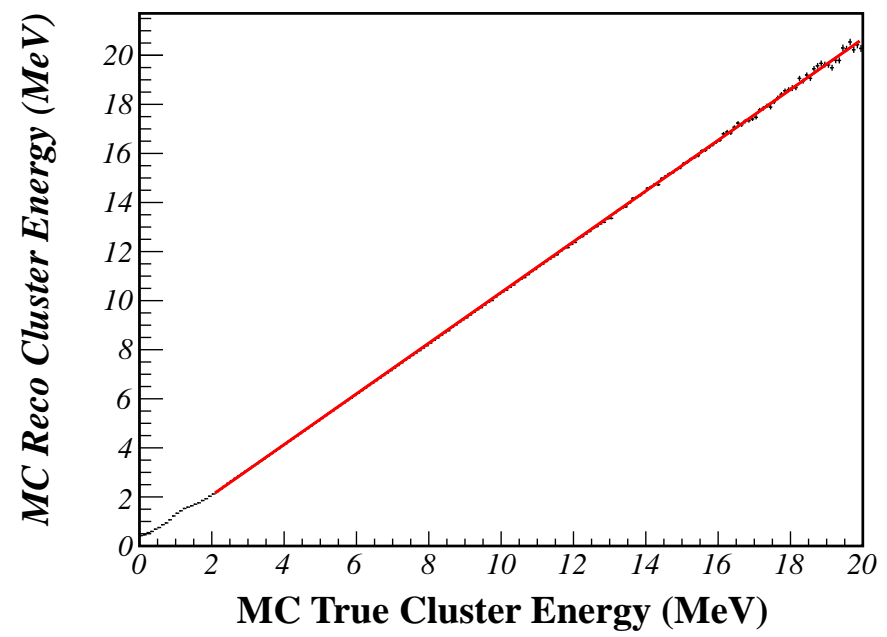

Figure 7.26: Average reconstructed energy vs. true energy of clusters on rock muon tracks for calibrating the detector energy scale. The red line is a straight-line fit with zero intercept. The slope of the fit is used to correct the MC peak reconstructed cluster energy. The non-linearity below $2 \mathrm{GeV}$ in true cluster energy is due to reconstruction bias. When the simulated muon energy deposition in a plane is small and the detector response fluctuates low, the resulting cluster tends not to be included in the track reconstruction. 


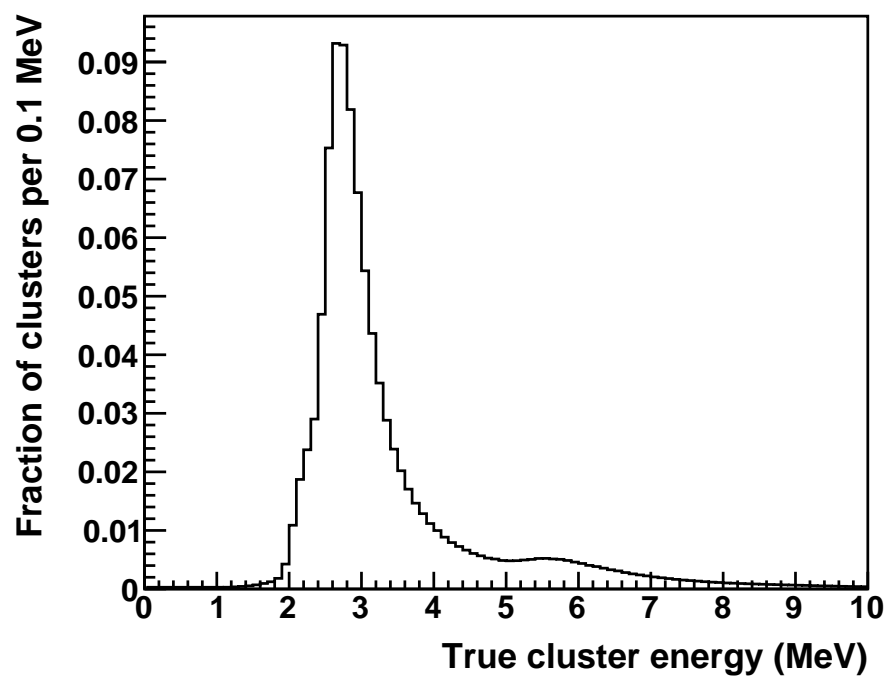

Figure 7.27: True cluster energy along rock muon tracks in the MINER $\nu \mathrm{A}$ MC. The peak is due to ionization, and the tail is primarily due to delta rays (knock-on electrons). The figure is from reference [102].

calibrate $Y$ to minimize effects from passive materials and muon energy loss. The peaks of the cluster PE distributions are found by fitting the distributions in the region above half-height with a fifth order polynomial. The calibrated light yield factor is calculated as

$$
Y=Y_{T} \frac{P_{d a t a}}{P_{M C}}
$$

where $P_{d a t a}$ and $P_{M C}$ are the peak cluster PE along rock muon tracks in the data and MC samples, respectively.

The data and MC distributions of cluster PE and energy along rock muon tracks after calibrating the detector energy scale and light yield factor are shown in Figure 7.29 .

The light level of the detector decreases over time due to scintillator aging. The 

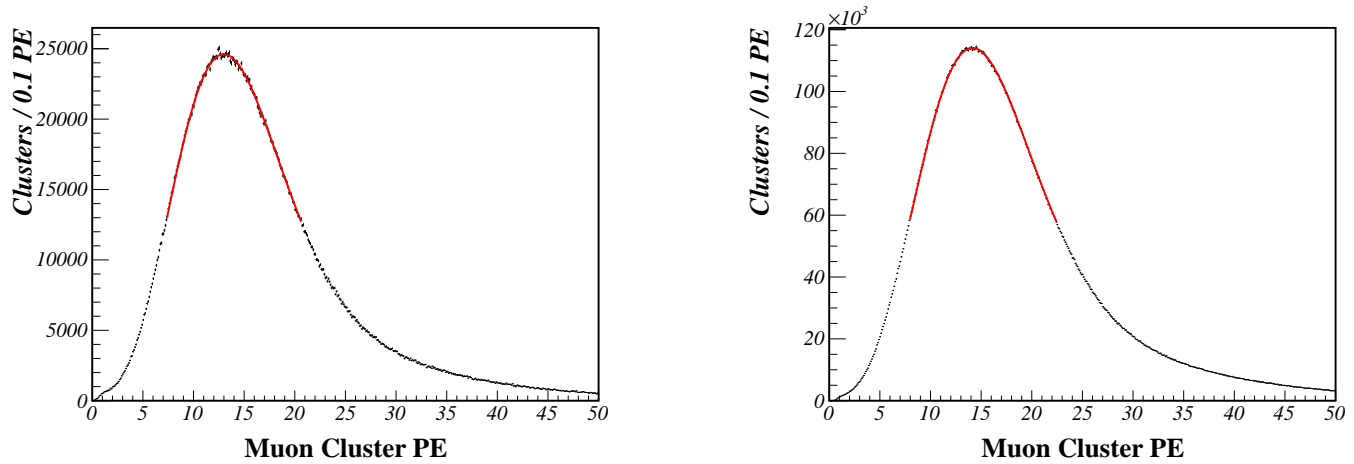

Figure 7.28: Cluster PE along rock muon tracks in data (left) and MC (right) for calibrating the light yield in the MC. The red line is a fifth order polynomial fit used to determine the peak of the distribution.
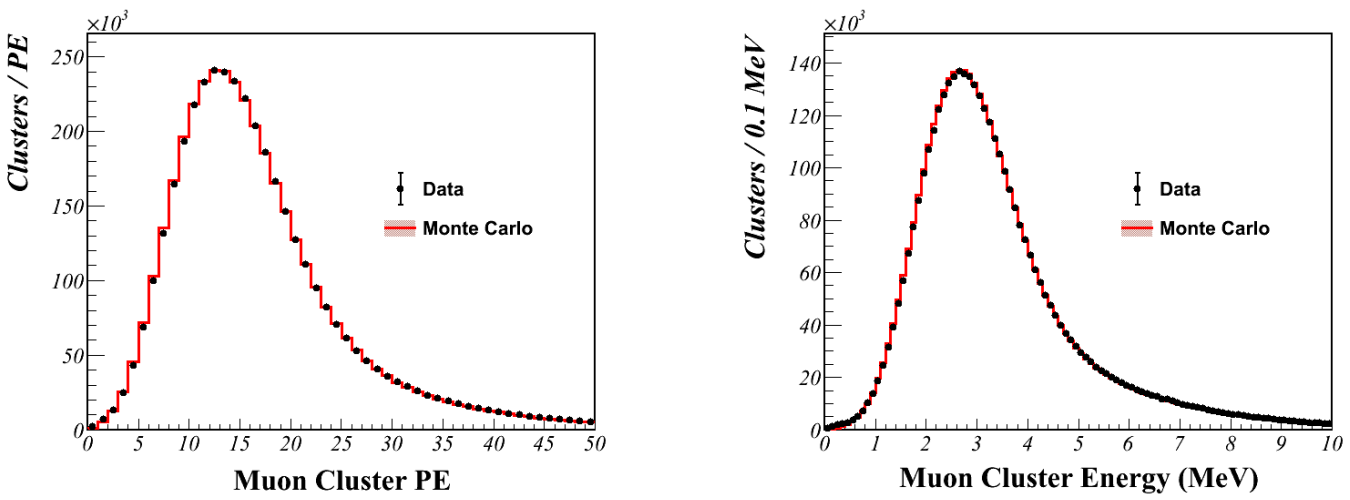

Figure 7.29: Cluster PE (left) and energy (right) along rock muon tracks in data and $\mathrm{MC}$ after calibrating the light yield in the MC and the detector energy scale

detector energy scale must compensate for the decreasing light level to give the correct energy. In addition, the MC must mimic the time dependence of the light level in the detector. This is achieved by calibrating $M$ and $Y$ once every $\sim 2$ days. Figure 7.30 shows the PE and calibrated energy of clusters on rock muon tracks vs. time during data taking in the LE beam configuration. While the light level dropped $\sim 13 \%$ over this 2-year period, calibrated cluster energy along rock muon tracks remained constant to within $0.1 \%$. The error on the detector energy scale is 
$\sim 2 \%$, which is dominated by the uncertainty on the amount of active scintillator per plane.
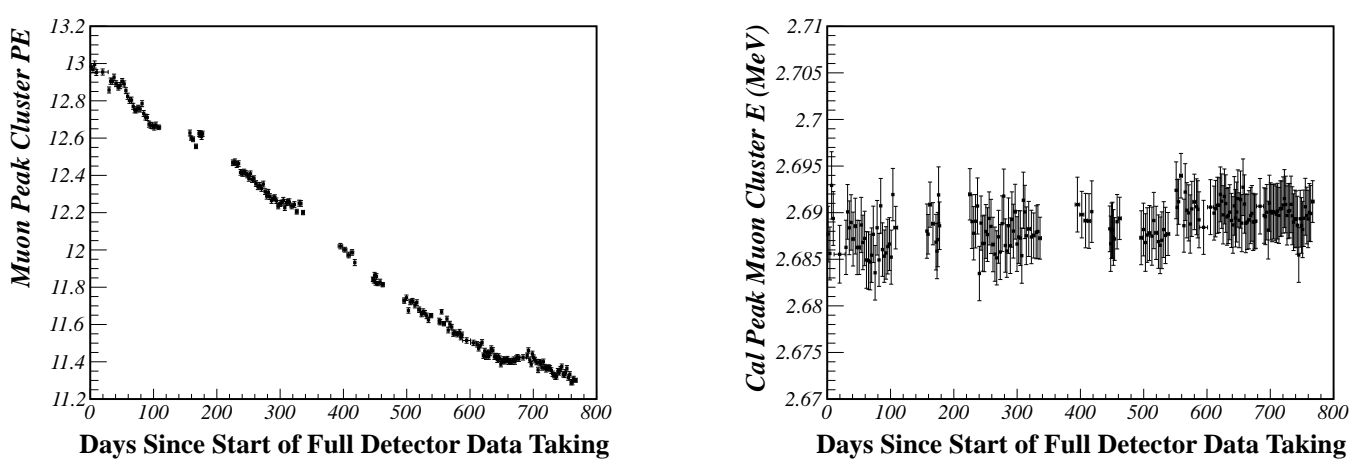

Figure 7.30: Peak cluster PE (left) and calibrated energy (left) vs. time along rock muon tracks in data. The cluster $\mathrm{PE}$ decreases in time due to scintillator aging, which is compensated by the calibrated detector energy scale. Peak calibrated cluster energy is constant to $0.1 \%$. The error bars are the error on the peak from the fifth order polynomial fit.

\subsubsection{Timing Corrections}

A set of timing corrections is used to convert the raw time of a hit, $T_{\text {raw }}$, to a calibrated time, $T_{c a l}$. For a hit with a fired discriminator, $T_{\text {raw }}$ is the system time that the discriminator fired. For a hit without a fired discriminator, $T_{\text {raw }}$ is the system time at the end of the hit's charge integration period. $T_{c a l}$ is calculated as

$$
T_{\text {cal }}=T_{\text {raw }}-T_{\text {path }}(c)-T_{\text {slew }}(P E)-T_{\text {offset }}(c),
$$

where the timing corrections $T_{\text {path }}(c), T_{\text {slew }}(P E)$, and $T_{\text {offset }}(c)$ depend on the optical readout channel, $c$, and $\mathrm{PE}$ of the hit. $T_{\text {path }}(c)$ is the propagation time of light in the strip's fiber to the photocathode. $T_{\text {path }}(c)$ is estimated using the speed of light in the WLS and clear fibers, which is $156 \mathrm{~mm} / \mathrm{ns}$ from a test stand mesure- 
ment, and the total fiber length between the point of energy deposition in the strip to the strip's photocathode. This estimation assumes that direct light, opposed to light reflected from the mirror end of the WLS fiber, generates the PE that fires the discriminator. Nominally, $T_{\text {path }}(c)$ is calculated to the length center of the strip. $T_{\text {path }}(c)$ is updated to the position of the energy deposition along the strip when the position is determined by the reconstruction. Time slewing, $T_{\text {slew }}(P E)$, is the time delay arising from the decay times of excited molecules in the fluors of the scintillator and WLS fiber. Since the hit time is set by the first photoelectron to fire the discriminator, time slewing is dependent on the amount of light generated inside the strip, and therefore the PE generated at the photocathode. The material properties of the scintillator and WLS fibers are assumed to be uniform across the detector. Therefore, time slewing is also assumed to be uniform across the detector. $T_{\text {offset }}(c)$ is the constant time offset of the readout channel due to signal propagation time in the electronics.

$T_{\text {slew }}(P E)$ and $T_{\text {offset }}(c)$ for all channels in the detector are measured using hit times along rock muon tracks, where only hits with a fired discriminator are used. The measurement is iterative since the time slewing and time offset of each hit are convolved. Eight iterations are used to measure $T_{\text {slew }}(P E)$ and $T_{\text {offset }}(c)$. The time slewing and time offset of each hit along a rock muon track is measured by comparing the hit time to the track reference time, $T_{\text {track }} . T_{\text {track }}$ is calculated from the corrected time of each hit, $T_{c o r}$, along the track. $T_{c o r}$ is calculated as

$$
T_{\text {cor }}=T_{\text {raw }}-T_{\text {path }}(c)-T_{\text {slew }}(P E)-T_{\text {offset }}(c)-T_{\text {tof }},
$$

where $T_{\text {tof }}$ is the time of flight of the muon from the start point of the track to the 
reconstructed $3 \mathrm{D}$ position of the hit. $T_{\text {path }}(c)$ is calculated from the $3 \mathrm{D}$ position of the hit. $T_{\text {slew }}(P E)$ and $T_{\text {offset }}(c)$ are measured in the previous iteration and are zero in the zeroth iteration. $T_{\text {track }}$ is the PE weighted iterative truncated mean $T_{\text {cor }}$ along the track.

The measured time slewing for a hit, $T_{\text {slew }}$, is calculated as

$$
T_{\text {slew }}=T_{\text {raw }}-T_{\text {offset }}(c)-T_{\text {path }}(c)-T_{\text {tof }}-T_{\text {track }},
$$

where $T_{\text {offset }}(c)$ is measured in the previous iteration and is zero in the zeroth iteration. $T_{\text {slew }}(P E)$ is assumed to be uniform across the detector and is measured in each iteration by binning the measured time slewing of all hits on all tracks by hit PE. Figure 7.31 shows the measured hit time slewing distribution in different $\mathrm{PE}$ bins. In each PE bin, peak time slewing is determined by fitting the distribution with a convolution of a Landau distribution and a Gaussian distribution. In each iteration, peak time slewing as a function of hit PE (Figure 7.31) is fit with a 3rd order polynomial in $\frac{1}{\sqrt{P E}}$. The fit gives the most likely time slewing as a function of hit $\mathrm{PE}$ and is used to calculate $T_{\text {slew }}(P E)$.

The measured time offset for a hit, $T_{\text {offset }}$, is calculated as

$$
T_{\text {offset }}=T_{\text {raw }}-T_{\text {slew }}(P E)-T_{\text {path }}(c)-T_{\text {tof }}-T_{\text {track }}
$$

where $T_{\text {slew }}(P E)$ is measured in the previous iteration and is zero in the zeroth iteration. Here, another assumption is made that the difference between time offsets of readout channels with the same high gain TriP-t is negligible. This assumption is motivated by test stand measurements of hit time by injecting charge into channels on FEBs. This assumption allows one $T_{\text {offset }}(c)$ to be measured for each group of 
Pulse height (PE)
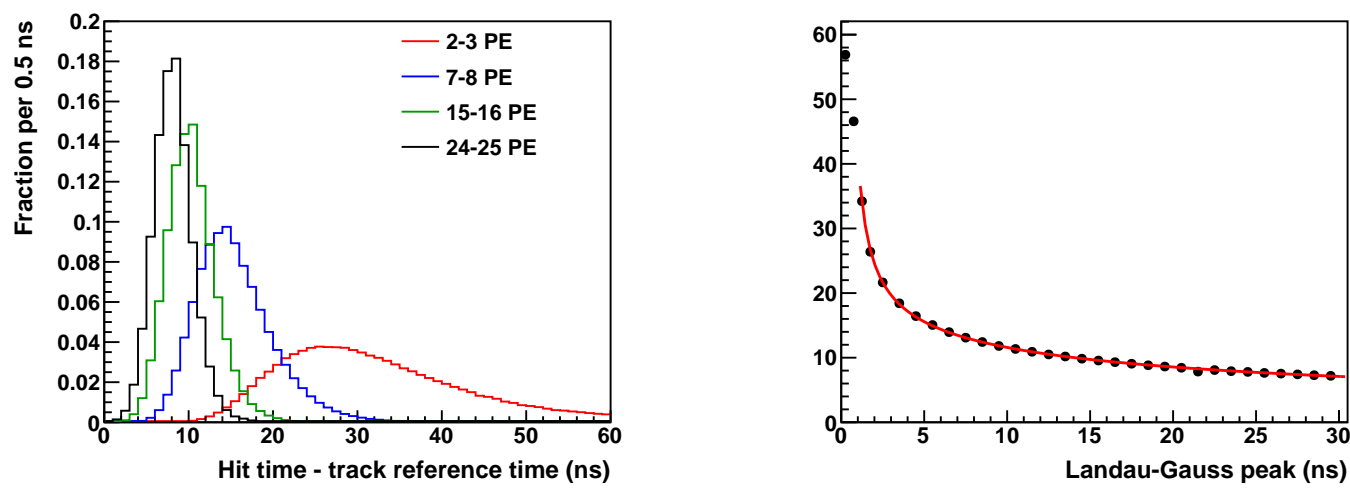

Figure 7.31: The measured time slewing for hits along rock muon tracks. The left plot shows the time slewing in different bins of hit PE. The right plot shows the peak time slewing as a function of hit PE, which is fit with a 3rd order polynomial in $\frac{1}{\sqrt{P E}}$ (red line). The figures are from reference [102].

16 readout channels with the same high gain TriP-t, referred to herein as a channel group. This leverages statistics to account for readout channels with low rock muon hit statistics, particularly those that serve strips near the edge of the detector. Figure 7.32 shows the measured hit time offset distribution, weighted by hit PE, for a channel group in four different iterations. In each iteration, the measured $T_{\text {of } f s e t}(c)$ for the channel group is the iterative truncated mean of the distribution. $T_{\text {offset }}(c)$ for each channel group converges after eight iterations (not to be confused with the iterations in calculating the truncated mean of the distribution). The measured channel time offsets are as large as 30 ns. The CRIMs synchronize the clocks on FEBs along the same chain to within one clock tick (9.4 ns). The remainder of the channel time offsets come from time offsets between FEB chains served by the same CROC, and time offsets between the CROCs.

The $T_{\text {cal }}$ resolution is a function of hit $\mathrm{PE}$ due to time slewing. The $T_{\text {cal }}$ resolution is measured using $T_{c a l}$ along rock muon tracks, where $T_{c a l}$ is corrected for muon time 


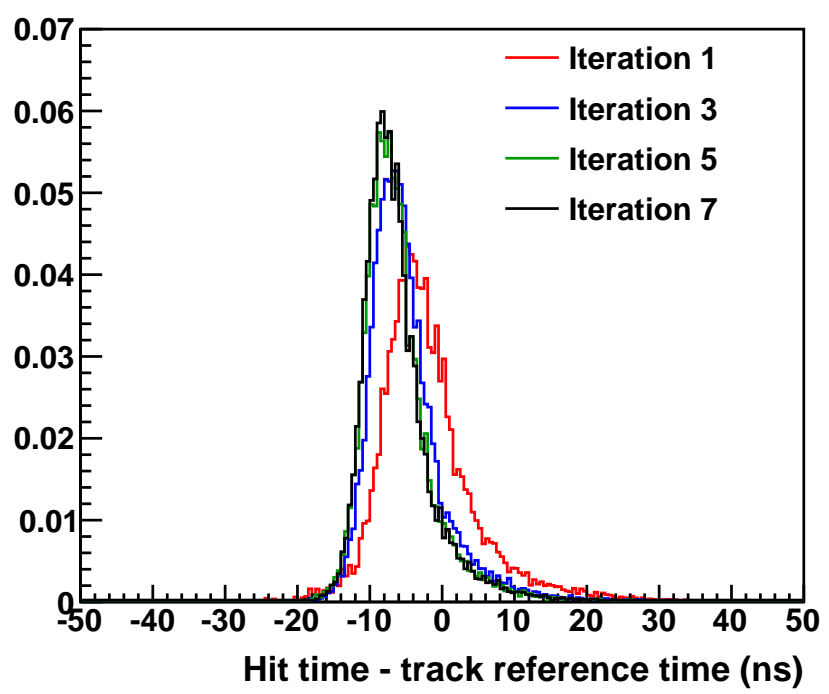

Figure 7.32: The measured hit time offset distribution for one group of 16 channels served by the same high gain TriP-t, where the measured time offset for each hit is weighted by the hit PE. The distribution is shown for four different iteractions. The figure is from reference [102].

of flight. The $T_{\text {cal }}$ resolution for each hit is measured as $T_{\text {cal }}-T_{\text {track }}$, where $T_{\text {track }}$ is the track reference time defined earlier. Figure 7.33 shows the $T_{\text {cal }}$ resolution for three bins in hit $\mathrm{PE}$. The $T_{\text {cal }}$ resolution improves with increasing $\mathrm{PE}$, and asymptotically approaches the quarter tick (2.4 ns) granularity of the FEBs.

Hardware (e.g. FEB, PMT) swaps and power cycles change the time offsets. $T_{\text {slew }}(P E)$ and $T_{\text {offset }}(c)$ are remeasured after each hardware swap and/or power cycle when rock muon statistics are sufficient. Measuring $T_{\text {slew }}(P E)$ and $T_{\text {offset }}(c)$ requires at least $25 \mathrm{k}$ rock muons. 


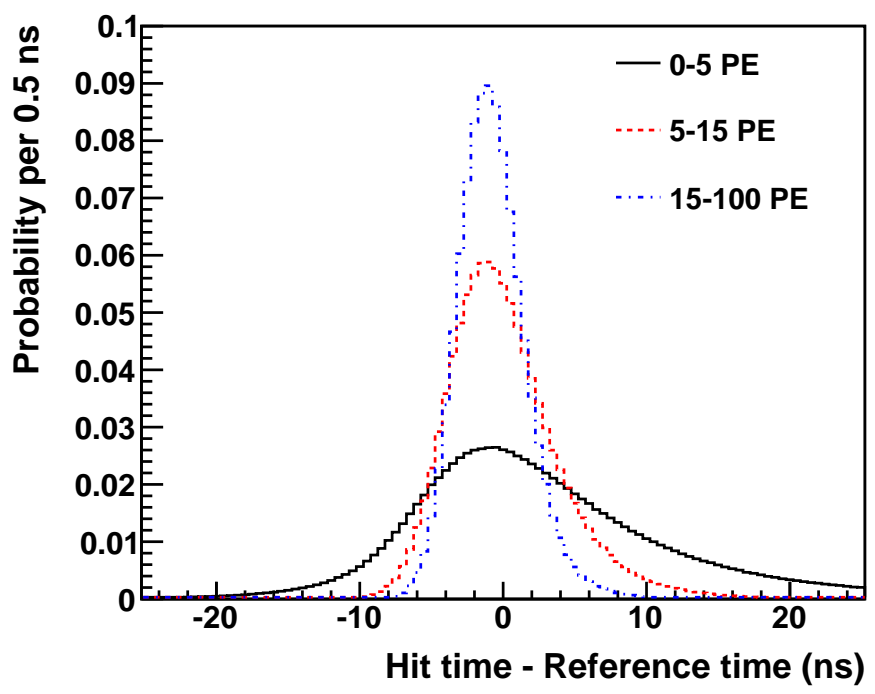

Figure 7.33: The $T_{\text {cal }}$ resolution of hits along rock muon tracks for three bins in hit $\mathrm{PE}$, where the calibrated hit time is corrected for muon time of flight. The reference time for each hit is the track reference time, $T_{\text {track }}$. The figure is from reference [102].

\subsubsection{Cross-Talk Measurement}

Cross talk is the leakage of light and/or charge between readout channels. The dominant sources of cross talk are the leakage of light between pixels on a photocathode and the leakage of charge between dynode chains in a PMT, where pixel cross talk is the larger effect. As discussed in Section 7.1.5, the weave of the ODU fibers in a PMT box routes adjacent strips to non-adjacent pixels on the photocathode, which enables identification of cross talk using the proximity of hits in the strips and on the photocathode pixels.

A measurement of the total cross talk on each PMT was made using rock muon tracks. A hit in the same time slice as a rock muon track that was not in a strip adjacent to a strip on the rock muon track, and was on a photocathode pixel adjacent 
to a pixel containing a hit on the rock muon track, was identified as cross talk (Figure 7.34). The cross talk ratio for each PMT was calculated as the ratio of the total PE of identified cross talk hits from the PMT to the total PE of hits on rock muon tracks read by the PMT. The measured cross talk ratios are used in simulating cross talk in the Monte Carlo simulation of the MINER $\nu$ A detector (Section 7.6.2).

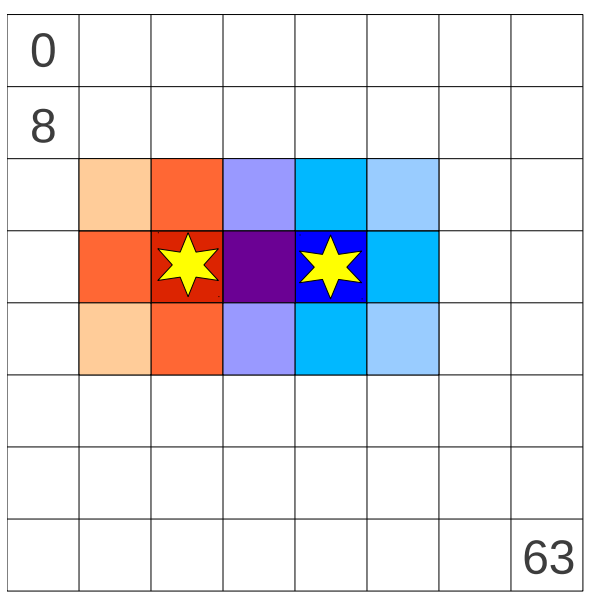

PMT pixels

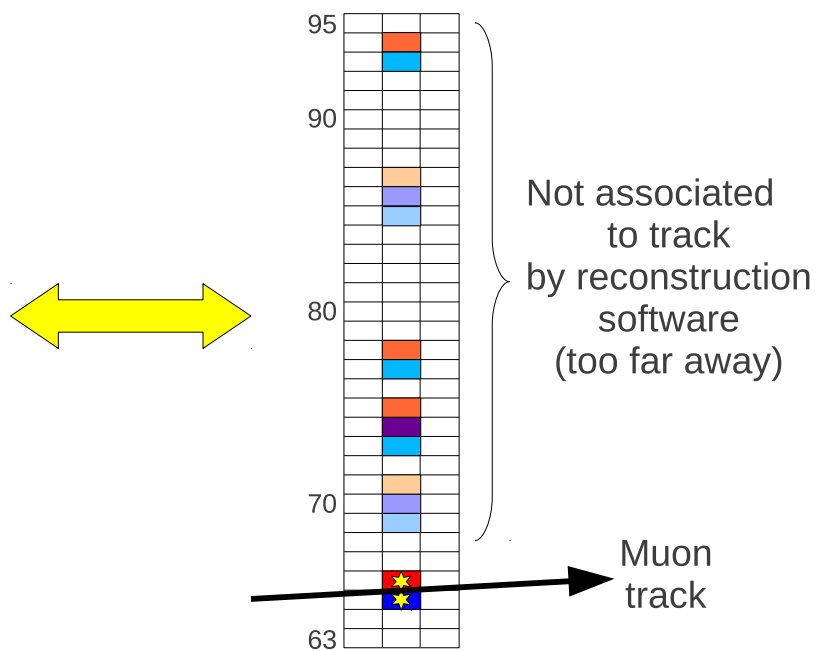

Scintillator strips

Figure 7.34: An illustration of cross talk from a muon track. A muon will usually deposit energy in two adjacent strips in a plane (right), which are routed to two non-adjacent pixels on a photocathode (left) by the ODU fiber weave. Cross talk on pixels adjacent to the pixels containing the hits from the muon will appear as hits in strips that are separated from the muon track. The figure is from reference [88].

\subsection{MINER $\nu \mathrm{A}$ Monte Carlo}

The MINER $\nu$ A Monte Carlo (MC) is a detailed simulation of the MINER $\nu$ A data. The MC simulates neutrino interactions in the MINER $\nu \mathrm{A}$ detector, the propagation of the resulting particles in the detector, and the response and readout of the de- 
tector. The MC is processed with the same reconstruction algorithms as the data. The MC is used in MINER $\nu$ A's neutrino cross section measurements for estimating backgrounds, experimental resolution, reconstruction efficiency, the efficiency of selecting neutrino interactions of interest, and systematic uncertainties.

A MC sample is simulated for each data sample (see Section 8.4). Detector response and event pile-up in each $\mathrm{MC}$ sample are data-driven, where each $\mathrm{MC}$ sample is informed by its respective data sample. Event pile-up varies with neutrino beam intensity. Detector response varies in time due to effects such as scintillator and PMT aging, changes in PMT voltages, and hardware swaps. Each MC sample mimics the detector response and pileup of its respective data sample at each point in time. Each MC sample is generated with $\sim 10 \times$ the statistics of its respective data sample so the MC statistical uncertainty on MINER $\nu$ A's cross section measurements is negligible.

The MC uses a solid model of the MINER $\nu \mathrm{A}$ detector which describes the geometry, position, orientation, and materials of all components in the detector. Herein, the MINER $\nu \mathrm{A}$ detector in the MC refers to this model.

\subsubsection{Physics Simulation}

The first stage of the MC is the simulation of neutrino interactions in the MINER $\nu \mathrm{A}$ detector by the GENIE neutrino event generator (see Section 4.3). The analysis presented in this thesis used GENIE release 2.6.2. For each simulated interaction, GENIE samples the energy, position, direction, and species of the incident neutrino from the NuMI beam flux prediction. GENIE then simulates an interaction on a nucleus or electron within the MINER $\nu \mathrm{A}$ detector. The scattering target and scattering process are determined from pre-calculated cross sections. 
The final state particles from the interaction simulated by GENIE are input to Geant4 [82, 83], which simulates the propagation of the final state particles in the MINER $\nu$ A detector. The MINER $\nu \mathrm{A}$ MC uses Geant4 version 9.4.p02. Geant4 simulates electromagnetic and hadronic (strong) interactions. The MINER $\nu$ A implementation of Geant4 uses the quark gluon string precompound (QGSP) model [103] coupled with the Bertini hadronic cascade model $[104,105]$ to simulate hadronic interactions. The largest uncertainties on the Geant4 simulation come from hadronic interactions. The hadrons produced in neutrino-nucleus interactions in MINER $\nu \mathrm{A}$ are primarily pions, protons, and neutrons. The uncertainties on the simulated response of the MINER $\nu \mathrm{A}$ detector to pions and protons are constrained by the MINER $\nu$ A test beam (Section 7.7). The uncertainties on the simulated response of the MINER $\nu \mathrm{A}$ detector to neutrons are constrained to external neutron scattering data (Section 8.13.5).

Simulated particles that exit the MINER $\nu$ A detector are input to the MINOS detector simulation. The partial detector MC includes a solid model of the ArgoNeuT detector situated between the MINER $\nu \mathrm{A}$ and MINOS detectors. The partial detector MC simulates energy loss and scattering of particles in ArgoNeuT, which is important for simulating muon reconstruction bias in the partial detector sample due to the presence of ArgoNeuT.

\subsubsection{Detector Simulation}

The MINER $\nu$ A detector simulation models the data readout gates described in Section 7.1.5. Each simulated readout gate contains exactly one simulated neutrino interaction in the MINER $\nu$ A detector. An optical model simulates the scintillation light generated by energy depositions in strips from Geant4 and the resulting PE 
generated at the PMT photocathode pixels. A PMT model simulates the amplification of PE in the dynode chains and cross talk between the pixels and dynode chains. A FEB model simulates the amplification, integration, and digitization of charge from the PMT anode as well as dead time. Finally, to simulate event pile-up effects and particles entering the MINER $\nu \mathrm{A}$ detector from neutrino interactions in the upstream rock of the detector hall, detector activity from a readout gate from the data sample the $\mathrm{MC}$ is simulating is overlaid onto the simulated gate. The simulated detector response is data driven so each $\mathrm{MC}$ sample mimics the detector response of its corresponding data sample at each point in time. This is accomplished using the calibrations for estimating hit PE and energy in the data sample in simulating the detector response. Simulated readout gates are then calibrated and reconstructed using the same calibrations and reconstruction algorithms used for the data.

\section{Optical Model}

The first stage of the detector simulation is the optical model, which predicts the PE generated at the photocathode pixel for an energy deposition in a strip. To calculate the scintillation light generated by a simulated energy deposition in a strip, the optical model uses Birk's law:

$$
\frac{L}{d x}=L_{0} \frac{d E / d x}{1+k_{B} d E / d x},
$$

where $L$ is the luminosity, $L_{0}$ is the luminosity at low specific ionization density, $E$ is the deposited energy, $x$ is the path length over which the energy was deposited, and $k_{B}$ is Birk's constant which determines the scintillator saturation for large, localized energy deposition. The value of $L_{0}$ used in the optical model is 8000 photons / MeV, 
which is corrected by the measured light yield factor (Section 7.5.7) as will shown shortly. The value of $k_{B}$ in the MC samples used in the analysis presented in this thesis was $0.133 \mathrm{~mm} / \mathrm{MeV}$. The MINER $\nu$ A test beam (Section 7.7) subsequently measured $k_{B}$ to be $0.0905 \pm 0.012 \mathrm{~mm} / \mathrm{MeV}$. The MC therefore over-predicted the scintillator saturation. This is accounted for in the systematic uncertainties on the measurements reported in this thesis (Section 8.13.5).

The optical model divides the calculated scintillation light into two "pulses", where each pulse contains half the scintillation light. One pulse is propagated directly to readout end of the strip, and the other pulse is propagated to the mirror end where it is reflected. The average PE generated at the photocathode from each pulse is calculated as

$$
\langle P E\rangle=L \times \frac{1}{A(c)} \times \frac{1}{S(c, t)} \times Y(t) \times \sigma(c),
$$

where $A(c)$ and $S(c, t)$ are the measured attenuation and strip response corrections used in estimating hit energy (Equation 7.3) in the data, respectively, $Y(t)$ is the light yield factor, and $\sigma(c)$ is a channel smearing factor. $A(c)$ is used to attenuate the simulated scintillation light in the strip, WLS fiber in the baggie, and clear fiber cable, where the attenuation is calculated to the position of the energy deposition along the strip. $S(c, t)$ is used to tune the response of the strip. $Y(t)$ corrects the light yield of the scintillator in the MC to match the observed light yield in the data sample the $\mathrm{MC}$ is simulating, and converts the scintillation light arriving at photocathode pixel to the generated number of photoelectrons. The time periods of $S(c, t)$ and $Y(t)$ are matched to the time of the overlaid data gate. $\sigma(c)$ for the readout channel is sampled from a gaussian distribution with a mean of zero and a 
1 sigma width of 0.0557 , and is fixed during the simulation. The $5.57 \%$ smearing represents the total uncertainty on the calibrations used in estimating hit energy in the data, which is dominated by the $3-5 \%$ statistical uncertainty on the measured PMT gain for each channel. The smearing was determined from the widths of the distributions of cluster energy on rock muon tracks in data and MC [106, 107].

The simulated PE for each pulse is sampled from a Poisson distribution with a mean equal to the average PE. The simulated time for each pulse is the sum of the time of the energy deposition and the transport time in the WLS and clear fibers.

\section{PMT Model}

The PMT model simulates the amplification of PE on a photocathode pixels in the dynode chains as well as cross talk between pixels and dynode chains. The PMT model first simulates cross talk from a photocathode pixel containing PE from a simulated energy deposition in a strip into the neighboring pixels. The cross talk on a neighboring pixel is sampled from a Poisson distribution with a mean tuned by the measured cross talk ratio for the PMT (see Section 7.5.9). The amplification of $\mathrm{PE}$ on each photocathode pixel is simulated using a statistical model of the dynode chain, where the average gain is set to the channel gain $G(c, t)$ measured from light injection data and used in estimating hit PE in data (see Section 7.5.3). The time period of $G(c, t)$ is matched to the time of the overlaid data gate. The PMT model also simulates cross talk between dynode chains using a model based on PMT bench tests performed by MINOS [108]. The simulated amplified charge at the PMT dynodes is input to the FEB model. A detailed description of the PMT model is given in reference [109]. 


\section{FEB Model}

The FEB model simulates the amplification, integration, and digitization of charge from the PMT anodes and dead time. The FEB model mimics the functions of the FEB. The FEB model first divides charge from a PMT anode amongst the low, medium, and high gain channels on the FEB channel and amplifies the charge in the gain channels. Discriminator fires on the high gain channels and the resulting charge integration periods and subsequent dead time periods are simulated. A charge pulse from a PMT anode arriving during a dead time period is masked and is not further simulated. Integrated charge is stored in an ADC block and is time stamped according to whether the discriminator fired. The time stamp is digitized into clock ticks and quarter ticks. Charge in the ADC blocks is digitized at the end of the simulated readout gate. Only simulated hits with an ADC count three standard deviations above the measured channel pedestal are retained, where one standard deviation is the RMS of the measured channel pedestal distribution (Section 7.5.1). This cut mimics the pedestal suppression in data. As usual, the time period for the measured channel pedestal is matched to the time of the overlaid data gate. A detailed description of the FEB model is given in reference [109].

\section{Data Overlay}

Each simulated readout gate contains exactly one simulated neutrino interaction in the MINER $\nu$ A detector. To simulate event pile-up effects such as lost detector activity due to dead time, time slices containing activity from more than one neutrino interaction, and particles entering the MINER $\nu \mathrm{A}$ detector from neutrino interactions in the upstream rock of the detector hall, activity from a readout gate in the data sample the MC is simulating is overlaid onto the simulated gate. Readout gates are 
selected randomly from the data sample, where each readout gates is weighted by the POT for its neutrino beam pulse. To minimize file size, only hits from the data gate occurring less than $50 \mathrm{~ns}$ before and less than $200 \mathrm{~ns}$ after the time of the simulated neutrino interaction are added to the simulated gate. Hits from either the simulation or the data overlay that fall within a dead time period in either the simulation or the data overlay are masked. The time of the overlaid data gate determines the time periods of the calibrations used in simulating the detector response.

\section{Timing Simulation}

Time slewing and the time offsets in the electronics channels are not modeled in the detector simulation. Therefore, unlike the data, the time slewing and time offset corrections are not applied to simulated hit times. What is done instead is a datadriven smearing of the simulated hit times during the calibration stage of the MC. The time smearing for a simulated hit with a given PE is sampled from the calibrated hit time resolution as a function of hit PE (Figure 7.35) measured from rock muons in data (Section 7.5.8). The hit time resolution after the calibration stage in data and $\mathrm{MC}$ match due to the data-driven time smearing and the data-driven detector response in the $\mathrm{MC}$ which governs the simulated hit $\mathrm{PE}$.

\subsection{The MINER $\nu$ A Test Beam}

The MINER $\nu \mathrm{A}$ test beam [110] is a program to measure the accuracy of the simulated response of the MINER $\nu \mathrm{A}$ detector to protons and charged pions. Neutrinonucleus interactions measured by MINER $\nu \mathrm{A}$ include quasi-elastic scattering, resonance production, and coherent pion production which produce a single proton 


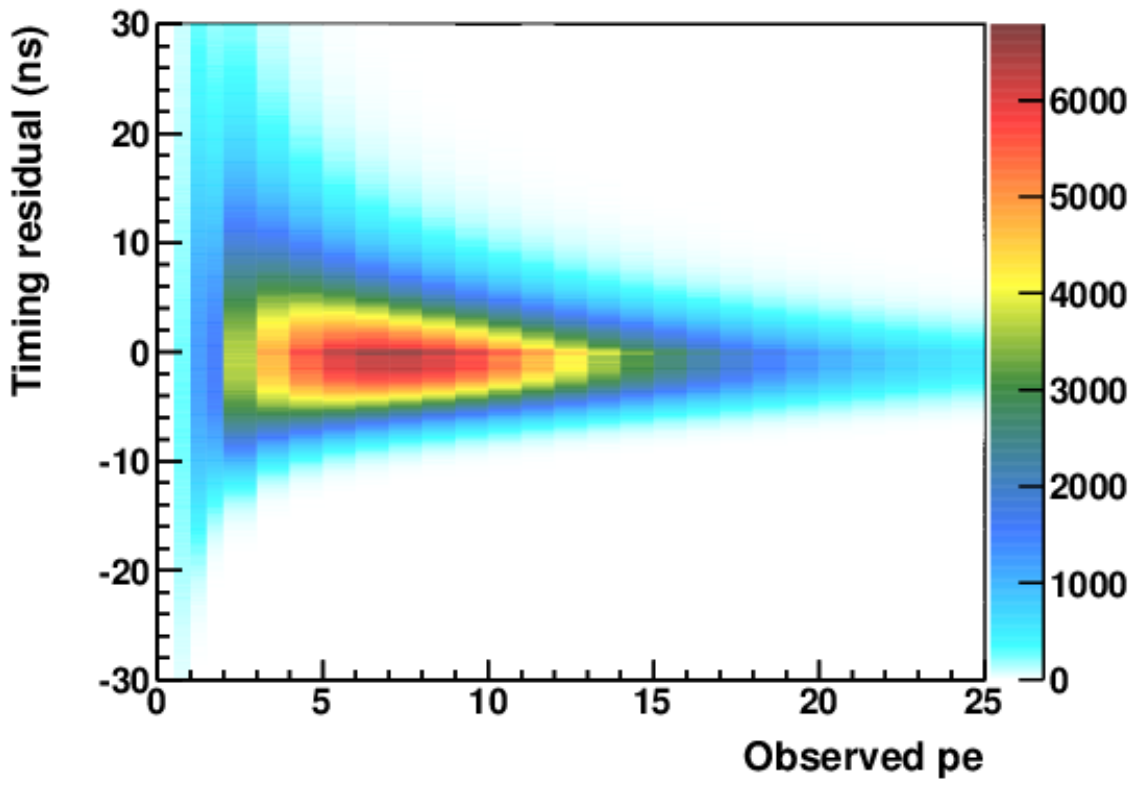

Figure 7.35: The calibrated hit time resolution as a function of hit PE measured from rock muons in data. The time smearing for simulated hits is sampled from this histogram. The figure is from reference [102].

and/or pion final state. Cross section measurements in MINER $\nu \mathrm{A}$ rely on the MC to estimate selection efficiency, kinematic resolution, and backgrounds, and these estimates often depend on the simulated response to protons and pions. The MINER $\nu \mathrm{A}$ test beam apparatus, located at the Fermilab Test Beam Facility, consists of a tertiary hadron beam and a small detector of similar design to the MINER $\nu$ A detector.

A diagram of the beam line of the MINER $\nu \mathrm{A}$ test beam is shown in Figure 7.36. The tertiary hadron beam is generated from a $16 \mathrm{GeV}$ pion beam, generated from $120 \mathrm{GeV}$ protons from the Main Injector, striking a copper target. Products from interactions in the target pass through a collimator which directs the products through a set of four wire chambers. The wire chambers measure the path of a particle. Two 
dipole bending magnets are located between the second and third wire chambers. Time of flight monitors are located upstream and downstream of the wire chambers. Time of flight and path information are used to distinguish particle species in the beam. The beam line delivers hadrons with energies ranging from 0.4 to $2.0 \mathrm{GeV}$ to the test beam detector.

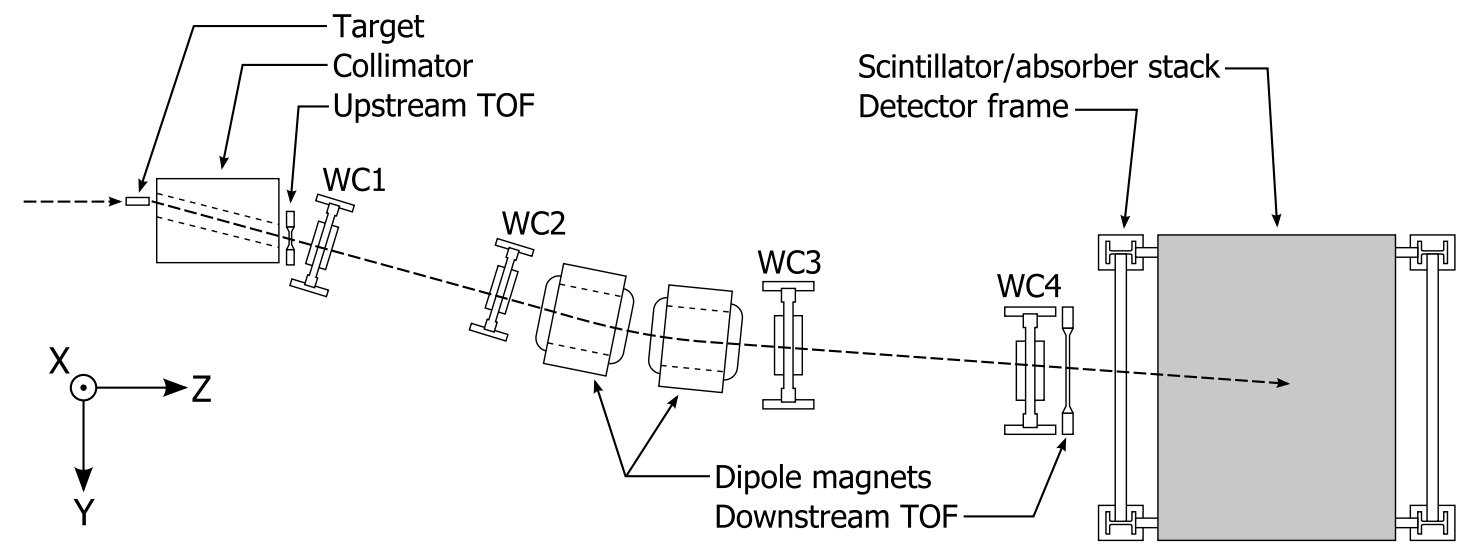

Figure 7.36: The MINER $\nu$ A test beam apparatus. The figure is from reference [110].

The test beam detector is a scaled down replica of the tracker region and downstream ECAL and HCAL of the MINER $\nu$ A detector. The scintillator strips, optical fiber, PMTs, and readout electronics of the test beam detector are identical to that of the MINER $\nu \mathrm{A}$ detector. The test beam detector contains 40 scintillator planes, each of which is approximately $1 \mathrm{~m} \times 1 \mathrm{~m}$ and contains 63 strips. The scintillator planes in the test beam detector are arranged in the same alternating $\mathrm{X}, \mathrm{U}$, and $\mathrm{V}$ orientations of the MINER $\nu \mathrm{A}$ detector (Figure 7.37). The test beam detector is operated in one of two configurations. In the "Tracker-ECAL" configuration, a 0.2 $\mathrm{cm}$ thick lead absorber layer sits in front of each of the 20 downstream planes. In the "ECAL-HCAL" configuration, a $0.2 \mathrm{~cm}$ thick lead absorber layer sits in front of each of the 20 upstream planes, and a $2.6 \mathrm{~cm}$ steel plate sits in front of each of 
the 20 downstream planes. The calibrations of the test beam detector are identical to that of the MINER $\nu \mathrm{A}$ detector, where calibrations determined using rock muons in the MINER $\nu \mathrm{A}$ detector are determined using cosmic ray muons in the test beam detector. The test beam and MINER $\nu \mathrm{A}$ detectors are simulated by the same software. Position, direction, and energy of pions and protons in the test beam MC are data-driven using data from the wire chambers. The reconstruction algorithms for the MINER $\nu \mathrm{A}$ detector are also used for the the test beam detector. Event displays of a proton and pion in the test beam detector are shown in Figure 7.38.

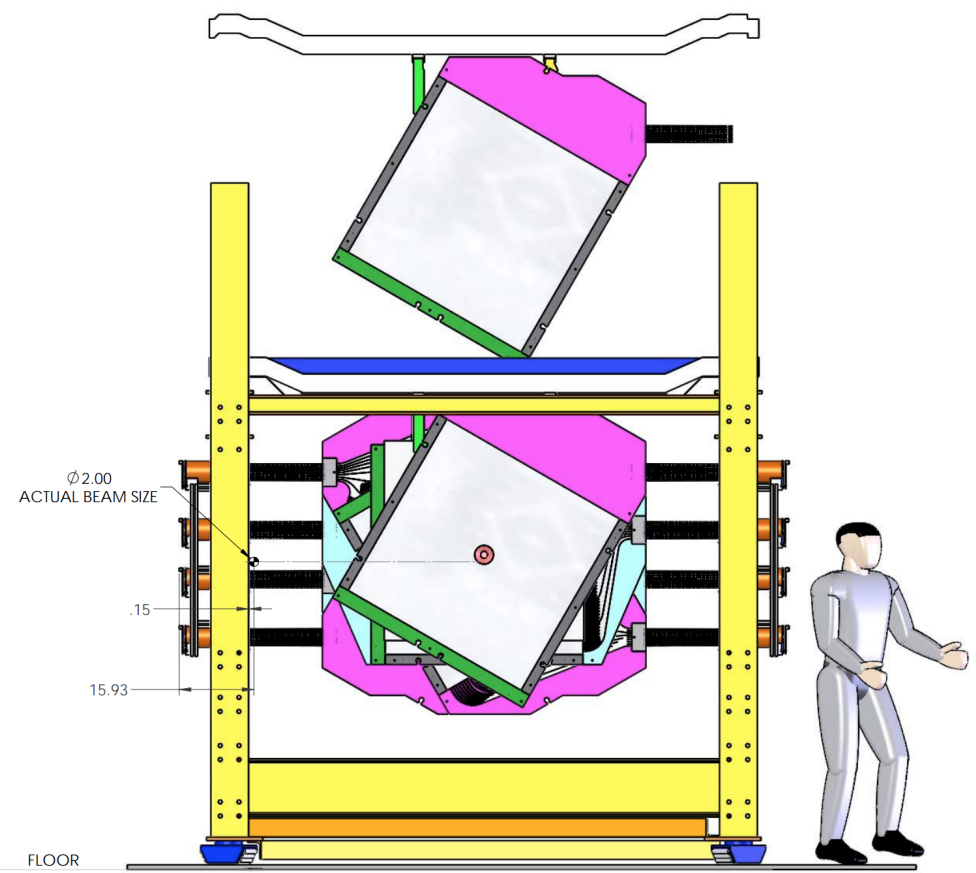

Figure 7.37: Drawing of the front view of the MINER $\nu \mathrm{A}$ test beam detector, where a plane is being lowered into place. The figure is from reference [110].

The saturation of the scintillator and the calorimetric response to protons and pions were measured in the test beam detector. The scintillator saturation was measured using a sample of protons that stopped within the detector. Protons leave 

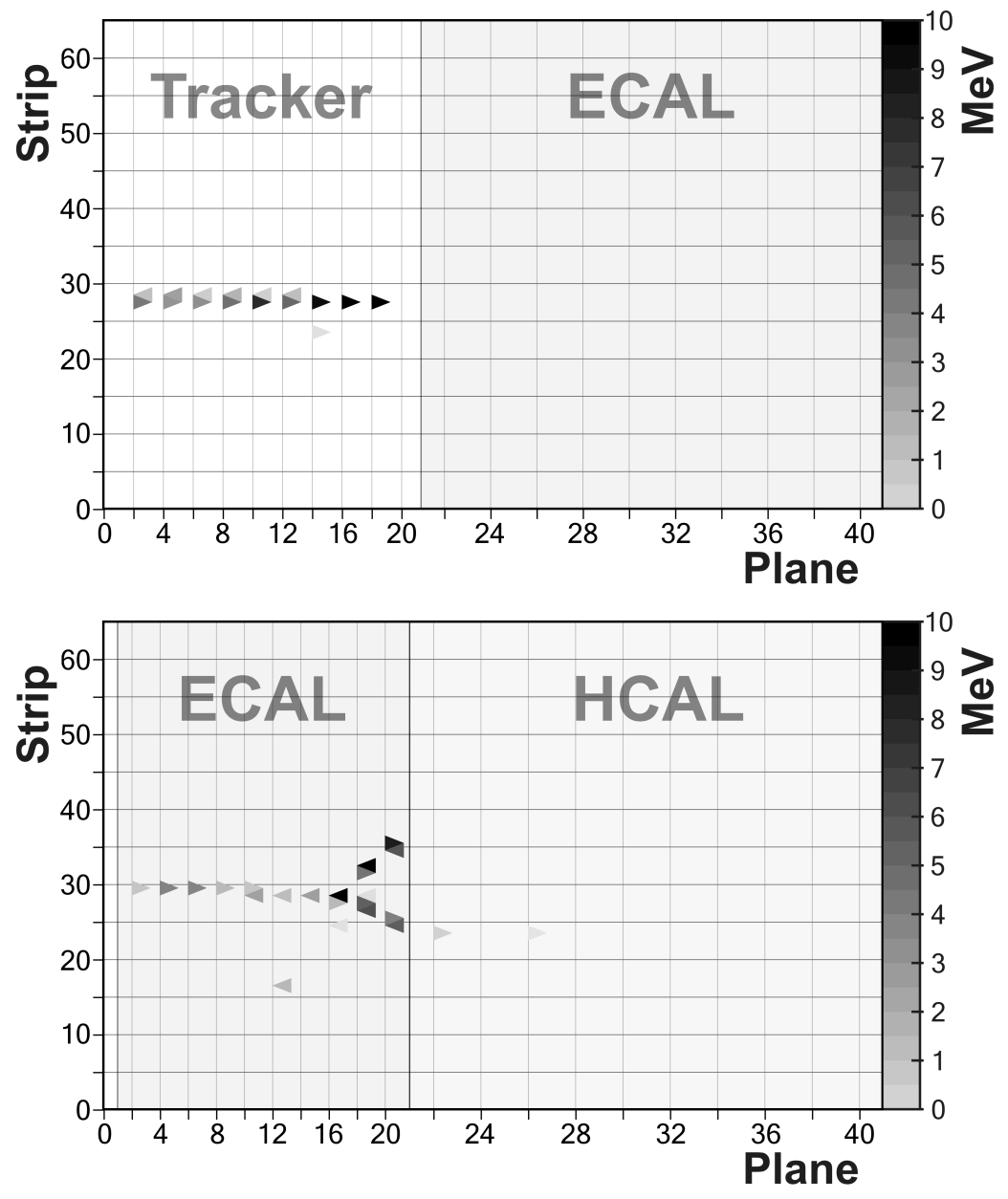

Figure 7.38: Event displays of a stopping proton (top) and an interacting pion (bottom) in the MINER $\nu \mathrm{A}$ test beam detector. Both event displays show only the $\mathrm{X}$ planes. The figure is from reference [110]. 
a large, dense energy deposit at the end of their path, which is accurately described by the Bethe-Bloch formula (Equation 7.1). The Birks' law parameterization of the scintillator saturation in the detector simulation (Equation 7.12) was tuned by comparing the energy deposited by stopping protons in the test beam detector data and MC. The calorimetric response (see Section 8.6) of the test beam detector to single protons and pions was measured in both the data and $\mathrm{MC}$ and compared to establish an uncertainty on the simulated response (Section 8.13.5) for use in MINER $\nu$ A's cross section measurements. 


\section{Chapter 8}

\section{Coherent Pion Production at $\operatorname{MINER} \nu \mathrm{A}$}

This chapter details the measurement of $\nu_{\mu}$ and $\bar{\nu}_{\mu}$ charged current coherent pion production on carbon from MINER $\nu \mathrm{A}$ data. The measurement involves reconstructing the coherent scattering kinematics for each interaction, isolating coherent scattering candidates, constraining the incoherent background, and extracting the coherent pion production cross sections. The evaluation of the systematic uncertainties on the measured cross sections is discussed in detail. The measured cross sections are compared to predictions by the Rein-Sehgal and Berger-Sehgal coherent pion production models. A search for $\nu_{\mu}$ and $\bar{\nu}_{\mu}$ charged current diffractive pion production on hydrogen, an unsimulated background and a possible contribution to the measured coherent cross sections, is also discussed. 


\subsection{Experimental Signature}

Coherent scattering produces a muon and charged pion in the forward direction while leaving the nucleus in its ground state. In MINER $\nu \mathrm{A}$, coherent scattering appears as two forward tracks originating from a common vertex with no additional visible energy near the vertex (Figure 8.1). The muon typically exits the downstream end of MINER $\nu \mathrm{A}$ and enters MINOS, producing a minimum ionizing track in both detectors. The pion produces a minimum ionizing track before stopping or interacting hadronically within MINER $\nu \mathrm{A}$. Visible energy near the vertex in addition to that from a minimum ionizing muon and pion is indicative of nuclear breakup in an incoherent interaction. In addition, the MINER $\nu \mathrm{A}$ detector enables reconstruction of the squared four momentum transfer to the nucleus $|t|$ (Section 8.6), which is necessarily small for the nucleus to remain intact. A forward muon track and pion track, no additional visible energy near the interaction vertex, and small- $|t|$ are model independent features of coherent scattering and are used in isolating coherent scattering interactions (Section 8.7).

\subsection{Fiducial Volume}

The fiducial volume is the region of the detector where interactions are selected for measuring the cross sections. The fiducial volume for this analysis is contained within the tracker (Figure 8.2). The fiducial volume boundaries are recessed from the boundaries of the tracker to minimize contamination from interactions on noncarbon nuclei (e.g. lead) in the adjacent sub-detectors (upstream nuclear targets region, side and downstream ECALs) that result from from mis-reconstructing the interaction vertex position. The fiducial volume spans the central 108 (56) scintil- 


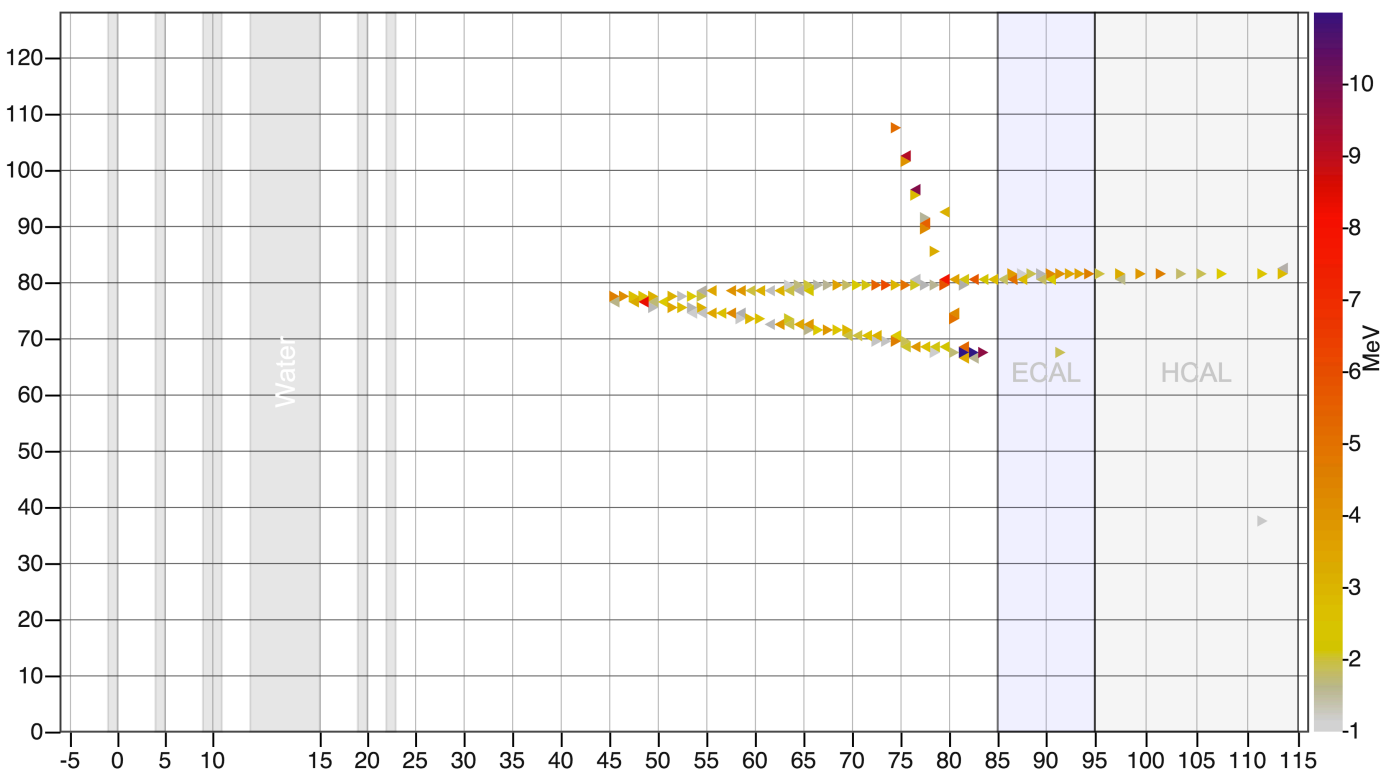

Figure 8.1: A data $\nu_{\mu}$ coherent scattering candidate.

lator planes of the tracker in the full (partial) detector, which excludes the 8 most upstream and 8 most downstream planes of the tracker. In the transverse (XY) plane, the fiducial volume edges are defined by a hexagon with an $85 \mathrm{~cm}$ apothem centered at the detector axis, which are recessed $\sim 10 \mathrm{~cm}$ from the inside edges of the side ECAL. The full (partial) detector fiducial volume mass is 5.47 (2.84) metric tons.

\subsection{Signal and Background Definitions}

Interactions in $\mathrm{MC}$ are categorized as either signal or background for estimating the kinematic resolution (Section 8.6) and selection efficiency (Section 8.9) for coherent scattering, and the selected background rate (Section 8.8). The MC signal and background categories are defined as follows, where $W$ is the invariant mass of the hadronic recoil: 

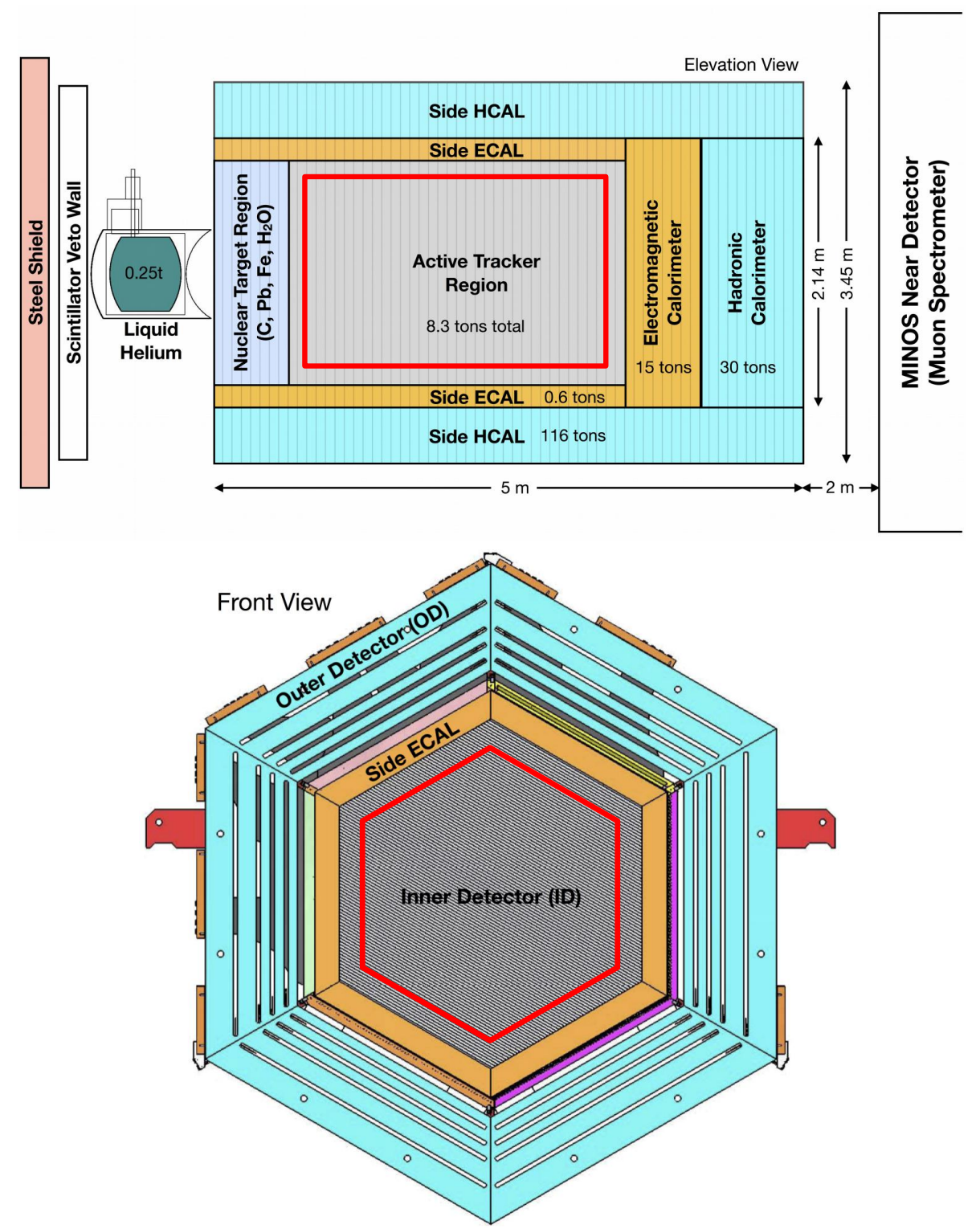

Figure 8.2: The fiducial volume illustrated by the red rectangle and hexagon (approximate scale) 
- Signal ("Coherent") - All interactions that produce a final state consisting of a muon, a charged pion, and the initial state nucleus. The only GENIE interactions that produce this final state are $\nu_{\mu}\left(\bar{\nu}_{\mu}\right)$ charged current coherent pion production interactions. Coherent interactions on non-carbon nuclei are categorized as signal rather than background to avoid dependence of the background prediction on the signal model. The measured cross sections are corrected for coherent interactions on non-carbon nuclei as described in Section 8.9.

- Charged Current Quasielastic ("QE") - $\nu_{\mu}\left(\bar{\nu}_{\mu}\right)$ charged current quasielastic interactions as modeled in GENIE.

- Non-Quasielastic, $\mathbf{W}<1.4 \mathrm{GeV}-\nu_{\mu}\left(\bar{\nu}_{\mu}\right)$ charged current interactions, excluding quasi-elastic, with true invariant mass $W<1.4 \mathrm{GeV}$. This category is primarily delta resonance production, but also includes non-resonant pion production.

- $1.4<\mathrm{W}<2.0 \mathrm{GeV}-\nu_{\mu}\left(\bar{\nu}_{\mu}\right)$ charged current interactions with true invariant mass $1.4<W<2.0 \mathrm{GeV}$. This is the transition region from delta resonance production to deep inelastic scattering.

- $\mathbf{W}>2.0 \mathrm{GeV}-\nu_{\mu}\left(\bar{\nu}_{\mu}\right)$ charged current interactions with true invariant mass $W>2.0 \mathrm{GeV}$. This is the deep inelastic scattering (DIS) region.

- Other - All other background interactions. This category is primarily wrong sign interactions (i.e. $\bar{\nu}_{\mu}$ instead of $\nu_{\mu}$ interactions and vise versa), but also includes non- $\nu_{\mu}\left(\bar{\nu}_{\mu}\right)$ and neutral current interactions. Wrong sign charged current coherent pion production interactions are included in this category 
but are a small contribution.

\subsection{Data and MC Samples}

This analysis uses MINER $\nu \mathrm{A}$ data taken in the LE FHC $\left(\nu_{\mu}\right)$ and $\mathrm{RHC}\left(\bar{\nu}_{\mu}\right)$ neutrino beam configurations. These data span the time period beginning November 2009 and ending April 2012. The data are divided into "playlists" (Table 8.1), each of which corresponds to neutrino beam and MINER $\nu$ A detector configurations. The Downstream 1 and 2 playlists were taken in the $\bar{\nu}_{\mu}$ beam configuration with the partial MINER $\nu$ A detector, where ArgoNeuT was situated between the MINER $\nu$ A and MINOS detectors. The MINER $\nu$ A 5 playlist was taken in the $\bar{\nu}_{\mu}$ beam configuration with the full MINER $\nu$ A detector. All other playlists were taken in the $\nu_{\mu}$ beam configuration with the full MINER $\nu \mathrm{A}$ detector. The neutrino flux for each playlist is normalized to the "protons on target" (POT) of the playlist, which is the number of protons incident on the NuMI target. The total POT for data taken in the $\nu_{\mu}\left(\bar{\nu}_{\mu}\right)$ beam configuration was $3.04 \times 10^{20}\left(2.00 \times 10^{20}\right)$.

\begin{tabular}{l|c|c|c} 
Playlist & Beam Configuration & Data POT & MC POT \\
\hline Downstream 1 & $\bar{\nu}_{\mu}$ & $4.157 \times 10^{19}$ & $2.776 \times 10^{20}$ \\
Downstream 2 & $\bar{\nu}_{\mu}$ & $5.301 \times 10^{19}$ & $2.454 \times 10^{20}$ \\
MINER $\nu$ A 1 & $\nu_{\mu}$ & $9.599 \times 10^{19}$ & $7.169 \times 10^{20}$ \\
MINER $\nu$ A 5 & $\bar{\nu}_{\mu}$ & $1.061 \times 10^{20}$ & $9.960 \times 10^{20}$ \\
MINER $\nu$ A 7 & $\nu_{\mu}$ & $2.873 \times 10^{18}$ & $7.920 \times 10^{19}$ \\
MINER $\nu$ A 9 & $\nu_{\mu}$ & $6.782 \times 10^{18}$ & $7.990 \times 10^{19}$ \\
MINER $\nu$ A 13B & $\nu_{\mu}$ & $1.792 \times 10^{19}$ & - \\
MINER $\nu$ A 13C & $\nu_{\mu}$ & $1.225 \times 10^{20}$ & $1.184 \times 10^{21}$ \\
MINER $\nu \mathrm{A} 13 \mathrm{D}$ & $\nu_{\mu}$ & $6.678 \times 10^{18}$ & - \\
MINER $\nu \mathrm{A} 13 \mathrm{E}$ & $\nu_{\mu}$ & $5.220 \times 10^{19}$ & - \\
& & &
\end{tabular}

Table 8.1: Data and MC samples 
A MC sample was generated for each data playlist with the exception of playlists MINER $\nu \mathrm{A} 13 \mathrm{~B}, 13 \mathrm{D}$, and 13E. The MINER $\nu \mathrm{A} 13$ playlists correspond to MINER $\nu \mathrm{A}$ detector configurations with different levels of helium in the helium target, the effects of which are irrelevant to this analysis. A MC sample was generated for MINER $\nu \mathrm{A}$ 13C, which is the MINER $\nu \mathrm{A} 13$ playlist with the largest data POT. Each MC sample was generated with the neutrino beam configuration of its playlist, and was overlaid with data from its playlist to mimic the pile-up effects in the data. The statistics of the MC samples, quantified in POT, are several times that of the data so that the statistical uncertainty on the $\mathrm{MC}$ predictions (e.g. background rate) is small relative to the systematic uncertainties (Section 8.13). The Downstream 1 and 2 MC samples were generated with the partial MINER $\nu \mathrm{A}$ detector and ArgoNeuT simulation.

High-statistics signal-only MC samples were generated for estimating the resolution of the reconstructed kinematics (Section 8.6), unfolding matrices (Section 8.9.2), and selection efficiency (Section 8.9.3) for $\nu_{\mu}$ and $\bar{\nu}_{\mu} \mathrm{CC}$ coherent scattering. Table 8.2 lists the beam and detector configurations, overlaid data, and number of events generated in the fiducial volume for each signal-only MC sample. The $\bar{\nu}_{\mu}$ partial detector sample was generated with the ArgoNeuT simulation.

\begin{tabular}{c|c|c|c} 
Beam Configuration & Detector & Data Overlay & Generated Fiducial Events \\
\hline $\bar{\nu}_{\mu}$ & Partial & Downstream 1 & 133,056 \\
$\nu_{\mu}$ & Full & MINER $\nu \mathrm{A} \mathrm{1}$ & 123,216 \\
$\bar{\nu}_{\mu}$ & Full & MINER $\nu \mathrm{A} 5$ & 90,277
\end{tabular}

Table 8.2: Signal-only MC samples. 


\subsection{Reweighting}

The MC was reweighted to apply constraints on the flux prediction and the rate of neutrino interactions with single pion final states in GENIE, to apply corrections to the pion angle distribution in delta resonance decay and the coherent pion production cross section in GENIE, and to correct the simulated MINOS muon tracking efficiency.

The MC was generated with the g4numi flux - the flux prediction for the nominal GEANT4 prediction of hadron production at the NuMI target. The MC was reweighted to reflect the flux prediction constrained by both external hadron production data (the ppfx flux) and the in situ $\nu+e$ elastic scattering measurement. This was done by weighting each neutrino interaction in MC by the ratio of the $\nu+e$ constrained ppfx flux to the g4numi flux for the $E_{\nu}$ of the interaction.

The GENIE prediction of single pion final states was constrained to re-analyzed $\nu_{\mu}$-deuterium scattering data [111]. The axial vector mass for resonant pion production $M_{A}^{R E S}$ and corrections to the resonant pion production and non-resonant single pion production normalizations in GENIE were extracted from a fit of GENIE to the re-analyzed data for single pion final states [112]. Table 8.3 lists the values extracted from the fit for GENIE 2.6.2. The default $M_{A}^{R E S}$ in GENIE is $1.12 \pm 0.22$ $\mathrm{GeV}$. Resonant interactions and non-resonant single pion production interactions in the $\mathrm{MC}$ were weighted to the values from the fit.

In simulating neutrino production of baryon resonances, GENIE simulates the decay of the baryon resonance in order to simulate final state interactions of the decay products within the nucleus. For simplicity in generating events, GENIE simulates isotropic decay of baryon resonances, where the decay products have a 


\begin{tabular}{l|c} 
Parameter & Value \\
\hline$M_{A}^{R E S}(\mathrm{GeV})$ & $0.94 \pm 0.05$ \\
Resonant Normalization Correction & $1.15 \pm 0.07$ \\
Non-Resonant $1 \pi$ Normalization Correction & $0.46 \pm 0.04$
\end{tabular}

Table 8.3: Parameters for single pion production in GENIE extracted from the fit of GENIE to re-analyzed $\nu_{\mu}$-deuterium scattering data.

uniform angle distribution in the center of mass frame of the resonance with respect to the angular momentum quantization axis of the resonance [113]. This deviates from the Rein-Sehgal model for resonance production utilized in GENIE, which predicts non-isotropic baryon resonance decay. For $\Delta \rightarrow N \pi$ delta resonance decays, the angle distribution of the pion is given by

$$
W_{\pi}(\cos \theta)=1-p\left(\frac{3}{2}\right) P_{2}(\cos \theta)+p\left(\frac{1}{2}\right) P_{2}(\cos \theta)
$$

where $\theta$ is the pion angle in the $\Delta$ center of mass frame with respect to the $\Delta$ angular quantization axis, $p\left(\frac{3}{2}\right)$ and $p\left(\frac{1}{2}\right)$ are coefficients for the $\frac{3}{2}$ and $\frac{1}{2}$ angular momentum states of the $\Delta$, respectively, and $P_{2}(\cos \theta)$ is the 2 nd order Legendre polynomial. For $\Delta \rightarrow N \pi$, GENIE simulates isotropic $\Delta$ decay with $p\left(\frac{3}{2}\right)=p\left(\frac{1}{2}\right)=$ 0.5 , whereas the Rein-Sehgal resonance production model predicts non-isotropic $\Delta$ decay with $p\left(\frac{3}{2}\right)=0.75$ and $p\left(\frac{1}{2}\right)=0.25$. For propagating the uncertainty from simulating isotropic $\Delta^{++} \rightarrow N \pi$ decay, GENIE provides event-by-event weights that warp the isotropic angle distribution of the decay pions to the non-isotropic ReinSehgal prediction. Using these weights, the pion angle distribution measured from MINER $\nu$ A data of $\nu_{\mu}$ charged current $\pi^{+}$production was shown to prefer the GENIE prediction for these processes with non-isotropic $\Delta^{++} \rightarrow N \pi$ decay per Rein-Sehgal [114]. The convention within MINER $\nu \mathrm{A}$, which is applied in this analysis, is to 
weight the isotropic pion angle distribution from $\Delta^{++} \rightarrow N \pi$ decays as generated by GENIE to half the non-isotropy predicted by the Rein-Seghal resonance production model.

The GENIE implementation of the Rein-Sehgal coherent scattering model uses data of the total and inelastic cross sections for pion-proton and pion-deuterium scattering to calculate the pion-nucleus elastic scattering cross section. GENIE 2.6.2, the version of GENIE used to generate the MC used in this analysis, contained an error in indexing the pion-proton and pion-deuterium cross section data tables. This error was corrected by weighting each coherent event in the $\mathrm{MC}$ by the ratio of its corrected to uncorrected GENIE coherent cross sections. Figure 8.3 illustrates the size of the correction as the ratio of the corrected to uncorrected generated event rate as a function of true $E_{\pi}$ for $\nu_{\mu}$ and $\bar{\nu}_{\mu}$ charged current coherent interactions on carbon in the fiducial volume.
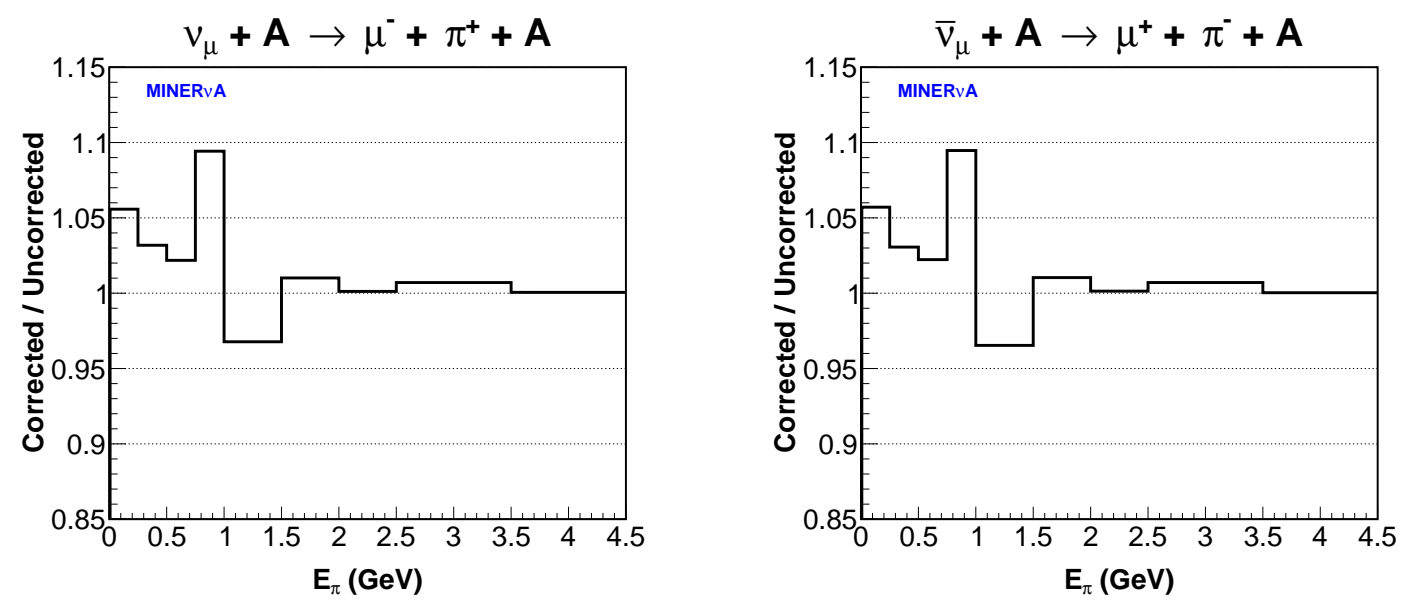

Figure 8.3: The ratio of the generated event rate corrected for the GENIE coherent cross section error to the uncorrected generated event rate as a function of true $E_{\pi}$ for $\nu_{\mu}$ (left) and $\bar{\nu}_{\mu}$ (right) charged current coherent interactions on carbon in the fiducial volume.

The efficiency of tracking the muon in MINOS differed in data and MC due 
to pile-up not being simulated in MINOS. The efficiency was measured in both data and MC by projecting muon tracks that exited MINER $\nu \mathrm{A}$ into MINOS and measuring the rate that the muon was reconstructed in MINOS. The efficiency was measured separately for muon momenta in MINOS $p_{\mu}^{M I N O S} \leq 3.0 \mathrm{GeV} / \mathrm{c}$ and $p_{\mu}^{M I N O S}>3.0 \mathrm{GeV} / \mathrm{c}$, where $p_{\mu}^{M I N O S}$ was estimated by the deflection of the muon in the downstream HCAL due to multiple scattering. The correction to the efficiency in $\mathrm{MC}$ is defined as the ratio of the total efficiency in data to $\mathrm{MC}$ and is listed for each playlist in Table 8.4. The efficiency corrections for the $\bar{\nu}_{\mu}$ partial detector playlists (Downstream 1 and 2) were not measured and were instead assumed to be equal to the $\bar{\nu}_{\mu}$ full detector playlist (MINER $\nu \mathrm{A} 5$ ). The difference in efficiency between data and $\mathrm{MC}$ was corrected by weighting each event in the $\mathrm{MC}$ by the efficiency correction.

\begin{tabular}{l|c|c} 
Playlist & $p_{\mu}^{M I N O S} \leq 3.0 \mathrm{GeV} / \mathrm{c}$ & $p_{\mu}^{M I N O S}>3.0 \mathrm{GeV} / \mathrm{c}$ \\
\hline MINER $\nu \mathrm{A} \mathrm{1}$ & $0.934 \pm 0.002$ & $0.982 \pm 0.001$ \\
MINER $\nu \mathrm{A} 5$ & $0.956 \pm 0.002$ & $0.989 \pm 0.001$ \\
MINER $\nu \mathrm{A} 7$ & $0.969 \pm 0.009$ & $0.994 \pm 0.002$ \\
MINER $\nu \mathrm{A} 9$ & $0.951 \pm 0.006$ & $0.994 \pm 0.002$ \\
MINER $\nu \mathrm{A}$ 13 & $0.942 \pm 0.002$ & $0.987 \pm 0.001$
\end{tabular}

Table 8.4: The measured corrections, with statistical uncertainty, to the MINOS muon tracking efficiency in $\mathrm{MC}$

The muon tracking efficiency in MINER $\nu \mathrm{A}$ was also measured in both data and $\mathrm{MC}$ by projecting muon tracks in MINOS into MINER $\nu \mathrm{A}$ and measuring the rate that the muon was reconstructed in MINER $\nu$ A. The efficiencies in data and MC were found to be consistent, as pile-up is simulated in MINER $\nu$ A. Therefore, no correction is made to the MINER $\nu \mathrm{A}$ muon tracking efficiency in MC. 


\subsection{Kinematics Reconstruction}

The kinematic quantities that characterize coherent scattering must be reconstructed for each event in order to both select coherent interactions from the data and to measure the cross sections. This requires measuring the direction and energy of both the muon and pion. From these quantities the remaining kinematics for coherent scattering can be calculated, which are the neutrino energy $E_{\nu}$, the squared four momentum transferred from the lepton system $Q^{2}$, and the squared four momentum transferred to the nucleus $|t|$.

The muon and pion directions are measured by their reconstructed track originating from the interaction vertex. The muon is identified by the track that exits the downstream end of MINER $\nu \mathrm{A}$ and is matched to a track in MINOS. The reconstructed interaction vertex position is defined as the position of the most upstream node of the MINER $\nu \mathrm{A}$ muon track, which is the best estimate of the interaction vertex position for coherent scattering. The pion track is the second track originating from the interaction vertex. Each track measures the 3D direction vector of its respective particle in each scintillator plane along the track. The direction of both the muon and pion are taken as the direction vector in the most upstream plane along their respective track. This gives the best measurement of the directions of the muon and pion since both are produced in the forward direction in coherent scattering and both scatter while traversing the detector material. The angle between the directions of the muon (pion) and incoming neutrino is denoted by $\theta_{\mu}$ $\left(\theta_{\pi}\right)$. The direction of the incoming neutrino is assumed to be parallel to neutrino beam axis.

The muon energy $E_{\mu}$ is reconstructed from the muon's tracks in MINER $\nu \mathrm{A}$ and 
MINOS. The energy of the muon at its entrance point to MINOS is reconstructed from the MINOS track's range (curvature in the magnetic field) if muon stops inside (exits) MINOS. This energy is added to the calculated muon energy loss by ionization along the MINER $\nu$ A track to give the reconstructed muon energy at the interaction vertex $E_{\mu}$. For the partial detector, the calculated muon energy loss by ionization in ArgoNeuT is included in $E_{\mu}$.

The pion energy $E_{\pi}$ is reconstructed by calorimetry. This approach is motivated by the majority of charged pions in MINER $\nu \mathrm{A}$ interacting hadronically with nuclei in the detector. These interactions produce a variety of secondary particles, each of which produces a different detector response. In particular, neutrons can be produced which, if they interact, only deposit a fraction of their energy in the form of scattered low energy protons and thereby constitute missing pion energy. Calorimetry corrects the visible energy from a pion and its secondary particles to give, on average, the correct $E_{\pi} . E_{\pi}$ is reconstructed under a coherent scattering hypothesis where all hadronic (i.e. non-muon) visible energy is assumed to result from the pion. To minimize sensitivity to mismodeled vertex activity in incoherent interactions, hadronic visible energy within $200 \mathrm{~mm}$ of the interaction vertex is excluded from the $E_{\pi}$ reconstruction and replaced with $60 \mathrm{MeV}$, which is an estimate of the average calorimetric energy deposited by a minimum ionizing pion along a $200 \mathrm{~mm}$ path in the tracker region. The reconstructed $E_{\pi}$ is calculated as

$$
E_{\pi}=60 \mathrm{MeV}+1.7 \sum_{i} E_{i}^{c o r}
$$

where $E_{i}^{c o r}$ is the visible energy corrected for passive materials in subdetector $i$. The scale factor 1.7 corrects for missing pion energy and is tuned using simulated coherent 
interactions. The subdetectors included in the sum of Equation 8.2 are the tracker, downstream and side ECALs, and downstream and side HCALs. The nuclear targets region is excluded from the sum since it is upstream of the fiducial volume and a pion from coherent scattering will deposit energy downstream of the interaction vertex. The corrected visible energy $E^{c o r}$ in each subdetector is calculated as

$$
E^{c o r}=\sum_{i} c_{i} E_{i}^{c l u s}
$$

where $E_{i}^{\text {clus }}$ is the visible energy of cluster $i$ in the subdetector and $c_{i}$ is the passive material correction for the cluster. The sum in Equation 8.3 is over all clusters in the subdetector beyond $200 \mathrm{~mm}$ from the interaction vertex and within -20 ns and +30 ns of the reconstructed interaction vertex time. The cluster time requirement minimizes contamination from pile-up. The passive material correction is calculated as

$$
c=\frac{E^{t o t}}{E^{v i s}}
$$

where $E^{\text {tot }}\left(E^{\text {vis }}\right)$ is the energy deposited by a minimum ionizing particle at normal incidence in all materials (active scintillator only) in a module or story. Here, normal incidence is defined for the side HCAL as perpendicular to the scintillator bars in the transverse (XY) plane, and perpendicular to the scintillator planes for all other subdetectors. The passive material corrections for each subdetector (Table 8.5) are calculated from the MC.

The reconstructed neutrino energy $E_{\nu}$ is calculated as

$$
E_{\nu}=E_{\mu}+E_{\pi}
$$




\begin{tabular}{l|c} 
Subdetector & Correction \\
\hline Tracker & 1.2 \\
Side ECAL - X Planes & 2.8 \\
Side ECAL - UV Planes & 4.4 \\
Downstream ECAL & 2.0 \\
Downstream HCAL & 10.3 \\
Side HCAL - Stories 1-3 & 21.7 \\
Side HCAL - Story 4 & 42.1
\end{tabular}

Table 8.5: Subdetector passive material corrections

where $E_{\mu}$ and $E_{\pi}$ are the reconstructed muon and pion energies, respectively. This calculation assumes zero energy transfer to the nucleus, which is a good approximation for coherent scattering since the nucleus remains in its ground state and the recoil of the nucleus is small due to small $|t|$.

The reconstructed squared four-momentum transferred to the nucleus $|t|$ is calculated as

$$
|t|=\left|\left(q-p_{\pi}\right)^{2}\right|=\left|\left(p_{\nu}-p_{\mu}-p_{\pi}\right)^{2}\right|
$$

where $p_{\nu}, p_{\mu}$, and $p_{\pi}$ are the reconstructed four-momentum vectors for the neutrino, muon, and pion, respectively. The reconstructed four-momentum vector for each particle is calculated from the particle's reconstructed energy and direction (as mentioned previously, the neutrino direction is assumed to be parallel to the neutrino beam axis). Likewise, the reconstructed squared four-momentum transferred from the lepton system $Q^{2}$ is calculated as

$$
Q^{2}=-q^{2}=\left(p_{\nu}-p_{\mu}\right)^{2}
$$

The resolution of the reconstructed interaction vertex position and reconstructed kinematic parameters for reconstructed $\nu_{\mu}$ and $\bar{\nu}_{\mu} \mathrm{CC}$ coherent $\mathrm{MC}$ interactions 
inside the fiducial volume are shown in Figures 8.4-8.9. For a reconstructed quantity $\xi_{\text {reco }}$ with true value $\xi_{\text {true }}$, the resolution is defined as $\xi_{\text {reco }}-\xi_{\text {true }}$, and the fractional resolution is defined as $\left(\xi_{\text {reco }}-\xi_{\text {true }}\right) / \xi_{\text {true }}$. The resolutions of the reconstructed kinematic parameters were quantified by a double gaussian fit and are listed in Tables 8.6-8.11. 

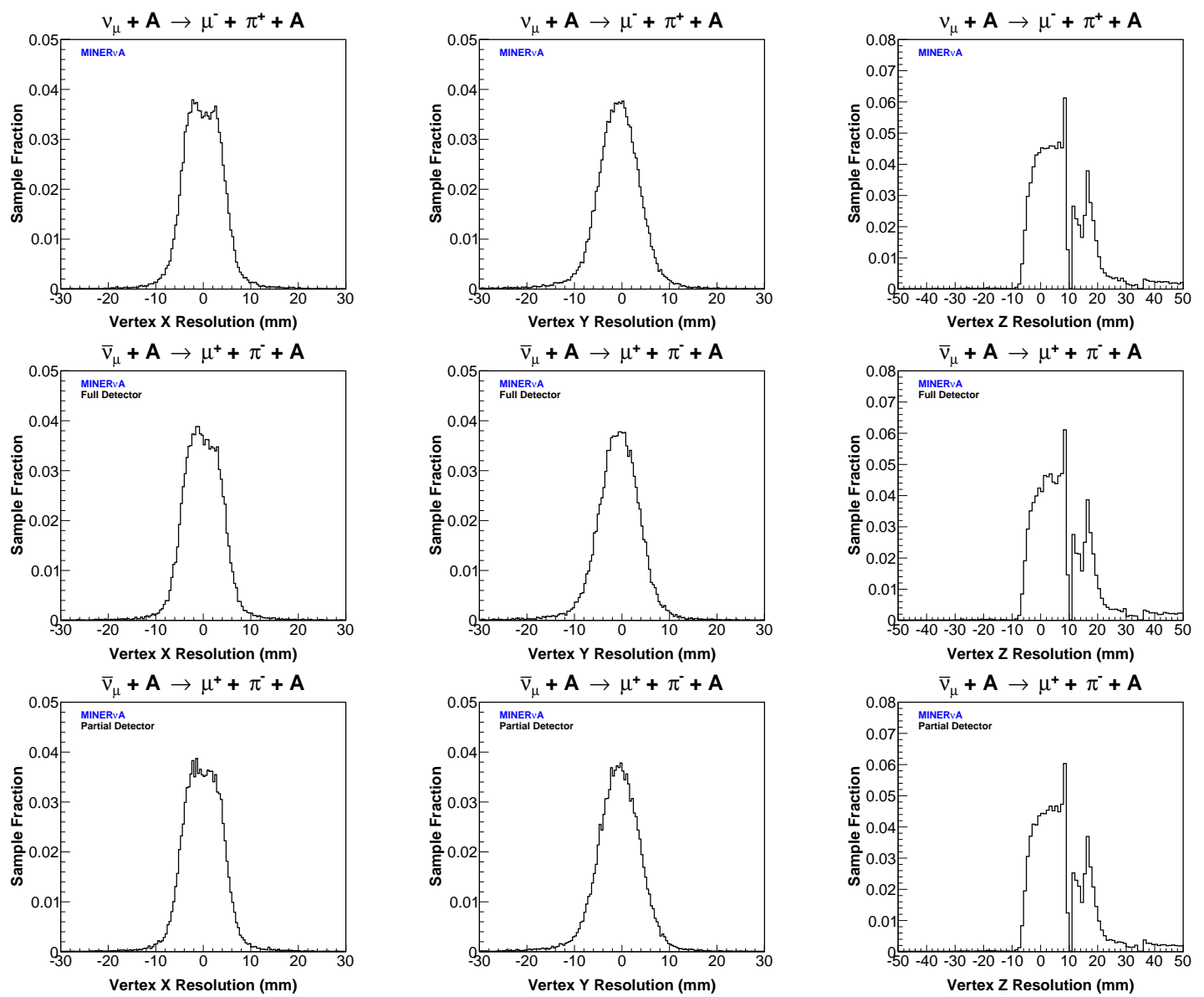

Figure 8.4: The resolution of the reconstructed interaction vertex $\mathrm{X}$ (left), $\mathrm{Y}$ (center), and Z (right) positions for reconstructed $\nu_{\mu}$ and $\bar{\nu}_{\mu} \mathrm{CC}$ coherent MC interactions inside the fiducial volume. The top plots are for $\nu_{\mu}$ interactions in the full detector, and the middle (bottom) plots are for $\bar{\nu}_{\mu}$ interactions in the full (partial) detector. The reconstructed interaction vertex position is defined as the position of the most upstream node of the muon track. The structure in the vertex $\mathrm{Z}$ position resolution is due to the $\mathrm{Z}$ position of the most upstream node of the muon track being defined as the $\mathrm{Z}$ position of the center of the plane containing the node coupled with the $\mathrm{Z}$ positions of the planes. 

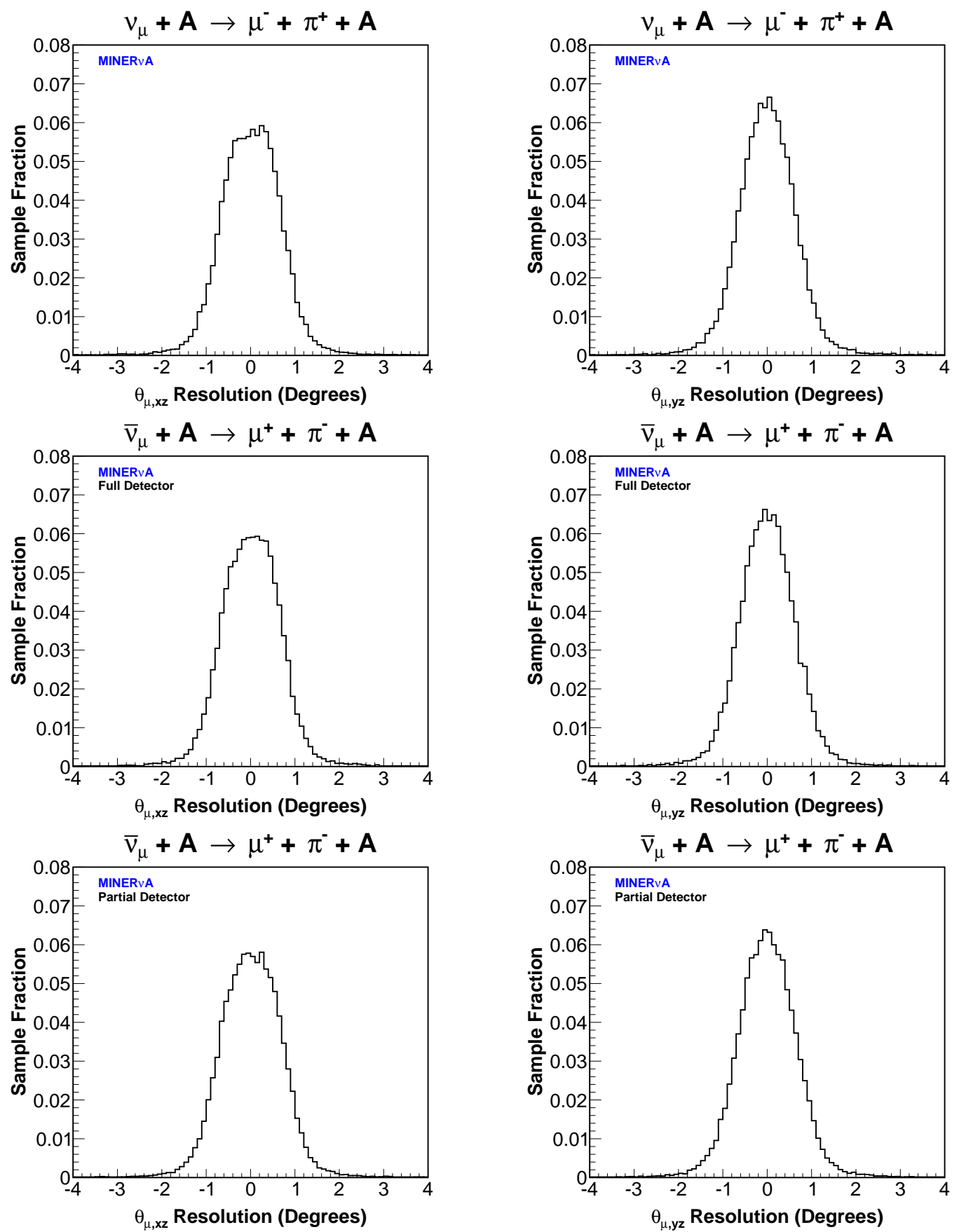

Figure 8.5: The reconstructed muon angle resolution in the XZ plane $\theta_{\mu, x z}$ (left) and YZ plane $\theta_{\mu, y z}$ (right) for reconstructed $\nu_{\mu}$ and $\bar{\nu}_{\mu} \mathrm{CC}$ coherent $\mathrm{MC}$ interactions inside the fiducial volume. The top plots are for $\nu_{\mu}$ interactions in the full detector, and the middle (bottom) plots are for $\bar{\nu}_{\mu}$ interactions in the full (partial) detector. 

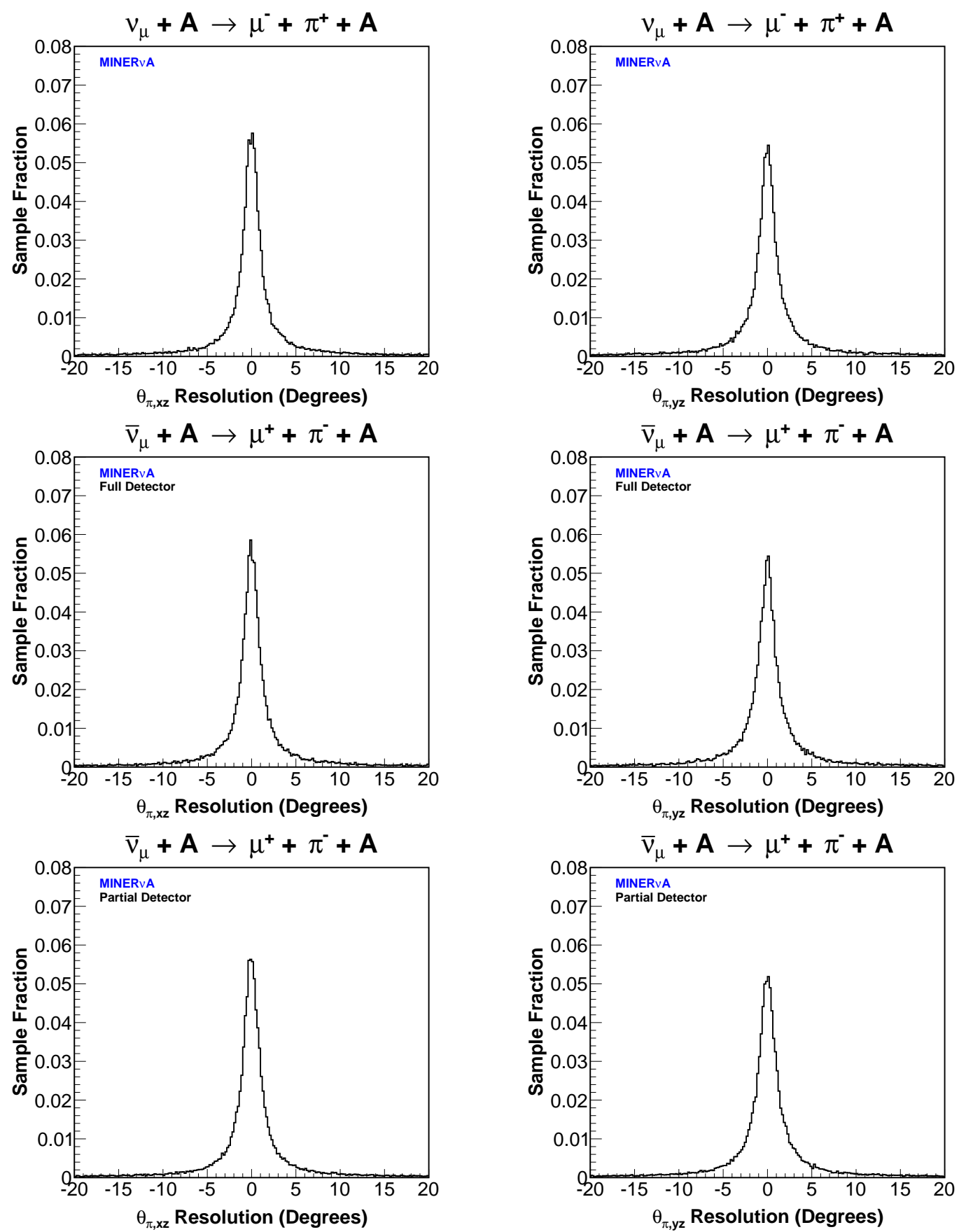

Figure 8.6: The reconstructed pion angle resolution in the XZ plane $\theta_{\pi, x z}$ (left) and YZ plane $\theta_{\pi, y z}$ (right) for reconstructed $\nu_{\mu}$ and $\bar{\nu}_{\mu} \mathrm{CC}$ coherent MC interactions inside the fiducial volume. The top plots are for $\nu_{\mu}$ interactions in the full detector, and the middle (bottom) plots are for $\bar{\nu}_{\mu}$ interactions in the full (partial) detector. 

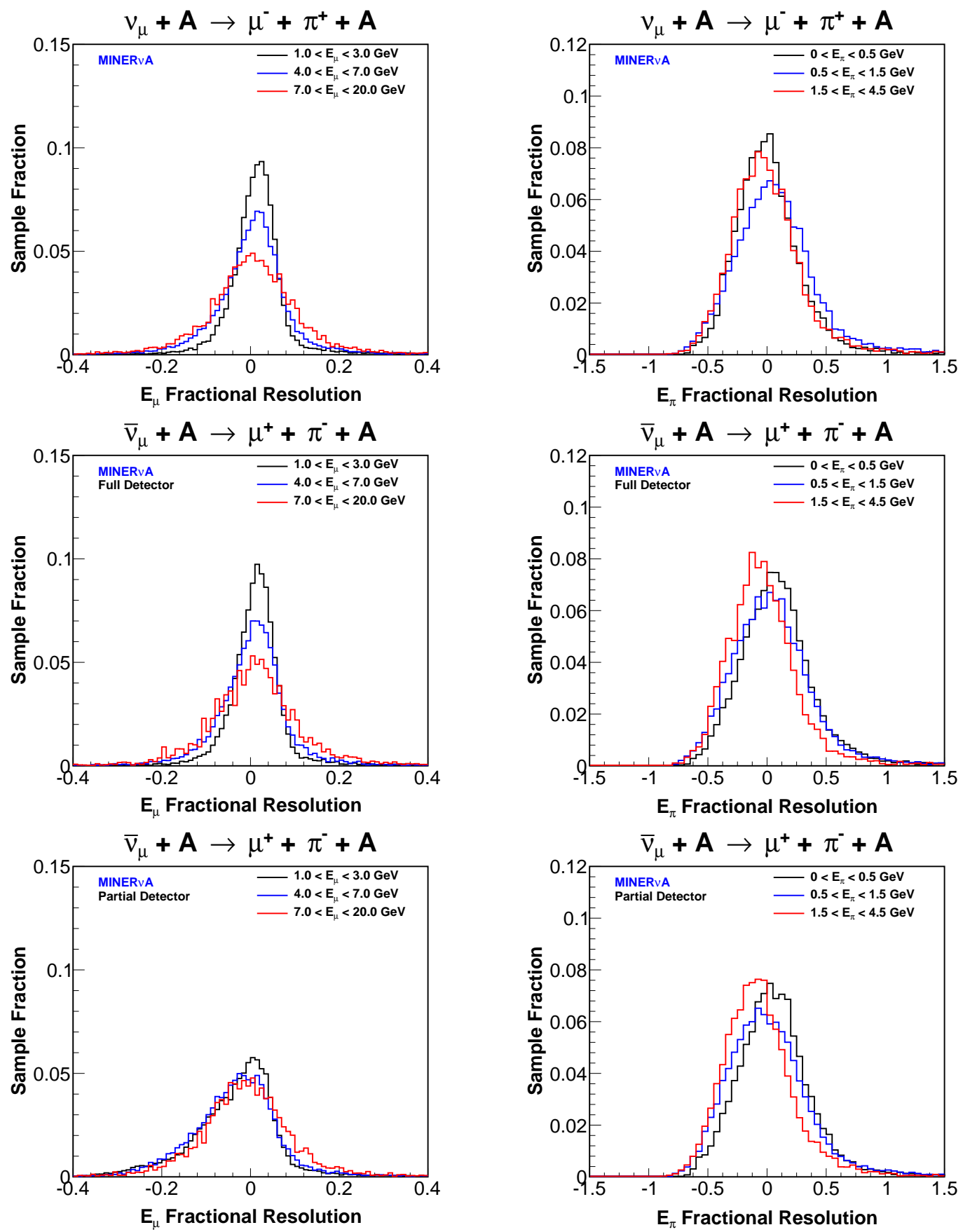

Figure 8.7: The reconstructed $E_{\mu}$ (left) and $E_{\pi}$ (right) fractional resolution for reconstructed $\nu_{\mu}$ and $\bar{\nu}_{\mu} \mathrm{CC}$ coherent $\mathrm{MC}$ interactions inside the fiducial volume. The top plots are for $\nu_{\mu}$ interactions in the full detector, and the middle (bottom) plots are for $\bar{\nu}_{\mu}$ interactions in the full (partial) detector. 

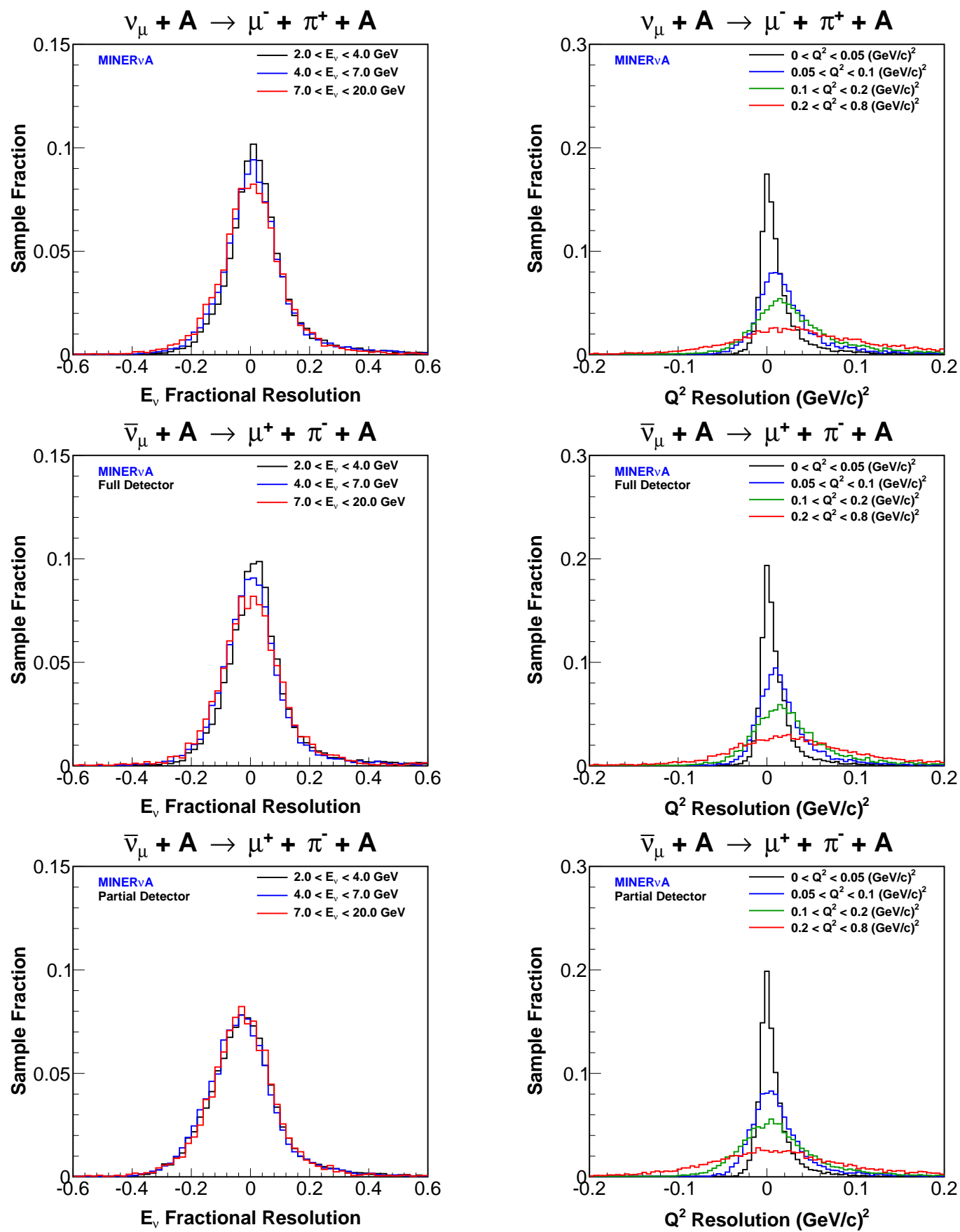

Figure 8.8: The reconstructed $E_{\nu}$ fractional resolution (left) and the reconstructed $Q^{2}$ resolution (right) for reconstructed $\nu_{\mu}$ and $\bar{\nu}_{\mu} \mathrm{CC}$ coherent $\mathrm{MC}$ interactions inside the fiducial volume. The top plots are for $\nu_{\mu}$ interactions in the full detector, and the middle (bottom) plots are for $\bar{\nu}_{\mu}$ interactions in the full (partial) detector. 

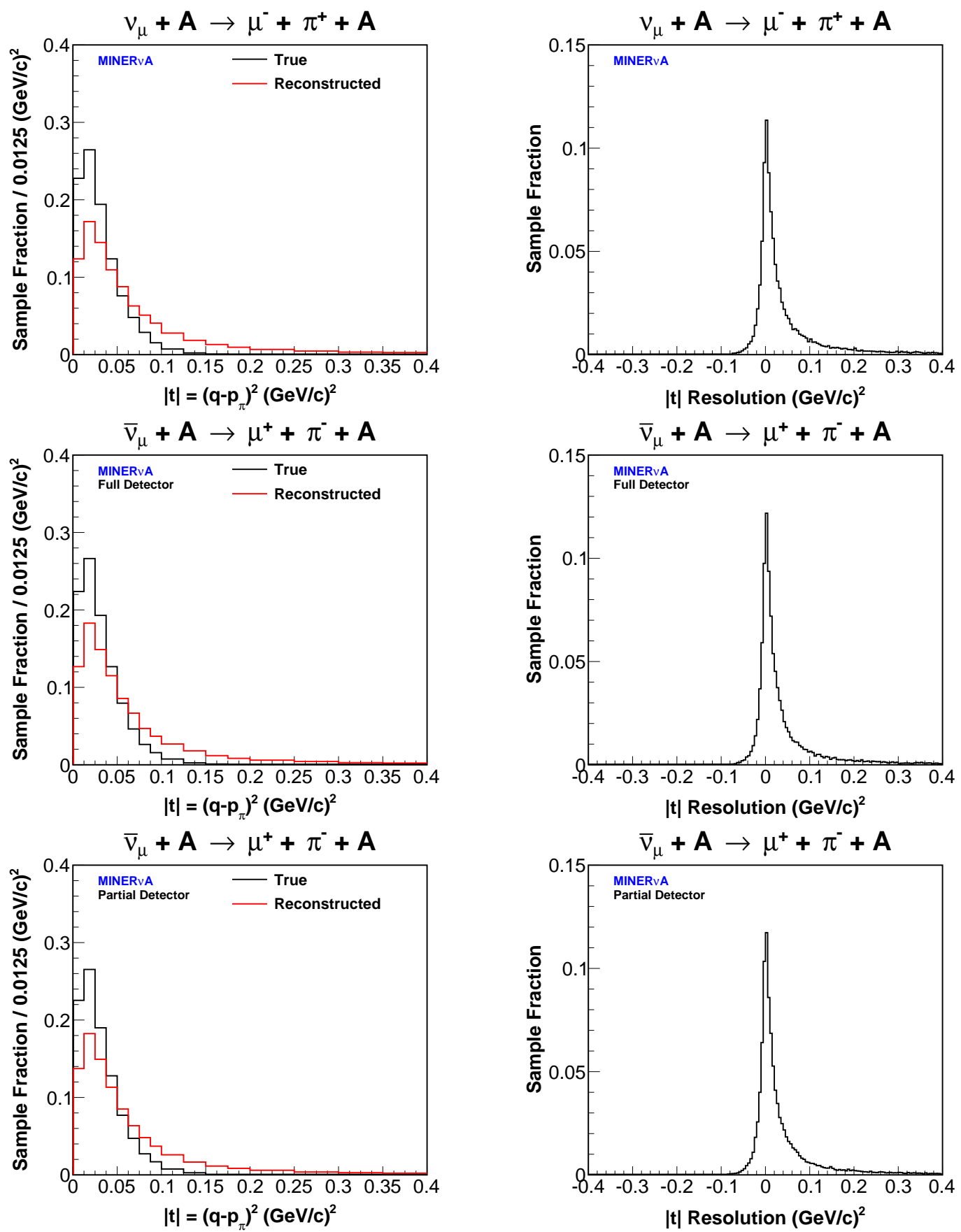

Figure 8.9: The reconstructed and true $|t|$ distributions (left) and the $|t|$ resolution (right) for reconstructed $\nu_{\mu}$ and $\bar{\nu}_{\mu} \mathrm{CC}$ coherent $\mathrm{MC}$ interactions inside the fiducial volume. The top plots are for $\nu_{\mu}$ interactions in the full detector, and the middle (bottom) plots are for $\bar{\nu}_{\mu}$ interactions in the full (partial) detector. 


\begin{tabular}{c|ccc|ccc|ccc} 
& \multicolumn{5}{|c}{$\nu_{\mu}$} & \multicolumn{3}{c}{$\bar{\nu}_{\mu}$ Full Detector } & \multicolumn{3}{c}{$\bar{\nu}_{\mu}$ Partial Detector } \\
Angle & $\sigma_{1}$ (Degrees) & $\sigma_{2}$ (Degrees) & $A_{2} / A_{1}$ & $\sigma_{1}$ (Degrees) & $\sigma_{2}$ (Degrees) & $A_{2} / A_{1}$ & $\sigma_{1}$ (Degrees) & $\sigma_{2}$ (Degrees) & $A_{2} / A_{1}$ \\
\hline$\theta_{\mu, x z}$ & 0.616 & 1.901 & 0.014 & 0.586 & 1.148 & 0.052 & 0.626 & 1.582 & 0.019 \\
$\theta_{\mu, y z}$ & 0.576 & 1.728 & 0.018 & 0.573 & 1.426 & 0.024 & 0.594 & 1.556 & 0.023 \\
$\theta_{\pi, x z}$ & 1.156 & 9.244 & 0.051 & 1.152 & 7.535 & 0.066 & 1.213 & 9.193 & 0.049 \\
$\theta_{\pi, y z}$ & 1.320 & 10.249 & 0.053 & 1.382 & 9.479 & 0.056 & 1.382 & 10.794 & 0.048
\end{tabular}

Table 8.6: The resolution of the muon and pion angles in the XZ and YZ planes for reconstructed $\nu_{\mu}$ and $\bar{\nu}_{\mu} \mathrm{CC}$ coherent MC interactions inside the fiducial volume. The resolution is quantified by a double-gaussian fit, where $A_{2} / A_{1}$ is the ratio of the gaussian normalizations.

\begin{tabular}{c|ccc|ccc|ccc} 
& \multicolumn{4}{|c|}{$\nu_{\mu}$} & \multicolumn{3}{c|}{$\bar{\nu}_{\mu}$ Full Detector } & \multicolumn{3}{c}{$\bar{\nu}_{\mu}$ Partial Detector } \\
$E_{\mu}(\mathrm{GeV})$ & $\sigma_{1}$ & $\sigma_{2}$ & $A_{2} / A_{1}$ & $\sigma_{1}$ & $\sigma_{2}$ & $A_{2} / A_{1}$ & $\sigma_{1}$ & $\sigma_{2}$ & $A_{2} / A_{1}$ \\
\hline $1-3$ & 0.038 & 0.132 & 0.050 & 0.037 & 0.109 & 0.071 & 0.042 & 0.108 & 0.460 \\
$3-7$ & 0.047 & 0.127 & 0.176 & 0.047 & 0.119 & 0.173 & 0.050 & 0.108 & 0.721 \\
$7-20$ & 0.071 & 0.173 & 0.151 & 0.073 & 0.198 & 0.093 & 0.078 & 0.208 & 0.074
\end{tabular}

Table 8.7: The $E_{\mu}$ fractional resolution for reconstructed $\nu_{\mu}$ and $\bar{\nu}_{\mu} \mathrm{CC}$ coherent $\mathrm{MC}$ interactions inside the fiducial volume. The fractional resolution is quantified by a double-gaussian fit, where $A_{2} / A_{1}$ is the ratio of the gaussian normalizations.

\begin{tabular}{c|ccc|ccc|ccc} 
& \multicolumn{4}{|c|}{$\nu_{\mu}$} & \multicolumn{3}{c}{$\bar{\nu}_{\mu}$ Full Detector } & \multicolumn{3}{|c}{$\bar{\nu}_{\mu}$ Partial Detector } \\
$E_{\pi}(\mathrm{GeV})$ & $\sigma_{1}$ & $\sigma_{2}$ & $A_{2} / A_{1}$ & $\sigma_{1}$ & $\sigma_{2}$ & $A_{2} / A_{1}$ & $\sigma_{1}$ & $\sigma_{2}$ & $A_{2} / A_{1}$ \\
\hline $0-0.5$ & 0.222 & 0.422 & 0.058 & 0.260 & 0.636 & 0.024 & 0.260 & 0.534 & 0.028 \\
$0.5-1$ & 0.285 & 0.614 & 0.032 & 0.288 & 0.733 & 0.021 & 0.283 & 0.544 & 0.038 \\
$1-1.5$ & 0.269 & 0.697 & 0.033 & 0.266 & 0.595 & 0.041 & 0.264 & 0.457 & 0.063 \\
$1.5-4.5$ & 0.241 & 0.613 & 0.026 & 0.236 & 0.741 & 0.027 & 0.237 & 0.411 & 0.061
\end{tabular}

Table 8.8: The $E_{\pi}$ fractional resolution for reconstructed $\nu_{\mu}$ and $\bar{\nu}_{\mu} \mathrm{CC}$ coherent $\mathrm{MC}$ interactions inside the fiducial volume. The fractional resolution is quantified by a double-gaussian fit, where $A_{2} / A_{1}$ is the ratio of the gaussian normalizations.

\begin{tabular}{c|ccc|ccc|ccc} 
& \multicolumn{4}{|c|}{$\nu_{\mu}$} & \multicolumn{3}{|c|}{$\bar{\nu}_{\mu}$ Full Detector } & \multicolumn{3}{|c}{$\bar{\nu}_{\mu}$ Partial Detector } \\
$E_{\nu}(\mathrm{GeV})$ & $\sigma_{1}$ & $\sigma_{2}$ & $A_{2} / A_{1}$ & $\sigma_{1}$ & $\sigma_{2}$ & $A_{2} / A_{1}$ & $\sigma_{1}$ & $\sigma_{2}$ & $A_{2} / A_{1}$ \\
\hline $2-4$ & 0.076 & 0.237 & 0.054 & 0.079 & 0.241 & 0.046 & 0.100 & 0.277 & 0.035 \\
$4-7$ & 0.082 & 0.246 & 0.060 & 0.081 & 0.247 & 0.048 & 0.096 & 0.210 & 0.078 \\
$7-20$ & 0.079 & 0.181 & 0.174 & 0.093 & 0.341 & 0.025 & 0.095 & 0.251 & 0.048
\end{tabular}

Table 8.9: The $E_{\nu}$ fractional resolution for reconstructed $\nu_{\mu}$ and $\bar{\nu}_{\mu}$ CC coherent $\mathrm{MC}$ interactions inside the fiducial volume. The fractional resolution is quantified by a double-gaussian fit, where $A_{2} / A_{1}$ is the ratio of the gaussian normalizations. 


\begin{tabular}{|c|c|c|c|c|c|c|c|c|c|}
\hline$Q^{2}(\mathrm{GeV} / \mathrm{c})^{2}$ & $\sigma_{1}(\mathrm{GeV} / \mathrm{c})^{2}$ & $\begin{array}{c}\nu_{\mu} \\
\sigma_{2}(\mathrm{GeV} / \mathrm{c})^{2}\end{array}$ & $A_{2} / A_{1}$ & $\begin{array}{r}\bar{\nu}_{\mu} \mathrm{I} \\
\sigma_{1}(\mathrm{GeV} / \mathrm{c})^{2} \\
\end{array}$ & $\begin{array}{l}\text { ull Detector } \\
\sigma_{2}(\mathrm{GeV} / \mathrm{c})^{2}\end{array}$ & $A_{2} / A_{1}$ & $\begin{array}{r}\bar{\nu}_{\mu} \mathrm{P} \\
\sigma_{1}(\mathrm{GeV} / \mathrm{c})^{2} \\
\end{array}$ & $\begin{array}{l}\text { artial Detector } \\
\sigma_{2}(\mathrm{GeV} / \mathrm{c})^{2}\end{array}$ & $A_{2} / A_{1}$ \\
\hline $0-0.05$ & 0.009 & 0.026 & 0.136 & 0.009 & 0.024 & 0.132 & 0.009 & 0.023 & 0.131 \\
\hline $0.05-0.1$ & 0.020 & 0.060 & 0.105 & 0.018 & 0.047 & 0.118 & 0.021 & 0.051 & 0.083 \\
\hline $0.1-0.2$ & 0.031 & 0.091 & 0.120 & 0.030 & 0.080 & 0.101 & 0.033 & 0.088 & 0.096 \\
\hline $0.2-0.8$ & 0.063 & 0.178 & 0.153 & 0.056 & 0.185 & 0.112 & 0.067 & 0.202 & 0.103 \\
\hline
\end{tabular}

Table 8.10: The $Q^{2}$ resolution for reconstructed $\nu_{\mu}$ and $\bar{\nu}_{\mu} \mathrm{CC}$ coherent $\mathrm{MC}$ interactions inside the fiducial volume. The resolution is quantified by a double-gaussian fit, where $A_{2} / A_{1}$ is the ratio of the gaussian normalizations.

\begin{tabular}{c|ccc} 
Sample & $\sigma_{1}(\mathrm{GeV} / \mathrm{c})^{2}$ & $\sigma_{2}(\mathrm{GeV} / \mathrm{c})^{2}$ & $A_{2} / A_{1}$ \\
\hline$\nu_{\mu}$ & 0.015 & 0.062 & 0.127 \\
$\bar{\nu}_{\mu}$ Full Detector & 0.014 & 0.054 & 0.132 \\
$\bar{\nu}_{\mu}$ Partial Detector & 0.014 & 0.052 & 0.153
\end{tabular}

Table 8.11: The $|t|$ resolution for reconstructed $\nu_{\mu}$ and $\bar{\nu}_{\mu} \mathrm{CC}$ coherent MC interactions inside the fiducial volume. The resolution is quantified by a double-gaussian fit, where $A_{2} / A_{1}$ is the ratio of the gaussian normalizations. 


\subsection{Event Selection}

This section describes the procedure for selecting coherent candidates (i.e. events that match the experimental signature for coherent scattering) from the data and $\mathrm{MC}$ for measuring the coherent scattering cross sections. The event selection procedure consists of a series of requirements, referred to as cuts, that correspond to the features of the experimental signature and determine whether each event is accepted or rejected. The MC is used to estimate the signal and background acceptance for each cut, and the background rate in the coherent candidate samples.

\subsubsection{Reconstruction and Fiducial Volume Cuts}

The event selection first requires each event have exactly one track in MINER $\nu \mathrm{A}$ that is matched to a track in MINOS. The matched tracks identify the muon since other particles tend to either stop or interact within MINER $\nu \mathrm{A}$. This requirement gives a sample $>99 \%$ pure in charged-current $\nu_{\mu} / \bar{\nu}_{\mu}$ events. In addition, the MINOS track enables determination of the muon charge and reconstruction of the muon energy.

Each event is required to have exactly one additional track originating at the interaction vertex and pointing in the forward direction. For coherent events, this track identifies the pion and measures its direction.

The interaction vertex is required to be located within the fiducial volume (Section 8.2). The interaction vertex is located at the origin of the muon track. For coherent events, the muon track origin gives the best estimate of the interaction vertex position.

Dead time can result in an interaction upstream of the fiducial volume faking an interaction inside fiducial volume. This occurs when a portion of the visible 
energy deposited inside the fiducial volume by a muon from an upstream interaction is lost due to dead time, resulting in the muon track originating inside the fiducial volume. While most of these "dead time" events are rejected by the requirement for a second track originating at the event vertex, a dead time cut is also applied to reject these events. For the dead time cut, the muon track is projected into the four planes immediately upstream of the event vertex. The event is rejected if two or more TriP-t chips reading out the strips intersected by the track projection underwent dead time. Dead time is modeled in the MC by overlaying the $\mathrm{MC}$ with data and simulating the charge integration period for the channels in the $\mathrm{MC}$ containing charge from the data overlay.

\subsubsection{Muon Charge Cut}

The muon charge is then used to select either $\nu_{\mu}$ or $\bar{\nu}_{\mu}$ charged current events. The muon charge is measured by the quantity $q / p$ extracted from the MINOS track fit, where $q$ and $p$ are the muon charge and momentum. $q / p$ is proportional to the reciprocal of the radius of curvature of the muon track in the magnetic field. Selected $\nu_{\mu}\left(\bar{\nu}_{\mu}\right)$ events have $q / p>0(q / p<0)$. Figure 8.10 shows the $q / p$ significance, defined as $q / p$ divided by the error on $q / p$ from the fit, distribution for events in the $\nu_{\mu}$ and $\bar{\nu}_{\mu}$ samples that pass the reconstruction and fiducial volume cuts. These distributions demonstrate that the background category "Other" is composed primarily of wrong-sign events. Prior to the $q / p$ cut, the $\bar{\nu}_{\mu}$ sample contains more wrong-sign $\left(\nu_{\mu}\right)$ events than $\bar{\nu}_{\mu}$ events. This is results from $\nu_{\mu}$ composing $15 \%$ of the RHC flux and charged current $\nu_{\mu}$ interactions producing a tracked hadron more often than charged current $\bar{\nu}_{\mu}$ interactions due to charge conservation. 

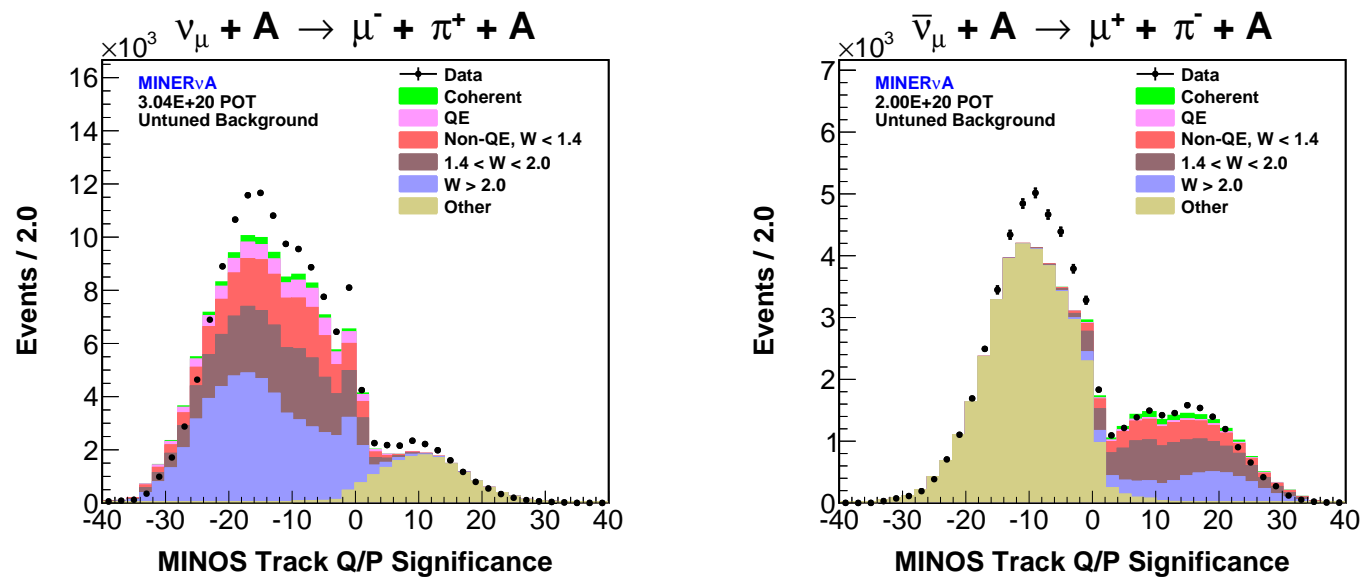

Figure 8.10: The $\nu_{\mu}$ (left) and $\bar{\nu}_{\mu}$ (right) $q / p$ significance of the matched MINOS track for events that pass the reconstruction and fiducial volume cuts. Selected $\nu_{\mu}$ $\left(\bar{\nu}_{\mu}\right)$ events have $q / p>0(q / p<0)$.

\subsubsection{Neutrino Energy Cut}

The neutrino energy of the $\nu_{\mu}$ and $\bar{\nu}_{\mu}$ samples (Figure 8.11) is restricted to $2.0<$ $E_{\nu}<20 \mathrm{GeV}$. Muons that originate in the tracker and are tracked in MINOS have $E_{\mu}>1.5 \mathrm{GeV}$. The $E_{\nu}>1.5 \mathrm{GeV}$ requirement therefore excludes mis-reconstructed muons. The $E_{\nu}<20 \mathrm{GeV}$ requirement excludes neutrinos resulting from kaon production at the NuMI target which is not well constrained. The $E_{\nu}$ cut also provides a well-defined neutrino energy range for comparing the measured coherent cross sections to model predictions.

\subsubsection{Proton Score Cut}

The visible energy along the hadron track (i.e. the non-muon track emerging from the event vertex) is analyzed to reject events with a tracked final state proton. The likelihood that the hadron track corresponds to a proton, referred to as the proton score, is calculated by comparing the visible energy in the clusters along the 

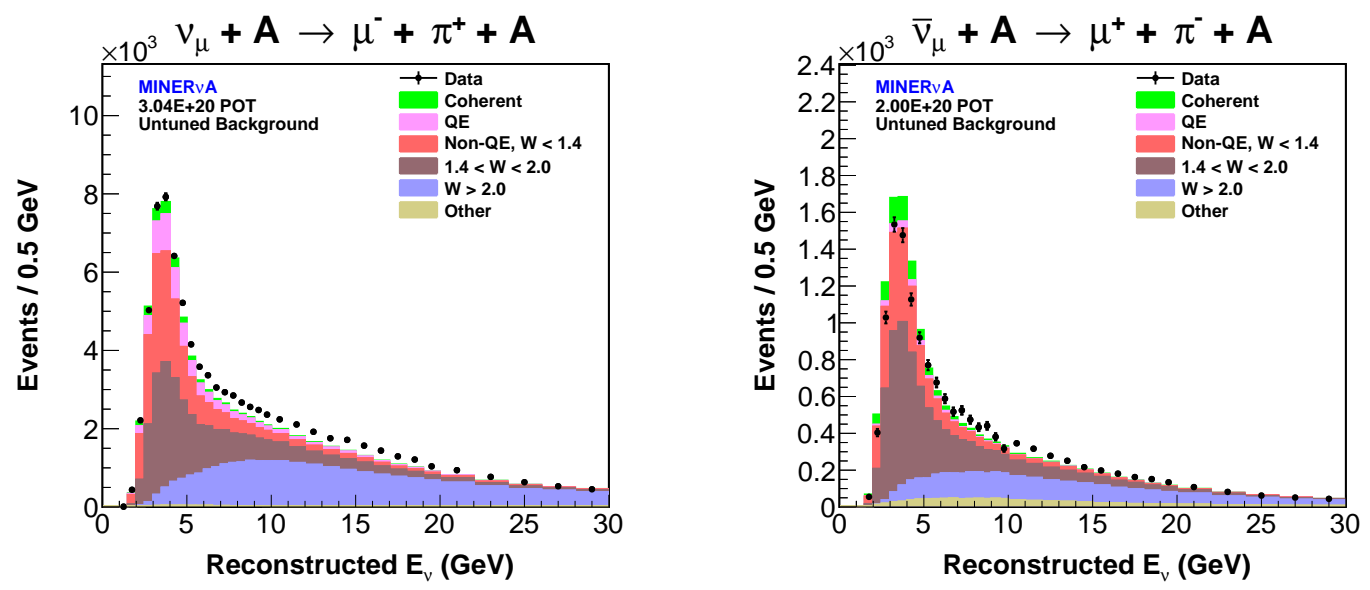

Figure 8.11: The $E_{\nu}$ distribution for events in the $\nu_{\mu}$ (left) and $\bar{\nu}_{\mu}$ (right) sample that pass all selection cuts up through the matched MINOS track $q / p$ cut. Events in the range $2.0<E_{\nu}<20 \mathrm{GeV}$ are selected.

hadron track to the predicted energy deposition by a stopping proton and pion. In predicting the energy deposition, the proton/pion originates (stops) at the begin (end) point of the hadron track. The predicted energy deposition in each cluster is the product of the average $d E / d x$ in the scintillator, calculated by the Bethe-Bloch equation, and the path length of the track in the scintillator. In calculating $d E / d x$, the pion/proton momentum at each cluster is estimated by range along the hadron track. The reduced $\chi^{2}$ for comparing the visible energy to the predicted pion/proton energy deposition in the clusters along the hadron track is calculated as

$$
\chi^{2}=\frac{1}{n} \sum_{i=1}^{n} \frac{\Delta E_{i}^{\text {meas }}-\Delta E_{i}^{\text {pred }}}{\sigma_{i}^{c o m b}}
$$

where $\Delta E_{i}^{\text {meas }}$ is the measured deposited energy (i.e. visible energy), $\Delta E_{i}^{\text {pred }}$ is the predicted energy deposition, and $\sigma_{i}^{\text {comb }}$ is the combined uncertainty on $\Delta E_{i}^{\text {meas }}$ and $\Delta E_{i}^{\text {pred }}$ in the $i^{\text {th }}$ cluster along the hadron track with $n$ total clusters. The combined uncertainty $\sigma_{i}^{c o m b}$ consists of range fluctuations on the calculated $d E / d x$, 
photo-statistical uncertainty on the measured and predicted deposited energy, and uncertainty on the path length of the track in the scintillator [115]. The proton score is calculated as

$$
\text { Proton Score }=1.0-\frac{\chi_{p}^{2}}{\sqrt{\left(\chi_{p}^{2}\right)^{2}+\left(\chi_{\pi}^{2}\right)^{2}}},
$$

where $\chi_{p}^{2}$ and $\chi_{\pi}^{2}$ are the reduced $\chi^{2}$ for the predicted proton and pion energy deposition, respectively.

A hadron track representing a particle that interacts in the detector may have one or more secondary tracks that emerge from its endpoint. The secondary tracks represent the scattered incident particle and/or particles produced in the interaction. For hadron tracks with exactly one secondary track, the proton score is calculated from the secondary track only, where it is assumed the secondary track represents a scattered proton/pion. The proton score is not calculated for hadron tracks with two or more secondary tracks, since it is ambiguous which secondary track corresponds to the scattered proton/pion. The proton score is also not calculated for hadron/secondary tracks associated with activity in the outer detector where the proton score is unreliable due to the thick layers of steel.

Figure 8.12 shows the proton score distribution for events in the $\nu_{\mu}$ and $\bar{\nu}_{\mu}$ samples that pass all cuts up through the $E_{\nu}$ cut, where the $\mathrm{MC}$ is categorized by the final state particle represented by the hadron track. Pions and protons are categorized by whether they stopped or interacted in the detector. The category "Other Particles" consists primarily of charged kaons and neutrons that interacted near the event vertex. The disagreement between the data and $\mathrm{MC}$ in the proton score distribution is attributed to the $\mathrm{MC}$ prediction of neutrino pion production, 
which is subsequently tuned to data (Section 8.8). The peak at 0.3 proton score is due to overlapping activity - clusters along the hadron track containing energy deposition from multiple particles. This is demonstrated by the peak in DIS events $(\mathrm{W}>2 \mathrm{GeV})$ at 0.3 proton score (Figure 8.12$)$. DIS interactions tend to produce multiple final state hadrons which lends to overlapping activity.

Events in the $\nu_{\mu}$ sample with proton score $<0.4$ are selected. Events where the proton score was not calculated are also selected. As shown in Figure 8.13, most events in the $\bar{\nu}_{\mu}$ sample with a tracked proton are rejected by the vertex energy cut (Section 8.7.5), since these events tend to have additional final state charged hadrons due to charge conservation. A proton score cut is not imposed on the $\bar{\nu}_{\mu}$ sample to maximize the signal selection efficiency and preserve statistics for the background tuning (Section 8.8).

\subsubsection{Vertex Energy Cut}

Coherent scattering produces a muon and charged pion in the forward direction while leaving the nucleus intact. The vertex energy (i.e. energy near the interaction vertex) of each event is required to be consistent with the energy deposited by a minimum ionizing muon and pion only. Additional energy indicates break up of the nucleus resulting from an incoherent interaction. Vertex energy $E_{v t x}$ is defined as the sum of the energies of clusters on the two vertex tracks within \pm 5 planes $(\sim 110$ $\mathrm{mm}$ in the longitudinal direction) from the event vertex, and clusters not on the two vertex tracks within \pm 5 planes and $200 \mathrm{~mm}$ in the transverse direction from the event vertex. In calculating $E_{v t x}$, the cluster energies are corrected for passive material and the on-track cluster energies are corrected for the angle between their respective track and the perpendicular to the scintillator planes. This correction 

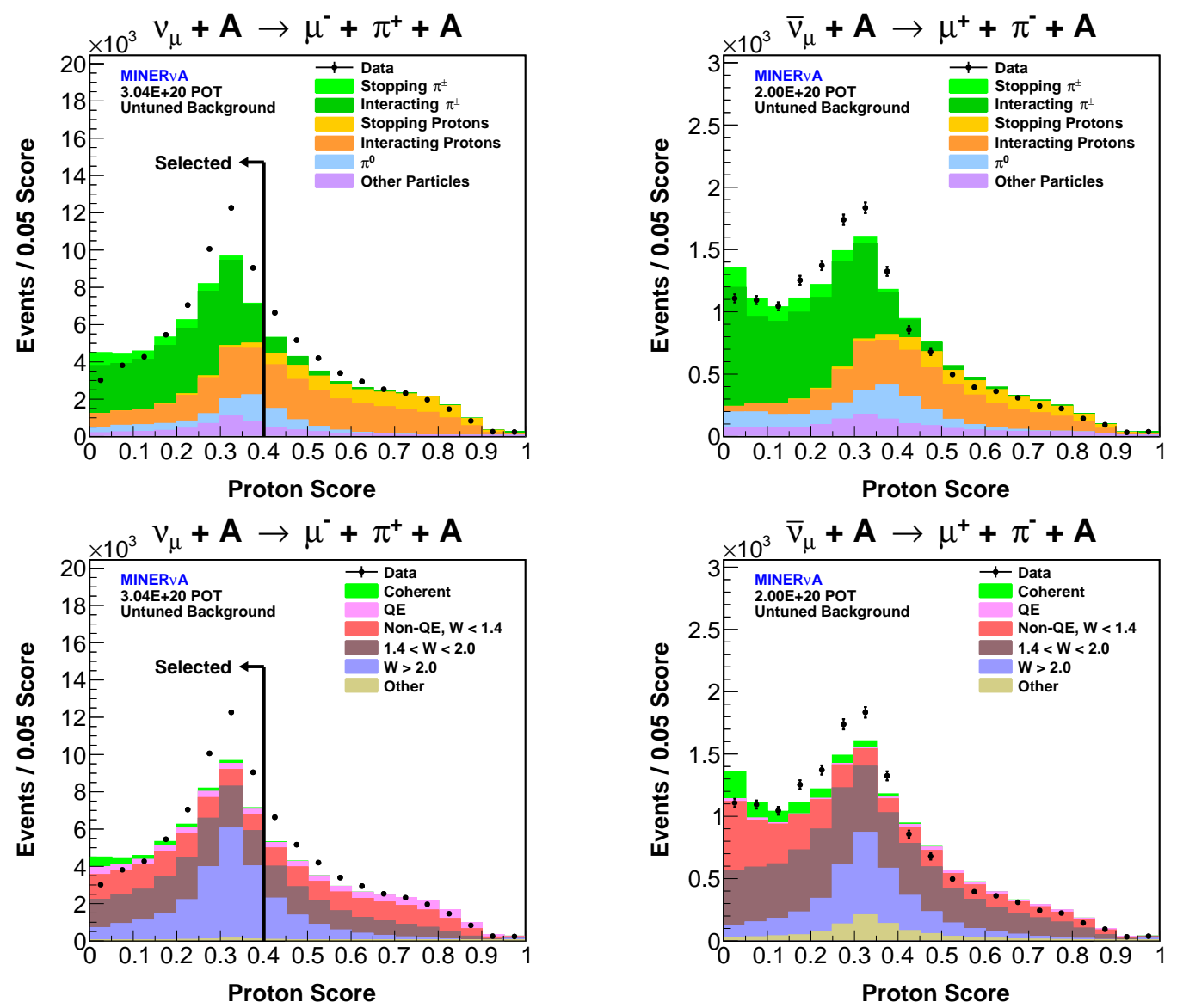

Figure 8.12: The proton score distribution for events in the $\nu_{\mu}$ (left) and $\bar{\nu}_{\mu}$ (right) sample that pass all selection cuts up through the $E_{\nu}$ cut. In the top plots, the MC is categorized by the final state particle represented by the hadron track. In the bottom plots, the MC is categorized by the neutrino interaction type. Events in the $\nu_{\mu}$ sample are required to have proton score $<0.4$. 

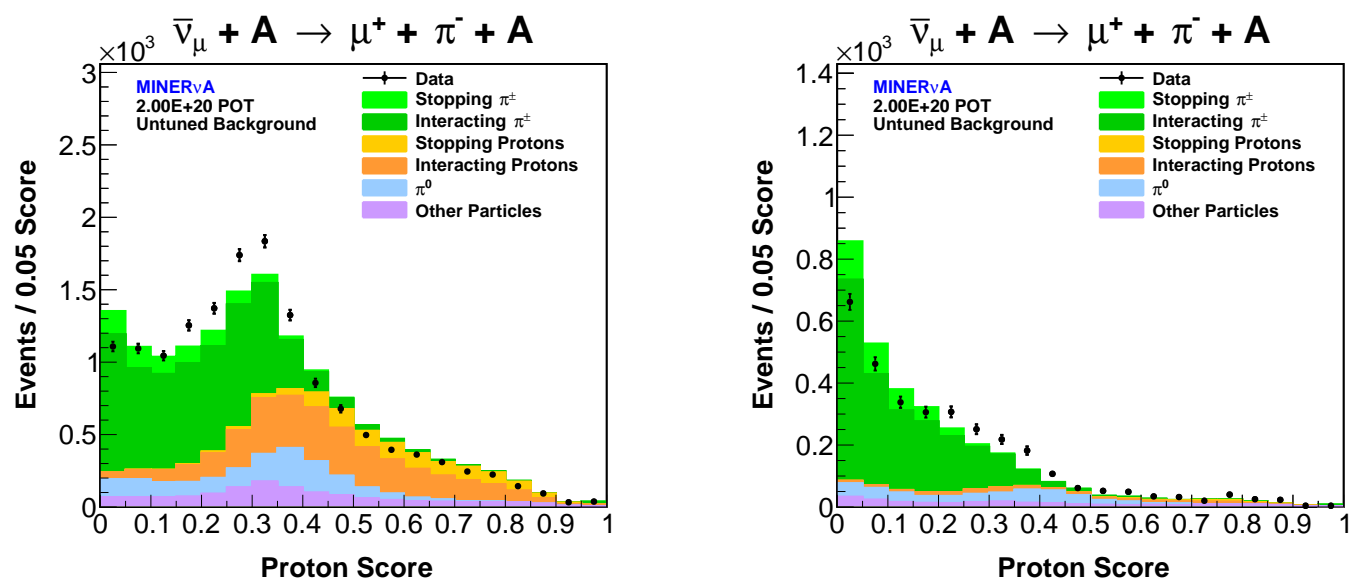

Figure 8.13: The left (right) plot shows the proton score distribution for events in the $\bar{\nu}_{\mu}$ sample that pass all selection cuts up through the $E_{\nu}$ (vertex energy) cut. A cut on proton score is not imposed on the $\bar{\nu}_{\mu}$ sample. The vertex energy cut rejects most events in the $\bar{\nu}_{\mu}$ sample with a tracked proton.

to normal incidence minimizes the dependence of the $E_{v t x}$ cut on the muon and pion kinematics. The \pm 5 plane longitudinal range was optimized to sample enough planes to smooth fluctuations in single plane energy deposition by the muon and pion while minimizing the amount of sampled material traversed by the pion, which in turn minimizes the loss in signal acceptance due to pion interactions which increase $E_{v t x}$. The $\pm 200 \mathrm{~mm}$ range in the transverse direction extends to the edge of the scintillator plane from the edge of the $850 \mathrm{~mm}$ fiducial volume apothem. Figure 8.14 shows the $E_{v t x}$ distribution for events in the $\nu_{\mu}\left(\bar{\nu}_{\mu}\right)$ sample that pass all selection cuts up through the proton score $\left(E_{\nu}\right)$ cut. Events with $30<E_{v t x}<70 \mathrm{MeV}$ are selected. Requiring $E_{v t x}>30 \mathrm{MeV}$ rejects events with a tracked $\gamma$ from the decay of a final state $\pi^{0}$, where the energy deposited by the $\gamma$ via $\gamma \rightarrow e^{+} e^{-}$is separated from the event vertex. 

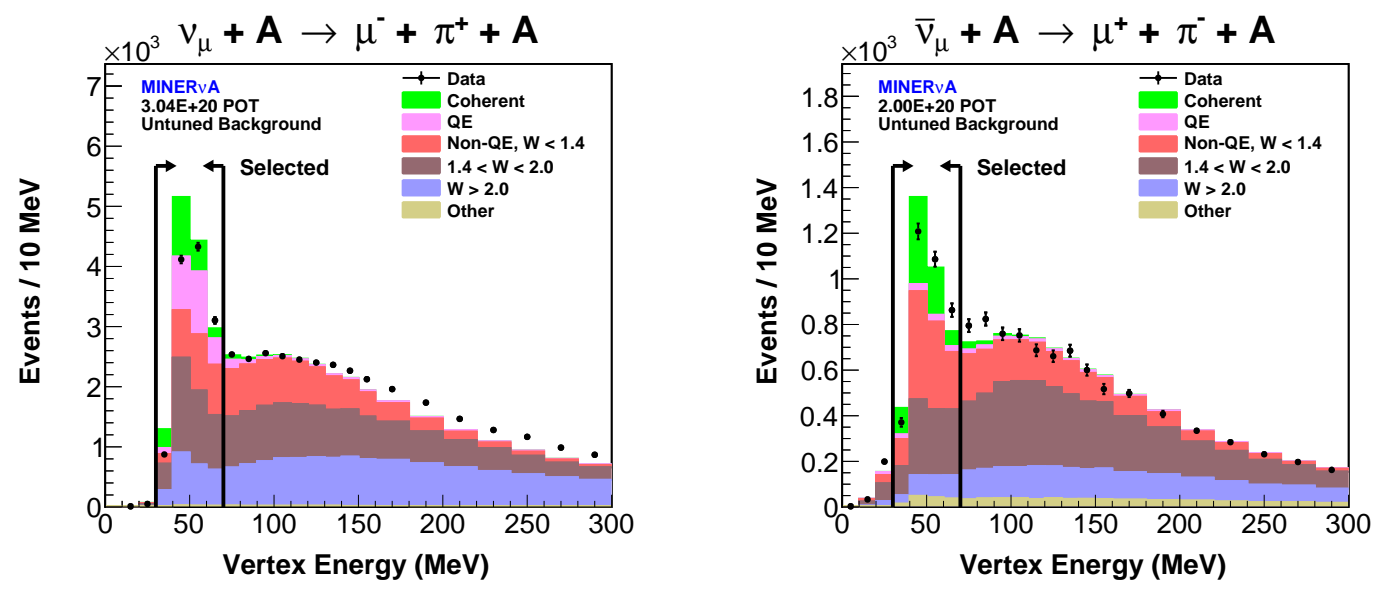

Figure 8.14: The left (right) plot shows the $E_{v t x}$ distribution for events in the $\nu_{\mu}$ $\left(\bar{\nu}_{\mu}\right)$ sample that pass all section cuts up through the proton score $\left(E_{\nu}\right)$ cut. Events in the range $30<E_{v t x}<70 \mathrm{MeV}$ are selected.

\subsection{6 $|t|$ Cut}

The final cut in the event selection is on the squared four-momentum transferred to the nucleus $|t|$, which is necessarily small for coherent scattering where the nucleus remains in its ground state. Large $|t|$ is indicative of nuclear break-up in incoherent interactions. Per Section 8.6, $|t|$ is calculated from the reconstructed four-momenta of the neutrino, muon, and pion. The $\nu_{\mu}$ and $\bar{\nu}_{\mu}|t|$ distributions with the tuned background prediction (Section 8.8) are shown in Figure 8.15 for events passing all cuts up through the $E_{v t x}$ cut. Events with $|t|<0.125(\mathrm{GeV} / \mathrm{c})^{2}$ are selected.

The coherent selection efficiency of the $|t|$ cut is dependent on the $|t|$ dependence of the coherent cross section, the reconstructed $|t|$ resolution, and the selected $|t|$ range. The coherent selection efficiency is an input to the measurement of the coherent cross sections and is estimated using coherent events in the MC (Section 8.9.3). A cut on $|t|$ can therefore introduce dependence of the measured cross sections on the $|t|$ dependence of the coherent cross section model in the MC. In the Rein-Sehgal 
and Berger-Sehgal coherent models, the $|t|$ dependence arises from the pion-nucleus elastic scattering cross section, which falls exponentially in $|t|$ as $\exp (-b|t|)$ where the exponential slope $b$ is a free parameter. In the MC, GENIE calculated the coherent scattering cross section on carbon using the Rein-Sehgal coherent model with an exponential slope $b \sim 40(\mathrm{GeV} / \mathrm{c})^{-2}$. Of the coherent events on carbon in the MC, 99\% had true $|t|<0.125(\mathrm{GeV} / \mathrm{c})^{2}$. The pion-carbon elastic scattering cross section in the Berger-Sehgal model was fit to pion-carbon elastic scattering data for incident pion kinetic energies $\lesssim 1 \mathrm{GeV}$, giving an exponential slope $\gtrsim 60(\mathrm{GeV} / \mathrm{c})^{-2}$ [48]. In addition, an exponential slope $\sim 60(\mathrm{GeV} / \mathrm{c})^{-2}$ was measured from data of $\pi^{+}$and $\pi^{-}$elastic scattering on carbon at $\sim 2 \mathrm{GeV} / \mathrm{c}$ incident pion momentum [116]. The pion-carbon elastic scattering data suggest the cross section for coherent scattering on carbon in the MC should fall faster in $|t|$, which would result in $>99 \%$ of $\mathrm{MC}$ coherent events on carbon having true $|t|<0.125(\mathrm{GeV} / \mathrm{c})^{2}$. Therefore, for the selected range $|t|<0.125(\mathrm{GeV} / \mathrm{c})^{2}$, the coherent selection efficiency of the $|t|$ cut is effectively independent of the $|t|$ dependence of the coherent cross section model in the MC and is dependent on the reconstructed $|t|$ resolution only.

\subsection{Background Tuning}

The $\nu_{\mu}$ and $\bar{\nu}_{\mu}$ coherent candidate samples contain incoherent backgrounds that must be subtracted from the data in order to measure the coherent cross sections. The $\mathrm{MC}$ is used to estimate the rate of the background coherent candidates, which can have large uncertainties due to uncertainties on the flux and underlying background interaction models. These uncertainties are minimized by tuning the MC estimate of the background rates to data in a sideband. 

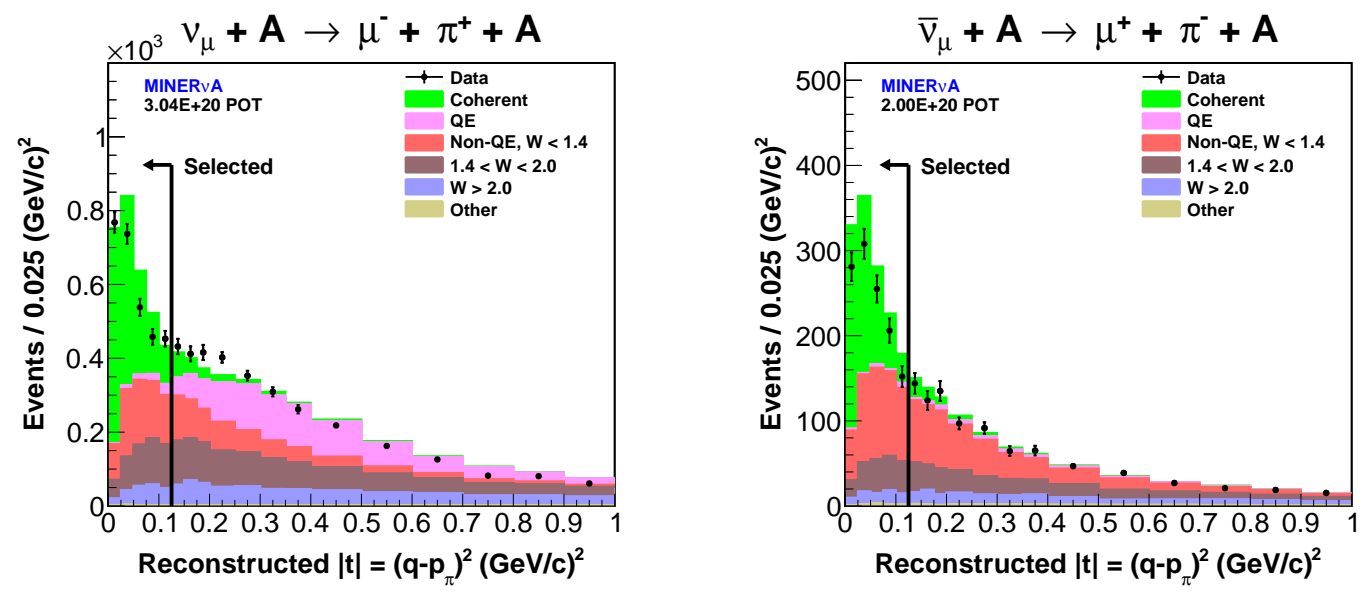

Figure 8.15: The $\nu_{\mu}$ (left) and $\bar{\nu}_{\mu}$ (right) $|t|$ distributions, after background tuning, for events passing all cuts up through the $E_{v t x}$ cut. Events with $|t|<0.125(\mathrm{GeV} / \mathrm{c})^{2}$ are selected.

The sideband is defined as events with $0.2<|t|<0.6(\mathrm{GeV} / \mathrm{c})^{2}$ that pass all selection cuts up through the vertex energy and proton score cuts (Figure 8.16). The requirement that events in the sideband pass the vertex energy cut minimizes sensitivity of the background tuning to mismodeled vertex activity (Section 8.13.3). This mismodeling will result in disagreement between data and the $\mathrm{MC}$ in the background acceptance of the vertex energy cut, and performing the background tuning after imposing the vertex energy cut will correct this disagreement.

The background tuning extracts a correction to the normalization of each background. These corrections are referred to as the background scale factors and are determined by varying the normalizations of the backgrounds in a fit of the total MC to data in the sideband. Choosing a sideband distribution for the background tuning that gives separation of the backgrounds facilitates the fit and reduces uncertainties on the background scale factors. The $\nu_{\mu}$ sideband $E_{\pi}$ distribution (Figure 8.17) gives separation of the resonance, transition, and DIS regions, while the $\nu_{\mu}$ sideband $Q^{2}$ distribution (Figure 8.17) gives separation of the resonance region and QE. The $\nu_{\mu}$ 

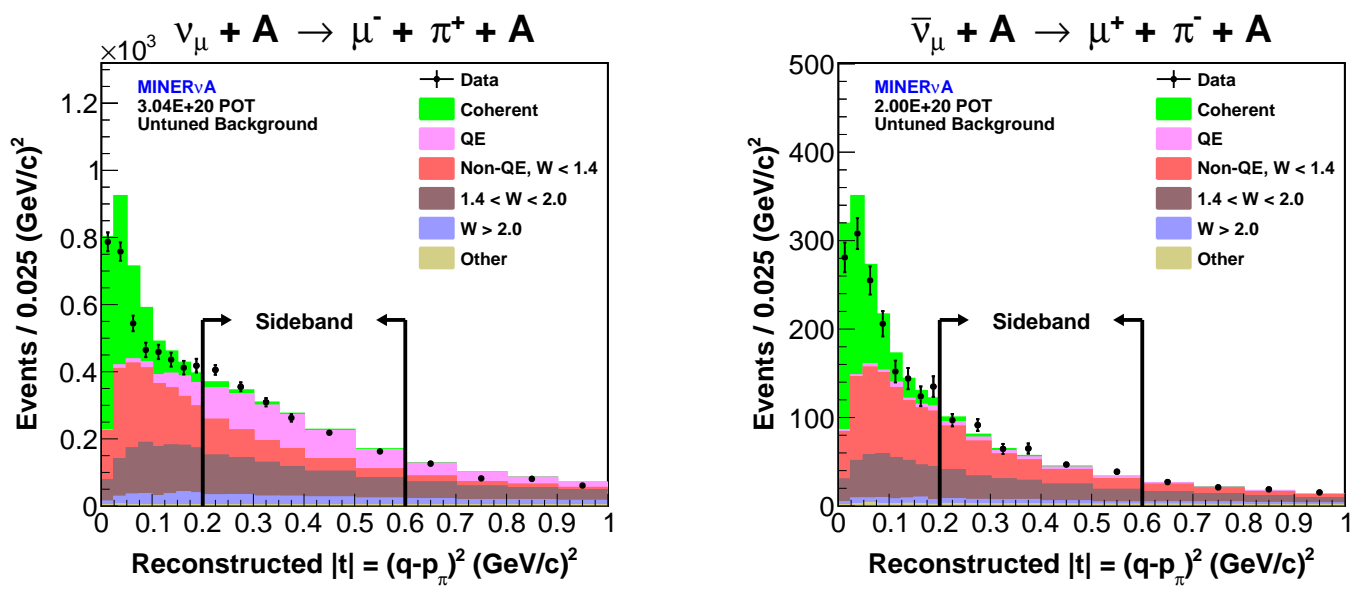

Figure 8.16: The $\nu_{\mu}$ (left) and $\bar{\nu}_{\mu}$ (right) $|t|$ distributions, before background tuning, for events that pass all selection cuts up through the vertex energy and proton score cuts. The sideband used for the background tuning are events with $0.2<|t|<0.6$ $(\mathrm{GeV} / \mathrm{c})^{2}$.

background tuning therefore is performed using the sideband $Q^{2}$ vs. $E_{\pi}$ distribution. The $\chi^{2}$ minimized in fitting the $\nu_{\mu}$ background scale factors is calculated as

$$
\chi^{2}=\sum_{i} \sum_{j} \frac{\left(N_{i j}^{\text {Data }}-\sum_{k} \alpha_{k} N_{i j k}^{M C}\right)^{2}}{\sum_{k} \alpha_{k} N_{i j k}^{M C}},
$$

where $i$ and $j$ are $E_{\pi}$ and $Q^{2}$ bins, respectively, $k$ is a MC event category, $\alpha_{k}$ is the scale factor for the normalization of category $k, N_{i j k}^{M C}$ is the number of MC events from category $k$ in $Q^{2}$ vs. $E_{\pi}$ bin $i j$, and $N_{i j}^{D a t a}$ is the number of data events in bin $i j$. Coherent scattering is a small contribution to the $\nu_{\mu}$ sideband and its scale factor is fixed to 1.0 in the fit. The background category "Other" is a small contribution to the $\nu_{\mu}$ sample and its scale factor is fixed to 1.0 to minimize the number of free parameters in the fit. The $\nu_{\mu}$ background scale factors extracted from the fit are listed in Table 8.12. The $\nu_{\mu}$ sideband $E_{\pi}$ and $Q^{2}$ distributions after applying the background scale factors to the MC are shown in Figure 8.17. 

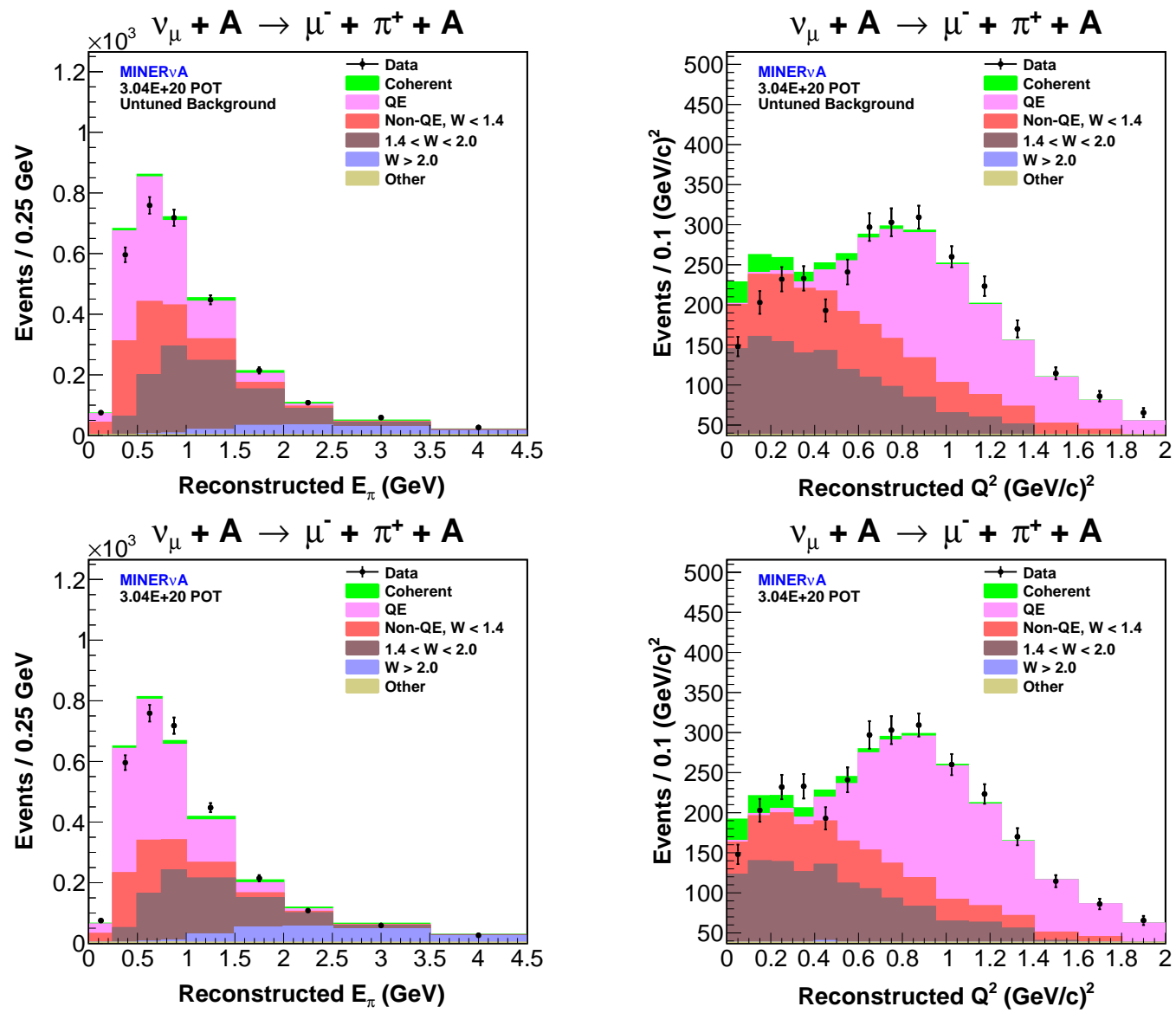

Figure 8.17: The $\nu_{\mu}$ sideband $E_{\pi}$ (left) and $Q^{2}$ (right) distributions before (top) and after (bottom) background tuning

The $\bar{\nu}_{\mu}$ background tuning is performed using the $\bar{\nu}_{\mu}$ sideband $E_{\pi}$ distribution (Figure 8.18), which gives separation between the resonance, transition, and DIS regions. Unlike the $\nu_{\mu}$ sample, QE is a small contribution to the $\bar{\nu}_{\mu}$ sample since the recoil neutron rarely produces a reconstructed track. The $\chi^{2}$ minimized in fitting the $\bar{\nu}_{\mu}$ background scale factors is calculated as

$$
\chi^{2}=\sum_{i} \frac{\left(N_{i}^{D a t a}-\sum_{k} \alpha_{k} N_{i k}^{M C}\right)^{2}}{\sum_{k} \alpha_{k} N_{i k}^{M C}},
$$



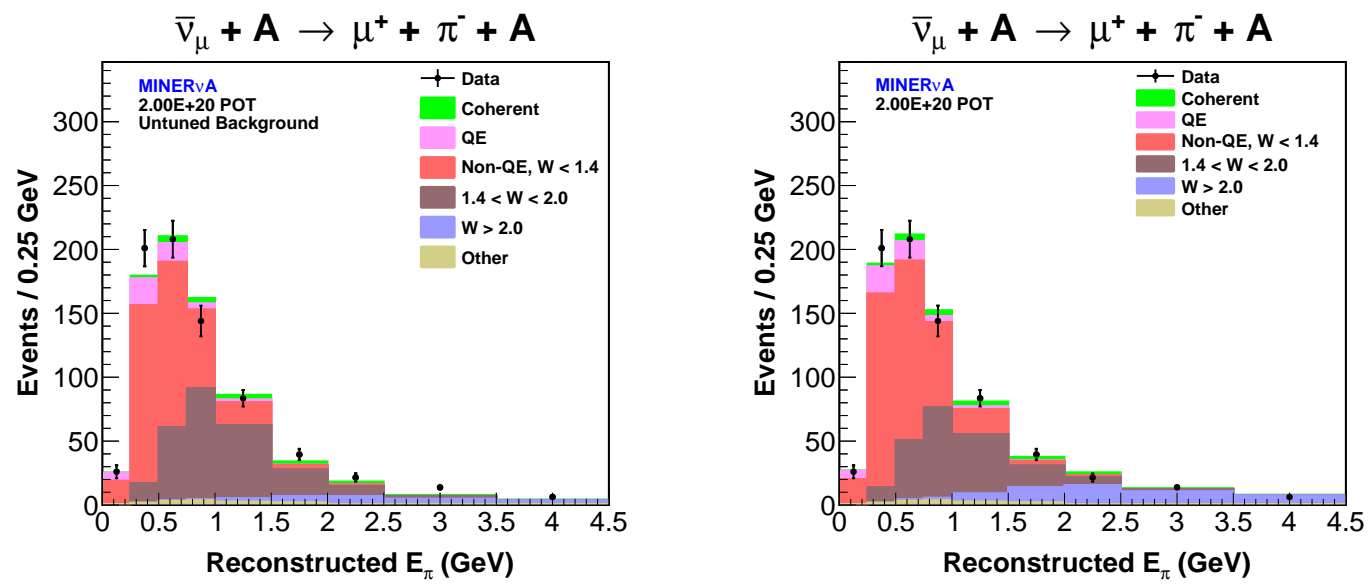

Figure 8.18: The $\bar{\nu}_{\mu}$ sideband $E_{\pi}$ distribution before (left) and after (right) background tuning

where $N_{i}^{\text {Data }}$ is the number of data events in $E_{\pi}$ bin $i, \alpha_{k}$ is the scale factor for the normalization of $\mathrm{MC}$ event category $k$, and $N_{i k}^{M C}$ is the number of $\mathrm{MC}$ events from category $k$ in bin $i$. The scale factors for coherent scattering and the background category "Other" are fixed to 1.0 in the fit for the reasons given for the $\nu_{\mu}$ background tuning. QE is a small contribution to the $\bar{\nu}_{\mu}$ sample and its scale factor is fixed to 1.0 to minimize the number of free parameters in the fit. The $\bar{\nu}_{\mu}$ background scale factors extracted from the fit are listed in Table 8.12. The $\bar{\nu}_{\mu}$ sideband $E_{\pi}$ after applying the background scale factors to the MC is shown in Figure 8.18.

\begin{tabular}{l|c|c} 
Background & $\nu_{\mu}$ Sample & $\bar{\nu}_{\mu}$ Sample \\
\hline Charged Current Quasielastic & $1.13 \pm 0.04$ & 1.0 (fixed) \\
Non-Quasielastic, $W_{\text {gen }}<1.4 \mathrm{GeV}$ & $0.73 \pm 0.08$ & $1.07 \pm 0.08$ \\
$1.4<W_{\text {gen }}<2.0 \mathrm{GeV}$ & $0.81 \pm 0.05$ & $0.79 \pm 0.09$ \\
$W_{\text {gen }}>2.0 \mathrm{GeV}$ & $1.7 \pm 0.20$ & $2.3 \pm 0.3$ \\
Other & 1.0 (fixed) & 1.0 (fixed)
\end{tabular}

Table 8.12: Background scale factors

It should be noted that the background tuning corrects the normalization, but 
not the kinematics, of each background. Mismodeling the kinematics of the backgrounds is a source of systematic uncertainty on the background prediction and the measured cross sections. The evaluation of this uncertainty is described in Section 8.13.

\subsubsection{Pion Angle Weighting}

Disagreement between data and MC remains in the $\nu_{\mu}$ and $\bar{\nu}_{\mu}$ sideband $\theta_{\pi}$ distributions after background tuning (Figure 8.19). This disagreement is corrected by weighting the total tuned background as a function of reconstructed $\theta_{\pi}$ (Figure 8.19). For each group of two or more consecutive bins in the sideband $\theta_{\pi}$ distribution with tuned MC above (below) the data beyond the data's $1 \sigma$ statistical uncertainty, the weighting decreases (increases) the group's total background by an amount equal to the group's total data-MC $1 \sigma$ disagreement. The $\nu_{\mu}$ and $\bar{\nu}_{\mu}$ sideband $\theta_{\pi}$ distributions after background tuning and $\theta_{\pi}$ weighting are shown in Figure 8.19. The $\theta_{\pi}$ weighting is applied to the tuned background in the coherent-like sample where the full difference from the $\theta_{\pi}$ weighting is applied as a systematic uncertainty (Section 8.13).

\subsection{Cross Section Extraction}

Measurements of the $\nu_{\mu}$ and $\bar{\nu}_{\mu}$ charged current coherent pion production cross sections were extracted from the data by subtracting the tuned background prediction from the selected data coherent candidates, unfolding to correct distortion of the kinematic distributions from reconstruction, correcting for the efficiency of selecting coherent interactions, normalizing to the neutrino flux, normalizing to the number 

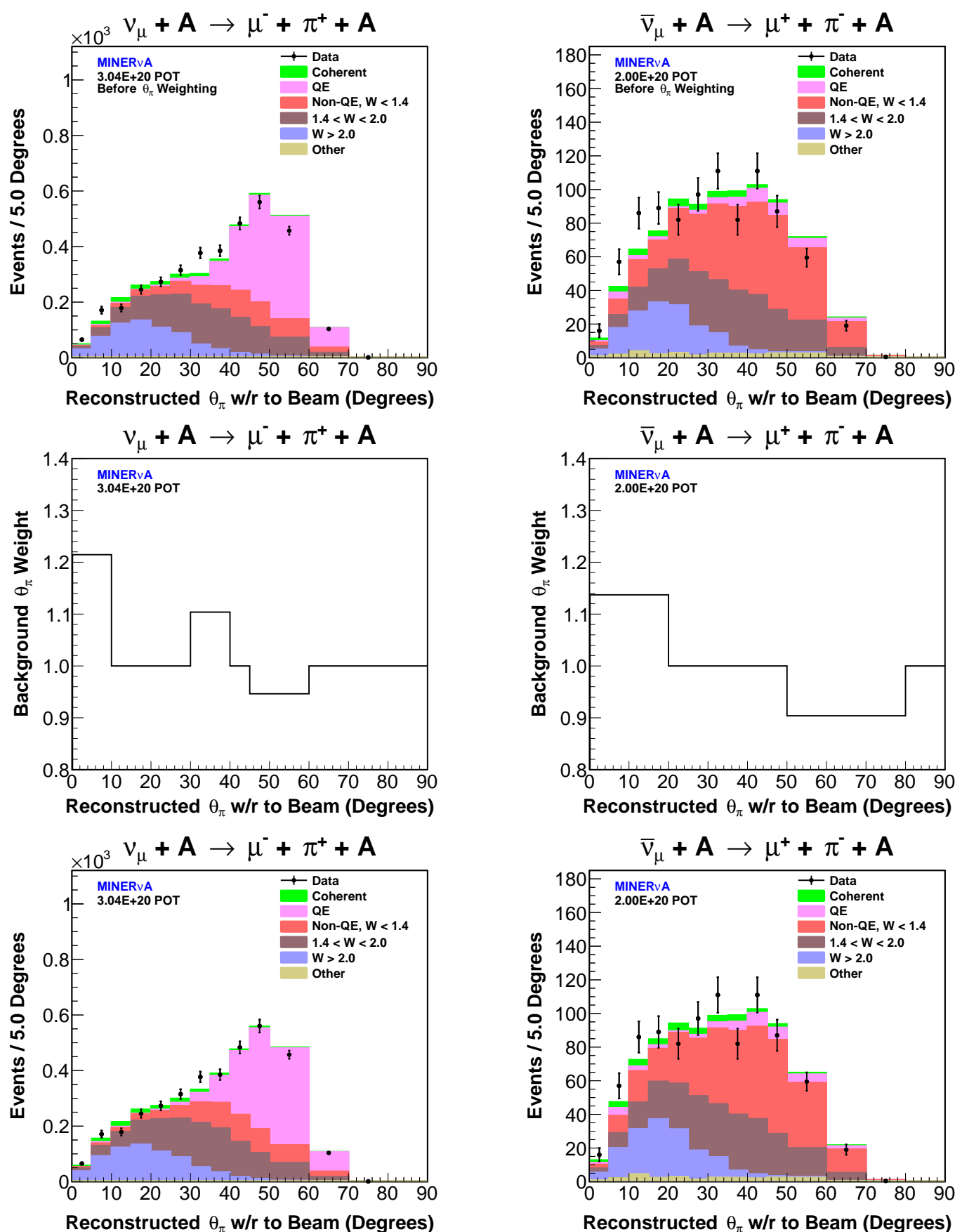

Figure 8.19: The top plots show the $\nu_{\mu}$ (left) and $\bar{\nu}_{\mu}$ (right) sideband $\theta_{\pi}$ distributions after background tuning. The middle plots show the $\theta_{\pi}$ weighting applied to the $\mathrm{MC}$ background to correct the disagreement between the data and the MC. The bottom plots show the sideband $\theta_{\pi}$ distributions after background tuning and $\theta_{\pi}$ weighting. 
of carbon nuclei in the fiducial volume, and correcting for non-carbon nuclei in the fiducial volume. The measured cross section in true $E_{\nu}$ bin $i$ was was calculated as

$$
\sigma_{i}=\frac{\beta \sum_{j} U_{i j}\left(N_{j}^{\text {data }}-N_{j}^{b k g d}\right)}{T \phi_{i} \epsilon_{i}}
$$

where $N_{j}^{\text {data }}$ is the number of data coherent candidates in reconstructed $E_{\nu}$ bin $j, N_{j}^{b k g d}$ is the tuned estimate of the number of background coherent candidates, $U_{i j}$ is the unfolding matrix element that estimates the signal contribution from reconstructed bin $j$ to true bin $i, \epsilon_{i}$ is the coherent selection efficiency, $\phi_{i}$ is the $\nu_{\mu} / \bar{\nu}_{\mu}$ flux, $T$ is the number of carbon nuclei in the fiducial volume, and $\beta$ is the correction to the coherent event rate for interactions on non-carbon nuclei. Similarly, the measured differential cross section in true bin $i$ of kinematic parameter $\xi$ was calculated as

$$
\left(\frac{d \sigma}{d \xi}\right)_{i}=\frac{\beta \sum_{j} U_{i j}\left(N_{j}^{d a t a}-N_{j}^{b k g d}\right)}{(\Delta \xi)_{i} T \Phi \epsilon_{i}}
$$

where $\Phi$ is the integrated $\nu_{\mu} / \bar{\nu}_{\mu}$ flux and $(\Delta \xi)_{i}$ is the bin width.

Cross sections for the $\bar{\nu}_{\mu}$ partial and full detector data sets (Section 8.4) were calculated separately and combined to account for the effect of ArgoNeuT on the reconstructed kinematics and selection efficiency. The combined $\bar{\nu}_{\mu}$ cross section was calculated as

$$
\sigma_{\bar{\nu}_{\mu}}=\frac{P_{p} T_{p} \sigma_{p}+P_{f} T_{f} \sigma_{f}}{P_{p} T_{p}+P_{f} T_{f}}=0.317 \sigma_{p}+0.683 \sigma_{f}
$$

where $P$ and $T$ are the POT (Section 8.4) and number of carbon nuclei in the fiducial volume (Section 8.9.5), respectively, and the subscripts $p$ and $f$ denote the partial and full detector data samples, respectively. This calculation accounts for 
the difference in the flux and fiducial mass between the two samples. The combined $\bar{\nu}_{\mu}$ differential cross sections were calculated in the same way.

The unfolding matrices and efficiency corrections for measuring the cross sections were estimated using coherent events in the $\mathrm{MC}$, where events with $E_{\pi}<0.5 \mathrm{GeV}$ were weighted by $50 \%$. This weighting is referred to as the signal model weighting. The justification for the signal model weighting and its effects on the measured cross sections are discussed in Section 8.10.

The following sections detail each step of the cross section calculation.

\subsubsection{Background Subtraction}

The first step in calculating the measured cross sections is to subtract the tuned estimate of the number of background coherent candidates $N^{b k d g}$ from the number of data coherent candidates $N^{d a t a}$. For a given kinematic parameter, $N_{j}^{b k d g}$ in reconstructed bin $j$ was calculated as

$$
N_{j}^{b k g d}=\sum_{k} \alpha_{k} N_{j k}^{M C}
$$

where $\alpha_{k}$ is the background scale factor (Section 8.8) for the $k^{t h}$ background and $N_{j k}^{M C}$ is the untuned MC prediction of the number of coherent candidates from the $k^{t h}$ background in reconstructed bin $j$. After background subtraction, the $\nu_{\mu}\left(\bar{\nu}_{\mu}\right)$ sample contains 1411 (481) coherent candidates. The kinematic distributions for the $\nu_{\mu}$ and $\bar{\nu}_{\mu}$ coherent candidate samples after background subtraction are shown in Figures 8.20-8.23. 

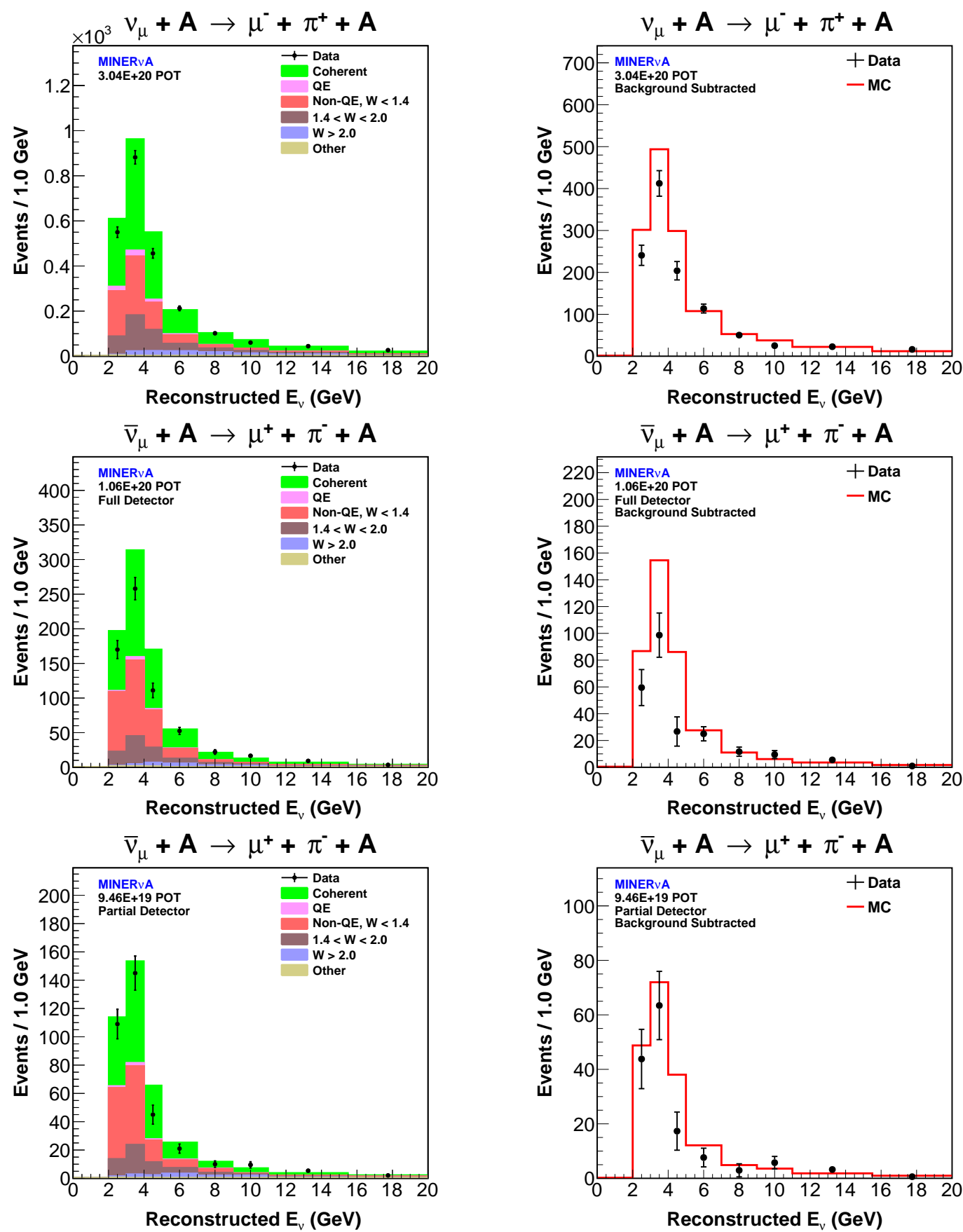

Figure 8.20: The $E_{\nu}$ distribution for the coherent candidate event samples before (left) and after (right) background subtraction for the $\nu_{\mu}$ (top), full detector $\bar{\nu}_{\mu}$ (middle), and parital detector $\bar{\nu}_{\mu}$ (bottom) samples 

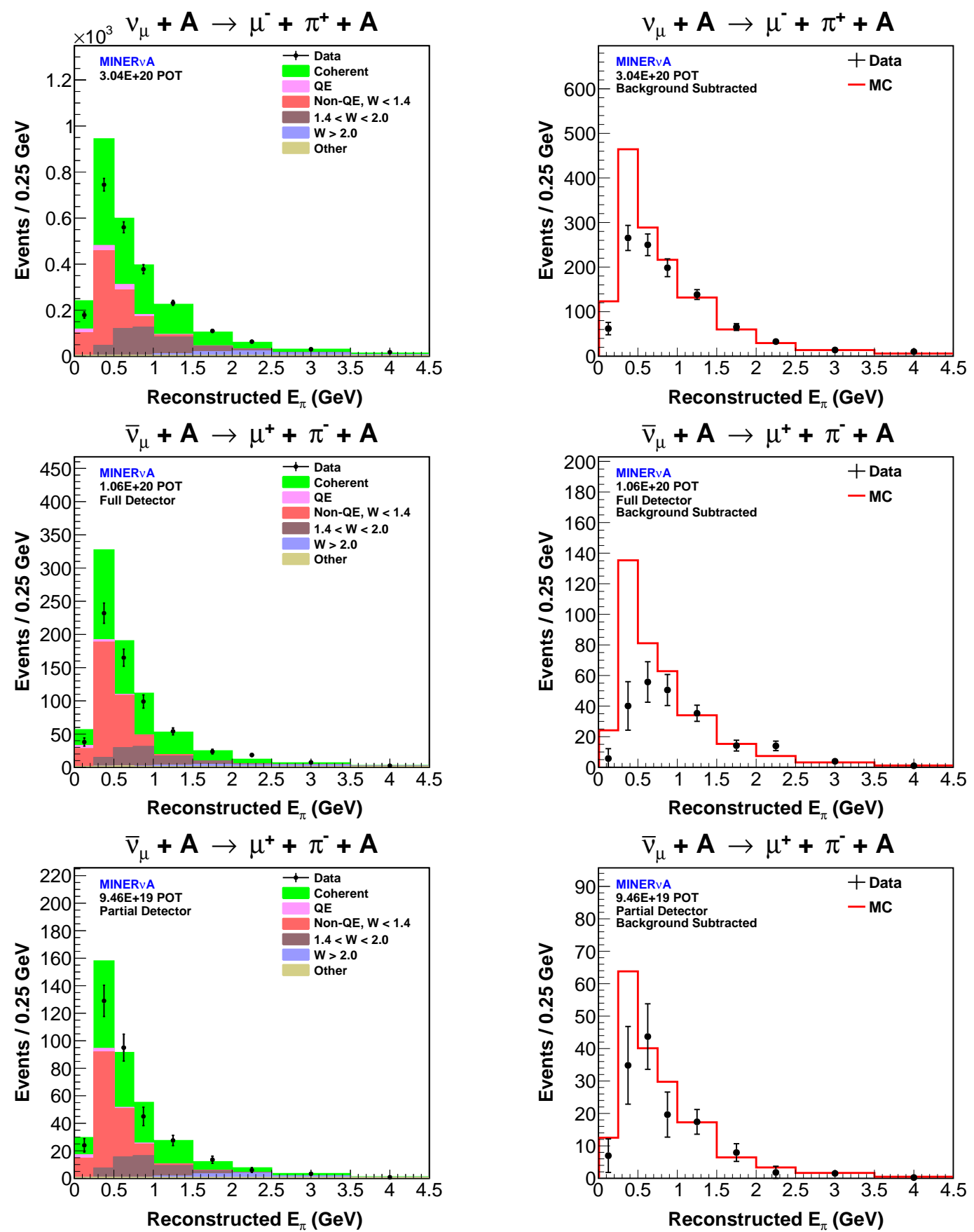

Figure 8.21: The $E_{\pi}$ distribution for the coherent candidate event samples before (left) and after (right) background subtraction for the $\nu_{\mu}$ (top), full detector $\bar{\nu}_{\mu}$ (middle), and parital detector $\bar{\nu}_{\mu}$ (bottom) samples 


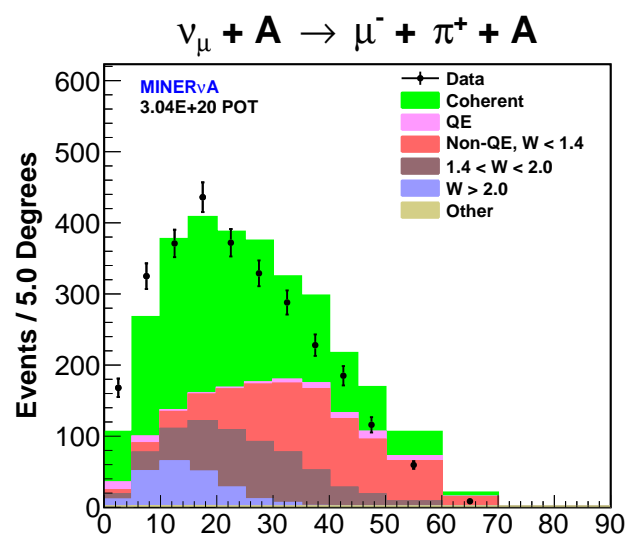

Reconstructed $\theta_{\pi} \mathbf{w} / \mathbf{r}$ to Beam (Degrees)

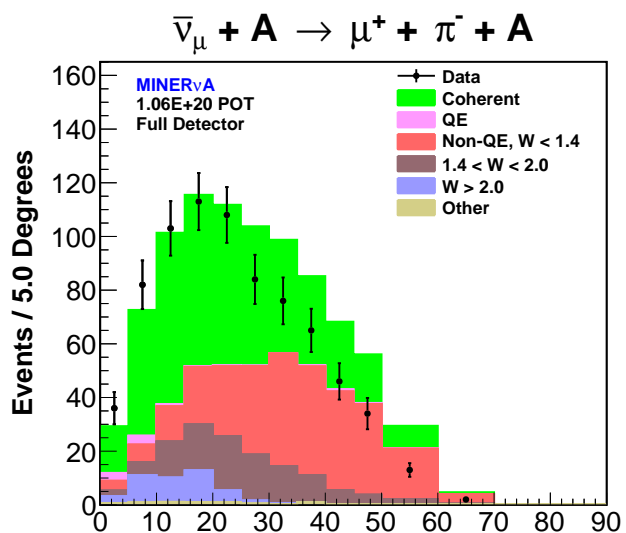

Reconstructed $\theta_{\pi} \mathbf{w} / \mathbf{r}$ to Beam (Degrees)

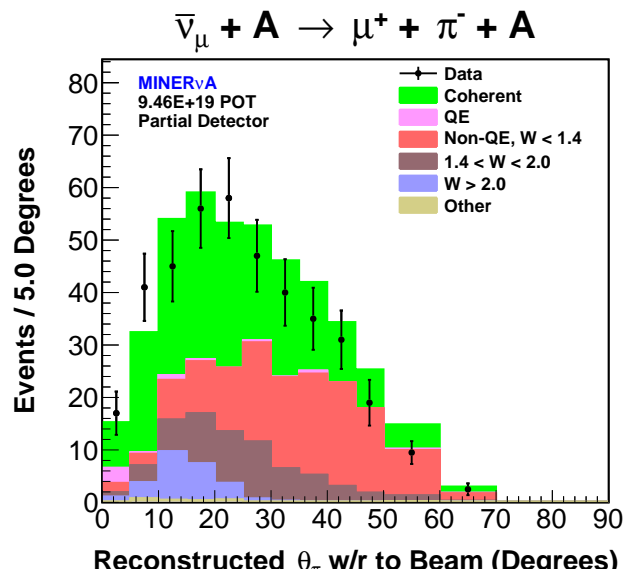

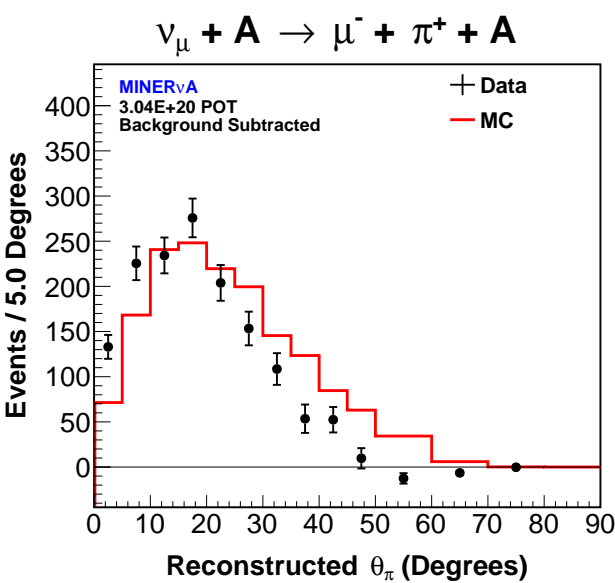
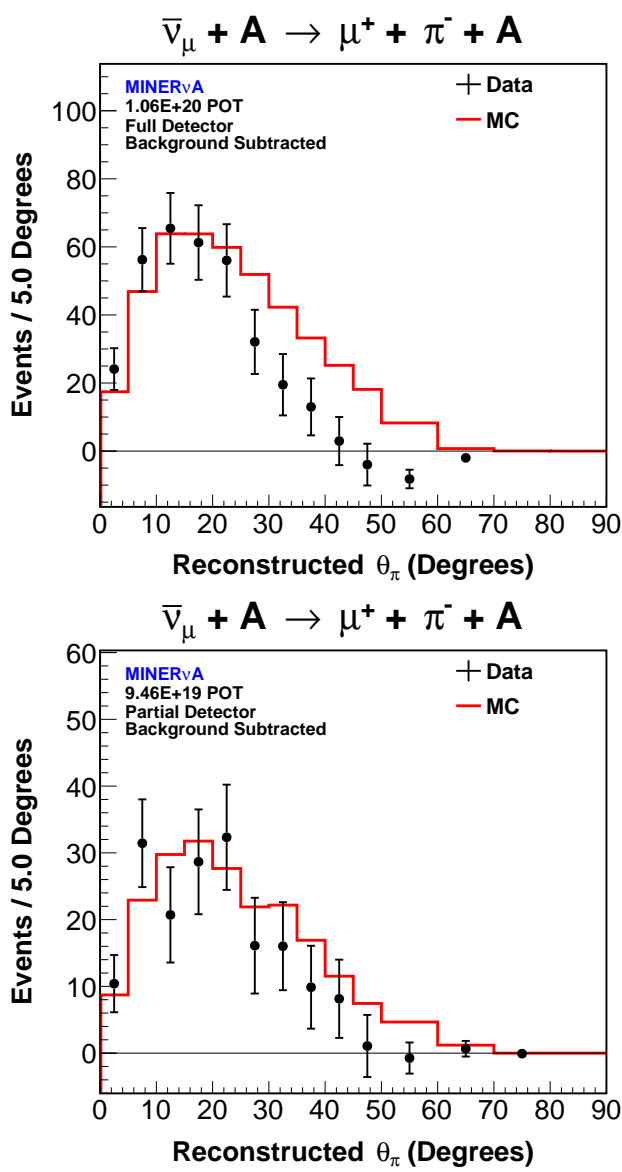

Figure 8.22: The $\theta_{\pi}$ distribution for the coherent candidate event samples before (left) and after (right) background subtraction for the $\nu_{\mu}$ (top), full detector $\bar{\nu}_{\mu}$ (middle), and parital detector $\bar{\nu}_{\mu}$ (bottom) samples 

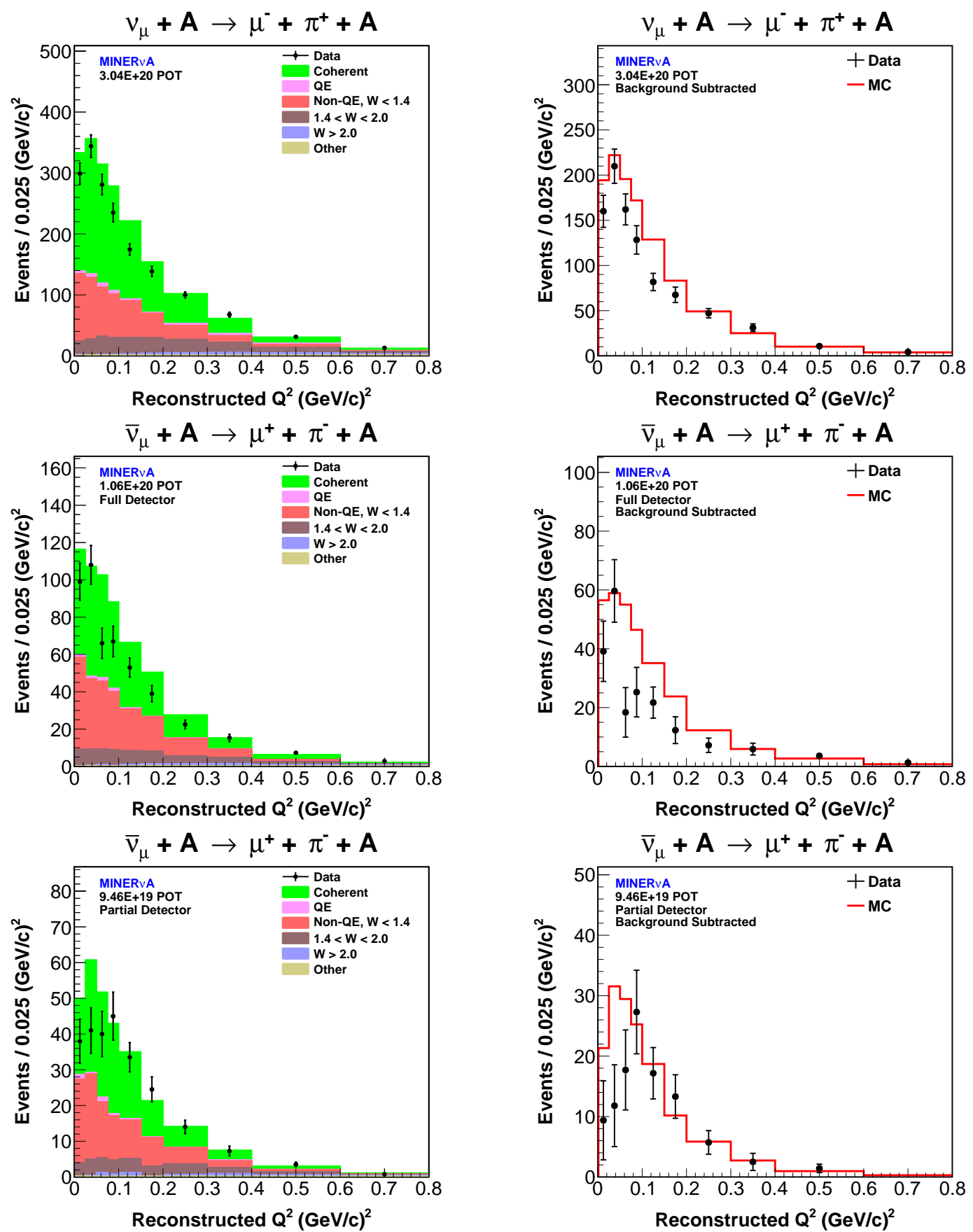

Figure 8.23: The $Q^{2}$ distribution for the coherent candidate event samples before (left) and after (right) background subtraction for the $\nu_{\mu}$ (top), full detector $\bar{\nu}_{\mu}$ (middle), and parital detector $\bar{\nu}_{\mu}$ (bottom) samples 


\subsubsection{Unfolding}

After background subtraction, the kinematic distributions are unfolded to correct for distortion resulting from reconstruction of the kinematic parameters. Given a distribution of event rates in bins of a reconstructed quantity, the unfolding estimates the event rates in the bins of the true value of that quantity. The distributions were unfolded using the iterative Bayesian unfolding method of D'Agostini [117] with 2 iterations. The method begins by using Bayes' theorem to estimate the unfolded distribution from the reconstructed distribution and an unfolding matrix. The unfolding matrix $U_{i j}$ estimates the contribution from true bin $i$ to reconstructed bin $j$. The unfolding matrices (Figures 8.24-8.27) were calculated from the signalonly MC samples as

$$
U_{i j}=\frac{N_{i j}^{s e l}}{\sum_{j} N_{i j}^{s e l}},
$$

where $N_{i j}^{s e l}$ is the number of coherent events passing all selection cuts in bin $i j$. The signal model weighting (Section 8.10) was applied in calculating the unfolding matrices. In each successive iteration the unfolded distribution is recalculated in the same way from the from the unfolded distribution resulting from the previous iteration. The unfolded kinematic distributions for the $\nu_{\mu}$ and $\bar{\nu}_{\mu}$ samples are shown in Figures 8.24-8.27.

The statistical uncertainty on the unfolded distribution increases with each unfolding iteration. The number of unfolding iterations was optimized to give adequate correction of the distortion while minimizing the increase in statistical uncertainty [118]. This was done using a mock data sample constructed from coherent events in the signal-only MC samples that pass all selection cuts. The mock data sample was scaled to the data event rate after background subtraction. For each kinematic 

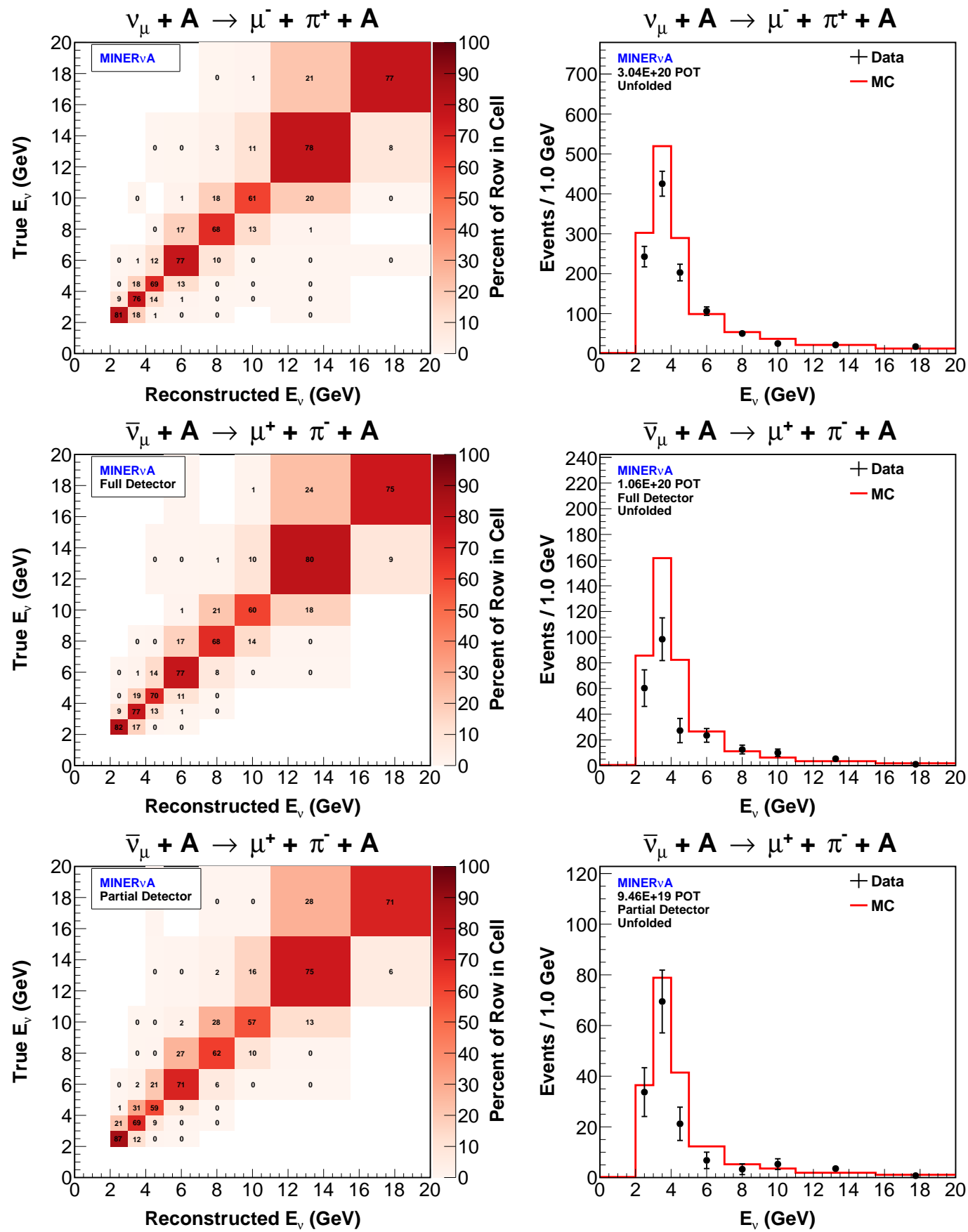

Figure 8.24: The $E_{\nu}$ unfolding matrix (left) and unfolded distribution (right) for the $\nu_{\mu}$ (top), full detector $\bar{\nu}_{\mu}$ (middle), and partial detector $\bar{\nu}_{\mu}$ (bottom) samples 

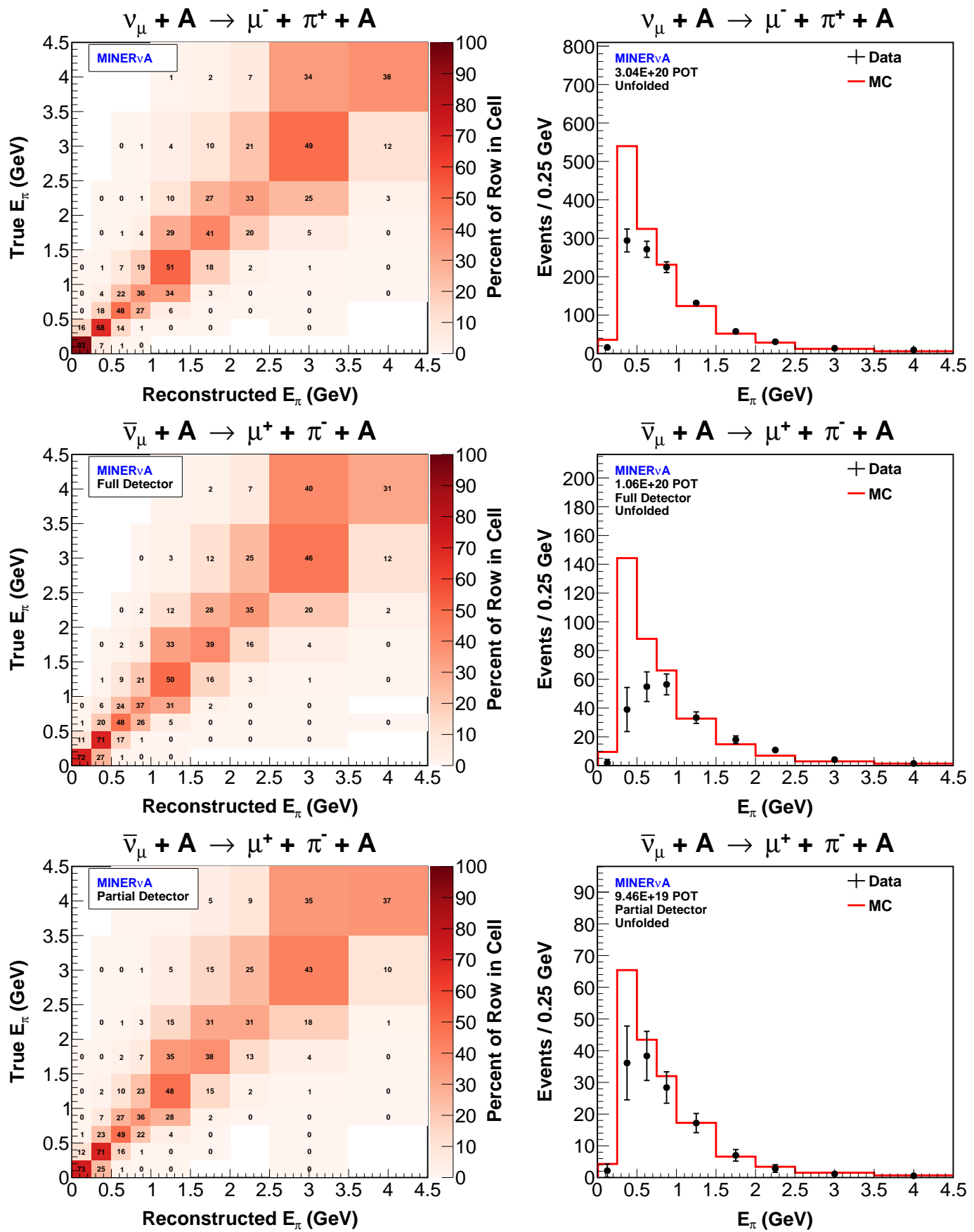

Figure 8.25: The $E_{\pi}$ unfolding matrix (left) and unfolded distribution (right) for the $\nu_{\mu}$ (top), full detector $\bar{\nu}_{\mu}$ (middle), and partial detector $\bar{\nu}_{\mu}$ (bottom) samples 

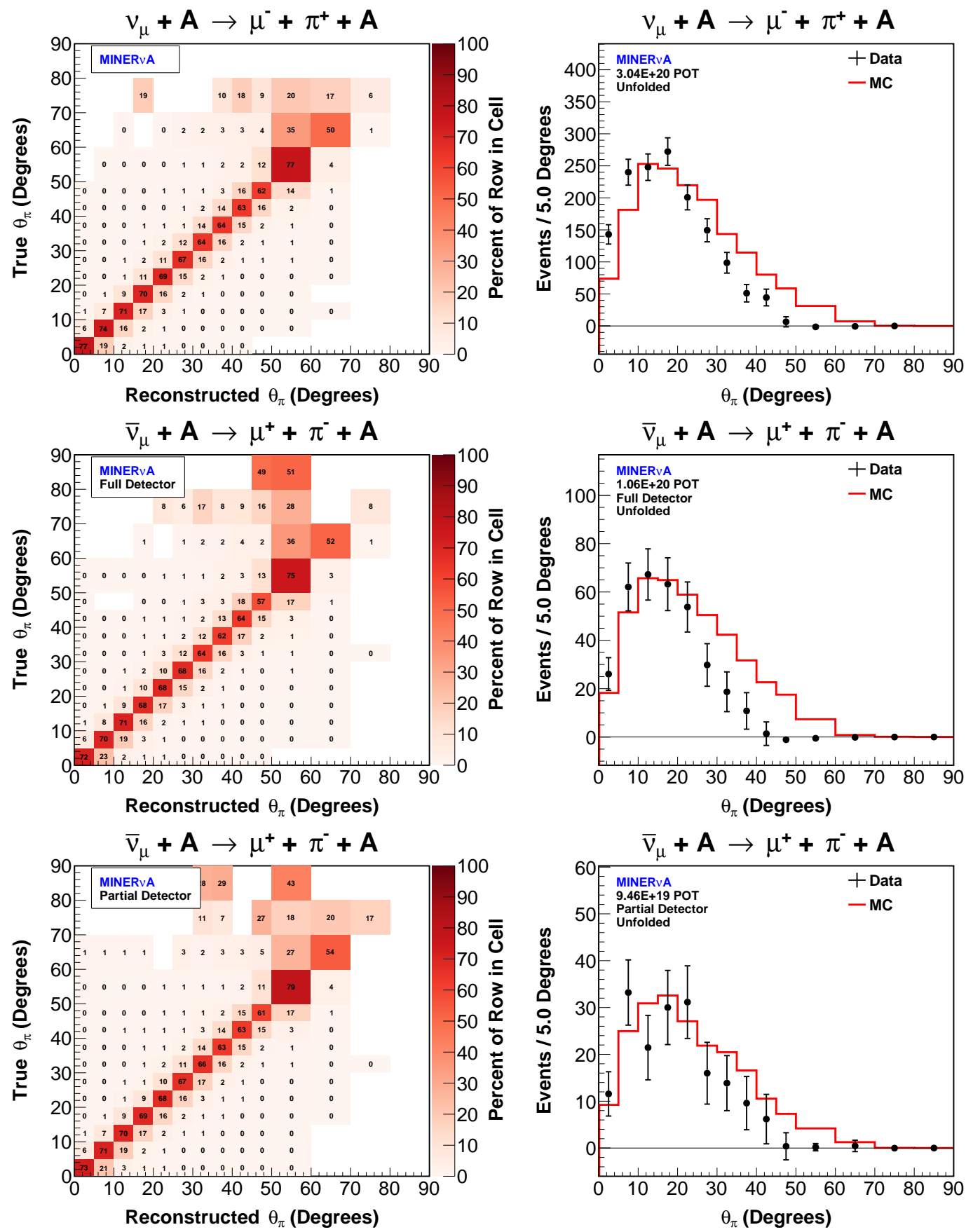

Figure 8.26: The $\theta_{\pi}$ unfolding matrix (left) and unfolded distribution (right) for the $\nu_{\mu}$ (top), full detector $\bar{\nu}_{\mu}$ (middle), and partial detector $\bar{\nu}_{\mu}$ (bottom) samples 

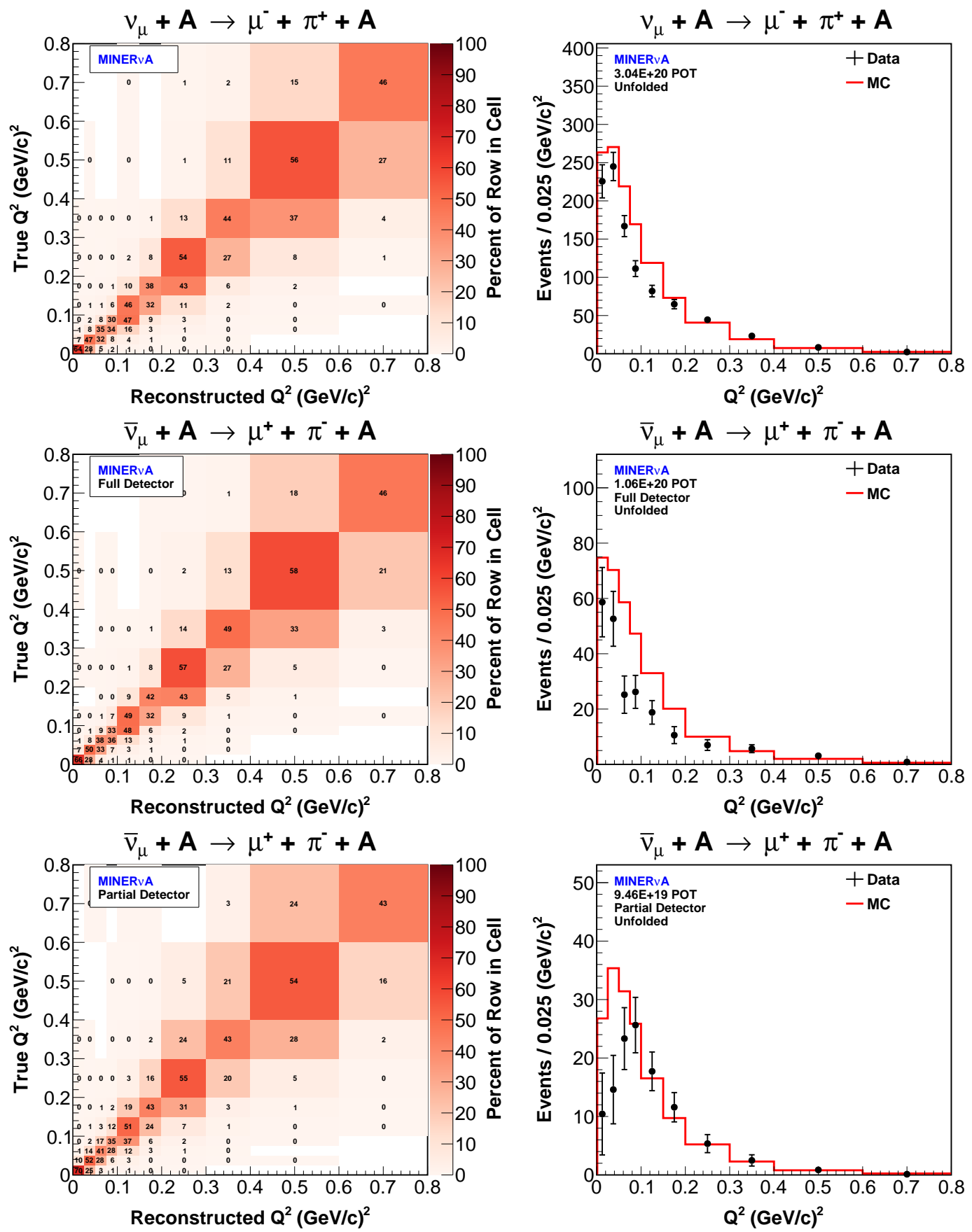

Figure 8.27: The $Q^{2}$ unfolding matrix (left) and unfolded distribution (right) for the $\nu_{\mu}$ (top), full detector $\bar{\nu}_{\mu}$ (middle), and partial detector $\bar{\nu}_{\mu}$ (bottom) samples 
parameter, the mock data sample was weighted so its reconstructed distribution was similar in shape to the data distribution after background subtraction. The reconstructed distributions of the mock data sample were unfolded up through 8 iterations. For each kinematic parameter in the $\nu_{\mu}$ and $\bar{\nu}_{\mu}$ mock data samples, the unfolded and true distributions agreed in each bin within the $1 \sigma$ statistical uncertainty after 2 iterations. The optimization study is illustrated in Figures 8.28-8.39. The unfolding results in correlations in the statistical uncertainty between bins. These correlations are included in a statistical covariance matrix, which is propagated to the measured cross section. 

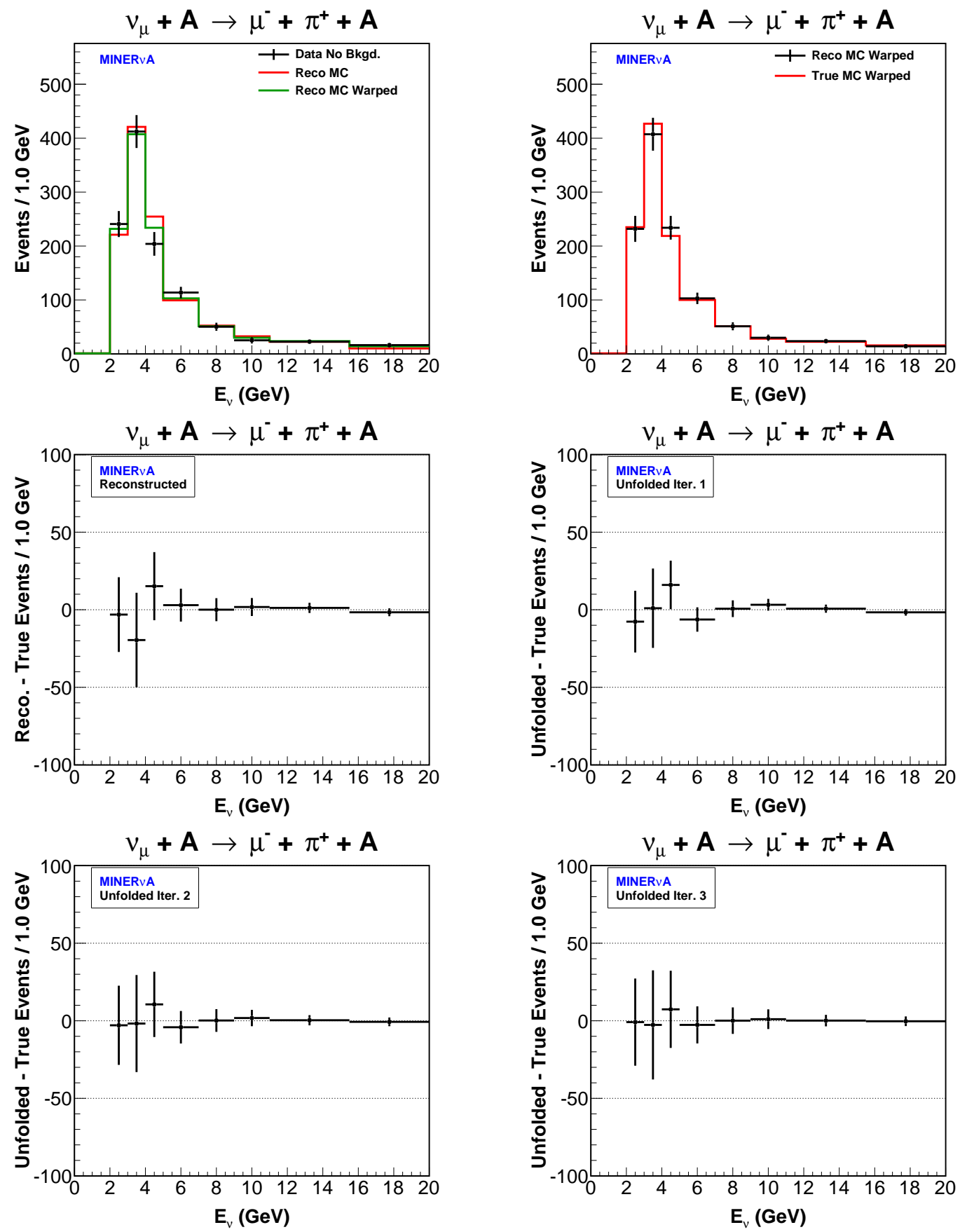

Figure 8.28: The $\nu_{\mu} E_{\nu}$ unfolding study. The upper-left plot shows the reconstructed $E_{\nu}$ distribution for the data after background subtraction and the mock data sample before and after warping to the data. The upper right plot shows the reconstructed and true $E_{\nu}$ distributions for the warped mock data sample, where the error bars are the statistical uncertainty. The remaining plots show the deviation from the true event rate with statistical uncertainty as a function of true $E_{\nu}$ for the warped mock data sample before unfolding (middle left) and after each of the first three unfolding iterations. 

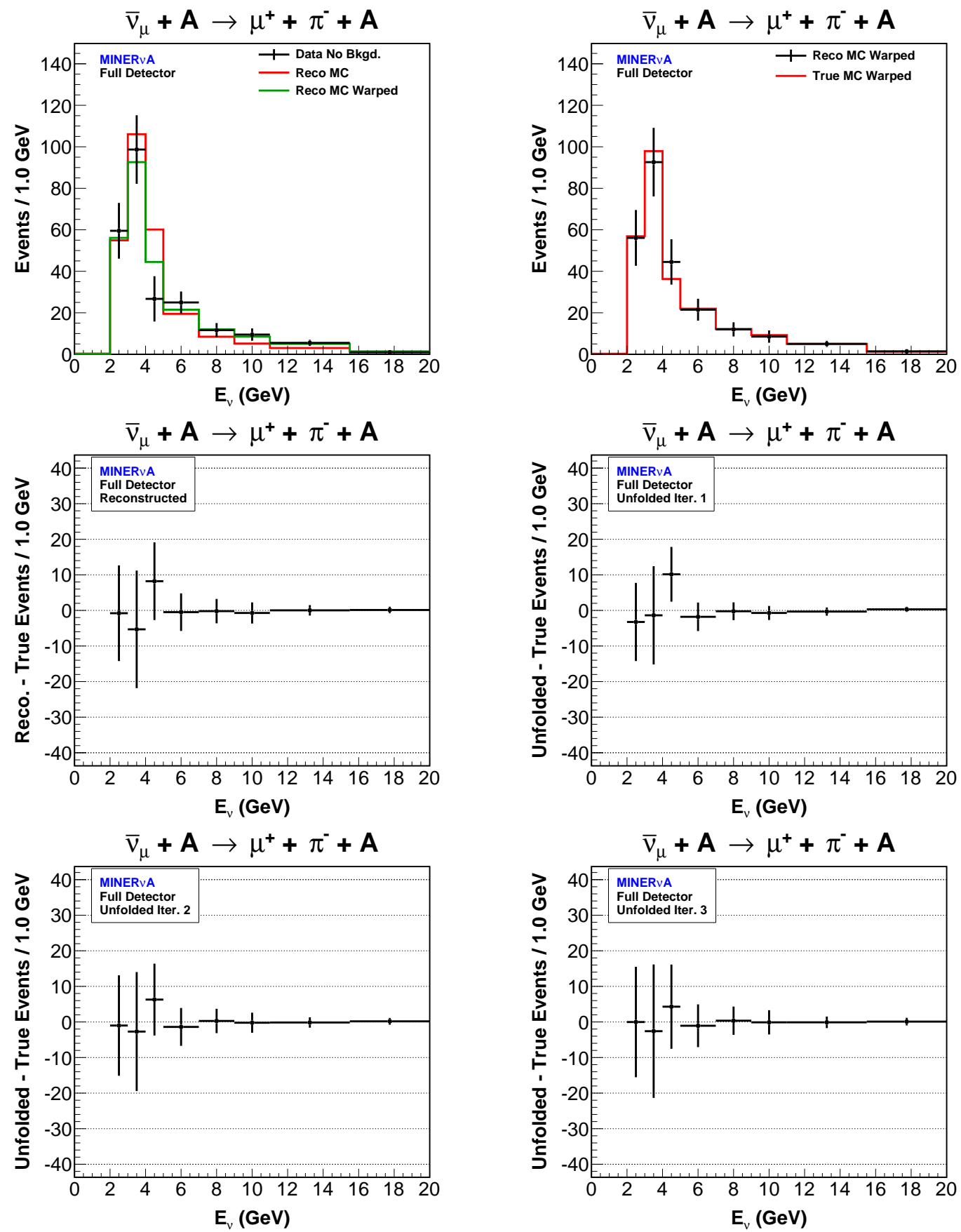

Figure 8.29: The full detector $\bar{\nu}_{\mu} E_{\nu}$ unfolding study. The upper-left plot shows the reconstructed $E_{\nu}$ distribution for the data after background subtraction and the mock data sample before and after warping to the data. The upper right plot shows the reconstructed and true $E_{\nu}$ distributions for the warped mock data sample, where the error bars are the statistical uncertainty. The remaining plots show the deviation from the true event rate with statistical uncertainty as a function of true $E_{\nu}$ for the warped mock data sample before unfolding (middle left) and after each of the first three unfolding iterations. 

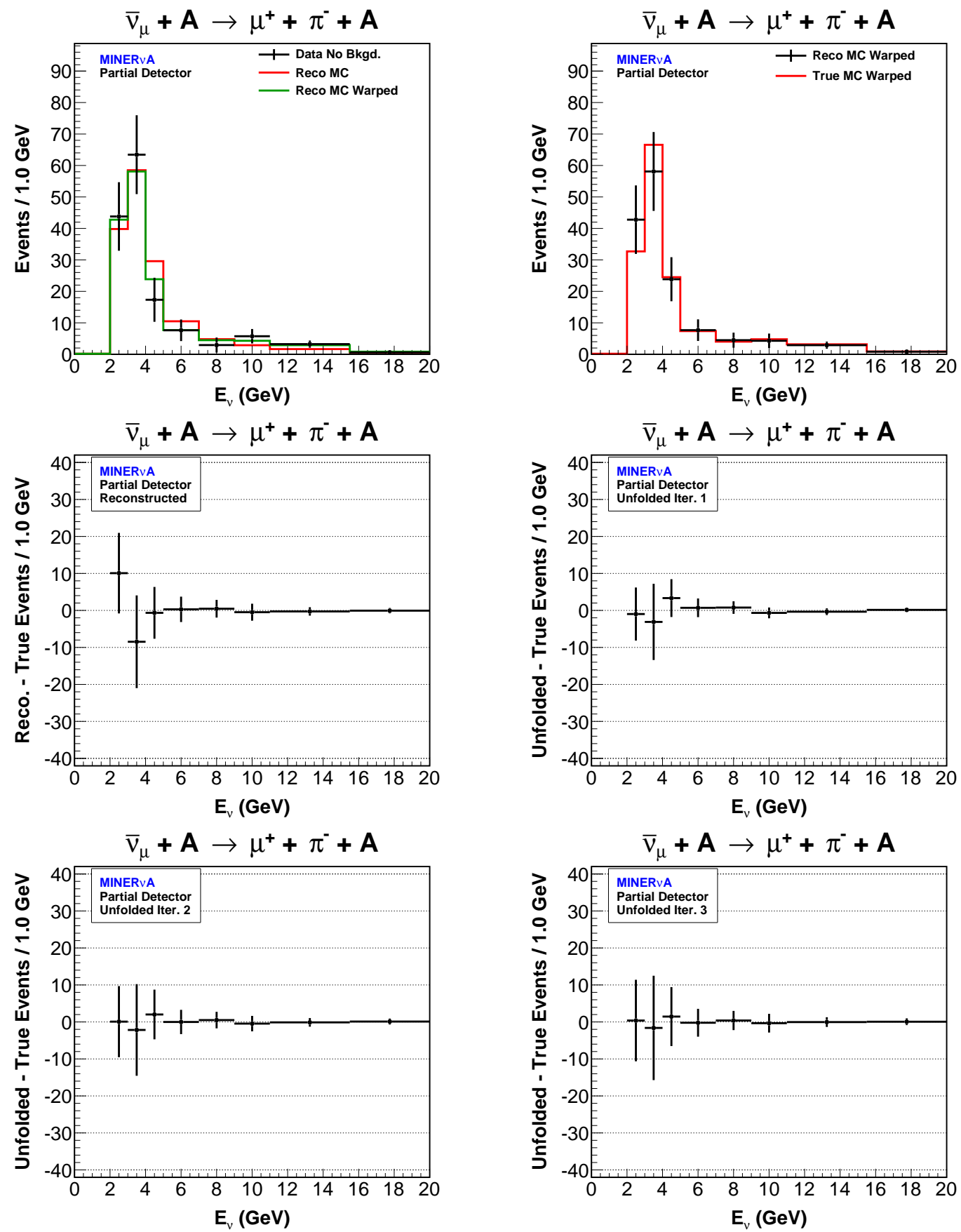

Figure 8.30: The partial detector $\bar{\nu}_{\mu} E_{\nu}$ unfolding study. The upper-left plot shows the reconstructed $E_{\nu}$ distribution for the data after background subtraction and the mock data sample before and after warping to the data. The upper right plot shows the reconstructed and true $E_{\nu}$ distributions for the warped mock data sample, where the error bars are the statistical uncertainty. The remaining plots show the deviation from the true event rate with statistical uncertainty as a function of true $E_{\nu}$ for the warped mock data sample before unfolding (middle left) and after each of the first three unfolding iterations. 

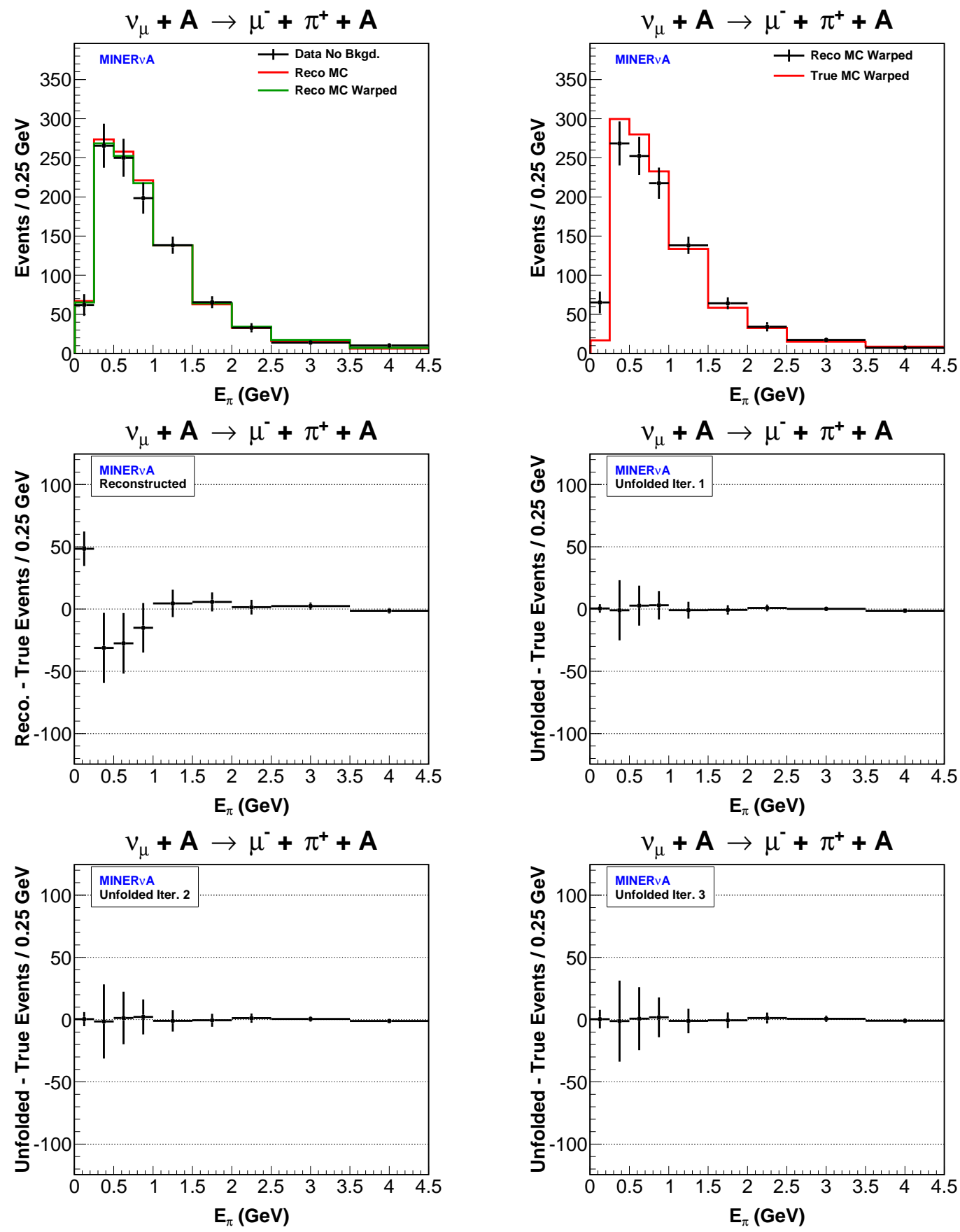

Figure 8.31: The $\nu_{\mu} E_{\pi}$ unfolding study. The upper-left plot shows the reconstructed $E_{\pi}$ distribution for the data after background subtraction and the mock data sample before and after warping to the data. The upper right plot shows the reconstructed and true $E_{\pi}$ distributions for the warped mock data sample, where the error bars are the statistical uncertainty. The remaining plots show the deviation from the true event rate with statistical uncertainty as a function of true $E_{\pi}$ for the warped mock data sample before unfolding (middle left) and after each of the first three unfolding iterations. 

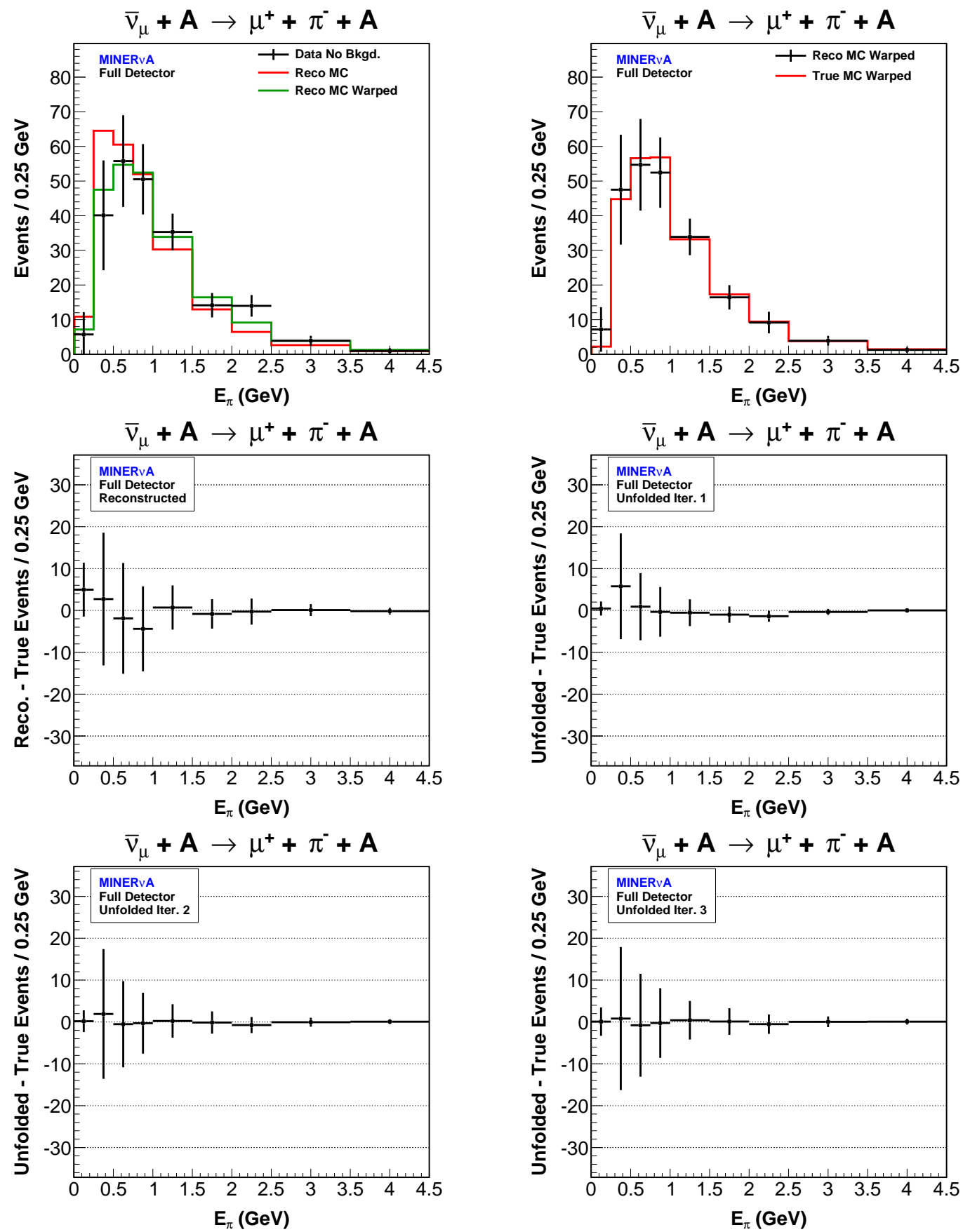

Figure 8.32: The full detector $\bar{\nu}_{\mu} E_{\pi}$ unfolding study. The upper-left plot shows the reconstructed $E_{\pi}$ distribution for the data after background subtraction and the mock data sample before and after warping to the data. The upper right plot shows the reconstructed and true $E_{\pi}$ distributions for the warped mock data sample, where the error bars are the statistical uncertainty. The remaining plots show the deviation from the true event rate with statistical uncertainty as a function of true $E_{\pi}$ for the warped mock data sample before unfolding (middle left) and after each of the first three unfolding iterations. 

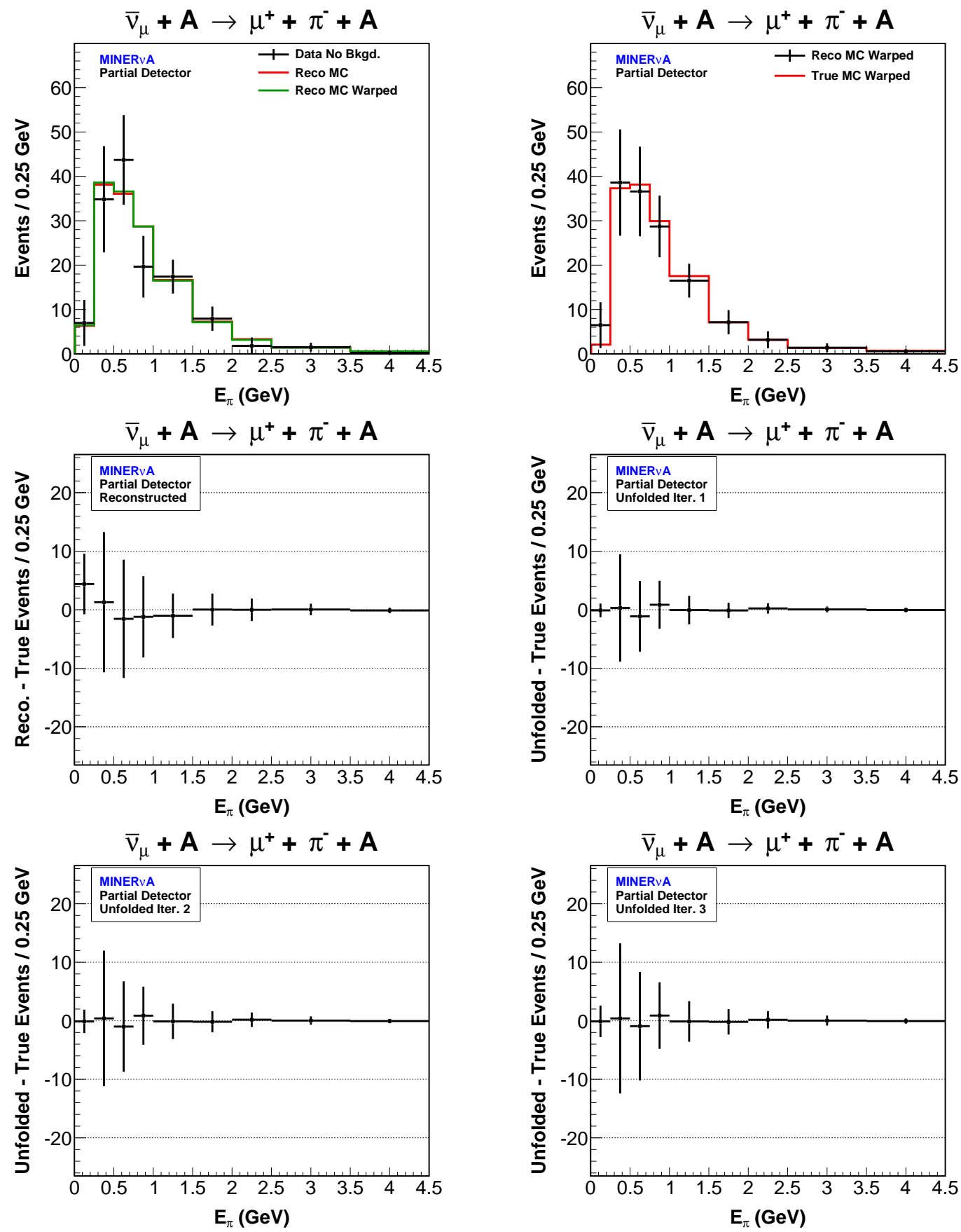

Figure 8.33: The partial detector $\bar{\nu}_{\mu} E_{\pi}$ unfolding study. The upper-left plot shows the reconstructed $E_{\pi}$ distribution for the data after background subtraction and the mock data sample before and after warping to the data. The upper right plot shows the reconstructed and true $E_{\pi}$ distributions for the warped mock data sample, where the error bars are the statistical uncertainty. The remaining plots show the deviation from the true event rate with statistical uncertainty as a function of true $E_{\pi}$ for the warped mock data sample before unfolding (middle left) and after each of the first three unfolding iterations. 

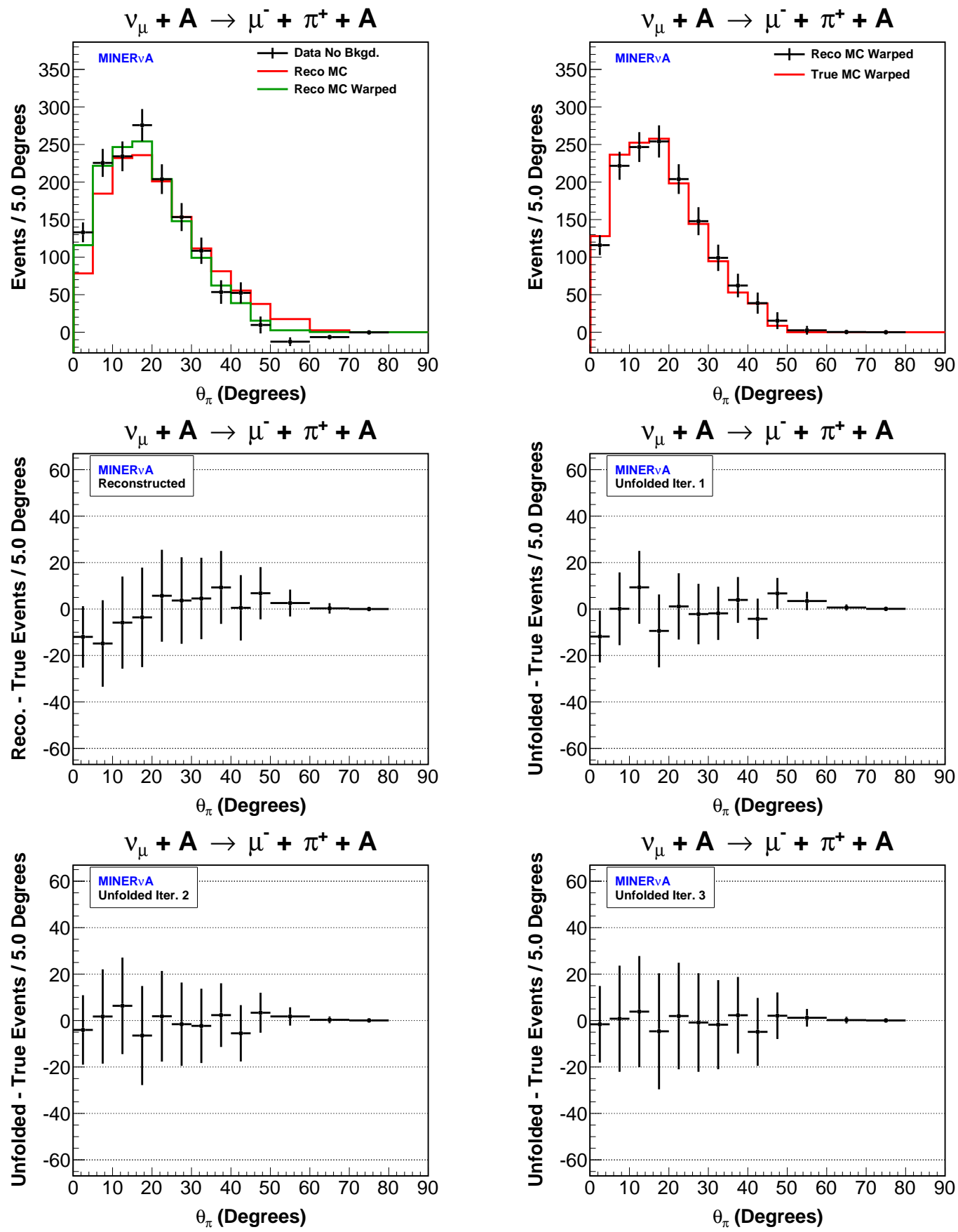

Figure 8.34: The $\nu_{\mu} \theta_{\pi}$ unfolding study. The upper-left plot shows the reconstructed $\theta_{\pi}$ distribution for the data after background subtraction and the mock data sample before and after warping to the data. The upper right plot shows the reconstructed and true $\theta_{\pi}$ distributions for the warped mock data sample, where the error bars are the statistical uncertainty. The remaining plots show the deviation from the true event rate with statistical uncertainty as a function of true $\theta_{\pi}$ for the warped mock data sample before unfolding (middle left) and after each of the first three unfolding iterations. 

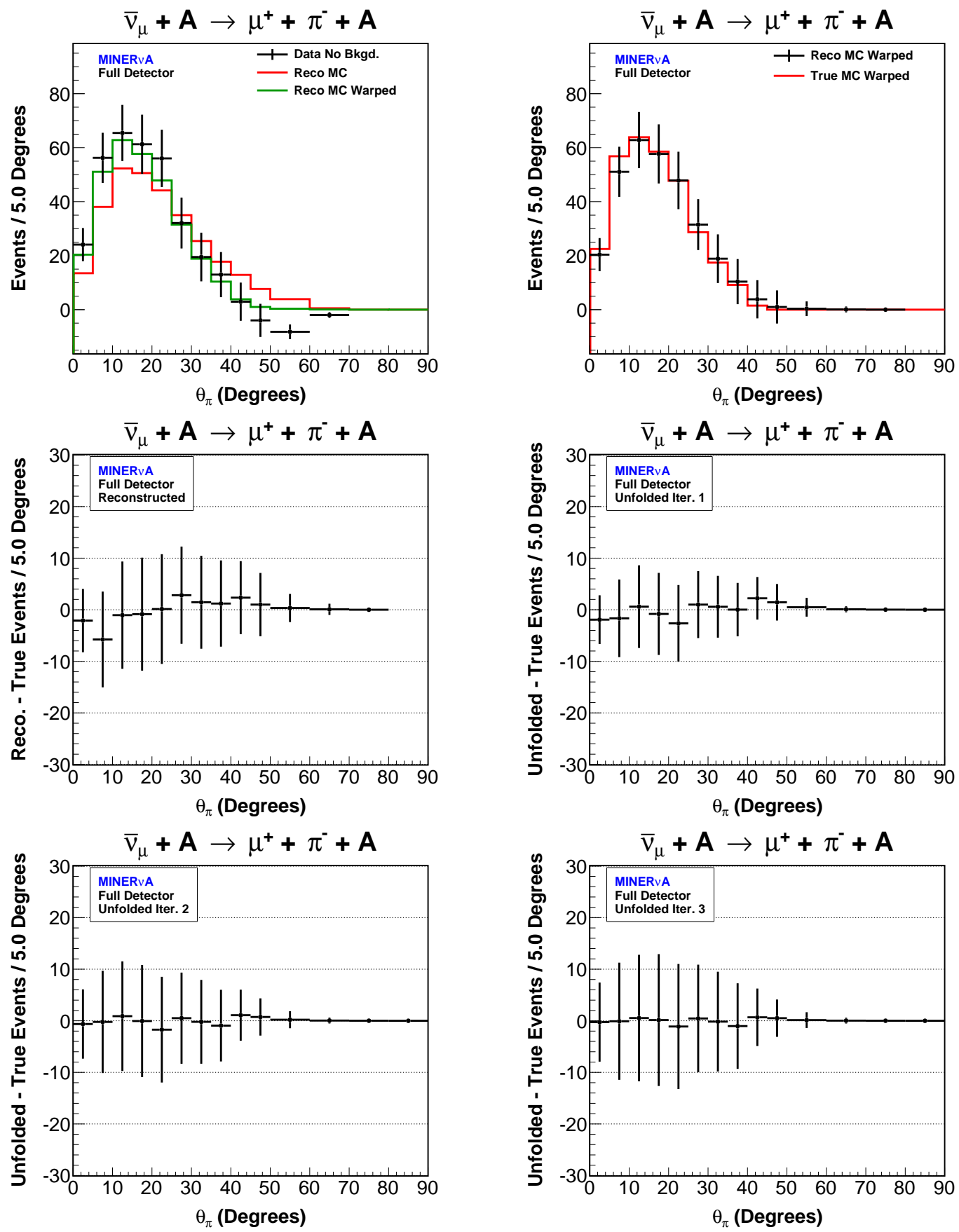

Figure 8.35: The full detector $\bar{\nu}_{\mu} \theta_{\pi}$ unfolding study. The upper-left plot shows the reconstructed $\theta_{\pi}$ distribution for the data after background subtraction and the mock data sample before and after warping to the data. The upper right plot shows the reconstructed and true $\theta_{\pi}$ distributions for the warped mock data sample, where the error bars are the statistical uncertainty. The remaining plots show the deviation from the true event rate with statistical uncertainty as a function of true $\theta_{\pi}$ for the warped mock data sample before unfolding (middle left) and after each of the first three unfolding iterations. 

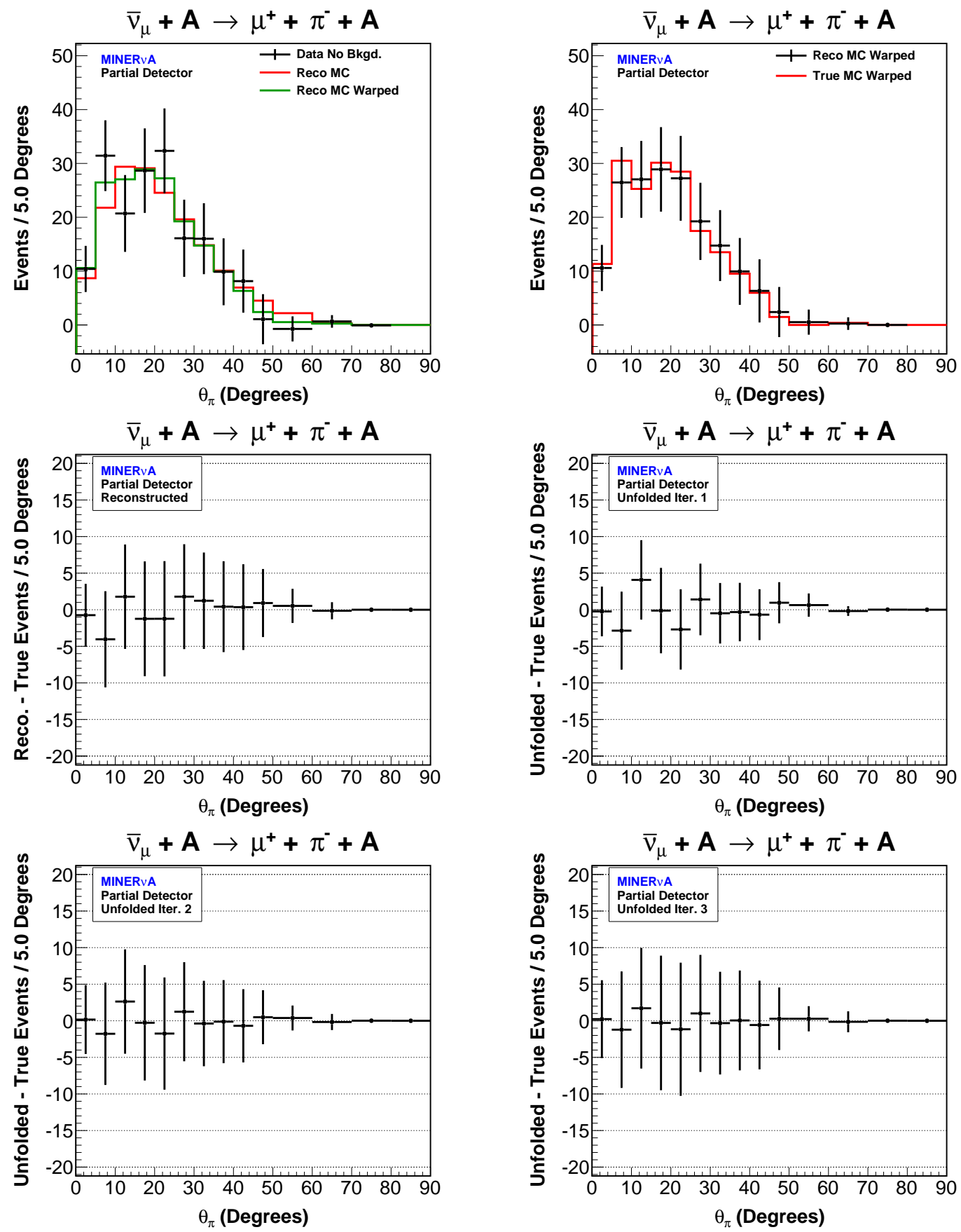

Figure 8.36: The partial detector $\bar{\nu}_{\mu} \theta_{\pi}$ unfolding study. The upper-left plot shows the reconstructed $\theta_{\pi}$ distribution for the data after background subtraction and the mock data sample before and after warping to the data. The upper right plot shows the reconstructed and true $\theta_{\pi}$ distributions for the warped mock data sample, where the error bars are the statistical uncertainty. The remaining plots show the deviation from the true event rate with statistical uncertainty as a function of true $\theta_{\pi}$ for the warped mock data sample before unfolding (middle left) and after each of the first three unfolding iterations. 

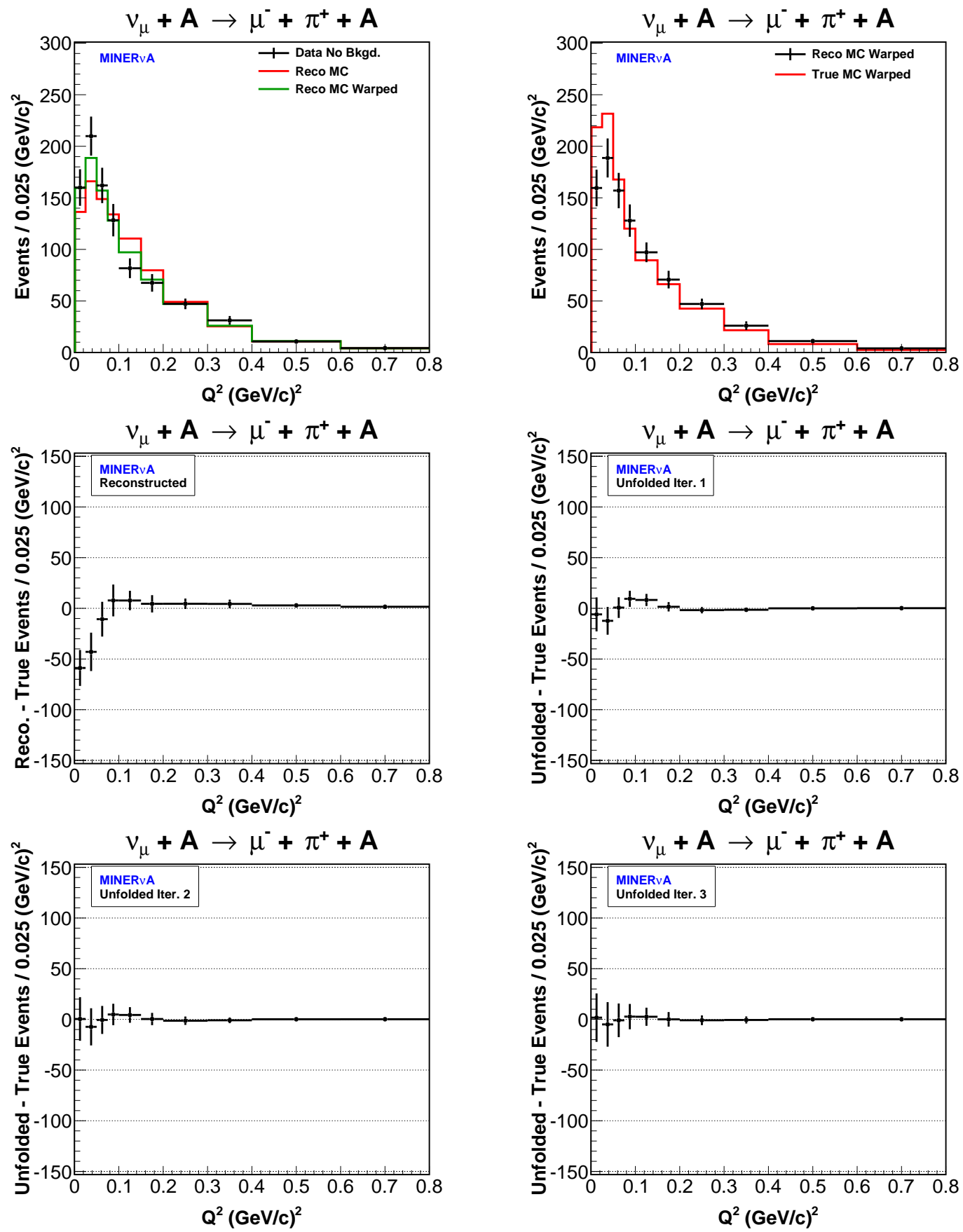

Figure 8.37: The $\nu_{\mu} Q^{2}$ unfolding study. The upper-left plot shows the reconstructed $Q^{2}$ distribution for the data after background subtraction and the mock data sample before and after warping to the data. The upper right plot shows the reconstructed and true $Q^{2}$ distributions for the warped mock data sample, where the error bars are the statistical uncertainty. The remaining plots show the deviation from the true event rate with statistical uncertainty as a function of true $Q^{2}$ for the warped mock data sample before unfolding (middle left) and after each of the first three unfolding iterations. 

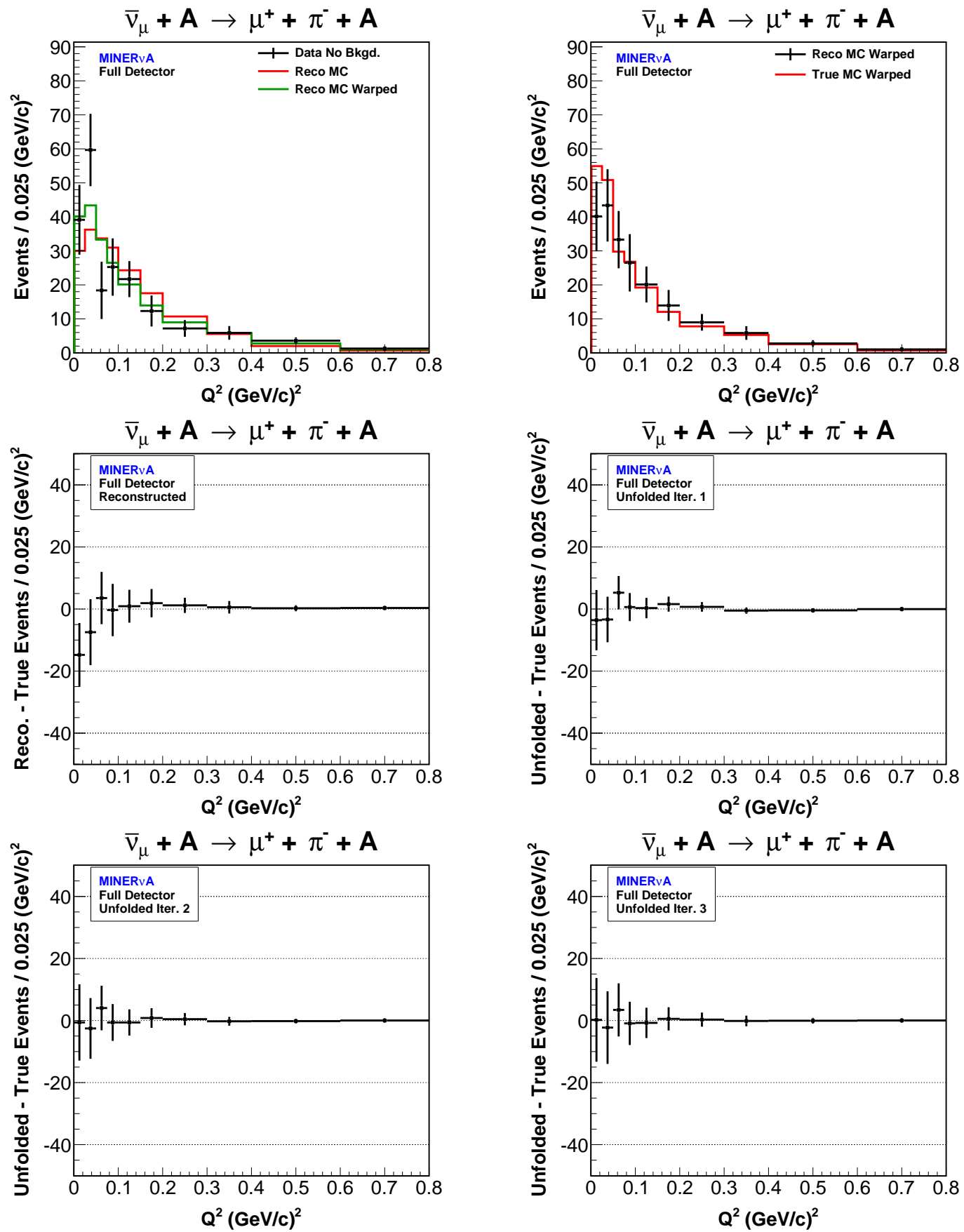

Figure 8.38: The full detector $\bar{\nu}_{\mu} Q^{2}$ unfolding study. The upper-left plot shows the reconstructed $Q^{2}$ distribution for the data after background subtraction and the mock data sample before and after warping to the data. The upper right plot shows the reconstructed and true $Q^{2}$ distributions for the warped mock data sample, where the error bars are the statistical uncertainty. The remaining plots show the deviation from the true event rate with statistical uncertainty as a function of true $Q^{2}$ for the warped mock data sample before unfolding (middle left) and after each of the first three unfolding iterations. 

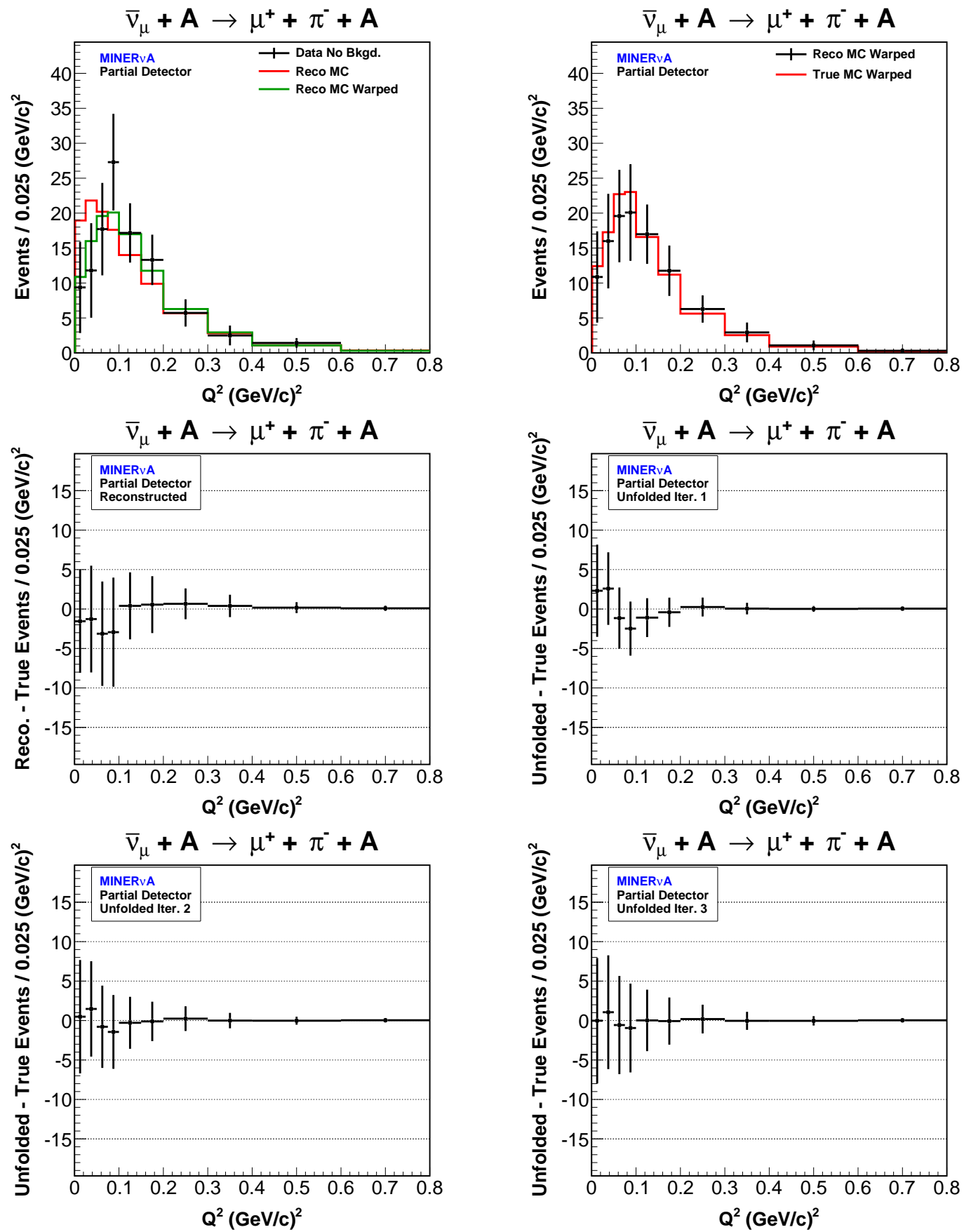

Figure 8.39: The partial detector $\bar{\nu}_{\mu} Q^{2}$ unfolding study. The upper-left plot shows the reconstructed $Q^{2}$ distribution for the data after background subtraction and the mock data sample before and after warping to the data. The upper right plot shows the reconstructed and true $Q^{2}$ distributions for the warped mock data sample, where the error bars are the statistical uncertainty. The remaining plots show the deviation from the true event rate with statistical uncertainty as a function of true $Q^{2}$ for the warped mock data sample before unfolding (middle left) and after each of the first three unfolding iterations. 


\subsubsection{Efficiency Correction}

After unfolding, the kinematic distributions are corrected for the coherent selection efficiency, which was estimated using coherent events from the signal-only MC samples. The efficiency in each true kinematic bin $\epsilon_{i}$ was calculated as

$$
\epsilon_{i}=\frac{N_{i}^{s e l}}{N_{i}^{g e n}},
$$

where $N_{i}^{g e n}$ is the number of coherent events generated inside the fiducial volume with true $E_{\nu}$ satisfying $2.0<E_{\nu}<20 \mathrm{GeV}$ in bin $i$, and $N_{i}^{\text {sel }}$ is the subset of those events that passed all selection cuts. The signal model weighting (Section 8.10) was applied in calculating the efficiency. The selection efficiency and efficiency corrected distribution for each kinematic parameter for the $\nu_{\mu}$ and $\bar{\nu}_{\mu}$ samples are shown in Figures 8.40-8.43. The total efficiency for the $\nu_{\mu}$ and $\bar{\nu}_{\mu}$ samples is $24-25 \%$.

The selection efficiency includes the acceptance of the reconstruction. The requirement that the muon be reconstructed in both MINER $\nu \mathrm{A}$ and MINOS limits $\theta_{\mu}$ of accepted events to $\theta_{\mu}<20$ degrees, and the minimum number of planes required to form a track limits $\theta_{\pi}$ of accepted events to $\theta_{\pi}<70$ degrees. Of the coherent events in the MC occurring inside the fiducial volume, 96\% have $\theta_{\mu}<20$ degrees and $\theta_{\pi}<70$ degrees. The $4 \%$ loss in the coherent candidate event rate due to the reconstruction is corrected in the efficiency correction. 

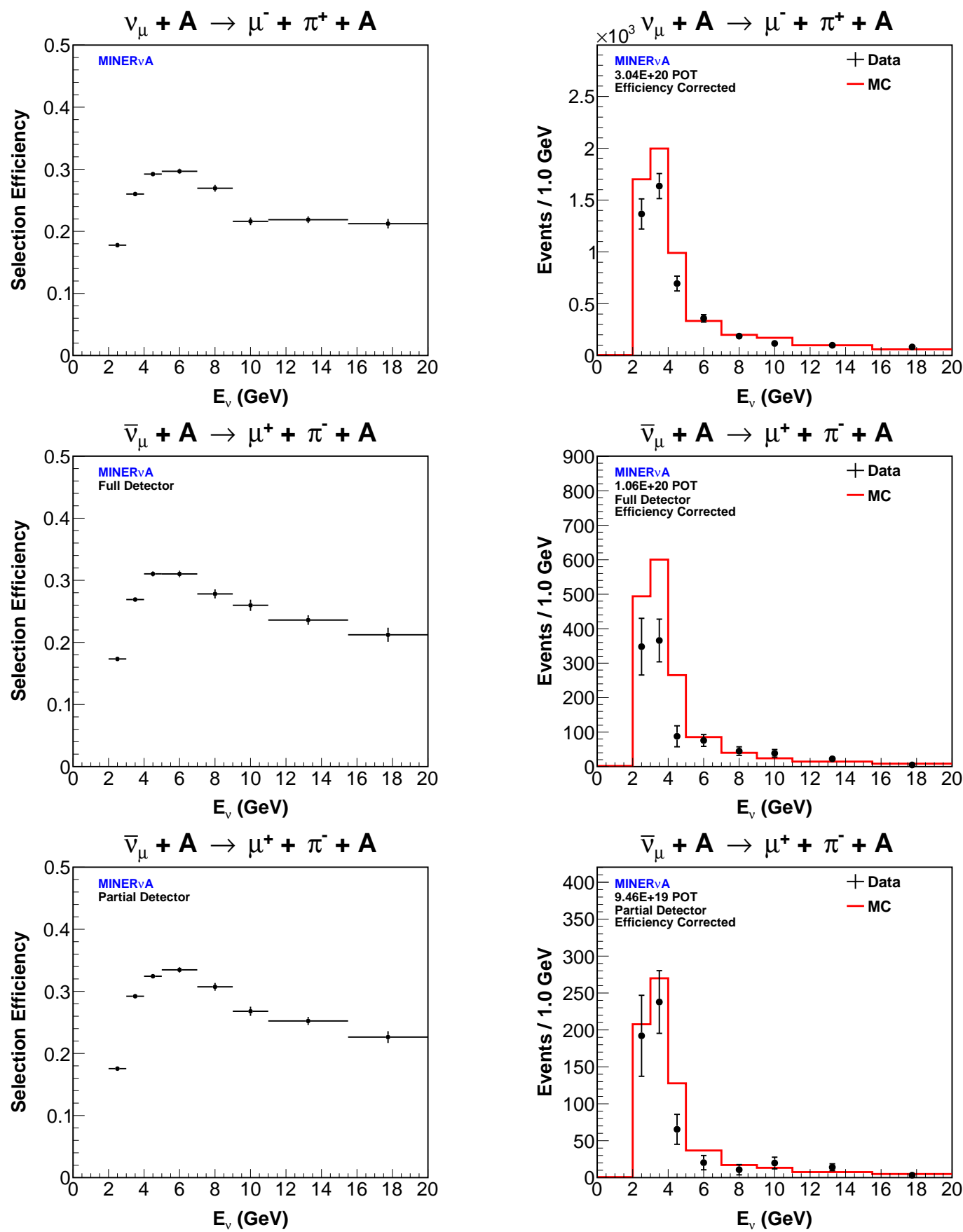

Figure 8.40: The selection efficiency as a function of $E_{\nu}$ (left) and the efficiency corrected $E_{\nu}$ distribution (right) for the $\nu_{\mu}$ (top), full detector $\bar{\nu}_{\mu}$ (middle), and partial detector $\bar{\nu}_{\mu}$ (bottom) samples 

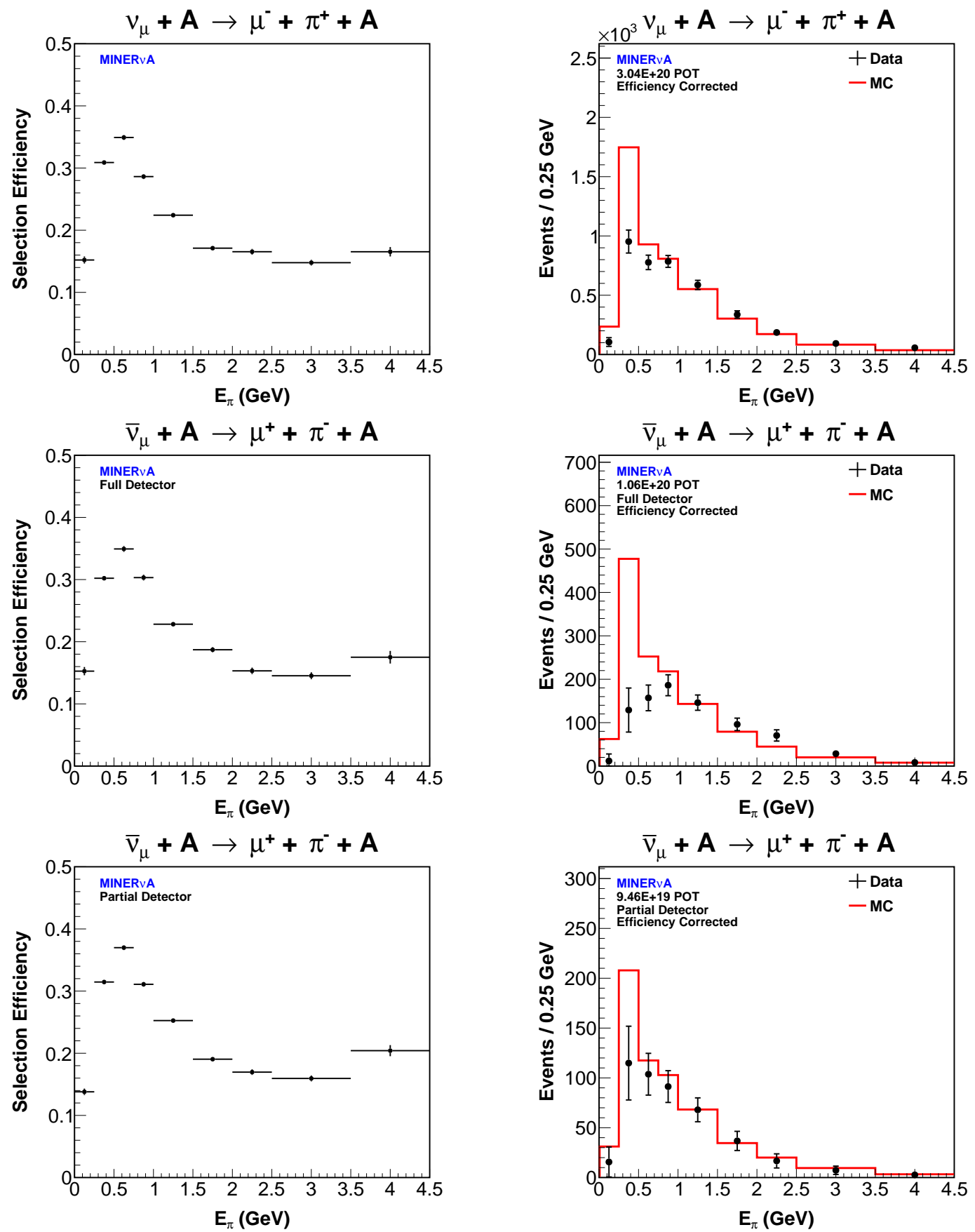

Figure 8.41: The selection efficiency as a function of $E_{\pi}$ (left) and the efficiency corrected $E_{\pi}$ distribution (right) for the $\nu_{\mu}$ (top), full detector $\bar{\nu}_{\mu}$ (middle), and partial detector $\bar{\nu}_{\mu}$ (bottom) samples 

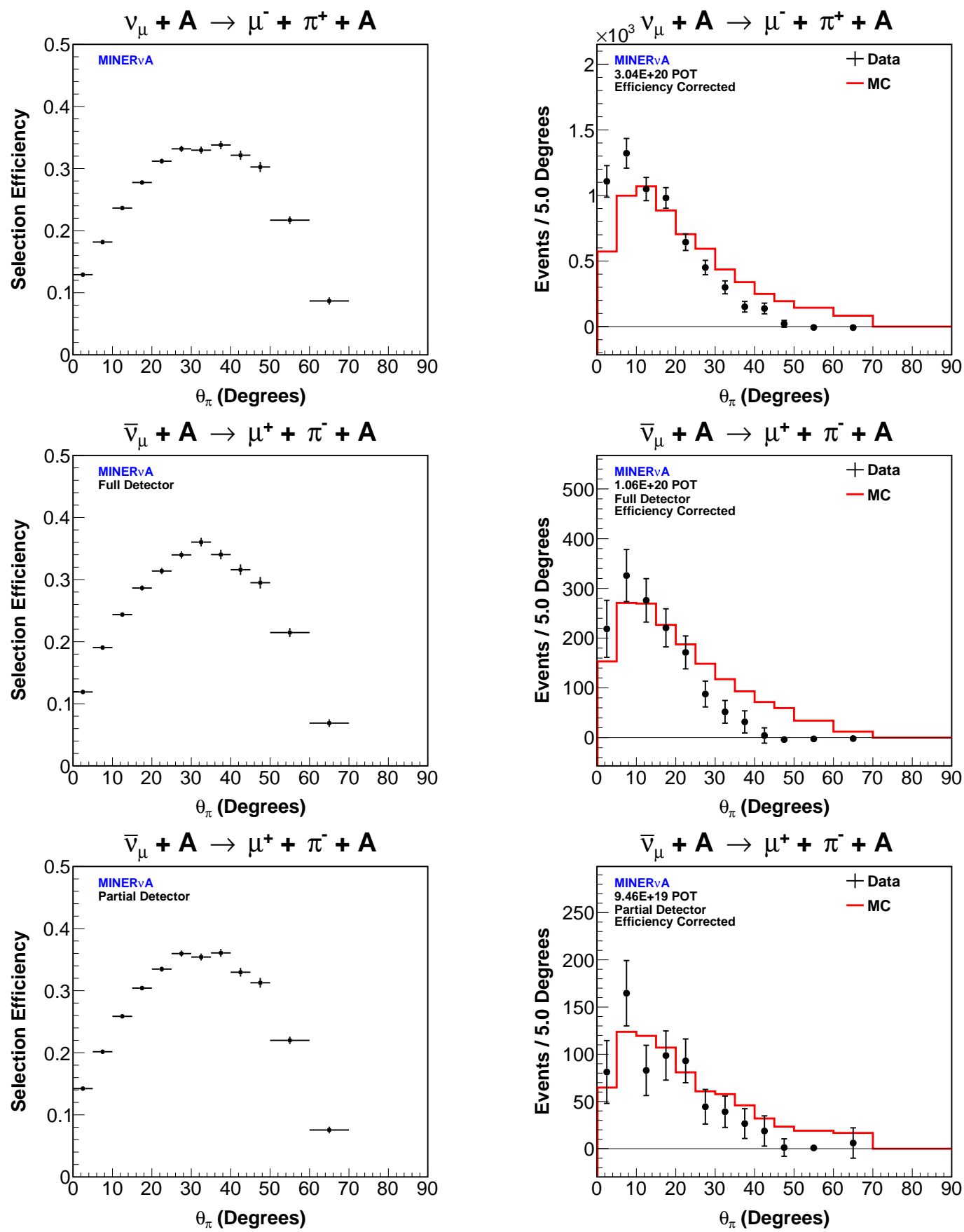

Figure 8.42: The selection efficiency as a function of $\theta_{\pi}$ (left) and the efficiency corrected $\theta_{\pi}$ distribution (right) for the $\nu_{\mu}$ (top), full detector $\bar{\nu}_{\mu}$ (middle), and partial detector $\bar{\nu}_{\mu}$ (bottom) samples 

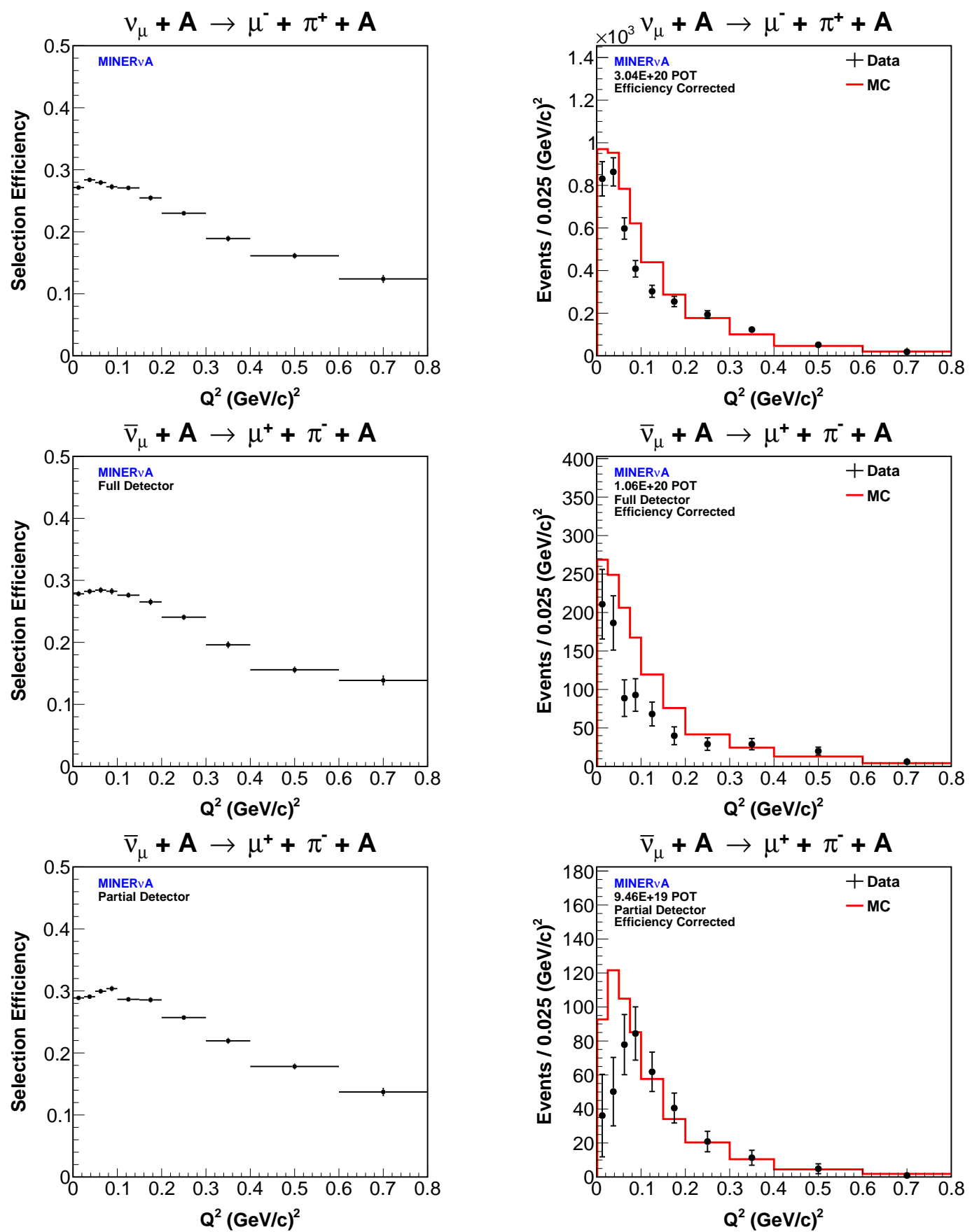

Figure 8.43: The selection efficiency as a function of $Q^{2}$ (left) and the efficiency corrected $Q^{2}$ distribution (right) for the $\nu_{\mu}$ (top), full detector $\bar{\nu}_{\mu}$ (middle), and partial detector $\bar{\nu}_{\mu}$ (bottom) samples 


\subsubsection{Flux Normalization}

The cross sections were normalized to the flux prediction scaled to the number of protons on target for each sample, where the flux prediction was tuned to external hadron production data (the ppfx flux) and constrained to the in situ $\nu+e^{-} \rightarrow \nu+e^{-}$ elastic scattering measurement. The cross section $\sigma_{i}$ in each $E_{\nu}$ bin $i$ was normalized to the flux integrated over the bin range (Table 8.13). The differential cross section $\left(\frac{d \sigma}{d \xi}\right)_{i}$ in each $\xi$ bin $i$ was normalized to the flux integrated over $2.0<E_{\nu}<20 \mathrm{GeV}$.

\begin{tabular}{c|c|c|c|c|c|c|c|c}
$E_{\nu}(\mathrm{GeV})$ & $2.0-3.0$ & $3.0-4.0$ & $4.0-5.0$ & $5.0-7.0$ & $7.0-9.0$ & $9.0-11.0$ & $11.0-15.5$ & $15.5-20.0$ \\
\hline$\left(\nu_{\mu} / \mathrm{cm}^{2} / \mathrm{POT}\right) \times 10^{-8}$ & 0.740 & 0.765 & 0.312 & 0.189 & 0.098 & 0.065 & 0.077 & 0.044 \\
$\left(\bar{\nu}_{\mu} / \mathrm{cm}^{2} / \mathrm{POT}\right) \times 10^{-8}$ & 0.662 & 0.644 & 0.244 & 0.129 & 0.055 & 0.033 & 0.035 & 0.018
\end{tabular}

Table 8.13: $\nu_{\mu}$ and $\bar{\nu}_{\mu}$ fluxes

\subsubsection{Target Number Normalization}

The measured cross sections were normalized to the number of carbon nuclei "targets" contained in the fiducial volume. The number of carbon nuclei was estimated using the detector geometry and material models, the latter of which was informed by the material assay. The full (partial) detector fiducial volume was estimated to contain $2.404 \times 10^{29}\left(1.246 \times 10^{29}\right)$ carbon nuclei.

The number of coherent candidates $N^{c o h}=N^{\text {data }}-N^{\text {bkgd }}$, where $N^{\text {data }}$ and $N^{b k g d}$ are the number of selected data and tuned background events, respectively, include coherent interactions on non-carbon nuclei inside the fiducial volume. Noncarbon coherent interactions were not included in $N^{\text {bkgd }}$ to avoid dependence of the background estimate on the coherent model (Section 8.3). Instead, $N^{\text {coh }}$ was corrected to the number of coherent candidates on carbon only $N_{c}^{c o h}$. The correction 
$\beta$ was calculated as

$$
\beta=\frac{N_{c}^{c o h}}{N^{c o h}}=\frac{\phi \epsilon_{c} \sigma_{c} T_{c}}{\sum_{i} \phi \epsilon_{i} \sigma_{i} T_{i}},
$$

where $\phi$ is the flux and $\epsilon_{i}, \sigma_{i}$, and $T_{i}$ are the coherent acceptance and selection efficiency, coherent cross section, and number of nuclei in the fiducial volume for nuclear species $i$. Assuming the coherent acceptance and selection efficiency is the same for all nuclear species and the coherent cross section scales with the nuclear mass number $A$ as $A^{1 / 3}[34]$,

$$
\beta \approx \frac{A_{c}^{1 / 3} T_{c}}{\sum_{i} A_{i}^{1 / 3} T_{i}}
$$

The sums in Equations 8.18 and 8.19 exclude hydrogen since coherent scattering, by definition, occurs on nuclei with multiple nucleons only. The estimated number of nuclei for each nuclear species in the full detector fiducial volume (Table 8.14) gives $\beta$ $=0.962$. This correction was applied to all measured cross sections (Equations 8.12 and 8.13).

\begin{tabular}{l|c|c} 
Nucleus & $A$ & $T$ \\
\hline${ }^{1} \mathrm{H}$ & 1.008 & $2.425 \times 10^{29}$ \\
${ }^{12} \mathrm{C}$ & 12.011 & $2.404 \times 10^{29}$ \\
${ }^{16} \mathrm{O}$ & 15.999 & $6.548 \times 10^{27}$ \\
${ }^{27} \mathrm{Al}$ & 26.982 & $3.175 \times 10^{26}$ \\
${ }^{28} \mathrm{Si}$ & 28.085 & $3.167 \times 10^{26}$ \\
${ }^{35} \mathrm{Cl}$ & 35.453 & $5.111 \times 10^{26}$ \\
${ }^{48} \mathrm{Ti}$ & 47.867 & $4.749 \times 10^{26}$
\end{tabular}

Table 8.14: The nuclear mass number $A$ and estimated number of nuclei $T$ for each nuclear species in the full detector fiducial volume, which spans the central 108 scintillator planes of the tracker and extends to the edges of a hexagon with an 850 $\mathrm{mm}$ apothem in the transverse (XY) plane (Section 8.2) 


\subsection{Signal Model Weighting}

Initial measurements of the cross sections (Figures 8.44-8.47) were made using unfolding matrices and efficiency corrections estimated from the unmodified GENIE Rein-Sehgal coherent model. The initial measurements revealed that GENIE overpredicts the production rate at low- $E_{\pi}$ and high- $\theta_{\pi}$. Better agreement between the GENIE prediction and the initial measurements was achieved by weighting the rate of interactions predicted by GENIE with $E_{\pi}<0.5 \mathrm{GeV}$ by $50 \%$ (Figures $8.44-$ 8.47). This weighting is referred to as the signal model weighting. The $\chi^{2}$ for the comparison of the initial measurement of each cross section to the nominal and weighted GENIE predictions is listed in Table 8.15, where the $\chi^{2}$ was calculated per Equation 8.20.
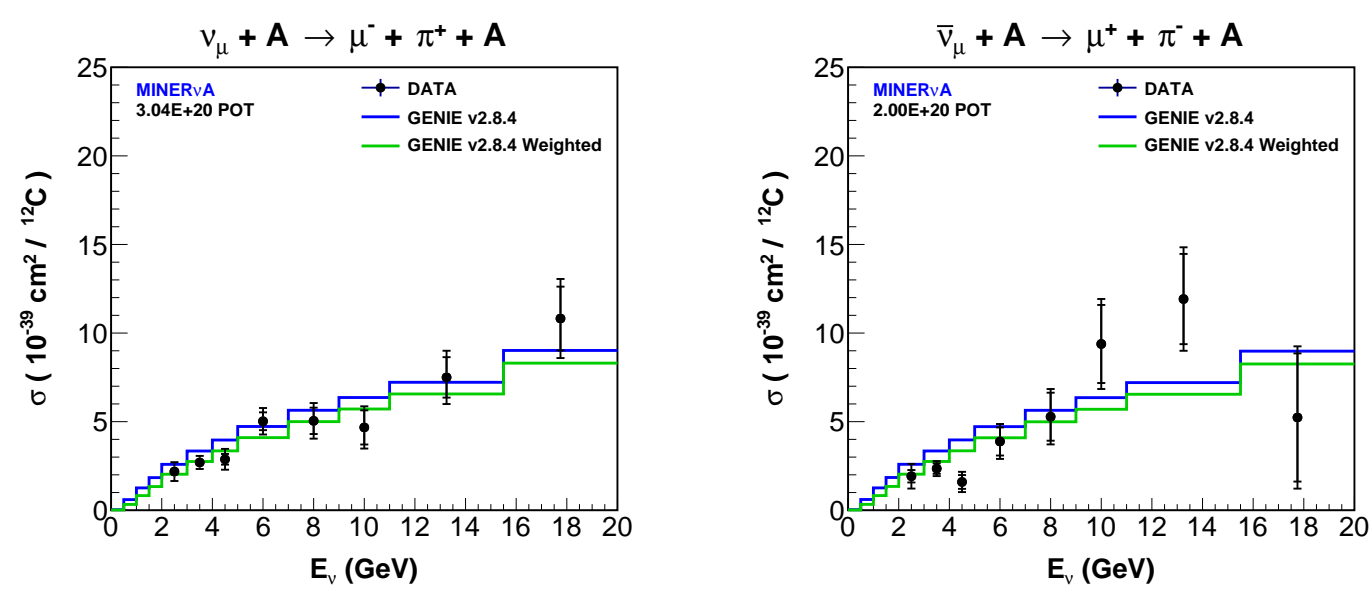

Figure 8.44: The initial measurements of the $\nu_{\mu}$ (left) and $\bar{\nu}_{\mu}$ (right) $\sigma\left(E_{\nu}\right)$ made without the signal model weighting, and the GENIE prediction with and without the signal model weighting

The unfolding matrices and efficiency corrections are dependent on the kinematics of the signal model. To minimize bias on the measured cross sections from the signal model, the signal model weighting was applied to coherent events in the MC, 

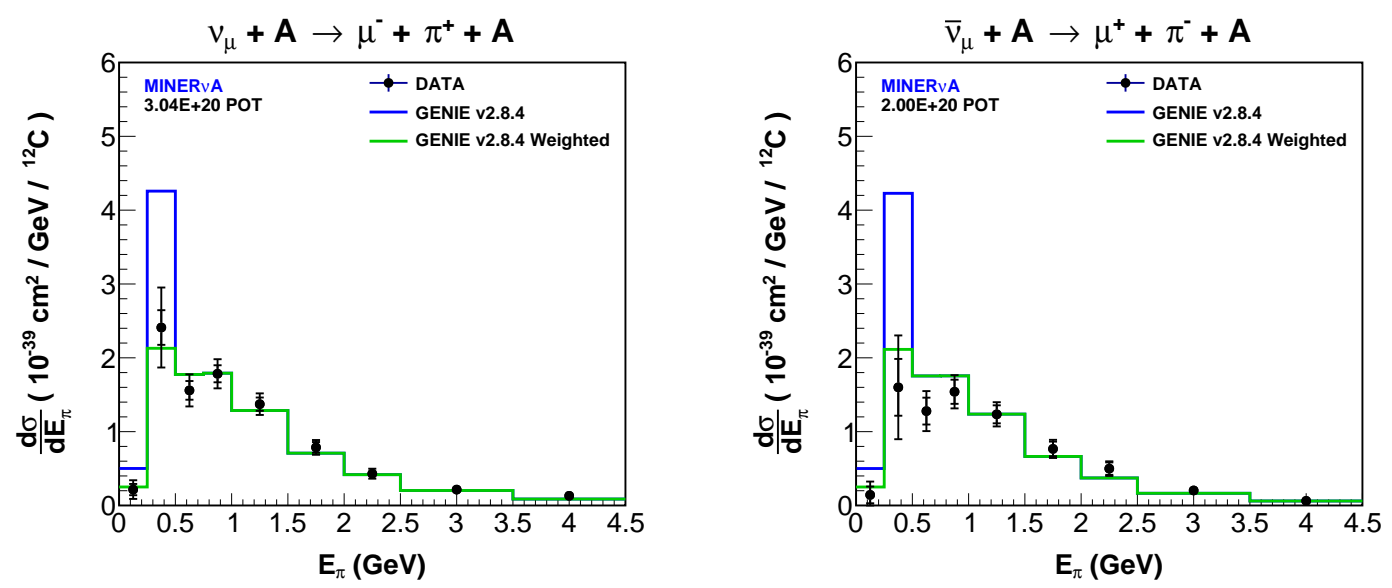

Figure 8.45: The initial measurements of the $\nu_{\mu}$ (left) and $\bar{\nu}_{\mu}$ (right) $\frac{d \sigma}{d E_{\pi}}$ made without the signal model weighting, and the GENIE prediction with and without the signal model weighting
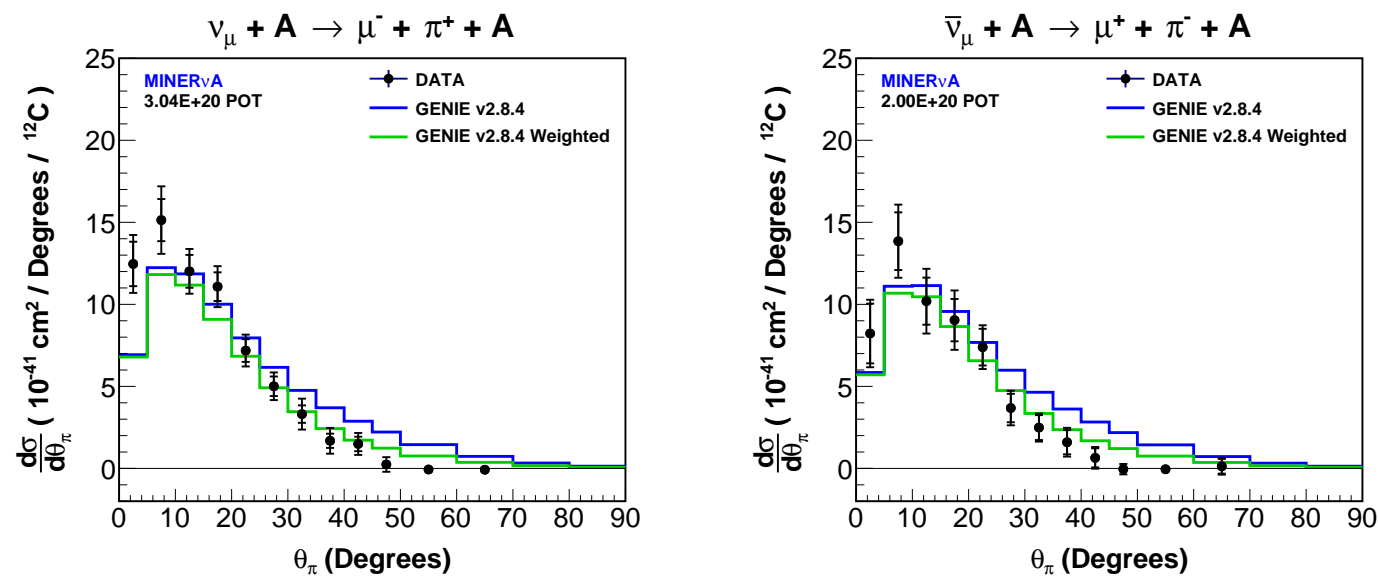

Figure 8.46: The initial measurements of the $\nu_{\mu}$ (left) and $\bar{\nu}_{\mu}$ (right) $\frac{d \sigma}{d \theta_{\pi}}$ made without the signal model weighting, and the GENIE prediction with and without the signal model weighting 

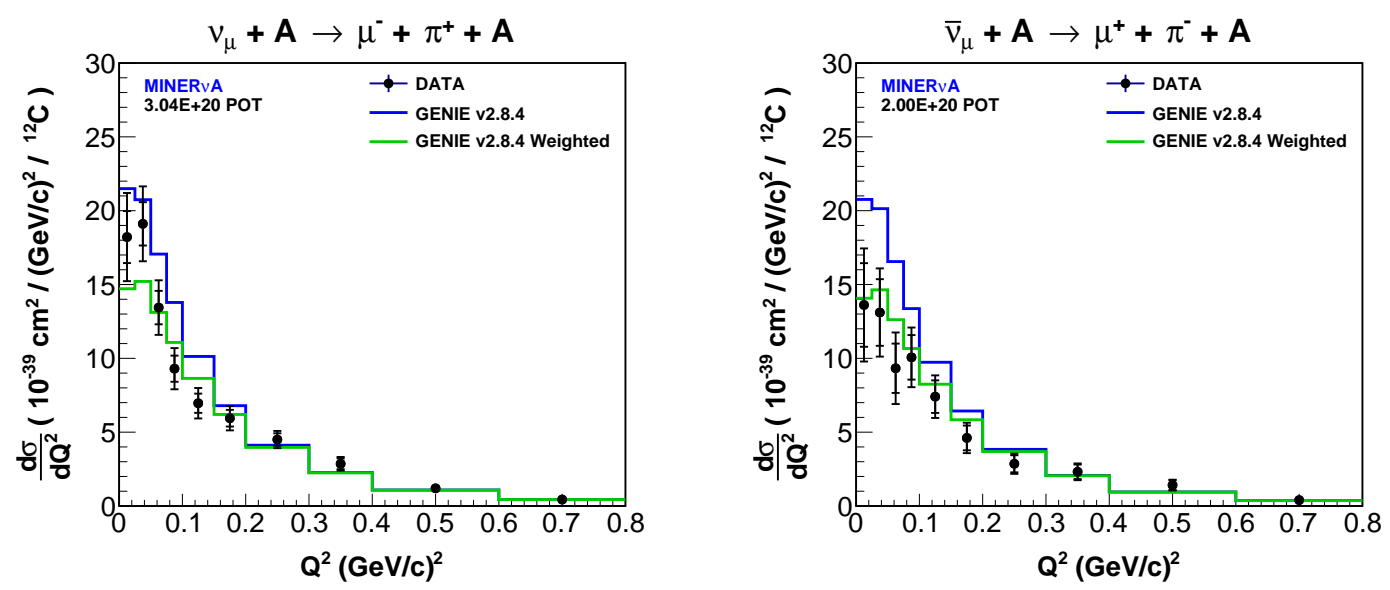

Figure 8.47: The initial measurements of the $\nu_{\mu}$ (left) and $\bar{\nu}_{\mu}$ (right) $\frac{d \sigma}{d Q^{2}}$ made without the signal model weighting, and the GENIE prediction with and without the signal model weighting

\begin{tabular}{c|cc|cc|c} 
& \multicolumn{3}{|c|}{$\nu_{\mu} \chi^{2}$} & \multicolumn{2}{|c}{$\bar{\nu}_{\mu} \chi^{2}$} \\
Cross Section & Rein-Sehgal & Rein-Sehgal Weighted & Rein-Sehgal & Rein-Sehgal Weighted & NDF \\
\hline$\sigma\left(E_{\nu}\right)$ & 11.8 & 7.5 & 27.0 & 17.6 & 8 \\
$d \sigma / d E_{\pi}$ & 22.4 & 13.5 & 16.6 & 7.1 & 9 \\
$d \sigma / d \theta_{\pi}$ & 1388.4 & 418.8 & 144.1 & 46.9 & 12 \\
$d \sigma / d Q^{2}$ & 19.2 & 15.4 & 16.8 & 10.0 & 10
\end{tabular}

Table 8.15: $\chi^{2}$ for the comparisons of the initial measured $\nu_{\mu}$ and $\bar{\nu}_{\mu}$ cross sections to the nominal and weighted GENIE Rein-Sehgal predictions

the unfolding matrices and efficiency corrections were re-estimated, and the cross sections were remeasured.

The effect of the signal model weighting on the measured cross sections is shown in Figures 8.48-8.51. The signal model weighting increased the measured $\nu_{\mu}$ and $\bar{\nu}_{\mu}$ cross sections at low- $E_{\nu}$, high- $\theta_{\pi}$, and low- $Q^{2}$. This kinematic region corresponds to the low- $E_{\pi}$ region suppressed by the signal model weighting. The MC coherent event rate and selection efficiency are maximal near near $E_{\pi}=0.5 \mathrm{GeV}$ (Figure 8.41). Therefore, the signal model weighting reduces the selection efficiency, and thereby 
the measured cross section, at low- $E_{\nu}$, high- $\theta_{\pi}$, and low- $Q^{2}$.

The signal model weighting reduced the measured $\nu_{\mu}$ and $\bar{\nu}_{\mu} \frac{d \sigma}{d E_{\pi}}$ by $\sim 10 \%$ in the 0.25-0.5 GeV bin while increasing the cross section in the adjacent bins by a similar amount (Figure 8.49). The signal model weighting did not change the selection efficiency as a function of $E_{\pi}$ since events in the numerator and denominator of the efficiency calculation (Equation 8.17) in each $E_{\pi}$ bin were weighted equally. Instead, the change to the measured $\frac{d \sigma}{d E_{\pi}}$ results from the change to the $E_{\pi}$ unfolding matrix. The nominal signal model predicts a large peak in the coherent event rate in the 0.25-0.5 GeV $E_{\pi}$ bin, and the resulting unfolding matrix predicts a net migration of events from this bin into the adjacent bins. The signal model weighting suppresses the predicted peak in the event rate and thereby the predicted amount of migration from the $0.25-0.5 \mathrm{GeV} E_{\pi}$ bin, resulting in the observed change to the measured $\nu_{\mu}$ and $\bar{\nu}_{\mu} \frac{d \sigma}{d E_{\pi}}$.
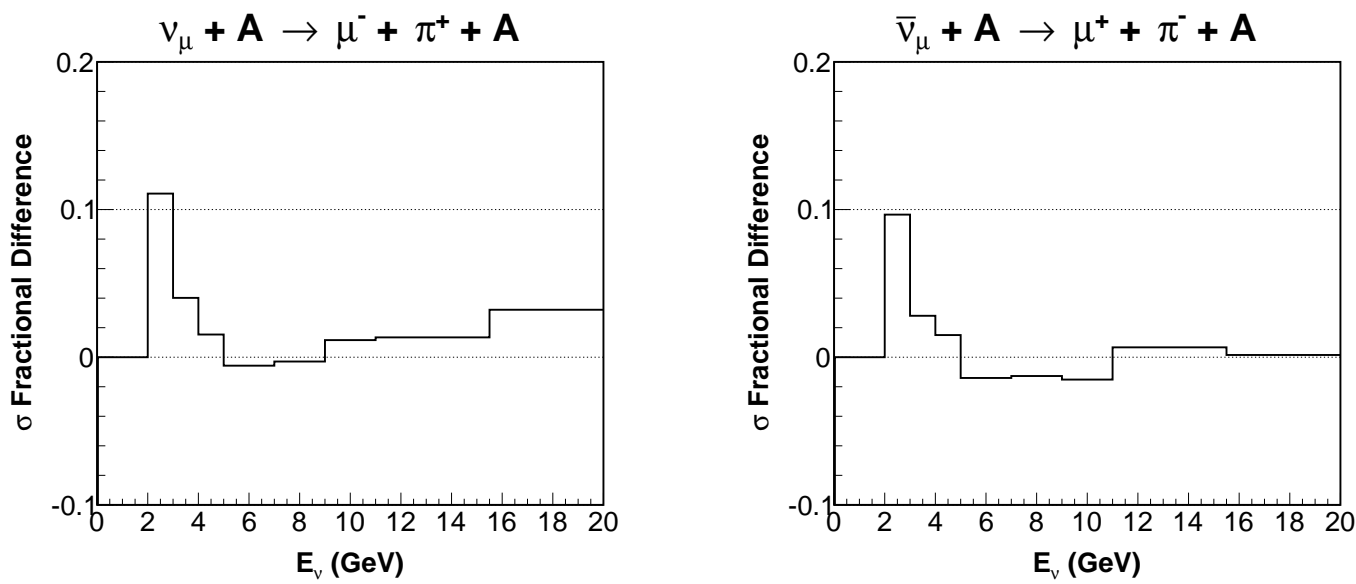

Figure 8.48: The effect of the signal model weighting on the measured $\nu_{\mu}$ (left) and $\bar{\nu}_{\mu}$ (right) $\sigma\left(E_{\nu}\right)$

In principle, the cross sections could be measured iteratively where the signal model weighting is corrected after each iteration. Given the improved agreement 

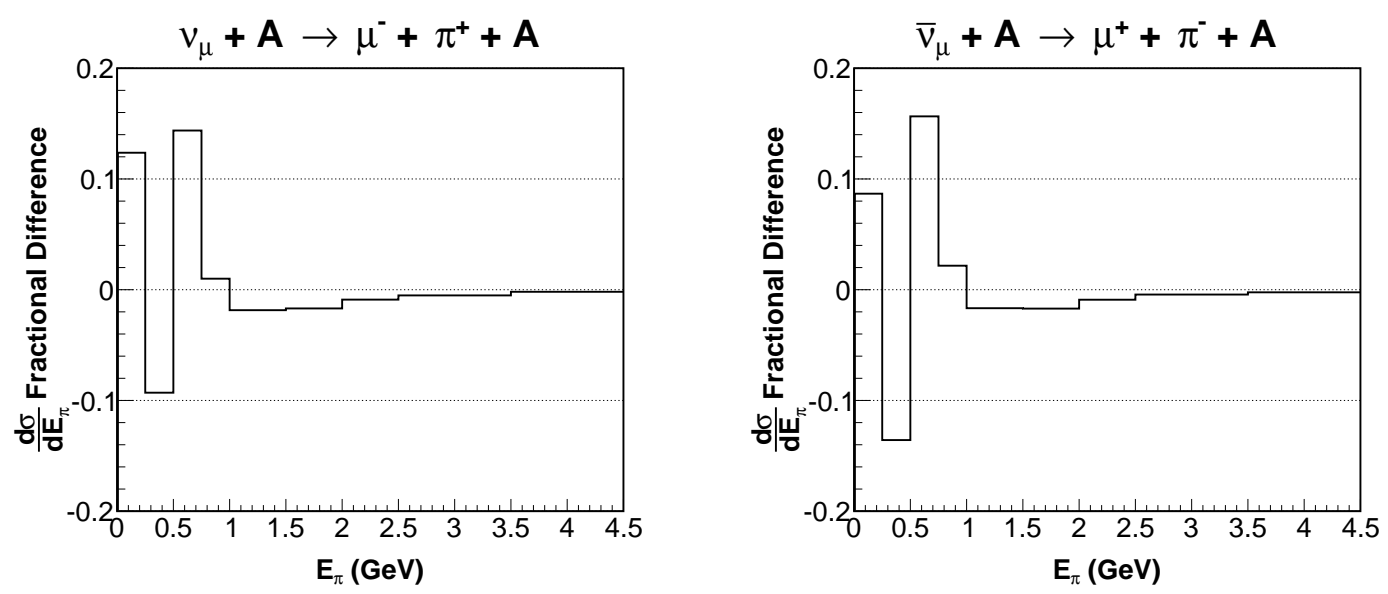

Figure 8.49: The effect of the signal model weighting on the measured $\nu_{\mu}$ (left) and $\bar{\nu}_{\mu}$ (right) $\frac{d \sigma}{d E_{\pi}}$
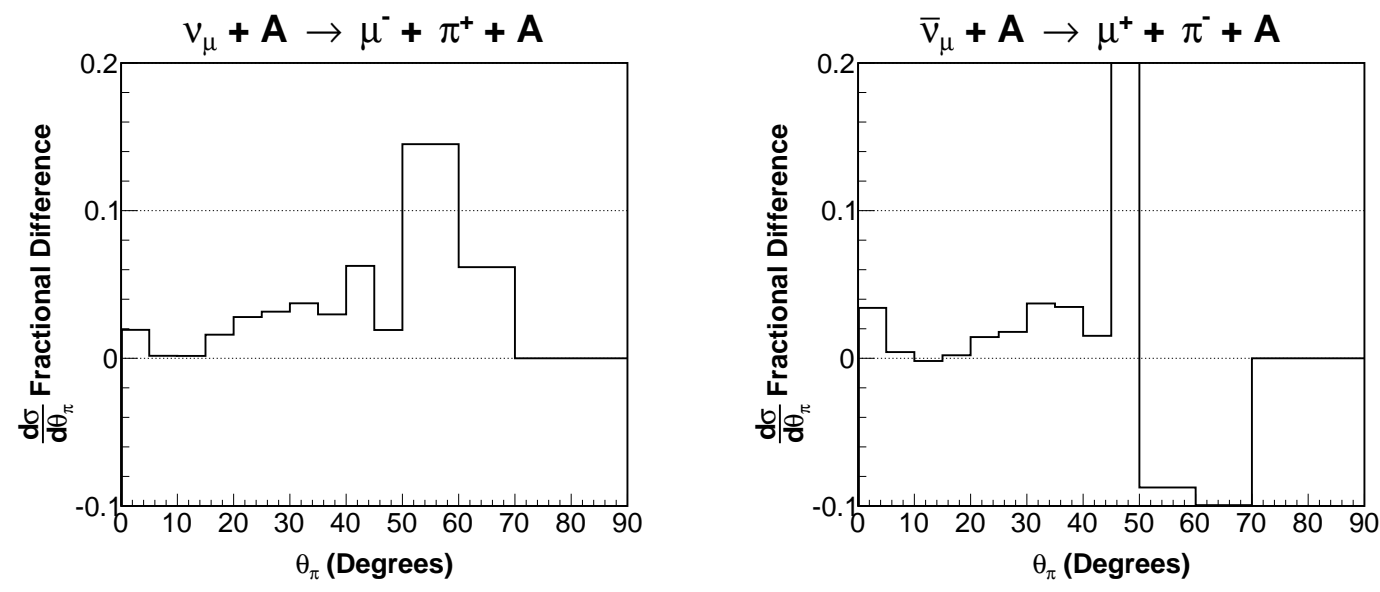

Figure 8.50: The effect of the signal model weighting on the measured $\nu_{\mu}$ (left) and $\bar{\nu}_{\mu}$ (right) $\frac{d \sigma}{d \theta_{\pi}}$ 

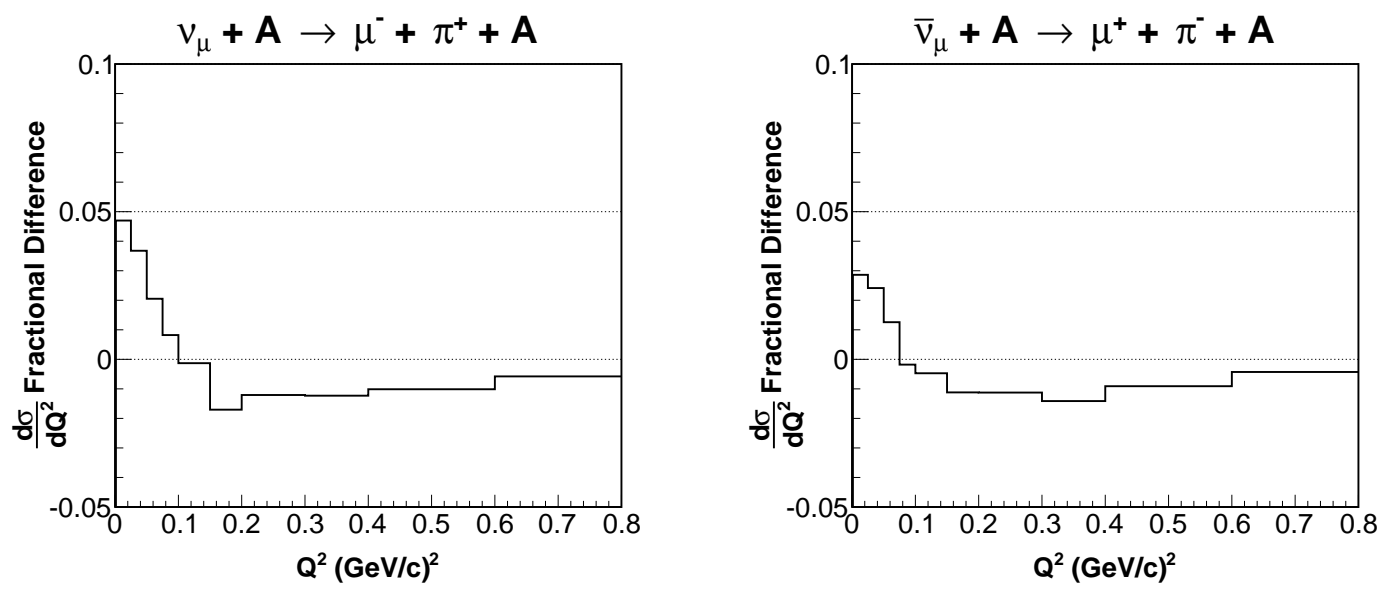

Figure 8.51: The effect of the signal model weighting on the measured $\nu_{\mu}$ (left) and $\bar{\nu}_{\mu}$ (right) $\frac{d \sigma}{d Q^{2}}$

between the initial measurements of the cross sections and the GENIE prediction after the signal model weighting, and the $<15 \%$ effect of the signal model weighting on the measured cross sections (excluding the change to $\frac{d \sigma}{d \theta_{\pi}}$ at high- $\theta_{\pi}$ where the cross section is small), a correction to the signal model weighting would be small compared to the initial $50 \%$ reduction in the predicted interaction rate for $E_{\pi}<$ $0.5 \mathrm{GeV}$. Furthermore, given the $<15 \%$ effect of the signal model weighting on the measured cross sections, a correction to the signal model weighting would result in a change to the measured cross sections that is much smaller than the total uncertainty on the measured cross sections (Section 8.13). Therefore, the final reported cross sections (Section 8.11) were measured with a single weighting of the signal model (50\% reduction in the predicted interaction rate for $E_{\pi}<0.5 \mathrm{GeV}$ ) and an uncertainty on the weighting was not included in the uncertainty on the cross sections. 


\subsection{Measured Cross Sections}

The measured $\nu_{\mu}$ and $\bar{\nu}_{\mu}$ cross sections with the GENIE Rein-Sehgal and BergerSehgal predictions are shown in Figures 8.52-8.55. Figure 8.56 shows the measured $\nu_{\mu}$ and $\bar{\nu}_{\mu} \sigma\left(E_{\nu}\right)$ compared with previous measurements at $E_{\nu}<20 \mathrm{GeV}$ and with the GENIE Rein-Sehgal model prediction. The signal model weighting (Section 8.10) was applied in calculating the unfolding matrices and efficiency corrections used to measure the cross sections. The $\chi^{2}$ for the comparison of each measured cross section to the GENIE Rein-Sehgal and Berger-Sehgal predictions is listed in Table 8.16. The $\chi^{2}$ was calculated as

$$
\chi^{2}=A C^{-1} A^{T}
$$

where $C$ is the bin-to-bin covariance matrix for the total uncertainty (statistical + total systematic) on the measured cross section, and the elements of the vector $A$ are

$$
A_{i}=\sigma_{i}^{\text {meas }}-\sigma_{i}^{\text {pred }}
$$

where $\sigma_{i}^{\text {meas }}$ and $\sigma_{i}^{\text {pred }}$ are the measured and predicted cross sections in bin $i$, respectively. The evaluation of the systematic uncertainties on the measured cross sections is detailed in Section 8.13.

The GENIE Rein-Sehgal and Berger-Sehgal predictions agree with the measured cross sections in normalization. The Berger-Sehgal normalization is lower than the Rein-Sehgal normalization due to the lower Berger-Sehgal pion-carbon elastic scattering cross section in the delta resonance region. Both predictions differ in shape from the measured $\frac{d \sigma}{d E_{\pi}}$ and $\frac{d \sigma}{d \theta_{\pi}}$, suggesting both models mis-model the pion-carbon elastic scattering cross section. Both predictions agree in shape with the measured $\frac{d \sigma}{d Q^{2}}$, which supports the axial vector dipole parameterization of the $Q^{2}$ dependence 
of the coherent cross section. The difference in shape between the full and partial detector $\bar{\nu}_{\mu} \frac{d \sigma}{d Q^{2}}$ (Figure 8.55) is discussed in Section 8.12.
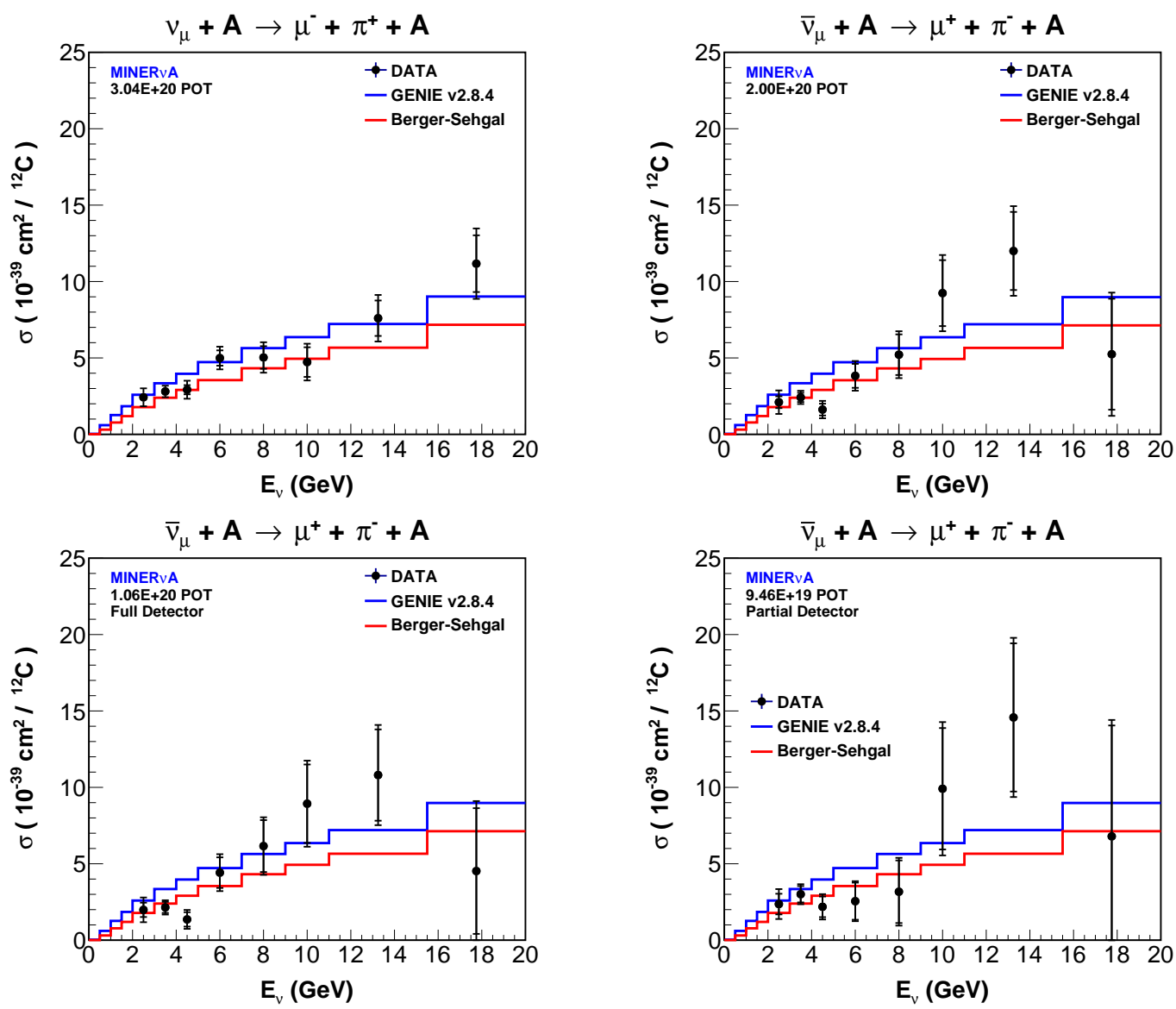

Figure 8.52: The measured $\sigma\left(E_{\nu}\right)$ for the $\nu_{\mu}$ (top left), combined $\bar{\nu}_{\mu}$ (top right), full detector $\bar{\nu}_{\mu}$ (bottom left), and partial detector $\bar{\nu}_{\mu}$ (bottom right) samples. The inner and outer error bars are the statistical and total (statistical + systematic) uncertainties, respectively.

\section{$8.12 \bar{\nu}_{\mu} Q^{2}$}

The $\frac{d \sigma}{d Q^{2}}$ measured from the $\bar{\nu}_{\mu}$ partial and full detector samples (Figure 8.55) differ in shape. While the full detector $\bar{\nu}_{\mu} \frac{d \sigma}{d Q^{2}}$ peaks near $0(\mathrm{GeV} / \mathrm{c})^{2}$, the partial detector 

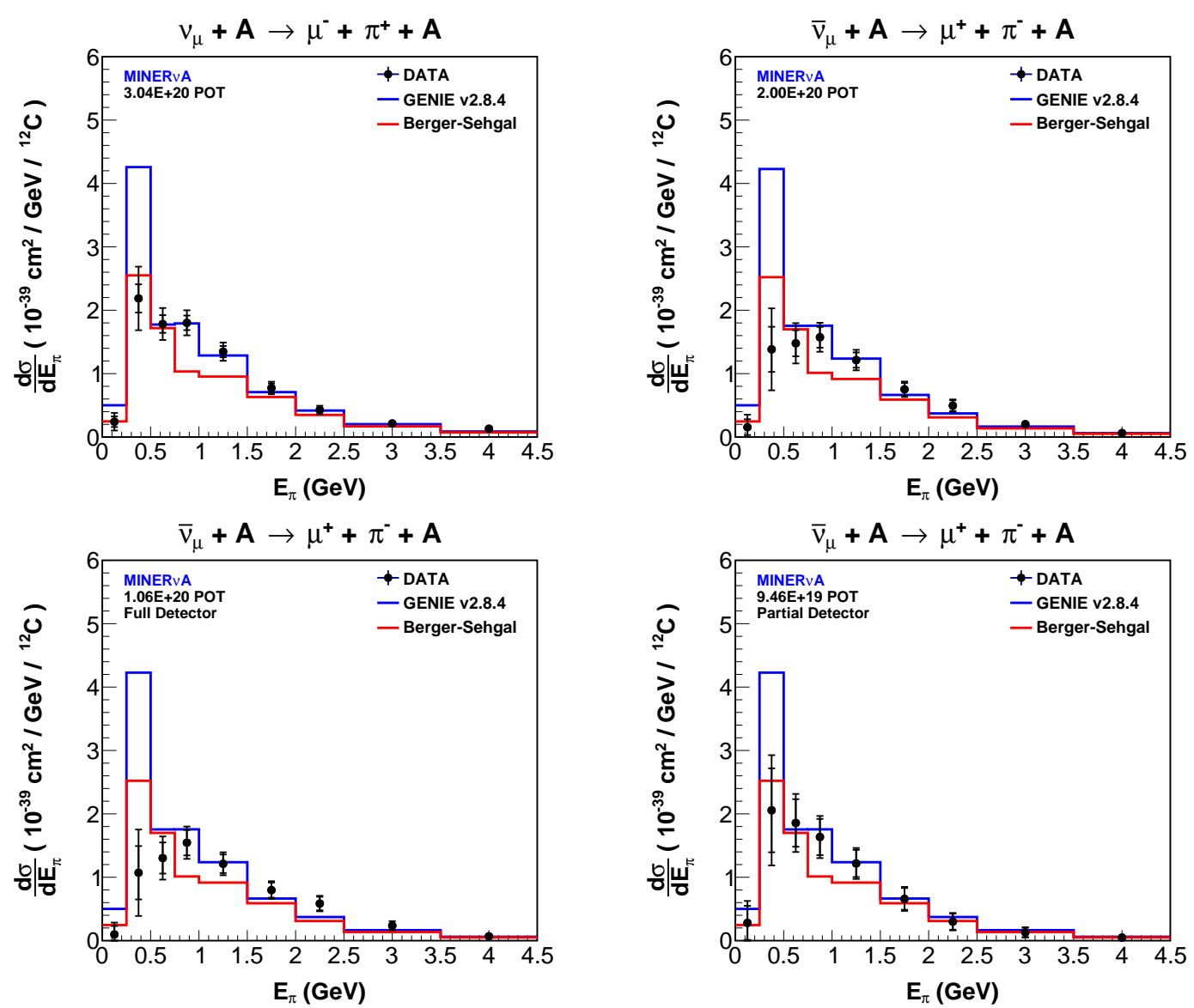

Figure 8.53: The measured $\frac{d \sigma}{d E_{\pi}}$ for the $\nu_{\mu}$ (top left), combined $\bar{\nu}_{\mu}$ (top right), full detector $\bar{\nu}_{\mu}$ (bottom left), and partial detector $\bar{\nu}_{\mu}$ (bottom right) samples. The inner and outer error bars are the statistical and total (statistical + systematic) uncertainties, respectively.

$\bar{\nu}_{\mu} \frac{d \sigma}{d Q^{2}}$ increases monotonically from $0(\mathrm{GeV} / \mathrm{c})^{2}$ before peaking near $0.1(\mathrm{GeV} / \mathrm{c})^{2}$. This shape difference is important since the PCAC coherent models predict the cross section is maximum at $Q^{2}=0$ when the final state muon mass is ignored.

The partial and full detector $\bar{\nu}_{\mu} \frac{d \sigma}{d Q^{2}}$ shape difference was studied to determine whether it is the result of statistical fluctuation or systematic bias in reconstructing $Q^{2}$ in either the partial or full detector $\bar{\nu}_{\mu}$ samples that is present in the data and not modeled in the MC. Table 8.17 lists for each measured cross section the statistical 

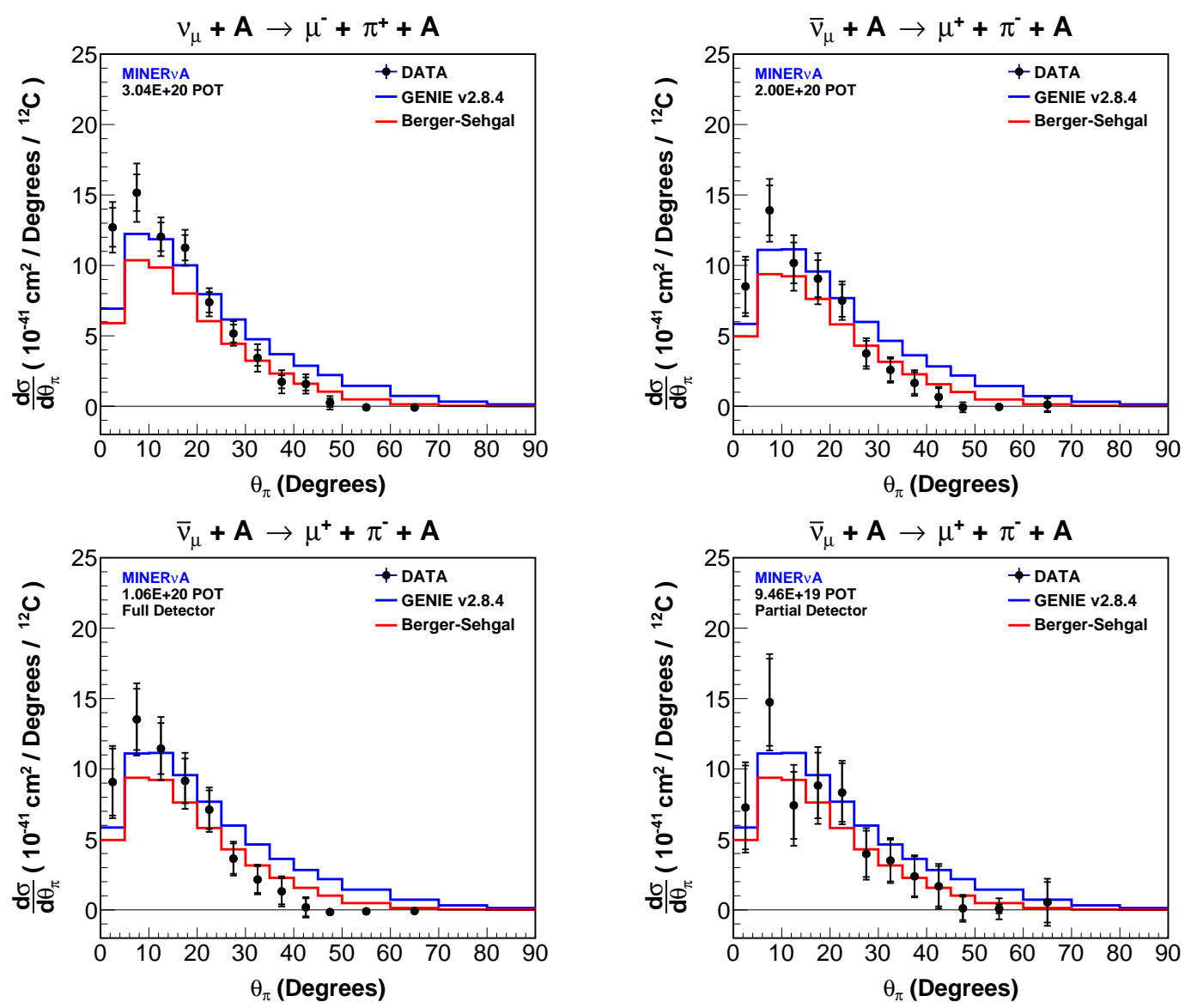

Figure 8.54: The measured $\frac{d \sigma}{d \theta_{\pi}}$ for the $\nu_{\mu}$ (top left), combined $\bar{\nu}_{\mu}$ (top right), full detector $\bar{\nu}_{\mu}$ (bottom left), and partial detector $\bar{\nu}_{\mu}$ (bottom right) samples. The inner and outer error bars are the statistical and total (statistical + systematic) uncertainties, respectively.

$\chi^{2}$, number of degrees of freedom (NDF), and $\chi^{2}$ probability for the partial and full detector $\bar{\nu}_{\mu}$ cross section difference. The statistical $\chi^{2}$ was calculated as

$$
\chi^{2}=A\left(C^{p}+C^{f}\right)^{-1} A^{T}
$$

where $C^{p}$ and $C^{f}$ are the bin-to-bin statistical covariance matrices for the partial and full detector $\bar{\nu}_{\mu}$ cross sections, respectively, and the elements of the vector $A$ 

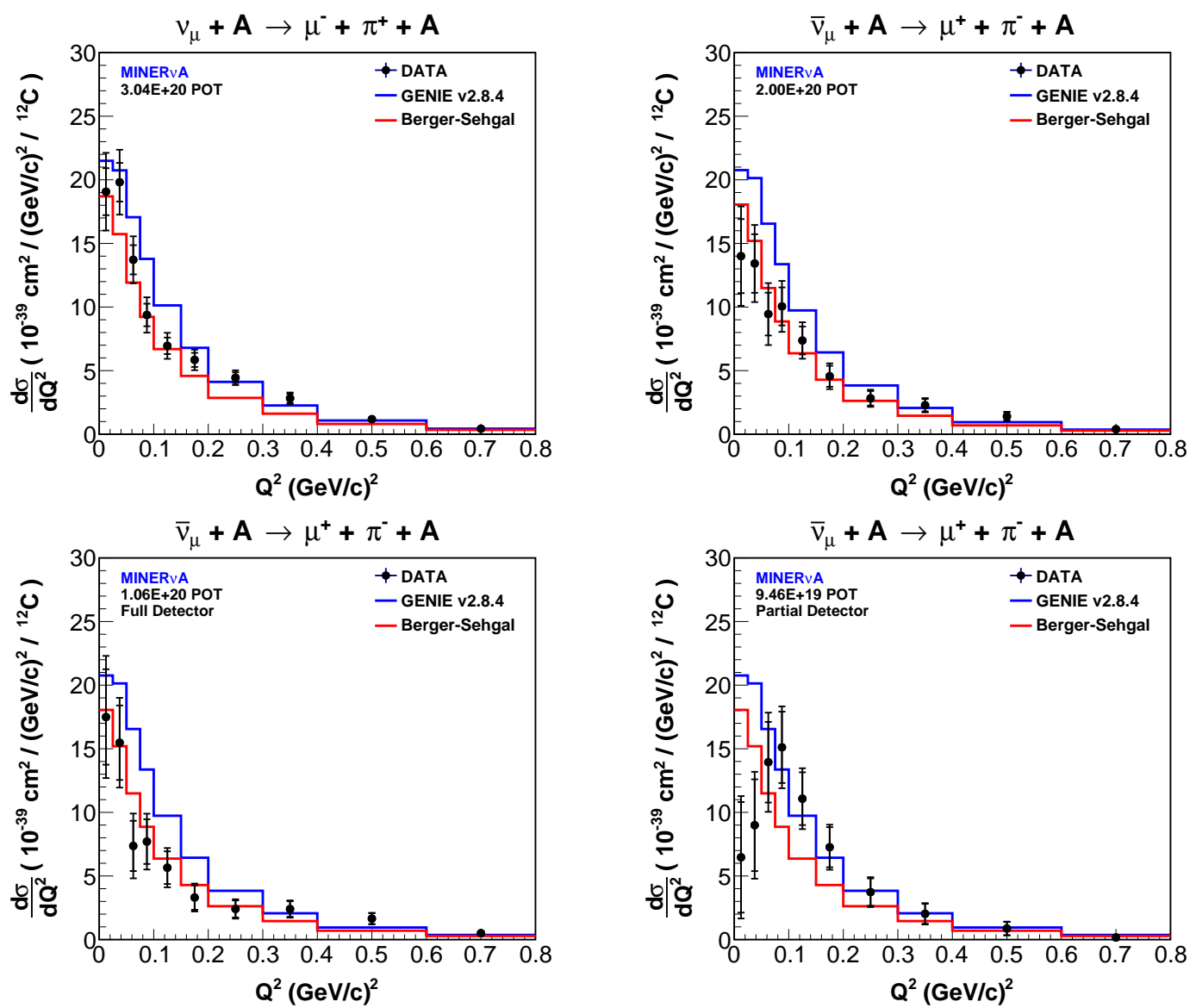

Figure 8.55: The measured $\frac{d \sigma}{d Q^{2}}$ for the $\nu_{\mu}$ (top left), combined $\bar{\nu}_{\mu}$ (top right), full detector $\bar{\nu}_{\mu}$ (bottom left), and partial detector $\bar{\nu}_{\mu}$ (bottom right) samples. The inner and outer error bars are the statistical and total (statistical + systematic) uncertainties, respectively. The difference in shape between the full and partial detector $\bar{\nu}_{\mu} \frac{d \sigma}{d Q^{2}}$ is discussed in Section 8.12. 

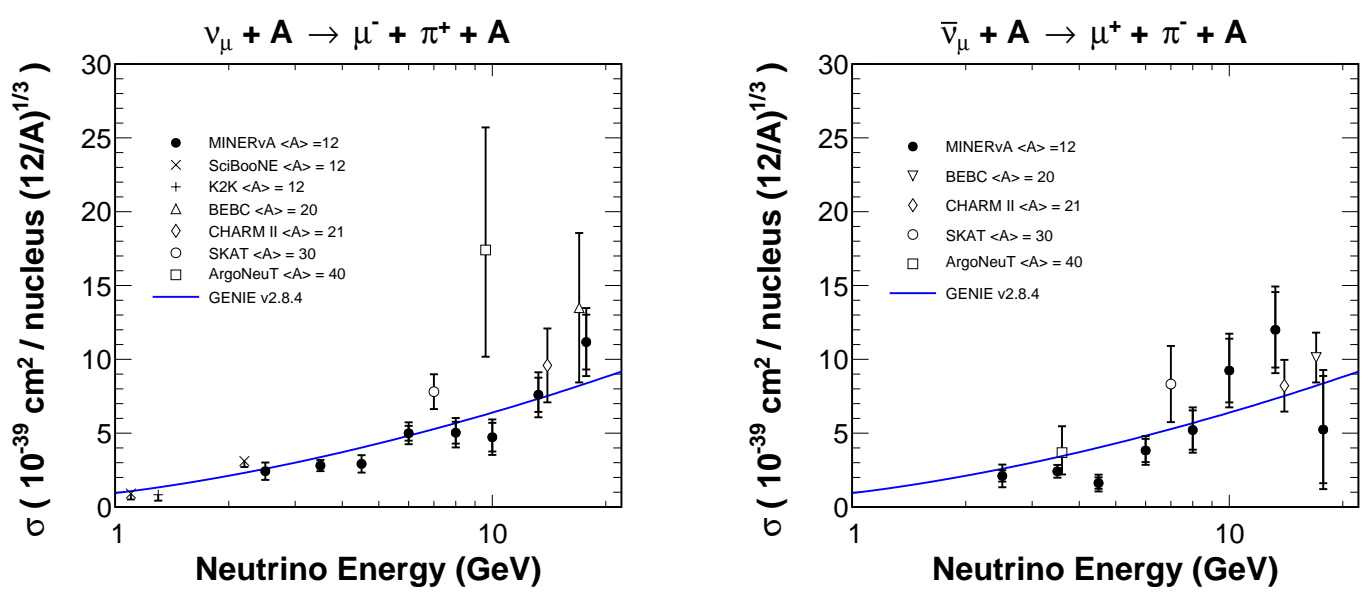

Figure 8.56: The measured $\sigma\left(E_{\nu}\right)$ for the $\nu_{\mu}$ (left) and combined $\bar{\nu}_{\mu}$ (right) samples compared with previous measurements $[60,65,67,70,71,72,73]$ at $E_{\nu}<20 \mathrm{GeV}$. The previous measurements were scaled to carbon using the $A^{1 / 3}$ dependence of $\sigma\left(E_{\nu}\right)$ predicted by the Rein-Sehgal model [34].

\begin{tabular}{c|cc|cc|c} 
& \multicolumn{2}{|c|}{$\nu_{\mu} \chi^{2}$} & \multicolumn{2}{|c|}{$\bar{\nu}_{\mu} \chi^{2}$} & \\
Cross Section & Rein-Sehgal & Berger-Sehgal & Rein-Sehgal & Berger-Sehgal & NDF \\
\hline$\sigma\left(E_{\nu}\right)$ & 10.6 & 9.6 & 25.6 & 14.7 & 8 \\
$d \sigma / d E_{\pi}$ & 46.2 & 58.7 & 35.8 & 40.4 & 9 \\
$d \sigma / d \theta_{\pi}$ & 1164.5 & 171.9 & 122.2 & 29.1 & 12 \\
$d \sigma / d Q^{2}$ & 19.3 & 13.5 & 16.2 & 11.4 & 10
\end{tabular}

Table 8.16: $\chi^{2}$ for the comparisons of the measured $\nu_{\mu}$ and $\bar{\nu}_{\mu}$ cross sections to the GENIE Rein-Sehgal and Berger-Sehgal predictions

are

$$
A_{i}=\sigma_{i}^{p}-\sigma_{i}^{f}
$$

where $\sigma_{i}^{p}$ and $\sigma_{i}^{f}$ are the partial and full detector $\bar{\nu}_{\mu}$ cross sections in bin $i$, respectively. The partial and full detector $\bar{\nu}_{\mu} \sigma\left(E_{\nu}\right), \frac{d \sigma}{d E_{\pi}}$, and $\frac{d \sigma}{d \theta_{\pi}}$ agree well within the statistical uncertainty. The largest difference is in $\frac{d \sigma}{d Q^{2}}$, where the difference between the partial and full detector measurements give a statistical $\chi^{2}$ of 18.0 for 10 degrees of freedom. However, the $\chi^{2}$ probability for the difference is 0.055 , which implies 
the difference is not statistically significant.

\begin{tabular}{c|ccc} 
Cross Section & $\chi^{2}$ & NDF & $\chi^{2}$ Probability \\
\hline$\sigma\left(E_{\nu}\right)$ & 5.4 & 8 & 0.714 \\
$d \sigma / d E_{\pi}$ & 7.6 & 9 & 0.574 \\
$d \sigma / d \theta_{\pi}$ & 6.9 & 12 & 0.864 \\
$d \sigma / d Q^{2}$ & 18.0 & 10 & 0.055
\end{tabular}

Table 8.17: The statistical $\chi^{2}$, number of degrees of freedom (NDF), and $\chi^{2}$ probability for the difference between the $\bar{\nu}_{\mu}$ cross sections measured from the partial and full detector samples

To determine whether the difference is due systematic bias in reconstructing $Q^{2}$, the reconstructed kinematic parameters from which the reconstructed $Q^{2}$ was calculated were compared between the partial detector $\bar{\nu}_{\mu}$ sample and the subset of the full detector $\bar{\nu}_{\mu}$ sample within the partial detector fiducial volume (for the remainder of this section, full detector $\bar{\nu}_{\mu}$ sample refers to this subset). Reconstructed $Q^{2}$ was calculated from the reconstructed $E_{\mu}, E_{\pi}$ (via $E_{\nu}=E_{\mu}+E_{\pi}$ ), and muon angle $\theta_{\mu}$ as

$$
Q^{2}=-\left(p_{\mu}-p_{\mu}\right)^{2}=2 E_{\nu}\left(E_{\mu}-\left|\vec{p}_{\mu}\right| \cos \theta_{\mu}\right),
$$

where $p_{\mu}$ and $p_{\mu}$ are the neutrino and muon four-momenta, respectively, and $\left|\vec{p}_{\mu}\right|$ is the magnitude of the muon three-momentum. The reconstructed $E_{\mu}, E_{\pi}$, and $\theta_{\mu}$ distributions for the partial and full detector $\bar{\nu}_{\mu}$ coherent candidate event samples are shown in Figures 8.57-8.60. The partial-to-full detector sample ratio for each reconstructed parameter in both the data and MC are shown in Figure 8.61, where the full detector sample in the ratio is scaled to the POT of the partial detector sample. The data $Q^{2}$ ratio exhibits a higher (or lower) event rate in the partial (full) detector sample for $0.075<Q^{2}<0.2(\mathrm{GeV} / \mathrm{c})^{2}$ beyond the $1 \sigma$ statistical uncertainty which is not present in the MC ratio. A difference in the reconstructed 
$E_{\mu}$ between the partial and full detector samples is expected due to energy loss and scattering of muons in ArgoNeuT. However, these effects are accounted for in the simulation of ArgoNeuT in the partial detector sample MC, and are therefore corrected in measuring $\frac{d \sigma}{d Q^{2}}$ from the partial detector sample. This is supported by the data and $\mathrm{MC} E_{\mu}$ ratios, which are similar in shape and agree within the statistical uncertainty. Differences between the partial and full detector samples in reconstructing $E_{\pi}$ and $\theta_{\mu}$ are not expected since these parameters are reconstructed using the MINER $\nu \mathrm{A}$ detector only. The $E_{\pi}$ and $\theta_{\mu}$ ratios in data and $\mathrm{MC}$ are mostly consistent (within the statistical uncertainty) with no difference between the full and partial detector samples in reconstructing $E_{\pi}$ and $\theta_{\mu}$. The shape of the data $E_{\pi}$ ratio suggests a low(or high)-bias in the reconstructed $E_{\pi}$ in the partial (full) detector sample, which would result in a low(high)-bias in the reconstructed $Q^{2}$. However, these biases in the reconstructed $Q^{2}$ are inconsistent with the shapes of the $\frac{d \sigma}{d Q^{2}}$ measured from the full and partial detector samples. Therefore, no evidence of systematic bias in reconstructing the kinematic parameters used to calculate the reconstructed $Q^{2}$ was found in the partial and full detector $\bar{\nu}_{\mu}$ samples.

A potential source of bias in reconstructing $\theta_{\mu}$, and thereby $Q^{2}$, is misalignment of the nominal neutrino beam direction (i.e. the beam direction in the geometry model). A measurement of the beam direction in data and MC was made from the reconstructed $\theta_{\mu}$ of inclusive $\nu_{\mu}$ and $\bar{\nu}_{\mu}$ charged current low- $\nu(\nu<0.5 \mathrm{GeV})$ events in the tracker [126][127], where $\nu$ is the energy of the hadronic recoil $(\nu=$ $E_{\nu}-E_{\mu}$ ). Low- $\nu$ events tend to have small- $\theta_{\mu}$ (due to low- $\nu$ requiring low- $Q^{2}$ ) and are therefore insensitive to the muon angle acceptance of the reconstruction. In the horizontal (XZ) and vertical (YZ) planes, the beam angle was measured by the peak of the reconstructed 2-D muon angle distribution. Table 8.18 lists the 

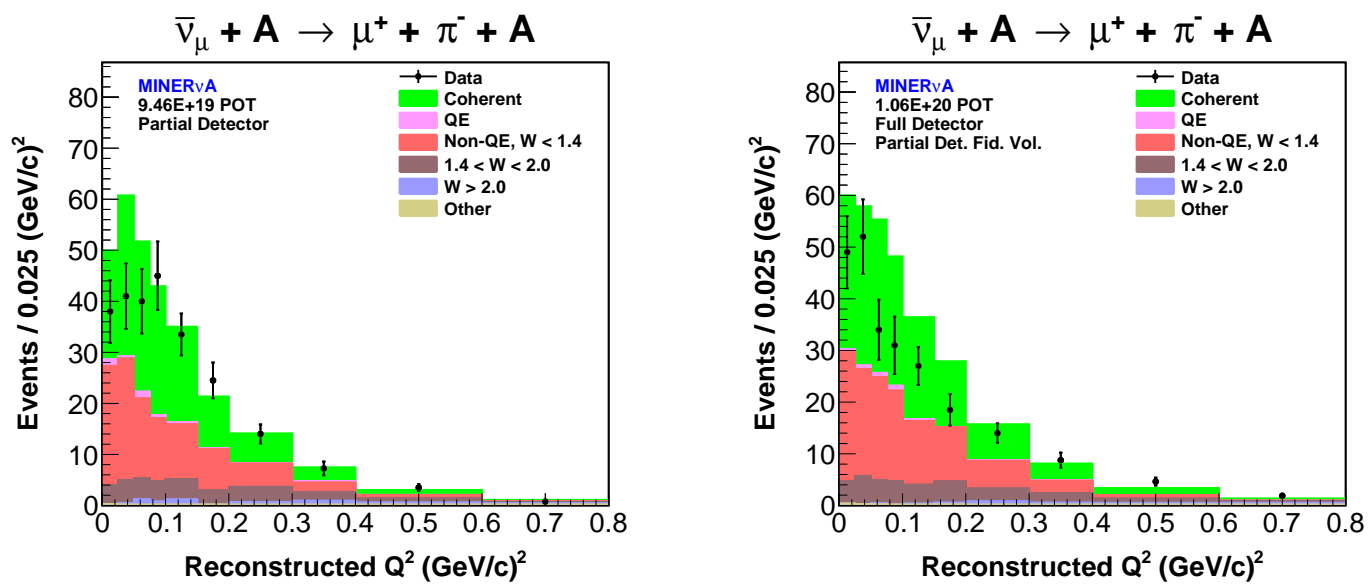

Figure 8.57: The reconstructed $Q^{2}$ distribution for the partial detector $\bar{\nu}_{\mu}$ coherent candidate sample (left) and the subset of the full detector $\bar{\nu}_{\mu}$ coherent candidate sample within the partial detector fiducial volume (right)
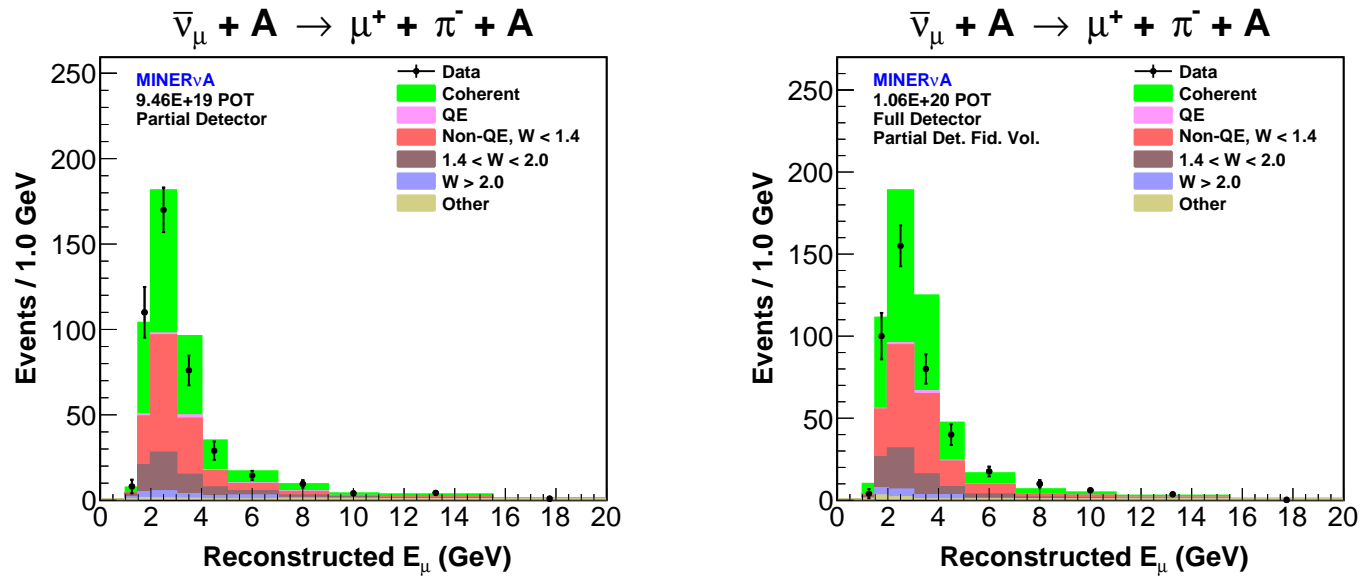

Figure 8.58: The reconstructed $E_{\mu}$ distribution for the partial detector $\bar{\nu}_{\mu}$ coherent candidate sample (left) and the subset of the full detector $\bar{\nu}_{\mu}$ coherent candidate sample within the partial detector fiducial volume (right) 

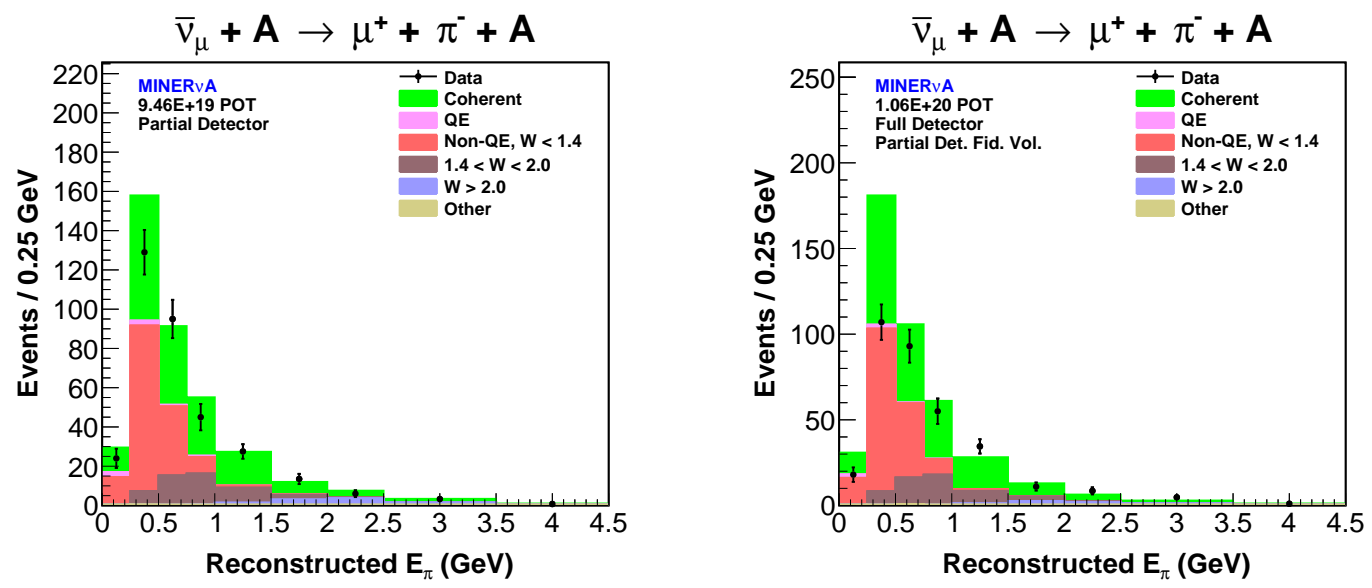

Figure 8.59: The reconstructed $E_{\pi}$ distribution for the partial detector $\bar{\nu}_{\mu}$ coherent candidate sample (left) and the subset of the full detector $\bar{\nu}_{\mu}$ coherent candidate sample within the partial detector fiducial volume (right)
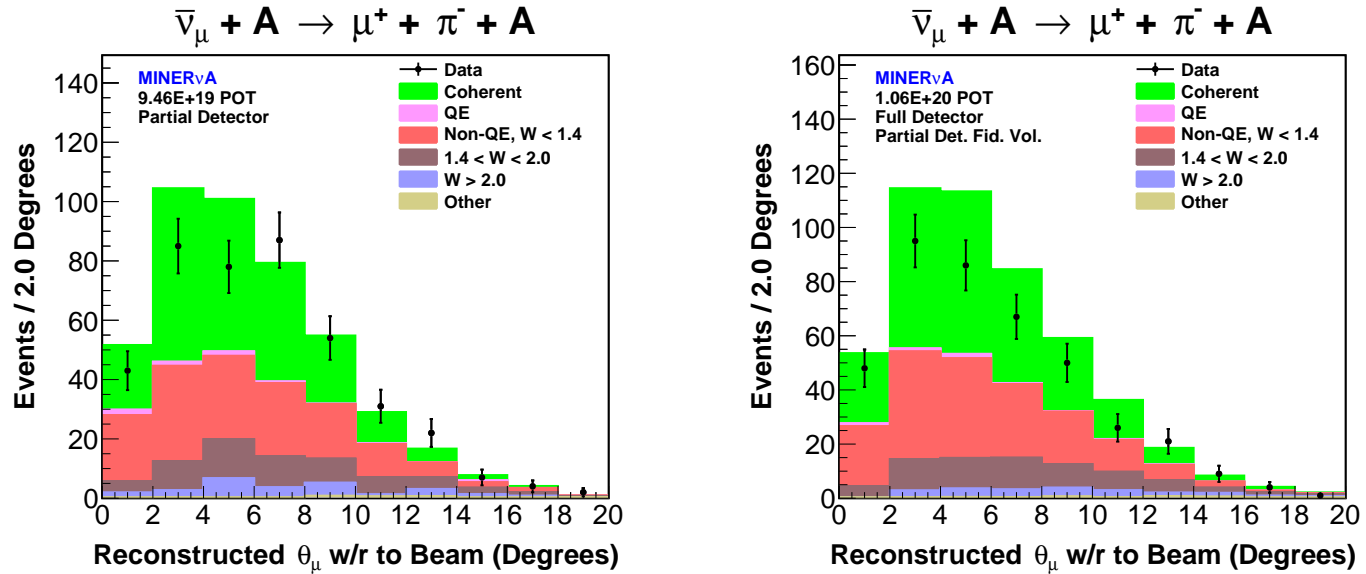

Figure 8.60: The reconstructed $\theta_{\mu}$ distribution for the partial detector $\bar{\nu}_{\mu}$ coherent candidate sample (left) and the subset of the full detector $\bar{\nu}_{\mu}$ coherent candidate sample within the partial detector fiducial volume (right) 

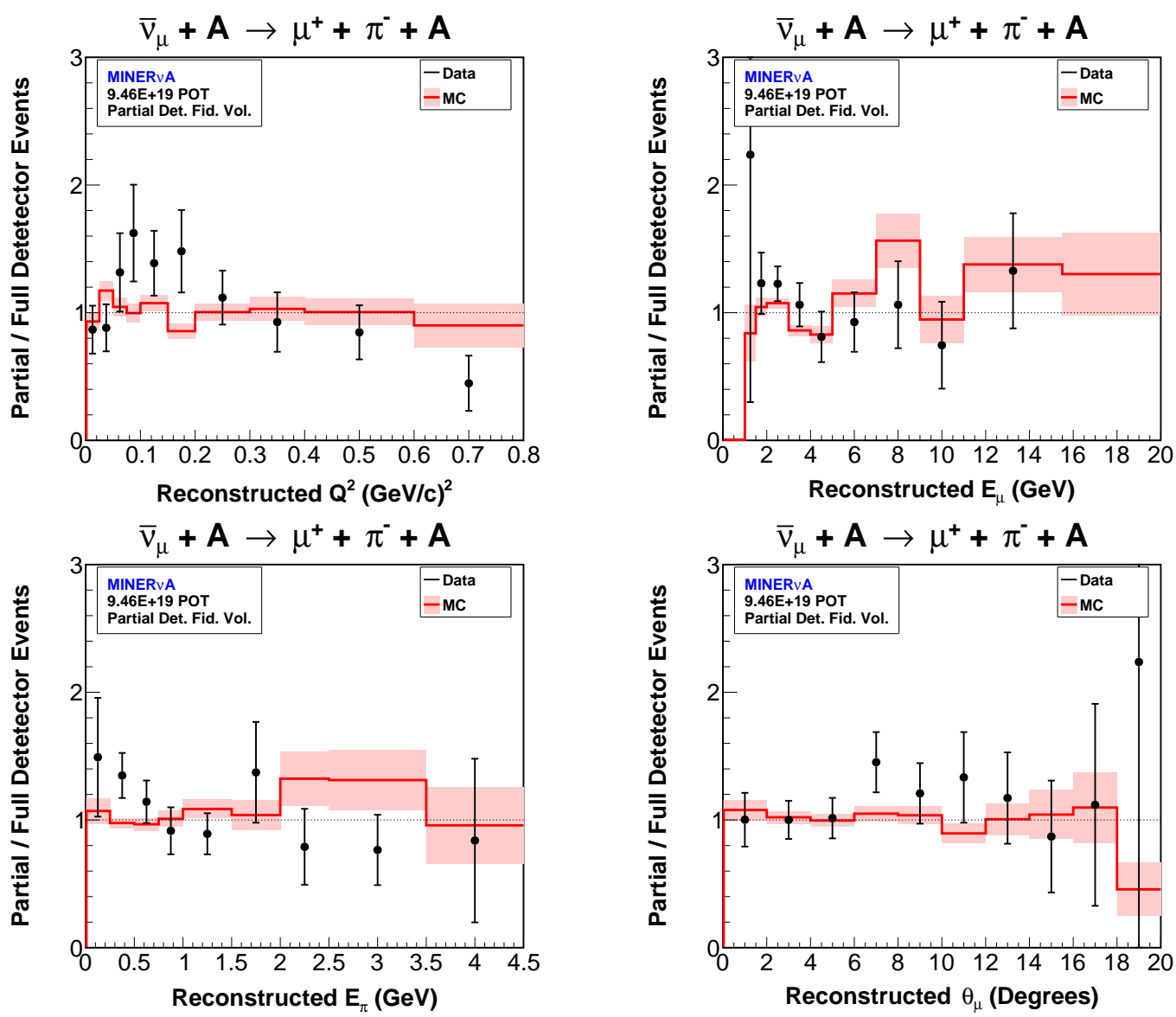

Figure 8.61: The ratio of the partial detector event rate to the full detector event rate for selected $\bar{\nu}_{\mu}$ coherent candidates (signal + background) in the data and MC as a function of the reconstructed $Q^{2}$ (top left), $E_{\mu}$ (top right), $E_{\pi}$ (bottom left) and $\theta_{\mu}$ (bottom right). The full detector event rate in the ratio is for the subset of the full detector candidates within the partial detector fiducial volume, and is scaled to the POT of the partial detector sample. The error bars on the data and the error band on the MC represent the statistical unceratinty. 
deviation of the measured and nominal beam angles in the $\mathrm{XZ}$ and $\mathrm{YZ}$ planes in partial and full detector data and MC. The measured and nominal beam angles agree within $\sim 3$ mrad, which is small compared to the 3.4 degree (59 mrad) angle between the nominal beam direction and the detector axis. This ruled out $Q^{2}$ bias from misalignment of the nominal beam direction. The measured and nominal beam angle deviations are included in the systematic uncertainty on the cross sections (Section 8.13.5).

\begin{tabular}{c|cc|cc} 
& \multicolumn{2}{|c|}{$\Delta \theta_{x z}(\mathrm{mrad})$} & \multicolumn{2}{c}{$\Delta \theta_{y z}(\mathrm{mrad})$} \\
Playlist & Data & $\mathrm{MC}$ & Data & $\mathrm{MC}$ \\
\hline Downstream 1 & $0.6 \pm 1.6$ & $-1.5 \pm 1.0$ & $3.5 \pm 1.5$ & $0.0 \pm 1.0$ \\
Downstream 2 & $-1.4 \pm 0.7$ & $-0.2 \pm 1.0$ & $1.1 \pm 0.7$ & $-0.5 \pm 1.0$ \\
MINER A 1 & $-0.1 \pm 1.0$ & $-1.4 \pm 0.4$ & $3.2 \pm 0.8$ & $0.4 \pm 0.3$
\end{tabular}

Table 8.18: The measured and nominal neutrino beam angle deviations in the $\mathrm{XZ}$ and YZ planes for the partial detector (Downstream 1 \& 2) and full detector (MINER $\nu \mathrm{A} 1)$ data and MC.

In summary, no evidence for systematic bias in reconstructing $Q^{2}$ in the partial and full detector $\bar{\nu}_{\mu}$ samples was found. The shape differences in the $\frac{d \sigma}{d Q^{2}}$ measured from the partial and full detector $\bar{\nu}_{\mu}$ samples were therefore attributed to statistical fluctuations.

\subsection{Systematic Uncertainties}

The cross section measurements relied on the MC to estimate the background, resolution of the kinematic parameters, signal selection efficiency, and the flux. Uncertainties on the predictions of the MC therefore result in uncertainties on the measured cross sections. These uncertainties were evaluated by varying the MC predictions and measuring the resulting change to the cross sections. Each system- 
atic uncertainty was calculated from a covariance matrix $C$ whose elements were were calculated as

$$
C_{i j}=\frac{1}{N} \sum_{k} \Delta \sigma_{i k} \Delta \sigma_{j k}
$$

where $\Delta \sigma_{i k}$ is the change to the cross section in bin $i$ for variation $k$, and $N$ is the number of variations from which the uncertainty was evaluated. The MC inputs to the measured cross sections were recalculated for each variation to the MC. These inputs were the tuned background prediction, the unfolding matrices, the signal selection efficiency, and the flux prediction.

The systematic uncertainties on the measured cross sections are shown in Figures 8.62-8.65. The interaction model and detector model uncertainties in these figures are decomposed into their constituent uncertainties in Sections 8.13.2 and 8.13.5, respectively. The fractional systematic uncertainties tend to be larger for the $\bar{\nu}_{\mu}$ cross sections than the $\nu_{\mu}$ cross sections. This is due to the larger background fraction in the selected $\bar{\nu}_{\mu}$ coherent candidate sample coupled with the systematic uncertainties on the background prediction.

The evaluation of the systematic uncertainties on the measured cross sections is detailed in the following sections.

\subsubsection{Flux}

The uncertainty on the flux prediction consists of uncertainties on the parameters in the flux MC that govern hadronic interactions in the NuMI target and downstream beamline materials, the magnetic focusing of hadrons emerging from the target, and the geometry model of the beamline components. The flux uncertainty was evaluated from a set of 100 alternate flux predictions. Each alternate flux prediction, referred 

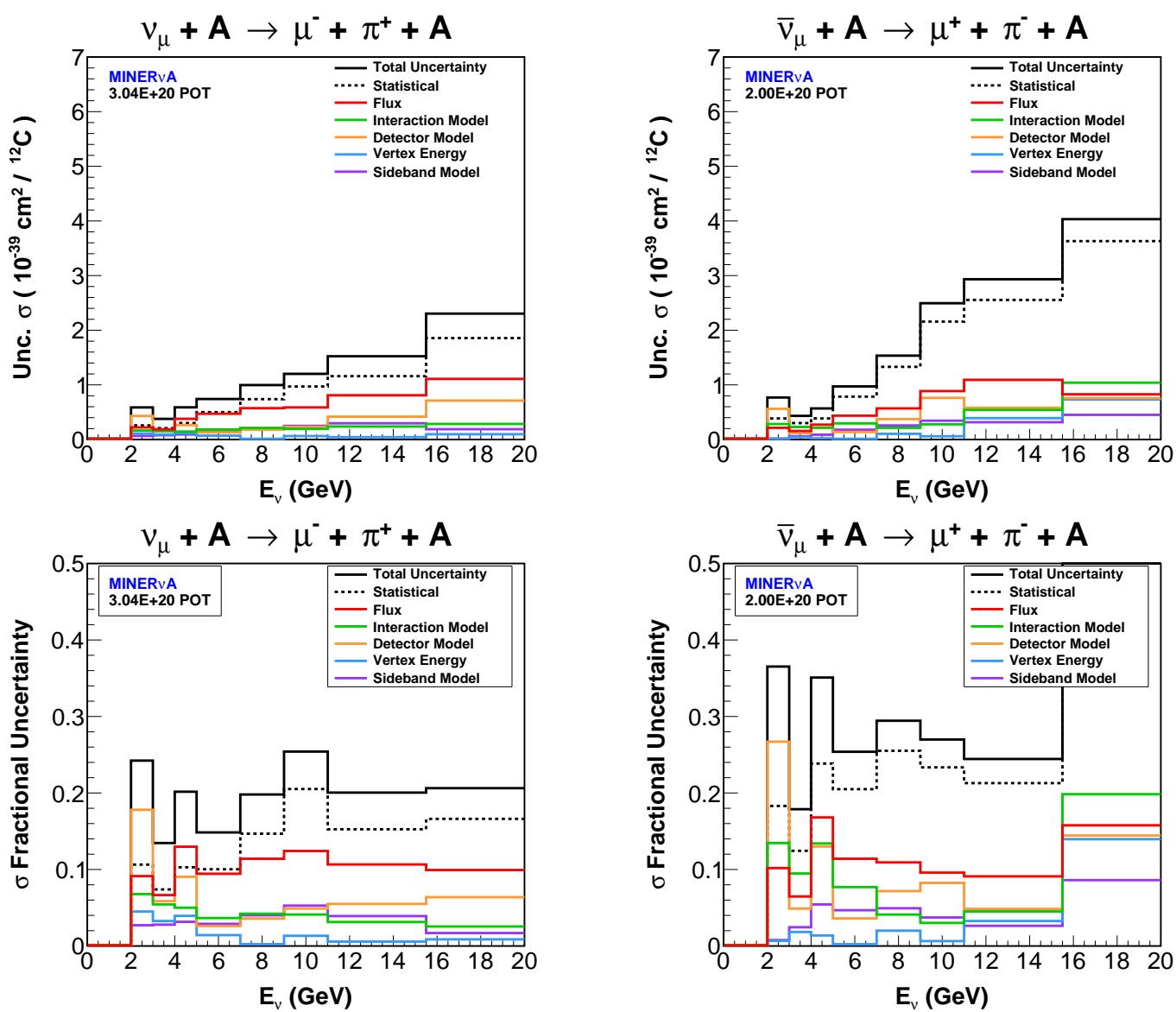

Figure 8.62: The statistical and systematic uncertainties on the measured $\nu_{\mu}$ (left) and $\bar{\nu}_{\mu}$ (right) $\sigma\left(E_{\nu}\right)$. The top (bottom) plots show the absolute (fractional) uncertainty.

to herein as a flux variation, was the result of simultaneously varying the parameters in the flux MC, where each parameter was varied by a random amount determined from a gaussian distribution centered on the nominal parameter value with a $1 \sigma$ width equal to the parameter uncertainty. To evaluate the flux uncertainty on the cross sections, the cross sections were remeasured for each flux variation and a covariance matrix for the set of flux variations was calculated for each cross section.

The fractional flux uncertainty is $6-16 \%$ for $\sigma\left(E_{\nu}\right)$ and $5-8 \%$ for the differential cross sections. The larger flux uncertainty on $\sigma\left(E_{\nu}\right)$ is due to shape distortions of 

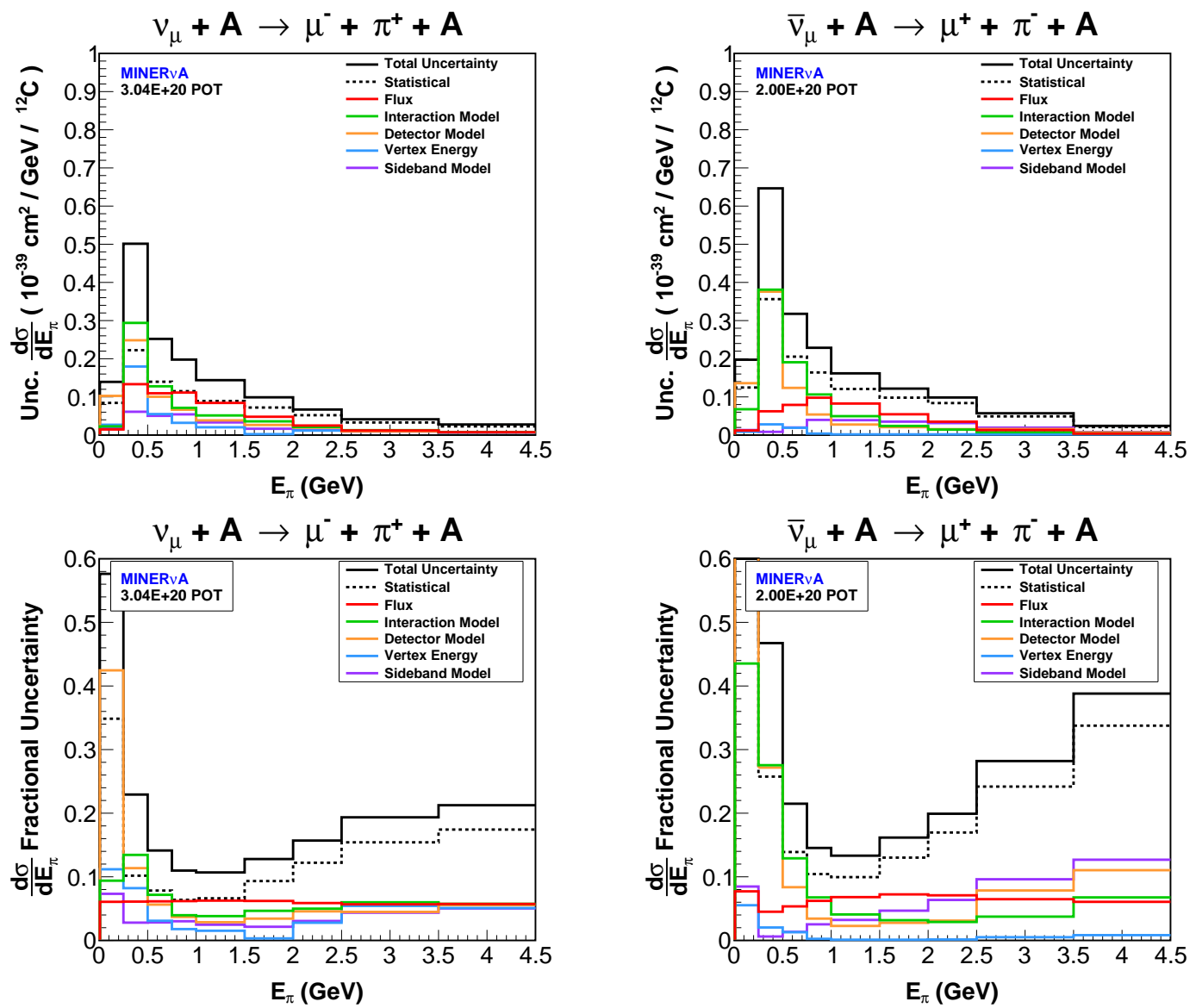

Figure 8.63: The statistical and systematic uncertainties on the measured $\nu_{\mu}$ (left) and $\bar{\nu}_{\mu}$ (right) $\frac{d \sigma}{d E_{\pi}}$. The top (bottom) plots show the absolute (fractional) uncertainty. In the $0-0.25 \mathrm{GeV}$ bin, the fractional uncertainty is large due to the small measured cross section.

the $E_{\nu}$ spectrum from the flux variations. This is illustrated in Figures 8.66-8.69, which show the effect of a single flux variation on the measurement of $\sigma\left(E_{\nu}\right)$ and the differential cross sections. For each kinematic parameter, the flux variation changes both the shape and normalization of the background prediction. The background tuning corrects the change to the normalization, but the shape distortion of the background $E_{\nu}$ spectrum largely remains. This is due to a combination of tuning the background in $E_{\pi}$ and $Q^{2}$ and the flux variation having the largest effect on the 

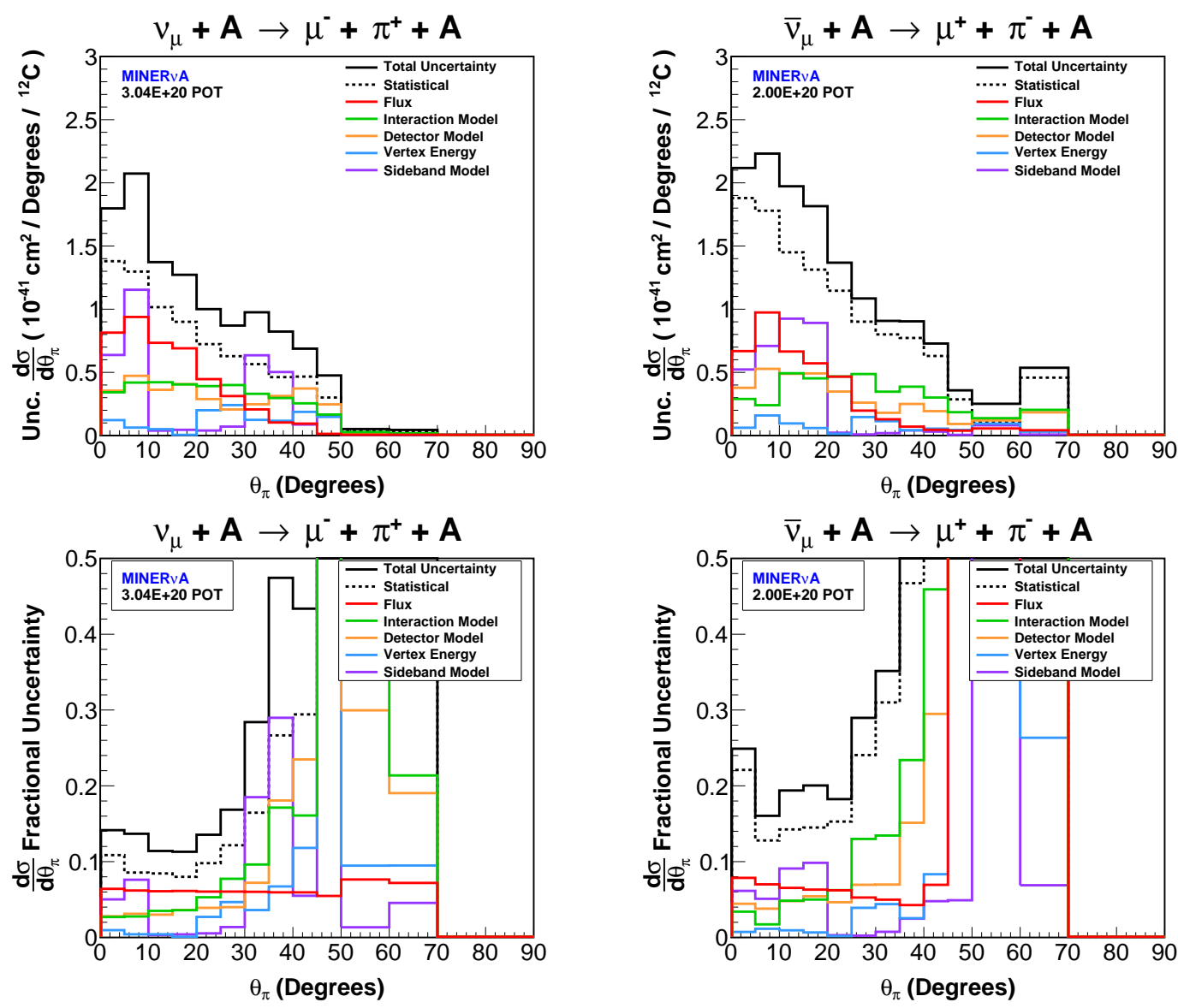

Figure 8.64: The statistical and systematic uncertainties on the measured $\nu_{\mu}$ (left) and $\bar{\nu}_{\mu}$ (right) $\frac{d \sigma}{d \theta_{\pi}}$. The top (bottom) plots show the absolute (fractional) uncertainty. At high- $\theta_{\pi}$, the fractional uncertainty is large due to the small measured cross section.

background shape in $E_{\nu}$. After background subtraction, the shape distortions of the coherent candidate sample and flux normalization $E_{\nu}$ spectra are anti-correlated, resulting in the larger $\sigma\left(E_{\nu}\right)$ shape distortion. The flux variation primarily affects the differential cross sections in normalization. This is due to the differential cross sections being normalized to the integrated flux and the flux variation having a smaller effect on the background shape. In summary, the flux variations affect both $\sigma\left(E_{\nu}\right)$ and the differential cross sections in normalization, but have a larger effect 

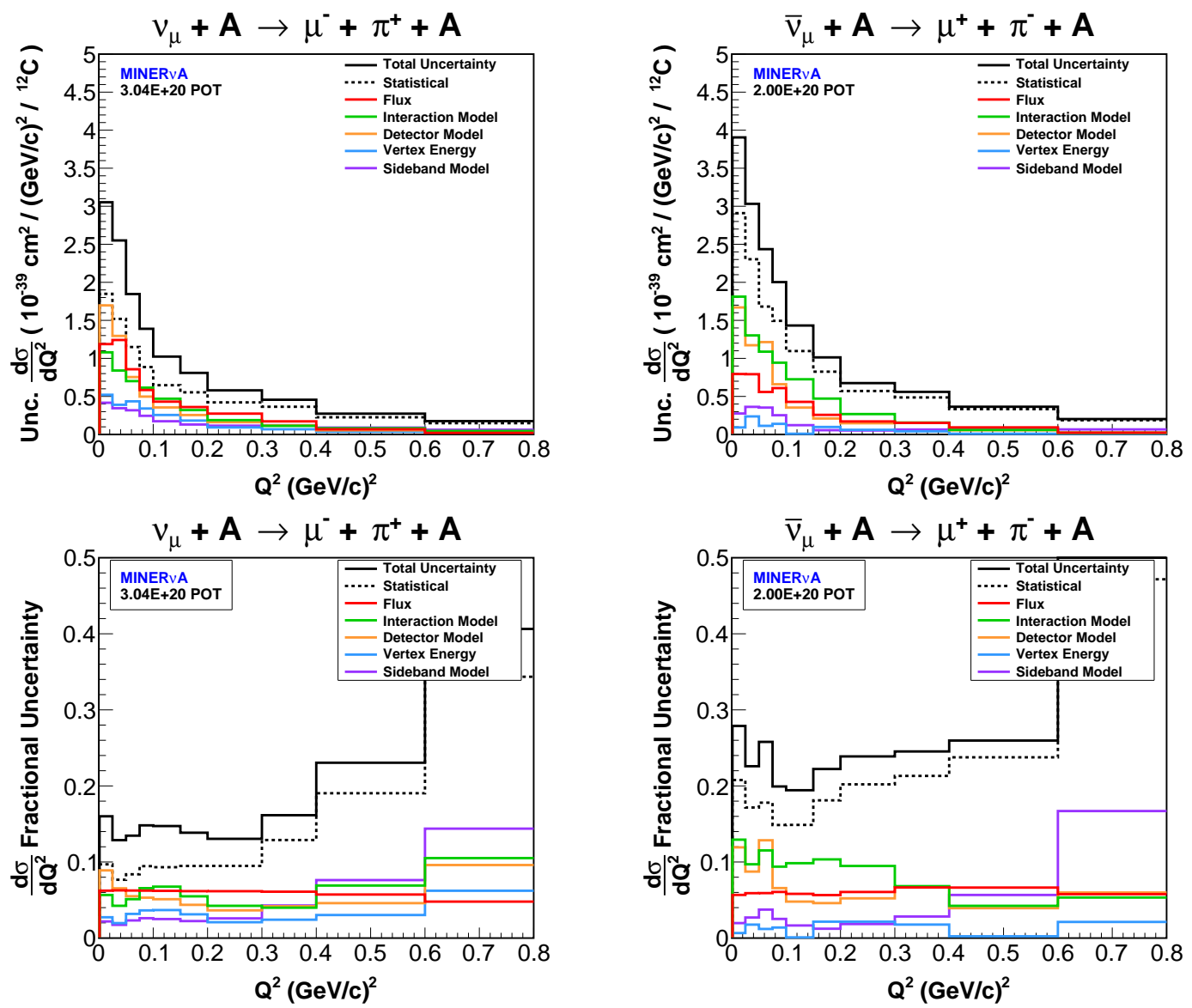

Figure 8.65: The statistical and systematic uncertainties on the measured $\nu_{\mu}$ (left) and $\bar{\nu}_{\mu}$ (right) $\frac{d \sigma}{d Q^{2}}$. The top (bottom) plots show the absolute (fractional) uncertainty.

on the $\sigma\left(E_{\nu}\right)$ shape, which results in the larger $\sigma\left(E_{\nu}\right)$ flux uncertainty.

The $\sigma\left(E_{\nu}\right)$ shape distortions from the flux variations also result in bin-to-bin anti-correlations in the $\sigma\left(E_{\nu}\right)$ flux uncertainty (Figure 8.109 in Section 8.14). This is illustrated in Figure 8.66, where the changes to $\sigma\left(E_{\nu}\right)$ at low- $E_{\nu}$ and high- $E_{\nu}$ from the single flux variation are anti-correlated. Bin-to-bin anti-correlations are not present in the flux uncertainty on the differential cross sections (Figures 8.111, 8.113, and 8.115 in Section 8.14). This is due to the flux variations primarily affecting the differential cross sections in normalization, which can be seen by the effect of the 
single flux variation on the differential cross sections in Figures 8.68-8.69.
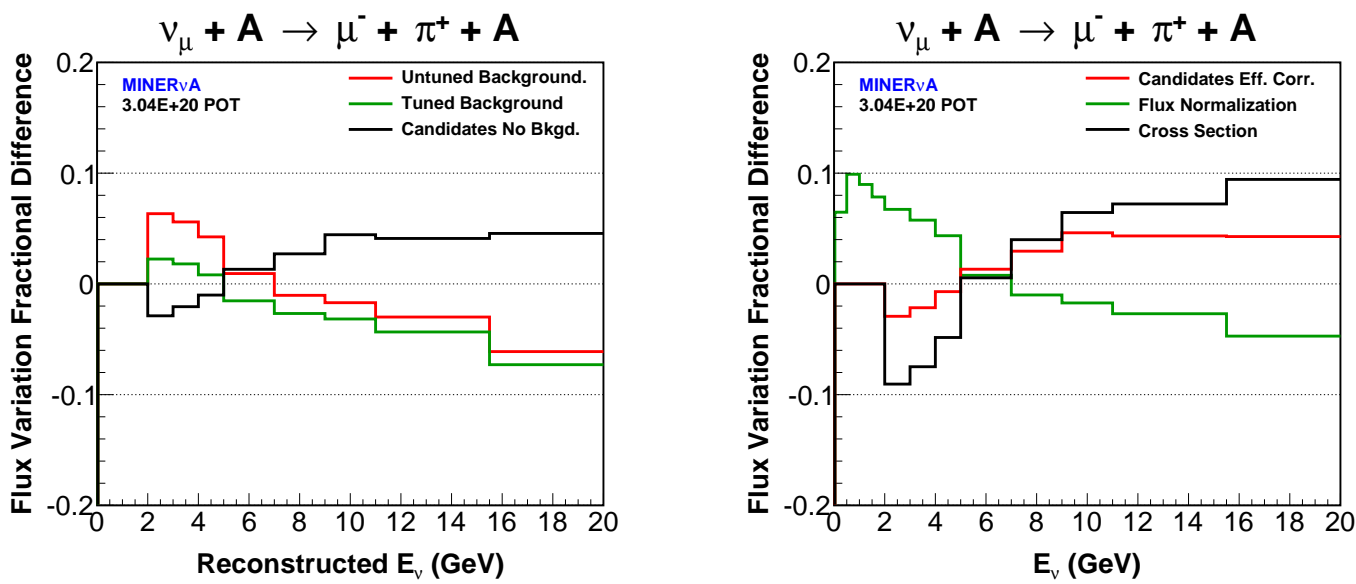

Figure 8.66: Left plot: the fractional difference on the $\nu_{\mu}$ reconstructed $E_{\nu}$ distribution due to a single flux variation for the untuned (red) and tuned (green) background predictions, and coherent candidate sample after background subtraction (black). Right plot: the fractional difference on the $\nu_{\mu}$ true $E_{\nu}$ distribution due to a single flux variation for the coherent candidate sample after the efficiency correction (red), flux normalization (green), and measured $\sigma\left(E_{\nu}\right)$ (black).
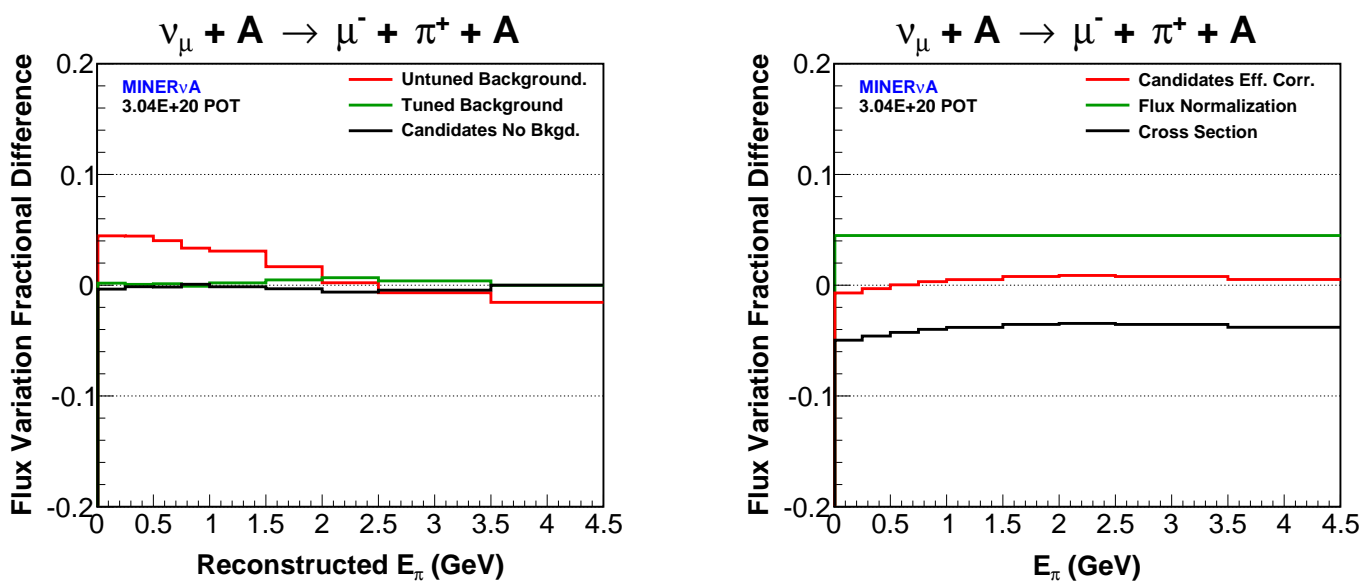

Figure 8.67: Left plot: the fractional difference on the $\nu_{\mu}$ reconstructed $E_{\pi}$ distribution due to a single flux variation for the untuned (red) and tuned (green) background predictions, and coherent candidate sample after background subtraction (black). Right plot: the fractional difference on the $\nu_{\mu}$ true $E_{\pi}$ distribution due to a single flux variation for the coherent candidate sample after the efficiency correction (red), flux normalization (green), and measured $\frac{d \sigma}{d E_{\pi}}$ (black). 

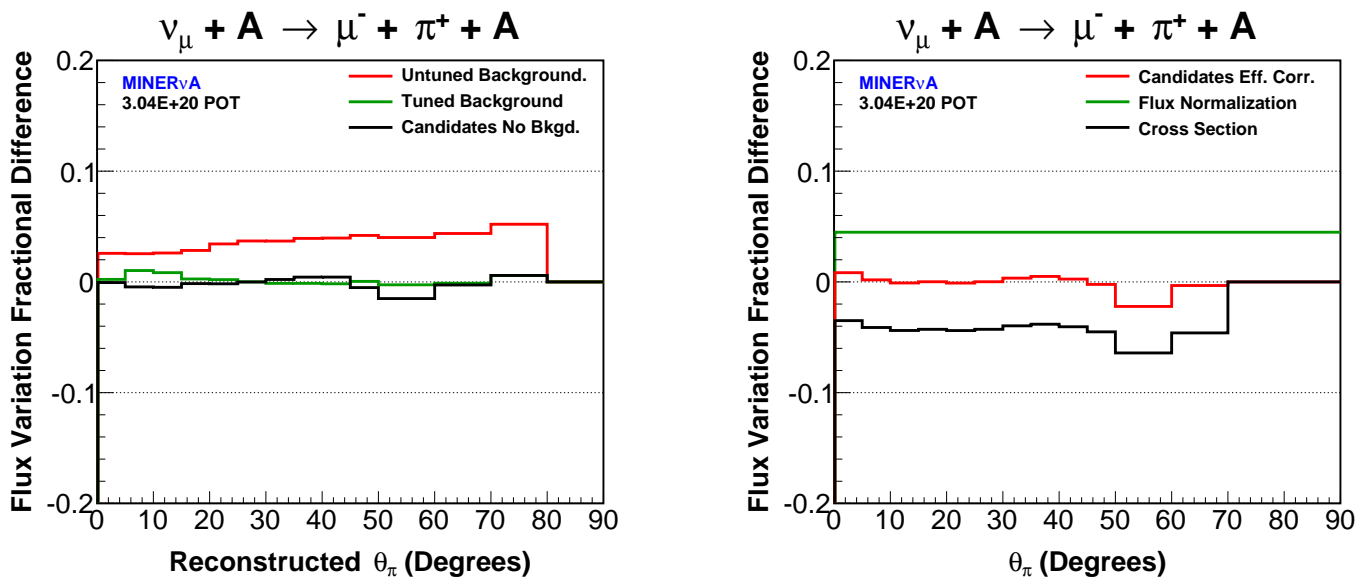

Figure 8.68: Left plot: the fractional difference on the $\nu_{\mu}$ reconstructed $\theta_{\pi}$ distribution due to a single flux variation for the untuned (red) and tuned (green) background predictions, and coherent candidate sample after background subtraction (black). Right plot: the fractional difference on the $\nu_{\mu}$ true $\theta_{\pi}$ distribution due to a single flux variation for the coherent candidate sample after the efficiency correction (red), flux normalization (green), and measured $\frac{d \sigma}{d \theta_{\pi}}$ (black).
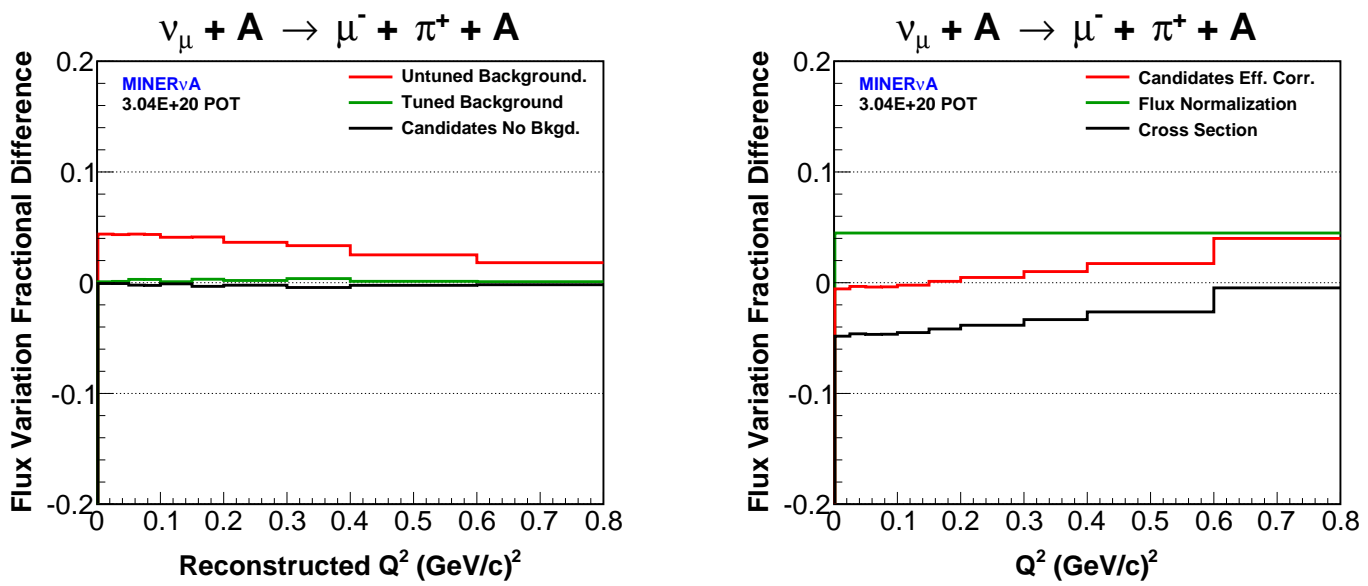

Figure 8.69: Left plot: the fractional difference on the $\nu_{\mu}$ reconstructed $Q^{2}$ distribution due to a single flux variation for the untuned (red) and tuned (green) background predictions, and coherent candidate sample after background subtraction (black). Right plot: the fractional difference on the $\nu_{\mu}$ true $Q^{2}$ distribution due to a single flux variation for the coherent candidate sample after the efficiency correction (red), flux normalization (green), and measured $\frac{d \sigma}{d Q^{2}}$ (black). 


\subsubsection{Neutrino Interaction Model}

The cross section measurements relied on the MC to predict the rate of the incoherent backgrounds. The measured cross sections are thereby subject to uncertainties on the underlying neutrino-nucleus interaction models in GENIE. For evaluating the effects of these uncertainties, GENIE provides event-by-event weights that modify the predictions of the cross section models and the model of final state interactions (FSI) - the interactions of final state particles within the target nucleus. These weights modify the normalization and/or shape (i.e. kinematic dependence) of the model predictions. The weights used to evaluate the neutrino-nucleus interaction model uncertainties on the measured cross sections correspond to $\pm 1 \sigma$ uncertainties on the GENIE model parameters. Table 8.19 lists the default $\pm 1 \sigma$ uncertainties on these parameters for the MINER $\nu \mathrm{A}$ implementation of GENIE 2.6.2 used in this analysis [119].

The GENIE prediction of single pion final states was fit to $\nu_{\mu}$ deuterium scattering data (Section 8.5). This fit resulted in refined values and reduced uncertainties for the axial vector mass for resonant pion production $M_{A}^{R E S}$, and the resonant pion production and non-resonant single pion production normalizations in GENIE. Furthermore, the uncertainty on the vector mass for resonant pion production $M_{V}^{R E S}$ was reduced from the GENIE default $\pm 10 \%$ to $\pm 3 \%$. This reduction was supported by comparisons of predicted and measured helicity amplitudes for resonance production in electron-nucleus scattering [120]. The reduced CC resonance production and CC non-resonant single pion production uncertainties are listed in Table 8.20.

The isotropic $\Delta^{++} \rightarrow N \pi$ decay in the MC was weighted to half the non-isotropy predicted by the Rein-Sehgal resonance production model (Section 8.5). The uncertainty on the decay isotropy applied to the measured cross sections was half the dif- 


\begin{tabular}{|c|c|}
\hline Parameter & Variation \\
\hline \multicolumn{2}{|l|}{ CC Quasielastic } \\
\hline Normalization & $+20 \%,-15 \%$ \\
\hline Axial vector mass (shape only) & $\pm 10 \%$ \\
\hline Vector form factor model (shape only) & BBBA to dipole parameterization \\
\hline Pauli suppression & $\pm 30 \%$ \\
\hline \multicolumn{2}{|l|}{ NC Elastic } \\
\hline Axial vector mass & $\pm 25 \%$ \\
\hline Strange axial form factor $\eta$ & $\pm 30 \%$ \\
\hline \multicolumn{2}{|l|}{ CC Resonance Production } \\
\hline Normalization & $\pm 20 \%$ \\
\hline Axial vector mass & $\pm 20 \%$ \\
\hline Vector mass & $\pm 10 \%$ \\
\hline \multicolumn{2}{|c|}{$C C \&$ NC Non-Resonant Pion Production } \\
\hline Normalization of $1 \pi$ final states from $\nu p / \bar{\nu} p$ & $\pm 50 \%$ \\
\hline Normalization of $1 \pi$ final states from $\nu n / \bar{\nu} n$ & $\pm 50 \%$ \\
\hline Normalization of $2 \pi$ final states from $\nu p / \bar{\nu} p$ & $\pm 50 \%$ \\
\hline Normalization of $2 \pi$ final states from $\nu n / \bar{\nu} n$ & $\pm 50 \%$ \\
\hline \multicolumn{2}{|l|}{ Deep Inelastic Scattering } \\
\hline Bodek-Yang model parameter $A_{H T}$ & $\pm 25 \%$ \\
\hline Bodek-Yang model parameter $B_{H T}$ & $\pm 25 \%$ \\
\hline Bodek-Yang model parameter $C_{V 1 u}$ & $\pm 30 \%$ \\
\hline Bodek-Yang model parameter $C_{V 2 u}$ & $\pm 40 \%$ \\
\hline \multicolumn{2}{|l|}{ Final State Interactions } \\
\hline Nucleon mean free path & $\pm 20 \%$ \\
\hline Nucleon charge exchange probability & $\pm 50 \%$ \\
\hline Nucleon elastic interaction probability & $\pm 30 \%$ \\
\hline Nucleon inelastic interaction probability & $\pm 40 \%$ \\
\hline Nucleon absorption probability & $\pm 20 \%$ \\
\hline Nucleon $\pi$-production probability & $\pm 20 \%$ \\
\hline$\pi$ mean free path & $\pm 20 \%$ \\
\hline$\pi$ charge exchange probability & $\pm 50 \%$ \\
\hline$\pi$ elastic interaction probability & $\pm 10 \%$ \\
\hline$\pi$ inelastic interaction probability & $\pm 40 \%$ \\
\hline$\pi$ absorption probability & $\pm 30 \%$ \\
\hline$\pi \pi$-production probability & $\pm 20 \%$ \\
\hline \multicolumn{2}{|c|}{ Hadronization and Resonance Decay } \\
\hline$x_{F}$ dependence for $N \pi$ final states in AGKY hadronization model & $\pm 20 \%$ \\
\hline Resonance $\rightarrow X+1 \gamma$ branching ratio & $\pm 50 \%$ \\
\hline Pion angular distribution in $\Delta^{++} \rightarrow N \pi$ & otropic $\rightarrow$ Rein-Sehgal parameterization \\
\hline
\end{tabular}

Table 8.19: The default $\pm 1 \sigma$ variations to the GENIE model parameters.

ference between the isotropic and non-isotropic predictions, where the isotropic and full non-isotropic predictions serve as the $\pm 1 \sigma$ variations to the half non-isotropic 


\begin{tabular}{lc} 
Parameter & Variation \\
\hline \multicolumn{2}{c}{$C C$ Resonance Production } \\
Normalization & $\pm 7 \%$ \\
Axial vector mass & $\pm 5 \%$ \\
Vector mass & $\pm 3 \%$ \\
\hline \multicolumn{2}{c}{$C C \&$ NC Non-Resonant Pion Production } \\
Normalization of $1 \pi$ final states from $\nu p / \bar{\nu} p$ & $\pm 4 \%$ \\
Normalization of $1 \pi$ final states from $\nu n / \bar{\nu} n$ & $\pm 4 \%$
\end{tabular}

Table 8.20: The reduced $\pm 1 \sigma$ variations to the $\mathrm{CC}$ resonance production and $\mathrm{CC}$ non-resonant single pion production model parameters in GENIE.

prediction. This is a reduction of the default GENIE uncertainty, which is the full difference between the isotropic and non-isotropic predictions.

The uncertainty on the measured cross sections does not include uncertainty on the signal model (Rein-Sehgal coherent model) in the MC. The MINER $\nu$ A implementation of GENIE 2.6.2 did not include event weights for uncertainties on the Rein-Sehgal coherent model. In principle, the uncertainty on the measured cross sections should include uncertainty on the signal model due to the signal model dependence introduced by the unfolding and efficiency correction. However, bias from the signal model was minimized by remeasuring the cross sections with the signal model weighted to the initial cross section measurements and, as a result, remaining bias should be small compared to the total uncertainty on the cross sections (Section 8.10).

The GENIE interaction model uncertainties on the measured cross sections are shown in Figures 8.70-8.97. 

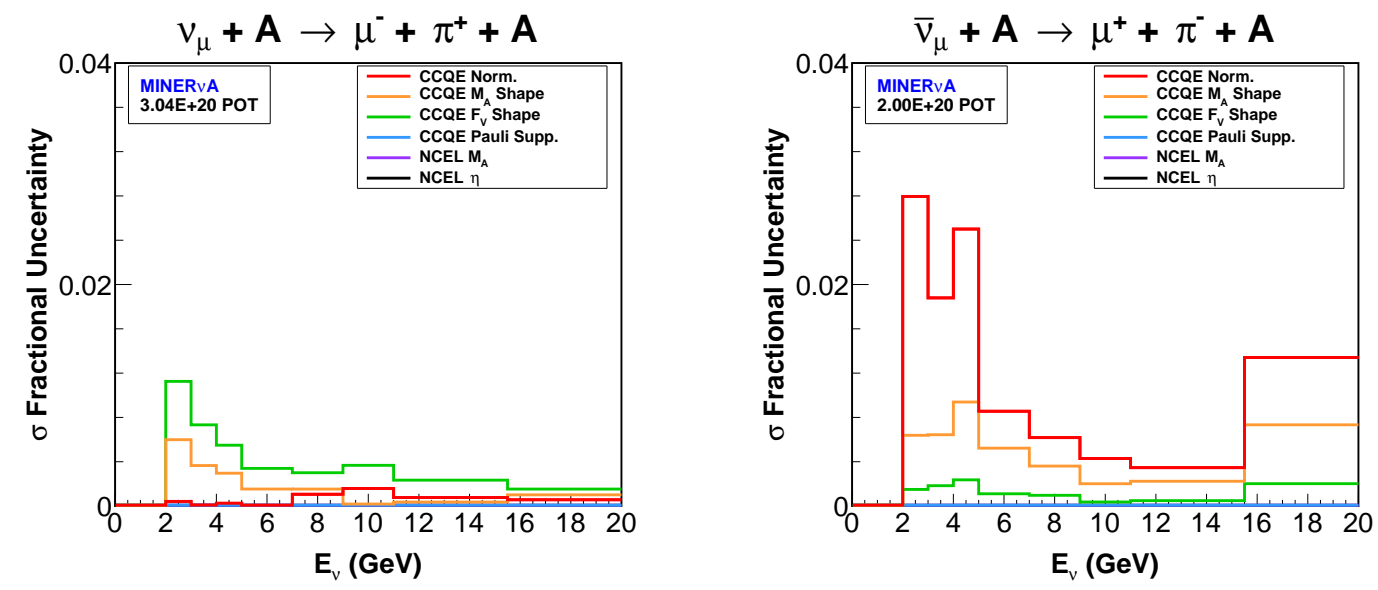

Figure 8.70: The GENIE charged current quasi-elastic (CCQE) and neutral current elastic (NCEL) interaction model uncertainties (Table 8.19) on the measured $\nu_{\mu}$ (left) and $\bar{\nu}_{\mu}$ (right) $\sigma\left(E_{\nu}\right)$.
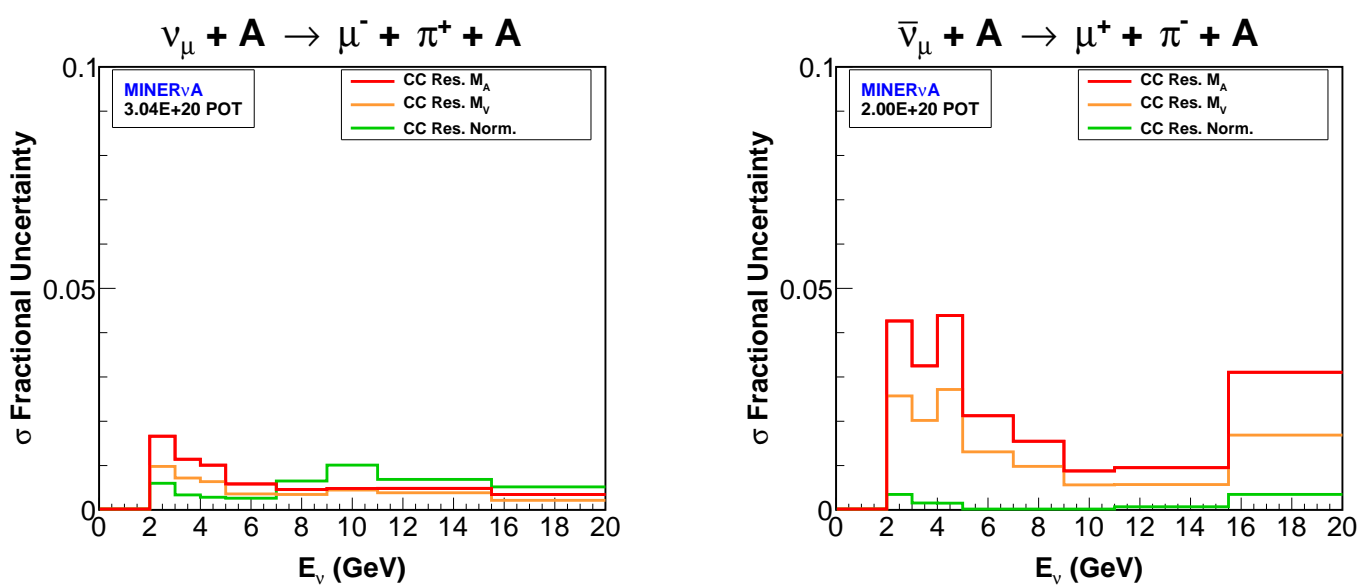

Figure 8.71: The GENIE charged current resonance model uncertainties (Tables 8.19 and 8.20) on the measured $\nu_{\mu}$ (left) and $\bar{\nu}_{\mu}$ (right) $\sigma\left(E_{\nu}\right)$. 

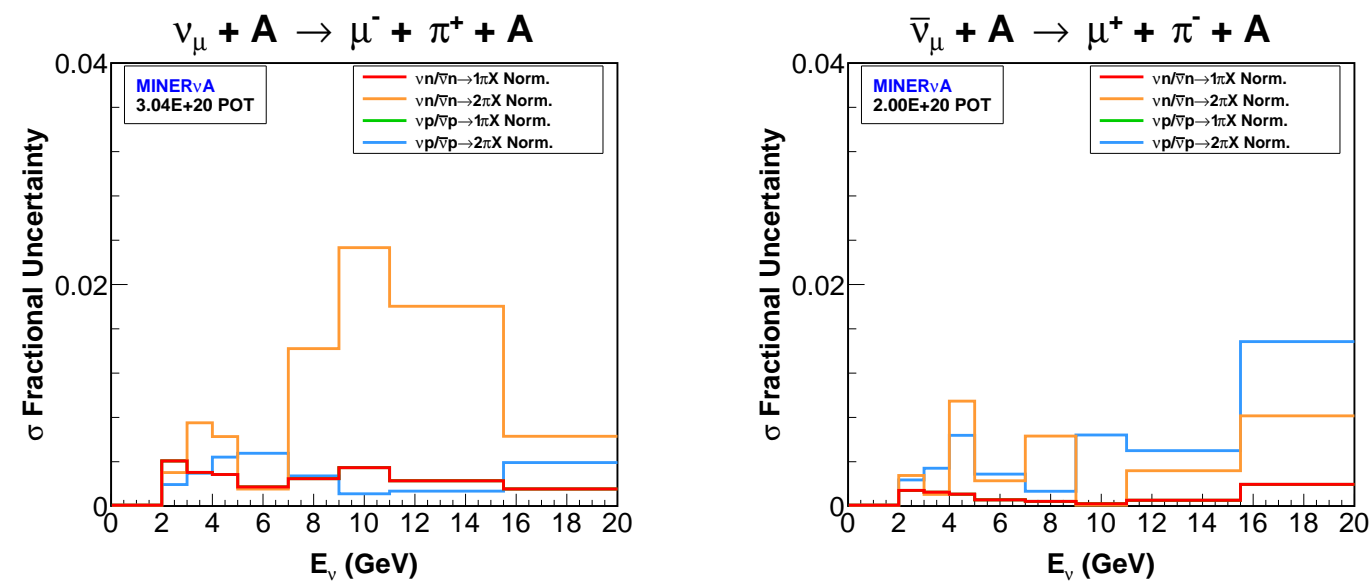

Figure 8.72: The GENIE charged and neutral current non-resonant pion production model uncertainties (Tables 8.19 and 8.20) on the measured $\nu_{\mu}$ (left) and $\bar{\nu}_{\mu}$ (right) $\sigma\left(E_{\nu}\right)$.
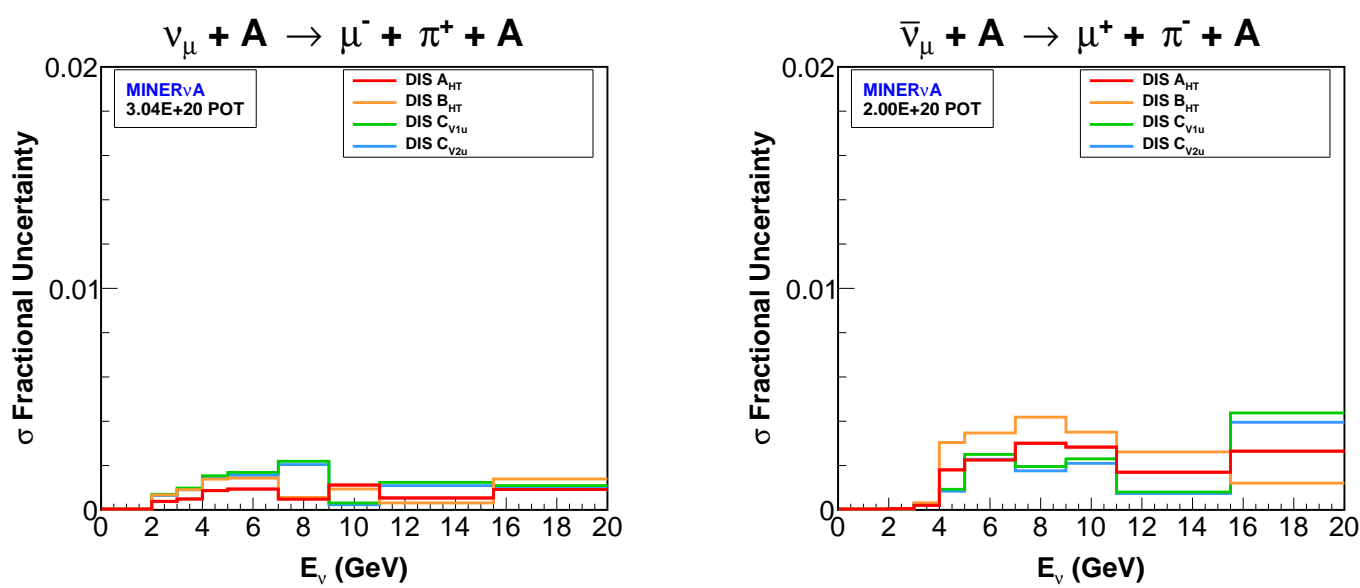

Figure 8.73: The GENIE deep inelastic scattering (DIS) model uncertainties (Table 8.19) on the measured $\nu_{\mu}$ (left) and $\bar{\nu}_{\mu}$ (right) $\sigma\left(E_{\nu}\right)$. 

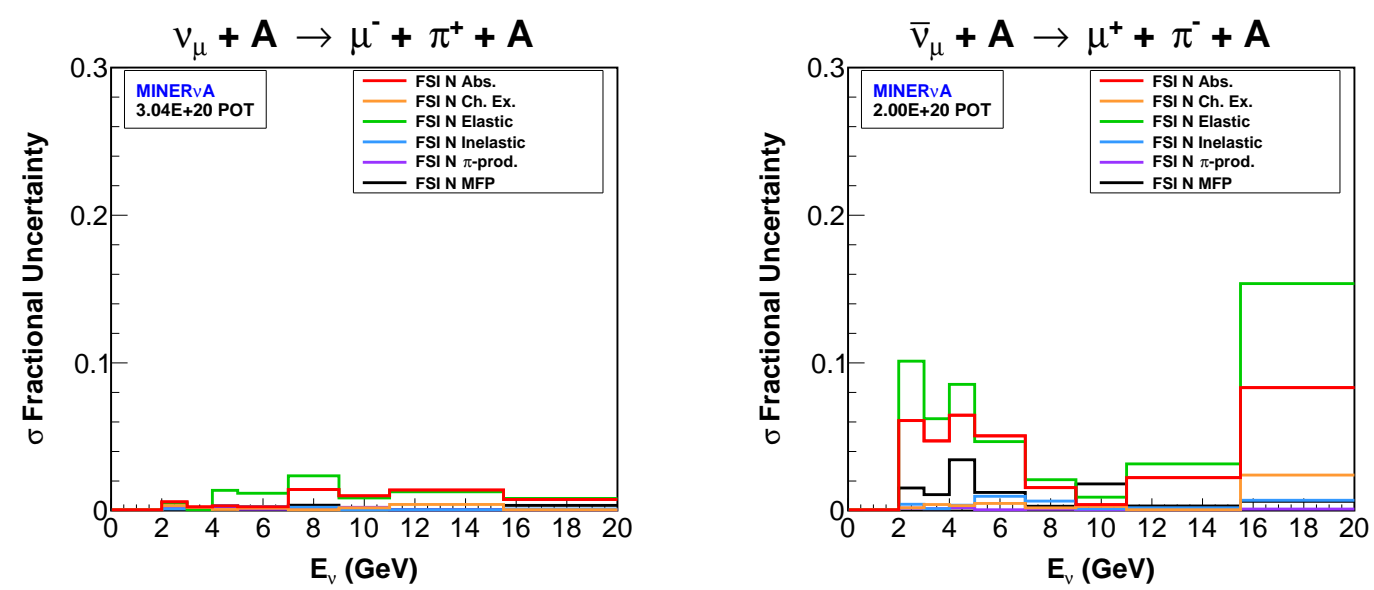

Figure 8.74: The GENIE nucleon final state interaction (FSI) model uncertainties (Table 8.19) on the measured $\nu_{\mu}$ (left) and $\bar{\nu}_{\mu}$ (right) $\sigma\left(E_{\nu}\right)$.
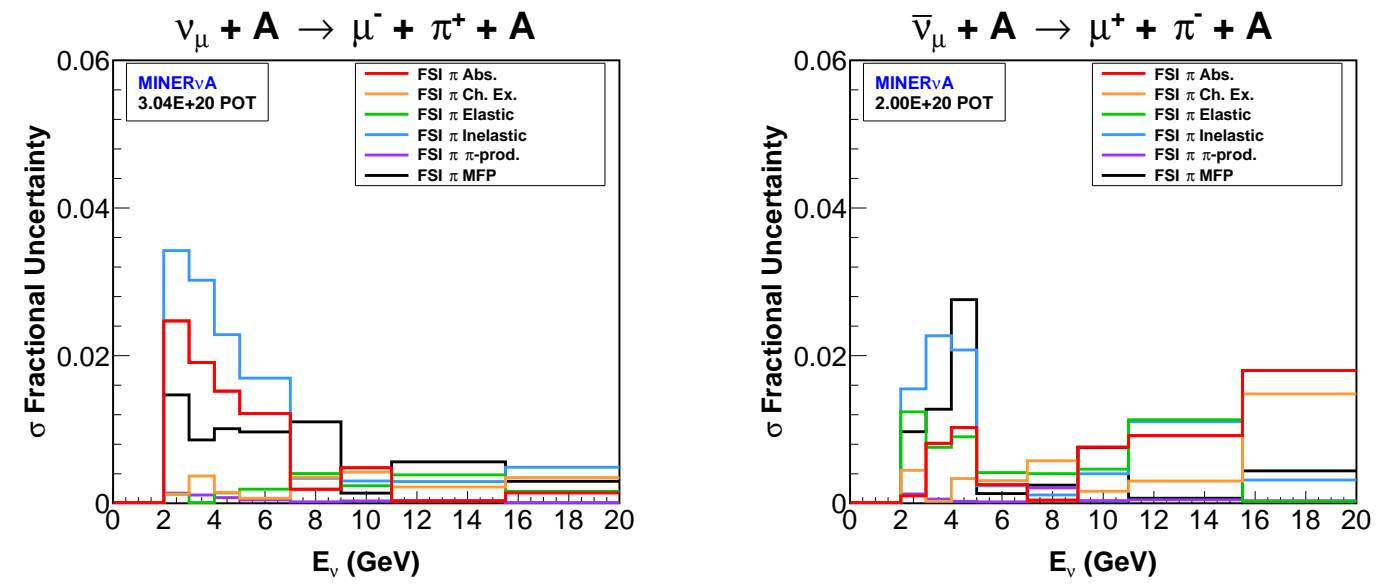

Figure 8.75: The GENIE pion final state interaction (FSI) model uncertainties (Table 8.19) on the measured $\nu_{\mu}$ (left) and $\bar{\nu}_{\mu}$ (right) $\sigma\left(E_{\nu}\right)$. 

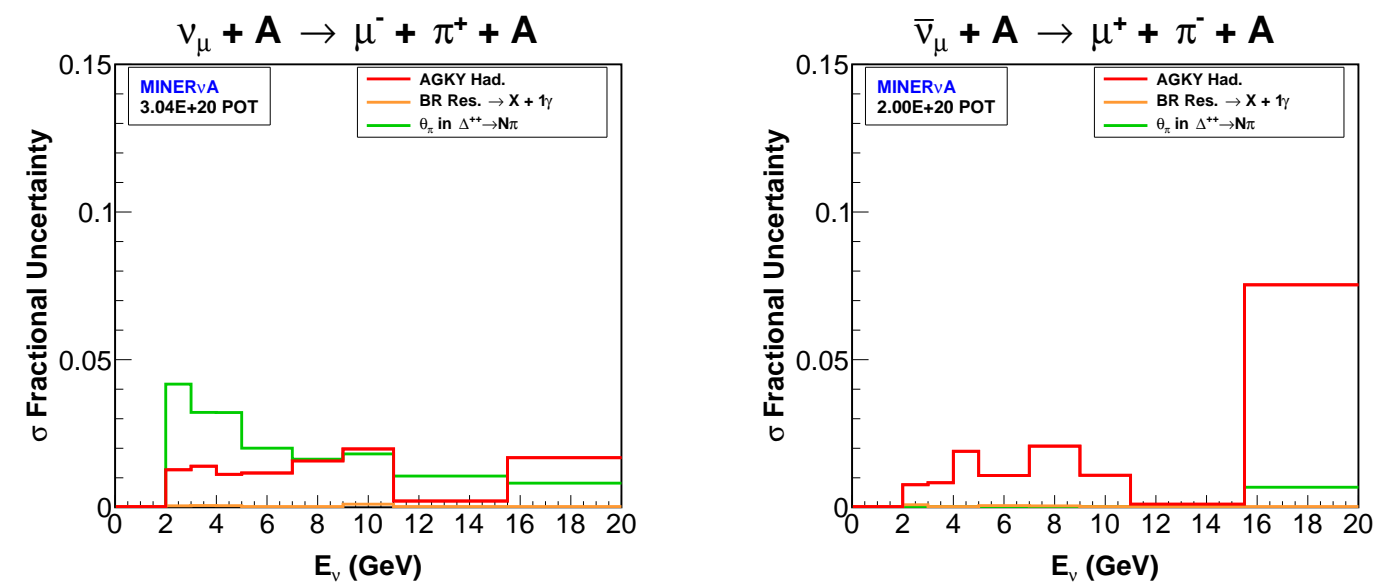

Figure 8.76: The GENIE hadronization and resonance decay model uncertainties (Table 8.19) on the measured $\nu_{\mu}$ (left) and $\bar{\nu}_{\mu}$ (right) $\sigma\left(E_{\nu}\right)$.
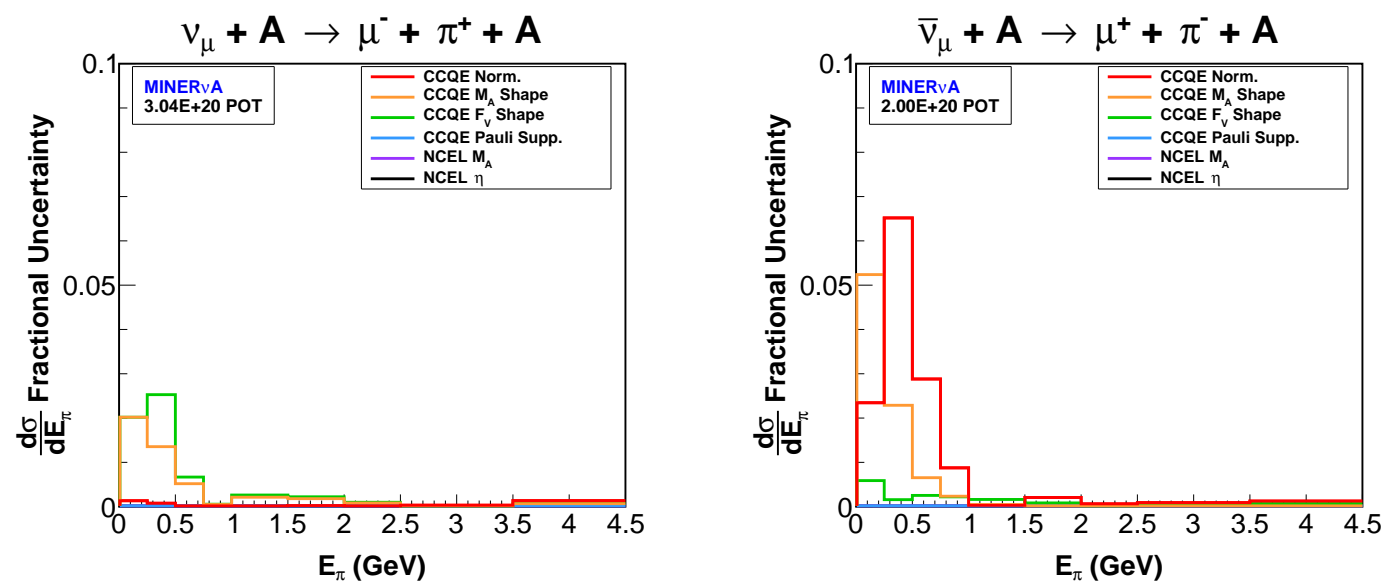

Figure 8.77: The GENIE charged current quasi-elastic (CCQE) and neutral current elastic (NCEL) interaction model uncertainties (Table 8.19) on the measured $\nu_{\mu}$ (left) and $\bar{\nu}_{\mu}$ (right) $\frac{d \sigma}{d E_{\pi}}$. 

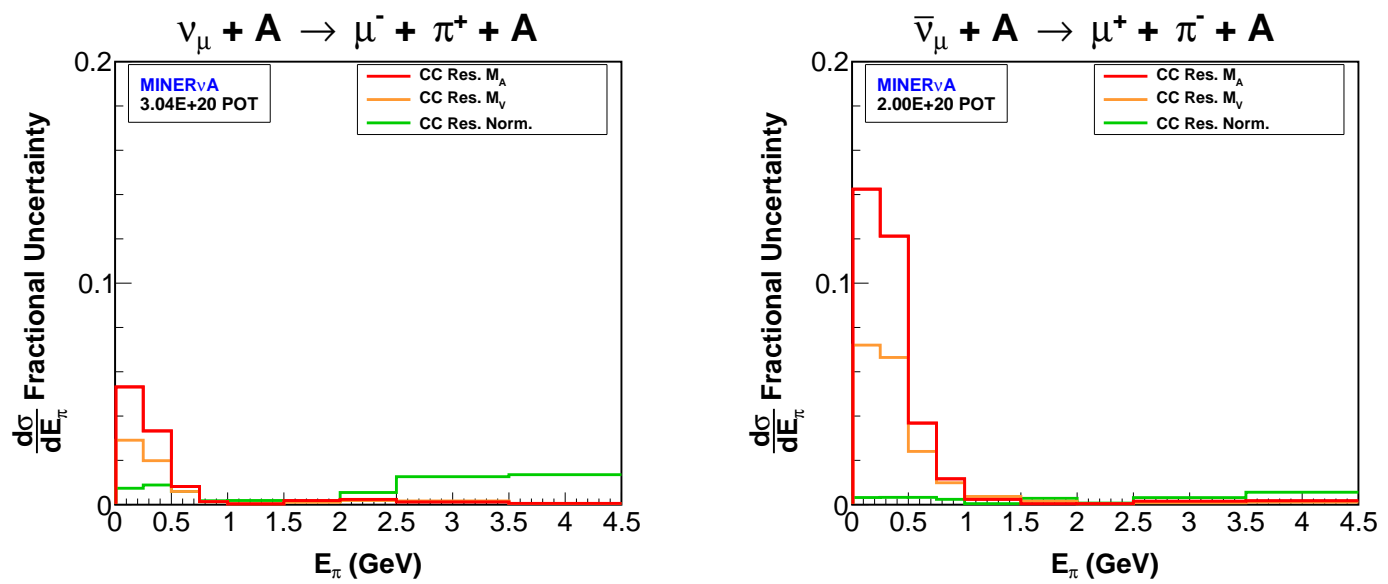

Figure 8.78: The GENIE charged current resonance model uncertainties (Tables 8.19 and 8.20) on the measured $\nu_{\mu}$ (left) and $\bar{\nu}_{\mu}$ (right) $\frac{d \sigma}{d E_{\pi}}$.
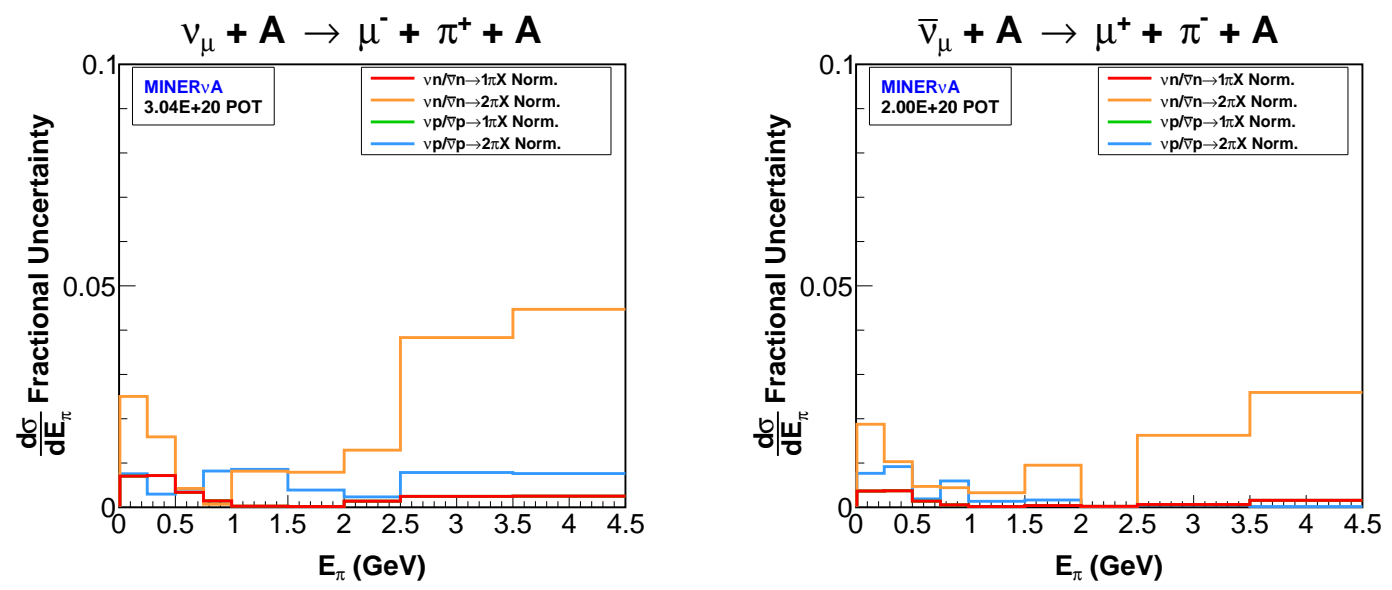

Figure 8.79: The GENIE charged and neutral current non-resonant pion production model uncertainties (Tables 8.19 and 8.20) on the measured $\nu_{\mu}$ (left) and $\bar{\nu}_{\mu}$ (right) $\frac{d \sigma}{d E_{\pi}}$. 

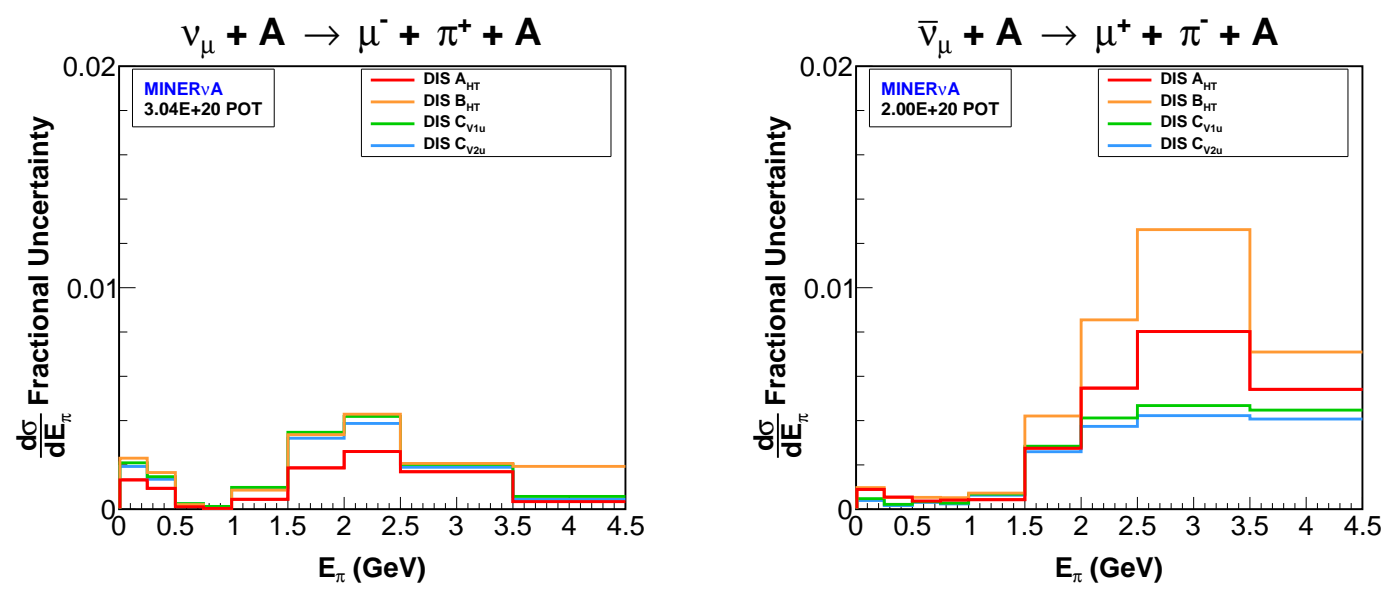

Figure 8.80: The GENIE deep inelastic scattering (DIS) model uncertainties (Table 8.19) on the measured $\nu_{\mu}$ (left) and $\bar{\nu}_{\mu}$ (right) $\frac{d \sigma}{d E_{\pi}}$.
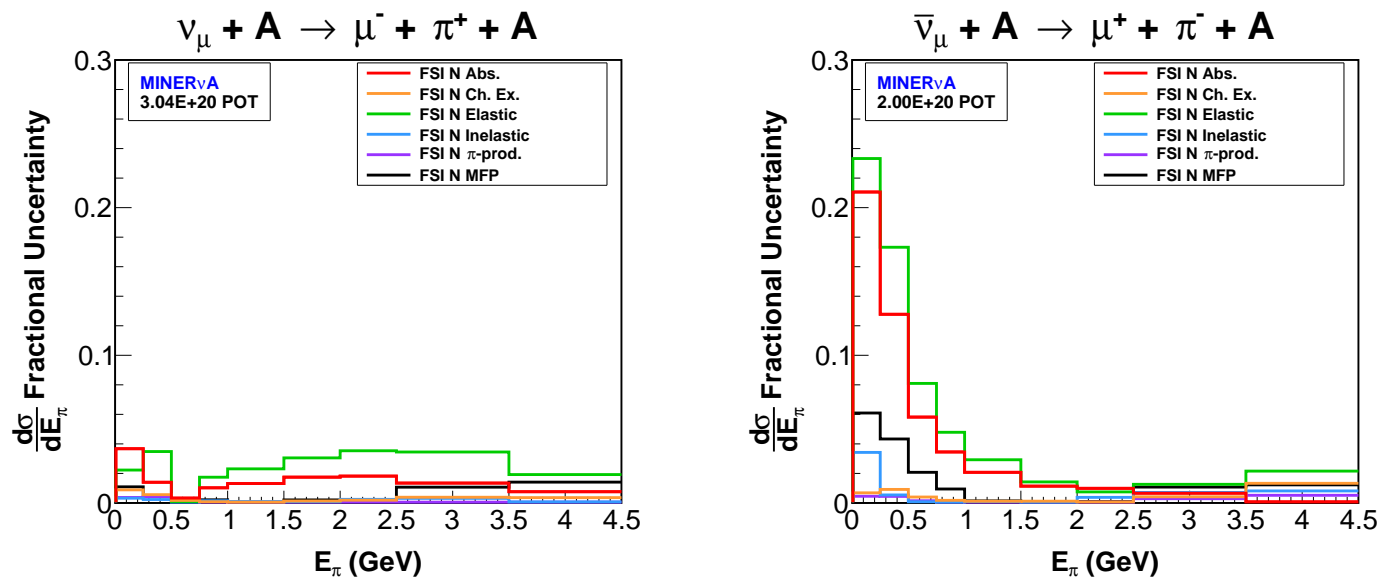

Figure 8.81: The GENIE nucleon final state interaction (FSI) model uncertainties (Table 8.19) on the measured $\nu_{\mu}$ (left) and $\bar{\nu}_{\mu}$ (right) $\frac{d \sigma}{d E_{\pi}}$. 

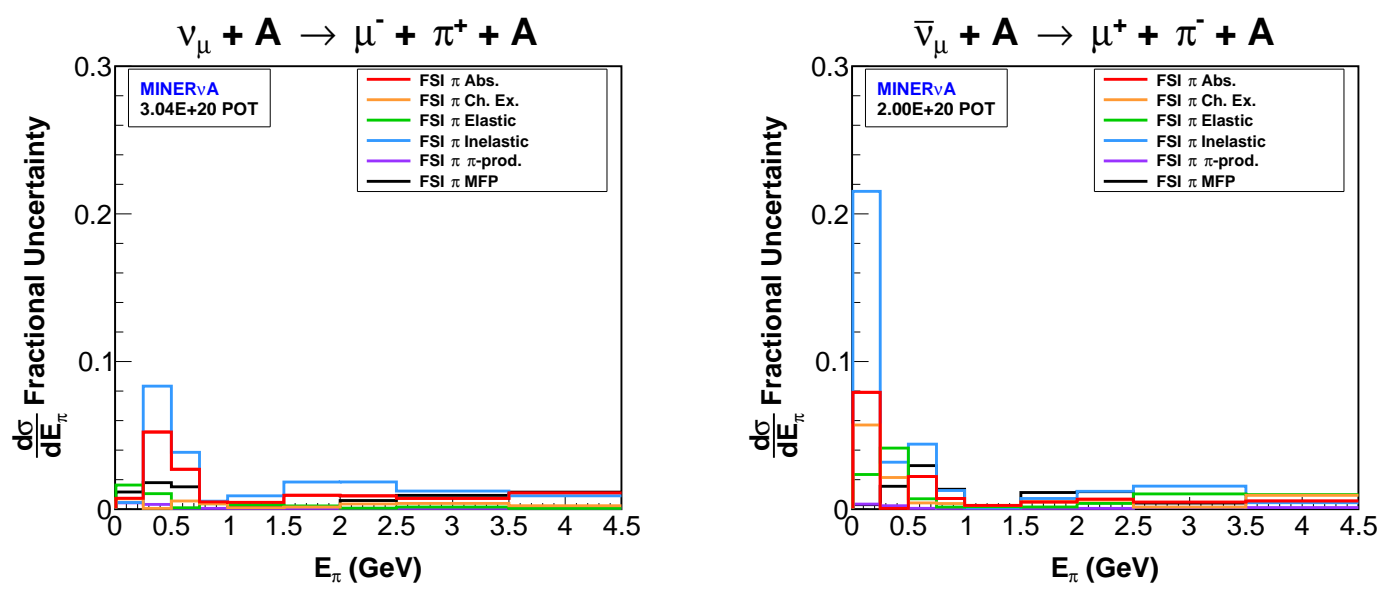

Figure 8.82: The GENIE pion final state interaction (FSI) model uncertainties (Table 8.19) on the measured $\nu_{\mu}$ (left) and $\bar{\nu}_{\mu}$ (right) $\frac{d \sigma}{d E_{\pi}}$.
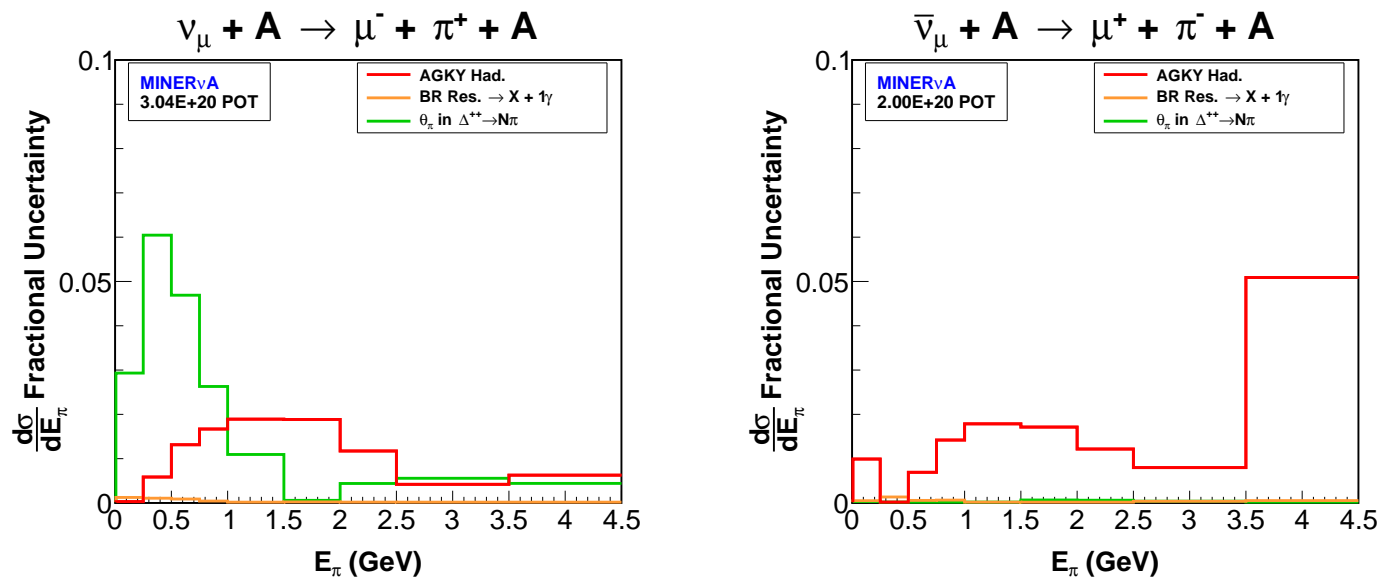

Figure 8.83: The GENIE hadronization and resonance decay model uncertainties (Table 8.19) on the measured $\nu_{\mu}$ (left) and $\bar{\nu}_{\mu}$ (right) $\frac{d \sigma}{d E_{\pi}}$. 

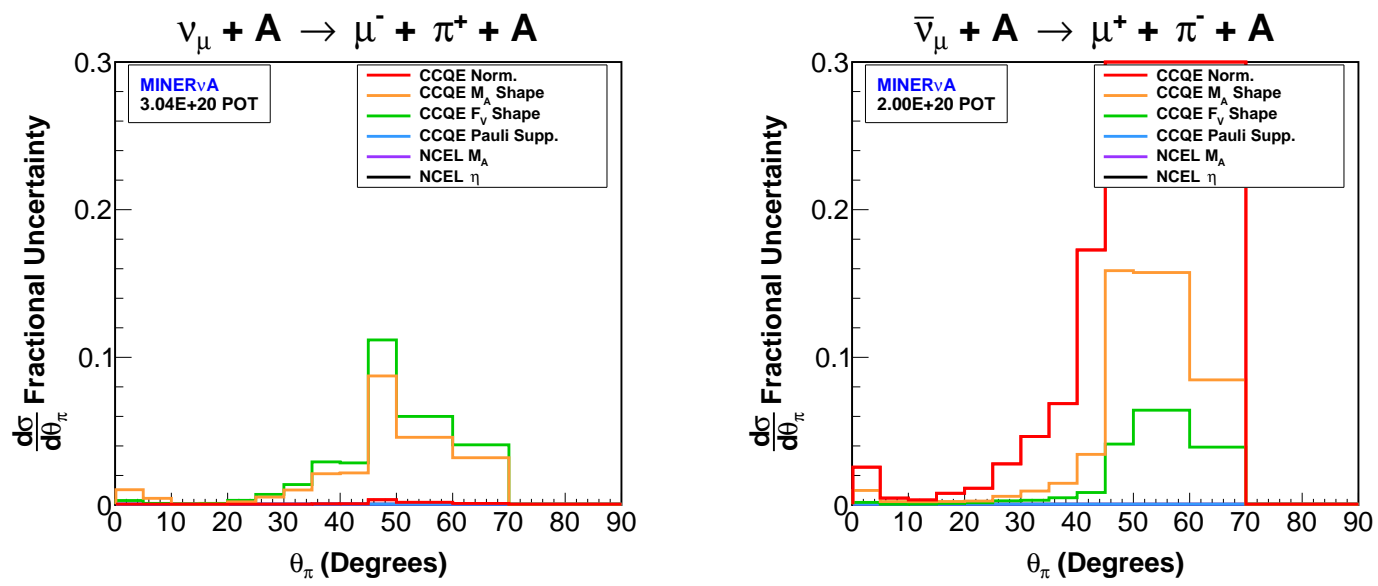

Figure 8.84: The GENIE charged current quasi-elastic (CCQE) and neutral current elastic (NCEL) interaction model uncertainties (Table 8.19) on the measured $\nu_{\mu}$ (left) and $\bar{\nu}_{\mu}$ (right) $\frac{d \sigma}{d \theta_{\pi}}$.
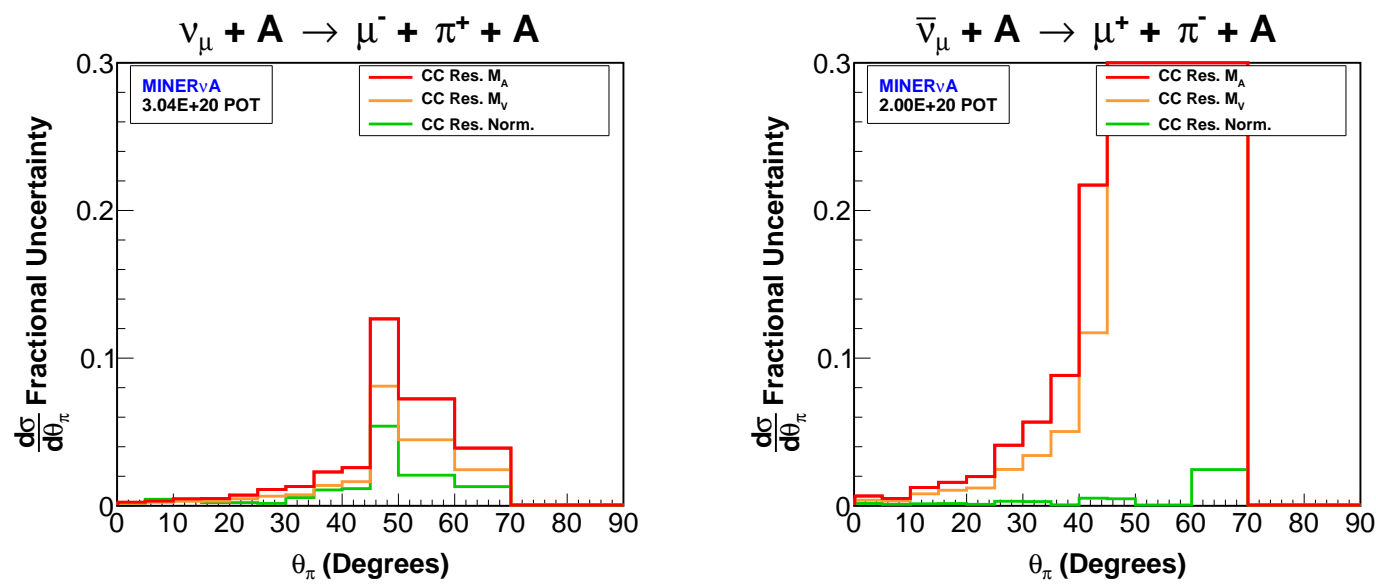

Figure 8.85: The GENIE charged current resonance model uncertainties (Tables 8.19 and 8.20) on the measured $\nu_{\mu}$ (left) and $\bar{\nu}_{\mu}$ (right) $\frac{d \sigma}{d \theta_{\pi}}$. 

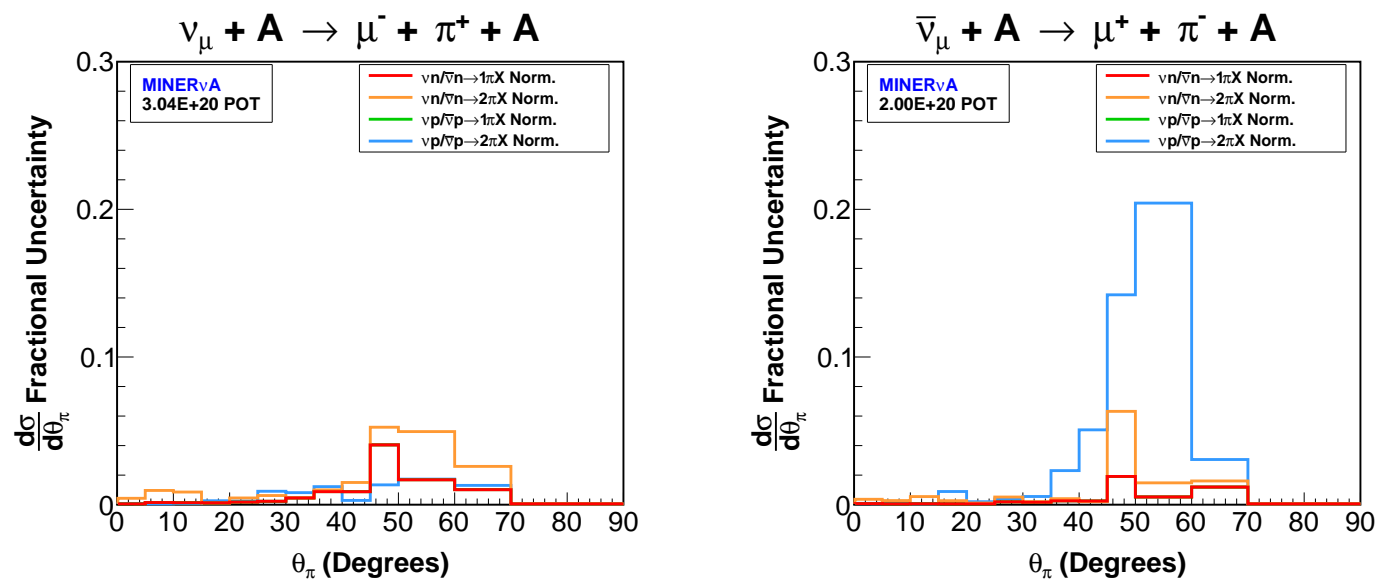

Figure 8.86: The GENIE charged and neutral current non-resonant pion production model uncertainties (Tables 8.19 and 8.20) on the measured $\nu_{\mu}$ (left) and $\bar{\nu}_{\mu}$ (right) $\frac{d \sigma}{d \theta_{\pi}}$.
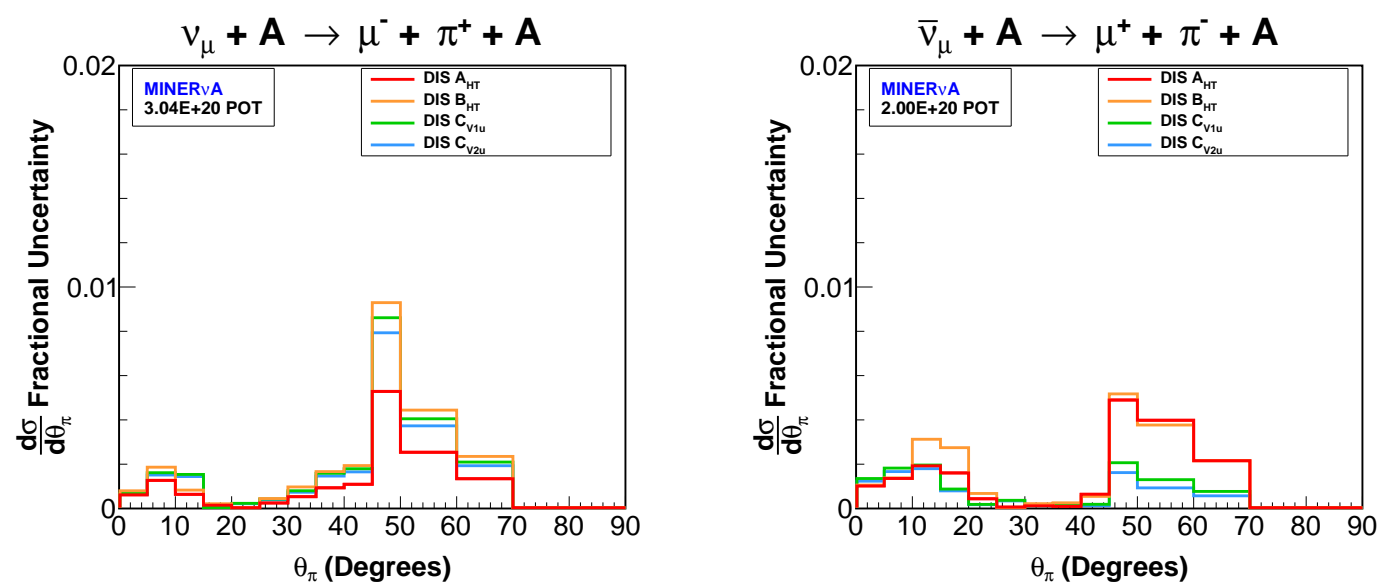

Figure 8.87: The GENIE deep inelastic scattering (DIS) model uncertainties (Table 8.19) on the measured $\nu_{\mu}$ (left) and $\bar{\nu}_{\mu}$ (right) $\frac{d \sigma}{d \theta_{\pi}}$. 

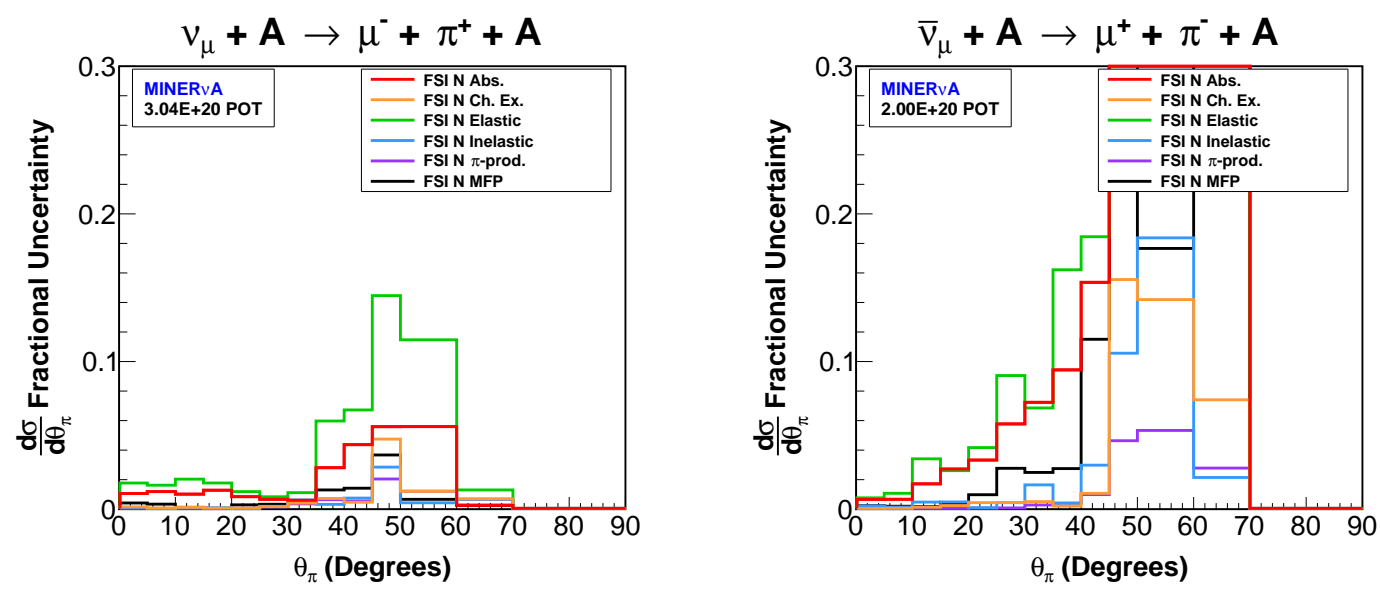

Figure 8.88: The GENIE nucleon final state interaction (FSI) model uncertainties (Table 8.19) on the measured $\nu_{\mu}$ (left) and $\bar{\nu}_{\mu}$ (right) $\frac{d \sigma}{d \theta_{\pi}}$.
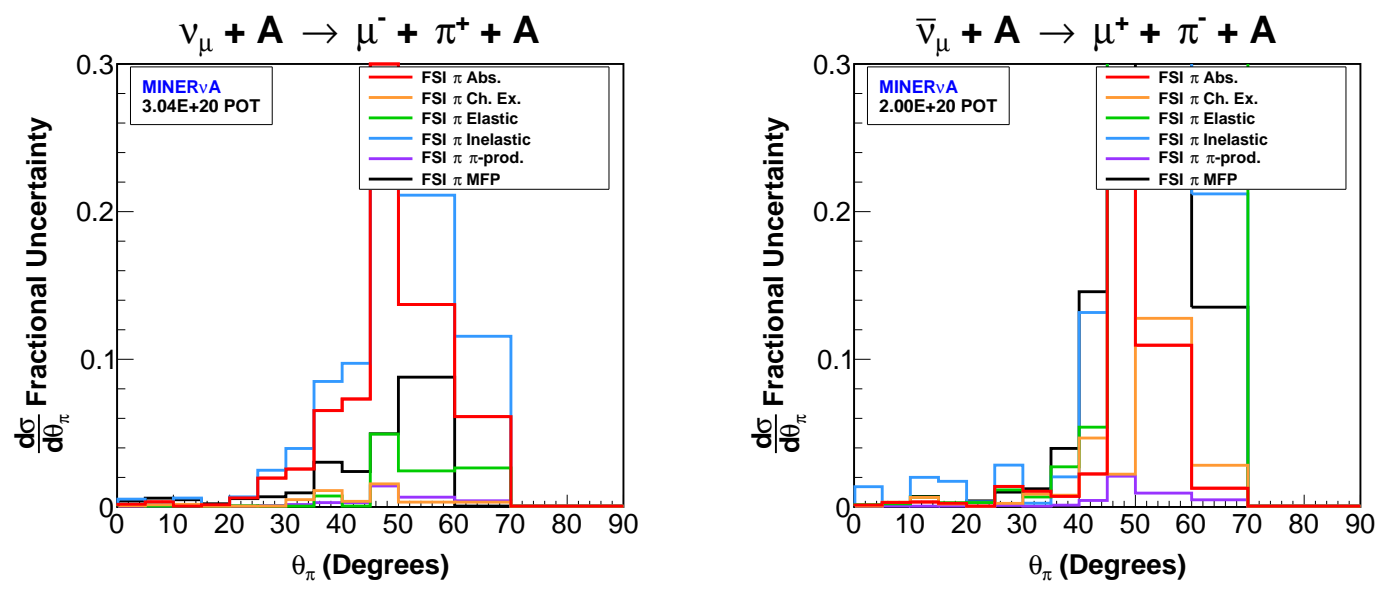

Figure 8.89: The GENIE pion final state interaction (FSI) model uncertainties (Table 8.19) on the measured $\nu_{\mu}$ (left) and $\bar{\nu}_{\mu}$ (right) $\frac{d \sigma}{d \theta_{\pi}}$. 

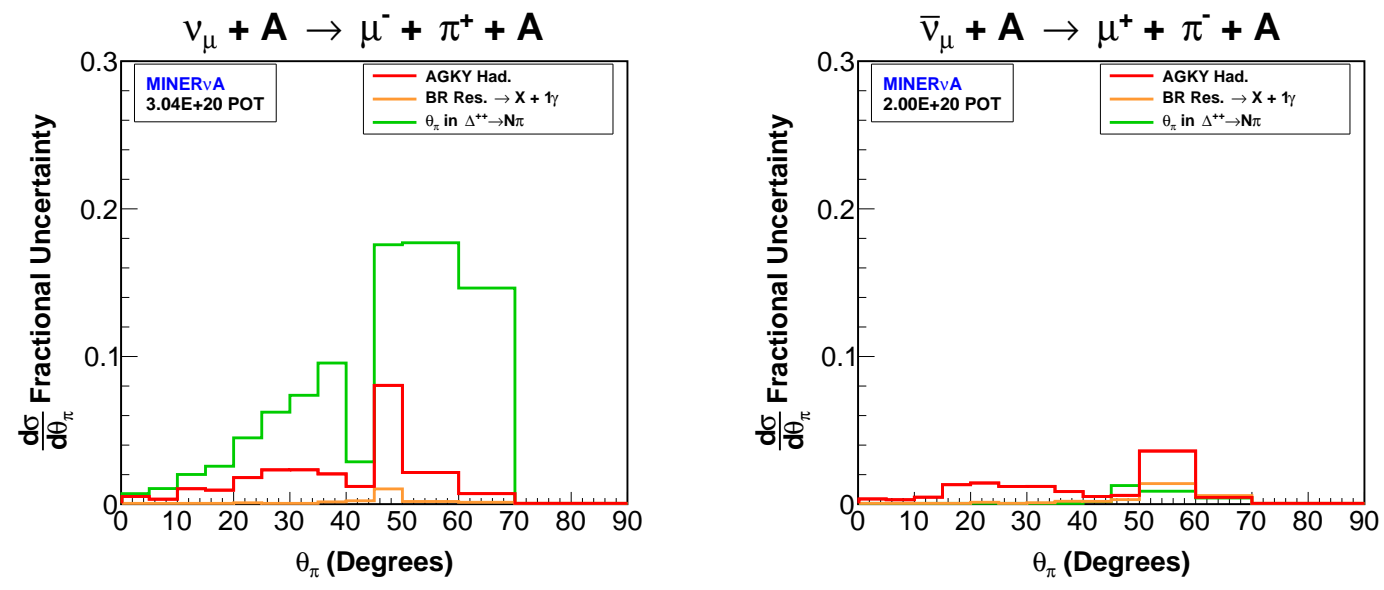

Figure 8.90: The GENIE hadronization and resonance decay model uncertainties (Table 8.19) on the measured $\nu_{\mu}$ (left) and $\bar{\nu}_{\mu}$ (right) $\frac{d \sigma}{d \theta_{\pi}}$.
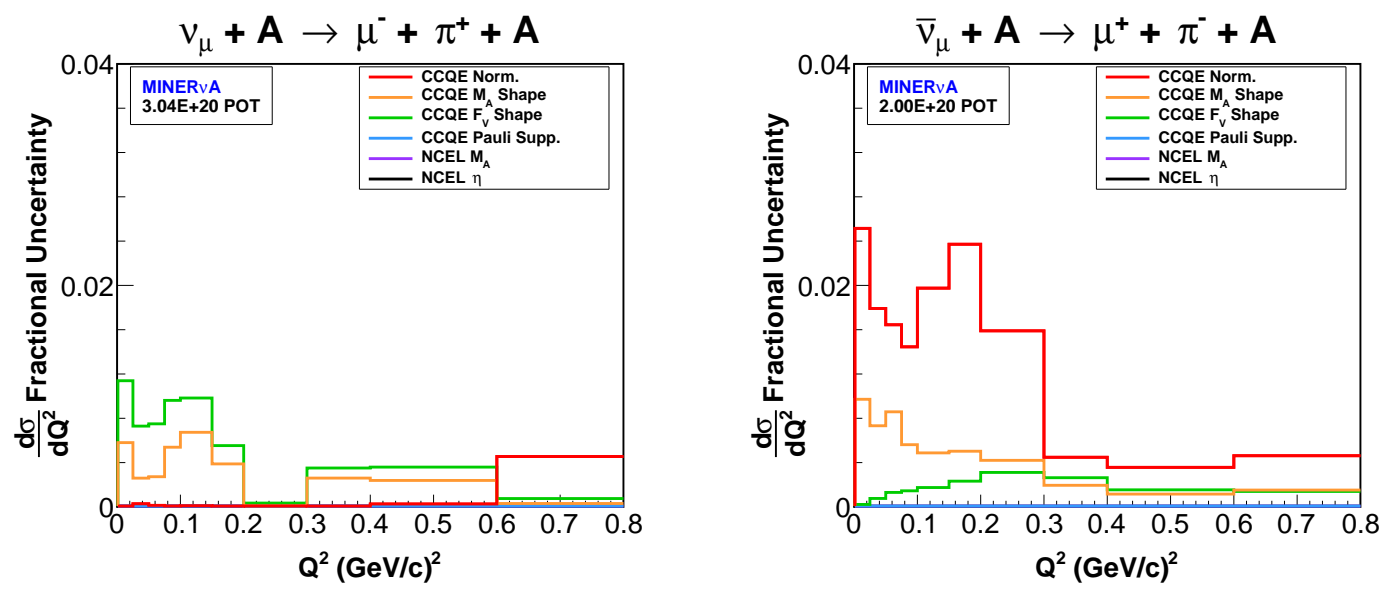

Figure 8.91: The GENIE charged current quasi-elastic (CCQE) and neutral current elastic (NCEL) interaction model uncertainties (Table 8.19) on the measured $\nu_{\mu}$ (left) and $\bar{\nu}_{\mu}$ (right) $\frac{d \sigma}{d Q^{2}}$. 

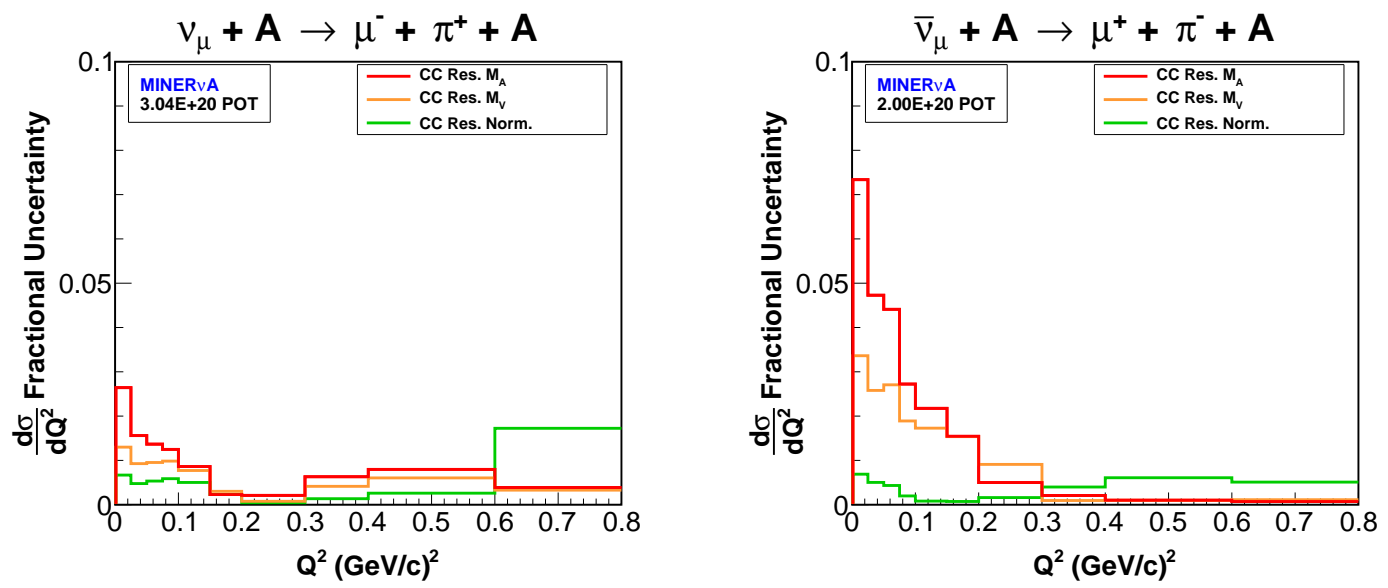

Figure 8.92: The GENIE charged current resonance model uncertainties (Tables 8.19 and 8.20) on the measured $\nu_{\mu}$ (left) and $\bar{\nu}_{\mu}$ (right) $\frac{d \sigma}{d Q^{2}}$.
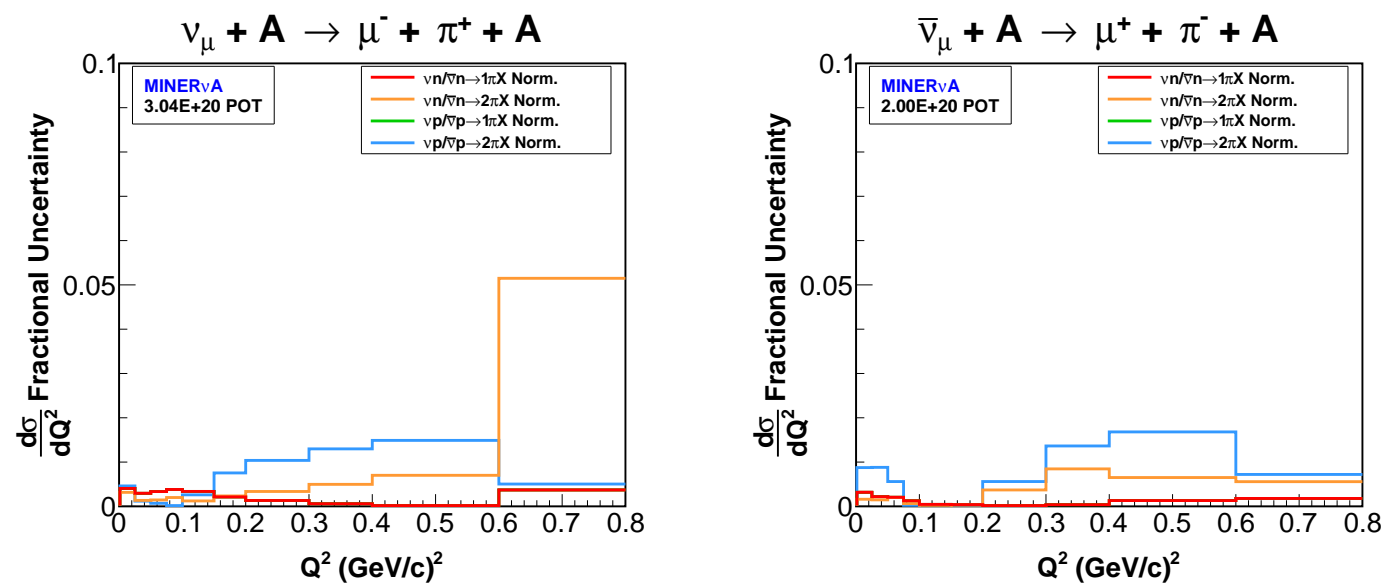

Figure 8.93: The GENIE charged and neutral current non-resonant pion production model uncertainties (Tables 8.19 and 8.20) on the measured $\nu_{\mu}$ (left) and $\bar{\nu}_{\mu}$ (right) $\frac{d \sigma}{d Q^{2}}$. 

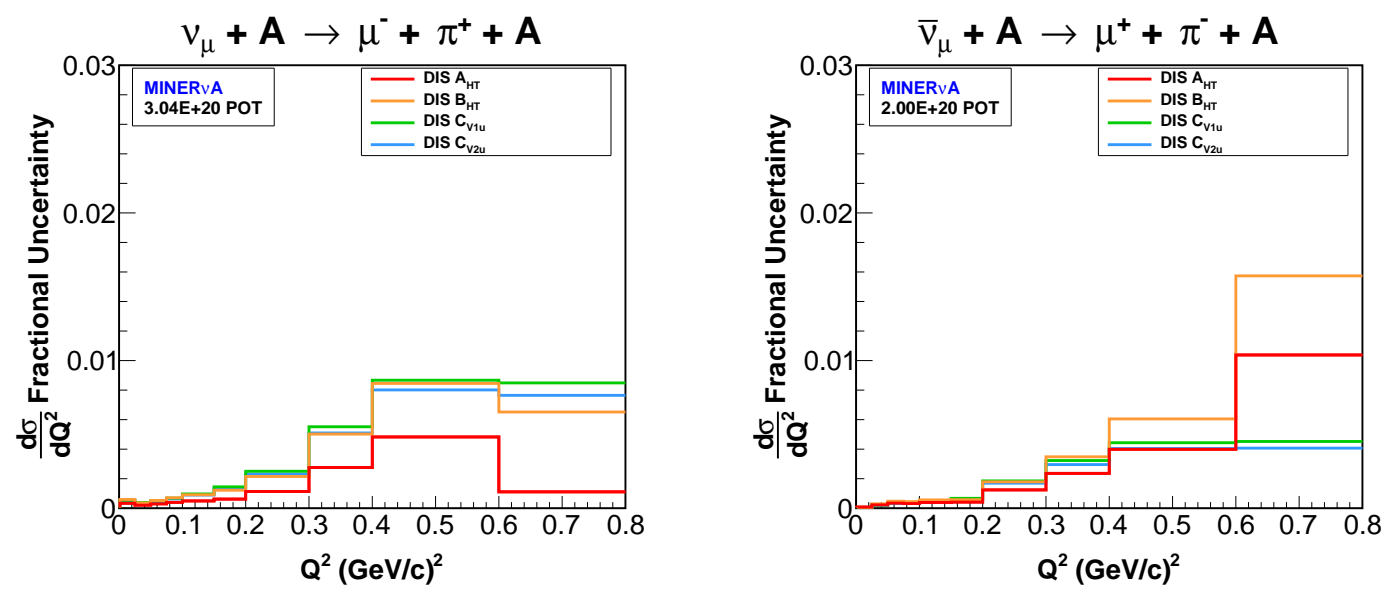

Figure 8.94: The GENIE deep inelastic scattering (DIS) model uncertainties (Table 8.19) on the measured $\nu_{\mu}$ (left) and $\bar{\nu}_{\mu}$ (right) $\frac{d \sigma}{d Q^{2}}$.
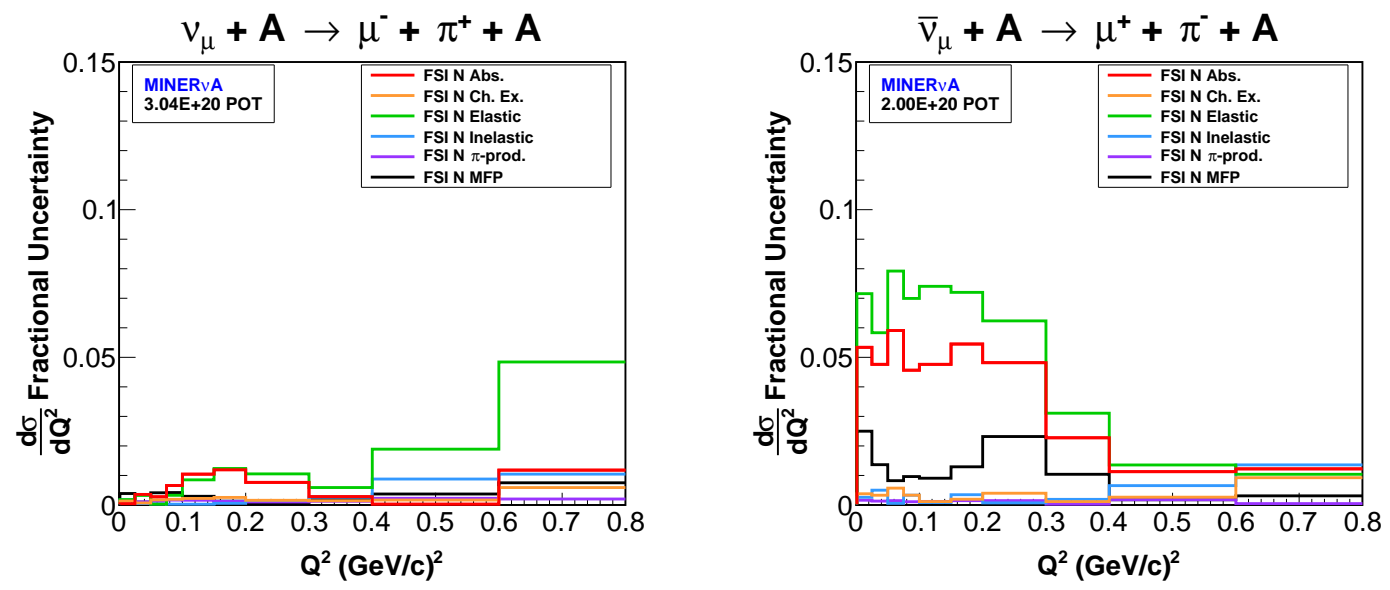

Figure 8.95: The GENIE nucleon final state interaction (FSI) model uncertainties (Table 8.19) on the measured $\nu_{\mu}$ (left) and $\bar{\nu}_{\mu}$ (right) $\frac{d \sigma}{d Q^{2}}$. 

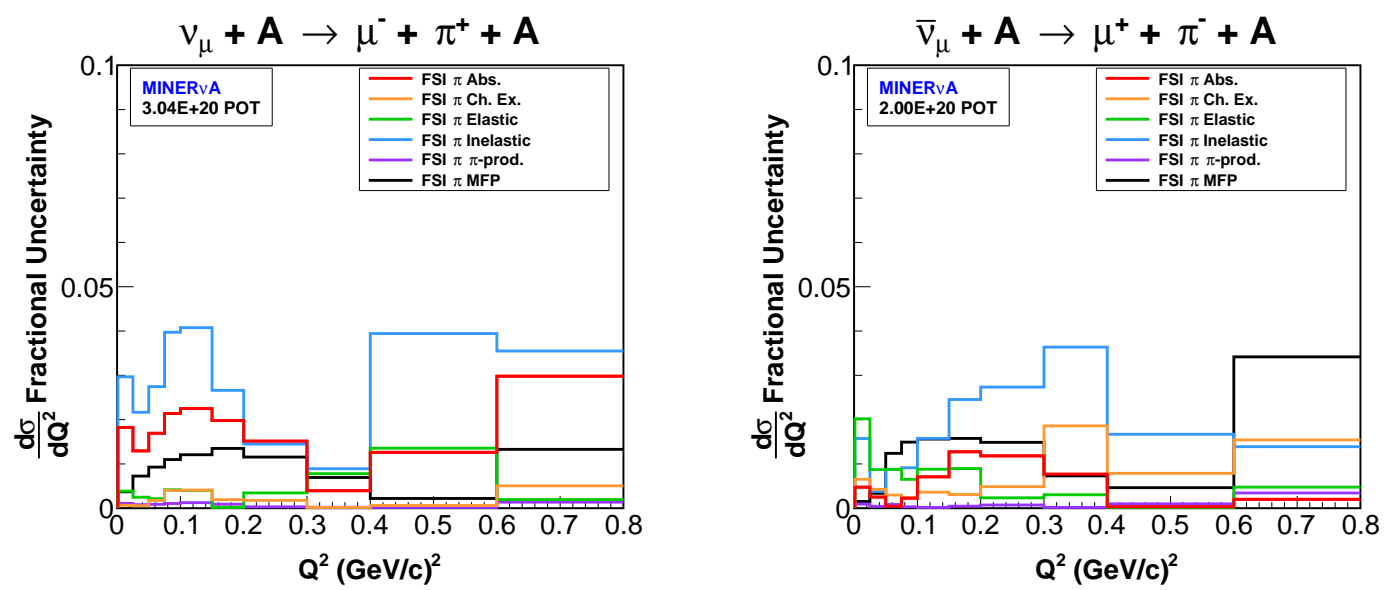

Figure 8.96: The GENIE pion final state interaction (FSI) model uncertainties (Table 8.19) on the measured $\nu_{\mu}$ (left) and $\bar{\nu}_{\mu}$ (right) $\frac{d \sigma}{d Q^{2}}$.
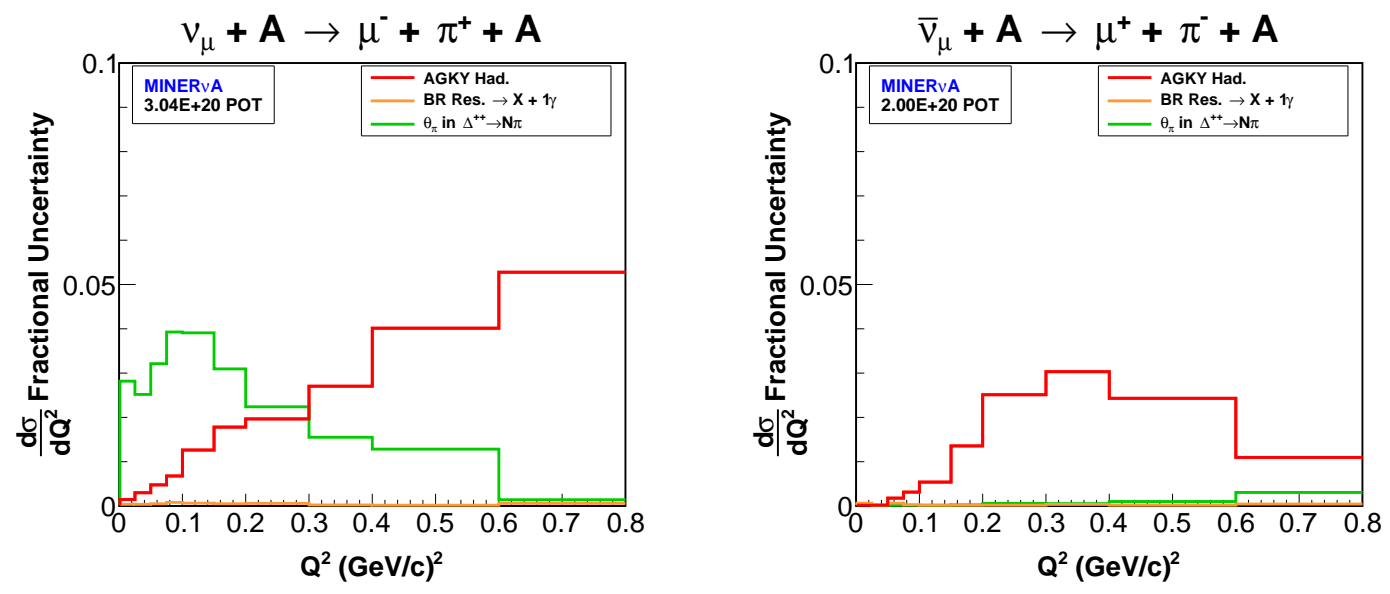

Figure 8.97: The GENIE hadronization and resonance decay model uncertainties (Table 8.19) on the measured $\nu_{\mu}$ (left) and $\bar{\nu}_{\mu}$ (right) $\frac{d \sigma}{d Q^{2}}$. 


\subsubsection{Vertex Energy}

For non-coherent interactions in the MC, the amount of visible energy near the event vertex is dependent upon the model of initial and final state nuclear effects in GENIE. Since vertex energy is used to reject non-coherent interactions, the predicted rate of background in the coherent candidate sample is sensitive to the modeling of these effects. Per Section 8.8, this sensitivity was minimized by tuning the background prediction to data after cutting on vertex energy. Uncertainties on the measured cross sections from modeling final state interactions in the nucleus were evaluated using the GENIE FSI weights.

The modeling of initial state nuclear effects in current neutrino-nucleus event generators is known to be incomplete. In particular, the version of GENIE used in generating the MC does not model scattering off correlated nucleon pairs, an effect observed in electron scattering data. The MINER $\nu \mathrm{A} \nu_{\mu} \mathrm{CCQE}$ results provide evidence for scattering off correlated nucleon pairs in neutrino-nucleus interactions [121]. This evidence was the result of an analysis of the visible energy near the event vertex of a $\nu_{\mu}$ CCQE-enhanced sample. A fit of the vertex energy distribution predicted by the GENIE-based MC to that of the data preferred the addition of a final state proton with kinetic energy less than $225 \mathrm{MeV}$ to $25 \%$ of the events in the MC. This implies the presence of charged current scattering off an initial state neutron-proton correlated pair resulting in the ejection of two final state protons from the nucleus.

An uncertainty was applied to the measured cross sections to account for the absence of modeling the scattering off correlated nucleon pairs in GENIE. Informed by the MINER $\nu \mathrm{A} \nu_{\mu}$ CCQE results, this uncertainty was evaluated by adding the vertex energy from a final state proton with kinetic energy less than $225 \mathrm{MeV}$ to the 
vertex energy of $25 \%$ of events in the MC where the neutrino scattered off a neutron. The additional vertex energy was sampled from a vertex energy distribution for a sample of simulated single protons originating in the tracker with a flat $25-225 \mathrm{MeV}$ kinetic energy spectrum. The uncertainty on the measured cross sections from the additional vertex energy is shown in Figures 8.62-8.65.

\subsubsection{Sideband Model}

The MC was weighted as a function of reconstructed $\theta_{\pi}$ to correct the disagreement between the data and $\mathrm{MC}$ in the sideband reconstructed $\theta_{\pi}$ distribution after tuning the background normalizations (Section 8.8.1). An uncertainty was applied to the background prediction that accounted for the extrapolation of the weighting from the sideband to the signal region. The size of the uncertainty was the full difference between the weighted and unweighed tuned background predictions. This uncertainty was propagated to the measured coherent cross sections (Figures 8.62-8.65).

\subsubsection{Detector Model}

The detector model uncertainties on the measured cross sections (Figures 8.988.101) consist of uncertainties on the simulation of particle propagation in the detectors, the particle response of the detectors, and the particle and kinematics reconstruction. The following sections detail the evaluation of the detector model uncertainties. 

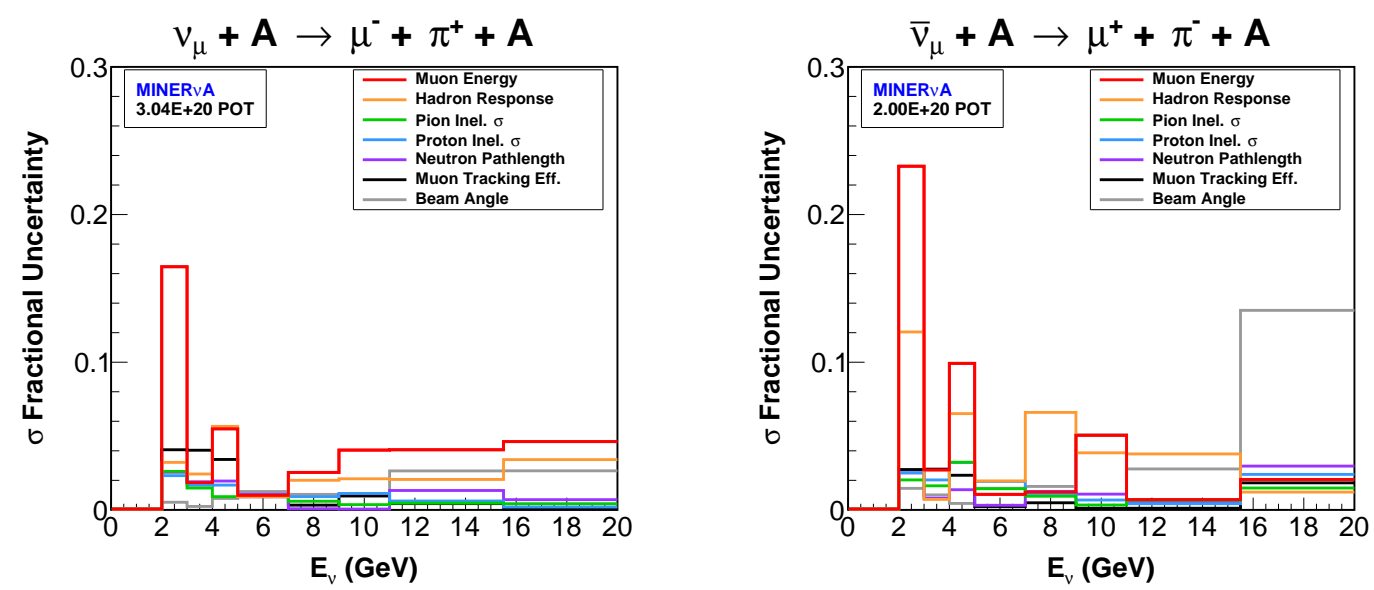

Figure 8.98: The fractional detector model uncertainties on the measured $\nu_{\mu}$ (left) and $\bar{\nu}_{\mu}$ (right) $\sigma\left(E_{\nu}\right)$.
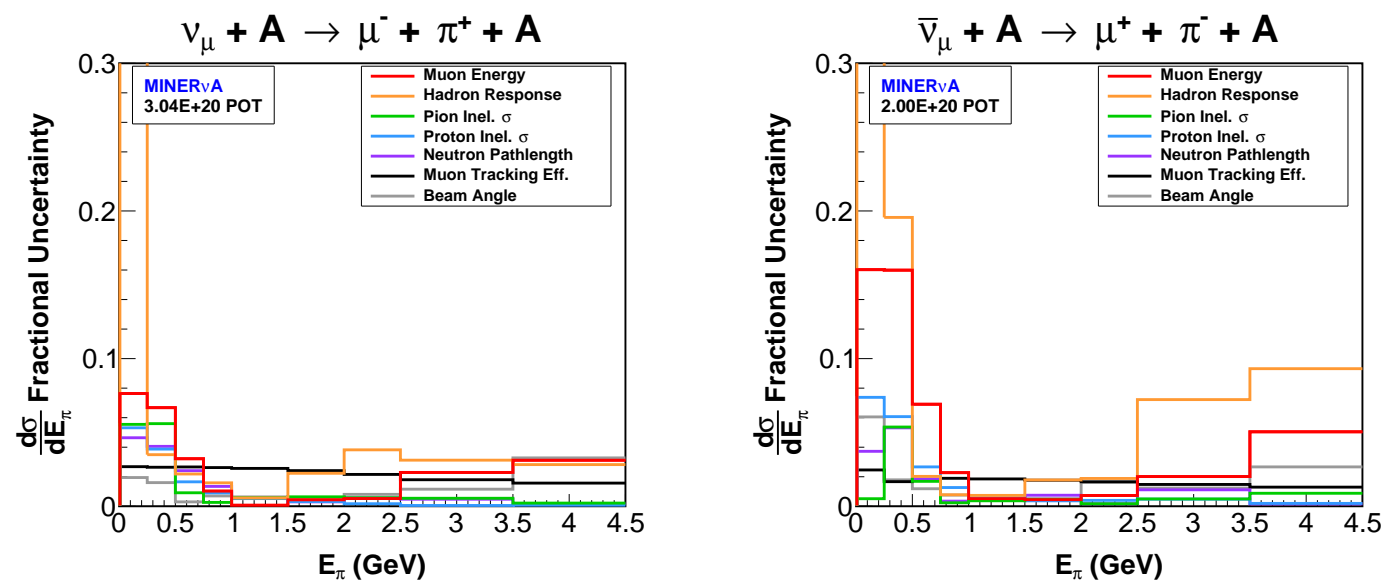

Figure 8.99: The fractional detector model uncertainties on the measured $\nu_{\mu}$ (left) and $\bar{\nu}_{\mu}$ (right) $\frac{d \sigma}{d E_{\pi}}$. 

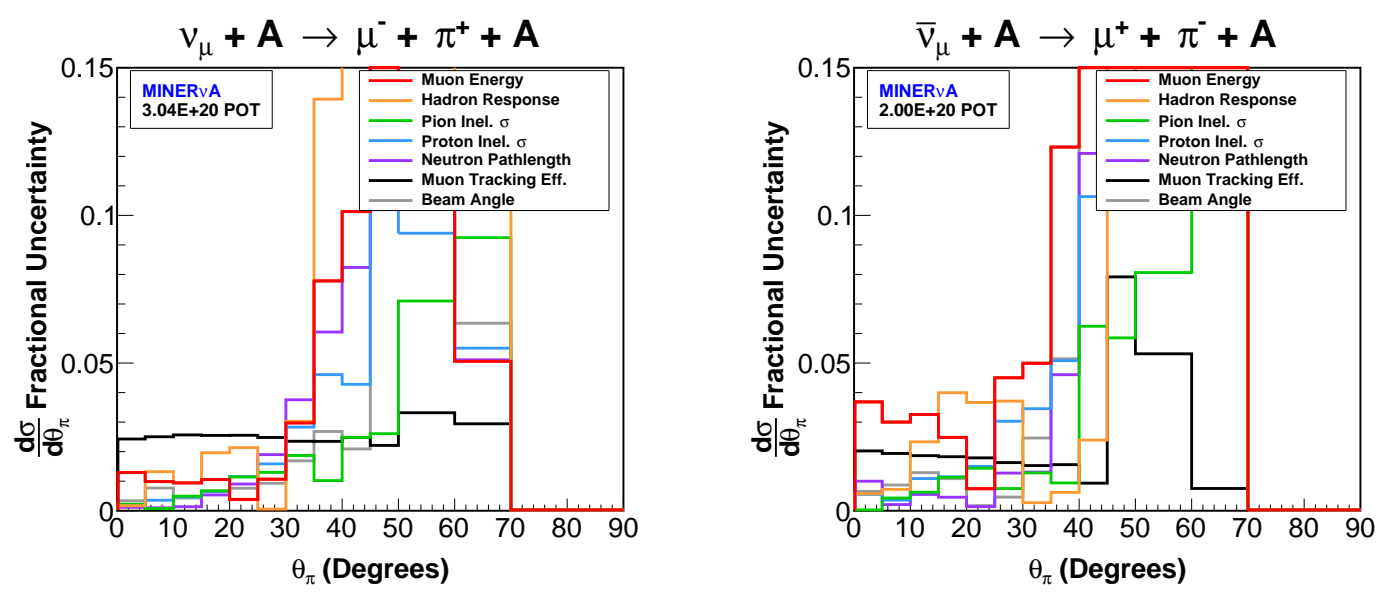

Figure 8.100: The fractional detector model uncertainties on the measured $\nu_{\mu}$ (left) and $\bar{\nu}_{\mu}$ (right) $\frac{d \sigma}{d \theta_{\pi}}$.
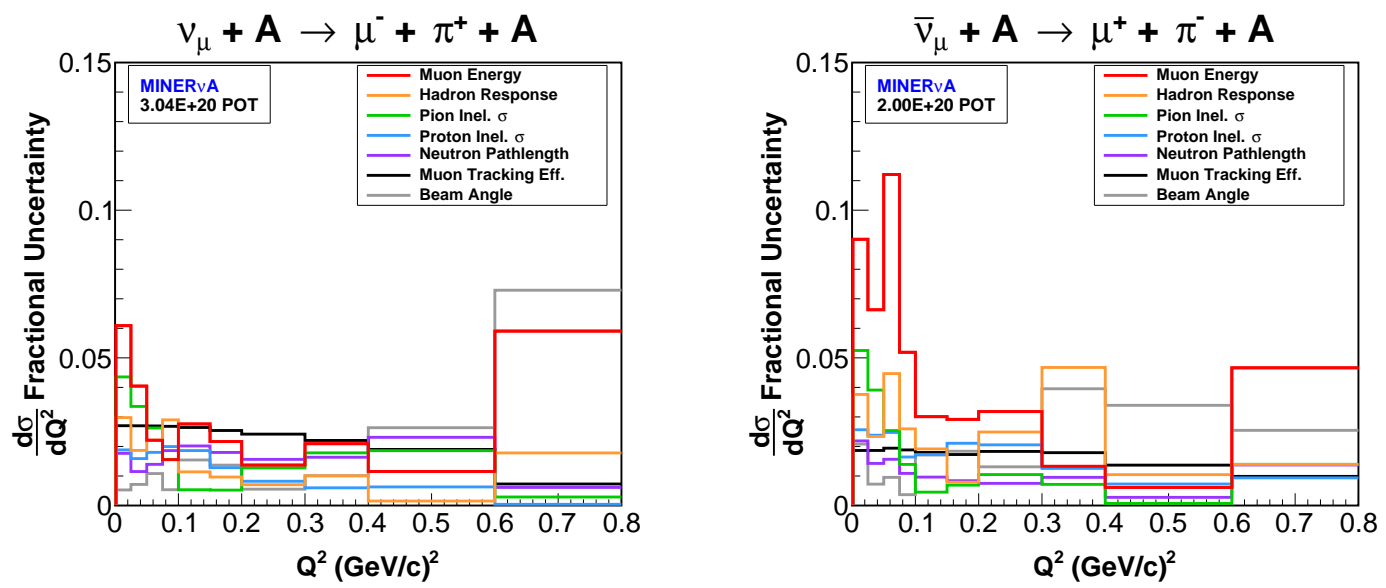

Figure 8.101: The fractional detector model uncertainties on the measured $\nu_{\mu}$ (left) and $\bar{\nu}_{\mu}$ (right) $\frac{d \sigma}{d Q^{2}}$. 


\section{Muon Tracking Efficiency}

Per Section 8.5, the MC was reweighted to correct the difference between MC and the data in the efficiency of tracking the muon in MINOS due to pile-up not being simulated in MINOS. An uncertainty on the correction to the MC tracking efficiency was included in the cross section results, which was evaluated by varying the size of the correction (Table 8.4) by $\pm 50 \%$.

\section{Muon Energy Scale}

Systematic uncertainty on the reconstructed muon energy affects the reconstructed values of other kinematic parameters, as well as the signal and background coherent candidate event rates via the cuts on $E_{\nu}$ and $|t|$. The muon energy uncertainty consists of uncertainties on the MINOS track momentum and the muon energy loss within MINER $\nu \mathrm{A}$.

The momentum of a tracked muon in MINOS is measured by either the range of the track or its curvature in the magnetic field, with the range measurement being more precise. The range measurement uncertainty was estimated to be $2 \%$, and consists of uncertainties on the MINOS geometry and material models, muon energy loss calculation, and track vertex reconstruction [89]. The curvature measurement uncertainty was estimated using a high-statistics sample of rock muons that enter the front face of MINOS and stop in its fully instrumented region. The $\frac{1}{P_{\text {curve }}}-\frac{1}{P_{\text {range }}}$ distribution (Figure 8.102) was measured in both data and MC, where $P_{\text {curve }}$ and $P_{\text {range }}$ are the curvature and range measurements for a single muon, respectively. The difference between the data and MC distribution means established a $0.6 \%(2.5 \%)$ uncertainty on the curvature measurement beyond the $2 \%$ uncertainty on the range measurement for muon momentum above (below) $1 \mathrm{GeV} / \mathrm{c}$. The uncertainties were 

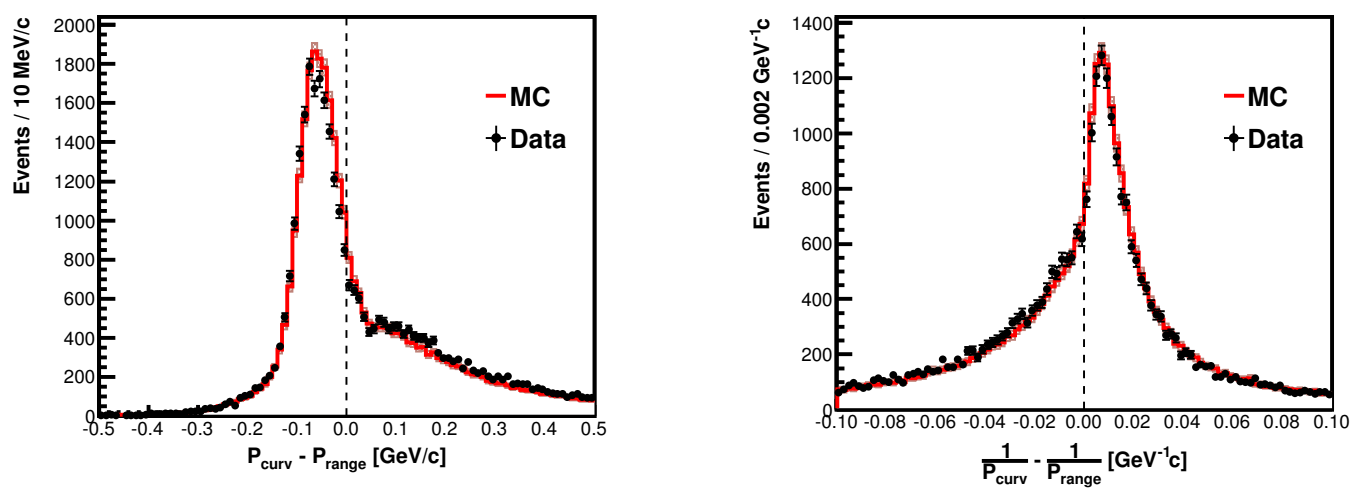

Figure 8.102: The $P_{\text {curve }}-P_{\text {range }}$ distribution (left) and $\frac{1}{P_{\text {curve }}}-\frac{1}{P_{\text {range }}}$ distribution (right) for $\nu_{\mu} \mathrm{CC}$ events in MINER $\nu \mathrm{A}$ where the muon stopped in the fully instrumented region of MINOS. $P_{\text {curve }}$ and $P_{\text {range }}$ are the momentum of the muon in MINOS reconstructed by the curvature and range of the MINOS muon track, respectively.

added in quadrature to give an estimated curvature measurement uncertainty of $2.1 \%(3.1 \%)$ for muon momentum above (below) $1 \mathrm{GeV} / \mathrm{c}$.

The uncertainty on the muon energy loss in MINER $\nu \mathrm{A}$ consisted of uncertainties on the MINER $\nu \mathrm{A}$ material assay and the Bethe-Bloch energy loss prediction. The effects of the material assay uncertainties were estimated by varying the detector mass in the muon energy loss calculation. The uncertainty on the Bethe-Bloch energy loss prediction was estimated by comparing the Bethe-Bloch muon range prediction to the Groom muon range tables [122] for the materials in the MINER $\nu \mathrm{A}$ detector. For muons originating in the tracker and exiting the back of MINER $\nu \mathrm{A}$, the effects of the material assay and Bethe-Bloch prediction uncertainties on the muon energy loss were estimated to be $11 \mathrm{MeV}$ and $30 \mathrm{MeV}$ on average, respectively.

For each event, the reconstructed muon energy uncertainty $\Delta E_{\mu}$ was the quadrature sum of the MINOS track momentum and MINER $\nu \mathrm{A}$ muon energy loss uncertainties. The muon energy uncertainty on the cross sections was evaluated by varying 

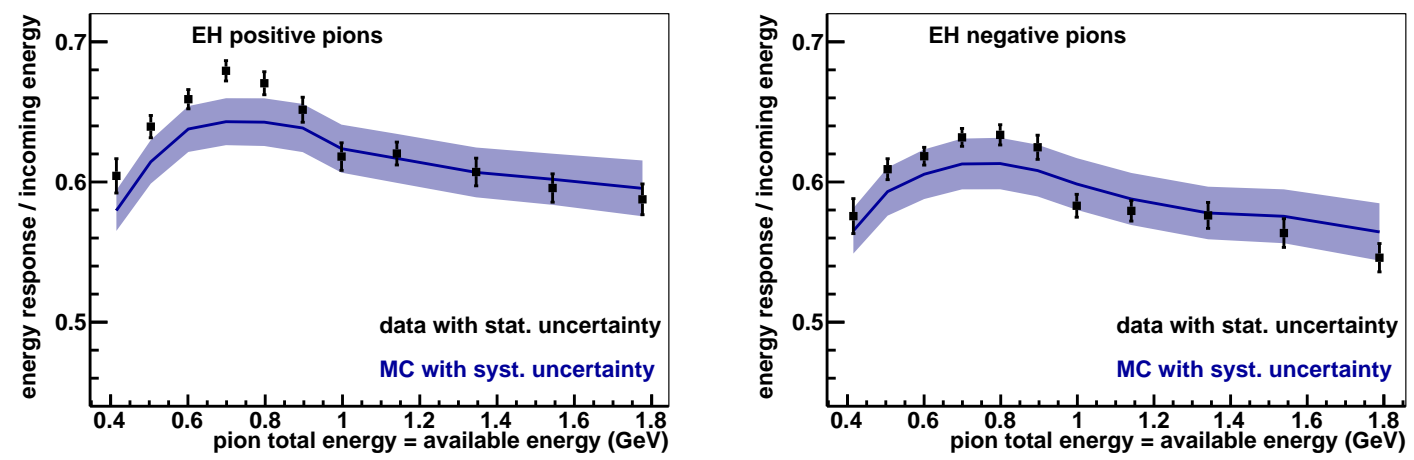

Figure 8.103: The mean ratio of visible energy to incoming energy for single $\pi^{+}$(left) and $\pi^{-}$(right) events in the MINER $\nu \mathrm{A}$ test beam EH configuration data and MC. The error on the data is the statistical uncertainty, and the error band on the MC is the systematic uncertainty consisting of beam line, detector, and event selection uncertainties.

the muon energy in the $\mathrm{MC}$ by $\pm \Delta E_{\mu}$

\section{Pion and Proton Response}

The calorimetric correction for reconstructing $E_{\pi}$ from the visible energy was tuned to the simulated response to pions. Uncertainty on the simulated response therefore affects the reconstructed event kinematics and the coherent candidate event rates. This uncertainty was constrained by measurements of the single pion and proton response in the MINER $\nu \mathrm{A}$ test beam data and $\mathrm{MC}$ [110]. The response for each pion and proton event was measured as the ratio of the visible energy to the incoming particle energy. The MC agreed with the data in the mean pion (proton) response to within 5\% (3\%) over the sampled incoming energy range (Figures 8.103 and 8.104). Using these results, the calorimetric response uncertainty on the cross sections was evaluated by varying the reconstructed $E_{\pi}$ in the $\mathrm{MC}$ by $\pm 5 \%( \pm 3 \%)$ for events with a tracked pion (proton). 


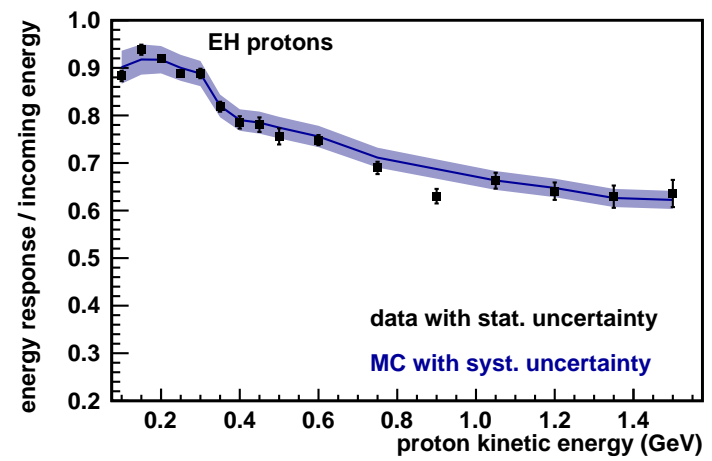

Figure 8.104: The mean ratio of visible energy to incoming energy for single proton events in the MINER $\nu \mathrm{A}$ test beam EH configuration data and MC. The error on the data is the statistical uncertainty, and the error band on the $\mathrm{MC}$ is the systematic uncertainty consisting of beam line, detector, and event selection uncertainties.

\section{Pion \& Proton Interaction Cross Section}

The interaction of pions and protons within MINER $\nu \mathrm{A}$ affects the pion/proton tracking efficiency and angular resolution, vertex energy, proton score, and calorimetric response. The uncertainty on the measured coherent cross sections due the uncertainty on the pion/proton interaction rate in the $\mathrm{MC}$ was evaluated by varying the rate of pion/proton inelastic scattering on carbon in the $\mathrm{MC}$ by $\pm 10 \%$. The size of the variation was determined from comparisons of Geant4 to hadron scattering data [123]. The inelastic scattering rate was varied using an event-by-event weighting technique [124], where a weight was calculated for each final state pion/proton. The weight for a pion/proton that interacted inelastically was calculated as

$$
W_{\text {inel }}=\frac{1-e^{-\rho x \sigma_{\text {inel }}(1+\delta)}}{1-e^{-\rho x \sigma_{\text {inel }}}},
$$


and the weight for a pion/proton that did not interact inelastically was calculated as

$$
W_{\text {non-inel }}=\frac{e^{-\rho x \sigma_{\text {inel }}(1+\delta)}}{e^{-\rho x \sigma_{\text {inel }}}}=e^{-\rho x \sigma_{\text {inel }} \delta}
$$

where $\rho$ is the average density of the scintillator planes, $x$ is the total path length of the particle, $\sigma_{\text {inel }}$ is the energy averaged pion/proton inelastic scattering cross section on carbon, and $\delta$ is the variation on the inelastic scattering rate. The energy averaged cross section was used in calculating the weights to account for the dependence of the cross section on the pion/proton energy, and was calculated as

$$
\sigma_{\text {inel }}=\frac{1}{E_{f}-E_{i}} \int_{E_{i}}^{E_{f}} \sigma_{\text {inel }}(E) d E,
$$

where $E_{i}$ and $E_{f}$ are the initial and final kinetic energy of the pion/proton, respectively, and $\sigma_{\text {inel }}(E)$ is a parameterization of the pion/proton inelastic scattering cross section on carbon from the hadron scattering data. The weight for each MC event was the product of the pion/proton weights. The event weights were normalized to preserve the total neutrino interaction rate for each MC event category.

The pion and proton calorimetric response uncertainties from the test beam measurements include uncertainties on the pion and proton interaction rates. The separate evaluation of the interaction rate uncertainties here double counts their contribution to the calorimetric response uncertainties. Since it is difficult in practice to isolate their contribution to the calorimetric response uncertainties, the pion and proton interaction rate uncertainties on the calorimetric response are conservatively double counted. 


\section{Neutron Path Length}

In MINER $\nu \mathrm{A}$, neutrons tend to either exit the detector without depositing any energy or deposit a small fraction of their energy via a hadronic interaction. The neutron interaction rate therefore has a small effect on the calorimetric response. Final state neutrons that promptly interact can affect the vertex energy and occasionally produce a tracked proton or pion. The neutron interaction rate therefore affects the predicted rate of background coherent candidates.

The uncertainty on the measured coherent cross sections due the uncertainty on the neutron interaction rate in the $\mathrm{MC}$ was evaluated by varying the neutron mean free path [125]. The mean free path was varied $\pm 25 \%$ for neutron kinetic energy below $40 \mathrm{MeV}, \pm 10 \%$ between 50 and $150 \mathrm{MeV}$, and $\pm 20 \%$ above $300 \mathrm{MeV}$. The variation in the undefined energy regions was interpolated. The amount of variation was determined from comparisons of GEANT4 and neutron scattering data [123]. A weight was calculated for each final state neutron, which depended on the path length of the neutron in the tracker region and whether the neutron interacted inside the tracker region. The weight applied to each MC event was the product of its neutron weights. The event weights were normalized to preserve the total neutrino interaction rate for each $\mathrm{MC}$ event category.

\section{Beam Direction}

In reconstructing our event kinematics we assumed the incoming neutrino direction was parallel to the neutrino beam axis. The measured cross sections are therefore sensitive to uncertainty on the beam direction in the geometry model. The measurement of the beam direction using the $\nu_{\mu}$ and $\bar{\nu}_{\mu}$ CC low- $\nu$ samples agreed with the beam direction in the geometry model to within $\sim 3 \mathrm{mrad}$ in both the vertical (YZ) 
and horizontal (XZ) planes (Section 8.12). Using these results, the beam direction uncertainty on the cross sections was evaluated by varying the beam direction \pm 3 mrad in both the vertical and horizontal planes.

\subsection{4 $\nu_{\mu}-\bar{\nu}_{\mu}$ Cross Section Comparisons}

The measured $\nu_{\mu}$ and $\bar{\nu}_{\mu}$ coherent cross sections are compared to test the prediction of the PCAC coherent models that the neutrino and antineutrino cross sections are equal for a particular $E_{\nu}$. This prediction results from the assumption of no VA interference in the scattering amplitude. The measured $\nu_{\mu}$ and $\bar{\nu}_{\mu} \sigma\left(E_{\nu}\right)$ can be compared directly in each $E_{\nu}$ bin. The measured $\nu_{\mu}$ and $\bar{\nu}_{\mu}$ differential cross sections cannot be compared directly since they are integrated over different $E_{\nu}$ spectra (Figure 8.105). The effect of the different $E_{\nu}$ spectra on the $\nu_{\mu}$ and $\bar{\nu}_{\mu}$ differential cross sections predicted by the GENIE implementation of the Rein-Sehgal coherent model is illustrated in Figure 8.106. The $\bar{\nu}_{\mu}$ flux is $\sim \frac{1}{2}$ the $\nu_{\mu}$ flux for $5<E_{\nu}<20$ $\mathrm{GeV}$, which results in a lower $\bar{\nu}_{\mu}$ cross section at high- $E_{\pi}$, low- $\theta_{\pi}$, and high- $Q^{2}$.

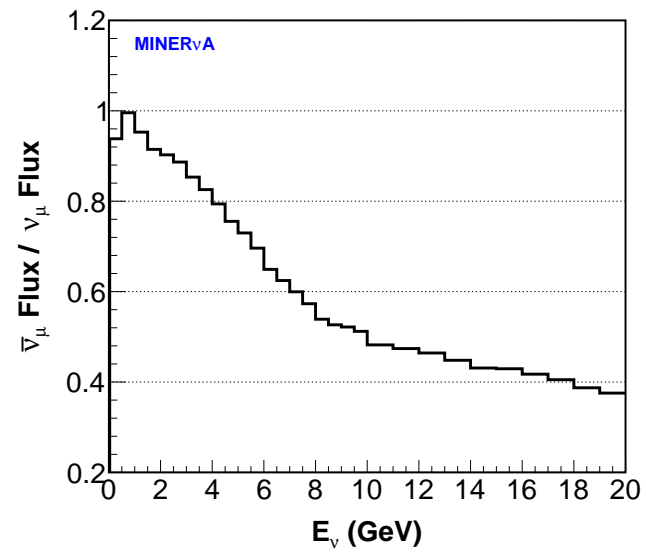

Figure 8.105: $\bar{\nu}_{\mu}$-to- $\nu_{\mu}$ flux prediction ratio 

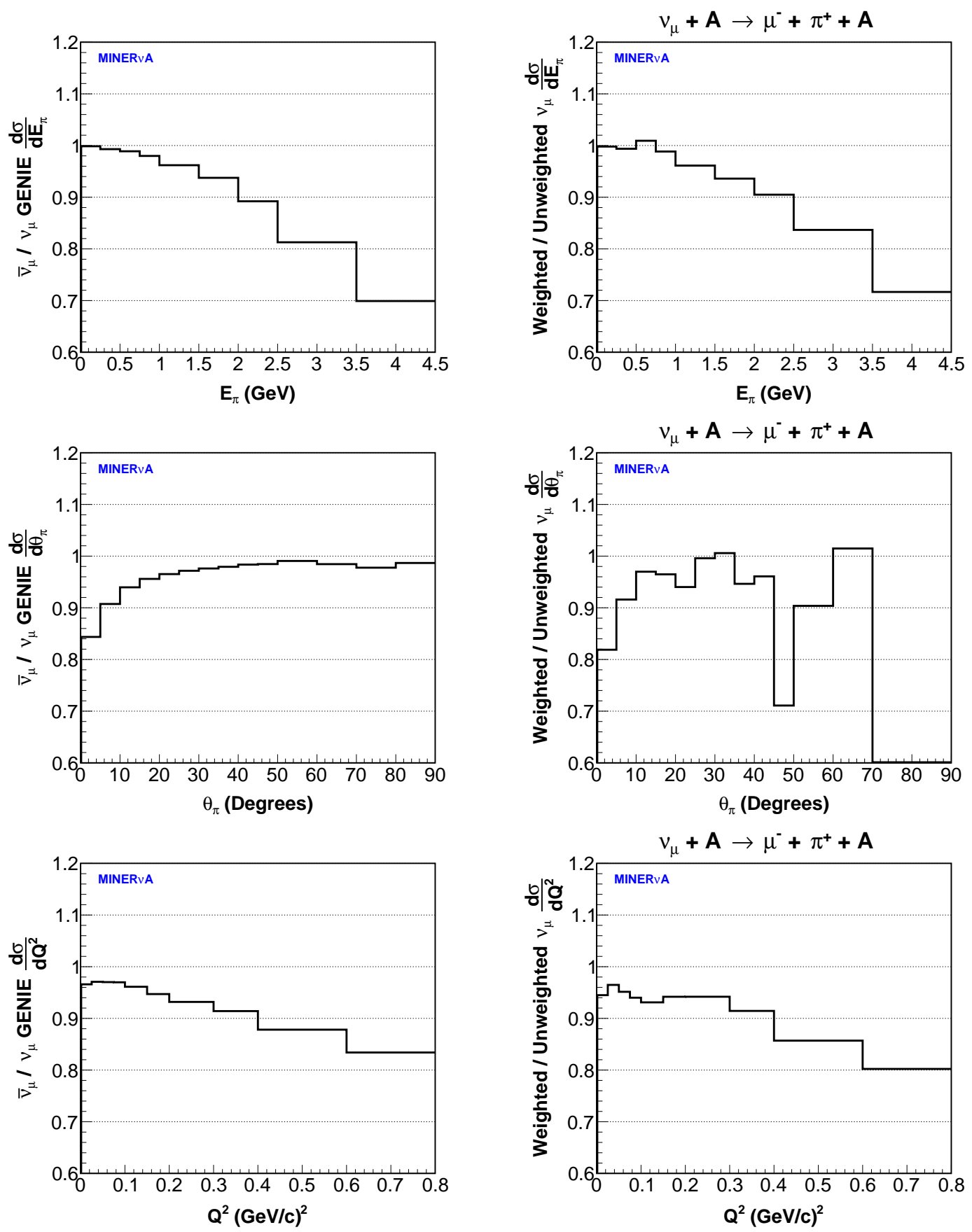

Figure 8.106: Left plots: the ratio of the coherent differential cross sections predicted by GENIE for the $\bar{\nu}_{\mu}$ flux to that for the $\nu_{\mu}$ flux. Right plots: the ratio of the measured $\nu_{\mu}$ coherent differential cross sections weighted to the $\bar{\nu}_{\mu}$ flux to the unweighted measured $\nu_{\mu}$ coherent differential cross sections. 
To compare the measured $\nu_{\mu}$ and $\bar{\nu}_{\mu}$ differential cross sections, the $\nu_{\mu}$ cross sections are weighted to the $\bar{\nu}_{\mu}$ flux spectrum. The $\nu_{\mu}$ cross sections are weighted instead of the $\bar{\nu}_{\mu}$ cross sections to minimize amplifying statistical fluctuations. Ideally, the weighted $\nu_{\mu}$ cross sections would be obtained by measuring the two-dimensional cross sections $\frac{d \sigma\left(E_{\nu}\right)}{d E_{\pi}}, \frac{d \sigma\left(E_{\nu}\right)}{d \theta_{\pi}}$, and $\frac{d \sigma\left(E_{\nu}\right)}{d Q^{2}}$, weighting the two-dimensional cross sections as a function of $E_{\nu}$ by the flux ratio $\phi^{\bar{\nu} \mu}\left(E_{\nu}\right) / \phi^{\nu_{\mu}}\left(E_{\nu}\right)$, and integrating over $E_{\nu}$. However, measuring the two-dimensional cross sections is precluded by the statistics of the coherent candidate event samples. The alternative approach employed was to weight the $\nu_{\mu}$ background subtracted coherent candidate event rates, unfolding matrices, efficiency corrections, and flux normalization to the $\bar{\nu}_{\mu}$ flux and remeasure the $\nu_{\mu}$ cross sections. The $\nu_{\mu}$ background subtracted coherent candidate event rate in reconstructed kinematic parameter bin $j$ was weighted as a function of reconstructed $E_{\nu}$ as

$$
N_{j}^{\text {data }}-N_{j}^{\text {bkgd }}=\sum_{k} W_{k}^{\text {reco }}\left(N_{j k}^{\text {data }}-N_{j k}^{b k g d}\right)
$$

where $W_{k}^{\text {reco }}$ is the weight for reconstructed $E_{\nu}$ bin $k . W_{k}^{\text {reco }}$ was calculated as

$$
W_{k}^{r e c o}=\sum_{m} U_{k m}^{\nu_{\mu}} \frac{\phi_{m}^{\bar{\nu}_{\mu}}}{\phi_{m}^{\nu_{\mu}}}
$$

where $\phi_{m}^{\bar{\nu}_{\mu}}$ and $\phi_{m}^{\nu_{\mu}}$ are the $\bar{\nu}_{\mu}$ and $\nu_{\mu}$ fluxes per POT in true $E_{\nu}$ bin $m$, respectively, and $U_{k m}^{\nu_{\mu}}$ is the $\nu_{\mu} E_{\nu}$ unfolding matrix element normalized to give the estimated fraction of selected coherent events in reconstructed bin $k$ originating from true bin $m$. The $\nu_{\mu}$ unfolding matrices and efficiency corrections were weighted to the $\bar{\nu}_{\mu}$ flux by weighting each event in the $\nu_{\mu}$ signal-only MC sample by the flux ratio $\phi^{\bar{\nu}_{\mu}} / \phi^{\nu_{\mu}}$ 
for the event $E_{\nu}$ and recalculating the unfolding matrices and efficiency corrections. The $\nu_{\mu}$ flux was weighted as a function of $E_{\nu}$ by the flux ratio $\phi^{\bar{\nu}_{\mu}}\left(E_{\nu}\right) / \phi^{\nu_{\mu}}\left(E_{\nu}\right)$ and integrated to give the flux normalization for the weighted $\nu_{\mu}$ differential cross sections. The fractional systematic uncertainty on the weighted $\nu_{\mu}$ differential cross sections was forced to be equal to that on the unweighted cross sections by preserving the fractional difference of each systematic variation in the weighting. The ratio of the weighted to unweighted $\nu_{\mu}$ differential cross sections is shown in Figure 8.106. For the remainder of this section, $\nu_{\mu}$ differential cross section refers to the $\nu_{\mu}$ differential cross section weighted to the $\bar{\nu}_{\mu}$ flux.
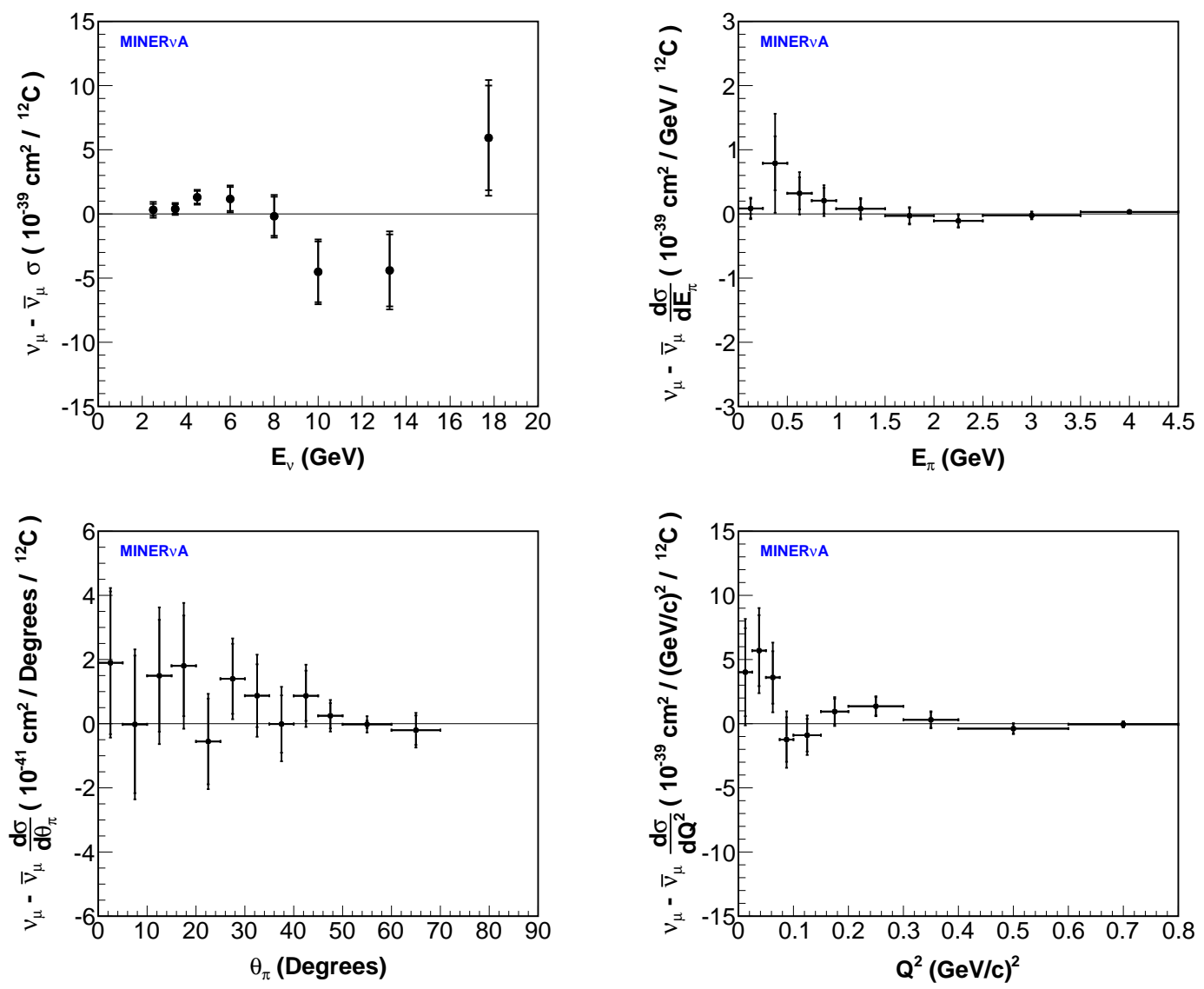

Figure 8.107: The measured $\bar{\nu}_{\mu}-\nu_{\mu}$ cross section differences 
The measured $\nu_{\mu}-\bar{\nu}_{\mu}$ cross section differences are shown in Figure 8.107. The uncertainties on the cross section differences were calculated from the combined statistical and systematic covariance matrices for the $\nu_{\mu}$ and $\bar{\nu}_{\mu}$ cross sections. The combined statistical covariance matrix is the sum of the $\nu_{\mu}$ and $\bar{\nu}_{\mu}$ statistical covariance matrices. The elements of the combined covariance matrix for each systematic uncertainty $C^{\text {sys }}$ were calculated as

$$
C_{i j}^{s y s}=C_{i j}^{\nu_{\mu}}+C_{i j}^{\bar{\nu}_{\mu}}-C_{i j}^{\nu_{\mu} \bar{\nu}_{\mu}}-C_{j i}^{\nu_{\mu} \bar{\nu}_{\mu}}
$$

where $C^{\nu_{\mu}}$ and $C^{\bar{\nu}_{\mu}}$ are the systematic covariance matrices for the $\nu_{\mu}$ and $\bar{\nu}_{\mu}$ cross sections, respectively, and $C^{\nu_{\mu} \bar{\nu}_{\mu}}$ is the joint systematic covariance matrix for the $\nu_{\mu}$ and $\bar{\nu}_{\mu}$ cross sections. The elements of $C^{\nu_{\mu} \bar{\nu}_{\mu}}$ were calculated as

$$
C_{i j}^{\nu_{\mu} \bar{\nu}_{\mu}}=\frac{1}{N} \sum_{k} \Delta \sigma_{i k}^{\nu_{\mu}} \Delta \sigma_{j k}^{\bar{\nu}_{\mu}}
$$

where $\Delta \sigma_{i k}^{\nu_{\mu}}\left(\Delta \sigma_{j k}^{\bar{\nu}_{\mu}}\right)$ is the change to the $\nu_{\mu}\left(\bar{\nu}_{\mu}\right)$ cross section in bin $i(j)$ for variation $k$, and $N$ is the number of variations from which the systematic uncertainty was evaluated. The $\chi^{2}$ for the deviation of each $\nu_{\mu}-\bar{\nu}_{\mu}$ cross section difference from zero (the prediction of the PCAC coherent models) are listed in Table 8.21 and were calculated as

$$
\chi^{2}=A C^{-1} A^{T},
$$

where $C$ is the total covariance (statistical + total systematic) matrix for the cross section difference and the elements of the vector $A$ are defined as

$$
A_{i}=\sigma_{i}^{\nu_{\mu}}-\sigma_{i}^{\bar{\nu}_{\mu}}
$$


where $\sigma_{i}^{\nu_{\mu}}$ and $\sigma_{i}^{\bar{\nu} \mu}$ are the $\nu_{\mu}$ and $\bar{\nu}_{\mu}$ cross sections in bin $i$. The $\chi^{2}$ probabilities (Table 8.21) imply the deviation of the $\nu_{\mu}-\bar{\nu}_{\mu}$ cross section differences from zero are not significant and are consistent with the prediction of the PCAC coherent models that the neutrino and antineutrino cross sections are equal.

\begin{tabular}{c|ccc} 
Cross Section & $\chi^{2}$ & NDF & $\chi^{2}$ Probability \\
\hline$\sigma E_{\nu}$ & 11.4 & 8 & 0.181 \\
$d \sigma / d E_{\pi}$ & 5.5 & 9 & 0.791 \\
$d \sigma / d \theta_{\pi}$ & 5.3 & 12 & 0.948 \\
$d \sigma / d Q^{2}$ & 11.2 & 10 & 0.341
\end{tabular}

Table 8.21: The $\chi^{2}$, number of degrees of freedom (NDF), and $\chi^{2}$ probability for the deviation of the measured $\nu_{\mu}-\bar{\nu}_{\mu}$ cross section differences from zero.

The correlation matrices for the flux and non-flux systematic uncertainties on the measured $\nu_{\mu}$ and $\nu_{\mu}$ cross sections are shown in Figures 8.109-8.116. The flux uncertainty on the $\nu_{\mu}$ and $\bar{\nu}_{\mu} \sigma\left(E_{\nu}\right)$ (Figure 8.109) contains bin-to-bin anti-correlations not present in the uncertainty on the predicted $\nu_{\mu}$ and $\bar{\nu}_{\mu}$ fluxes (Figure 8.108). The origin of these anti-correlations is explained in Section 8.13.1. 

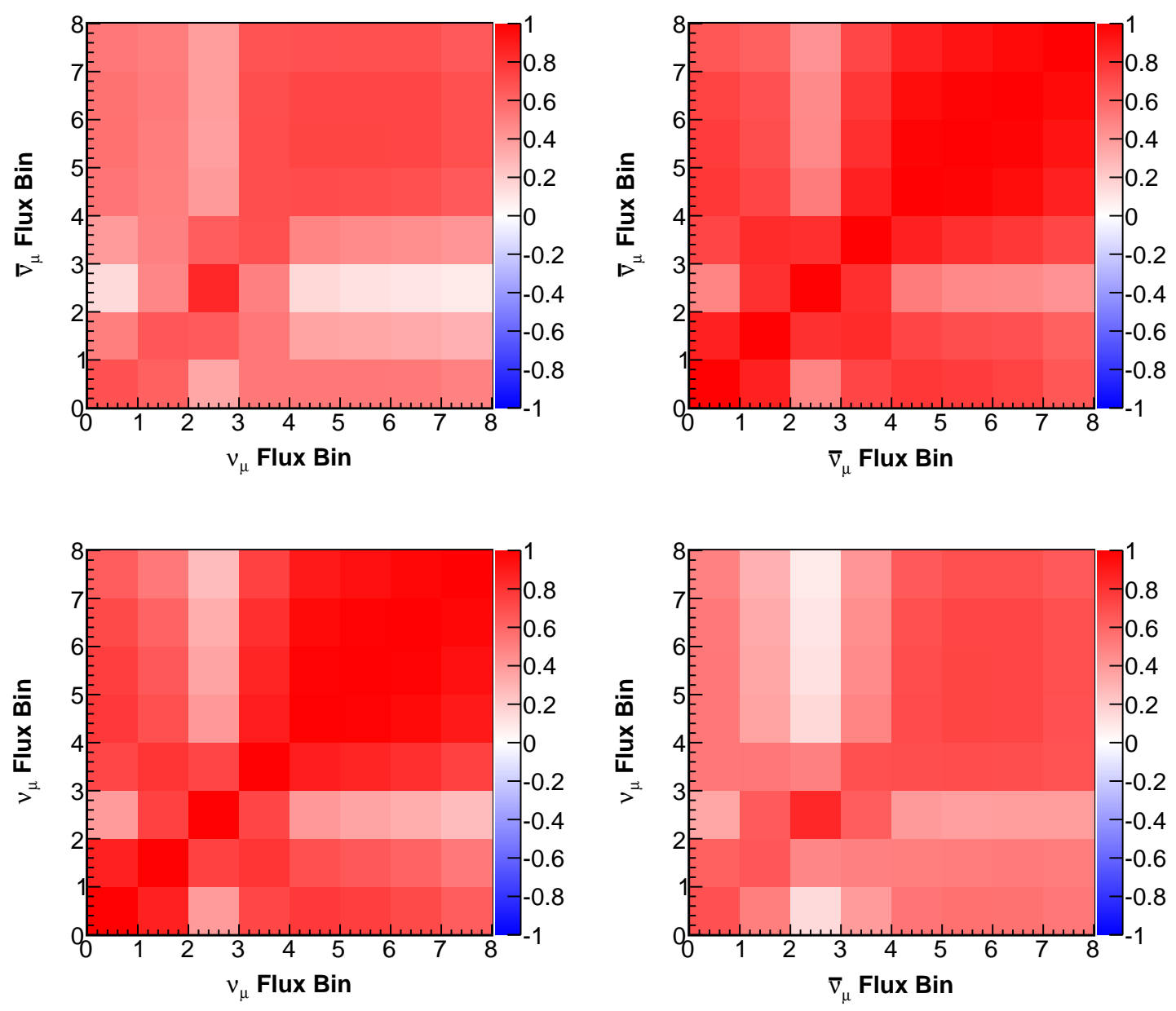

Figure 8.108: The correlation matrices for the uncertainty on the $\nu_{\mu}$ and $\bar{\nu}_{\mu}$ flux predictions in the measured $\sigma\left(E_{\nu}\right)$ bins 

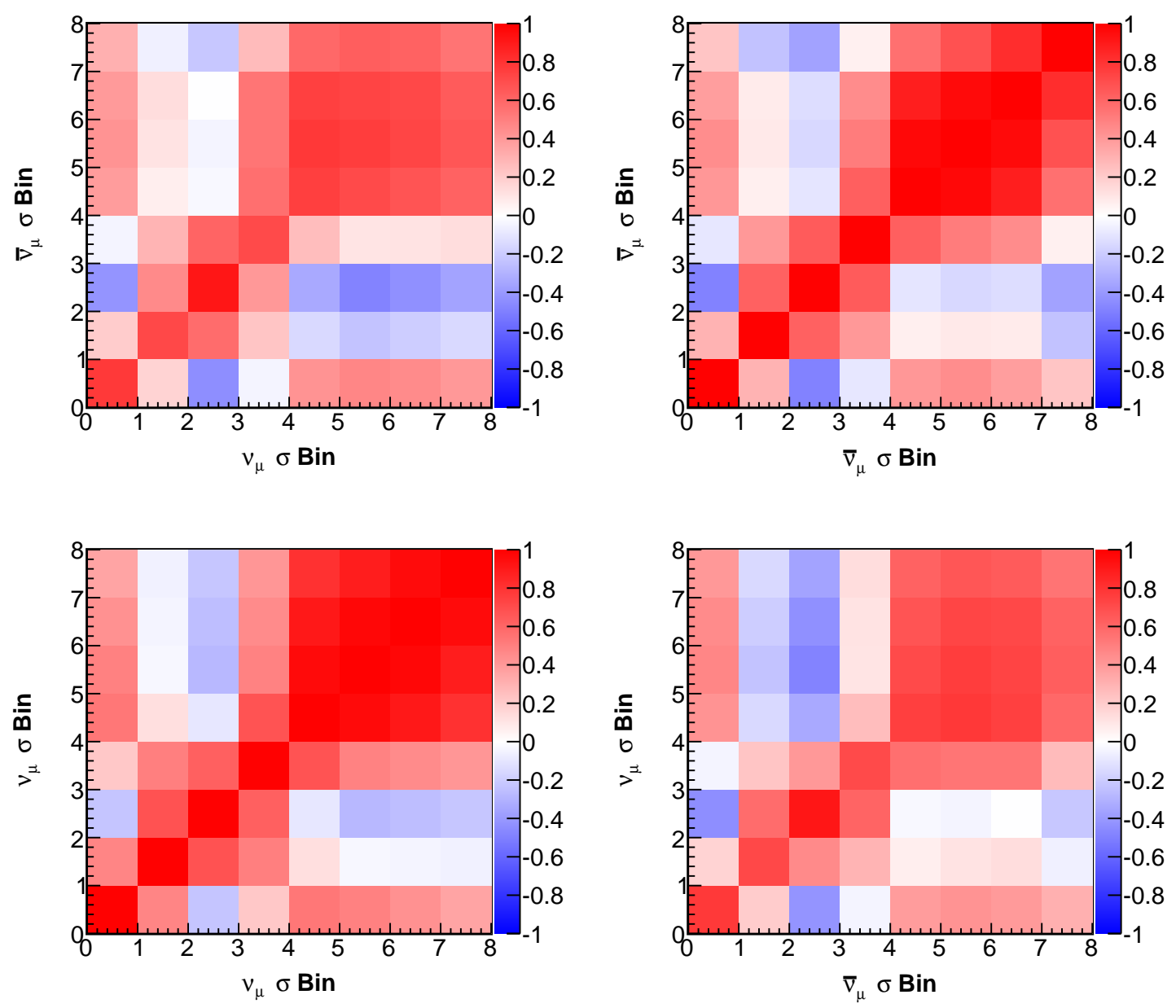

Figure 8.109: The correlation matrices for the flux uncertainty on the measured $\nu_{\mu}$ and $\bar{\nu}_{\mu} \sigma\left(E_{\nu}\right)$ 

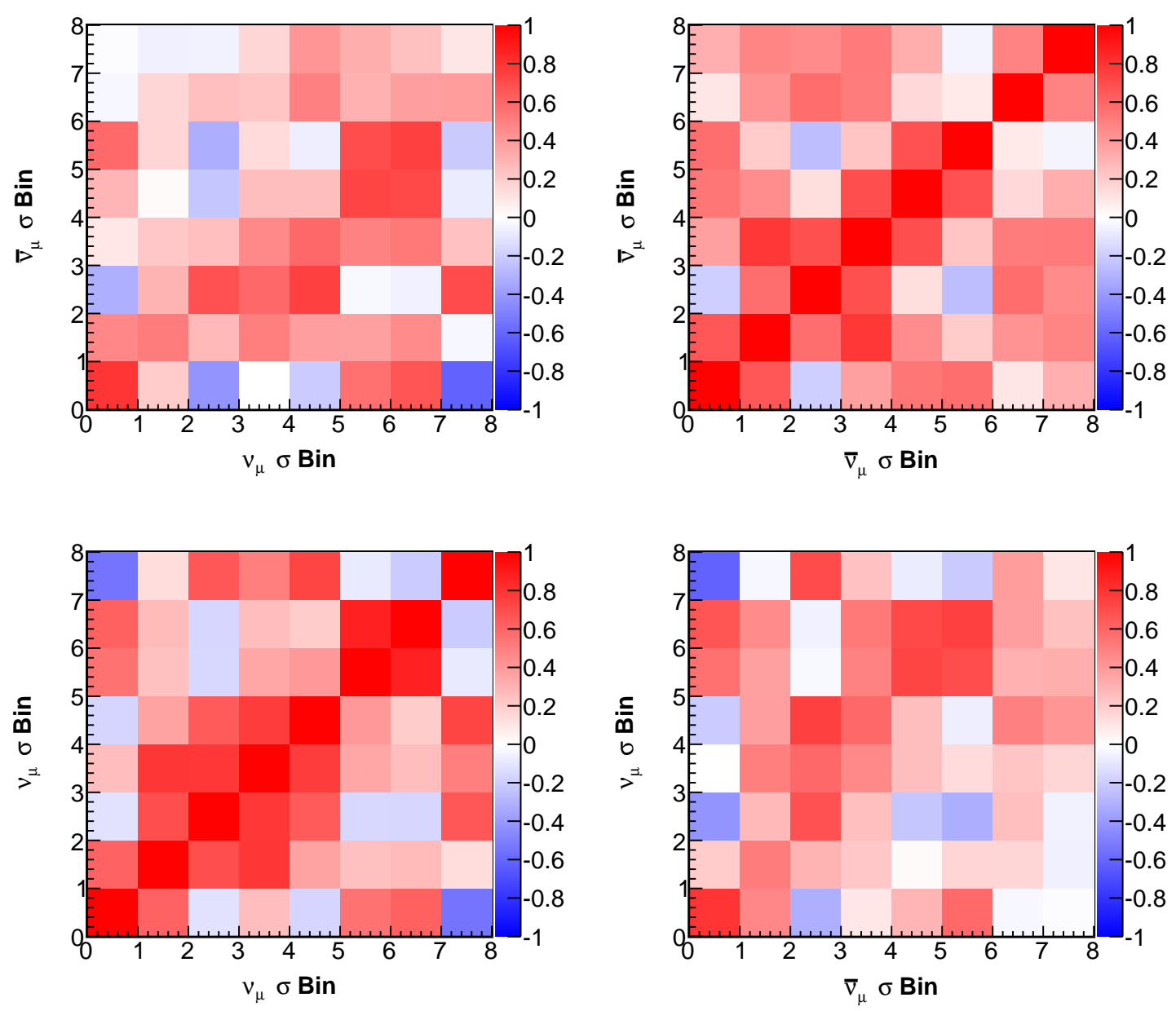

Figure 8.110: The correlation matrices for the non-flux systematic uncertainties on the measured $\nu_{\mu}$ and $\bar{\nu}_{\mu} \sigma\left(E_{\nu}\right)$ 

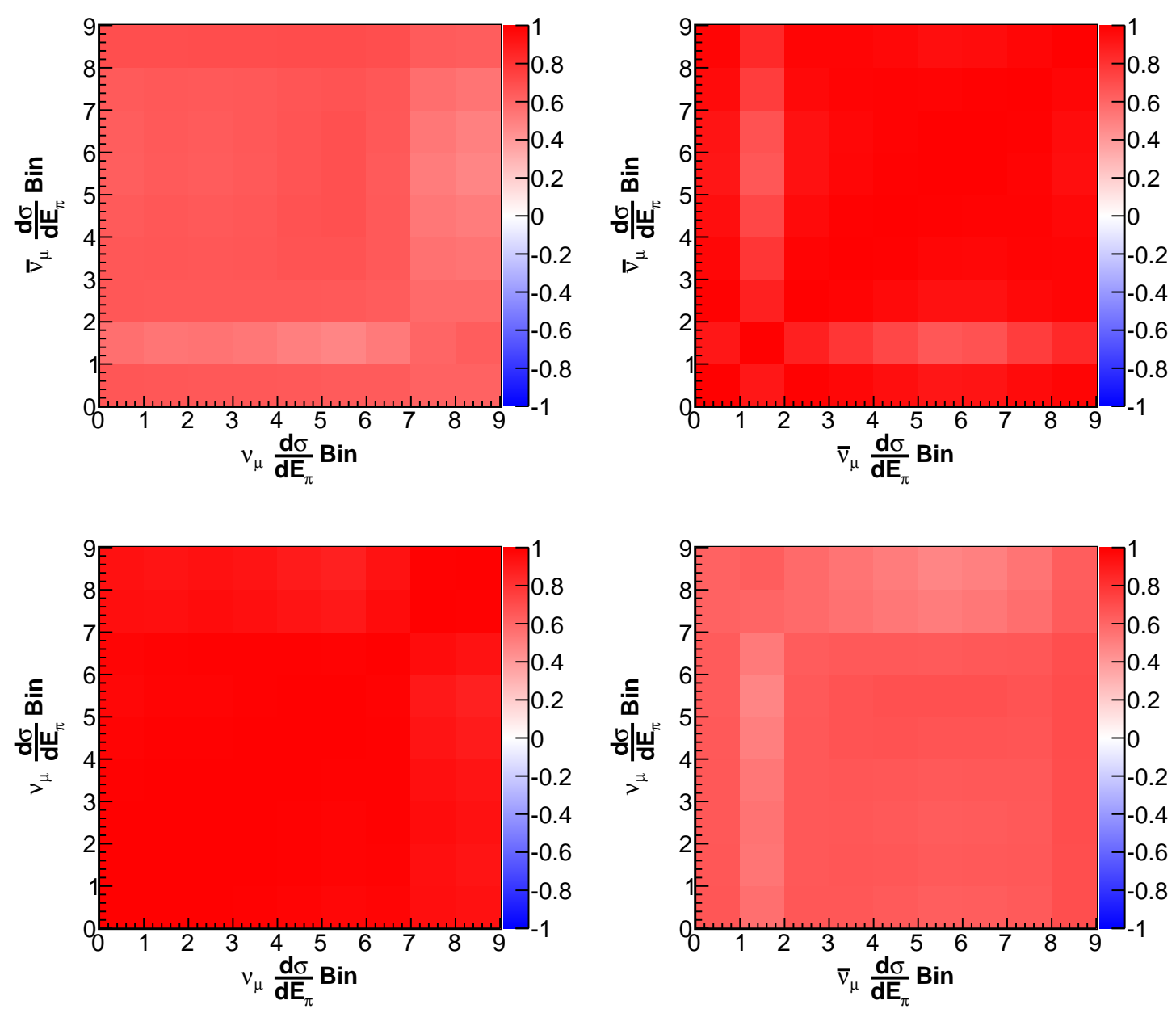

Figure 8.111: The correlation matrices for the flux uncertainty on the measured $\nu_{\mu}$ and $\bar{\nu}_{\mu} \frac{d \sigma}{d E_{\pi}}$ 

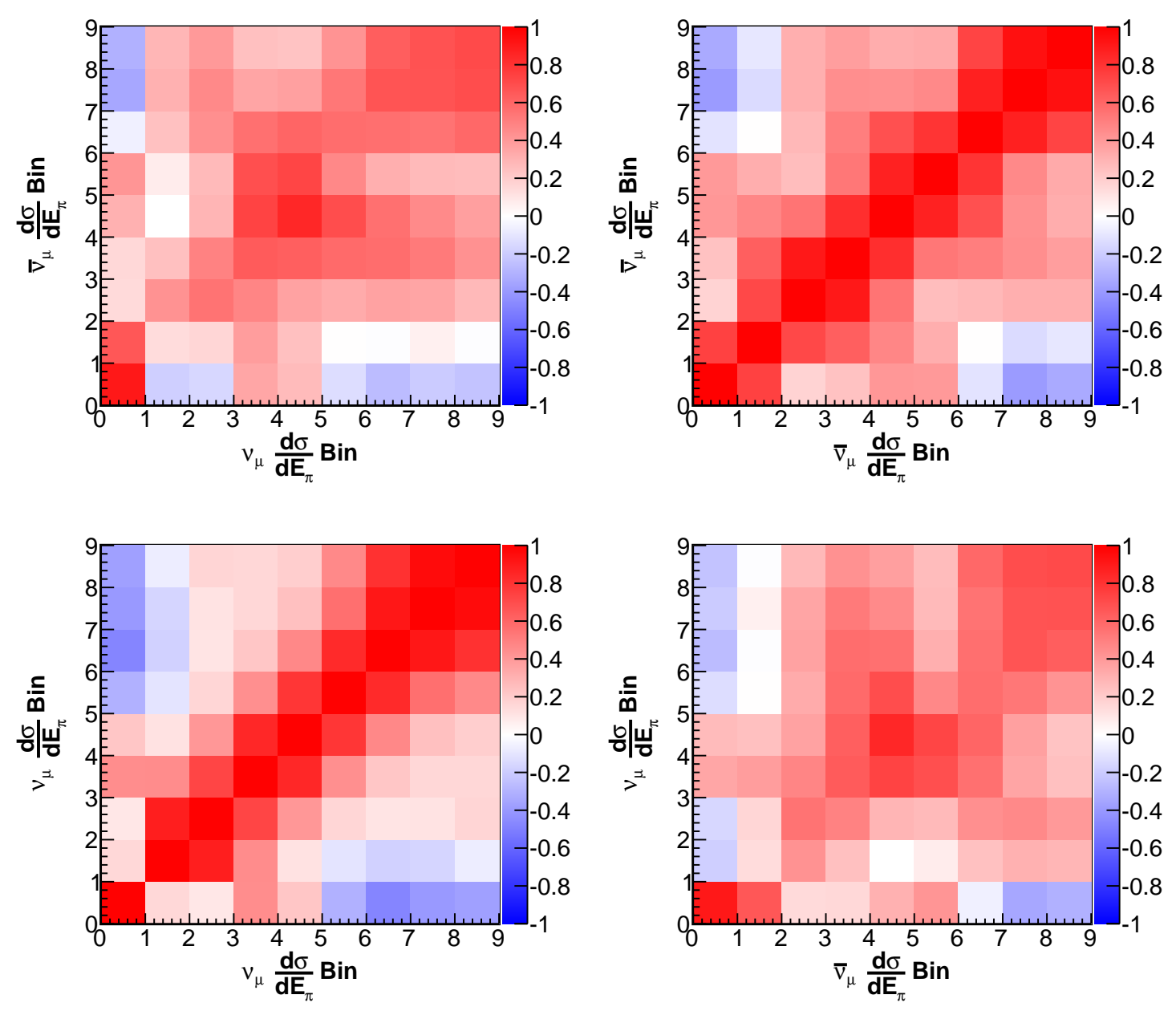

Figure 8.112: The correlation matrices for the non-flux systematic uncertainties on the measured $\nu_{\mu}$ and $\bar{\nu}_{\mu} \frac{d \sigma}{d E_{\pi}}$ 

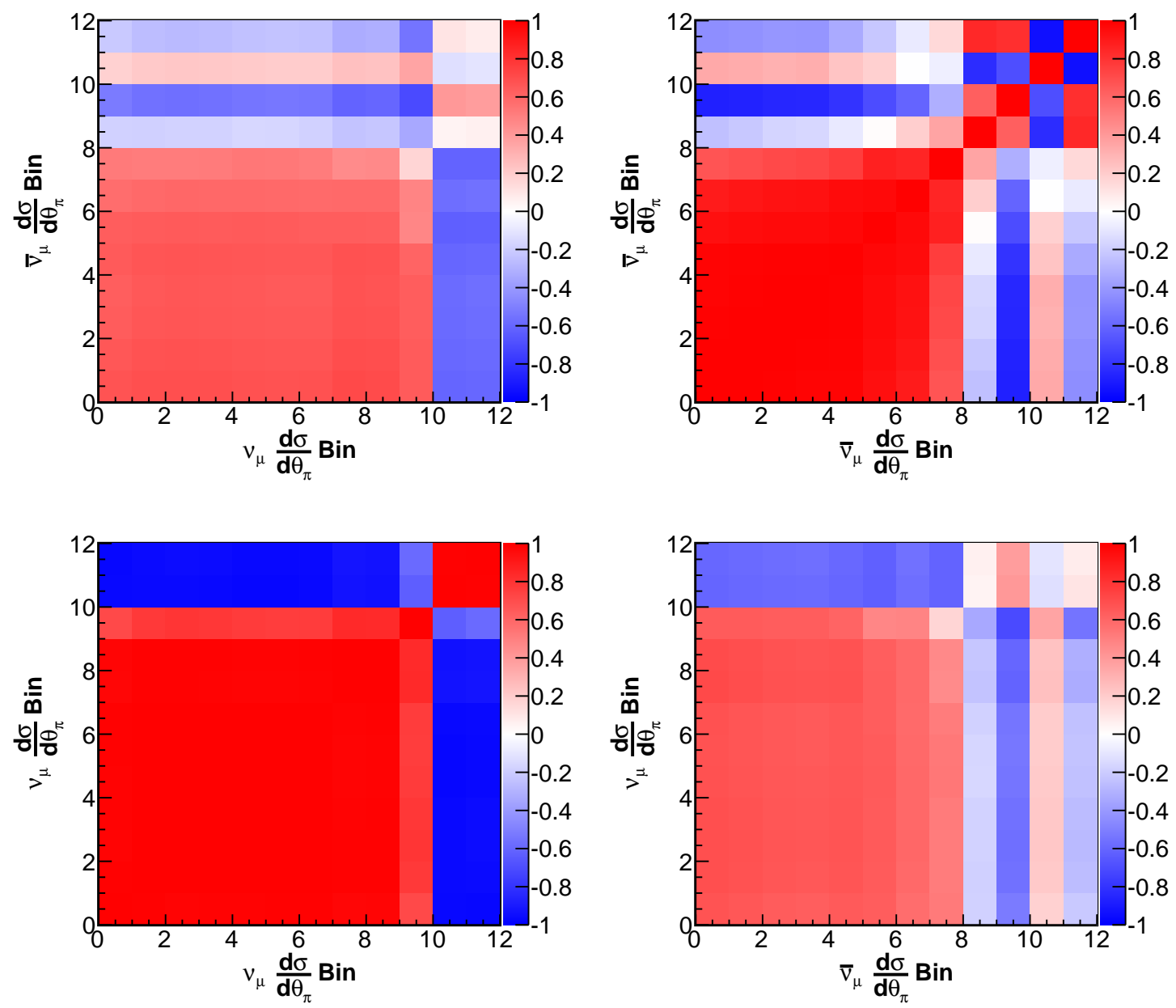

Figure 8.113: The correlation matrices for the flux uncertainty on the measured $\nu_{\mu}$ and $\bar{\nu}_{\mu} \frac{d \sigma}{d \theta_{\pi}}$ 

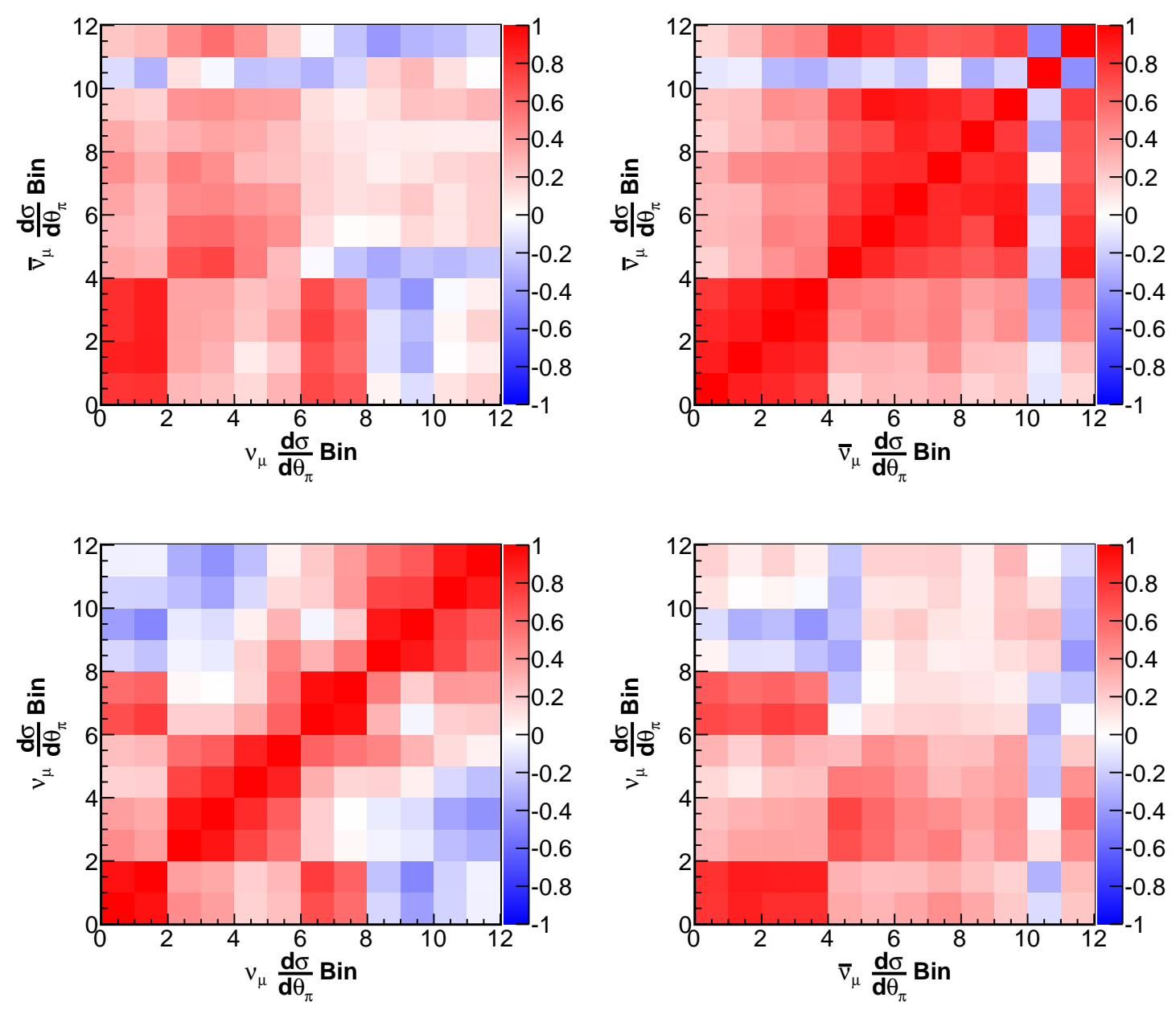

Figure 8.114: The correlation matrices for the non-flux systematic uncertainties on the measured $\nu_{\mu}$ and $\bar{\nu}_{\mu} \frac{d \sigma}{d \theta_{\pi}}$ 

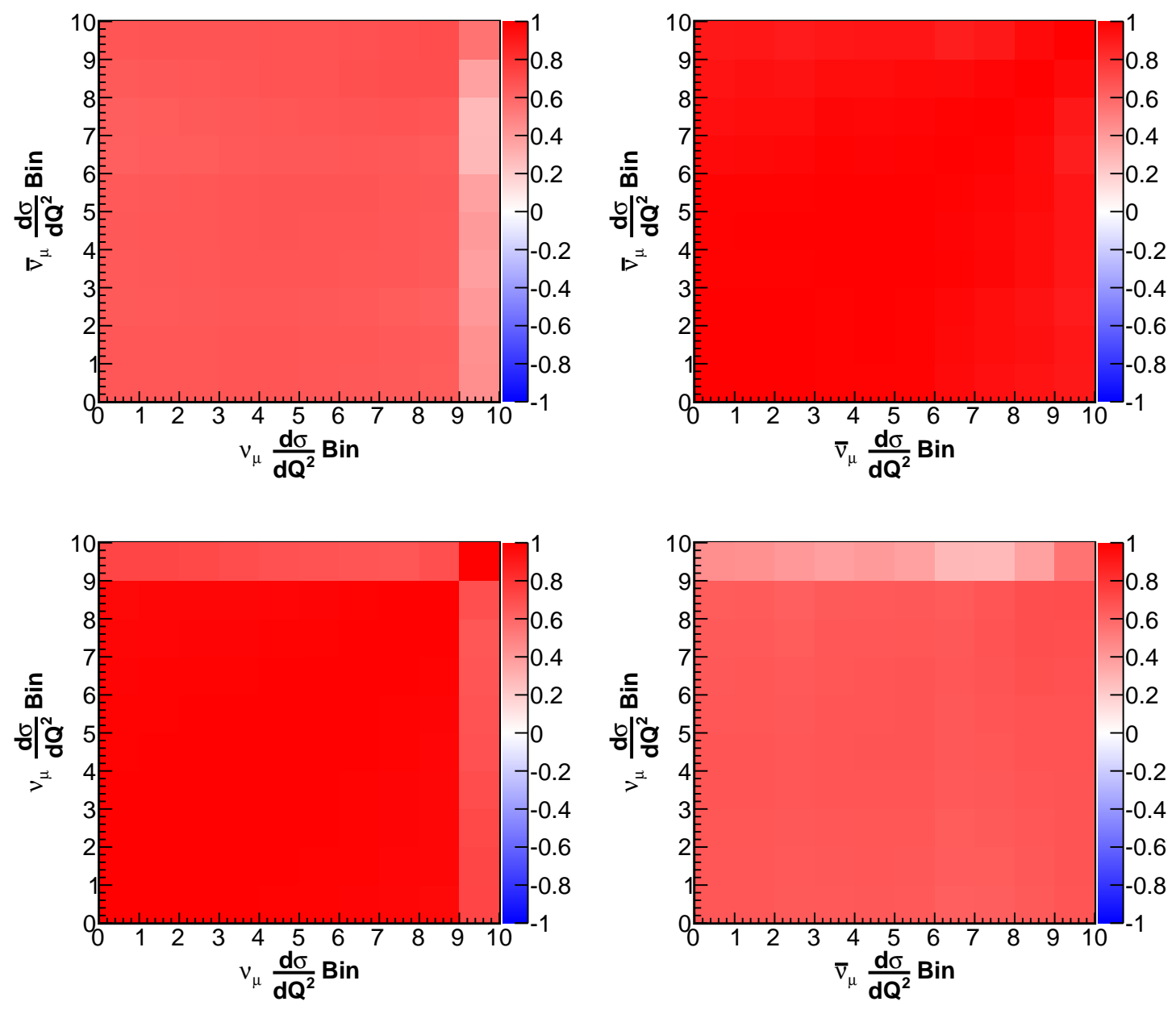

Figure 8.115: The correlation matrices for the flux uncertainty on the measured $\nu_{\mu}$ and $\bar{\nu}_{\mu} \frac{d \sigma}{d Q^{2}}$ 

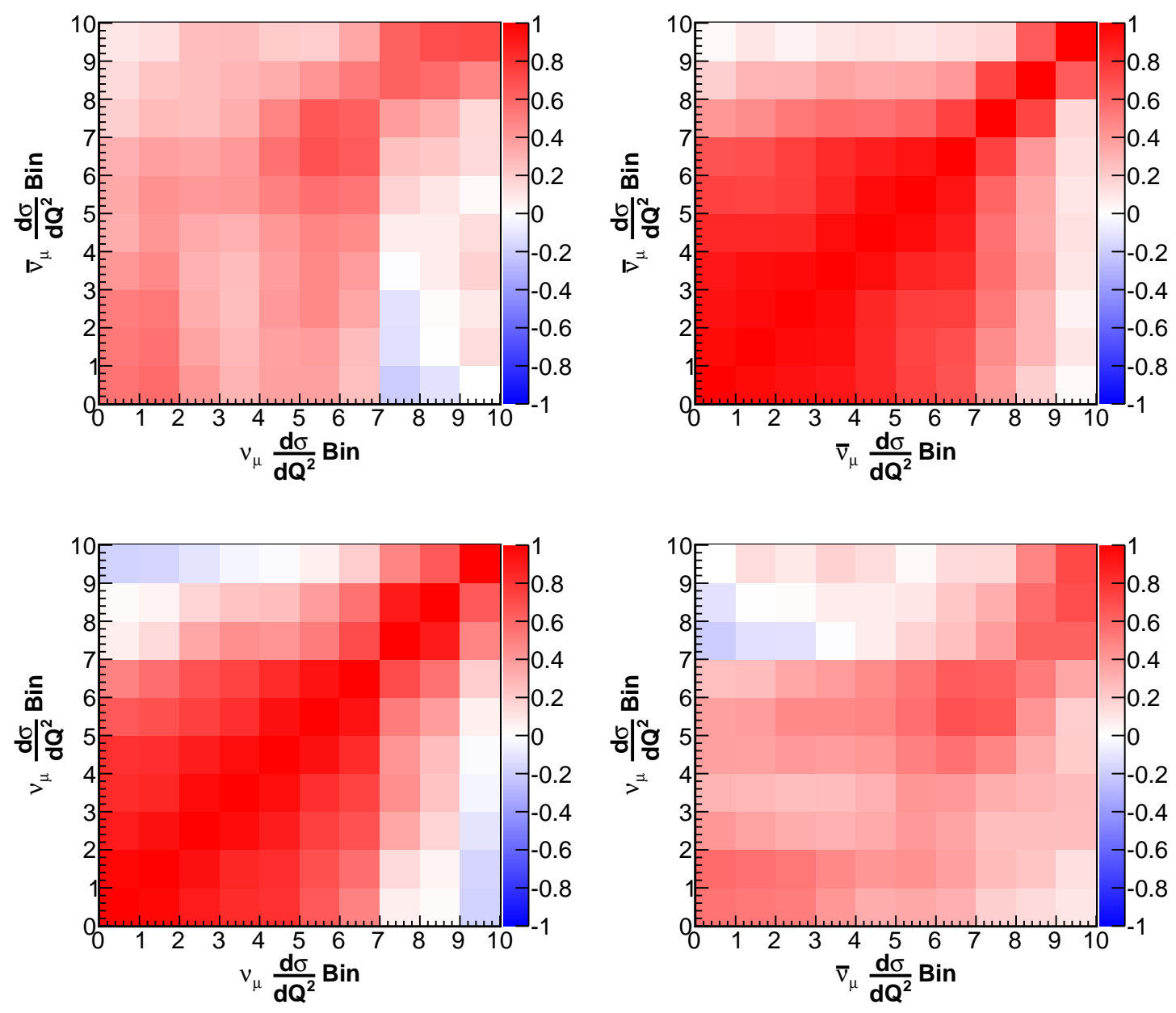

Figure 8.116: The correlation matrices for the non-flux systematic uncertainties on the measured $\nu_{\mu}$ and $\bar{\nu}_{\mu} \frac{d \sigma}{d Q^{2}}$ 


\subsection{Contribution From Diffractive Scattering}

Diffractive pion production on free protons, referred to herein as diffractive scattering, is a process analogous to coherent scattering that produces a muon and charged pion in the forward direction while leaving the proton in its ground state. In the PCAC picture of diffractive scattering (Figure 8.117), the intermediate weak boson fluctuates to a pion, which scatters elastically off the target proton. Diffractive scattering is indistinguishable from coherent scattering when the recoil proton is below detection threshold. A $\nu_{\mu} / \bar{\nu}_{\mu}$ charged current sample in the tracker may contain diffractive scattering interactions since the MINER $\nu$ A scintillator $(\mathrm{CH})$ contains equal numbers of carbon nuclei and free protons. Diffractive scattering was not simulated in the MC used to predict the non-coherent background. The measured coherent cross sections may therefore contain a contribution from diffractive scattering.

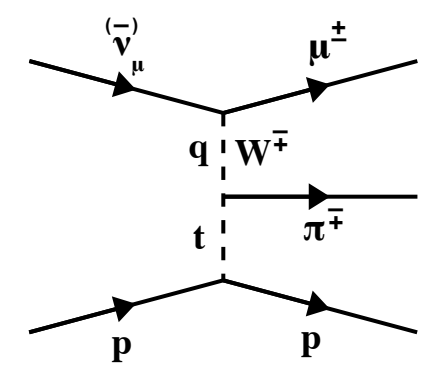

Figure 8.117: Diffractive pion production off a free proton

An important distinction between coherent and diffractive scattering is the $|t|$ dependence of the cross sections. In the PCAC picture, the $|t|$-dependence comes 
from the pion-proton/nucleus elastic scattering cross section, which is predicted to fall exponentially with $|t|$ as $\exp (-b|t|)$. The exponential slope $b$ is given by

$$
b=\frac{1}{3} R_{0}^{2} A^{2 / 3}
$$

where $R_{0} \sim 1 \mathrm{fm}$ is the nuclear length scale and $\mathrm{A}$ is the number of nucleons in the target. The predicted exponential slope for coherent scattering on carbon $(A=12)$ is $\sim 40(\mathrm{GeV} / \mathrm{c})^{-2}$, and the predicted exponential slope for diffractive scattering $(A=1)$ is $\sim 8(\mathrm{GeV} / \mathrm{c})^{-2}$. The diffractive cross section therefore falls more slowly with $|t|$ than the coherent cross section. The squared four-momentum exchanged with the target proton in diffractive scattering $|t|_{\text {diff }}$ is related to the recoil proton kinetic energy $T_{p}$ as

$$
\begin{aligned}
|t|_{\text {diff }} & =\left|\left(p_{\nu}-p_{\mu}-p_{\pi}\right)^{2}\right| \\
& =\left|\left(p_{p, f}-p_{p, i}\right)^{2}\right| \\
& =\left|m_{p}^{2}+m_{p}^{2}-2 E_{p, i} E_{p, f}-\vec{p}_{p, i} \cdot \vec{p}_{p, f}\right| \\
& =\left|2 m_{p}\left(m_{p}-E_{p, f}\right)\right| \\
& =2 m_{p} T_{p},
\end{aligned}
$$

where $p_{\nu}$ is the neutrino four-momentum, $p_{\mu}$ is muon four-momentum, $p_{\pi}$ is the pion four-momentum, $p_{p, i}$ and $p_{p, f}$ are the target (initial state) and recoil (final state) proton four-momentum, $m_{p}$ is the proton mass, $E_{p, i}$ and $E_{p, f}$ are the target and recoil proton energy, $\vec{p}_{p, i}$ and $\vec{p}_{p, f}$ are the target and recoil proton three-momentum, and the target proton is assumed to be on-shell and at rest $\left(E_{p, i}=m_{p},\left|\vec{p}_{p, i}\right|=0\right)$. The amount of energy deposited in the detector by the recoil proton from a diffractive 
scattering interaction, which determines whether the interaction is accepted or rejected by the vertex energy cut, is dependent on $T_{p}$. Diffractive interactions accepted by the vertex energy cut are therefore restricted to small- $T_{p}$, and equivalently small$|t|$. It will be shown that the small- $|t|$ diffractive acceptance in conjunction with the slowly falling $|t|$-dependence of the diffractive cross section results in a small contribution to the measured coherent cross sections.

The following describes the method for estimating the contribution to the measured coherent cross sections from diffractive scattering by estimating the diffractive acceptance as a function of $|t|$ and diffractive scattering cross section as a function of $|t|, \frac{d \sigma}{d|t|}$. A search for the presence of diffractive scattering within the selected coherent candidate sample by looking for ionization from the recoil proton near the event vertex is also presented.

\subsubsection{Diffractive Acceptance}

The first step in estimating the diffractive acceptance is to estimate the relative diffractive-to-coherent acceptance of the vertex energy cut. The acceptance of the vertex energy cut will differ for diffractive and coherent scattering due to the energy deposited by the recoil proton from diffractive scattering, which is dependent on $T_{p}$. The relative diffractive-to-coherent acceptance of the vertex energy cut is therefore a function of $|t|$ since $T_{p}$ is proportional to $|t|$.

The relative diffractive-to-coherent acceptance of the vertex energy cut was estimated using a distribution of vertex energy deposited by recoil protons as a function of $T_{p}$, which was estimated from a simulation of single protons originating in the fiducial volume and isotropic in direction, and a coherent $\mathrm{MC}$ sample passing all selection cuts up to the vertex energy cut (Section 8.7). For each event the 
vertex energy from a recoil proton with kinetic energy $T_{p}$ was sampled from the distribution and added to the vertex energy of the event. The relative diffractive-tocoherent acceptance (Figure 8.118) was calculated as the ratio of the vertex energy cut acceptance with to without added vertex energy as a function of $|t|$, which was calculated event-by-event from $T_{p}$ (Equation 8.36). This estimation assumes the acceptance of the proton score cut (Section 8.7.4) is the same for diffractive and coherent scattering. The vertex energy cut acceptance is insensitive to differences between diffractive and coherent scattering in the muon and pion kinematics since the energy deposited by the muon and pion is corrected to normal incidence in the vertex energy calculation (Section 8.7.5).

Per the estimated relative diffractive-to-coherent acceptance of the vertex energy cut, the vertex energy cut rejects nearly all diffractive events with $|t|>0.125$ $(\mathrm{GeV} / \mathrm{c})^{2}$. Since the $|t|$ cut selects events with reconstructed $|t|<0.125(\mathrm{GeV} / \mathrm{c})^{2}$, the acceptance of the $|t|$ cut is approximately the same for diffractive and coherent scattering after cutting on vertex energy. The absolute diffractive acceptance as a function of true $|t|$ can therefore be estimated by weighting the relative diffractiveto-coherent acceptance of the vertex energy cut by the total selection efficiency for coherent scattering as a function of true $|t|$ (Figure 8.118). It should be noted that the coherent acceptance is non-zero above $|t|=0.125(\mathrm{GeV} / \mathrm{c})^{2}$ due to the reconstructed $|t|$ resolution.

The diffractive scattering contribution to the sideband $\left(0.2<|t|<0.6(\mathrm{GeV} / \mathrm{c})^{2}\right)$ is negligible since the vertex energy cut rejects nearly all diffractive events with $|t|>$ $0.125(\mathrm{GeV} / \mathrm{c})^{2}$. Therefore, diffractive scattering is neglected in tuning the GENIE prediction of the incoherent backgrounds (Section 8.8). 

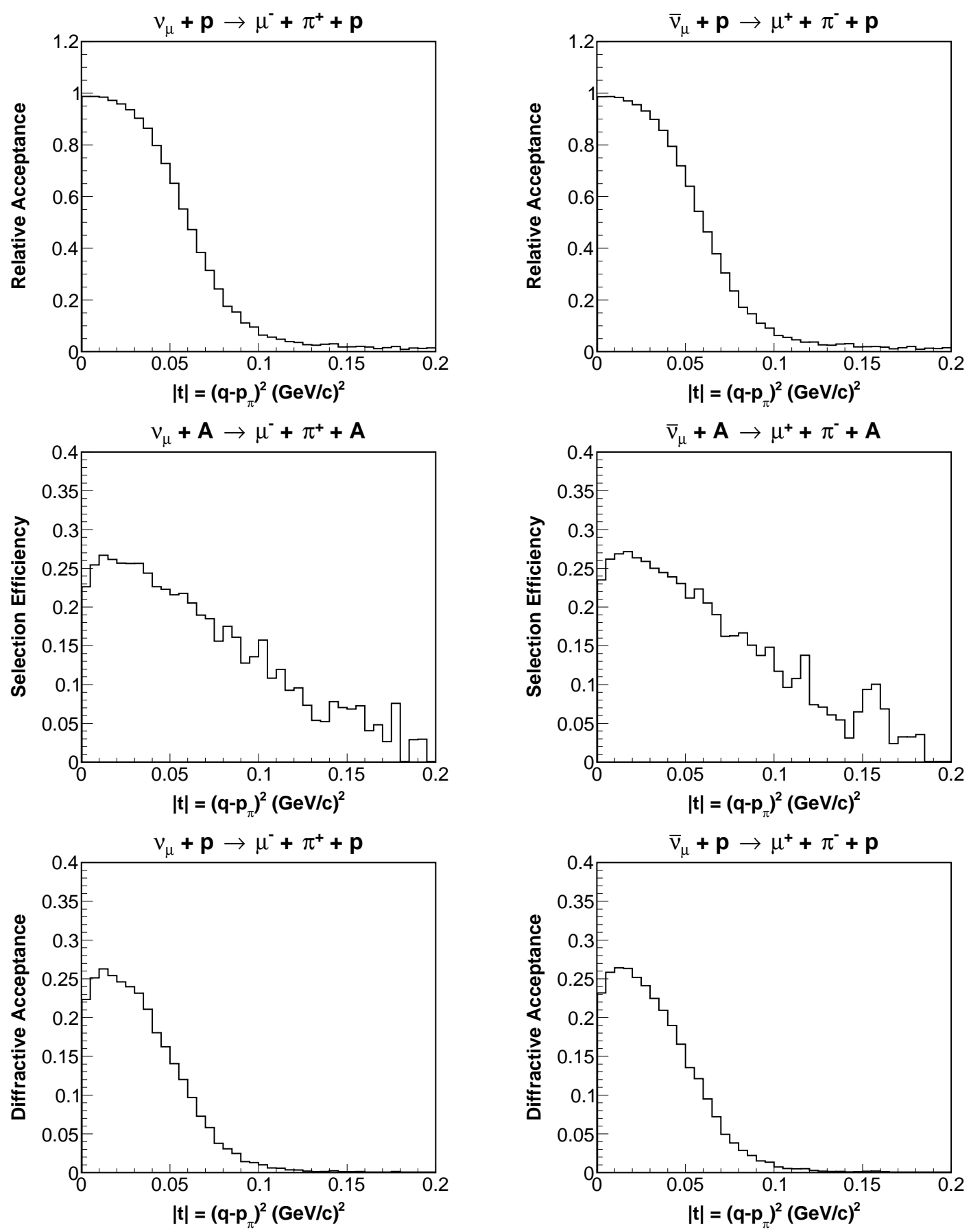

Figure 8.118: The $\nu_{\mu}$ (left) and $\bar{\nu}_{\mu}$ (right) estimated relative diffractive-to-coherent acceptance (top), coherent acceptance (middle), and absolute diffractive acceptance (bottom) as a function of $|t|$. The absolute diffractive acceptance was estimated by weighting the coherent acceptance by the relative diffractive-to-coherent acceptance. 


\subsubsection{Diffractive Cross Section Calculation}

With the estimated diffractive acceptance as a function of $|t|$, the diffractive scattering contribution to the measured coherent cross sections can be estimated from the predicted diffractive scattering cross section as a function of $|t|, \frac{d \sigma}{d|t|}$. However, an exclusive calculation of the diffractive cross section valid in the kinematic region of the measured coherent cross sections does not yet exist. The PCAC-based calculation of diffractive scattering by Rein [128] is valid only for $W>2 \mathrm{GeV}$, since the interference with $p \pi$ final states from neutrino resonance production must be calculated for $W<2 \mathrm{GeV}$. For diffractive scattering at small-|t|, $W>2 \mathrm{GeV}$ corresponds to $E_{\pi}>1.5 \mathrm{GeV}$, which covers only the high- $E_{\pi}$ phase space of the measured coherent cross sections. There is no microphysical calculation of diffractive scattering at MINER $\nu \mathrm{A}$ energies.

An estimate of the diffractive cross section was extracted from a calculation of inclusive $\nu_{\mu} p \rightarrow \mu^{-} \pi^{+} p$ and $\bar{\nu}_{\mu} p \rightarrow \mu^{+} \pi^{-} p$ on free protons by Kopeliovich et al. [129] that uses Adler's PCAC relation and pion-nucleus scattering data. Relative to the GENIE prediction, the Kopeliovich calculation exhibits a low- $|t|$ enhancement that falls exponentially in $|t|$. The difference includes all low- $|t|$ enhancements, including that from diffractive scattering, not present in GENIE. The low- $|t|$ enhancement was extracted from the Kopeliovich calculation and served as the estimated diffractive cross section.

The diffractive and non-diffractive components of the Kopeliovich calculation were estimated by fitting the Kopeliovich $\frac{d \sigma}{d\left(|t|-|t|_{\text {min }}\right)}$ with the GENIE prediction plus an exponential term (Figure 8.119). $|t|_{\text {min }}$ is the minimum $|t|$ for diffractive 
scattering to occur and is defined as

$$
|t|_{\min }=\frac{\left(Q^{2}+m_{\pi}^{2}\right)^{2}-\left[\sqrt{\lambda\left(W^{2},-Q^{2}, m_{n}^{2}\right)}-\sqrt{\lambda\left(W^{2}, m_{\pi}^{2}, m_{n}^{2}\right)}\right]^{2}}{4 W^{2}}
$$

where $m_{\pi}$ and $m_{n}$ are the pion and nucleon masses and $\lambda(a, b, c)=a^{2}+b^{2}+c^{2}-2 a b-$ $2 a c-2 b c$. The Kopeliovich cross section was fit as a function of $|t|-|t|_{\text {min }}$ since, for diffractive scattering, $\frac{d \sigma}{d|t|}$ will deviate from an exponential at low $|t|$ due to $|t|_{\text {min }}$ suppression, whereas $\frac{d \sigma}{d\left(|t|-|t|_{\text {min }}\right)}$ will not have this effect. Both the normalization and slope of the exponential term were varied in the fit. Differences between the Kopeliovich and GENIE predictions of the non-diffractive processes were accounted for by dividing the GENIE prediction into regions $W<1.4 \mathrm{GeV}$ and $W>1.4$ $\mathrm{GeV}$, due to their shape differences in $|t|$, and varying their normalizations in the fit. The fit range was $0<|t|-|t|_{\text {min }}<0.25(\mathrm{GeV} / \mathrm{c})^{2}$, which covers the range of the estimated diffractive acceptance. The Kopeliovich $\frac{d \sigma}{d\left(|t|-|t|_{\min }\right)}$ was fit for $E_{\nu}$ $=4.0 \mathrm{GeV}$, which is near the average $E_{\nu}$ of the neutrino flux used for measuring the coherent cross sections. The exponential normalization, exponential slope, and GENIE normalization scale factors extracted from the fit are listed in Table 8.22.

\begin{tabular}{l|c|c} 
Fit Parameter & $\nu_{\mu}$ & $\bar{\nu}_{\mu}$ \\
\hline Exponential Normalization $\left(10^{-39} \mathrm{~cm}^{2} /(\mathrm{GeV} / \mathrm{c})^{2} / \mathrm{H}\right.$ atom $)$ & 13.8 & 5.7 \\
Exponential Slope $(\mathrm{GeV} / \mathrm{c})^{-2}$ & 11.1 & 8.3 \\
GENIE $W<1.4 \mathrm{GeV}$ Normalization Scale Factor & 0.62 & 0.42 \\
GENIE $W>1.4 \mathrm{GeV}$ Normalization Scale Factor & 0.30 & 0.33
\end{tabular}

Table 8.22: Kopeliovich $\frac{d \sigma}{d\left(|t|-|t|_{\text {min }}\right)}$ fit parameters for $E_{\nu}=4.0 \mathrm{GeV}$.

The diffractive $\frac{d \sigma}{d|t|}$ is needed in order to account for the diffractive acceptance, estimated as a function of $|t|$, in estimating the diffractive contribution to the measured coherent cross sections. The diffractive acceptance could not instead be estimated 

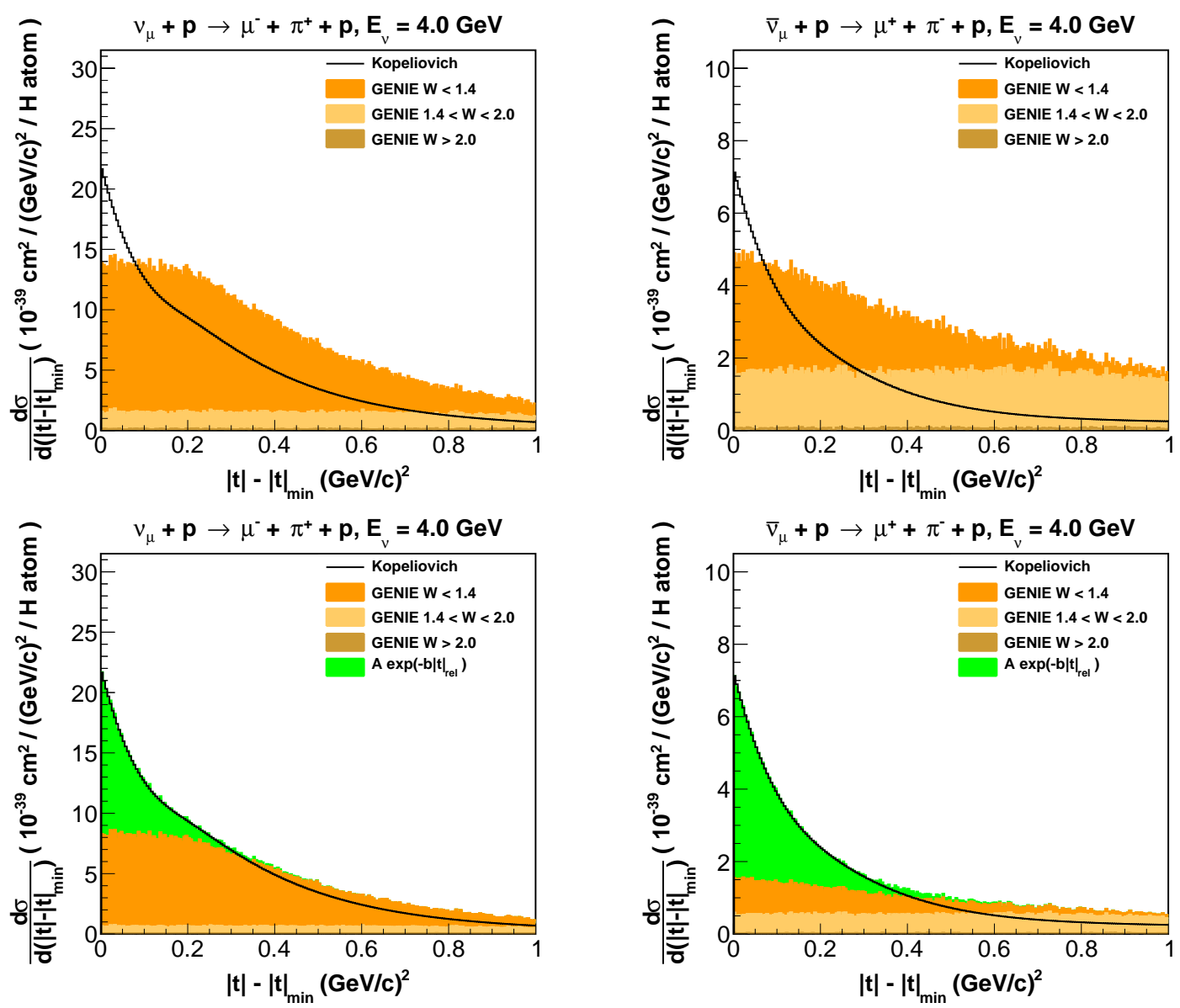

Figure 8.119: The $\nu_{\mu}$ (left) and $\bar{\nu}_{\mu}$ (right) Kopeliovich and GENIE predicted $\frac{d \sigma}{d\left(|t|-|t|_{\text {min }}\right)}$ for $E_{\nu}=4.0 \mathrm{GeV}$ before (top) and after (bottom) fitting the Kopeliovich prediction. The fit includes an exponential term representing the diffractive $\frac{d \sigma}{d\left(|t|-|t|_{\min }\right)}$. 
as a function of $|t|-|t|_{\text {min }}$ since calculating $|t|_{\text {min }}$ requires knowing the diffractive $Q^{2}$ and $W$ event-by-event. The diffractive $\frac{d \sigma}{d|t|}$ (Figure 8.121) was estimated by subtracting the GENIE $\frac{d \sigma}{d|t|}$ from the Kopeliovich $\frac{d \sigma}{d|t|}$ for $E_{\nu}=4.0 \mathrm{GeV}$ (Figure 8.120), where the GENIE $\frac{d \sigma}{d|t|}$ was scaled by the normalization scale factors extracted from the fit to the Kopeliovich $\frac{d \sigma}{d\left(|t|-|t|_{\text {min }}\right)}$.
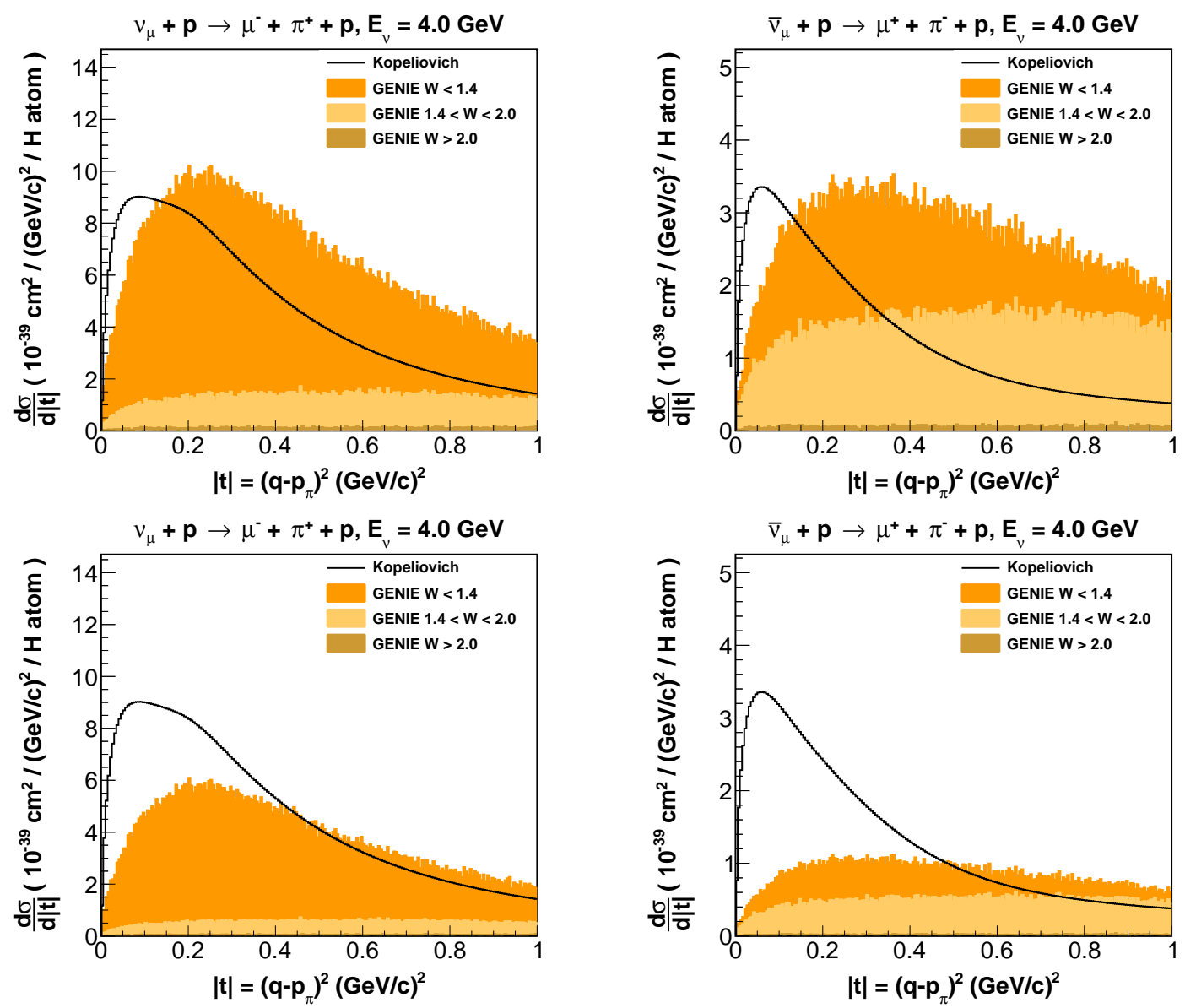

Figure 8.120: The $\nu_{\mu}$ (left) and $\bar{\nu}_{\mu}$ (right) Kopeliovich and GENIE predicted $\frac{d \sigma}{d|t|}$ for $E_{\nu}=4.0 \mathrm{GeV}$ before (top) and after (bottom) applying the GENIE normalization scale factors extracted from the fit to the Kopeliovich $\frac{d \sigma}{d\left(|t|-|t|_{\text {min }}\right)}$.

The $\nu_{\mu}$ and $\bar{\nu}_{\mu}$ diffractive cross sections at $E_{\nu}=4 \mathrm{GeV}$ (Table 8.23) were obtained by integrating the exponential extracted from the fit to the Kopeliovich $\frac{d \sigma}{d\left(|t|-|t|_{\text {min }}\right)}$. 

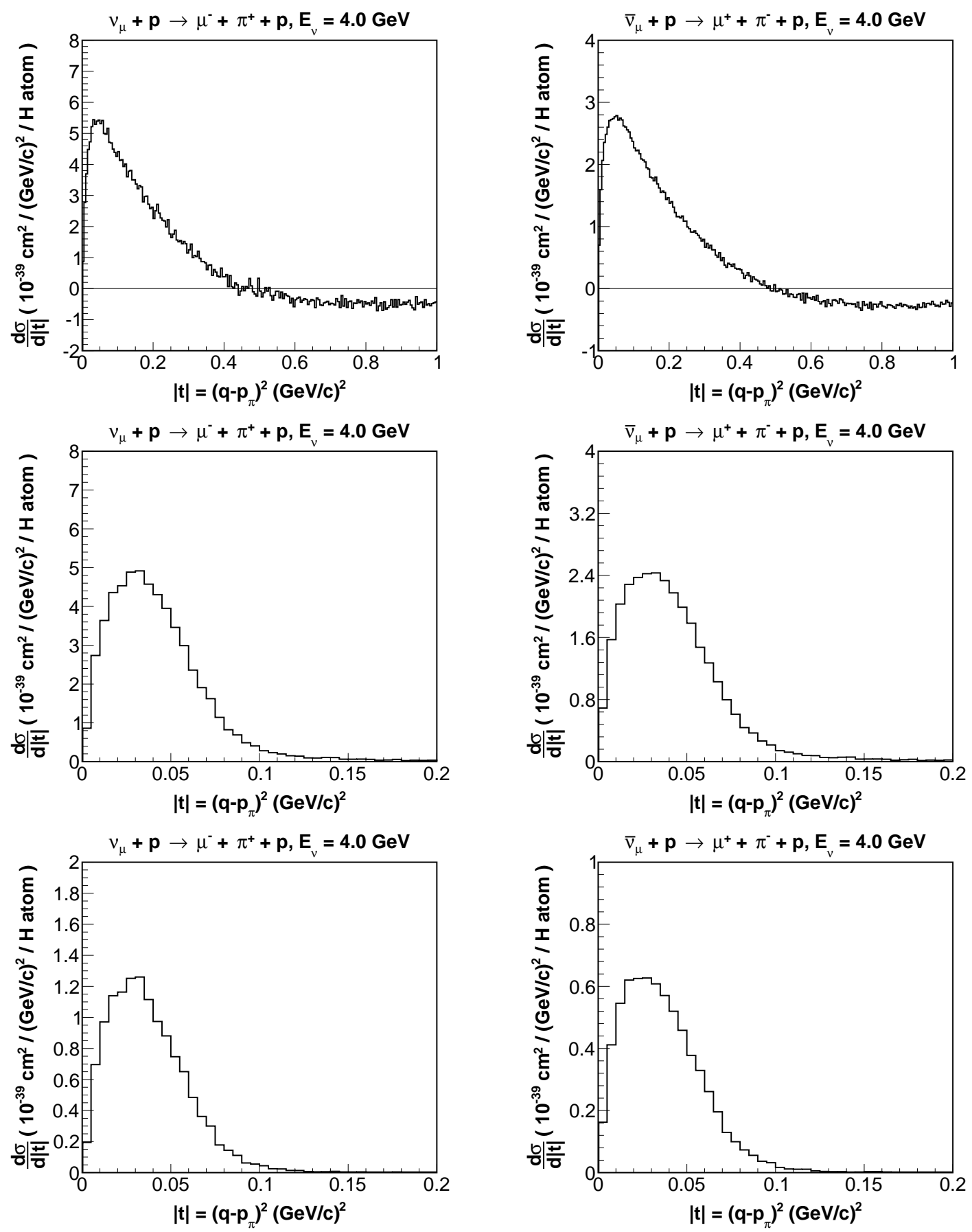

Figure 8.121: The estimated $\nu_{\mu}$ (left) and $\bar{\nu}_{\mu}$ (right) diffractive $\frac{d \sigma}{d|t|}$, diffractive $\frac{d \sigma}{d|t|}$ weighted as a function of $|t|$ by the relative diffractive-to-coherent acceptance (middle), and $\frac{d \sigma}{d|t|}$ weighted as a function of $|t|$ by the absolute diffractive acceptance. 
The $\nu_{\mu}\left(\bar{\nu}_{\mu}\right)$ diffractive cross section is $34 \%$ (19\%) of the GENIE coherent cross section on carbon at $E_{\nu}=4 \mathrm{GeV}$ (Table 8.23), which is reduced to $8 \%$ (4\%) by the diffractive acceptance. The acceptance reduced diffractive cross sections (Table 8.23) were calculated by weighting the diffractive $\frac{d \sigma}{d|t|}$ by the relative diffractive-to-coherent acceptance of the vertex energy cut as a function of $|t|$ (Figure 8.121) and integrating over $|t|$. When measuring the coherent cross sections, the rate of the accepted diffractive events is corrected by the coherent selection efficiency, where the primary difference between the coherent and diffractive selection efficiencies comes from the acceptance of the vertex energy cut. The reported diffractive scattering contribution to the measured $\nu_{\mu}\left(\bar{\nu}_{\mu}\right)$ coherent cross sections is $8 \%(4 \%)$. The measured coherent cross sections are not corrected for this possible contribution.

\begin{tabular}{l|c|c}
$\sigma\left(10^{-39} \mathrm{~cm}^{2} /\right.$ atom $)$ & $\nu_{\mu}$ & $\bar{\nu}_{\mu}$ \\
\hline Diffractive on H & $1.24(0.34)$ & $0.69(0.19)$ \\
Acceptance Reduced Diffractive on $\mathrm{H}$ & $0.28(0.08)$ & $0.15(0.04)$ \\
GENIE Coherent on ${ }^{12} \mathrm{C}$ & 3.64 & 3.64
\end{tabular}

Table 8.23: The estimated diffractive cross section, the estimated acceptance reduced diffractive cross section, and the GENIE coherent cross section at $E_{\nu}=4.0$ $\mathrm{GeV}$. The numbers in the parentheses are the fraction of the coherent cross section.

\subsubsection{Diffractive Search}

The search for diffractive interactions within the selected coherent candidate samples looks for ionization from the recoil proton near the event vertex. Accepted diffractive interactions are estimated to have $|t| \lesssim 0.1(\mathrm{GeV} / \mathrm{c})^{2}$, corresponding to a recoil proton with $T_{p} \lesssim 50 \mathrm{MeV}$ and range $\lesssim 2 \mathrm{~cm}$ in the scintillator. The search region for the recoil proton ionization extends \pm 2 planes (34 $\mathrm{mm}$ of scintillator) in the longitudinal direction, and \pm 2 strip widths (66 $\mathrm{mm}$ of scintillator) in the 
transverse direction, from the event vertex. For selected diffractive interactions, the recoil proton is identified by a large energy deposition in a single strip inside the search region. Figure 8.122 shows the distribution of maximum vertex strip energy (MVSE), defined for each event as the largest amount of visible energy in a single strip inside the search region, for the $\nu_{\mu}$ and $\bar{\nu}_{\mu}$ selected coherent-like samples. The region of large MVSE where the MC coherent contribution is small is indicative of ionization in addition to that from a muon and pion only and is analyzed for the presence of diffractive interactions. This is done by adding a simulated diffractive sample to the $\mathrm{MC}$ and fitting the diffractive sample normalization.

As mentioned previously, diffractive scattering was not simulated in the MC. Instead, a stand-in diffractive MC sample was constructed from MC interactions that pass all selection cuts and have a final state consisting exactly of a muon, a charged pion, and a proton. To represent the diffractive $|t|$-dependence and acceptance, the diffractive MC sample was weighted (Figure 8.123) as a function of $|t|$ (calculated from the proton kinetic energy) to the shape of the diffractive $\frac{d \sigma}{d|t|}$ weighted by the absolute diffractive acceptance (Figure 8.121). An important feature of the diffractive $\mathrm{MC}$ is the simulation of final state particles depositing energy in the same strip(s) which directly affects the MVSE.

The MVSE distribution was tested for the presence of diffractive scattering by adding the diffractive MC to the existing background tuned MC (Figure 8.124) and fitting the diffractive normalization. The fit was performed in the region $16<\mathrm{MVSE}$ $<40 \mathrm{MeV}$ where the coherent contribution is small. The $\chi^{2}$ in the fit was calculated as

$$
\chi^{2}=A C^{-1} A^{T},
$$



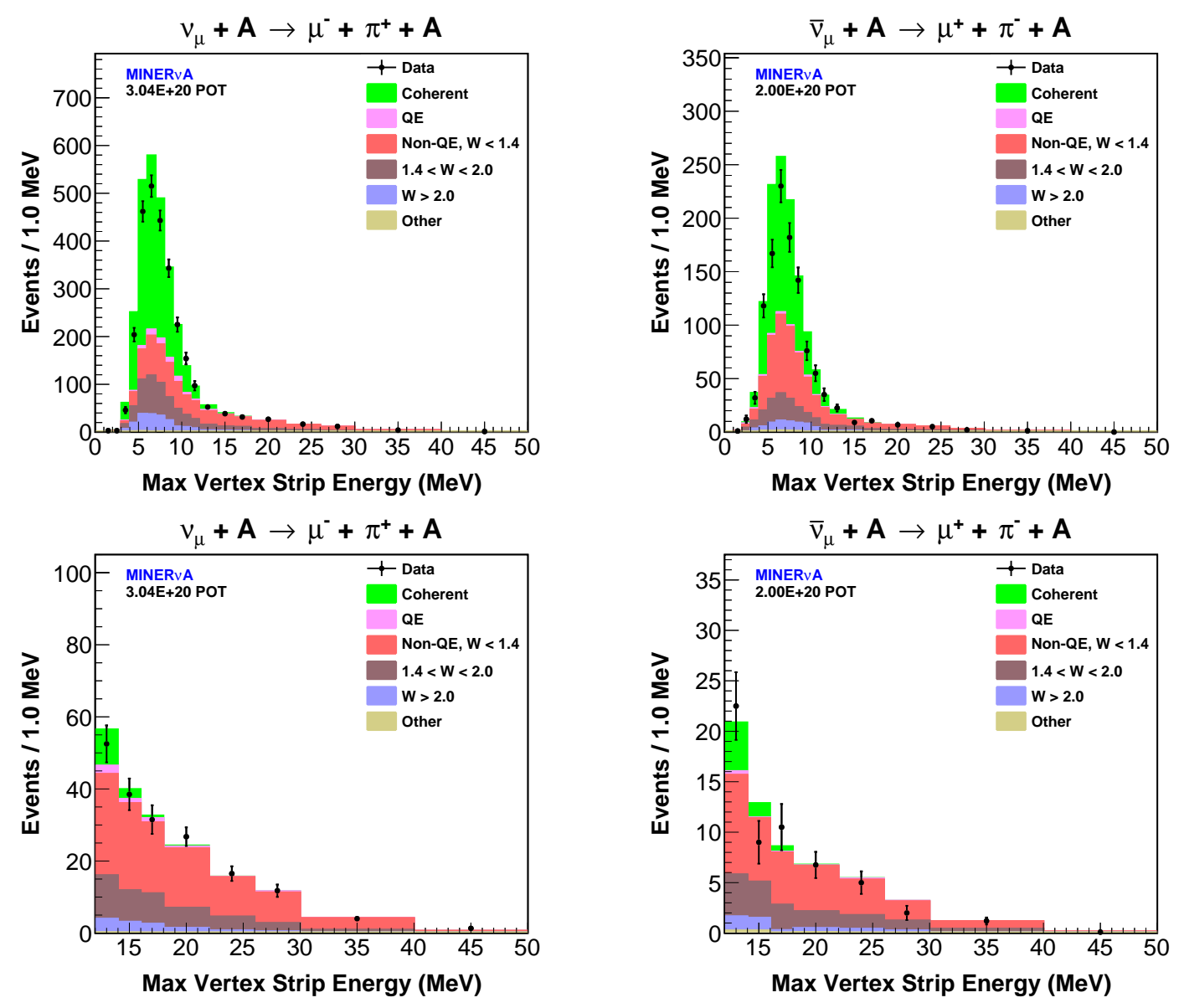

Figure 8.122: The maximum vertex strip energy (MVSE) distribution for the $\nu_{\mu}$ (left) and $\bar{\nu}_{\mu}$ (right) selected coherent candidate samples. The bottom plots show the high-MVSE region examined for the presence of diffractive scattering. The nondiffractive background normalizations are tuned.

where $C$ is the total covariance (statistical + systematic) matrix for the MVSE distribution in the fit region, and

$$
A_{i}=N_{i}^{d a t a}-N_{i}^{M C}-N_{i}^{d i f f}
$$

where $N_{i}^{\text {data }}, N_{i}^{M C}$, and $N_{i}^{\text {diff }}$ are the data, non-diffractive MC, and diffractive MC event rates, respectively, in MVSE bin $i$ within the fit region. The non-diffractive MC 

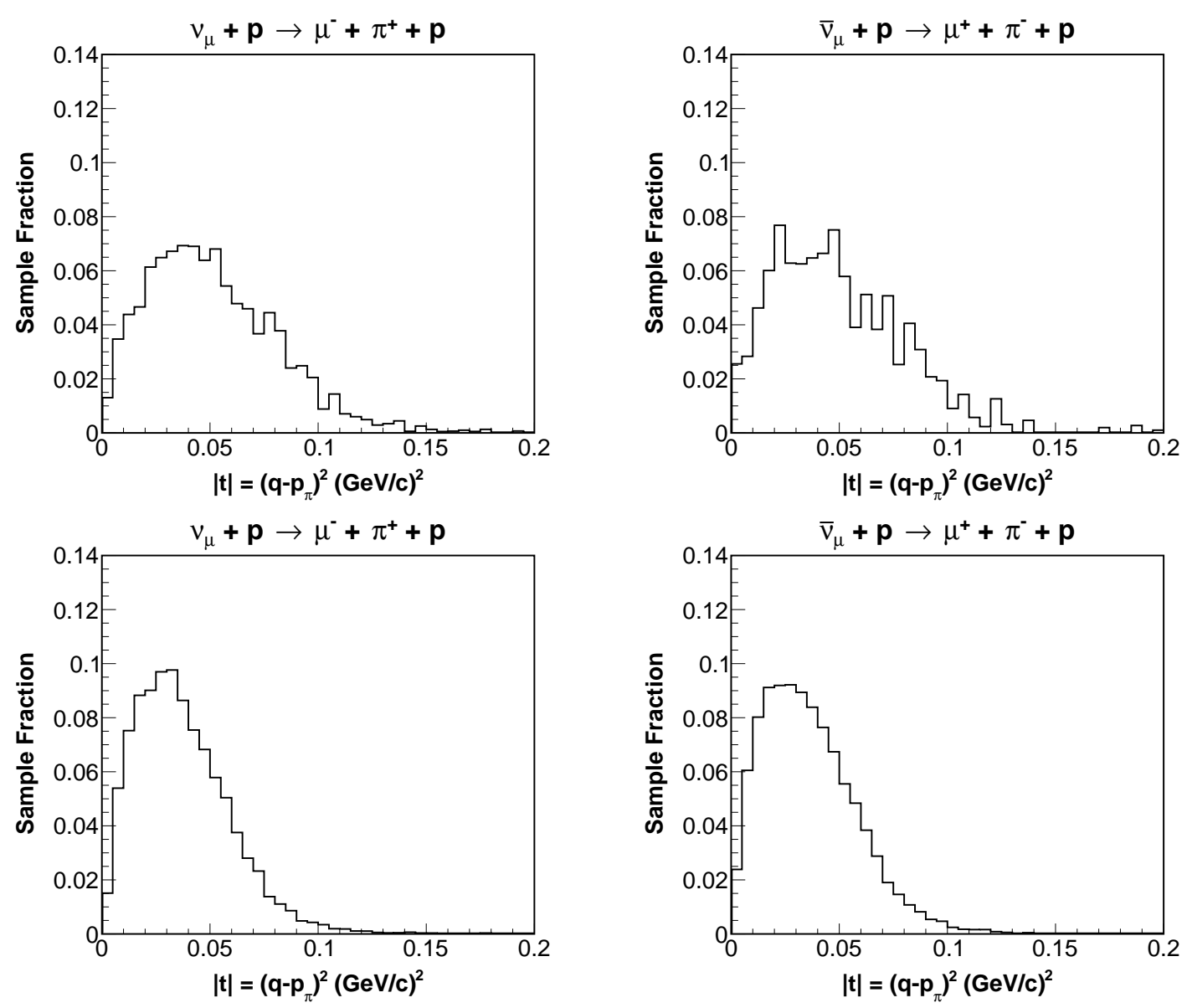

Figure 8.123: The $\nu_{\mu}$ (left) and $\bar{\nu}_{\mu}$ (right) diffractive MC $|t|$ distributions before (top) and after (bottom) weighting to the shape of the diffractive $\frac{d \sigma}{d|t|}$ weighted by the absolute diffractive acceptance.

event rate was held constant in the fit. The $\nu_{\mu}\left(\bar{\nu}_{\mu}\right)$ relative diffractive-to-coherent normalization, defined as the ratio of the diffractive and coherent MC integrated event rates, extracted from the fit is $+0.01 \pm 0.08(-0.03 \pm 0.09)$, which is consistent with the estimated $8 \%$ (4\%) diffractive contribution to the measured coherent cross sections. 

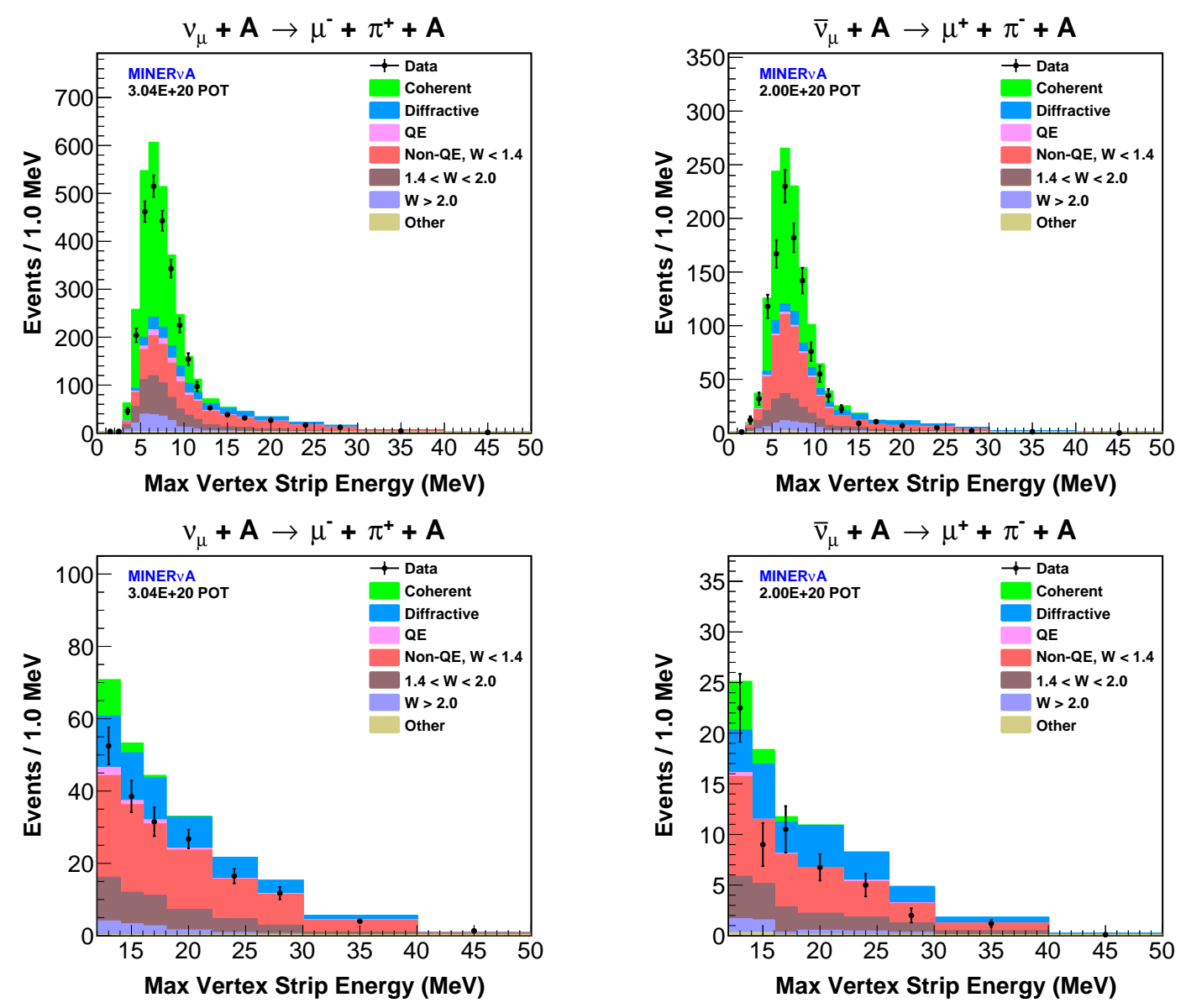

Figure 8.124: The maximum vertex strip energy (MVSE) distribution for the $\nu_{\mu}$ (left) and $\bar{\nu}_{\mu}$ (right) selected coherent-like samples. The bottom plots show the highMVSE region examined for the presence of diffractive scattering. The non-diffractive background normalizations are tuned. The added diffractive MC sample shown is for 0.2 relative diffractive-to-coherent normalization, which is clearly inconsistent with the data. The fitted $\nu_{\mu}\left(\bar{\nu}_{\mu}\right)$ relative diffractive-to-coherent normalization is $+0.01 \pm 0.08(-0.03 \pm 0.09)$. 


\subsubsection{Diffractive Scattering Conclusions}

The diffractive scattering contribution to the measured $\nu_{\mu}\left(\bar{\nu}_{\mu}\right)$ coherent cross sections is estimated to be $8 \%(4 \%)$, which is supported by the search for diffractive events in the selected coherent-like sample. The small contribution is the result of the $|t|$-dependence of the diffractive scattering cross section in conjunction with the vertex energy cut acceptance for diffractive scattering. The diffractive scattering cross section falls more slowly with $|t|$ than the coherent scattering cross section, and the vertex energy cut accepts only low- $|t|$ diffractive events due to the recoil proton ionization, thereby excluding $\sim 80 \%$ of the diffractive cross section. The measured coherent cross sections are not corrected for the possible contribution from diffractive scattering. 


\section{Chapter 9}

\section{Conclusions}

Neutral current (NC) and charged current (CC) neutrino-nucleus coherent pion production are important backgrounds to neutrino oscillation measurements. Current and future long baseline oscillation experiments, which aim to determine the neutrino mass hierarchy and measure the difference between neutrino and antineutrino oscillations, operate at neutrino energies in the range $1.0 \lesssim E_{\nu} \lesssim 10 \mathrm{GeV}$. Measurements of coherent pion production at these energies are needed to constrain models of coherent pion production used by oscillation experiments.

Ideally, coherent interactions are isolated using only model independent features of coherent scattering, which are the production of a lepton and pion in the forward direction and the nucleus remaining in its initial state. The squared four-momentum transferred to the nucleus, $|t|$, is necessarily small for the nucleus to remain intact and is a strong indicator of coherent scattering. $|t|$ cannot be measured for $\mathrm{NC}$ coherent scattering since the final state lepton is not observed. Since $|t|$ is not available, NC coherent pion production measurements make assumptions about the pion kinematics in isolating $\mathrm{NC}$ coherent interactions and have large uncertainties 
as a result. With a capable detector, the kinematics of CC coherent interactions, including $|t|$, can be measured completely. This enables measurements of CC coherent pion production to be made without assumptions of the coherent scattering kinematics, which then allows tests of the kinematic predictions of coherent models. This situation increases the importance of CC coherent pion production measurements which, in the PCAC picture of coherent scattering, provide a constraint on the $\mathrm{NC}$ reaction.

Neutrino oscillation experiments use the coherent pion production model by Rein and Sehgal. The predictions of the Rein-Sehgal model are in good agreement with early measurements of $\nu_{\mu}$ and $\bar{\nu}_{\mu} \mathrm{CC}$ coherent pion production, the majority of which were made a $E_{\nu} \gtrsim 10 \mathrm{GeV}$. Recently, the K2K and SciBooNE experiments found no evidence for $\nu_{\mu} \mathrm{CC}$ coherent pion production at $E_{\nu} \lesssim 2 \mathrm{GeV}$, and reported upper limits on the cross section that are lower than the Rein-Sehgal model prediction. Neither K2K nor SciBooNE were able to measure $|t|$. The non-observation of CC coherent pion production $E_{\nu} \lesssim 2 \mathrm{GeV}$ forced the T2K experiment, which operates at $E_{\nu} \sim 1 \mathrm{GeV}$, to apply a $100 \%$ uncertainty on the predicted $\mathrm{CC}$ coherent pion production rate in their oscillation measurements.

This thesis reported measurements of $\nu_{\mu}$ and $\bar{\nu}_{\mu} \mathrm{CC}$ coherent pion production on carbon from MINER $\nu \mathrm{A}$ data. The cross sections were measured for neutrino energies in the range $2.0<E_{\nu}<20 \mathrm{GeV}$, where $\left\langle E_{\nu}\right\rangle \approx 4 \mathrm{GeV}$. The measurements were made by isolating coherent interactions using the model independent experimental signature of $\mathrm{CC}$ coherent scattering, which consists of a charged lepton and pion in the forward direction, no evidence of nuclear breakup, and small $|t|$. In addition, the data-driven constraint on the background prediction was designed to minimize dependence on modeling nuclear effects which are poorly understood. Unambiguous 
signals of $\nu_{\mu}$ and $\bar{\nu}_{\mu} \mathrm{CC}$ coherent pion production above the predicted background were observed at small $|t|$. The cross sections $\sigma\left(E_{\nu}\right), \frac{d \sigma}{d Q^{2}}, \frac{d \sigma}{d E_{\pi}}$, and $\frac{d \sigma}{d \theta_{\pi}}$ were measured for both $\nu_{\mu}$ and $\bar{\nu}_{\mu} \mathrm{CC}$ coherent pion production. $E_{\nu}, Q^{2}, E_{\pi}, \theta_{\pi}$, and $|t|$ characterize the kinematics of coherent scattering completely.

The measured cross sections were compared to the Rein-Sehgal and BergerSehgal coherent models, which are both based on Adler's PCAC theorem. The Rein-Sehgal model calculates the pion-nucleus elastic scattering cross section from pion-nucleon scattering data. The Berger-Sehgal model calculates the pion-nucleus scattering cross section from pion-carbon elastic scattering data and scales the cross section to other nuclei. Both the Rein-Sehgal and Berger Sehgal predictions agree with the measured $\nu_{\mu}$ and $\bar{\nu}_{\mu} \sigma\left(E_{\nu}\right)$ within the uncertainty. For both $\nu_{\mu}$ and $\bar{\nu}_{\mu}$, the measured $\frac{d \sigma}{d E_{\pi}}\left(\frac{d \sigma}{d \theta_{\pi}}\right)$ exhibits a harder (more forward) spectrum than the ReinSehgal and Berger-Sehgal predictions, which suggests that both models miscalculate the pion-nucleus elastic scattering cross section. The Rein-Sehgal predictions were brought into better agreement with the measured $\frac{d \sigma}{d E_{\pi}}$ and $\frac{d \sigma}{d \theta_{\pi}}$ by weighting the predicted rate of interactions with $E_{\pi}<500 \mathrm{MeV}$ by $50 \%$. For both $\nu_{\mu}$ and $\bar{\nu}_{\mu}$, the Rein-Sehgal and Berger-Sehgal predictions are similar in shape to the measured $\frac{d \sigma}{d Q^{2}}$, which supports the axial vector dipole parameterization of the $Q^{2}$ dependence of the coherent cross section.

PCAC coherent models assume coherent scattering is a purely axial vector process. PCAC coherent models therefore predict equal cross sections for neutrinos and antineutrinos. To test this prediction, the measured $\nu_{\mu}$ and $\bar{\nu}_{\mu}$ cross sections were compared, where the $\nu_{\mu}$ differential cross sections were weighted to the $\bar{\nu}_{\mu}$ flux. No significant differences between the $\nu_{\mu}$ and $\bar{\nu}_{\mu}$ cross sections were observed.

Since the MINER $\nu \mathrm{A}$ scintillator contains equal numbers of carbon and hydrogen 
atoms, diffractive pion production on hydrogen is a possible contribution to the measured cross sections. Diffractive pion production is indistinguishable from coherent pion production when the recoil proton is undetected. A search for diffractive pion production within the coherent pion production candidate samples was performed by looking for ionization from a recoil proton near the interaction vertex. Neither the $\nu_{\mu}$ nor $\bar{\nu}_{\mu}$ coherent candidate samples exhibited evidence for a diffractive contribution. The absence of diffractive pion production in the coherent pion candidate samples is understood from the diffractive acceptance of the vertex energy cut and the $|t|$ dependence of the diffractive cross section.

The measurements reported in this thesis are a significant addition to the world data set of neutrino-nucleus coherent pion production. The measurements provide constraints on not only the total rate, but also the kinematics, of coherent pion production. The measurements can be used to benchmark coherent pion production models, from production threshold to neutrino energies up to $20 \mathrm{GeV}$, whose calculations are important inputs to neutrino oscillation measurements. 


\section{Bibliography}

[1] Particle Data Group (K.A. Olive et al.), Chin. Phys. C, 38, 090001 (2014).

[2] F. Halzen and D. Martin, "Quarks and Leptons: An Introductory Course in Modern Particle Physics", John Wiley and Sons, Inc. (1984).

[3] K.S. McFarland, (2008) arXiv:0804.3899 [hep-ex]

[4] D. Griffith, "Introduction to Elementary Particles", John Wiley and Sons, Inc. (1987).

[5] G. Arnison et al., [UA1 Collab.], Phys. Lett. B122, 103 (1983).

[6] G. Arnison et al., [UA1 Collab.], Phys. Lett. B126, 398 (1983).

[7] G. Aad et al., [ATLAS Collab.], Phys. Lett. B716, 1 (2012).

[8] S. Chatrchyan et al., [CMS Collab.], Phys. Lett. B716, 30 (2012).

[9] J.H. Christenson et al., Phys. Rev. Lett. 13, 138 (1964).

[10] B. Aubert et al., (BABAR Collaboration), Phys. Rev. Lett. 87, 091801 (2001).

[11] K. Abe et al., (Belle Collaboration), Phys. Rev. Lett. 87, 091802 (2001).

[12] A.D. Sakharov, Sov. Phys. JETP Lett. 5, 24 (1967).

[13] Y. Fukuda et al. (Super-Kamiokande Collaboration) Phys. Rev. Lett. 81, 1562 (1998).

[14] B. Kayser, arXiv:0804.1497 [hep-ph] (2008).

[15] LBNE Collaboration (C. Adams et al.), arXiv:1307.7335 [hep-ex] (2013).

[16] NOvA Collaboration (D.S. Ayers et al.), "The NOvA Technical Design Report," FERMILAB-DESIGN-2007-01, (2007). 
[17] JUNO Collaboration (Fengpeng An et al.), J.Phys. G43 (2016) no.3, 030401.

[18] T2K Collaboration (K. Abe et al.), Prog. Theor. Exp. Phys. (2015) 043C01.

[19] J. A. Formaggio and G. P. Zeller, Rev. Mod. Phys. 84, 1307 (2012).

[20] S. Adler, Phys. Rev. B135, 963 (1964).

[21] T2K Collaboration (K. Abe et al.), Phys. Rev. D91, 072010 (2015).

[22] NOvA Collaboration (P. Adamson et al.), Phys. Rev. Lett. 116, 151806 (2016).

[23] C. Llewellyn Smith, Phys. Rept. 3, 261 (1972).

[24] C. Giunti and C. W. Kim, "Fundamentals of Neutrino Physics and Astrophysics," Oxford University Press, (2007).

[25] V. Bernard, L. Elouadrhiri, and U.G. Meissner, J. Phys., G28, R1-R35 (2002).

[26] M. L. Goldberger and S. B.Treiman, Phys. Rev. 5, 1178-1184 (1958).

[27] M. Day and K.S. McFarland, Phys. Rev. D86 053003 (2012).

[28] D. Rein and L.M. Sehgal, Ann. Phys. 133, 79-153 (1981).

[29] GENIE collaboration, C. Andreopoulos et al., Nucl. Inst. Meth. A614, 87 (2010).

[30] R. Smith and E. Moniz, Nucl. Phys. B43, 605 (1972).

[31] A. Bodek, H. Budd, and M. Christy, Eur. Phys. J. C71, 1726 (2011).

[32] G. A. Fiorentini et al., Phys. Rev. Lett. 111, (2013) 022502.

[33] A. Bodek, U. K. Yang, J. Phys. G29, 1899 (2003).

[34] D. Rein and L. M. Sehgal, Nucl. Phys. B223, 29 (1983).

[35] H. Abramowicz, Int. J. Mod. Phys. A, 15, 495 (2000).

[36] F. E. Low, Phys. Rev. D12, 163 (1975).

[37] S. Nussinov, Phys. Rev. Lett. 34, 1286 (1975).

[38] K. Lackner, Nucl.Phys. B153, 526 (1979).

[39] S. Gerstein and Y. Zeldovich, Sov. Phys. JETP, 2, 576 (1956).

[40] R. P Feynman and M. Gell-Mann, Phys. Rev. 109, 193 (1958). 
[41] M.Gell-Mann and M. Levy, Nuovo Cim., 16, 705 (1960).

[42] D. Rein and L. M. Sehgal, Phys. Lett. B657, 207 (2007).

[43] A. A. Belkov and B. Z. Kopeliovich, Sov. J. Nucl. Phys. 46, 499 (1987).

[44] B. Z. Kopeliovich, Nucl. Phys. Proc. Suppl. 139, 219 (2005).

[45] S. S. Gershtein, Yu. Y. Komachenko and M. Y. Khlopov, Sov. J. Nucl. Phys. 32, 861 (1980).

[46] Yu. Y. Komachenko and M. Y. Khlopov, Yad. Fiz. 45, 467 (1987).

[47] E. A. Paschos, A. Kartavtsev and G. J. Gounaris, Phys. Rev. D74, 054007 (2006).

[48] Ch. Berger and L. Sehgal, Phys. Rev. D79, 053003 (2009).

[49] E. A. Paschos and D. Schalla, Phys. Rev. D80, 033005 (2009).

[50] A. Higuera and E. Paschos, Eur. Phys. J. Plus 129, 43 (2014).

[51] S. L. Adler, arXiv:hep-ph/0505177.

[52] S. Singh, M. Sajjad Athar, and S. Ahmad, Phys. Rev. Lett. 96, 241801 (2006).

[53] L. Alvarez-Ruso, L. Geng, S. Hirenzaki, and M. Vicente Vacas, Phys. Rev. C75, 055501 (2007).

[54] J. Amaro, E. Hernandez, J. Nieves, and M. Valverde, Phys. Rev. D79, 013002 (2009).

[55] T. Leitner, U. Mosel, and S. Winkelmann, Phys. Rev. C79, 057601 (2009).

[56] E. Hernandez, J. Nieves, and M. Vicente-Vacas, Phys. Rev. D80, 013003 (2009).

[57] H. Faissner et al., Phys. Lett. B125, 230 (1983).

[58] E. Isiksal et al., Phys. Rev. Lett. 52, 1096 (1984).

[59] F. Bergsma et al. (CHARM Collaboration), Phys. Lett. B157, 469 (1985).

[60] H. J. Grabosch et al. (SKAT Collaboration), Z. Phys. C31, 203 (1986).

[61] C. Baltay et al., Phys. Rev. Lett. 57, 2629 (1986).

[62] C.T. Kullenberg, S. R. Mishra, et al. (NOMAD Collaboration), Phys. Lett. B682, 177 (2009). 
[63] Y. Kurimoto et al. (SciBooNE Collaboration), Phys. Rev. D81, 111102 (R) (2010).

[64] A.A. Aguilar-Arevalo et al. (MiniBooNE Collaboration), Phys. Lett. B664, 41 (2008).

[65] P. Marage et al. (BEBC WA59 Collaboration), Z Phys. C31, 191 (1986).

[66] V.V. Ammosov et al., Sov, J. Nucl. Phys. 45, 1029 (1987).

[67] P. Marage et al. (BEBC WA59 Collaboration), Z. Phys. C43, 523 (1989).

[68] M Aderholz et al., Phys. Rev. Lett. 63, 2349 (1989).

[69] S. Willocq et al., Phys. Rev. D47, 2661 (1993).

[70] P. Vilain et al. (CHARM-II Collaboration), Phys. Lett. B313, 267 (1993).

[71] M. Hasegawa et al. (K2K Collaboration), Phys. Rev. Lett. 95, 252301 (2005).

[72] K. Hiraide et al. (The SciBooNE Collaboration), Phys. Rev. D78, 112004 (2008).

[73] R. Acciarri et al. (ArgoNeuT Collaboration), Phys. Rev. Lett. 113, 261801 (2014).

[74] A. Higuera, A. Mislivec, et al. (MINER $\nu$ A Collaboration), Phys. Rev.Lett. 113, 261802 (2014).

[75] P. Adamson et al., Nucl. Inst. Meth. A806, 279 (2016), 1507.06690.

[76] Fermilab's Accelerator Complex, http://www.fnal.gov/pub/science/ particle-accelerators/accelerator-complex.html, 2016.

[77] J. Thompson, FNAL Report No. FERMILAB-TM-1909, 1994.

[78] NuMI technical design handbook, http://www-numi.fnal.gov/numwork/tdh/ tdh_index.html, 2002.

[79] R. Zwaska et al., Nucl. Inst. Meth. A568, 548 (2006).

[80] L. Aliaga et al., arXiv:1607.00704 [hep-ex]

[81] L. Fields, MINER $\nu$ A DocDB 11938, version 3, 2016, http://minerva-docdb. fnal.gov: $8080 / c g i-b i n / S h o w D o c u m e n t ? d o c i d=11938 \& v e r s i o n=3$

[82] S. Agostinelli et al., Nucl. Inst. Meth. A506, 250 (2003). 
[83] J. Allison et al., IEEE Transactions on Nuclear Science 53 No. 1, 270 (2006).

[84] R. P. Feynman, Phys. Rev. Lett. 23, 1415 (1969).

[85] C. Alt et al. (NA49), Eur. Phys. J. C49, 897 (2007).

[86] D. S. Barton et al., Phys. Rev. D27, 2580 (1983).

[87] J. Park et al., Phys. Rev. D93, 112007 (2016).

[88] The MINER $\nu$ A Collaboration (L. Aliaga et al.), Nucl. Inst. Meth. A743, 130 (2014).

[89] The MINOS Collaboration (D. G. Michael et al.), Nucl. Inst. Meth. A596, 190 (2008).

[90] A. Pla-Dalmau, A. D. Bross, K. L. Mellott, FNAL Report No. FERMILABConf-99/095 (1999).

[91] MINER $\nu$ A Technical Design Report, FERMILAB-DESIGN-2006-01 (2006).

[92] N. Tagg et al. (The MINER $\nu$ A Collaboration), Nucl. Inst. Meth. 676, 44 (2012).

[93] G. N. Perdue et al., Nucl. Inst. Meth. A694, 179 (2012).

[94] J. Estrada, C. Garcia, B. Hoeneisen, and P. Rubinov, "MCM II and the TriP Chip", Aug 2002, FERMILAB-TM-2226.

[95] P. Adamson et al. (MINOS Collaboration), Phys. Rev. D81, 072002 (2010).

[96] The ArgoNeuT Collaboration (C. Anderson et al.), JINST 7, P10019 (2012).

[97] The MINOS Collaboration, P-875: "A Long-baseline Neutrino Oscillation Experiment at Fermilab", Technical report, 105 (1996).

[98] A. Mislivec, MINER $\nu$ A DocDB 4191, version 1, 2009, http://minerva-docdb. fnal.gov: $8080 /$ cgi-bin/ShowDocument?docid=4191\&version=1

[99] A. Mislivec, MINER $\nu$ A DocDB 4368, version 1, 2009, http://minerva-docdb. fnal.gov: $8080 /$ cgi-bin/ShowDocument?docid=4368\&version=1

[100] A. Mislivec, MINER $\nu \mathrm{A}$ DocDB 4475, version 1, 2009, http: $/ /$ minerva-docdb.fnal.gov:8080/cgi-bin/ShowDocument?docid= $4475 \&$ version $=1$

[101] R. Luchsinger and C. Grab, Comp. Phys. Comm. 76, 263 (1993). 
[102] C. M. Marshall, Ph.D. thesis, University of Rochester (2016).

[103] A.B. Kaidalov, Phys. Lett. B116, 459 (1982).

[104] H. W. Bertini et al., Nucl. Inst. Meth. 66, 29 (1968).

[105] H. W. Bertini and P. Guthrie, Nucl. Phys. A169, 43 (1971).

[106] A. Mislivec, MINER $\nu$ A DocDB 7897, version 1, 2012, http: //minerva-docdb.fnal.gov:8080/cgi-bin/ShowDocument?docid= $7897 \&$ version $=1$

[107] A. Mislivec, MINER $\nu$ A DocDB 8087, version 1, 2012, http: //minerva-docdb.fnal.gov:8080/cgi-bin/ShowDocument?docid= $8087 \&$ version $=1$

[108] A. Cabrera, A. D. Santo, P. S. Miyagawa, N. Tagg, and A. Weber, Oxford Report No. NuMI-NOTE-SCINT-934, 2003 (unpublished), MINOS Doc-DB 934 .

[109] J. Wolcott, Ph.D. Thesis, University of Rochester, FERMILAB-THESIS-201526 (2015).

[110] The MINER $\nu$ A Collaboration (L. Aliaga et al.), Nucl. Inst. Meth. A789, 28 (2015).

[111] C. Wilkinson et al., Phys. Rev. D90, 112017 (2014).

[112] P. Rodrigues, C. Wilkinson, and K. McFarland, arXiv:1601.01888 [hep-ex]

[113] The GENIE Neutrino Monte Carlo Generator, http://genie.hepforge.org/ manuals/

[114] B. Eberly, MINER $\nu$ A DocDB 9850, version 1, 2014, http://minerva-docdb. fnal.gov: 8080/cgi-bin/ShowDocument?docid=9850\&version=1

[115] T. Walton, Ph.D. thesis, Hampton U. (2014).

[116] B. Gobbi, W. Hakel, J. L. Rosen, and S. Shapiro, Phys. Rev. Lett. 29, 1278 (1972).

[117] G. D'Agostini, Nucl. Inst. Meth. A362, 487 (1995).

[118] A. Mislivec, MINER $\nu$ A DocDB 11666, version 3, 2015, http: //minerva-docdb.fnal.gov:8080/cgi-bin/ShowDocument?docid= 11666\&version $=3$ 
[119] H. Gallagher, MINER $\nu$ A DocDB 7451, version 2, 2012, http: //minerva-docdb.fnal.gov:8080/cgi-bin/ShowDocument?docid= $7451 \& v e r s i o n=2$

[120] L. Tiator, D. Drechsel, S. Kamalov et al., Eur. Phys. J. A19, 55 (2004).

[121] G. A. Fiorentini et al. (MINER $\nu$ A Collaboration), Phys. Rev. Lett. 111, (2013) 022502.

[122] D.E. Groom, N.V. Mokhov, and S.I. Striganov, Atomic Data and Nuclear Data Tables, 78(2), 183-356, (2001).

[123] J.P. Velasquez, S.A. Dytman, and A.M. Gago, MINER $\nu$ A DocDB 8249, version 1, 2012, http://minerva-docdb.fnal.gov:8080/cgi-bin/ ShowDocument?docid=8249\&version $=1$

[124] B. Eberly, MINER $\nu$ A DocDB 9523, version 1, 2014, http://minerva-docdb. fnal.gov:8080/cgi-bin/ShowDocument?docid=9523\&version=1

[125] L. Fields and K.S. McFarland, MINER $\nu$ A DocDB 7518, version 3, 2012, http://minerva-docdb.fnal.gov:8080/cgi-bin/ShowDocument? docid=7518\&version $=3$

[126] J. Devan, MINER $\nu$ A DocDB 9471, version 4, 2013, http://minerva-docdb. fnal.gov:8080/cgi-bin/ShowDocument?docid=9471\&version=4

[127] J. Devan, MINER $\nu$ A DocDB 12324, version 1, 2016, http://minerva-docdb. fnal.gov: $8080 /$ cgi-bin/ShowDocument?docid=12324\&version=1

[128] D. Rein, Nucl. Phys. B278, 61 (1986)

[129] B. Z. Kopeliovich, "NP: Neutrinoproduction of pions off nuclei," private communication, http://www.fis.utfsm.cl/np/ 


\title{
Appendix: The MINER $\nu$ A Collaboration
}

D.A. Martinez Caicedo, M.F. Carneiro, H. da Motta, A. Ghosh, K. Hurtado Centro Brasileiro de Pesquisas Físicas

\author{
D.W. Schmitz \\ University of Chicago
}

J. Mousseau, H. Ray, D. Rimal, M.Wospakrik

University of Florida

L. Bellantoni, M. Betancourt, L. Fields, D.A. Harris, M. Kiveni, J.G. Morfín, J.

Osta, G.N. Perdue, L. Rakotondravohitra

Fermilab

A. Bravar

University of Geneva

J. Felix, A. Higuera, M.A. Ramirez, E. Valencia, G. Zavala

Universidad de Guanajuato

T. Walton

Hampton University

E. Maher

Massachusetts College of Liberal Arts 


\author{
C.E. Patrick \\ Northwestern University \\ H. Schellman \\ Oregon State University \\ N. Tagg \\ Otterbein University
}

S.A. Dytman, B. Eberly, C.L. McGivern, B. Messerly, D. Naples, V. Paolone, L. Ren

University of Pittsburgh

E. Endress, A.M. Gago

Pontificia Universidad Catolica del Peru

A. Bercellie, A. Bodek, H. Budd, T. Cai, J. Chvojka, G.A. Díaz, R. Fine, T. Golan, J. Kleykamp, S. Manly, C.M. Marshall, K.S. McFarland, A.M. McGowan,

A. Mislivec, J. Park, P.A. Rodrigues, D. Ruterbories, J. Wolcott

University of Rochester

Nuruzzaman, R.D. Ransome, B.G. Tice

Rutgers University

O. Altinok, H. Gallagher, T. Le, W.A. Mann

Tufts University

C. Simon

University of California at Irvine

R. Gran

University of Minnesota at Duluth

C.J. Solano Salinas

Universidad Nacional de Ingeniería

W.K. Brooks, R.Galindo, J. Miller

Universidad Técnica Federico Santa María

L. Aliaga, J. Devan, M. Kordosky, J.K. Nelson, A. Norrick, D. Zhang 
College of William and Mary 